\title{
Plantwide Energy Assessment of a Sugarcane Farming and Processing Facility
}

\author{
Lee A. Jakeway \\ Principal Investigator \\ Hawaiian Commercial \& Sugar Company \\ and \\ Scott Q. Turn and Vheissu I. Keffer \\ Hawaii Natural Energy Institute \\ and \\ Charles M. Kinoshita \\ College of Tropical Agriculture and Human Resources \\ University of Hawaii
}

February 2006

DOE Award No. DE-FC36-03ID14454

Hawaiian Commercial \& Sugar Co.

P.O. Box 266

Puunene, HI 96784 


\section{Table of Contents}

Executive Summary........................................................

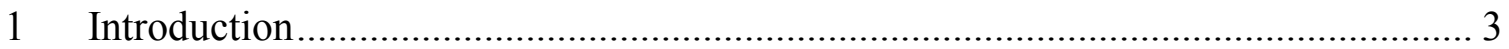

1.1 Description of HC\&S Co. Operations .................................................. 3

1.2 Description of Project Tasks ................................................................... 4

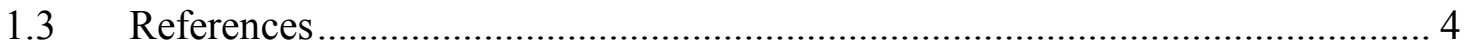

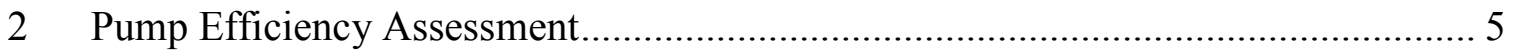

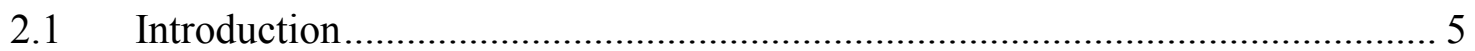

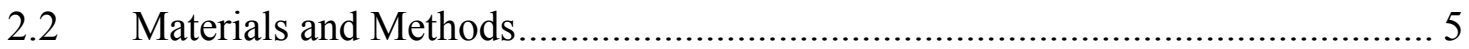

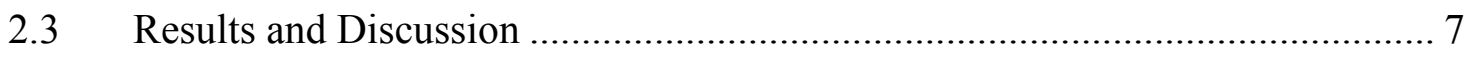

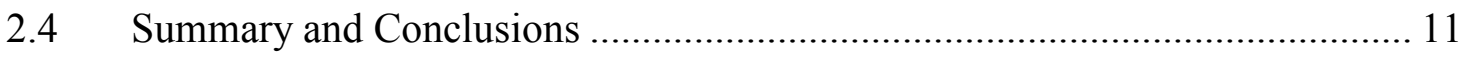

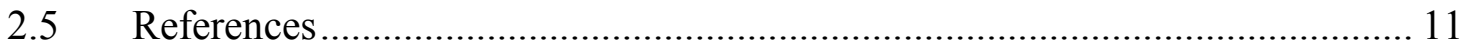

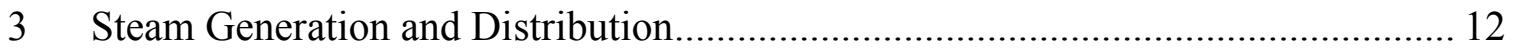

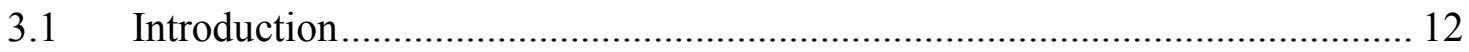

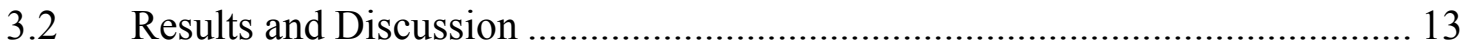

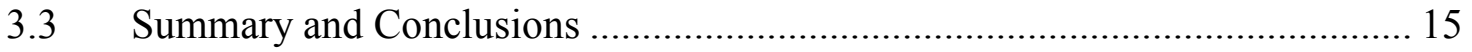

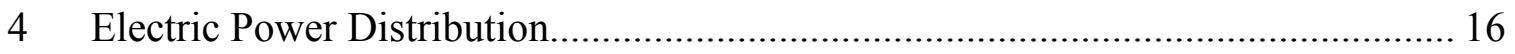

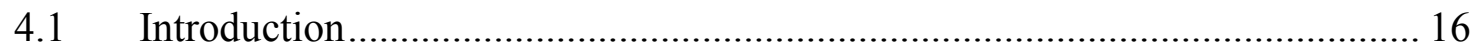

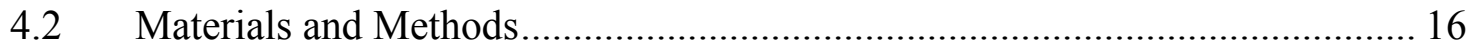

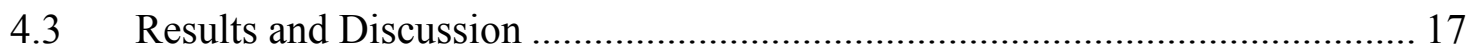

4.4 Summary and Conclusions ..................................................................... 18

5 Summary of Savings Opportunities ................................................................ 19

5.1 Savings Summary and Discussion .............................................................. 19

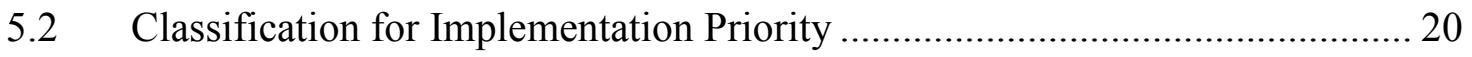

5.3 Environmental Impacts .......................................................................... 20

5.4 Discussion of Project Accomplishments Versus Stated Goals and Objectives 22

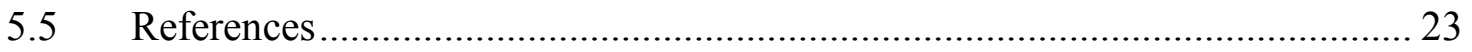

\section{Appendices}

Appendix A: PSAT Results

Appendix B: University of Hawaii Reports

Appendix C: Steam Line Insulation Survey Results 


\section{EXECUTIVE SUMMARY}

A plantwide energy assessment was performed at Hawaiian Commercial \& Sugar Co., an integrated sugarcane farming and processing facility. This investigation was performed using the internal resources of HC\&S with research collaboration from the University of Hawaii's, Hawaii Natural Energy Institute, School of Ocean and Earth Sciences and Technology and the College of Tropical Agriculture and Human Resources. The UH research collaborators focused on the generation and use of steam in the sugar factory, essential to all cane sugar factory operations for generating electricity, operating mechanical equipment, and evaporating cane juice to produce raw sugar.

There were four main tasks performed for the plantwide energy assessment: 1) pump energy assessment in both field and factory operations, 2) steam generation assessment in the power production operations, 3) steam distribution assessment in the sugar manufacturing operation, and 4) electric power distribution assessment of the company system grid. The technical and economic results from the tasks should prove useful to other cane sugar operations that employ cogeneration in their operations, especially where excess electricity generated is sold to the electric utility. Demand for energy produced from biomass resources such as sugarcane bagasse may increase in the future due to government incentives created to encourage the production of more energy from renewable sources to reduce greenhouse gas emissions in the electric utility sector.

The energy savings identified in each of these tasks were summarized in terms of fuel savings, electricity savings, or opportunity revenue that potentially exists mostly from increased electric power sales to the local electric utility. The results of this investigation revealed eight energy saving projects that can be implemented at HC\&S. These eight projects are summarized in Table 1 with accompanying data for fuel savings and opportunity revenue. The combined annual energy savings indicate the potential for over $\$ 1.5$ million in fuel savings, $22,337 \mathrm{MWh}$ equivalent electricity savings, and over $\$ 4.3$ million in opportunity revenue derived mostly from additional electricity sales to the local electric utility based on electricity rates paid by the electric utility in the last quarter of 2005. About two-thirds of the savings were derived from the first four projects listed.

Table 1. Summary of Annual Fuel, Electricity, and Opportunity Cost Savings

\begin{tabular}{|c|c|c|c|c|c|}
\hline \multirow[b]{2}{*}{ Project } & \multicolumn{2}{|c|}{ Fuel Savings (tons) } & \multirow{2}{*}{$\begin{array}{l}\text { Fuel Value } \\
(\$ k)\end{array}$} & \multirow{2}{*}{$\begin{array}{c}\text { Electricity } \\
\text { Savings (MWh) }\end{array}$} & \multirow{2}{*}{$\begin{array}{l}\text { Opportunity } \\
\text { Revenue (\$k) }\end{array}$} \\
\hline & Coal & Bagasse & & & \\
\hline 1. Field pumps efficiency & 247 & 2,392 & $\$ 74$ & 1,243 & $\$ 639$ \\
\hline 2. Factory pumps efficiency & 569 & 5,505 & $\$ 169$ & 2,861 & $\$ 504$ \\
\hline 3. Steam generation & 830 & 8,988 & $\$ 269$ & 4,554 & $\$ 802$ \\
\hline 4. Second vapor use & 1,750 & 16,936 & $\$ 521$ & 5,928 & $\$ 1,043$ \\
\hline 5. Flashing condensates & 663 & 6,418 & $\$ 197$ & 2,246 & $\$ 395$ \\
\hline 6. Steam line insulation & 701 & 6,788 & $\$ 209$ & 3,528 & $\$ 621$ \\
\hline 7. Capacitor installation & 113 & 1,095 & $\$ 34$ & 569 & $\$ 100$ \\
\hline 8. Transformer replacement & 280 & 2,708 & $\$ 83$ & 1,407 & $\$ 248$ \\
\hline Totals & 5,153 & $\mathbf{5 0 , 8 2 9}$ & $\$ 1,555$ & 22,337 & $\$ 4,352$ \\
\hline
\end{tabular}


If all the energy saving projects were implemented and the energy savings were realized as less fuel consumed, there would be several associated environmental benefits. Fewer air pollutants would be emitted into the atmosphere such as particulate matter, NOx, and SOx. As HC\&S is already a significant user of renewable biomass fuel in its operations, the projected reductions in air pollutants and emissions will not be as great compared to if only coal fuel were used for example. Nevertheless, the combined air pollutant and emissions reduction from the fuel mix used in this study indicated there would be 146 less tons annually of regulated air pollutants emitted to the atmosphere having a total monetary value of $\$ 7,558$ based on 2005 data. Also, since less coal will be used as supplemental fuel, there is the potential for reducing atmospheric $\mathrm{CO}_{2}$ emissions by 12,733 tons. Even if there are no realized fuel savings because steam and electricity can be used for other purposes at $\mathrm{HC} \& \mathrm{~S}$, there will be less air pollutants and emissions per unit of fuel consumed if these energy saving projects are implemented.

A win-win situation exists for HC\&S and for the public when energy efficiency improvements are implemented. For HC\&S, more energy can be produced per unit of fuel, thus reducing operating costs. For the public, there will be fewer air pollutants produced as a result of combustion of fuels along with less greenhouse gas emissions in the form of atmospheric $\mathrm{CO}_{2}$ produced by combustion of fossil fuels. $\mathrm{HC} \& \mathrm{~S}$ will also continue to be a significant producer of electricity produced from renewable biomass energy for the island of Maui. 


\section{Introduction}

\subsection{Description of HC\&S Co. Operations}

The operations of Hawaiian Commercial \& Sugar Co. (HC\&S) consist of sugarcane farming, raw sugar and molasses manufacturing, and energy production. HC\&S is a subsidiary of Alexander \& Baldwin (A\&B) Inc. A total of 37,000 acres are farmed in the central valley on the Island of Maui to support daily production of up to 1000 tons of raw sugar, 300 tons of molasses, and 650 megawatt-hours (MWh) of electricity. Declining commodity markets for raw sugar require HC\&S to improve its productivity and reduce costs while attempting to develop new revenue streams.

HC\&S currently uses $50-75 \%$ of the energy it produces. Renewable energy sources include sugarcane bagasse (the fibrous biomass residue remaining after sugar is extracted from cane) and power generated by hydro-turbines located strategically in the HC\&S surface water ditch system. Supplemental fuels such as imported coal and oil are used as fuel in steam boilers to meet energy requirements. Steam and electric power are used to operate the manufacturing facility and power plant. A significant amount of electric power is also required to operate irrigation pumps located throughout the farm area. Electricity that is not used by the company is sold to the local utility under a firm power contract that requires 12 megawatts (MW) during peak hours of 7 a.m. to 9 p.m. and 8 MW during off-peak hours of 9 p.m. to 7 a.m. and on Sundays. Any reduction in energy use for operations therefore becomes an opportunity for increased electricity sales (opportunity revenue) to the utility or a reduction in fuel use.

The opportunity revenue lost as a result of inefficient energy use can be quite substantial due to higher cost of electricity and fuel in Hawaii compared with other regions of the United States. The electricity tariff rates paid for power sales to the local electric utility were $\$ 0.176 / \mathrm{kWh}$ in the fourth quarter of 2005 . A capacity payment of $\$ 0.017 / \mathrm{kWh}$ is also paid if all power deliveries are met. The electric utilities in Hawaii depend on fossil fuels, most of which is imported oil [1], for about $93 \%$ of their energy needs. The electricity tariff rate paid to HC\&S for power sales is highly correlated to costs paid for imported oil by the utility.

The fuels used to operate the boilers at HC\&S are bagasse, imported coal, fuel oil, and a small amount of used vegetable oil. Bagasse is a byproduct of raw sugar manufacturing so its cost is relatively negligible although it can be argued that the monetary value of bagasse fuel is the same as coal on an equivalent Btu basis. On a wet mass basis, it takes about 3 tons of bagasse to provide the same fuel heating value as 1 ton of coal. The coalequivalent fuel value of bagasse is used in this report to calculate the monetary fuel savings. The cost for coal fuel used in this report was $\$ 70$ per ton wet basis $(7.40 \%$ moisture). 


\subsection{Description of Project Tasks}

The Plantwide Assessment Project at HC\&S was undertaken to identify energy saving opportunities in both the farming and processing operations for sugarcane. HC\&S is unique among US cane sugar producers in that it is an integrated sugarcane grower and processor. As a result, the operations of $\mathrm{HC} \& \mathrm{~S}$ involve all aspects of growing and processing sugarcane, making the scope of investigation for energy savings quite broad.

This investigation was broken into four main tasks for energy saving opportunities: 1) pump energy assessment in both field and factory operations, 2) steam generation assessment in the electric power production operations, 3) steam distribution assessment in the sugar manufacturing operation, and 4) assessment of the electric power distribution system.

This report is presented in three main sections with results reported by both HC\&S and outside collaborators. The pump energy assessment section presents measured pump efficiency data collected on targeted field and factory pumps. The steam generation and steam distribution assessments are combined into one section as most of this work was performed by the University of Hawaii research collaborators who were contracted to participate in this investigation. Their reports are attached as appendices to this report. HC\&S conducted an internal review of insulation savings in the power plant area. The electric power distribution assessment results were also conducted by HC\&S personnel and are summarized in a separate section of this report.

Finally, a summary of energy saving opportunities for all of the areas investigated is given in the last section of this report. The results are quantified in terms of amount of potential fuel savings, electric power savings, or opportunity revenue from electric power sales. Each energy efficiency project was also prioritized for implementation based on estimated savings and capital costs. A discussion is also presented on the actual accomplishments achieved against the goals and objectives that were originally stated for this investigation.

\subsection{References}

1. Hawaiian Electric Company, Inc. (n.d.). Renewable energy: about our fuel mix. Retrieved December 27, 2005 from http://www.heco.com 


\section{Pump Efficiency Assessment}

\subsection{Introduction}

Irrigation water for $\mathrm{HC} \& \mathrm{~S}$ is supplied mostly by watershed surface runoff and is delivered by the A\&B subsidiary, East Maui Irrigation Co., through a network of ditches and stream diversions. The collected water is eventually conveyed via four main irrigation ditches, supplying water to the HC\&S sugarcane farm area. A representation of the irrigation system is given in Figure 2-1. Irrigation water is also supplemented by water pumped from sixteen deep wells located throughout the farm area. The pumped water from these wells is slightly brackish as there is some mixing of fresh water with sea-water at depths below sea level and as such is less desirable compared to surface water.

Well water is finally delivered to the ditch system through 38 primary and booster pumps. The power requirement for these pumps ranges from $40 \mathrm{hp}$ to $2000 \mathrm{hp}$. Also, since HC\&S uses drip irrigation for most of its irrigation operations, there are approximately 150 smaller booster pumps needed to provide adequate pressure to operate the drip irrigation systems. These pumps range from $2.5 \mathrm{hp}$ to $100 \mathrm{hp}$ power rating.

During the dry summer months, there is heavy reliance on pumps to supply adequate irrigation water to meet the crop's irrigation requirements. Annual energy requirement to operate irrigation pumps ranges from 30,000 to 45,000 MWh depending on weather conditions. As HC\&S is required to provide $12 \mathrm{MW}$ of electric power to the local electric utility during peak daytime hours, there is often not enough generation capacity to meet both the utility electricity requirement and irrigation power requirement during dry periods. As a result, pumps are operated at night when the utility requires only 8 MW of export electricity and $4 \mathrm{MW}$ of power are available for pumping between the hours of 9 p.m. to 7 a.m. This situation places increased demands on the pump maintenance crew to start pumps in the evening after 9 p.m. and to stop pumps in the morning before 7 a.m.

\subsection{Materials and Methods}

Pump efficiency testing was performed according to standards prescribed for testing of centrifugal pumps by the American Society of Mechanical Engineers and the Hydraulic Institute $[1,2]$. Calibrated pressure gages were used to obtain pump inlet and outlet pressures. As prescribed by the aforementioned standards, it was noted during the pump test if valves located on the outlet end of the pump controlled flow. It was also noted if other pumps were operating in parallel with the pump-motor combination being measured. 


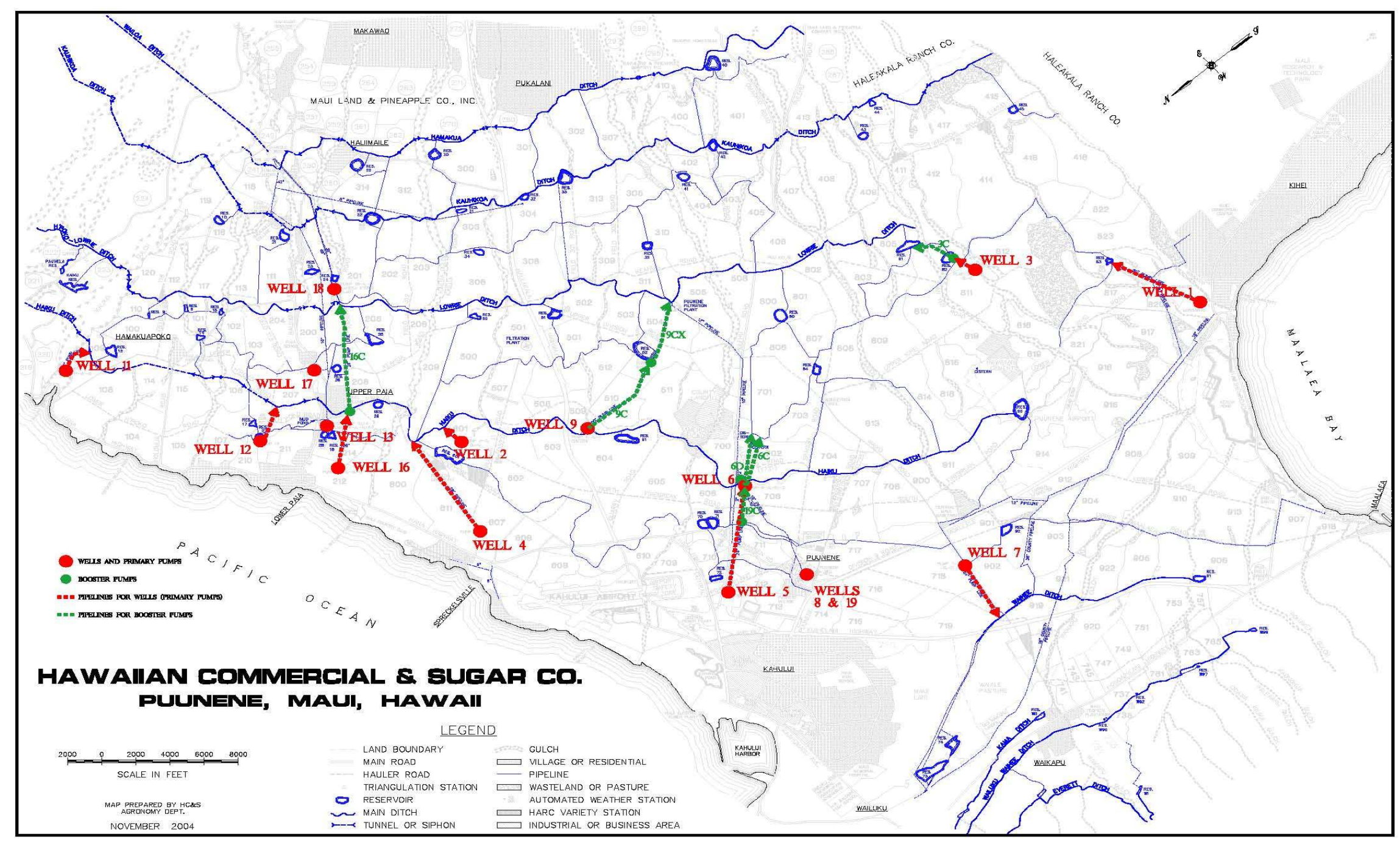

Figure 2-1. Map of farm area showing irrigation system network 
Pump flow was measured where possible with a portable flow-meter (Controlotron, System 1010). The flow-meter could measure water flow using the transit time method, but in difficult conditions could also measure flow using the Doppler method that was a feature of the flow-meter. The transit time method depends on sonic transmit signals sent by a transducer traveling though the liquid and arriving at the receiver transducer without excessive attenuation. Liquids that contain an excess of gas bubbles or mineral solids are better applications for the Doppler method for measuring flow.

Electricity consumption was determined using analog readings from the motor control center for voltage, amperage, and power when available. When these readings were not available, a portable meter (General Electric Multilin Power Quality Meter, Model 501) was used to obtain the necessary electrical readings.

Data were entered into a program referred to as the Pump Systems Assessment Tool (PSAT 2004), provided by the Department of Energy Office of Industrial Technologies. Once pump head and motor electrical data were entered, the program determined pump system efficiency and provided optimization ratings for the pump system. The program also quantified energy savings in terms of annual energy units and cost savings based on annual operating hours and electricity costs. The program could also provide energy consumption information for the condition using design head and flow parameters, considered the most optimal conditions for the pump/motor combination.

Not all pumps could be measured within the survey time period. As a result, pumps were selected according to their annual usage and power rating as it was believed these pumps would provide the greatest saving opportunities.

\subsection{Results and Discussion}

A complete listing of the PSAT output results for the pumps tested is provided in the Appendix. A summary of measured heads and flows compared to design heads and flows for the pumps tested is provided in Table 2-1. Results for measured head readings were in close agreement with design heads. In cases where the measured heads were significantly higher than the design heads, a control valve was throttling the outlet flow. The same cannot be said for the measured pump flows compared to the design pump flows. The measured flows were found to be on average significantly lower than the specified pump flows at the design head, especially for the factory pumps.

A distinction was made between the field pumps and factory pumps because different goals apply. The goal for field pumps is to maximize the flow output of these pumps to increase the irrigation water. The goal for most of the factory pumps is to supply the precise amount of water needed for the process.

The projected energy savings and opportunity revenue for the pumps measured in this survey are presented in Table 2-2. As the goal for the field pumps was to maximize pump flow, power requirement for design head and flow conditions could actually 
increase from measured conditions. The electrical power requirement under optimal conditions was determined from PSAT 2004 by using the design head and flow values for the pump measured. As a result, energy requirements for optimal conditions increased in some cases. This goal was also applied to pumps used to provide cooling water to the turbine condensers in the factory division (Pump Nos. 8A, 8B, 8D, 19A, 19B). The opportunity revenue was determined by projecting the revenue that would be realized if the potential electric power savings were instead sold to the utility.

The increase in annualized flow for the optimized pump system was projected for the field pumps only based on annual operating hours. This projection was not performed for the booster pumps as these provide no incremental increase in the water that is pumped from the ground. Once this value is known, a projection was made on the increased sugar yield expected from the increased irrigation water application amount. The sugar yield projection was not performed for the factory pumps as the optimization goal is different for these pumps. Results for the field pumps showed that significant savings could be realized if pump efficiencies were improved for Pump Nos. 19C4, 18A\&B, 9C, and 12.

Other distinctions noted between the field pumps and factory pumps were the operating hours were higher for the factory pumps. Even though the power ratings were lower for the factory pumps, the high operating hours associated with the factory pumps gave higher projected energy and cost savings. The largest energy savings were associated with the vertical pump system (Pump Nos. 6170, 6163, 6166, 6168) used to pump warm condenser cooling water to a cooling spray pond. The PSAT results showed that the flow output for these pumps could be provided by a $125 \mathrm{hp}$ motor instead of the existing 250 hp rated motor.

As was mentioned earlier, power available for pumping is limited during dry periods due to power sales requirements to the utility company. Currently, field pumps are manually started and stopped at the pump station. The majority of pumps are split case horizontal pumps that need priming before starting. Starting of pumps is conducted manually because of pump priming and other operational issues that require physical presence to protect equipment. Shutting down pumps in a controlled manner can be achieved by radio signal to a programmable controller at the pump station from a master station located at the factory power plant.

The cost to install electric or hydraulically operated stop valves was estimated to range from $\$ 20,000$ to $\$ 60,000$ per pump unit. Additional solenoid valves would be needed to secure auxiliary cooling water at a cost of about $\$ 1000$ per pump. An initiative is currently under way to convert all field pumps so these can be stopped automatically. The plan is to convert two pump stations per year. The operations impact would be more labor hours will then be dedicated to pump repair and maintenance. Automation would also allow slightly longer pump operating time during the off-peak hours between 9 p.m. to 7 a.m. 
Table 2-1. Comparison of Measured Versus Design Heads and Flows

\begin{tabular}{|c|c|c|c|c|c|c|}
\hline Pump ID & $\begin{array}{c}\text { Measured } \\
\text { Head (ft) }\end{array}$ & Design Head (ft) & $\begin{array}{c}\% \text { Measured } \\
\text { Head of Design } \\
\text { Head }\end{array}$ & $\begin{array}{c}\begin{array}{c}\text { Measured Flow } \\
(\mathrm{gpm})\end{array} \\
\end{array}$ & Design Flow (gpm) & $\begin{array}{l}\text { \% Measured Flow } \\
\text { of Design Flow }\end{array}$ \\
\hline \multicolumn{7}{|c|}{ Field Pumps } \\
\hline $19 \mathrm{C} 1$ & 107 & 111 & $96.4 \%$ & 3,595 & 3,475 & $103.5 \%$ \\
\hline $19 \mathrm{C} 2$ & 107 & 111 & $96.4 \%$ & 3,595 & 3,475 & $103.5 \%$ \\
\hline $19 \mathrm{C} 3$ & 103 & 120 & $85.8 \%$ & 4,861 & 6,850 & $71.0 \%$ \\
\hline $19 \mathrm{C} 4$ & 102 & 120 & $85.0 \%$ & 4,861 & 6,850 & $71.0 \%$ \\
\hline $6 \mathrm{~A}$ & 185 & 192 & $96.4 \%$ & 5,650 & 7,000 & $80.7 \%$ \\
\hline $6 \mathrm{~B}$ & 196 & 195 & $100.3 \%$ & 9,554 & 9,700 & $98.5 \%$ \\
\hline $6 \mathrm{C}$ & 129 & 132 & $97.8 \%$ & 5,342 & 7,000 & $76.3 \%$ \\
\hline $11 \mathrm{~A}$ & 273 & 270 & $101.1 \%$ & 2,527 & 2,750 & $91.9 \%$ \\
\hline 17 & 339 & 334 & $101.5 \%$ & 6,848 & 8,100 & $84.5 \%$ \\
\hline $18 \mathrm{~A}$ & 499 & 500 & $99.9 \%$ & 9,032 & 10,500 & $86.0 \%$ \\
\hline $18 \mathrm{~B}$ & 512 & 517 & $99.1 \%$ & 9,358 & 10,500 & $89.1 \%$ \\
\hline $16 \mathrm{~A}$ & 271 & 295 & $91.8 \%$ & 8,379 & 8,400 & $99.8 \%$ \\
\hline $16 \mathrm{D}$ & 244 & 280 & $87.1 \%$ & 5,913 & 6,000 & $98.6 \%$ \\
\hline $16 \mathrm{C}$ & 374 & 295 & $126.8 \%$ & 4,564 & 6,000 & $76.1 \%$ \\
\hline $9 \mathrm{~A}$ & 209 & 217 & $96.4 \%$ & 8,796 & 10,500 & $83.8 \%$ \\
\hline $9 \mathrm{C}$ & 185 & 195 & $95.0 \%$ & 8,514 & 9,750 & $87.3 \%$ \\
\hline $9 \mathrm{CX}$ & 162 & 180 & $89.9 \%$ & 5,044 & 6,950 & $72.6 \%$ \\
\hline 12 & 266 & 280 & $94.8 \%$ & 4,920 & 6,000 & $82.0 \%$ \\
\hline $7 \mathrm{~A}$ & 151 & 160 & $94.2 \%$ & 9,016 & 10,400 & $86.7 \%$ \\
\hline $3 \mathrm{~A}$ & 375 & 390 & $96.1 \%$ & 6,490 & 7,300 & $88.9 \%$ \\
\hline $3 B$ & 365 & 390 & $93.7 \%$ & 6,420 & 7,300 & $87.9 \%$ \\
\hline 1 & 194 & 196 & $99.0 \%$ & 3,259 & 4,000 & $81.5 \%$ \\
\hline \multicolumn{7}{|c|}{ Factory Pumps } \\
\hline $19 \mathrm{~A}$ & 119 & 120 & $98.9 \%$ & 4,036 & 4,900 & $82.4 \%$ \\
\hline $19 \mathrm{~B}$ & 115 & 120 & $95.6 \%$ & 3,784 & 4,900 & $77.2 \%$ \\
\hline $8 \mathrm{~A}$ & 108 & 117 & $92.1 \%$ & 2,846 & 3,475 & $81.9 \%$ \\
\hline $8 \mathrm{~B}$ & 108 & 117 & $92.5 \%$ & 3,023 & 3,475 & $87.0 \%$ \\
\hline $8 D$ & 108 & 117 & $92.1 \%$ & 2,177 & 3,475 & $62.6 \%$ \\
\hline 7717 & 150 & 110 & $136.1 \%$ & 1383 & 2500 & $55.3 \%$ \\
\hline 6170 & 63 & 100 & $63.4 \%$ & 4750 & 8000 & $59.4 \%$ \\
\hline 6163 & 59 & 100 & $58.8 \%$ & 4500 & 8000 & $56.3 \%$ \\
\hline 6166 & 50 & 100 & $49.6 \%$ & 4500 & 8000 & $56.3 \%$ \\
\hline 6168 & 70 & 100 & $70.4 \%$ & 4500 & 8000 & $56.3 \%$ \\
\hline 6639 & 128 & 110 & $116.4 \%$ & 4526 & 6500 & $69.6 \%$ \\
\hline
\end{tabular}


Table 2-2. Projected Energy and Cost Savings

\begin{tabular}{|c|c|c|c|c|c|c|}
\hline Pump ID & \begin{tabular}{|c|} 
Existing \\
Motor Rated \\
HP
\end{tabular} & $\begin{array}{c}\text { Measured } \\
\text { Motor/Pump } \\
\text { Efficiency (\%) }\end{array}$ & $\begin{array}{c}\text { Annual } \\
\text { Operating hrs. }\end{array}$ & $\begin{array}{c}\text { Energy Savings } \\
\text { (MWh) }\end{array}$ & $\begin{array}{l}\text { Opportunity } \\
\text { Revenue (\$) }\end{array}$ & $\begin{array}{c}\text { Incremental } \\
\text { Increase in } \\
\text { Pumped Water } \\
\text { (MGPY) }\end{array}$ \\
\hline \multicolumn{7}{|c|}{ Field Pumps } \\
\hline $19 \mathrm{C} 1$ & 125 & $80.4 \%$ & 3,504 & 43 & $\$ 7,603$ & $\mathrm{NA}$ \\
\hline $19 \mathrm{C} 2$ & 125 & $80.4 \%$ & 3,504 & 43 & $\$ 7,603$ & NA \\
\hline $19 \mathrm{C3}$ & 250 & $61.8 \%$ & 8,646 & -160 & $-\$ 28,195$ & $\mathrm{NA}$ \\
\hline $19 \mathrm{C} 4$ & 250 & $50.7 \%$ & 8,646 & 142 & $\$ 24,904$ & $\mathrm{NA}$ \\
\hline $6 \mathrm{~A}$ & 450 & $65.0 \%$ & 2,041 & 52 & $\$ 9,082$ & 165 \\
\hline $6 B$ & 600 & $76.7 \%$ & 990 & 72 & $\$ 12,602$ & 9 \\
\hline $6 \mathrm{C}$ & 300 & $76.5 \%$ & 2,041 & -45 & $-\$ 7,850$ & $\mathrm{NA}$ \\
\hline $11 \mathrm{~A}$ & 200 & $76.9 \%$ & 2,505 & 56 & $\$ 9,891$ & 34 \\
\hline 17 & 800 & $72.6 \%$ & 1,752 & 83 & $\$ 14,590$ & 132 \\
\hline $18 \mathrm{~A}$ & 1500 & $74.6 \%$ & 2,453 & 155 & $\$ 27,227$ & 216 \\
\hline $18 \mathrm{~B}$ & 2000 & $71.3 \%$ & 894 & 117 & $\$ 20,522$ & 61 \\
\hline $16 \mathrm{~A}$ & 700 & $78.4 \%$ & 1,577 & 52 & $\$ 9,222$ & 2 \\
\hline $16 \mathrm{D}$ & 600 & $57.4 \%$ & 876 & 111 & $\$ 19,501$ & 5 \\
\hline $16 \mathrm{C}$ & 700 & $67.1 \%$ & 526 & 62 & $\$ 10,912$ & $\mathrm{NA}$ \\
\hline $9 \mathrm{~A}$ & 800 & $67.2 \%$ & 1,498 & 75 & $\$ 13,253$ & 153 \\
\hline $9 \mathrm{C}$ & 800 & $59.6 \%$ & 1,419 & 159 & $\$ 27,949$ & NA \\
\hline $9 C X$ & 300 & $71.7 \%$ & 534 & -26 & $-\$ 4,541$ & NA \\
\hline 12 & 600 & $58.8 \%$ & 1,901 & 126 & $\$ 22,141$ & 123 \\
\hline $7 \mathrm{~A}$ & 600 & $70.0 \%$ & 438 & 11 & $\$ 1,971$ & 36 \\
\hline $3 A$ & 900 & $70.9 \%$ & 1,393 & 84 & $\$ 14,819$ & 68 \\
\hline $3 \mathrm{~B}$ & 900 & $75.8 \%$ & 1,910 & -14 & $-\$ 2,376$ & 101 \\
\hline 1 & 250 & $68.4 \%$ & 2,970 & $\underline{45}$ & $\$ 7,920$ & 132 \\
\hline \multicolumn{4}{|c|}{ Factory Pumps } & 1,243 & $\$ 218,750$ & 1,236 \\
\hline $19 \mathrm{~A}$ & 200 & $58.8 \%$ & 8,585 & 222 & $\$ 39,054$ & NA \\
\hline $19 B$ & 200 & $57.5 \%$ & 8,585 & 202 & $\$ 35,587$ & $\mathrm{NA}$ \\
\hline $8 \mathrm{~A}$ & 150 & $70.3 \%$ & 8,629 & -11 & $-\$ 1,971$ & $\mathrm{NA}$ \\
\hline $8 \mathrm{~B}$ & 150 & $69.8 \%$ & 8,287 & 42 & $\$ 7,357$ & $\mathrm{NA}$ \\
\hline $8 \mathrm{D}$ & 150 & $51.1 \%$ & 788 & 6 & $\$ 1,038$ & NA \\
\hline 7717 & 125 & $69.3 \%$ & 8,672 & 195 & $\$ 34,250$ & $\mathrm{NA}$ \\
\hline 6170 & 250 & $39.2 \%$ & 7,008 & 611 & $\$ 107,589$ & $\mathrm{NA}$ \\
\hline 6163 & 250 & $66.6 \%$ & 7,008 & 156 & $\$ 27,403$ & NA \\
\hline 6166 & 250 & $26.8 \%$ & 7,008 & 814 & $\$ 143,264$ & $\mathrm{NA}$ \\
\hline 6168 & 250 & $51.8 \%$ & 7,008 & 373 & $\$ 65,613$ & $\mathrm{NA}$ \\
\hline 6639 & 250 & $70.4 \%$ & 7,008 & 252 & $\$ 44,352$ & $\mathrm{NA}$ \\
\hline & & & & 2,861 & $\$ 503,536$ & \\
\hline
\end{tabular}




\subsection{Summary and Conclusions}

The results produced from pump efficiency measurements performed on selected field and factory pumps show that significant savings can be achieved by optimizing the pump/motor systems. The largest savings opportunities appear initially to come from repairing or replacing the factory pumps that have low efficiencies. However, when the potential revenue from increased cane yield is added to the opportunity revenue from the field pumps, the overall opportunity revenue will be greater for the field pumps.

The results are summarized in Table 2-3 where both pump efficiency improvements and opportunity revenue are presented using the assumption that 1 million gallons of irrigation water applied to the developing sugarcane crop will yield the equivalent of 1 ton of sugar. The potential gross revenue that HC\&S can realize from the increased sugar yield should be about $\$ 340 /$ ton based on 2005 operating results. This figure does not take into account any expenses for processing the additional sugar in the factory nor does it include any byproduct credits from additional bagasse fuel or molasses production. The results presented in Table 2-3 show total opportunity revenue of over \$1.1 million with a significant portion of this revenue derived from crop yield improvement from increased irrigation water. Given the importance of sugar yields to the operation, emphasis should be placed on improving field pumps to original specification. The measured flow results indicate there is potential for increasing pump flow either by rebuilding or by replacing the pump. Automating field pump shut-downs should provide more pumping time opportunity during the "off-peak" hours when $4 \mathrm{MW}$ is available for irrigation pumping.

Table 2-3 Pump Efficiency Improvement Opportunity Revenue Summary

\begin{tabular}{|c|c|c|c|}
\hline Division & $\begin{array}{c}\text { Pump Efficiency } \\
\text { Improvements } \\
(\$ 1000)\end{array}$ & $\begin{array}{c}\text { Crop Yield } \\
\text { Improvement } \\
(\$ 1000)\end{array}$ & $\begin{array}{c}\text { Total Opportunity } \\
\text { Revenue } \\
(\$ 1000)\end{array}$ \\
\hline Field & $\$ 219$ & $\$ 420$ & $\$ 639$ \\
\hline Factory & $\$ 504$ & NA & $\$ 504$ \\
\hline
\end{tabular}

It should be kept in mind that the savings opportunities described here apply to only those pumps measured in this survey. Other opportunities exist in improving pump efficiencies for the smaller drip pumps that require on average about $1.5 \mathrm{MW}$ daily to operate. An ongoing drip pump rebuilding program is already in place at HC\&S. Also, there are numerous other pumps used in the factory to convey a variety of fluids, specifically cane juice, syrup, and molasses. As these pumps are utilized throughout the grinding season (about 260 days in length) they also warrant efficiency checks.

\subsection{References}

1. The American Society of Mechanical Engineers. 1991. Centrifugal pumps performance test codes, ASME PTC 8.2-1990.

2. Hydraulic Institute. 2000. Centrifugal pump tests. ANSI/HI 1.6-2000. 


\section{Steam Generation and Distribution}

\subsection{Introduction}

HC\&S utilizes cogeneration for the simultaneous production of raw sugar and electric power. Efficient generation and use of steam is very important to any well run sugar factory, particularly if power sales and fuel savings are valued. This task was actually composed of two sub-tasks: 1) steam generation assessment in the power production operations, and 2) steam distribution assessment in the sugar manufacturing operation. The scope of work for these two sub-tasks required outside research collaboration from the University of Hawaii to assess the steam generation and distribution of steam for processing and power generation. The reports produced by the research collaborators are included in the Appendix. A brief overview and summary of their results are presented in this chapter.

Three boilers are used to generate steam at the HC\&S Puunene sugar factory. These boilers are all grate-fired, stoker-type units. Boilers 1 and 2 are identical units and operate at 900 psia steam pressure with rated capacities of $120 \mathrm{klb}$ steam per hour each. Boiler 3 operates at 425 psia steam pressure and is rated at $290 \mathrm{klb}$ steam per hour. All three boilers are able to use multiple types of fuel. The major fuel used is biomass in the form of sugarcane bagasse. Supplementary fuels used are coal, fuel oil, and a minor amount of used cooking oil. A breakdown of the fuels used for the operation in 2004 on a percentage heating value basis is given in Table 3-1.

Table 3-1. Boiler Fuels Used (MMBtu input basis) in 2004

\begin{tabular}{|c|r|c|}
\hline Fuel Type & MMBtu Fuel Input & \% of Total \\
\hline No.6 fuel oil & 18,724 & $0.3 \%$ \\
\hline Diesel, No. 2 & 41,461 & $0.7 \%$ \\
\hline Vegetable oil & 11,087 & $0.2 \%$ \\
\hline Coal & $1,188,985$ & $20.1 \%$ \\
\hline Bagasse & $\underline{4,662,816}$ & $78.7 \%$ \\
\hline Totals & $5,923,073$ & \\
\hline \multicolumn{2}{|r}{}
\end{tabular}

Boiler efficiency tests were conducted by the University of Hawaii on bagasse and coal fuel for Boilers 1 and 2 and for bagasse, coal, and fuel oil for Boiler 3. A full description of the materials and methods used are provided in their report in the Appendix.

The sugar factory steam use assessment was performed by the UH research collaborators as well. A factory steam balance was established and then modeling software was applied to assess the entire process and identify areas where improvements might be made. The modeling software used was the Advanced System for Process ENgineering (ASPEN) PLUS ${ }^{\circledR}$ commercial software package form Aspen Technology Inc. (Cambridge, MA). Further analysis was performed using a pinch analysis program, Aspen Pinch. 
An internal missing insulation survey was conducted by HC\&S in the Puunene power plant. The annual heat loss was determined and was converted to equivalent lost fuel or steam used for power generation. An internal review was also conducted on the integration of a standby turbogenerator referred to as TG3 into normal operations. This turbogenerator has been used as a standby generator because of efficiency and reliability issues that need to be addressed internally within HC\&S. The potential energy savings from integrating TG3 into normal operations will not be quantified in the results section but will only be discussed briefly as an opportunity that deserves further investigation pending internal action taken by HC\&S.

\subsection{Results and Discussion}

Material and methods and complete results obtained from the UH research collaborators are provided in their reports included in the Appendix. A brief summary of their results is discussed in this section. Since bagasse and coal fuel are the major boiler fuels used at HC\&S to generate steam, discussion will focus only on potential fuel savings using these fuels.

The UH research collaborators described opportunities for boiler efficiency gains by reducing excess air and flue gas temperature. Boiler efficiencies for the three boilers ranged from $63.2 \%$ to $67.2 \%$ on bagasse fuel and from $76.1 \%$ to $82.4 \%$ on coal fuel. Boiler 2 had consistently lower efficiency for both fuels. It was projected that if only a $1 \%$ improvement were made in boiler efficiency using coal fuel on all three boilers, then about 9.5 tons (dry basis) of coal could be saved per day using $100 \%$ coal fuel. Similarly, a $1 \%$ improvement in boiler efficiency could save 21.5 tons (dry basis) of bagasse fuel per day using $100 \%$ bagasse fuel. In reality, a mixture of these fuels are used throughout the grinding period. No specific recommendations were provided by the UH researchers on how to improve boiler operation procedures.

About the same time results were being collected by the UH research collaborators, Alstom Power, Inc. was commissioned by HC\&S to make recommendations on how to improve boiler stoker operations and reduce the particulate matter (PM) emissions when firing coal in Boiler No. 3. Although this study was not part of the scope of work of this project, the observations made by the consultant seemed to be consistent with the observations made by the UH research collaborators. Furthermore, specific recommendations were made to improve boiler operations. Some of these recommendations were:

- Install new over-fire air systems on all boilers to improve bagasse combustion

- Have dedicated mechanical feeders and distributors for bagasse and coal fuel

- Calibrate oxygen sensors and tie readings into the boiler control system

- Modify or replace the forced draft fan on Boiler 2

- Improve undergrate air distribution on Boiler 3

- Scribe and automate air bypass dampers on Boiler 3 to bypass air past the air heater when firing coal fuel 
Steam use for manufacturing raw sugar was modeled as described earlier. A pinch analysis was conducted to identify significant energy saving opportunities in the sugar factory, most notably in the boiling house operations. Steam consumption for evaporating sugarcane juice and boiling sugar was determined to be in the range of 800$850 \mathrm{lbs}$ steam per ton cane. As is mentioned in the report by UH researchers in the Appendix, experts predict that this steam usage figure can be reduced to $650 \mathrm{lbs}$ steam per ton cane or less. Reducing the steam-to-cane ratio can make more steam available for other processes or for electricity generation and export power sales.

The two greatest steam savings opportunities identified that were deemed possible for implementation were: 1) operating the pan boiling system on second evaporator cell vapor rather than first evaporator cell vapor, and 2) increasing use of condensate flash to all evaporator cells to save steam. The combined effects of these two improvements would reduce steam-to-cane ratio by $90 \mathrm{lbs}$ steam per ton cane and could increase electricity generation by $1.31 \mathrm{MW}$ if saved steam was instead fully condensed in the largest turbogenerator referred to as TG4. Capital expense would be required to change heat exchange surface areas in the evaporator train, the pans, and also to increase cooling water supply and pumping capacity in the evaporator train condenser. Other steam saving opportunities were identified in the modeling effort, but were not deemed possible within the operational constraints of the sugar factory. It should also be mentioned that the two steam saving opportunities mentioned were previously tried by HC\&S and were later abandoned because of negative impacts to the boiling house operation. The full modifications to the boiling house described in the UH report in the Appendix must be implemented in order to realize the potential savings described.

There are other steam saving opportunities that are known internally within HC\&S that were not investigated by the UH research collaborators as their scope of work involved using only the two full-time operating turbogenerators, TG4 and TG5. An operating scenario is possible where TG3 can be operated in conjunction with TG5 using the 425 psia extraction steam from TG5 to operate TG3. The extraction steam level would have to increase from TG5, thereby reducing the amount of steam condensed by TG5. With the integration of TG3 into regular operations, preliminary indications are that $2 \mathrm{MW}$ more of electricity could be generated from $30 \mathrm{klbs}$ of steam from the boilers. This would mean eliminating the practice of passing steam through pressure reducing valves (PRVs) and operating the boilers near maximum capacity during peak periods. However, as mentioned earlier, there are operation reliability issues associated with TG3 and up to now this TG has only been used intermittently. Therefore, this opportunity will not be treated as a firm opportunity until the operation reliability issues are addressed internally by $\mathrm{HC} \& \mathrm{~S}$.

Results of the internal investigation conducted by HC\&S of missing steam pipe insulation in the power plant are provided in the Appendix. The survey indicated that there is an estimated annual heat loss of 71,543 MMBtu from un-insulated steam lines in the power plant. Prevention of this heat loss would translate into either fuel savings or more energy from steam to perform work. 


\subsection{Summary and Conclusions}

The combined steam generation and distribution savings are summarized in Table 3-2 for each of the major steam energy saving areas identified. Savings are presented in terms of annual fuel value savings and steam quantity savings. The fuel dollar value for bagasse was determined by using an equivalent coal fuel value assuming that if bagasse were not available then coal fuel would have to be used. Annual fuel savings (in terms of tons wet basis) for coal and bagasse were based partly on the fuel use data for 2004 showing nearly $80 \%$ of fuel requirement provided by bagasse and about $20 \%$ from coal. The fuel and steam savings from boiler efficiency improvement were based on the annual fuel amount consumed for both coal and bagasse in 2004 and then determining the annual amount of fuel saved if a $1 \%$ improvement in boiler efficiency was obtained. Fuel and steam savings obtained from utilizing second vapor and increasing use of condensate flash in the factory were based on 260 operating days. The projected saving from insulating steam lines in the power plant was based on 347 operating days.

The greatest energy saving opportunity identified was utilizing second evaporator cell steam vapor to operate the pans in the boiling house. However, improved insulation of steam lines in the power plant is believed to provide the shortest payback.

Table 3-2. Summary of Steam Savings Opportunities

\begin{tabular}{|c|c|c|c|c|}
\hline \multirow[b]{2}{*}{ Project } & \multicolumn{2}{|c|}{ Fuel Savings } & \multirow[b]{2}{*}{$\begin{array}{l}\text { Fuel Value } \\
\qquad(\$ 1000)\end{array}$} & \multirow[b]{2}{*}{$\begin{array}{c}\text { Annual Steam Savings } \\
(\mathrm{klbs} / \mathrm{yr})\end{array}$} \\
\hline & $\begin{array}{c}\text { Coal } \\
\text { (tons, wb) }\end{array}$ & $\begin{array}{l}\text { Bagasse } \\
\text { (tons, wb) }\end{array}$ & & \\
\hline 1. Boiler efficiency & 830 & 8,988 & 269 & 67,628 \\
\hline 2. Second vapor use & 1,750 & 16,936 & 521 & 130,700 \\
\hline 3. Flash condensates & 663 & 6,418 & 197 & 49,528 \\
\hline 4. Pipe insulation & 701 & 6,788 & 209 & 52,383 \\
\hline
\end{tabular}




\section{Electric Power Distribution}

\section{1 $\underline{\text { Introduction }}$}

The electrical distribution system used at HC\&S resembles that of a small utility grid. HC\&S generates most of its own electric power for its operations and sells surplus electricity to the local electric utility. The maximum voltage used for the transmission distribution lines is 23 kilovolts. Voltages are stepped down from this level using electrical distribution substations in order to operate electrically driven equipment. Fifteen sub stations handle all incoming or outgoing electricity. The transmission and distribution system is key to providing electric power to operate the various irrigation pumps located throughout the farm area. The system also provides the means of distributing electric power generated from the hydro-turbines installed in the irrigation ditch system.

An ongoing program is in place at HC\&S to install capacitors on motors greater than 30 hp to improve the power factor. Power factor improvements will reduce the reactive power requirement to operate electrical equipment.

Transformers with large $\mathrm{kVA}$ ratings require electrical energy to remain activated. This electricity used to maintain activation is referred to as "no-load loss" and is actually electrical energy converted to heat. As these transformers are necessary for electricity distribution, the transformers operate on a continuous basis during the year. Some older transformers on the $\mathrm{HC} \& \mathrm{~S}$ grid system have already been replaced through an ongoing replacement program with proven energy savings. Replacement or consolidation of other older transformers could produce significant electrical energy savings.

\subsection{Materials and Methods}

Electrical test equipment was used to assess electric motors to determine the amount of capacitance needed to correct power factor. The portable Multilin Power Quality meter mentioned previously for pump efficiency testing was one of the instruments used for this purpose.

To determine transformer no-load-loss, instrumentation was used to measure current and voltage on the primary side and secondary side of the transformer. A Sensorlink Model No. 8-020 was used to measure amperage (0-2000 amps range) and a Hubbell-Chance, Model No. 62NCM, voltmeter was used to measure voltage (0-40 kilovolts range). As the transformers measured in this assessment all were associated with the well pumps, these readings were usually taken as part of the pump efficiency testing procedures.

Other power factor improvements that are possible to implement on the electrical distribution system are mentioned here, but estimates of potential annual energy savings were not determined. A quotation was obtained for a synchronous electric motor at Well 3 that is located at the extreme end of the distribution system. Synchronous electric 
motors are able to generate vars (reactive power) that would support voltage levels at extreme ends of the distribution system. The quotation for one synchronous electric motor with a motor control center was about \$186k in 2004.

Another method to increase reactive power production is to operate an additional steamdriven turbogenerator at the Puunene power plant. This possibility exists if TG3 (normally used as a standby generator) is run in conjunction with TG5. The operation of TG3 could provide more reactive power to the $23 \mathrm{kV}$ electrical system and improve the overall voltage regulation and reserve capacity of the system according to an internal power system study that was commissioned by HC\&S in 2002. The integration of TG3 into regular operations was discussed briefly in the previous section for potential steam saving opportunities.

Considerable work was performed by HC\&S personnel during 2005 on electrical distribution capital improvement projects. One of these projects was replacing the TG4 power management control system at the beginning of the year that cost over $\$ 800 \mathrm{k}$. It is believed this upgrade contributed to over $8000 \mathrm{MWh}$ of electricity sales above the 2005 operating plan for a total of 96,294 MWh of electricity sold to the electric utility in 2005. This incremental amount of electricity sold to the utility was worth over $\$ 1.4$ million in additional revenue using the fourth quarter 2005 tariff rate for electricity sold. However, not all of the additional revenue noted can be attributed to the upgrading of the TG4 power management control system because of favorable hydro-power generation that enabled an additional $2 \mathrm{MW}$ of electric power to be sold to the utility during peak hours.

\section{3 $\quad \underline{\text { Results and Discussion }}$}

The ongoing program of installing capacitors on electric motors greater than $30 \mathrm{hp}$ in the factory area has already demonstrated electrical savings. Approximately 50\% of the motors have been outfitted with capacitors and the reactive power savings was determined to be 26.67 kilovars. Using a system power factor of 0.925 in the factory area, this is equivalent to $569 \mathrm{MWh}$ annual electricity savings. Assuming the remaining motors in the factory larger than $30 \mathrm{hp}$ will be equipped with capacitors the potential future savings should be of the same magnitude.

As was mentioned previously, older transformers for well pumps were replaced and produced significant reductions in no-load losses. The average reduction in no-load loss was about $75 \%$ for two instances. In both cases there was a payback period of less than one year. Other candidate well pump transformer replacements are listed in Table 4-1. The measured no-load loss and projected annual electricity savings with a $75 \%$ reduction in no-load loss are also listed for these candidate transformer replacements. These results show that the transformers located at Wells 3 and 7 will provide the greatest potential savings. 
Table 4-1. Candidate Electrical Transformer Replacement

\begin{tabular}{|c|c|c|c|}
\hline Well No. & $\begin{array}{c}\text { Annual No-Load-Loss } \\
(\mathrm{MWh})\end{array}$ & $\begin{array}{c}\text { Potential Savings @ } \\
75 \% \text { Reduction }(\mathrm{MWh})\end{array}$ & $\begin{array}{c}\text { Opportunity Revenue } \\
(\$)\end{array}$ \\
\hline 6 & 169 & 127 & $\$ 22,296$ \\
\hline 11 & 87 & 65 & $\$ 11,471$ \\
\hline 7 & 750 & 562 & $\$ 98,975$ \\
\hline 3 & 870 & 653 & $\$ 114,911$ \\
\hline
\end{tabular}

\section{4 $\quad$ Summary and Conclusions}

A summary of annual electricity savings and opportunity revenue are presented in Table 4-2 for the projects investigated. Opportunity revenue could be realized if the electricity saved were sold instead to the local electric utility. The greatest potential for annual savings appears to come from electric transformer replacement.

Table 4-2. Summary of Electric Distribution System Savings

\begin{tabular}{|l|c|c|}
\hline Project & $\begin{array}{c}\text { Annual Electricity } \\
\text { Savings (MWh) }\end{array}$ & $\begin{array}{c}\text { Opportunity Revenue } \\
(\$)\end{array}$ \\
\hline Capacitor installation & 569 & $\$ 100,186$ \\
\hline Transformer replacement & 1,407 & $\$ 247,653$ \\
\hline
\end{tabular}




\section{Summary of Savings Opportunities}

\subsection{Savings Summary and Discussion}

Energy savings can be expressed either in terms of potential fuel savings, electrical energy savings, or opportunity revenue from electricity sales. These expressions of energy savings are not mutually exclusive where assumptions were made to perform the conversions between these terms. The fuel saving values represent a more conservative estimate of potential monetary value whereas the opportunity revenue from electricity sales will provide the highest monetary value. A more straightforward approach for fuel savings would be to use fuel heating value savings only (MMBtu), but then no distinction could be made between saved bagasse and coal fuel amounts to project reductions in air pollutants and emissions. If electrical energy savings were determined directly, then equivalent fuel savings were determined from the average annual steam requirement to produce electricity from the cogeneration system of HC\&S (this study used an annual average of 14,849 lbs steam per MWh). Fuel usage in 2004 indicated that nearly $20 \%$ of fuel input heat value was provided by coal fuel and $80 \%$ from bagasse. Knowing boiler efficiencies for each of these fuels ( $65 \%$ on bagasse, $80 \%$ on coal), the steam produced from each fuel could be estimated. Once this information was known, fuel quantities were determined along with their monetary value. In the case where fuel savings were determined directly for the steam generation investigation, a reverse procedure was used to determine equivalent electricity potential. Overall, applying the same criteria to compare the various energy savings from each project should put into perspective their relative potential energy savings. A summary of the results is presented in Table 5-1.

As mentioned previously, the tariff rate used for electricity sales was $\$ 176 / \mathrm{MWh}$, the avoided energy cost that the electric utility paid HC\&S in the fourth quarter of 2005. There is also a capacity payment of about $\$ 17 / \mathrm{MWh}$, but this was not included in the opportunity revenue projections as it is unknown if the electricity that would be sold to the utility would be included as dispatched power that is eligible for capacity payment.

From the data presented in Table 5-1, the major energy saving opportunities appears to come from pump efficiency improvements (field and factory) and steam efficiency improvements. It should be noted that the opportunity revenue for the field pumps includes about $\$ 420 \mathrm{k}$ from increased projected sugar production from increased pump water flow. The actual electrical energy savings is greater for pumps that were measured in the factory area. The combined opportunity revenue for the first four projects alone listed in Table 5-1 amounts to nearly $\$ 3$ million annually. The more conservative fuel cost savings showed over $\$ 1.5$ million in combined fuel cost savings for all projects in the form of coal and bagasse fuel. 
Table 5-1. Summary of Annual Fuel, Electricity Savings, and Opportunity Revenue

\begin{tabular}{|c|c|c|c|c|c|}
\hline \multirow[b]{2}{*}{ Project } & \multicolumn{2}{|c|}{ Fuel Savings (tons) } & \multirow{2}{*}{$\begin{array}{l}\text { Fuel Value } \\
(\$ \mathrm{k})\end{array}$} & \multirow{2}{*}{$\begin{array}{c}\text { Electricity } \\
\text { Savings (MWh) }\end{array}$} & \multirow{2}{*}{$\begin{array}{l}\text { Opportunity } \\
\text { Revenue (\$k) }\end{array}$} \\
\hline & Coal & Bagasse & & & \\
\hline 1. Field pumps efficiency & 247 & 2,392 & $\$ 74$ & 1,243 & $\$ 639 *$ \\
\hline 2. Factory pumps efficiency & 569 & 5,505 & $\$ 169$ & 2,861 & $\$ 504$ \\
\hline 3. Steam generation & 830 & 8,988 & $\$ 269$ & 4,554 & $\$ 802$ \\
\hline 4. Second vapor use & 1,750 & 16,936 & $\$ 521$ & 5,928 & $\$ 1,043$ \\
\hline 5. Flashing condensates & 663 & 6,418 & $\$ 197$ & 2,246 & $\$ 395$ \\
\hline 6. Steam line insulation & 701 & 6,788 & $\$ 209$ & 3,528 & $\$ 621$ \\
\hline 7. Capacitor installation & 113 & 1,095 & $\$ 34$ & 569 & $\$ 100$ \\
\hline 8. Transformer replacement & 280 & 2,708 & $\$ 83$ & 1,407 & $\$ 248$ \\
\hline Totals & 5,153 & 50,829 & $\$ 1,555$ & 22,337 & $\$ 4,352$ \\
\hline
\end{tabular}

* Includes $\$ 420 \mathrm{k}$ opportunity revenue from increased sugar yields due to increased pump flow

\subsection{Classification for Implementation Priority}

Each of the projects identified in this investigation were prioritized as a planning guide for future implementation. The results of this exercise are summarized in Table 5-2. Capital costs are categorized as either high capital costs requiring over $\$ 500 \mathrm{k}$, medium capital cost requiring $\$ 100$ to $\$ 500 \mathrm{k}$, and low capital cost requiring less than $\$ 100 \mathrm{k}$. Factors considered for implementation priority (classified as high, medium, or low) were potential annual fuel savings, expected payback for the initial capital cost, and operational considerations. For example, even though the annual fuel savings value is potentially high for use of second vapor steam from the evaporator station to the boiling pans, implementation of this project must be weighed against capital costs and the risk of using lower temperature steam that could possibly slow the processing rate of pan boiling operations if modifications to the evaporator cells are not fully implemented. If these projects were currently being implemented under ongoing energy efficiency improvement programs at HC\&S, this is also indicated in the table.

Table 5-2. Classification of Priority Implementation

\begin{tabular}{|l|c|c|c|c|}
\hline Project & $\begin{array}{c}\text { Fuel Value } \\
(\$ \mathrm{k})\end{array}$ & $\begin{array}{c}\text { Expected } \\
\text { Capital Cost }\end{array}$ & $\begin{array}{c}\text { Implementation } \\
\text { Priority }\end{array}$ & $\begin{array}{c}\text { Being } \\
\text { Implemented? }\end{array}$ \\
\hline 1. Field pumps efficiency & $\$ 74$ & $\$ 100-500 \mathrm{k}$ & High & Yes \\
\hline 2. Factory pumps efficiency & $\$ 169$ & $\$ 100-500 \mathrm{k}$ & High & No \\
\hline 3. Steam generation & $\$ 269$ & $\$ 100-500 \mathrm{k}$ & High & No \\
\hline 4. Second vapor use & $\$ 521$ & $>\$ 500 \mathrm{k}$ & Medium & No \\
\hline 5. Flashing condensates & $\$ 197$ & $\$ 100-500 \mathrm{k}$ & High & No \\
\hline 6. Steam line insulation & $\$ 209$ & $\$ 100-500 \mathrm{k}$ & High & Yes \\
\hline 7. Capacitor installation & $\$ 34$ & $<\$ 100 \mathrm{k}$ & High & Yes \\
\hline 8. Transformer replacement & $\$ 83$ & $\$ 100-500 \mathrm{k}$ & High & No \\
\hline
\end{tabular}

\subsection{Environmental Impacts}

Reduced emissions can be determined directly if energy savings are expressed in terms of equivalent fuel savings. If the energy is used instead to produce more steam and electricity for HC\&S operations, then there will be less air pollutants and emissions produced per unit of fuel consumed. Additionally, there would be reduced emissions for 
electricity generation on the island of Maui because the utility company would not need to burn as much fossil fuel for its electrical generation. A summary of reduced air emissions potential by HC\&S if all energy saving projects were implemented are provided in Table 5-3 for known air pollutants that are monitored in annual stack compliance tests. For NOx, SOx, VOC, and PM, fees are paid to the State of Hawaii for each ton of these pollutants. In 2005, these fees amounted to $\$ 51.83 /$ ton. Therefore a reduction in fuel usage translates directly into air emission fee savings that are quantified in Table 5-3.

Table 5-3. Annual Air Pollutant Reductions from Fuel Savings (tons)

\begin{tabular}{|c|c|c|c|c|c|c|}
\hline Project & NOx & sox & $\mathrm{CO}$ & VOC & PM & PM10 \\
\hline 1. Field pumps efficiency & 3.3 & 1.4 & 26.2 & 0.9 & 1.2 & 1.1 \\
\hline 2. Factory pumps efficiency & 7.7 & 3.1 & 60.4 & 2.0 & 2.7 & 2.5 \\
\hline 3. Steam generation & 12.0 & 4.6 & 98.6 & 3.3 & 4.3 & 3.9 \\
\hline 4. Second vapor use & 23.6 & 9.6 & 185.8 & 6.3 & 8.4 & 7.6 \\
\hline 5. Flashing condensates & 8.9 & 3.7 & 70.4 & 2.4 & 3.2 & 2.9 \\
\hline 6. Steam line insulation & 9.5 & 3.9 & 74.5 & 2.5 & 3.4 & 3.0 \\
\hline 7. Capacitor installation & 1.6 & 1.1 & 13.7 & 3.0 & 0.5 & 0.6 \\
\hline 8. Transformer replacement & 3.8 & 1.5 & 29.7 & 1.0 & 1.4 & 1.2 \\
\hline Totals & 70.4 & 28.9 & 559.3 & 21.4 & 25.1 & 27.8 \\
\hline Tons subject to fees & \multicolumn{6}{|c|}{145.8} \\
\hline Avoided emission fees & \multicolumn{6}{|c|}{$\$ 7,558$} \\
\hline
\end{tabular}

HC\&S uses primarily biomass fuel for its operations, but a significant amount of coal is also used. Biomass, a renewable fuel, will not contribute net carbon dioxide to the atmosphere. However, coal is used as supplemental fuel and accounts for about $20 \%$ of the fuel input at HC\&S. Coal combustion emits carbon dioxide to the atmosphere, contributing to greenhouse gases. According to EPA data in 2003 the electric utility sector emitted on average 205.9 lbs of carbon dioxide into the atmosphere for every million Btu of coal combusted [1]. Knowing the equivalent amount of coal fuel saved in this study, one can estimate the amount of reduced carbon dioxide emissions entering the atmosphere. A summary of potential reductions in $\mathrm{CO}_{2}$ emissions is summarized in Table 5-4 for each of the energy saving projects. Although there is no monetary penalty currently paid for $\mathrm{CO}_{2}$ emissions, coal usage is reported annually to the Energy Information Administration by HC\&S to estimate greenhouse gas emissions in the United States.

Table 5-4. $\mathrm{CO}_{2}$ Emissions Reduction from Coal Fuel (tons)

\begin{tabular}{|l|c|}
\hline Project & $\mathbf{C O}_{\mathbf{2}}$ Reduction (tons) \\
\hline 1. Field pumps efficiency & 611 \\
\hline 2. Factory pumps efficiency & 1,406 \\
\hline 3. Steam generation & 2,050 \\
\hline 4. Second vapor use & 4,324 \\
\hline 5. Flashing condensates & 1,639 \\
\hline 6. Steam line insulation & 1,733 \\
\hline 7. Capacitor installation & 279 \\
\hline 8. Transformer replacement & 691 \\
\hline \multicolumn{2}{|c|}{ Totals } \\
\hline
\end{tabular}




\subsection{Discussion of Project Accomplishments Versus Stated Goals and Objectives}

The Statement of Objectives identified in the project proposal related to the four main tasks: 1) Pump Energy Assessment and Management, 2) Steam Generation Efficiency Assessment, 3) Steam Distribution Efficiency Assessment, and 4) Electric Power Distribution System Efficiency Assessment. Progress on these tasks was reported in Quarterly Progress Reports during the entire project period.

The Pump Energy Assessment task originally focused on field irrigation pumps. Data collected from these pump/motor combinations were analyzed using the DOE/OIT Pump Assessment Tool (PSAT) that was updated in 2004. Pumps that operated in the factory were also measured during the project period and revealed some significant energy saving opportunities. Although not all pumps could be measured in both the field and factory areas, the largest pumps were the focus as these require significant electric power. One of the objectives stated under this task was to spend effort on determining what the requirements would be for more automatic operation in starting and stopping well pumps used for irrigation operations. Further investigation revealed that automating pump shut off was possible, but starting pumps was more complicated because of priming requirements and other operating issues requiring physical presence for equipment protection. As a result, the investigative effort was confined to automatic pump shut downs that could help in operations where pumps need to go down quickly in order to supply electricity to the electric utility at the beginning of the peak demand period.

The Steam Generation Assessment task was performed entirely by the University of Hawaii research collaborators. The UH team was able to monitor boiler efficiency and analyze combustion gas concentrations with their portable test equipment and were able to provide valuable insights where inefficiencies existed in boiler operations. During the investigation period, an outside consultant was hired by HC\&S to address a specific boiler operating matter that was outside the scope of this study. Specific recommendations were made by the consultant for modifications to boiler operations. The observations made by the consultant were consistent with the observations made by the UH team and so it would appear that the recommendations provided by the consultant would be beneficial towards improving boiler efficiencies.

The Steam Distribution Efficiency Assessment task was performed mostly by the UH research collaborators. The application of the ASPEN modeling software to the factory process steam balance was useful in identifying steam saving opportunities. The UH team was also specific on what exactly the saving opportunities were and provided some discussion on what modifications would be required to implement these changes. The application of the ASPEN modeling software precluded the use of the OIT Steam System Scoping Tool that was originally mentioned in the Statement of Objectives for the project. Regarding the work required by HC\&S for steam savings, we relied on the work of an outside contractor to perform the missing insulation steam line survey and projected potential heat loss savings. The steam trap survey mentioned in the Statement of Objectives to be performed by HC\&S ultimately was not performed. If this task were 
performed the results would not have been presented in terms of steam savings, rather in terms of improving steam quality before going to steam turbines to perform work.

The Electric Power Distribution Assessment task was presented in the Statement of Objectives as a task that would capitalize on a power system study that had been performed by an outside consultant in 2002 to recommend appropriate relay settings for electrical system protection. The goals mentioned in the Statement of Objectives described capitalizing on this work to develop a coordinated load shedding strategy and to conduct a critical review of current flows in the electric distribution system. Where transformers were under loaded, the plan was to consolidate load, where possible, to improve efficiencies. The task as it was presented in the Statement of Objectives appeared to require outside resources to help complete yet no amount was budgeted for this work. We proceeded to perform this task using HC\&S internal capabilities only. As a result, this task focused more on replacing inefficient transformers with high no-load losses and to make power factor corrections where possible in the electrical distribution system.

Giving priority to this project activity was challenging given the pressures of conducting normal operations at HC\&S. As a result, this work could have been performed in a shorter time period had additional outside consultants and contractors been used to perform more of the required task work, similar to how University of Hawaii research collaborators were incorporated before the project commenced for the steam generation and distribution tasks. As the potential energy savings quantified in this report are substantial, it is believed that identifying and implementing more energy saving projects will become a higher priority at HC\&S.

\section{5 $\underline{\text { References }}$}

1. United States Environmental Protection Agency. 2005. Inventory of U.S. Greenhouse Gas Emissions and Sinks: 1990-2003. EPA 430-R-05-003, Washington, DC. 


\section{APPENDIX A}

\section{PSAT Results}




\section{This PSAT2004 analysis was printed at 9:12 AM on Saturday, January 07, 2006}

\section{Condition A}

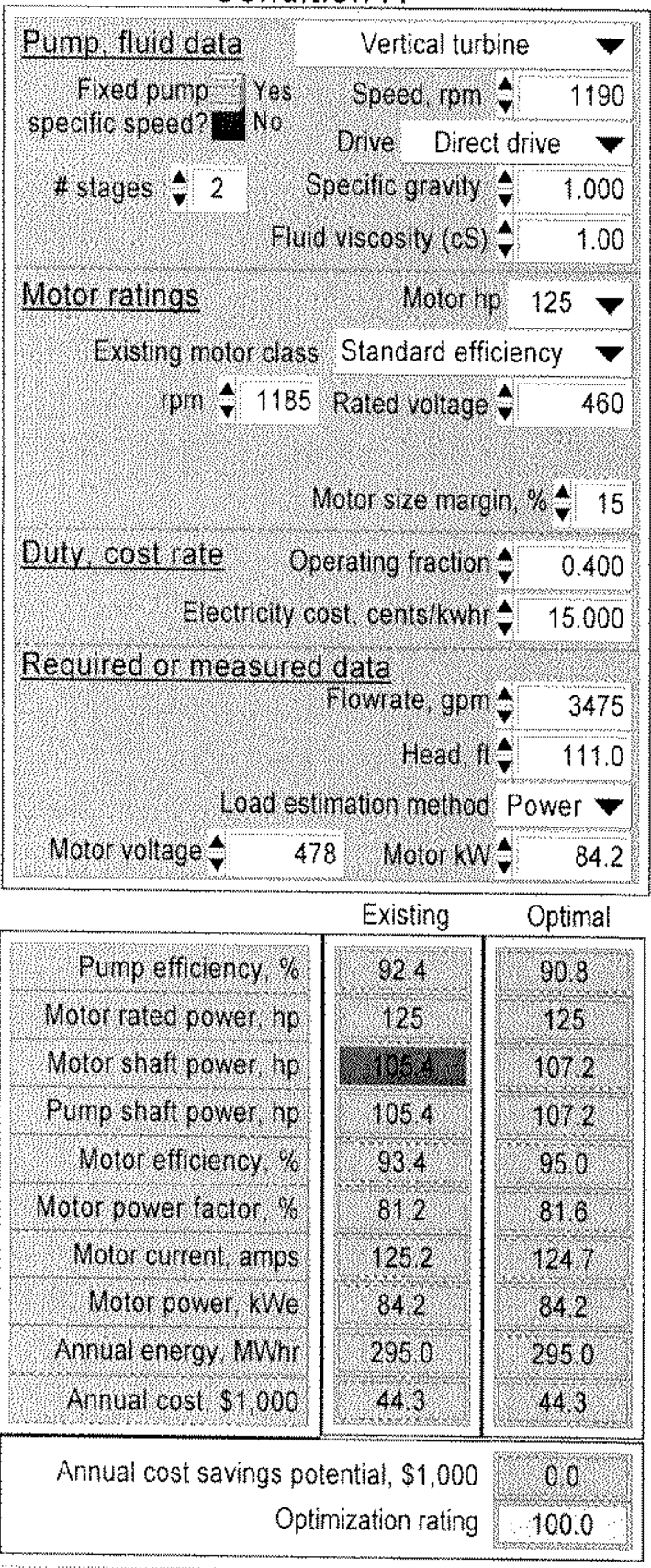

Condition B

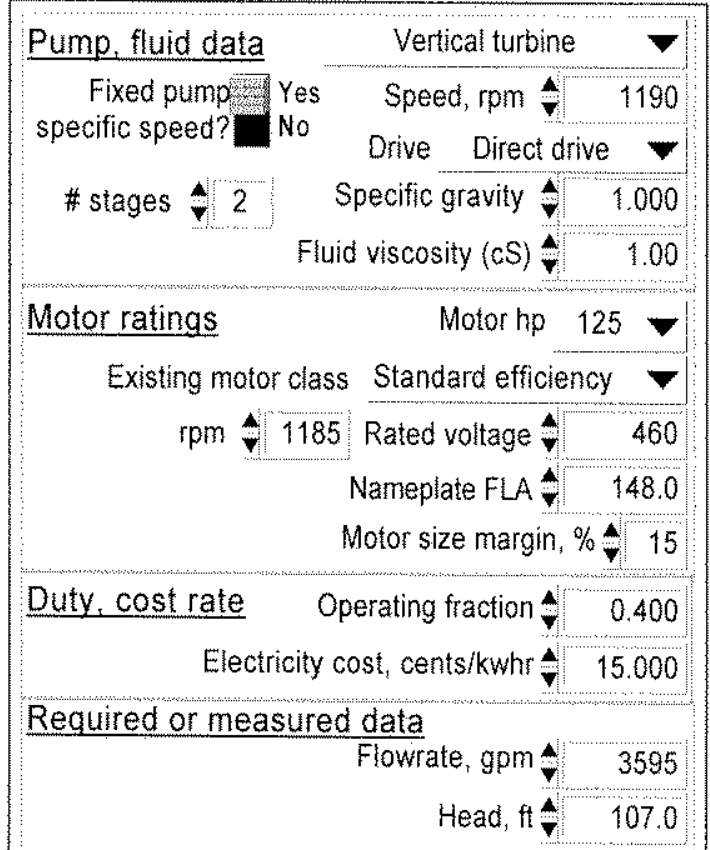

Load estimation method Current $\boldsymbol{\nabla}$

Motor voltage $\frac{4}{\mathbf{v}} \quad 478$ Motor amps $\frac{4}{\mathbf{v}} \quad 138.0$

\begin{tabular}{|c|c|c|}
\hline & Existing & Optimal \\
\hline Pump efficiency, $\%$ & 80.4 & 90.7 \\
\hline Motor rated power, hp & 125 & 125 \\
\hline Motor shaft power, hp & 120.9 & 107.1 \\
\hline Pump shaft power, hp & 120.9 & 107.1 \\
\hline Motor efficiency, $\%$ & 93.4 & 95.0 \\
\hline Motor power factor, \% & 84.5 & 81.5 \\
\hline Motor current, amps & 138.0 & 124.5 \\
\hline Motor power, kWe & 96.5 & 84.1 \\
\hline Annual energy, MWhr & 338.2 & 294.6 \\
\hline Annual cost, $\$ 1,000$ & 50.7 & 44.2 \\
\hline
\end{tabular}

Annual cost savings potential, $\$ 1,000$ Optimization rating

\section{Condition A Notes}

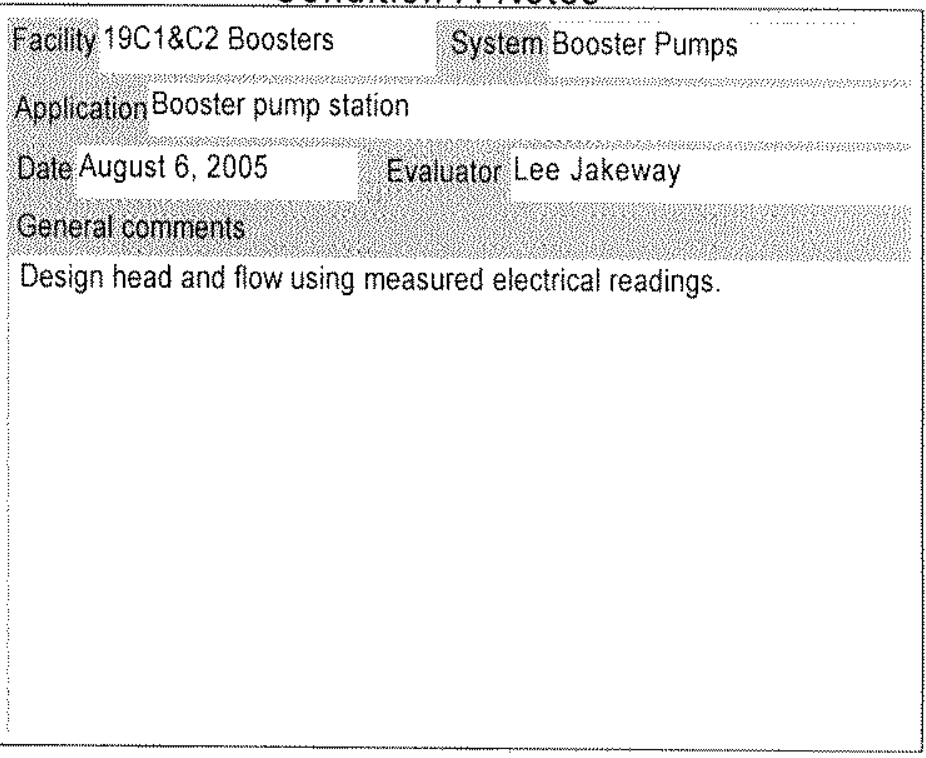

\section{Condition B Notes}

Facility 19C1\&C2 Boosters System Booster Pumps

Application Booster pump station

Date August 6, $2005 \quad$ Evaluator Lee Jakeway

General comments

Flow reading taken using transit time method and Controlotron flow meter taken on common header for $19 \mathrm{C}: \& \mathrm{C} 2$ outlet flow. Actual flow was for both pumps running, $19 \mathrm{C} 1 \& \mathrm{C} 2$. Flowrate used was half of this value. Head estimated from pump curve for measured flow. 


\section{This PSAT2004 analysis was printed at 9:17 AM on Saturday, January 07, 2006}

Condition A

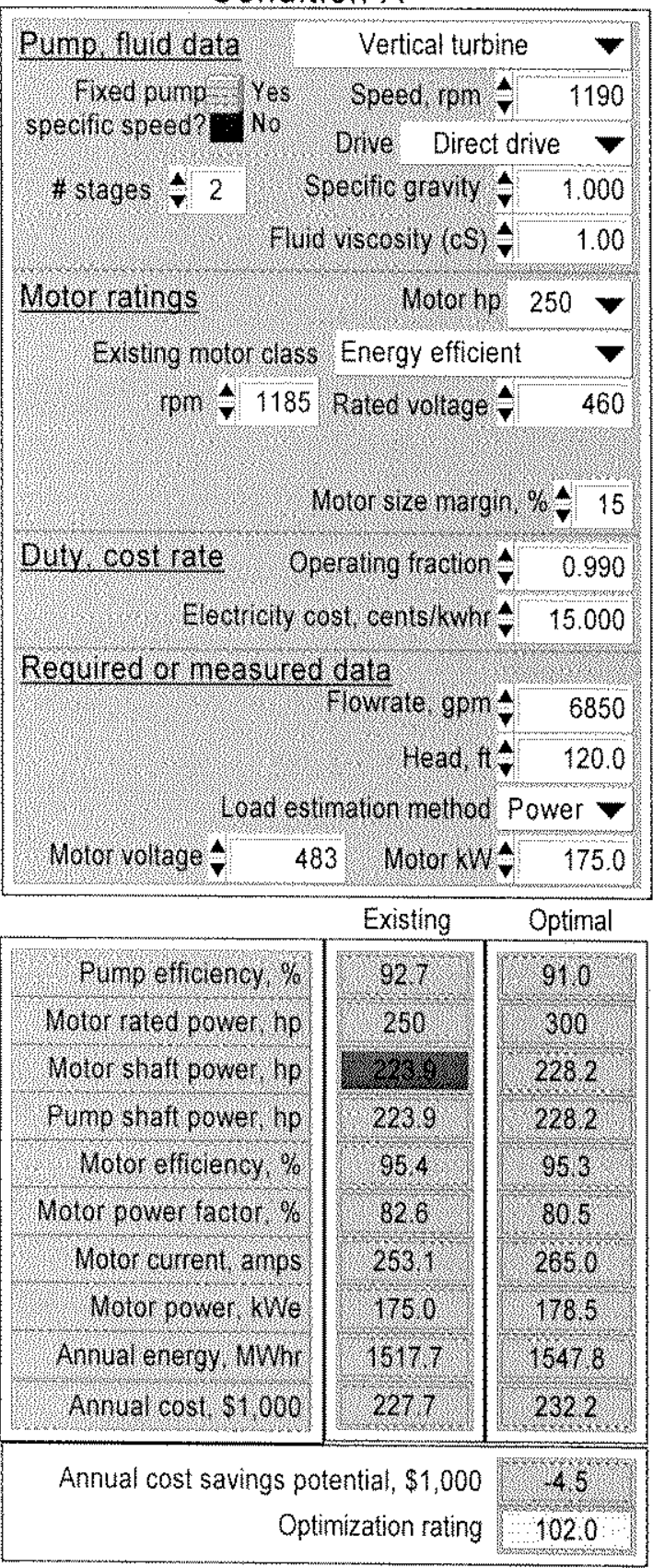

Condition B

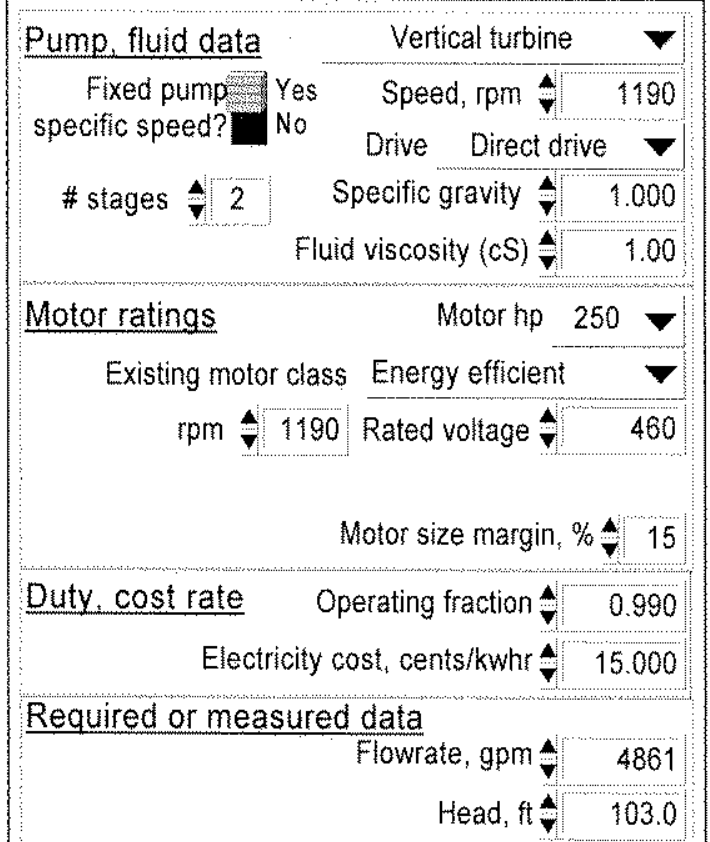

Load estimation method Power -

Motor voltage $4 \quad 487 \quad$ Motor $=20100$

\begin{tabular}{|c|c|c|}
\hline & Existing & Optimal \\
\hline Pump efficiency, $\%$ & 61.8 & 90.7 \\
\hline Motor rated power; hp & 250 & 200 \\
\hline Motor shaft power, hp & 204.5 & 139.3 \\
\hline Pump shaft power, hp & 204.5 & 139.3 \\
\hline Motor efficiency, $\%$ & 95.3 & 95.0 \\
\hline Motor power factor, \% & 81.2 & 77.9 \\
\hline Motor current, amps & 233.7 & 166.4 \\
\hline Motor power, kWe & 160.0 & 109.3 \\
\hline Annual energy, MWhr & 1387.6 & 947.9 \\
\hline Annual cost, $\$ 1,000$ & 208.1 & 142.2 \\
\hline \multicolumn{2}{|c|}{ Annual cost savings potential, $\$ 1,000$} & 65.9 \\
\hline \multicolumn{2}{|c|}{ Optimization rating } & 68.3 \\
\hline
\end{tabular}

\section{Condition A Notes}

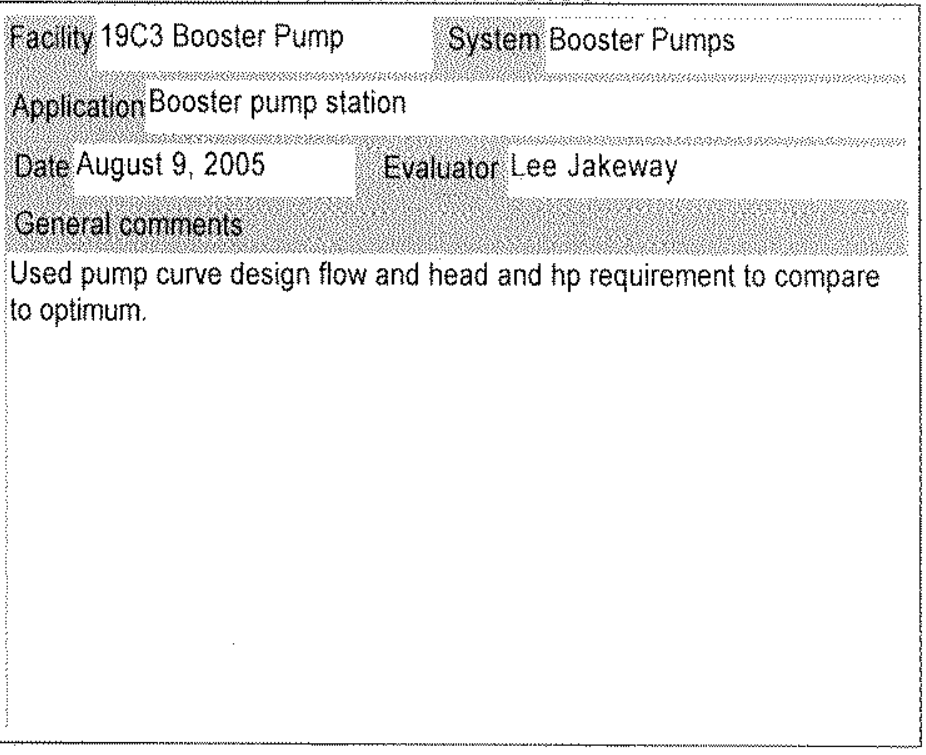

\section{Condition B Notes}

Facility $19 \mathrm{C} 3$ Booster Pump System Booster Pumps

Application Booster pump station

Date August 9, $2005 \quad$ Evaluator Lee Jakeway

General comments

Used estimate of 7 MGD for flow based on opearations accounting.

Loss coefficienct used for check valve and gate valve combination.

Pump leakage noticed when measured. 


\section{This PSAT2004 analysis was printed at 9:19 AM on Saturday, January 07, 2006}

Condition A

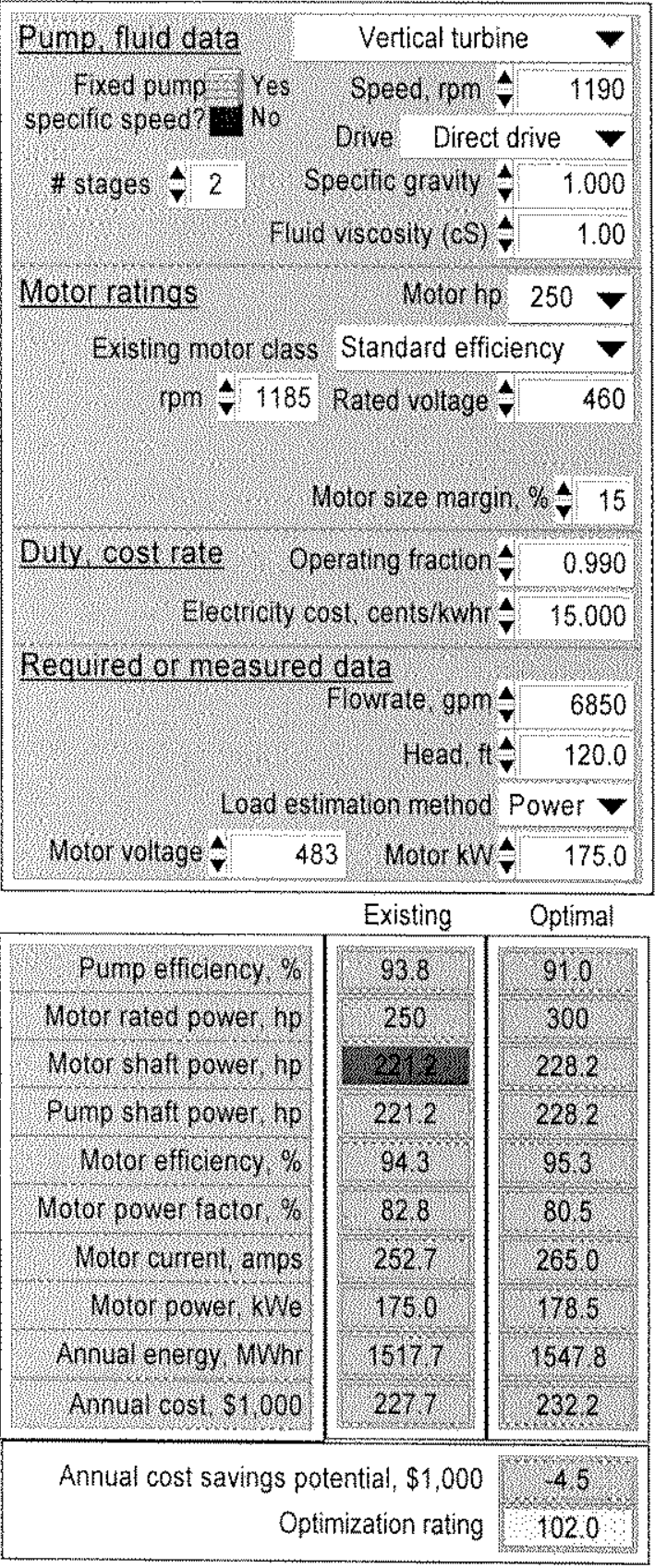

Condition B

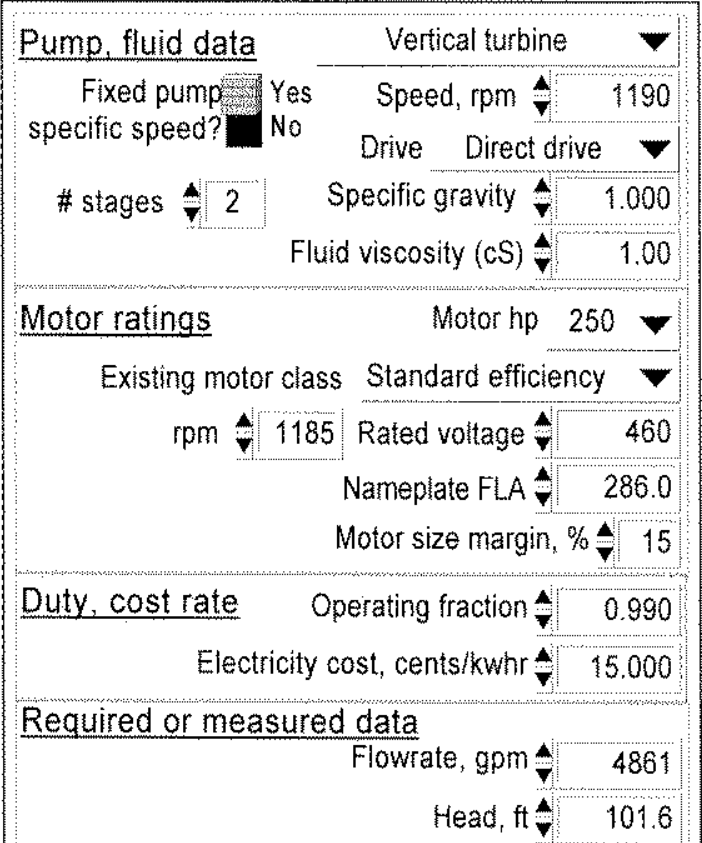

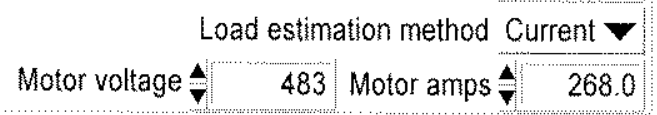

\begin{tabular}{|c|c|c|}
\hline & Existing & Optimal \\
\hline Pump efficiency $\%$ & 50.7 & 90.7 \\
\hline Motor rated power, hp & 250 & 200 \\
\hline Motor shaft power, hp & 246.1 & 137.5 \\
\hline Pump shaft power, hp & 246.1 & 137.5 \\
\hline Motor efficiency, $\%$ & 94.2 & 95.0 \\
\hline Motor power factor, $\%$ & 86.9 & 78.0 \\
\hline Motor current, amps & 268.0 & 165.4 \\
\hline Motor power, kWe & 194.8 & 107.9 \\
\hline Annual energy, MWhr & 1689.3 & 935.8 \\
\hline Annual cost, $\$ 1,000$ & 253.4 & 140.4 \\
\hline
\end{tabular}

Annual cost savings potential, $\$ 1,000$ Optimization rating

\section{Condition A Notes}

Facily 19C4 Booster Pump System Booster Pumps

Application Booster pump station

Date August 9,2005 Evaluator Lee Jakeway

General comnents

Used pump chart design flow and head and hp requirement to compare to optimum.

\section{Condition B Notes}

Facility 19C4 Booster Pump System Booster Pumps

Application Booster pump station

Date August 9,2005 Evaluator Lee Jakeway

General comments

Used estimate of 7 MGD for flow based on opearations accounting.

Loss coefficienct used for check valve and gate valve combination.

Pump leakage and noisy operation when measured. 
This PSAT2004 analysis was printed at 9:41 AM on Saturday, January 07, 2006

Condition A

\begin{tabular}{|c|c|c|}
\hline Pump, fuld data & End suction ANS & SIIAPI $\nabla$ \\
\hline Fred punp y yes & Speed rom & 1180 \\
\hline specilo speed int & Drve Direct & drive \\
\hline trstages: 1 & pecitic gravily & 1.000 \\
\hline 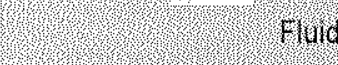 & viscosity $(\cos )$ & 1.00 \\
\hline Motor ratings & Notor ho & $450 \nabla$ \\
\hline Existing notor class & Standard effici & iency $\nabla$ \\
\hline rom 41180 & Rated voltage & 2300 \\
\hline & Nanepiare F( A & 1020 \\
\hline & Moror size nargin & 2. \\
\hline Duty. cost rate & rerating ractions & 0.230 \\
\hline Electricity o & ost ceris kwhir & 15.000 \\
\hline Required or neasured & data & \\
\hline & Gloware, gons & 7000 \\
\hline & read is & 192.0 \\
\hline (load est & mation nethod & Current $\nabla$ \\
\hline Moror voltage & 50 Motor anps s & 910 \\
\hline & Existing & Optimal \\
\hline Punp efficiency. \% & 83.6 & 890 \\
\hline Motor raved power ho & 450 & 450 \\
\hline Molor shatt power hp & 縟 & 2774 \\
\hline Pump shat power hp & 406.1 & 3774 \\
\hline Nolor etriclency. $6 \%$ & 947 & 957 \\
\hline Molor power facror, or & 86.3 & 835 \\
\hline Notor current, amps & 910 & 86.6 \\
\hline Nowor power , we & 3198 & 2942 \\
\hline Annual energy whri & 6448 & 6027 \\
\hline Annual cost 51000 & 96.6 & 889 \\
\hline Annual cost savings & tential, $\$ 1,000$ & 7 \\
\hline & timization rating & 920 \\
\hline
\end{tabular}

Condition B

\begin{tabular}{|c|c|}
\hline & \\
\hline \multirow{3}{*}{\multicolumn{2}{|c|}{ 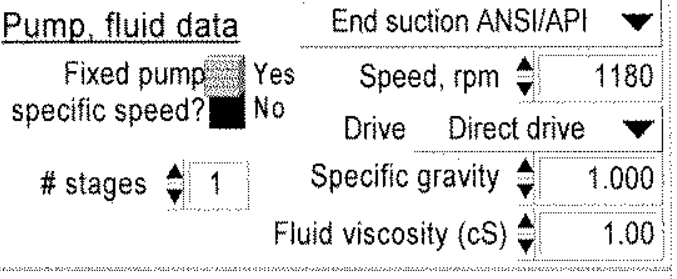 }} \\
\hline & \\
\hline & \multirow[t]{2}{*}{1.000} \\
\hline & \\
\hline \multirow{5}{*}{ ng mo } & 450 \\
\hline & iciency \\
\hline & 2300 \\
\hline & 102.6 \\
\hline & $\forall 15$ \\
\hline \\
\hline & \\
\hline \\
\hline & \\
\hline \\
\hline
\end{tabular}

Load estimation method Current -

Motor voltage $\mathbb{*} \quad 2350$ Motor amps $* \quad 91.0$

\begin{tabular}{|c|c|c|}
\hline & Existing & Optimal \\
\hline Pump efficiency $\%$ & 65.0 & 89.4 \\
\hline Motor rated power, $h p$ & 450 & 350 \\
\hline Motor shaft power, ho & 406.1 & 295.3 \\
\hline Pump shaft power, hp & 406.1 & 295.3 \\
\hline Motor efficiency, \% & 94.7 & 95.5 \\
\hline Motor power factor, $\%$ & 86.3 & 83.0 \\
\hline Motor current, amps & 91.0 & 68.2 \\
\hline Motor power, kWe & 319.8 & 230.5 \\
\hline Annual energy, MWhr & 644.3 & 464.3 \\
\hline Annual cost, $\$ 1,000$ & 96.6 & 69.7 \\
\hline \multicolumn{2}{|c|}{ Annual cost savings potential, $\$ 1,000$} & 27.0 \\
\hline \multicolumn{2}{|c|}{ Optimization rating } & 72.1 \\
\hline
\end{tabular}

\section{Condition A Notes}

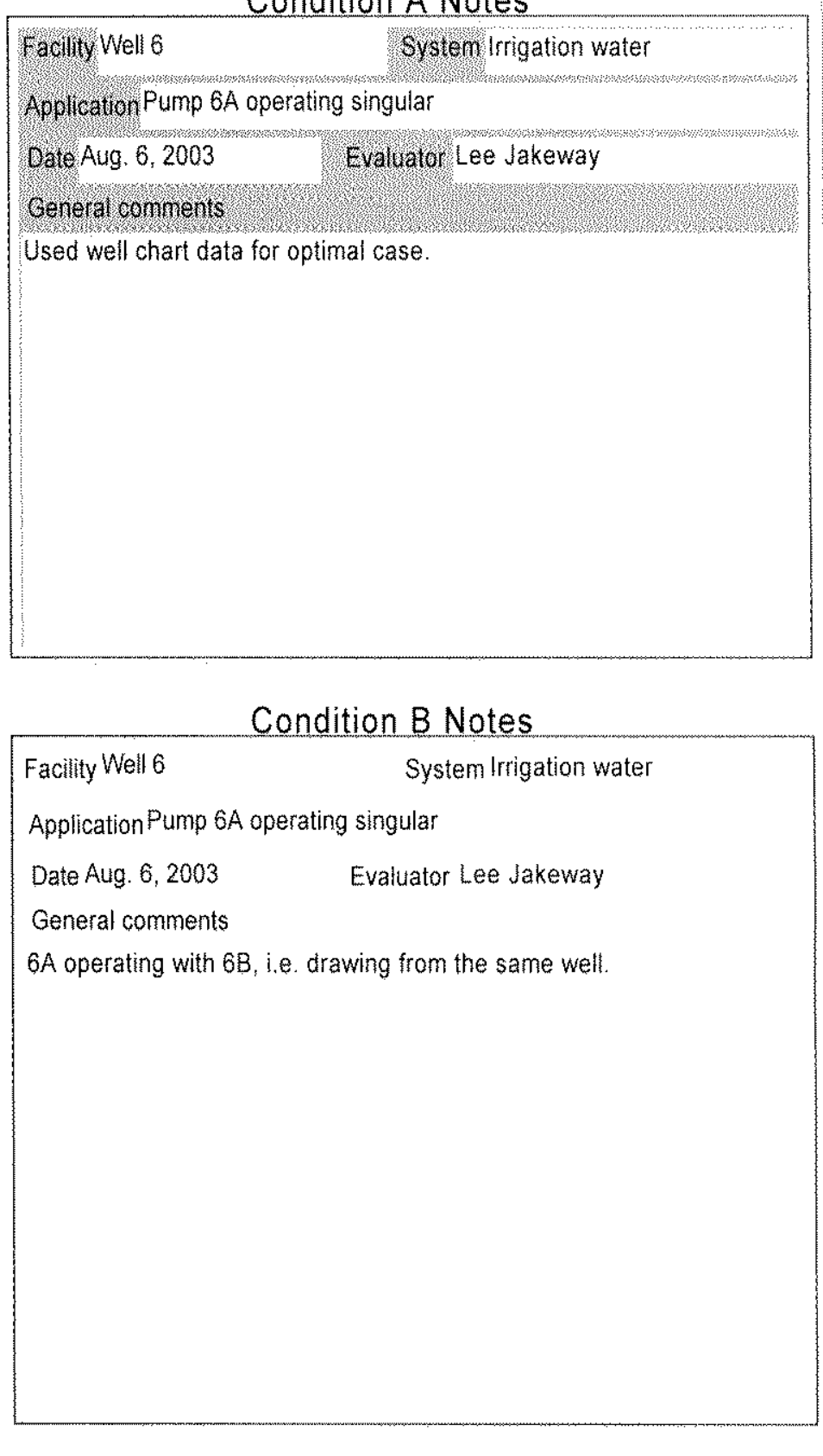


This PSAT2004 analysis was printed at 9:46 AM on Saturday, January 07, 2006

Condition A

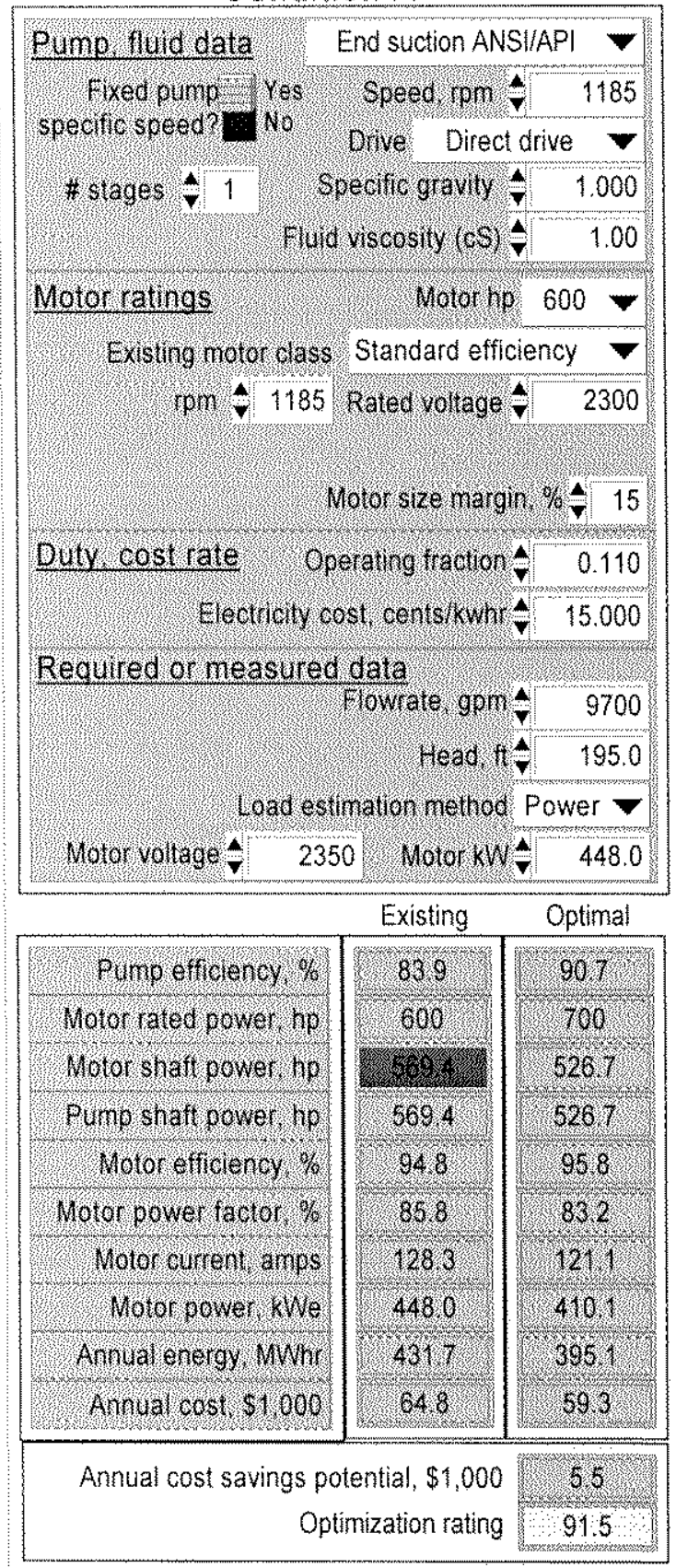

Condition B

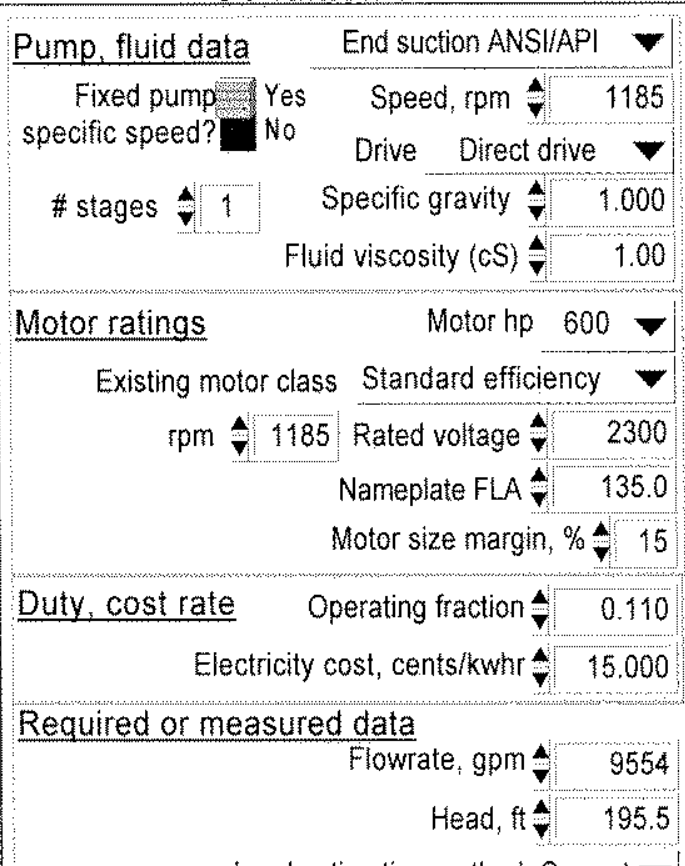

Load estimation method Current $\boldsymbol{\nabla}$

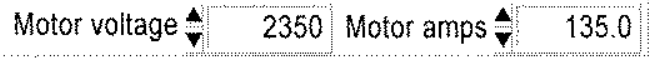

\begin{tabular}{|c|c|c|}
\hline & Existing & Optimal \\
\hline Pump efficiency, $\%$ & 76.7 & 90.7 \\
\hline Motor rated power, hp & 600 & 600 \\
\hline Motor shaft power, hp & 614.6 & 520.3 \\
\hline Pump shaft power, hp & 614.6 & 520.3 \\
\hline Motor efficiency, $\%$ & 94.7 & 95.8 \\
\hline Motor power factor, \% & 88.1 & 84.5 \\
\hline Motor current, amps & 135.0 & 117.7 \\
\hline Motor power, kWe & 484.3 & 404.9 \\
\hline Annual energy, MWhr & 466.7 & 390.1 \\
\hline Annual cost, $\$ 1,000$ & 70.0 & 58.5 \\
\hline \multicolumn{2}{|c|}{ Annual cost savings potential, $\$ 1,000$} & 11.5 \\
\hline \multicolumn{2}{|c|}{ Optimization rating } & 83.6 \\
\hline
\end{tabular}

\section{Condition A Notes}

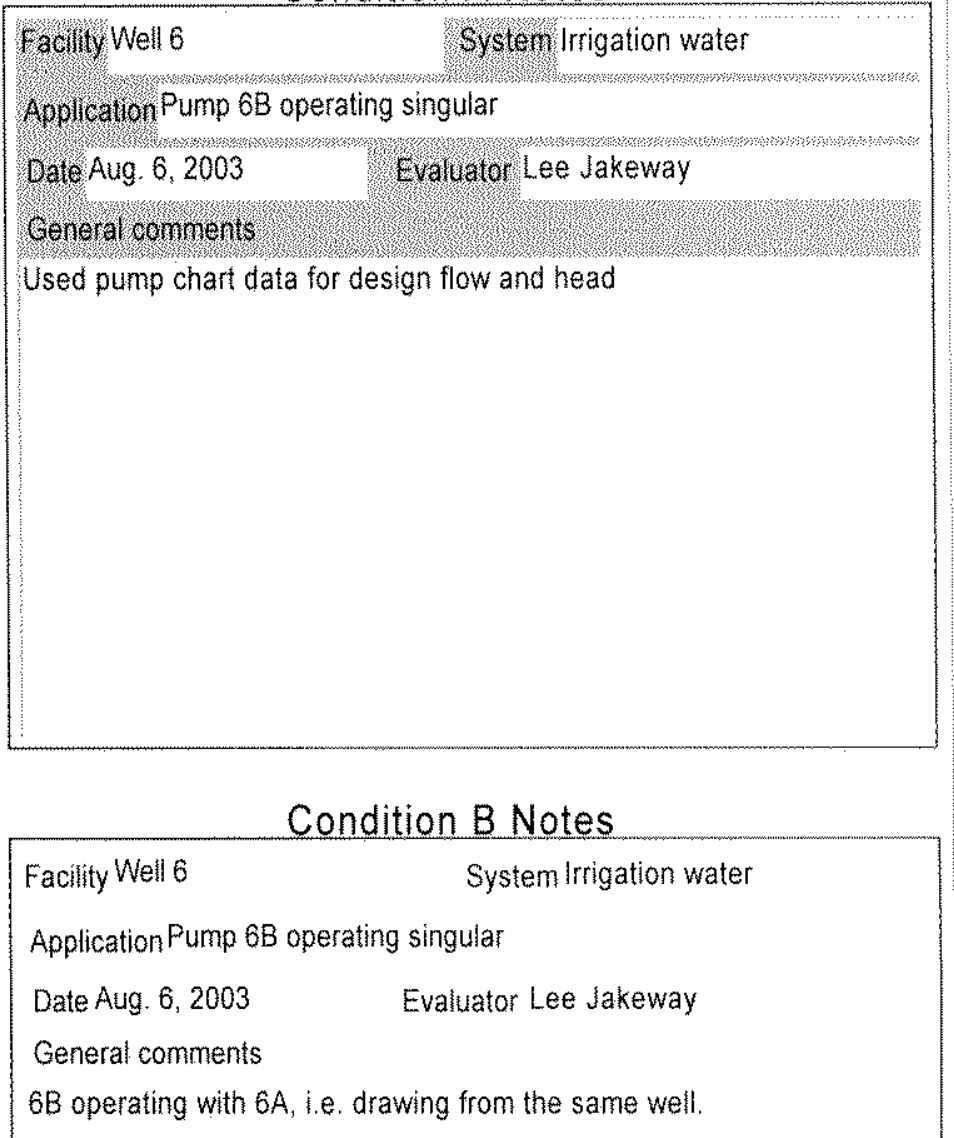

Date Aug. 6, 2003 Evaluator Lee Jakeway

Ceneral comments

Used pump chart data for design flow and head

$6 \mathrm{~B}$ operating with $6 \mathrm{~A}$, i.e. drawing from the same well 


\section{This PSAT2004 analysis was printed at 9:48 AM on Saturday, January 07, 2006}

\section{Condition A}

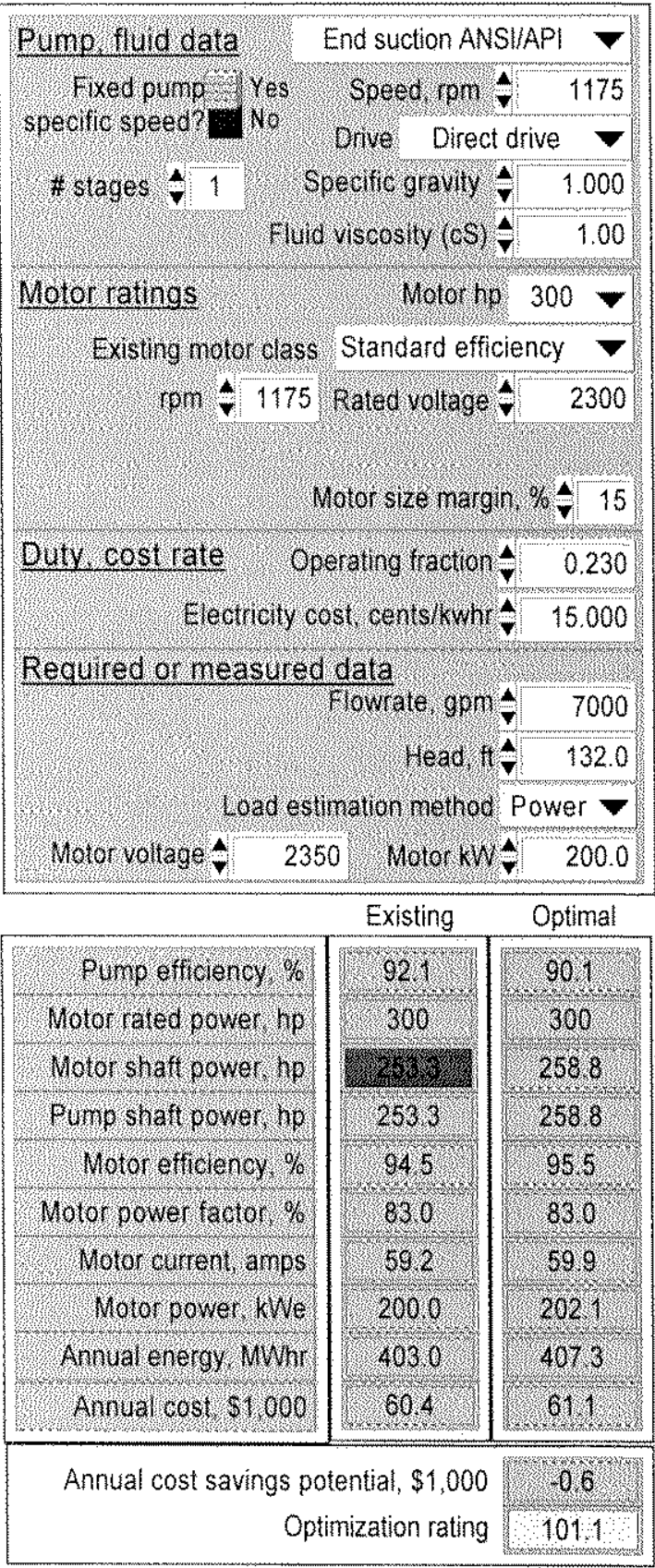

\section{Condition B}

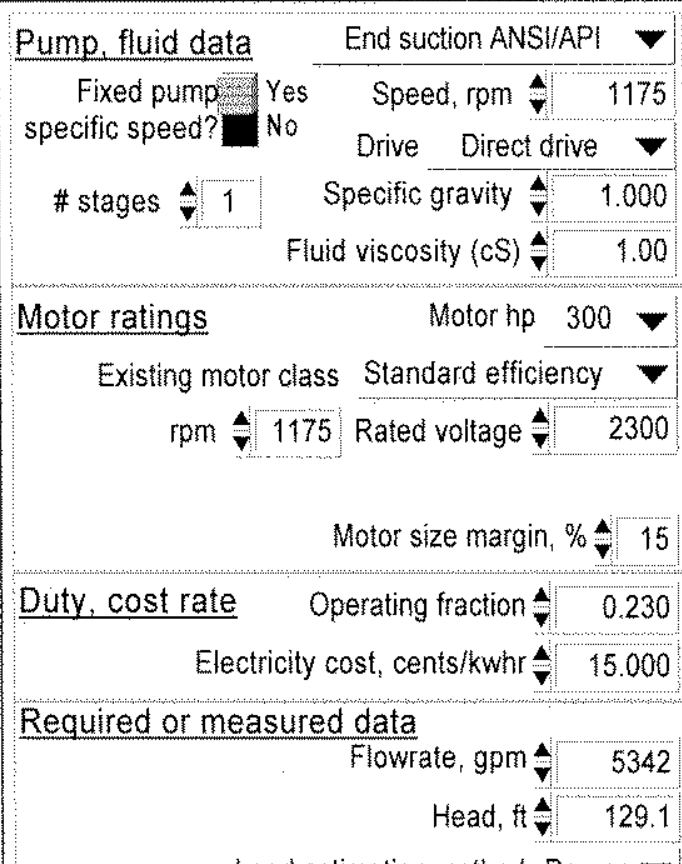

Load estimation method Power $\nabla$

Motor voltage $\frac{7}{7} 2350 \quad$ Motor 160.0

\begin{tabular}{|c|c|c|}
\hline & Existing & Optimal \\
\hline Pump efficiency, $\%$ & 76.5 & 89.6 \\
\hline Motor rated power, hp & 300 & 250 \\
\hline Motor shaft power, hp & 227.8 & 194.3 \\
\hline Pump shaft power, hp & 227.8 & 194.3 \\
\hline Motor efficiency, \% & 94.4 & 95.3 \\
\hline Motor power factor, $\%$ & 81.7 & 81.5 \\
\hline Motor current, amps & 54.1 & 45.9 \\
\hline Motor power, kWe & 180.0 & 152.0 \\
\hline Annual energy, MWhr & 362.7 & 306.3 \\
\hline Annual cost, $\$ 1,000$ & 54.4 & 45.9 \\
\hline
\end{tabular}

Annual cost savings potential, $\$ 1,000$ Optimization rating

\section{Condition A Notes}

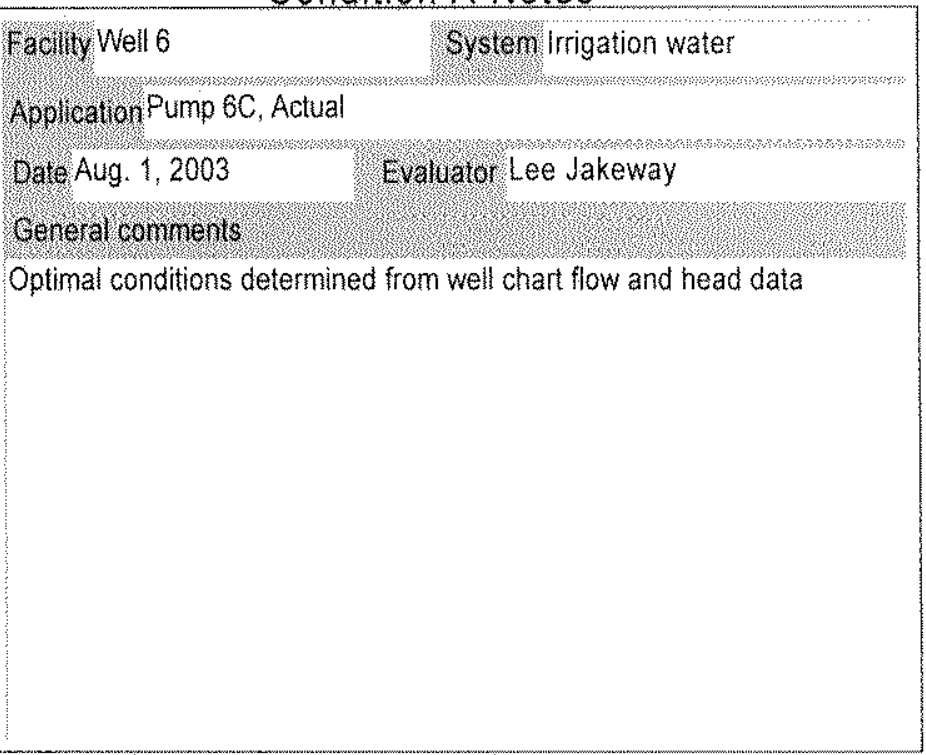

\section{Condition B Notes}

\section{Facility Well $6 \quad$ System Irrigation water}

Application Pump 6C, Actual

Date Aug. 1, $2003 \quad$ Evaluator Lee Jakeway

General comments

$6 \mathrm{C}$ booster pump operating with $6 \mathrm{~A}$. Motor nameplate data not very clear so approximating this informatio based on pump report data. Also, using

board reading power for load estimation method 


\section{This PSAT2004 analysis was printed at 9:49 AM on Saturday, January 07, 2006}

Condition A

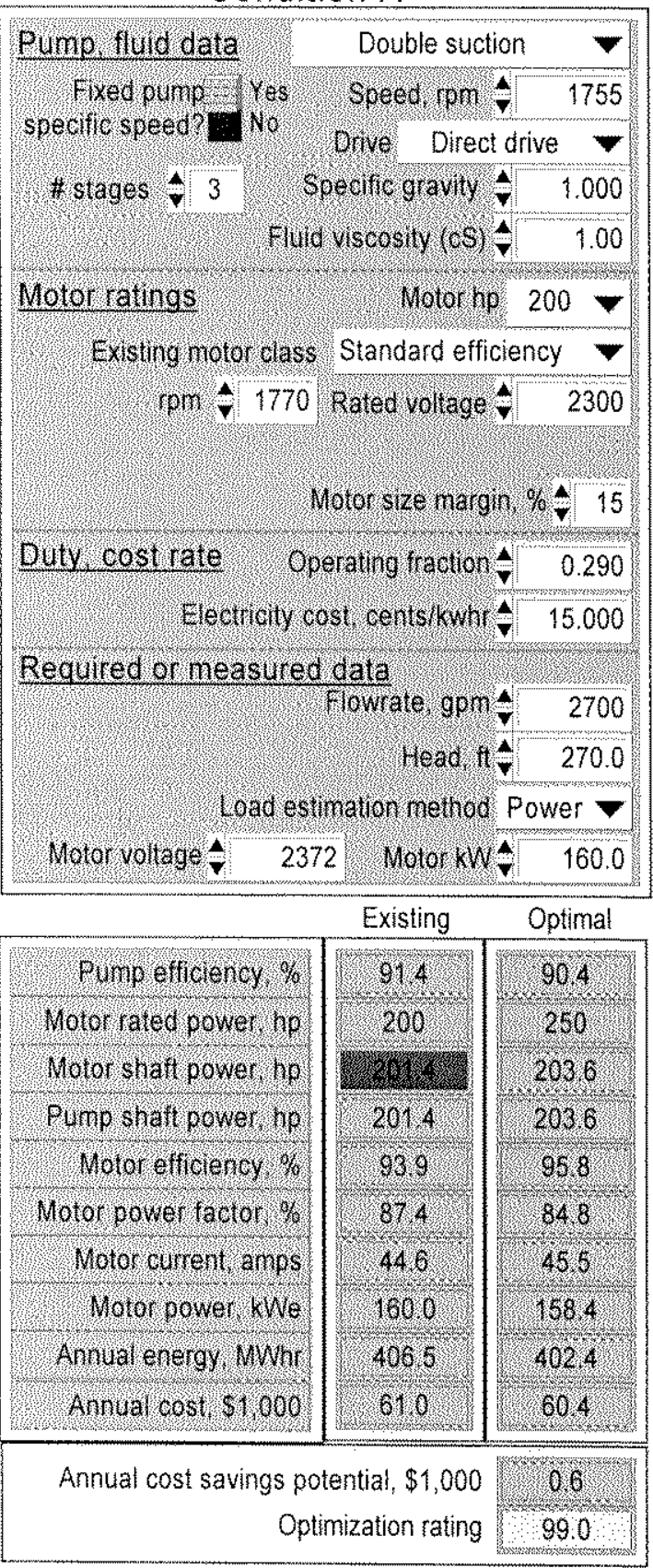

Condition B

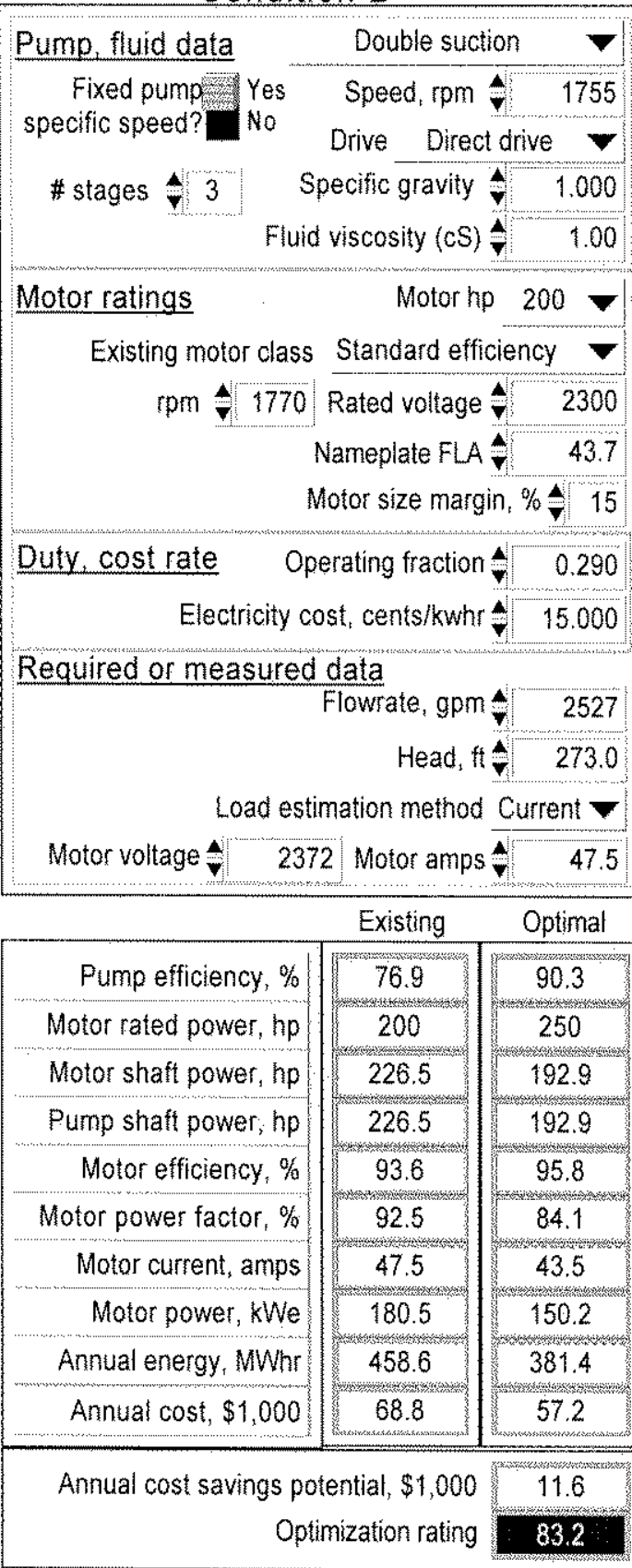

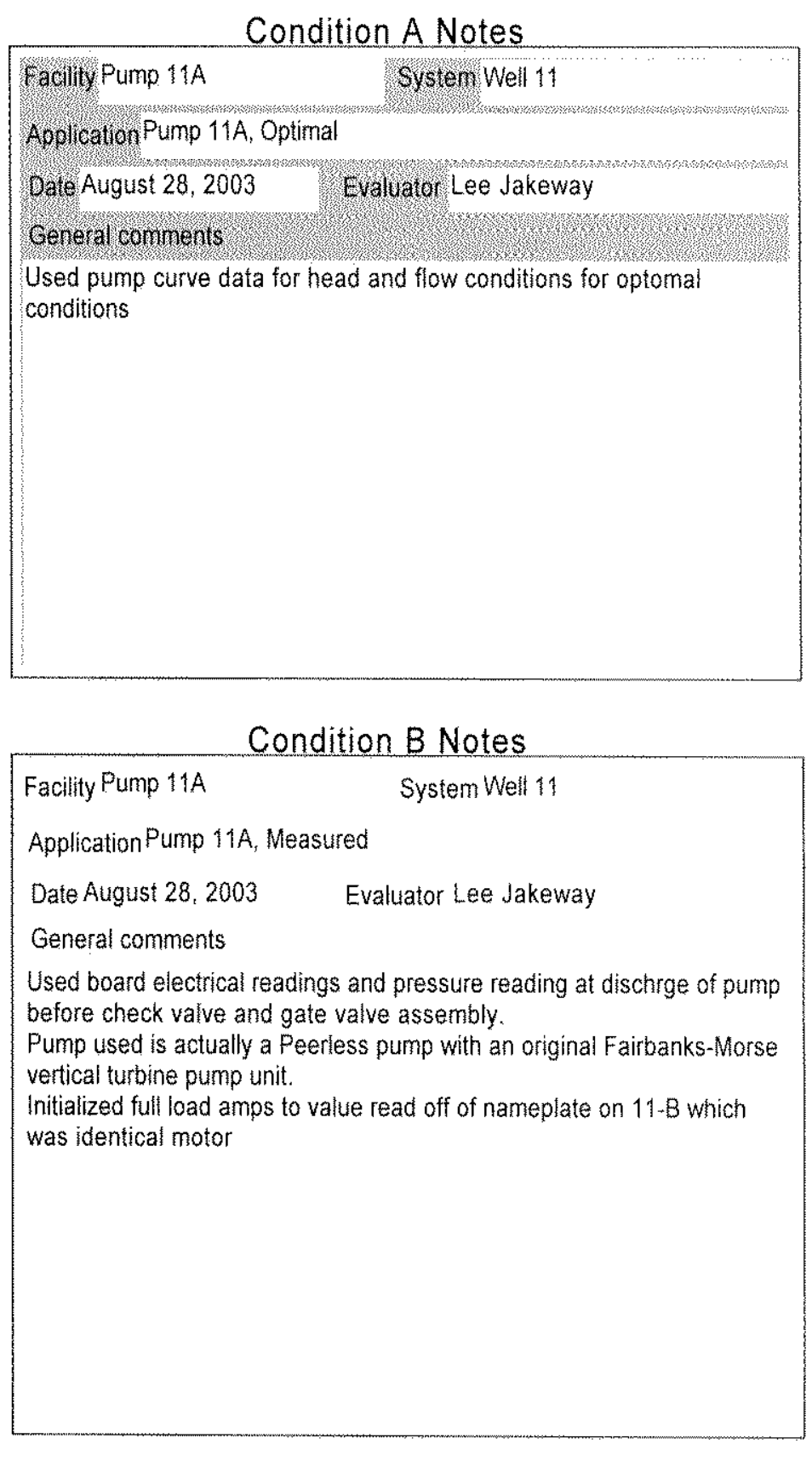


This PSAT2004 analysis was printed at 9:54 AM on Saturday, January 07, 2006

\section{Condition A}

\begin{tabular}{|c|c|}
\hline End suction ANSI/API $\mathbf{v}$ & \\
\hline Fixed pump= Yes & \\
\hline Drive Direct drive $\nabla$ & \\
\hline Specific gravity $\boldsymbol{\|} 1.000$ & \\
\hline Fluid viscosity $(c S) \div$ & \\
\hline Motor ratings & \\
\hline Existing motor class Standard efficiency $\vec{\nabla}$ & \\
\hline$p_{n} \geqslant 1200$ Rated voltage $\mathbf{T} 2300$ & \\
\hline Motor size margin. $\% \div 15$ & \\
\hline Operating raction $\hat{\boldsymbol{T}}$ & \\
\hline Electricity cost centsikwhr $\boldsymbol{v}$ & \\
\hline Required or measured data & \\
\hline flowrate gpmi & \\
\hline Head $1 \div$ & \\
\hline Load estination method Power $\boldsymbol{\nabla}$ & \\
\hline 2477 Motor kW & \\
\hline
\end{tabular}

\begin{tabular}{|c|c|c|}
\hline & Existing & Optimal \\
\hline funp efficiency of & 896 & 904 \\
\hline Motor rated power hp & 800 & 900 \\
\hline Molor shatt power, ho & Y. & 7558 \\
\hline Pump shat power hp & 7636 & $7558^{\circ}$ \\
\hline Molor efficiency, \% & 949 & 960 \\
\hline Molor power facror, 0 \% & 865 & 83.8 \\
\hline Motor current, anps & 1617 & 163,3 \\
\hline Molor power kwe & 600,0 & $587 T^{2}$ \\
\hline Arnual energy, WWh' & 10512 & 10286 \\
\hline Annual cost. 51.000 & 73.6 & 720 \\
\hline
\end{tabular}

Annual cost savings potential, $\$ 1,000$

Optimization rating

\section{Condition B}

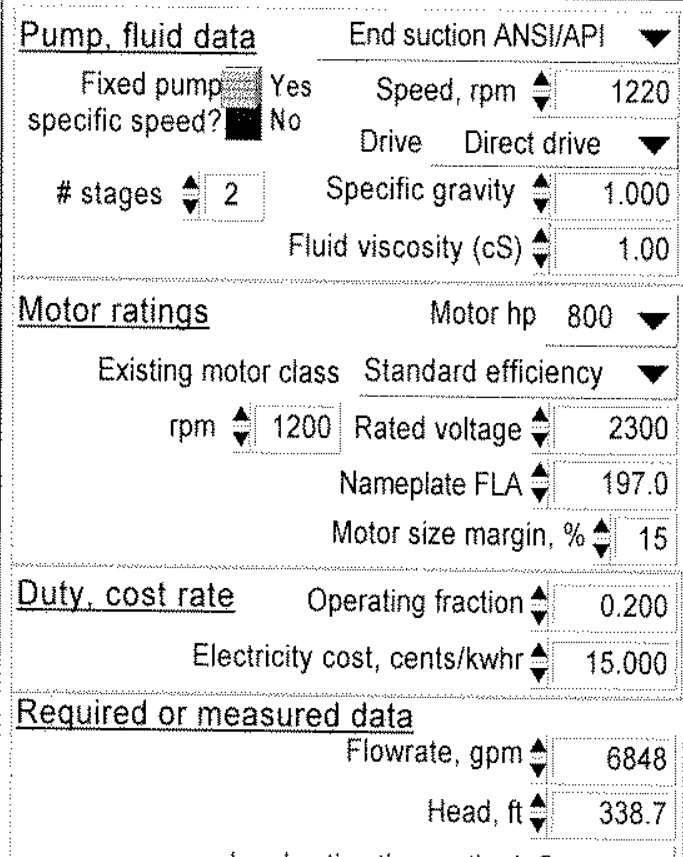

Load estimation method Current $\boldsymbol{\nabla}$

Motor voltage $\mathbf{v} \quad 2477$ Motor amps $\mathbf{v} \mid 183.0$

\begin{tabular}{|c|c|c|}
\hline & Existing & Optimal \\
\hline Pump efficiency, $\%$ & 72.6 & 90.0 \\
\hline Motor rated power, hp & 800 & 800 \\
\hline Motor shaft power, hp & 806.5 & 650.9 \\
\hline Pump shaft power, hp & 806.5 & 650.9 \\
\hline Motor efficiency, $\%$ & 94.8 & 95.9 \\
\hline Motor power factor, $\%$ & 80.8 & 83.0 \\
\hline Motor current, amps & 183.0 & 142.1 \\
\hline Motor power, kWe & 634.4 & 506.0 \\
\hline Annual energy, MWhr & 1111.5 & 886.6 \\
\hline Annual cost, $\$ 1,000$ & 166.7 & 133.0 \\
\hline
\end{tabular}

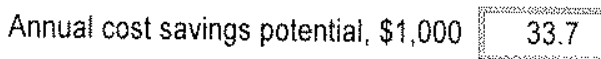
Optimization rating

\section{Condition A Notes}

Facily Pump 17 System Irigation Water

Apolication Pump 17, Optimal

Date July 18, $2002 \quad$ Evaluator Lee Jakeway

Ceneral comments

Used well chart flow and head values

\section{Condition B Notes}

\section{Facility Pump $17 \quad$ System Irrigation Water}

Application Pump 17, Measured

Date July $18,2002 \quad$ Evaluator Lee Jakeway

General comments

Pump 17 measured values, synchronous motor used here. Flow

measured by a combination of open ditch flow and pipe flow to Res.

26. because could not get flow reading on pipe in well shaft 


\section{This PSAT2004 analysis was printed at 9:56 AM on Saturday, January 07, 2006}

Condition A

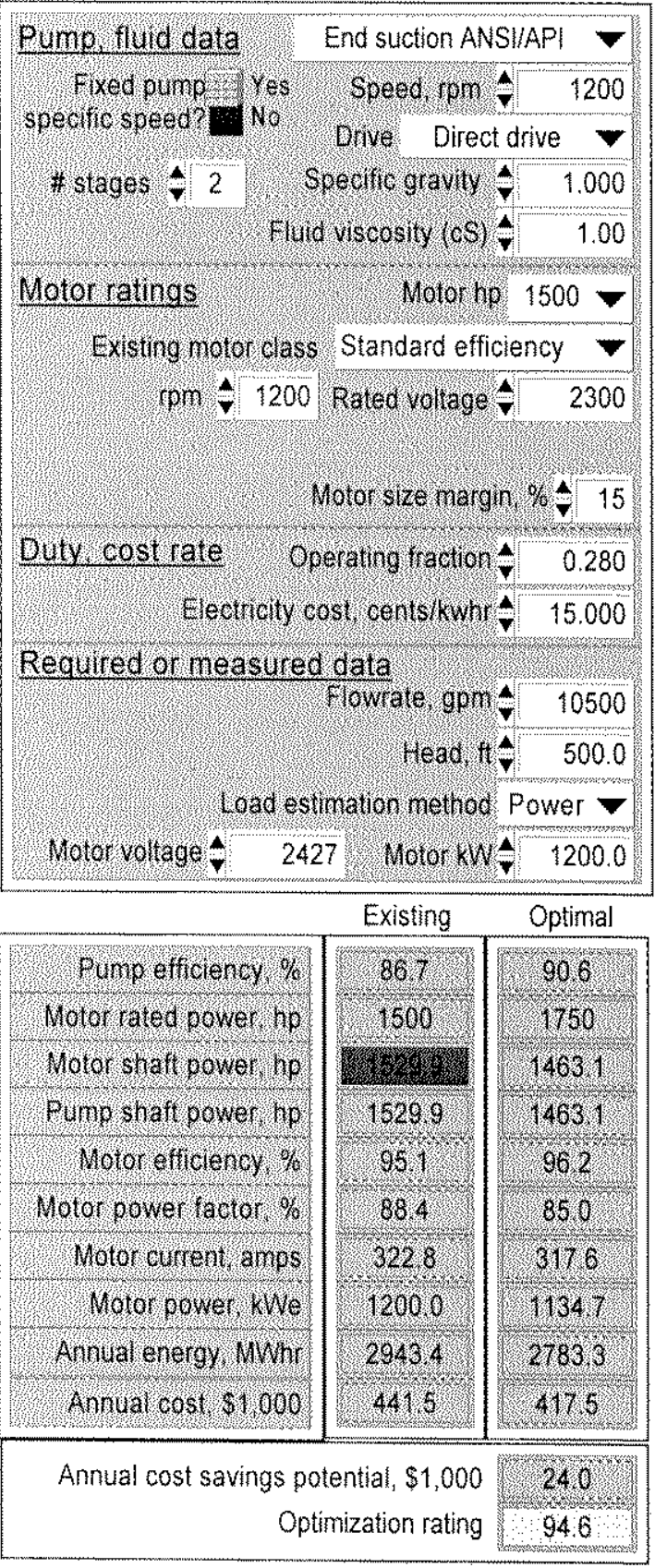

Condition B

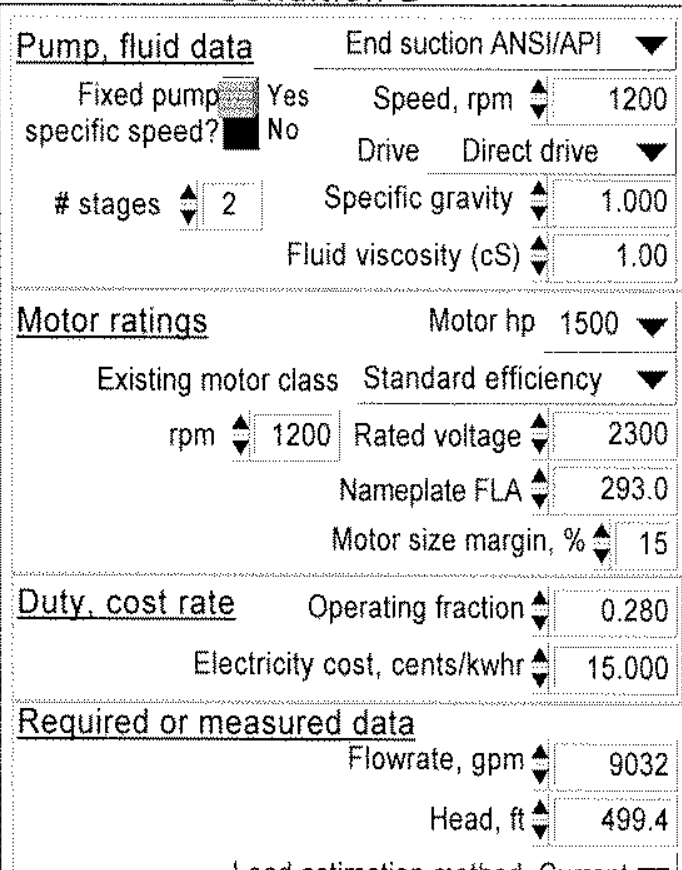

Load estimation method Current $\boldsymbol{\gamma}$

\begin{tabular}{ll|l|l|} 
Motor voltage $\sqrt{\boldsymbol{v}} 2427$ & Motor amps & $\mathbf{4}$ & 281.0 \\
\hline
\end{tabular}

\begin{tabular}{|c|c|c|}
\hline & Existing & Optimal \\
\hline Pump efficiency, $\%$ & 74.6 & 90.3 \\
\hline Motor rated power, hp & 1500 & 1500 \\
\hline Motor shaft power, hp & 1527.2 & 1261.8 \\
\hline Pump shaft power, hp & 1527.2 & 1261.8 \\
\hline Motor efficiency, $\%$ & 95.1 & 96.1 \\
\hline Motor power factor, $\%$ & 101.4 & 85.0 \\
\hline Motor current, amps & 281.0 & 274.0 \\
\hline Motor power, kWe & 1197.8 & 978.8 \\
\hline Annual energy, MWhr & 2938.0 & 2400.8 \\
\hline Annual cost, $\$ 1,000$ & 440.7 & 360.1 \\
\hline \multicolumn{2}{|c|}{ Annual cost savings potential, $\$ 1,000$} & 80.6 \\
\hline \multicolumn{2}{|c|}{ Optimization rating } & 81.7 \\
\hline
\end{tabular}

Condition A Notes

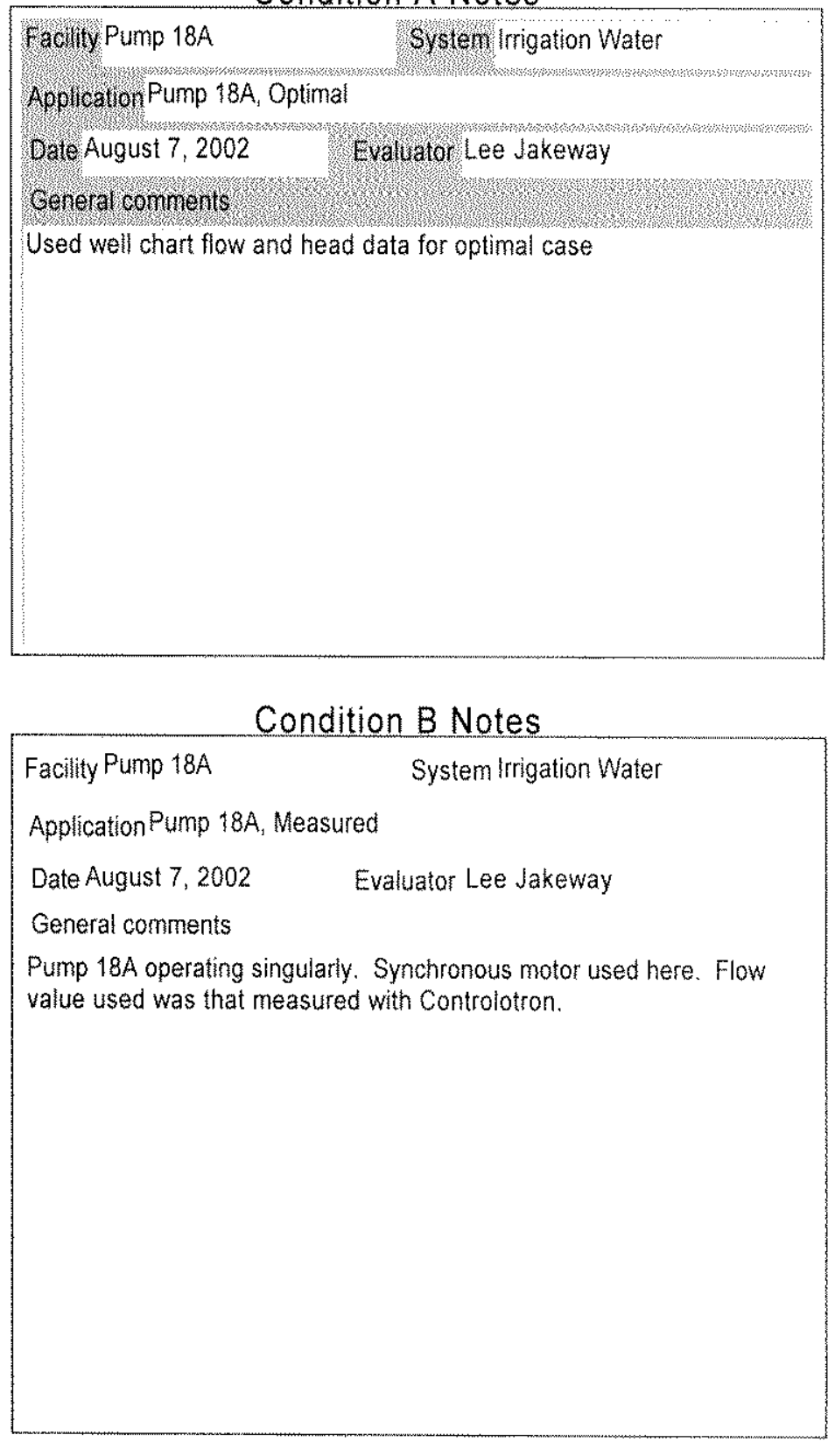




\section{This PSAT2004 analysis was printed at 9:56 AM on Saturday, January 07, 2006}

\section{Condition A}

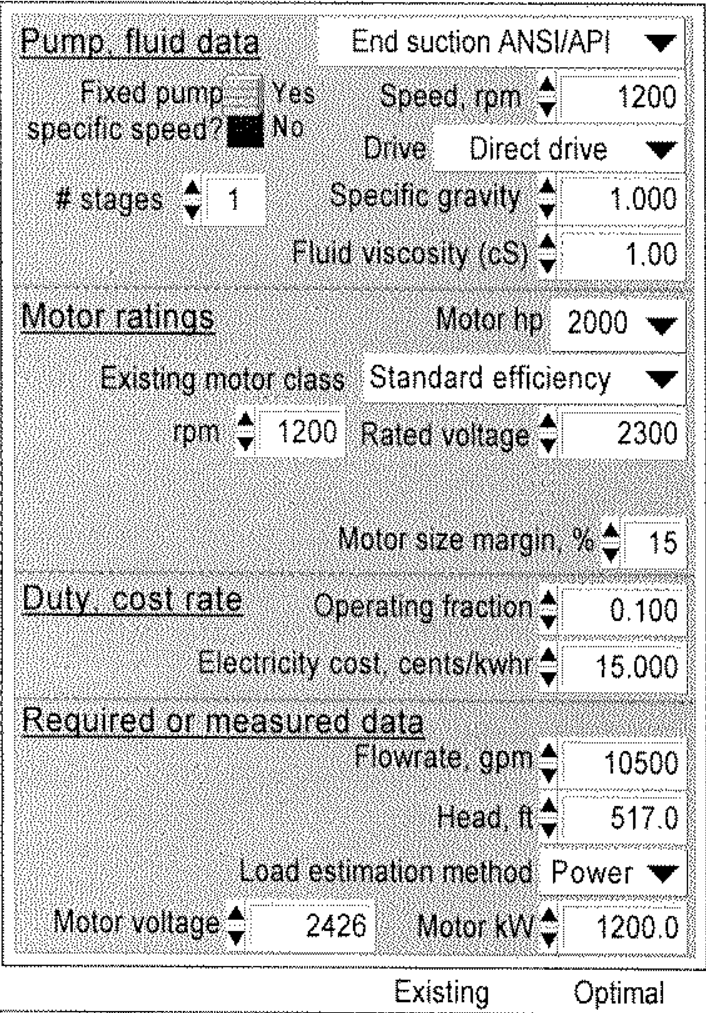

\begin{tabular}{|c|c|c|}
\hline & Existing & Optima \\
\hline Pump efficency o/ & 802 & 882 \\
\hline Molor rated power, no & 2000 & 2000 \\
\hline Motor stiatt power, he & (1) & 15542 \\
\hline pump shat power hp & 15367 & 15542 \\
\hline Motor efficiency of & 055 & 061 \\
\hline Molor power ractor, of & 6 & 839 \\
\hline Notor current amps & 841,1 & 3420 \\
\hline Nolor pover kne & 1200.0 & 1205.6 \\
\hline Annual energy MWh & 10512 & $1056 \%$ \\
\hline Annual cost 51,000 & $167 \%$ & 1584 \\
\hline
\end{tabular}

Annual cost savings potential, $\$ 1,000$ Optimization rating

\section{Condition B}

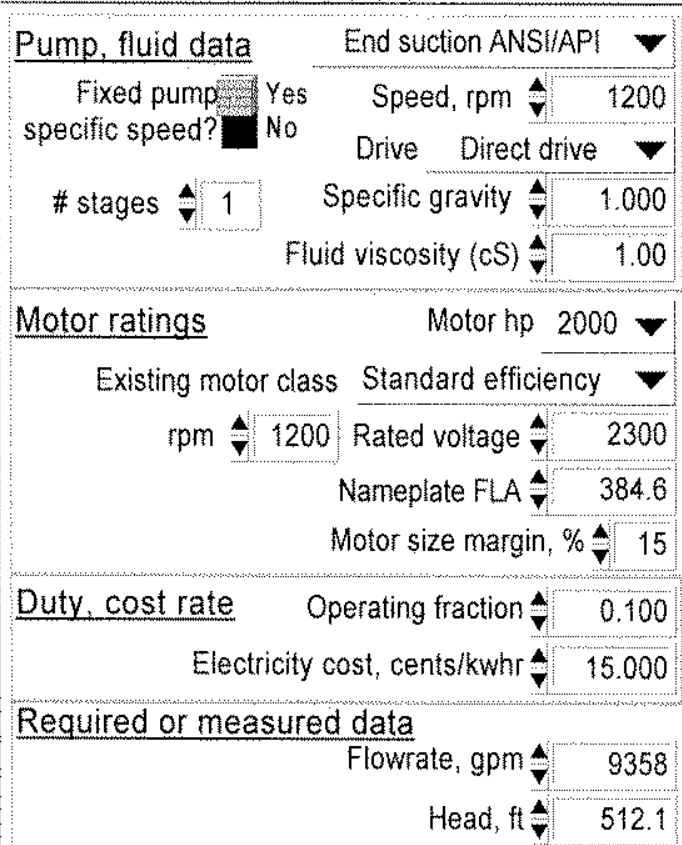

Motor voltage 42426 Motor amps 318.0

Existing Optimal

\section{Pump efficiency, $\%$}

Motor rated power, hp

Motor shaft power, hp

Pump shaft power, hp

Motor efficiency, \%

Motor power factor, \%

Motor current, amps

Motor power, kWe

Annual energy, MWhr

Annual cost, $\$ 1,000$

\begin{tabular}{|c|}
\hline 71.3 \\
\hline 2000 \\
\hline 1698.1 \\
\hline 1698.1 \\
\hline 95.5 \\
\hline 99.3 \\
\hline 318.0 \\
\hline 1326.6 \\
\hline 1162.1 \\
\hline 174.3 \\
\hline
\end{tabular}

Annual cost savings potential, $\$ 1,000$ Optimization rating

\section{Condition A Notes}

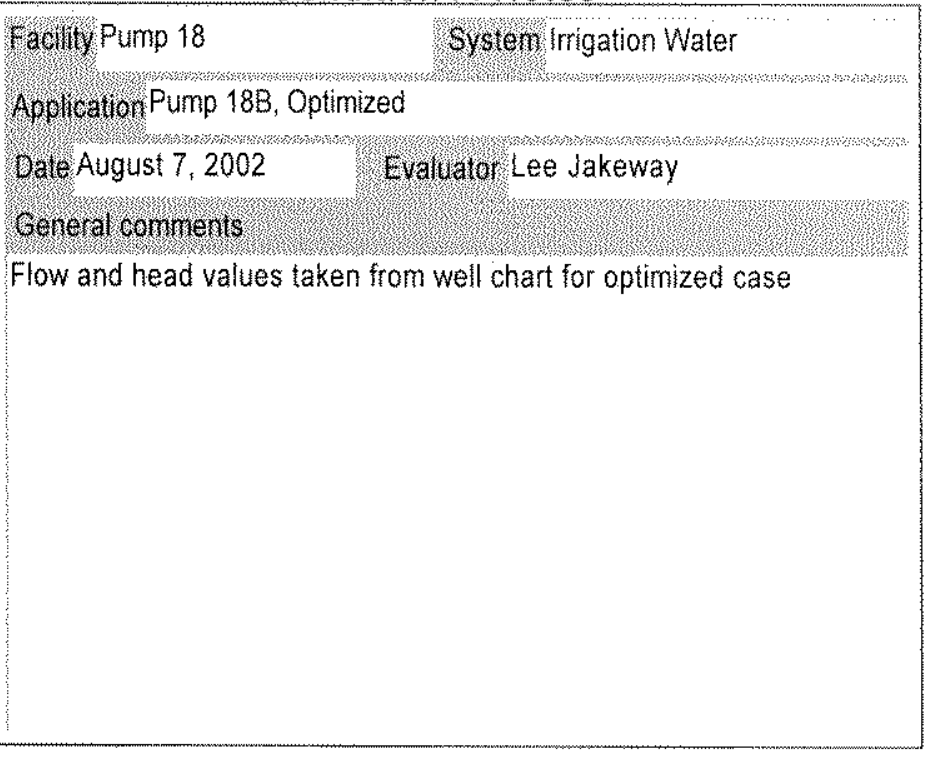

\section{Condition B Notes}

\section{Facility Pump $18 \quad$ System irrigation Wate}

Application Pump 18B, Measured

Date August 7,2002 Evaluator Lee Jakeway

General comments

Pump 18 measured values for flow and head operating singularly. Flow was measured with Panametric flowmeter, but adjusted for

Controlotron value. 


\section{This PSAT2004 analysis was printed at 10:40 AM on Saturday, January 07, 2006}

Condition A

\begin{tabular}{|c|c|}
\hline End suction ANSIIAPI $\mathbf{v}$ & \\
\hline Fixed punp $=$ Yes $\quad$ Speed rpm $\rightarrow 1200$ & \\
\hline Drive Direct drive $\nabla$ & \\
\hline Specific gravity $\mathbb{\nabla} \quad 1.000$ & \\
\hline Fud viscosity (cs) & \\
\hline Notor ho $700 \mathbf{\gamma}$ & \\
\hline Existing motor class Standard efficiency $\nabla$ & \\
\hline rpm 71200 Rated voltage $\div 2300$ & \\
\hline Motor size nargin, 7,415 & \\
\hline Operaling traction $\mid$ & \\
\hline Electricity cost, centsikwhr $\uparrow 15.000$ & \\
\hline Required or measured data & \\
\hline flowrate gpn: & \\
\hline Head $t \geqslant$ & \\
\hline Load estimation method Power $\nabla$ & \\
\hline Motor voltage 42400 Motor $\mathrm{WW}=$ & \\
\hline
\end{tabular}

\begin{tabular}{|c|c|c|}
\hline & Existing & Optimal \\
\hline Punp eficiency \% & 80.6 & 80,7 \\
\hline Motor rated power hp & 700 & 900 \\
\hline Motor shat power hp & S & 6977 \\
\hline Pump shatt power, hp & $6980^{\circ}$ & 6977 \\
\hline Notor efficiency, ga: & 94.8 & 959 \\
\hline Molor power tactor, & 868 & 83,3 \\
\hline Moior current, amps & 152,5 & 156.6 \\
\hline Motor power. We & 5500 & 542,4 \\
\hline Annual energy, Whir & 8672 & 8552 \\
\hline Annual cost, St $000^{\circ}$ & 1301 & 128.3. \\
\hline
\end{tabular}

Annual cost savings potential, $\$ 1,000$ Optimization rating
Condition B

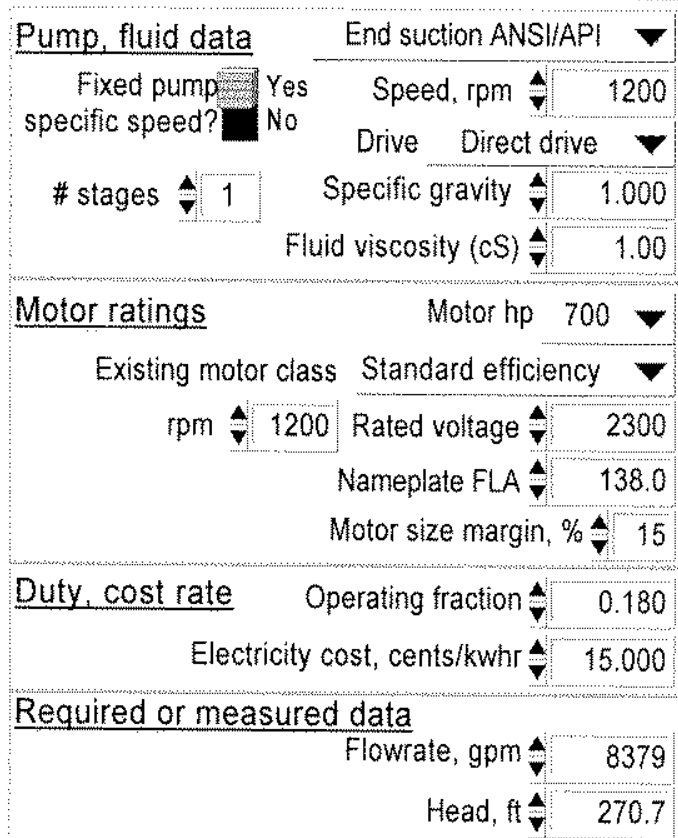

Load estimation method Current $\mathbf{-}$

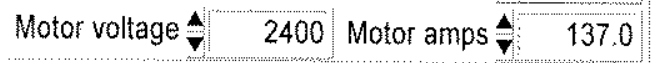

\begin{tabular}{|c|c|c|}
\hline & Existing & Optimal \\
\hline Pump efficiency, $\%$ & 78.4 & 89.9 \\
\hline Motor rated power, hp & 700 & 800 \\
\hline Motor shaft power, hp & 730.7 & 637.1 \\
\hline Pump shaft power, hp & 730.7 & 637.1 \\
\hline Motor efficiency, $\%$ & 94.7 & 95.9 \\
\hline Motor power factor, $\%$ & 101.1 & 83.5 \\
\hline Motor current, amps & 137.0 & 142.7 \\
\hline Motor power, kWe & 575.6 & 495.4 \\
\hline Annual energy, MWhr & 907.6 & 781.1 \\
\hline Annual cost, $\$ 1,000$ & 136.1 & 117.2 \\
\hline
\end{tabular}

Annual cost savings potential, $\$ 1,000$ Optimization rating
Condition A Notes

Facily Pump 16A
Application Pump 16A, measured
Date July 12,2002
General comments
Optimal conditions determined from well chart data
Evaluator Lee Jakeway
Condition B Notes
System Irrigation Water
Application Pump 16A, measured
Date July $12,2002 \quad$ Evaluator Lee Jakeway
General comments
Pump 16 A operating singularly pumping to Haiku ditch.




\section{This PSAT2004 analysis was printed at 10:41 AM on Saturday, January 07, 2006}

\section{Condition A}

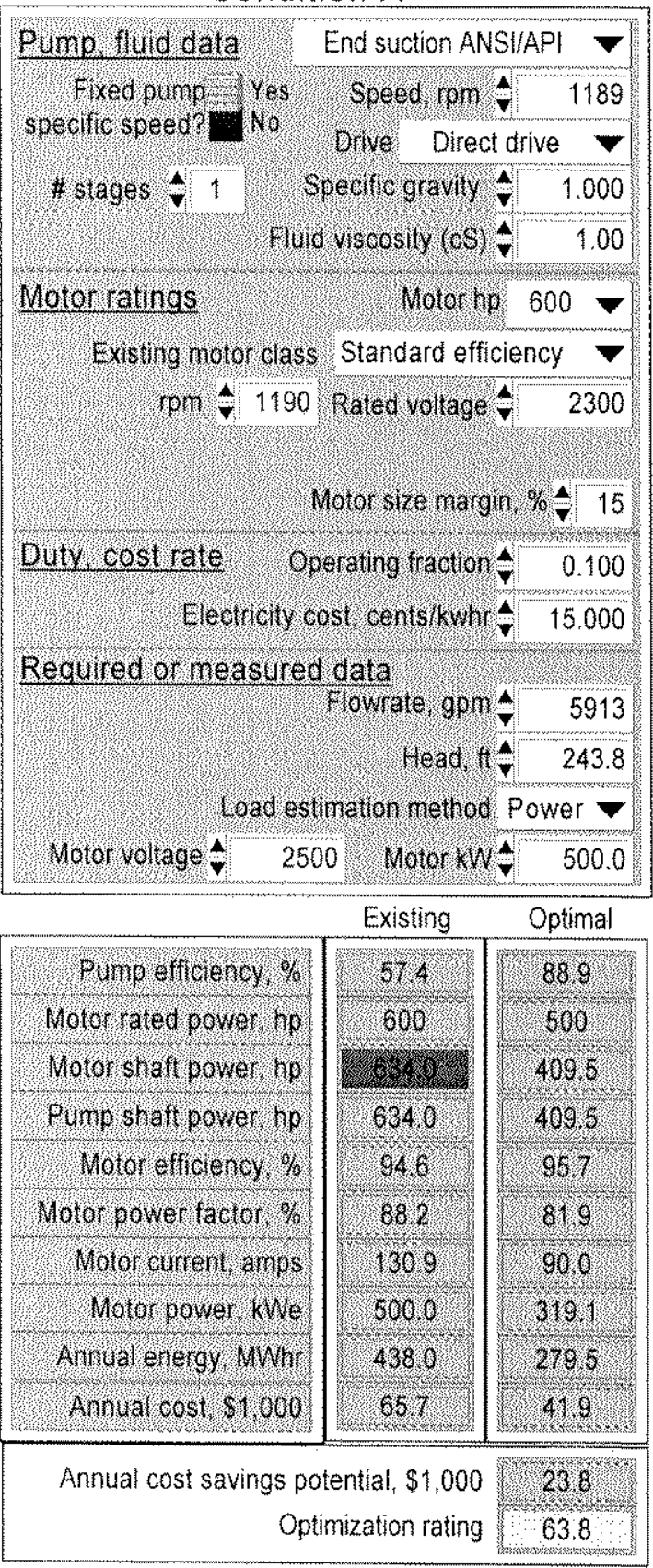

Condition B

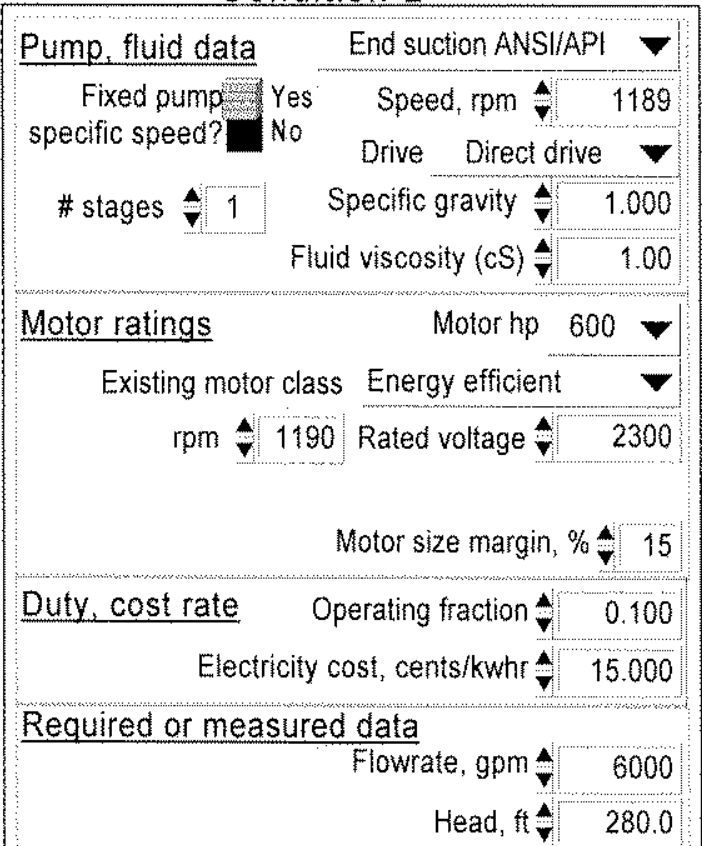

Load estimation method Power $\boldsymbol{\nabla}$

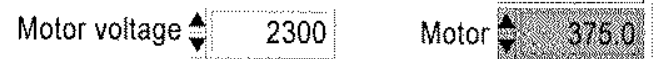

\begin{tabular}{|c|c|c|}
\hline & Existing & Optimal \\
\hline Pump efficiency, $\%$ & 88.1 & 88.4 \\
\hline Motor rated power, hp & 600 & 600 \\
\hline Motor shaft power, hp & 481.4 & 479.7 \\
\hline Pump shaft power, hp & 481.4 & 479.7 \\
\hline Motor efficiency, $\%$ & 95.8 & 95.8 \\
\hline Motor power factor, $\%$ & 84.3 & 84.3 \\
\hline Motor current, amps & 111.6 & 111.2 \\
\hline Motor power, kWe & 375.0 & 373.5 \\
\hline Annual energy, MWhr & 328.5 & 327.2 \\
\hline Annual cost, $\$ 1,000$ & 49.3 & 49.1 \\
\hline
\end{tabular}

Annual cost savings potential, $\$ 1,000$ Optimization rating

\section{Condition A Notes}

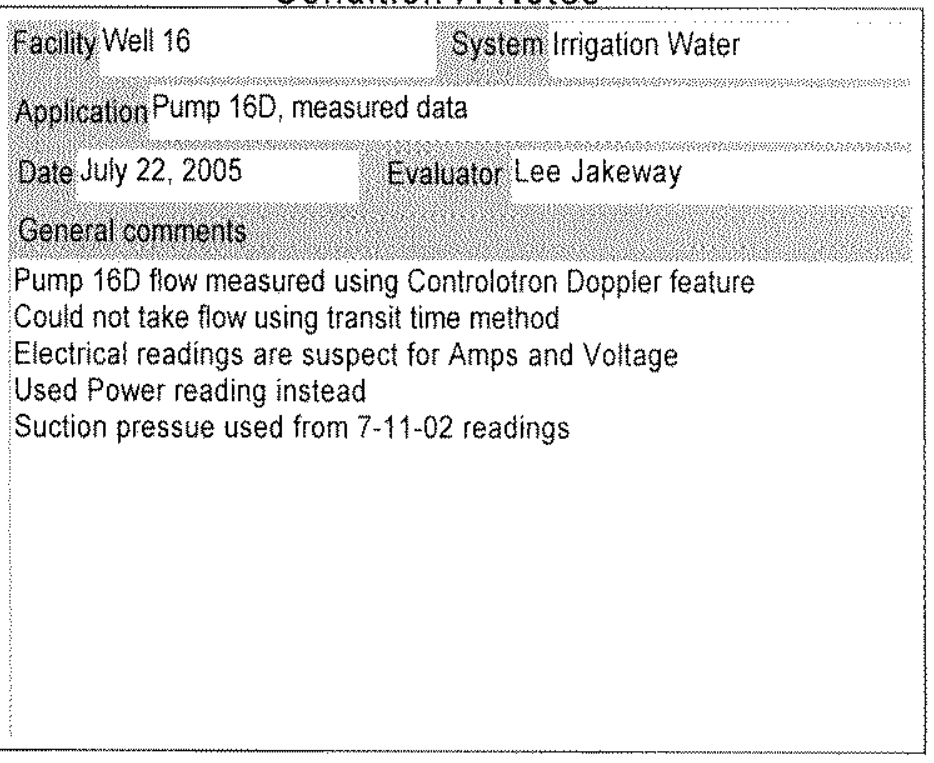

\section{Condition B Notes}

Facility Well $16 \quad$ System irrigation Water

Application Pump 160, optimized for design flow and head

Date July 22, 2005

Evaluator Lee Jakeway

General comments

Hypothetical optimal conditions using design flow and head 


\section{This PSAT2004 analysis was printed at 10:41 AM on Saturday, January 07, 2006}

Condition A

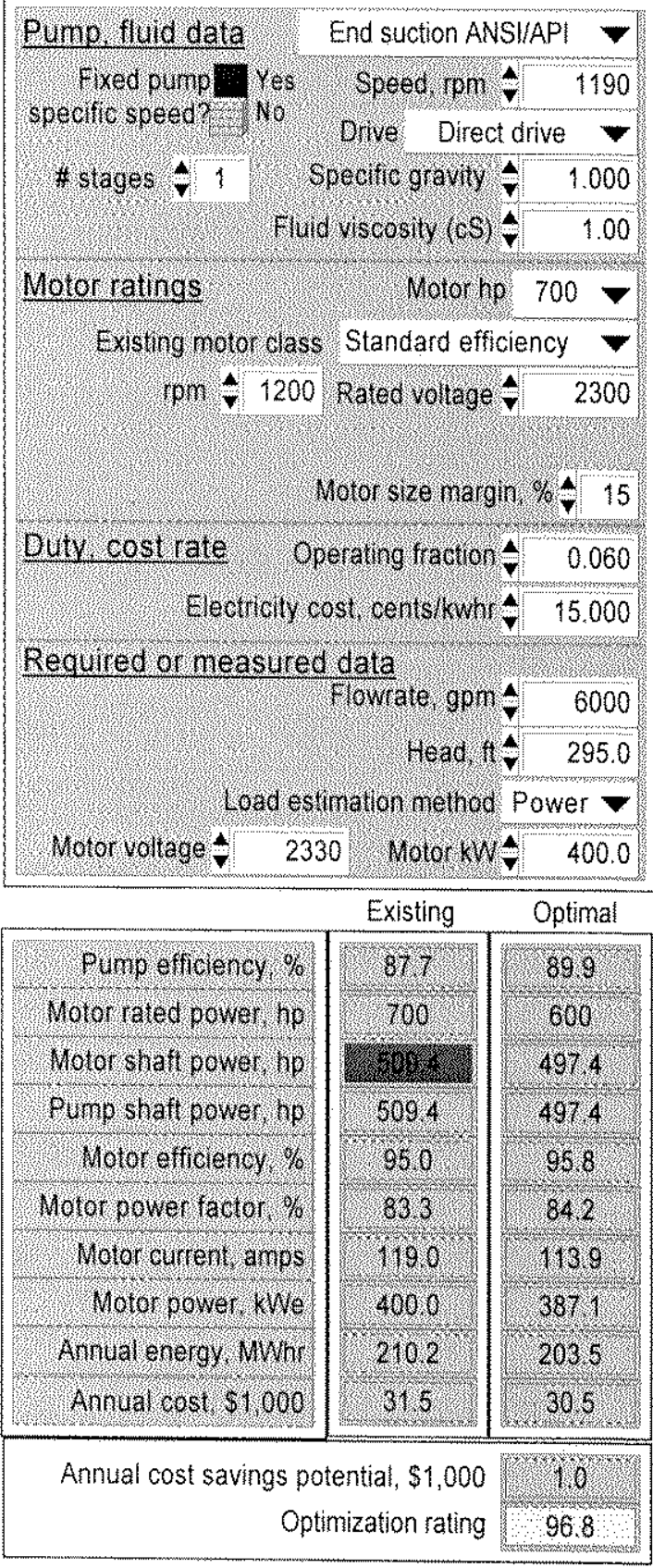

\section{Condition B}

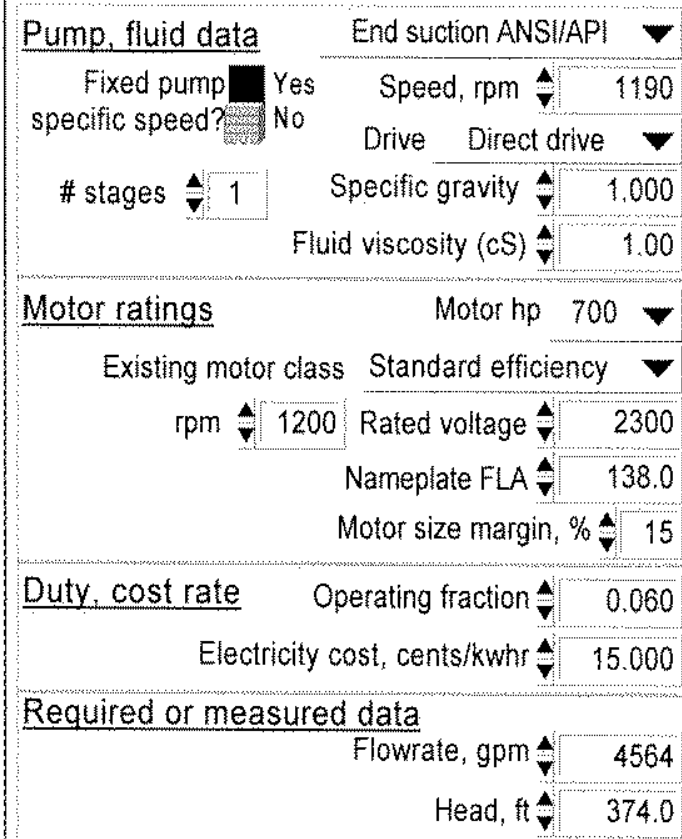

Load estimation method Current $\boldsymbol{\nabla}$

Motor voltage $\frac{\hat{\boldsymbol{v}}}{2330}$ Motor amps $\hat{\boldsymbol{v}} \quad 126.0$

\begin{tabular}{|c|c|c|}
\hline & Existing & Optimal \\
\hline Pump efficiency, $\%$ & 67.1 & 89.3 \\
\hline Motor rated power, hp & 700 & 600 \\
\hline Motor shaft power, hp & 642.7 & 482.6 \\
\hline Pump shaft power, hp & 642.7 & 482.6 \\
\hline Motor efficiency, $\%$ & 94.9 & 95.8 \\
\hline Motor power factor, \% & 99.3 & 83.9 \\
\hline Motor current, amps & 126.0 & 110.9 \\
\hline Motor power, kWe & 505.1 & 375.8 \\
\hline Annual energy, MWhr & 265.5 & 197.5 \\
\hline Annual cost, $\$ 1,000$ & 39.8 & 29.6 \\
\hline \multicolumn{2}{|c|}{ Annual cost savings potential, $\$ 1,000$} & 10.2 \\
\hline \multicolumn{2}{|c|}{ Optimization rating } & 74.4 \\
\hline
\end{tabular}

\section{Condition A Notes}

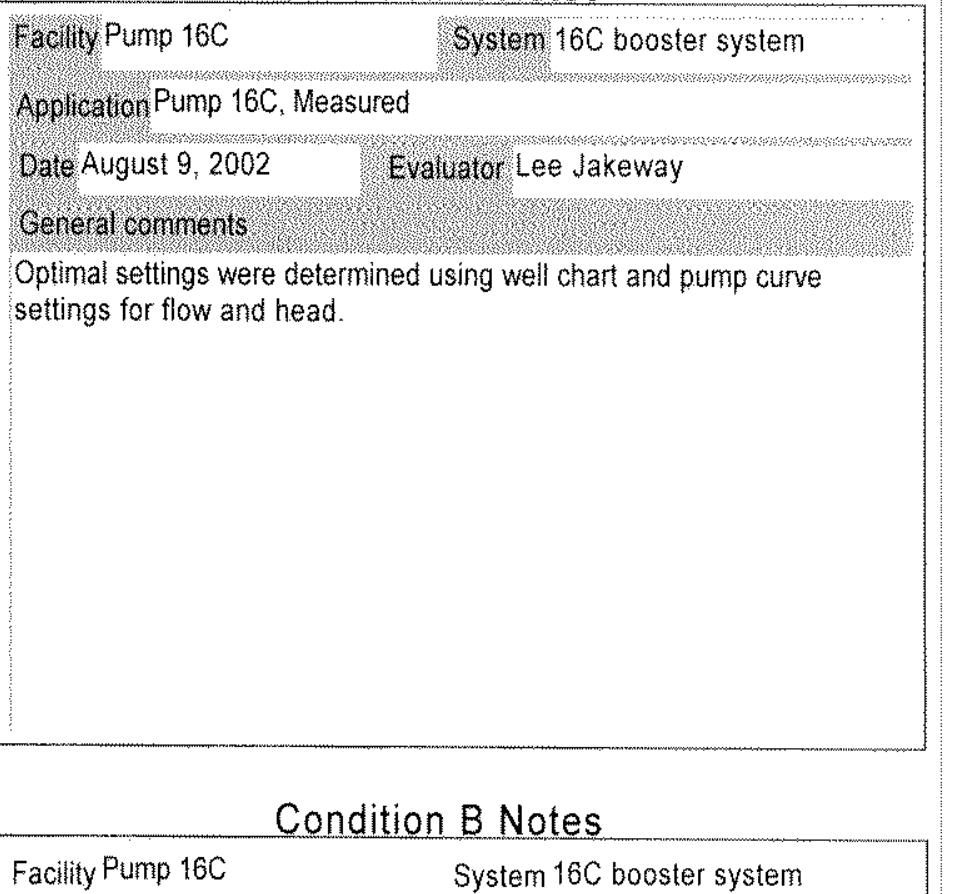

Application Pump 16C, Measured

Date August 9,2002 Evaluator Lee Jakeway

General comments

$16 \mathrm{C}$ booster pump operating with 160 supply pump. Outlet valve on pump discharge was $75 \%$ closed. 


\section{This PSAT2004 analysis was printed at 10:43 AM on Saturday, January 07, 2006}

\section{Condition A}

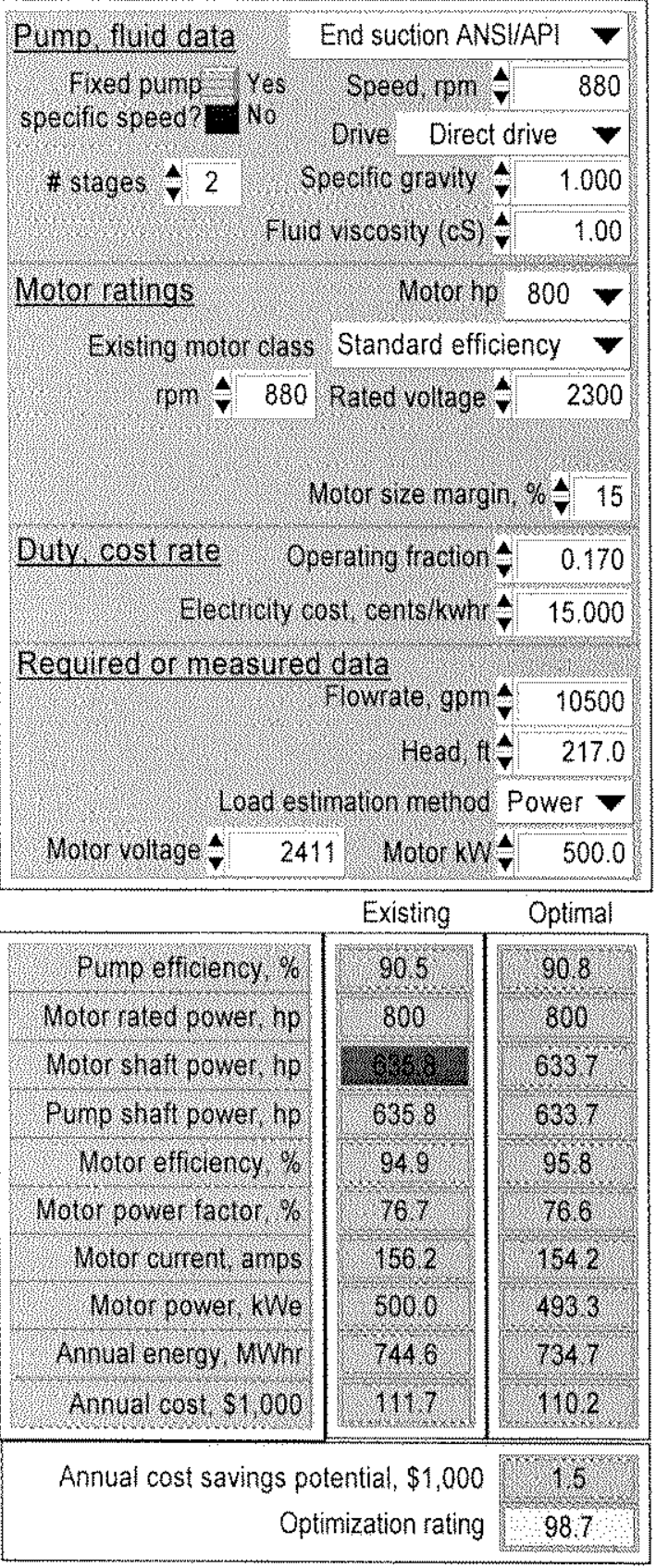

Condition B

\begin{tabular}{|c|c|c|c|c|}
\hline Pump, fluid data & & End su & on ANSIIAPI & $1 \%$ \\
\hline Fixed pump & Yes & Spee & , rpm $⿻$ & 880 \\
\hline speciflc speed? & & Drive & Direct drive & $\nabla$ \\
\hline \# stages $=2$ & & Specific & ravity $\hat{\mathbf{v}}$ & 1.000 \\
\hline & & jid visco & (cS) $\mathbb{7}$ & 1.00 \\
\hline
\end{tabular}

Motor ratings Motor hp $800 \nabla$

Existing motor class Standard efficiency $\mathrm{rpm} * 880$ Rated voltage $\sqrt{2300}$ Nameplate FLA $\overline{\mathbf{v}} \quad 180.0$ Motor size margin, \% $\mathbf{*} 15$ Duty, cost rate Operating fraction $\mathbf{v} \quad 0.170$ Electricity cost, cents/kwhr 15.000 Required or measured data

$$
\begin{aligned}
& \text { Flowrate, gpm * } 8796 \\
& \text { Head, } \mathrm{ft} \boldsymbol{\nabla} \quad 209.1
\end{aligned}
$$

Load estimation method Current $\mathbf{\nabla}$

\begin{tabular}{|c|c|c|}
\hline & Existing & Optimal \\
\hline Pump efficiency, $\%$ & 67.2 & 90.5 \\
\hline Motor rated power, $h p$ & 800 & 600 \\
\hline Motor shaft power, hp & 691.1 & 513.0 \\
\hline Pump shaft power, hp & 691.1 & 513.0 \\
\hline Motor efficiency, $\%$ & 94.8 & 95.6 \\
\hline Motor power factor, $\%$ & 85.1 & 77.7 \\
\hline Motor current, amps & 153.0 & 123.3 \\
\hline Motor power, $\mathrm{kWe}$ & 543.9 & 400.1 \\
\hline Annual energy, MWhr & 810.0 & 595.8 \\
\hline Annual cost, $\$ 1,000$ & 121.5 & 89.4 \\
\hline
\end{tabular}

Motor voltage $\boldsymbol{\nabla} \quad 2411$ Motor amps $\boldsymbol{\nabla} \quad 153.0$

Annual cost savings potential, $\$ 1,000$ Optimization rating

\begin{tabular}{|c|c|}
\hline Facily Well 9 & Systen Irrigation Water \\
\hline \multicolumn{2}{|c|}{ Apolicator Pump 9A, optimized } \\
\hline Date August 1, 2002 & Evaluator Lee Jakeway \\
\hline Generalcomnens & \\
\hline
\end{tabular}

\section{Condition A Notes}

Optimal conditions determined from well chart head and flow values

\section{Condition B Notes}

\section{Facility Well $9 \quad$ System Irrigation Water}

Application Pump 9A, measured

Date August 1, $2002 \quad$ Evaluator Lee Jakeway

General comments

Actual fiow could not be obtained for 9A. Pump 9A flow was based on flow measurement obtained from $9 \mathrm{C}$ when $9 \mathrm{~A}$ was running in series with this. Flow was adjusted uowards based on higher electrical readings when $9 \mathrm{~A}$ was running singularly. 


\section{This PSAT2004 analysis was printed at 10:44 AM on Saturday, January 07, 2006}

\section{Condition A}

\begin{tabular}{|c|c|c|}
\hline Pump fluid data & End suction ANS & $|A P| \nabla$ \\
\hline Fixed pump = Yes & speed ron $\mathbf{v}$ & 885 \\
\hline specific speed? No & Drive Direct & Irive $\nabla$ \\
\hline \# stages $\mathbf{V} 2$ & Specific gravity $=$ & 1.000 \\
\hline Fluig & discosity (cs) & 1.00 \\
\hline Motor ratings & Motor hip & $800 \vee$ \\
\hline Existing motor class & is Standard effici & ency $\nabla$ \\
\hline ppm 4890 & 0 Rated voltage $\mathbf{Y}$ & 2300 \\
\hline & Notor size nargin & $\% \leqslant 15$ \\
\hline Duty cost rate & Pperating traction & 0.160 \\
\hline Electricity o & cost cenisikwh & 15.000 \\
\hline Required or measured & d data & \\
\hline & Flowrate goms & 9750 \\
\hline & Head 14 & 195.0 \\
\hline Load est & stimation nethod $F$ & ower $\nabla$ \\
\hline Molor voltage $\widehat{\mathbf{T}} \quad 242$ & 427 Wotor kW & 400.0 \\
\hline & Existing & Optimal \\
\hline Pump efficiency \% & 94.5 & 907 \\
\hline Motor rated power, hp & 800 & 700 \\
\hline Motor shaff power hp & 25 & 5294 \\
\hline Punp shaft pover hp & 5083 & 5294 \\
\hline Motor efficiency, 60 & 948 & 957 \\
\hline Motor power factor, & 711 & 752 \\
\hline Notor current amos & 133.9 & 1306 \\
\hline Moror pover kwe & 4000 & 4127 \\
\hline Annual energy, MWhr & 5606 & 578.4 \\
\hline Annual cost, 51 000 & 841 & 86.8 \\
\hline Annual cost savings po & otential, $\$ 1,000$ & 27 \\
\hline & timization rating & 103.2 \\
\hline
\end{tabular}

\section{Condition B}

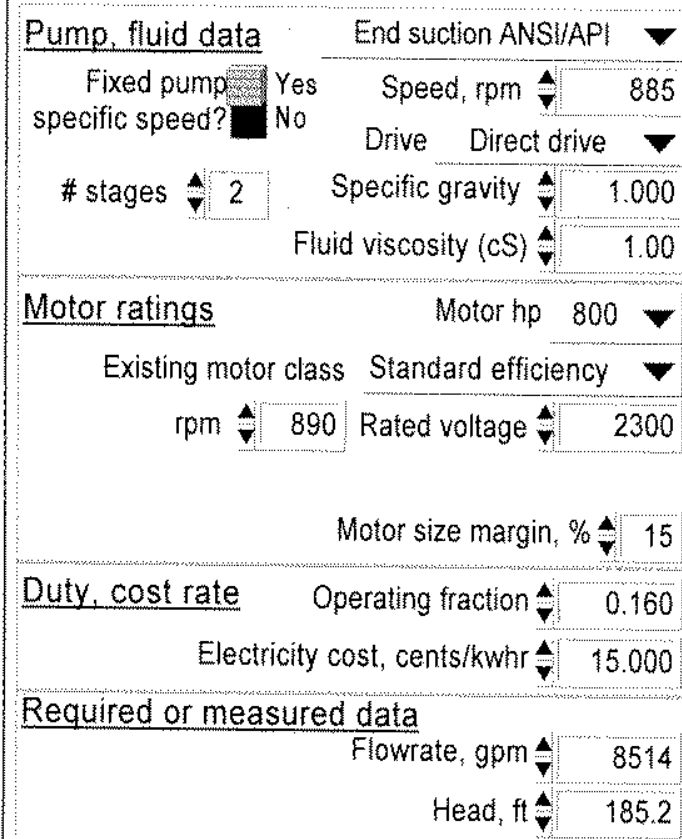

Load estimation mathod Power

$\begin{array}{lll}\text { Motor voltage } & \mathbf{7} 2427 \quad \text { Motor } 5260\end{array}$

\begin{tabular}{|c|c|c|}
\hline & Existing & Optimal \\
\hline Pump efficiency, $\%$ & 59.6 & 90.5 \\
\hline Motor rated power, hp & 800 & 600 \\
\hline Motor shaft power, hp & 668.6 & 440.1 \\
\hline Pump shaft power, hp & 668.6 & 440.1 \\
\hline Motor efficiency, $\%$ & 94.8 & 95.5 \\
\hline Motor power factor, $\%$ & 77.6 & 74.2 \\
\hline Motor current, amps & 161.2 & 110.2 \\
\hline Motor power, kWe & 526.0 & 343.6 \\
\hline Annual energy, MWhr & 737.2 & 481.5 \\
\hline Annual cost, $\$ 1,000$ & 110.6 & 72.2 \\
\hline
\end{tabular}

Annual cost savings potential, $\$ 1,000$ Optimization rating

\section{Condition A Notes}

Facilty Pump $9 \mathrm{C}$ Systen Irigation Water

Application Well water pumping

Date August 1, 2002 Evaluator Lee Jakeway

Gereral comnents

Optimal conditions using well chart head and flow.

\section{Condition B Notes}

\section{Facility Pump $9 \mathrm{C} \quad$ System Irrigation Water}

Application Well water pumping

Date August 1, $2002 \quad$ Evaluator Lee Jakeway

Generai comments

Pump $9 \mathrm{C}$ working in series with $9 \mathrm{~A}$ and pumping all water to Res. 52. 


\section{This PSAT2004 analysis was printed at 10:44 AM on Saturday, January 07, 2006}

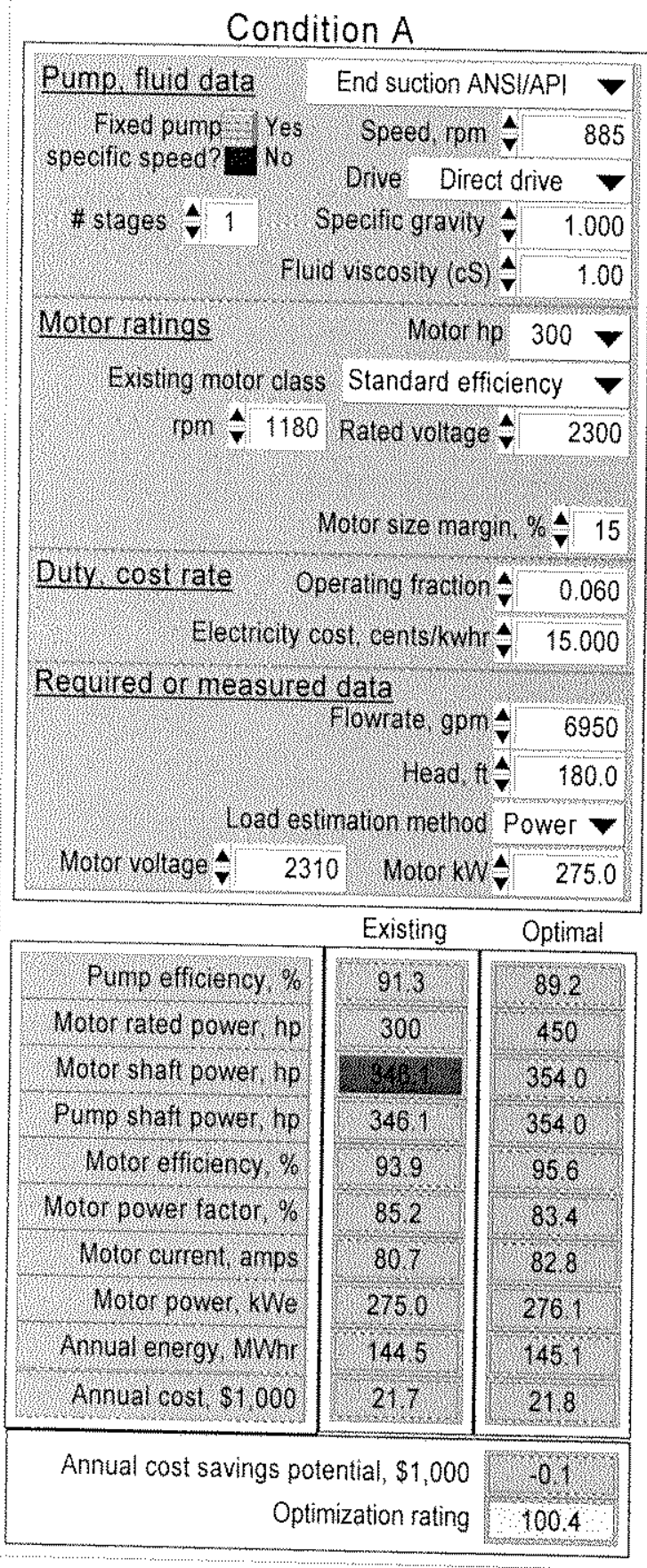

\section{Condition B}

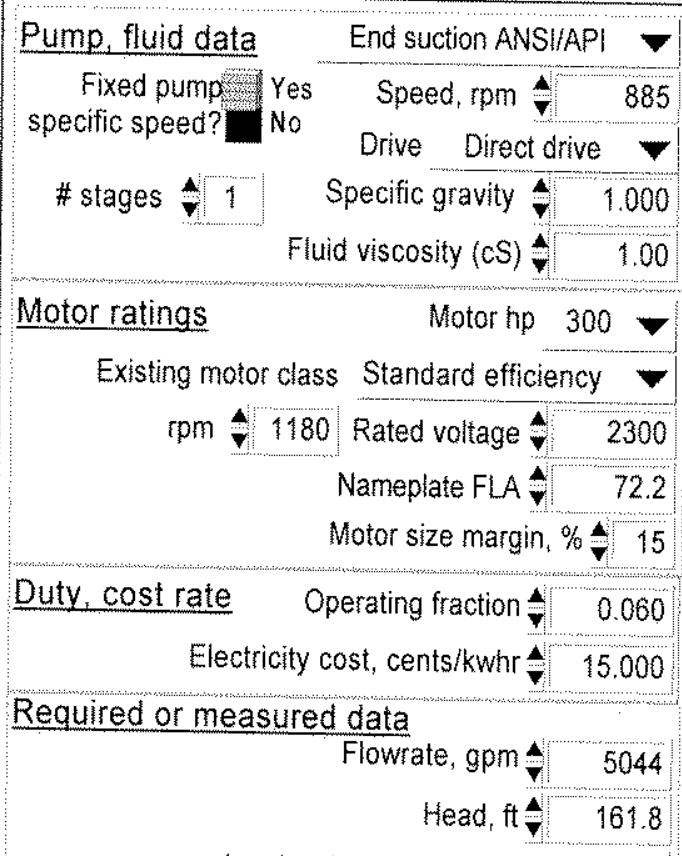

Load estimation method Current $\boldsymbol{\nabla}$

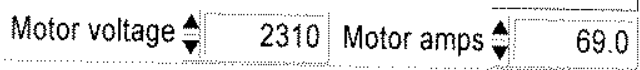

\begin{tabular}{|c|c|c|}
\hline & Existing & Optimal \\
\hline Pump efficiency, $\%$ & 71.7 & 88.2 \\
\hline Motor rated power, hp & 300 & 300 \\
\hline Motor shaft power, hp & 287.3 & 233.5 \\
\hline Pump shaft power, hp & 287.3 & 233.5 \\
\hline Motor efficiency, $\%$ & 94.4 & 95.4 \\
\hline Motor power factor, \% & 82.2 & 82.4 \\
\hline Motor current, amps & 690 & 55.4 \\
\hline Motor power, kWe & 227.0 & 182.6 \\
\hline Annual energy, MWhr & 119.3 & 96.0 \\
\hline Annual cost, $\$ 1,000$ & 17.9 & 14.4 \\
\hline \multicolumn{2}{|c|}{ Annual cost savings potential, $\$ 1,000$} & 3.5 \\
\hline \multicolumn{2}{|c|}{ Optimization rating } & 80.4 \\
\hline
\end{tabular}

\section{Condition A Notes}

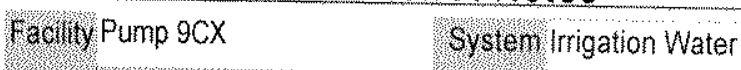

Apolication $9 \mathrm{CX}$ measured

Date August 1, $2002 \quad$ Evaluator Lee Jakeway

General comments

Optimal conditions using well chart head and flow data

\section{Condition B Notes}

\section{Facility Pump $9 \mathrm{CX} \quad$ System Irrigation Water}

Application $9 \mathrm{CX}$ measured

Date August 1, $2002 \quad$ Evaluator Lee Jakeway

General comments

Pump $9 \mathrm{CX}$ working in series with $9 \mathrm{~A} \& \mathrm{C}$ pumping. $9 \mathrm{CX}$ discharge is at

Lowrie ditch 
This PSAT2004 analysis was printed at 10:46 AM on Saturday, January 07, 2006

\section{Condition A}

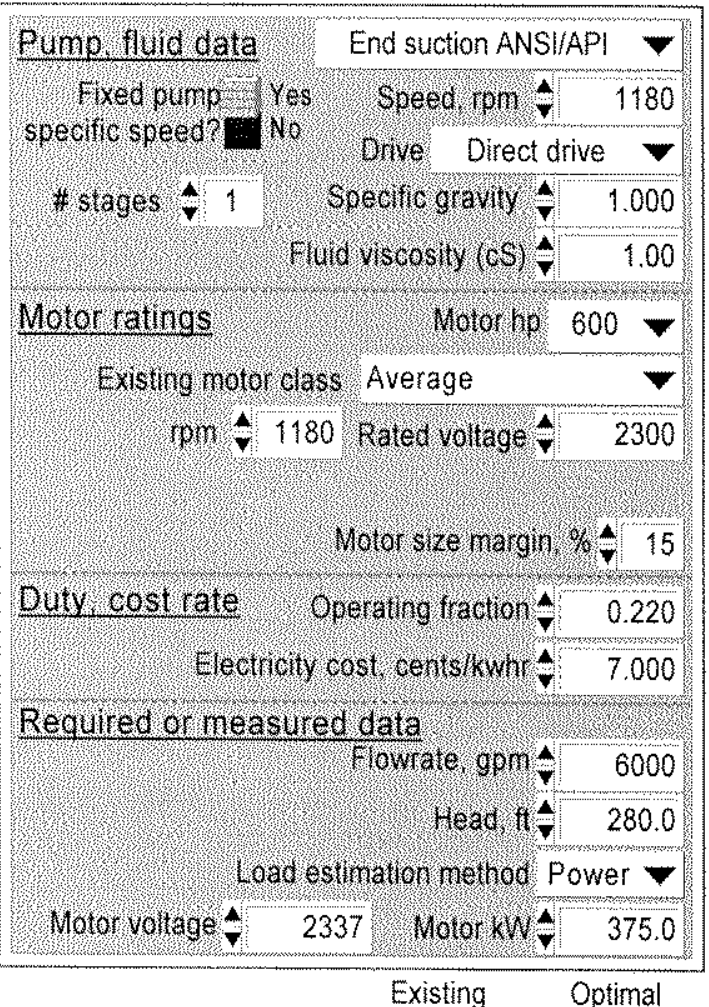

\begin{tabular}{|c|c|c|}
\hline & Existing & Optimal \\
\hline Pump efroiency, of & $888^{2}$ & 88.4 \\
\hline Molor rated power hp & 600 & 600, \\
\hline Molor shat power: hp & Hris & 1800 \\
\hline Pump shaft pover hp & 479.3 & 480.0 \\
\hline Notor efriciency \% & 953 & 958 \\
\hline Moror power ractor 6 & 839 & 838 \\
\hline Molor current, amps & 1104 & 1102 \\
\hline Molor pover Ne & 375.0 & 3737 \\
\hline Arnual energy, WWhr & 122,7 & 7202 \\
\hline Annual cost, 51,000 & 506 & 504 \\
\hline
\end{tabular}

Annual cost savings potential, $\$ 1,000$ Optimization rating

\section{Condition B}

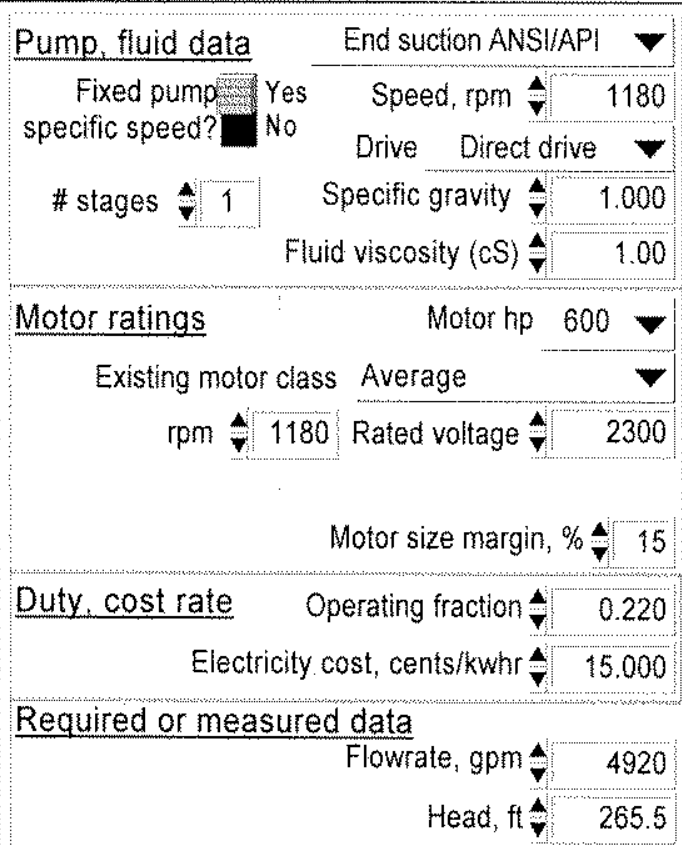

Load estimation method Power $\mathbf{v}$

Motor voltage $\frac{7}{7} 2337$ Motor 4300

\begin{tabular}{|c|c|c|}
\hline & Existing & Optimal \\
\hline Pump efficiency, $\%$ & 58.8 & 87.6 \\
\hline Motor rated power, hp & 600 & 450 \\
\hline Motor shaft power, hp & 561.0 & 376.4 \\
\hline Pump shaft power, hp & 561.0 & 376.4 \\
\hline Motor efficiency, \% & 95.3 & 95.7 \\
\hline Motor power factor, $\%$ & 85.5 & 83.6 \\
\hline Motor current, amps & 126.9 & 86.7 \\
\hline Motor power, kWe & 439.0 & 293.5 \\
\hline Annual energy, MWhr & 846.0 & 565.5 \\
\hline Annual cost, $\$ 1,000$ & 126.9 & 84.8 \\
\hline
\end{tabular}

Annual cost savings potential, $\$ 1,000$ Optimization rating

\section{Condition A Notes}

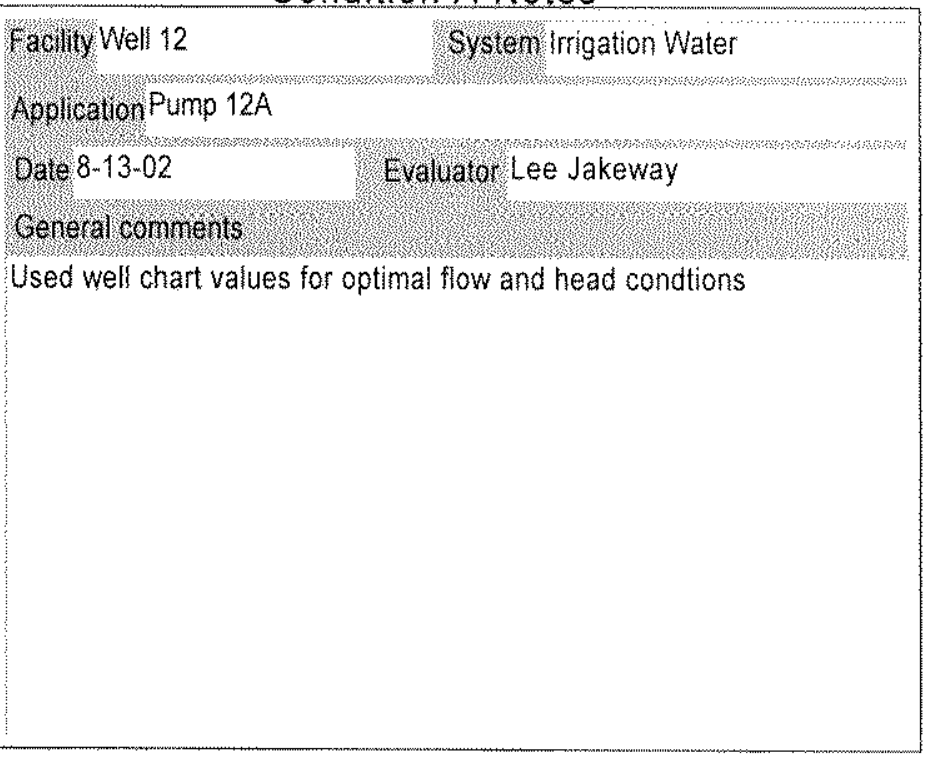

\section{Condition B Notes}

Facility Well $12 \quad$ System Irrigation Water

Application Pump $12 \mathrm{~A}$

Date 8-13-02 Evaluator Lee Jakeway

\section{General comments}

Pump measurements were made with butterfly valve only $5 / 8$ of the way open. Reported done this way because of low sump level and to limit amperage draw on pump. 


\section{This PSAT2004 analysis was printed at 10:47 AM on Saturday, January 07, 2006}

Condition A

\begin{tabular}{|c|c|c|}
\hline \multirow{3}{*}{$\begin{array}{l}\text { Pump, fluid data } \\
\text { Fixed pump } \\
\text { spedif speed? Yes }\end{array}$} & \multicolumn{2}{|c|}{ End suction ANSI/API } \\
\hline & \multicolumn{2}{|l|}{ Speed.$p m \rightarrow$} \\
\hline & \multicolumn{2}{|c|}{ Drive Direct drive } \\
\hline Specific gravity $\mathbb{\top} 1.000$ & \multicolumn{2}{|c|}{ Soecific oravity 4} \\
\hline \multicolumn{3}{|c|}{ Finid viscosity (cS) $\mathbf{T}$} \\
\hline \multicolumn{3}{|c|}{ Motor ratings $\quad$ Motor ho 600 V } \\
\hline \multicolumn{3}{|c|}{ Existing motor class Standard efficiency } \\
\hline ppm -720 & \multicolumn{2}{|c|}{ Rated voltage $\mathbf{v} \quad 230$} \\
\hline & \multicolumn{2}{|c|}{ Motor size nargin $\% \cup 1$} \\
\hline \multicolumn{3}{|l|}{ Duty cost rate } \\
\hline \multicolumn{3}{|c|}{ Electricity cost cents ikwhr $\boldsymbol{\nabla} 15,000$} \\
\hline \multirow{5}{*}{\multicolumn{3}{|c|}{ 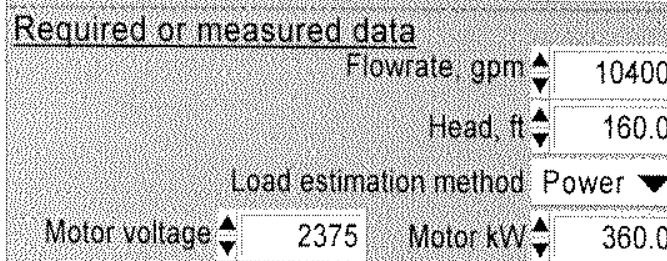 }} \\
\hline & & \\
\hline & & \\
\hline & & \\
\hline & & \\
\hline \multicolumn{3}{|c|}{ Existing } \\
\hline Pump efficiency \% & 923 & 908 \\
\hline Motor rated power hp & 600 & 600 \\
\hline Nolor shaft power. np & 48 & 4629 \\
\hline Pump shatt power 1 p & 4553 & 4629 \\
\hline Motor efficency $\%$ & 94.4 & 95.3 \\
\hline Moror power actor \% & 71.8 & 72.2 \\
\hline Motor current amps & 121.9 & 1219 \\
\hline Notor power k we & 560,0 & 3623 \\
\hline Annual energy MWh & 157.7 & 1587 \\
\hline Annual cost $\$ 1,000$ & 23.7 & 238 \\
\hline Annual cost savings pot & otential, $\$ 1,000$ & -0.1 \\
\hline & timization rating & 100.6 \\
\hline
\end{tabular}

Condition B

\begin{tabular}{|c|c|}
\hline \multirow{4}{*}{$\begin{array}{l}\frac{\text { Pump, fluid data }}{\text { Fixed pump }} \\
\text { specific speed? } \\
\text { \# stages } \sqrt{2}\end{array}$} & End suction ANSIIAPI $\boldsymbol{\nabla}$ \\
\hline & Speed, rpm $\leqslant$ \\
\hline & Drive Direct drive $\nabla$ \\
\hline & Specific gravity $\mathbf{\nabla} \quad 1.000$ \\
\hline & uid viscosity $(\mathrm{CS}) \stackrel{4}{\mathbf{V}}$ \\
\hline Motor ratings & Motor ho $600 \nabla$ \\
\hline Existing motor clas & ss Standard efficiency $\boldsymbol{\nabla}$ \\
\hline $\mathrm{rpm} \mathbf{7} 720$ & 0 Rated voltage $\frac{\nabla}{\nabla}$ \\
\hline & Nameplate FLA $\hat{\boldsymbol{\nabla}} \quad 148.0$ \\
\hline & Motor size margin, \% 15 \\
\hline Duty cost rate & Operating fraction $\mathbf{v}$ \\
\hline Electricity & cost, cents/kwhr $\mathbb{\nabla} 15.000$ \\
\hline Required or measure & ed data \\
\hline & Flowrate, gpm 4 \\
\hline & Head, $\mathrm{ft} \widehat{\mathbf{\nabla}} \quad 150.7$ \\
\hline
\end{tabular}

Load estimation method Current $\mathbf{v}$

\begin{tabular}{ll|l|l|}
\hline Motor voltage & $\boldsymbol{*}$ & 2375 & Motor amps \\
$\mathbf{v}$ & 123.0
\end{tabular}

\begin{tabular}{|c|c|c|}
\hline & Existing & Optimal \\
\hline Pump efficiency, \% & 70.0 & 90.6 \\
\hline Motor rated power, hp & 600 & 450 \\
\hline Motor shaft power, hp & 490.2 & 378.8 \\
\hline Pump shaft power, hp & 490.2 & 378.8 \\
\hline Motor efficiency, \% & 94.3 & 95.3 \\
\hline Motor power factor, $\%$ & 76.7 & 73.5 \\
\hline Motor current, amps & 123.0 & 98.0 \\
\hline Motor power, kWe & 388.0 & 296.5 \\
\hline Annual energy, MWhr & 169.9 & 129.8 \\
\hline Annual cost, $\$ 1,000$ & 25.5 & 19.5 \\
\hline \multicolumn{2}{|c|}{ Annual cost savings potential, $\$ 1,000$} & 6.0 \\
\hline \multicolumn{2}{|c|}{ Optimization rating } & 76.4 \\
\hline
\end{tabular}

\section{Condition A Notes}

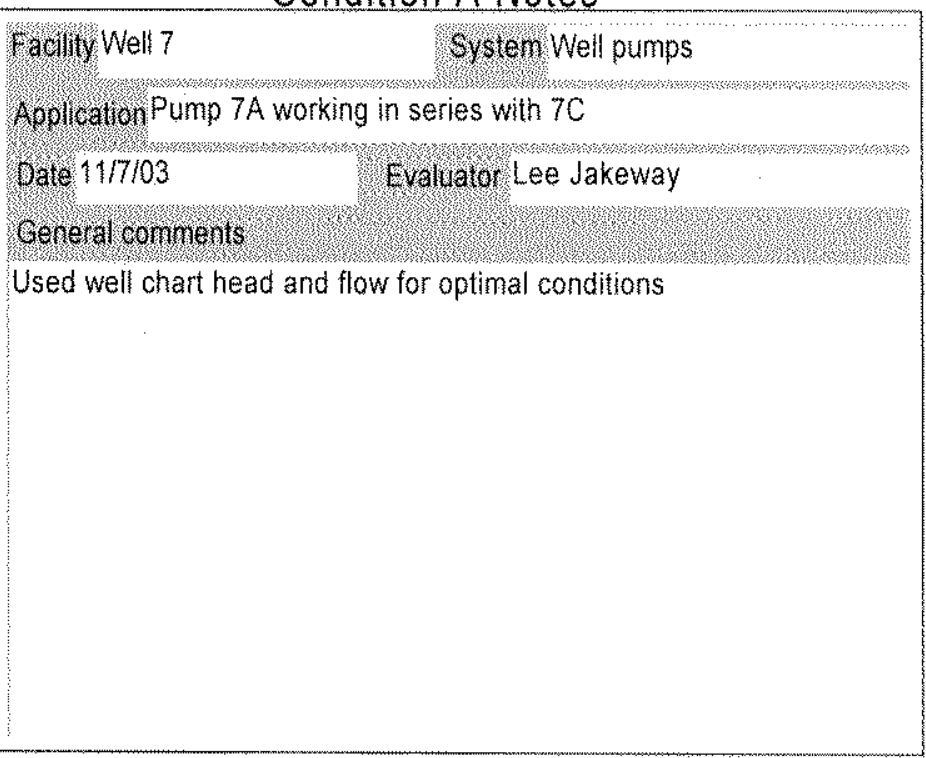

\section{Condition B Notes}

\section{Facilify Well $7 \quad$ System Well pumps}

Application Pump $7 \mathrm{~A}$ working in series with $7 \mathrm{C}$

Date $11 / 7103 \quad$ Evaluator Lee Jakeway

General comments

Pump $7 \mathrm{~A}$ and $7 \mathrm{C}$ were operating together. Flow reading was obtained at bottom of well shaft on $32^{\prime \prime}$ dia steel pipe with Panametric flow meter. 


\section{This PSAT2004 analysis was printed at 10:48 AM on Saturday, January 07, 2006}

\section{Condition A}

\begin{tabular}{|c|c|c|}
\hline Pump. lluod data & End suction ANS & SIIAPI \\
\hline Fixed pump Yus & speed ron & 1185 \\
\hline 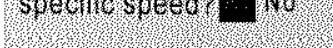 & Drve Direct & t drive \\
\hline Hrtages 42 & pecifo gravity & 1.000 \\
\hline PIIT & drscosiv $(\operatorname{cs})$ & A 1.00 \\
\hline Motor ratings & Motor 10 & $900 \nabla$ \\
\hline Existing notor class & Standard effic & ciency $\nabla$ \\
\hline $10 m \div 1187$ & Rated voltage & 2300 \\
\hline & Woror size nargin & W. \\
\hline Dut cost rate & ferathg ractions & 0.160 \\
\hline Elecricity & ost, centsukwir & 15.000 \\
\hline Required or neasures & data & \\
\hline & flowrate ghn & 7300 \\
\hline & rear 4 & 390.0 \\
\hline Load es & matron method & Power $\mathbf{F}$ \\
\hline Nolorvoltage $\mathbf{v} \quad 23$ & Moror kW & 650.0 \\
\hline & Existing & Optimal \\
\hline Pump efficiency, o\% & 868 & 900 \\
\hline Molor rated power ho & 900 & 1000 \\
\hline Motor shaft power, hp & 4 & 7986 \\
\hline Punp shatt power np & 8281 & 1986 \\
\hline Motor eficrency 9 & 96. & 060 \\
\hline Molor power tactor for & 608 & 846 \\
\hline Motor current, amps & 1850 & 1801 \\
\hline Motor power. kWe & 6500 & 620.4 \\
\hline Annual energy WWris & 9110 & 8605 \\
\hline Annual cost. 81,000 & 1367 & 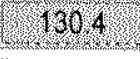 \\
\hline Annual cost savings po & tential, $\$ 1,000$ & 62 \\
\hline & imization rating & 95.4 \\
\hline
\end{tabular}

Condition B

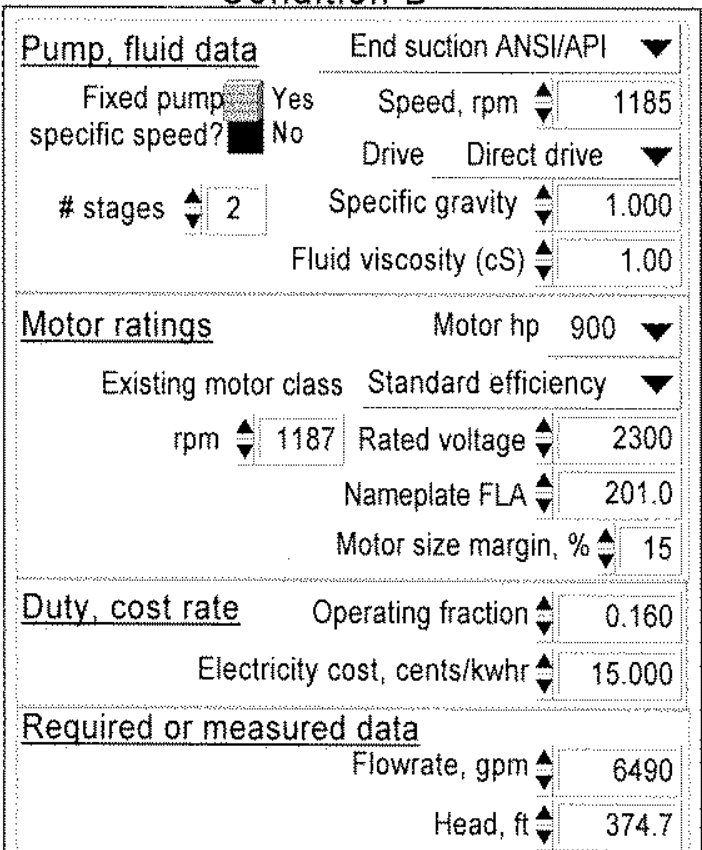

Load estimation method Current $\mathbf{\nabla}$

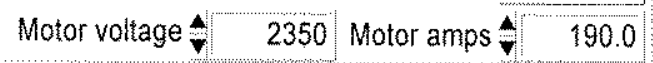

\begin{tabular}{|c|c|c|}
\hline & Existing & Optimal \\
\hline Pump efficiency, $\%$ & 70.9 & 89.8 \\
\hline Motor rated power, $h p$ & 900 & 800 \\
\hline Motor shaft power, hp & 866.3 & 684.0 \\
\hline Pump shaft power, hp & 866.3 & 684.0 \\
\hline Motor efficiency, $\%$ & 95.0 & 95.9 \\
\hline Motor power factor, $\%$ & 88.0 & 85.0 \\
\hline Motor current, amps & 190.0 & 153.7 \\
\hline Motor power, kWe & 680.4 & 531.6 \\
\hline Annual energy, MWhr & 953.7 & 745.1 \\
\hline Annual cost, $\$ 1,000$ & 143.0 & 111.8 \\
\hline \multicolumn{2}{|c|}{ Annual cost savings potential, $\$ 1,000$} & 31.3 \\
\hline \multicolumn{2}{|c|}{ Optimization rating } & 78.1 \\
\hline
\end{tabular}

\section{Condition A Notes}

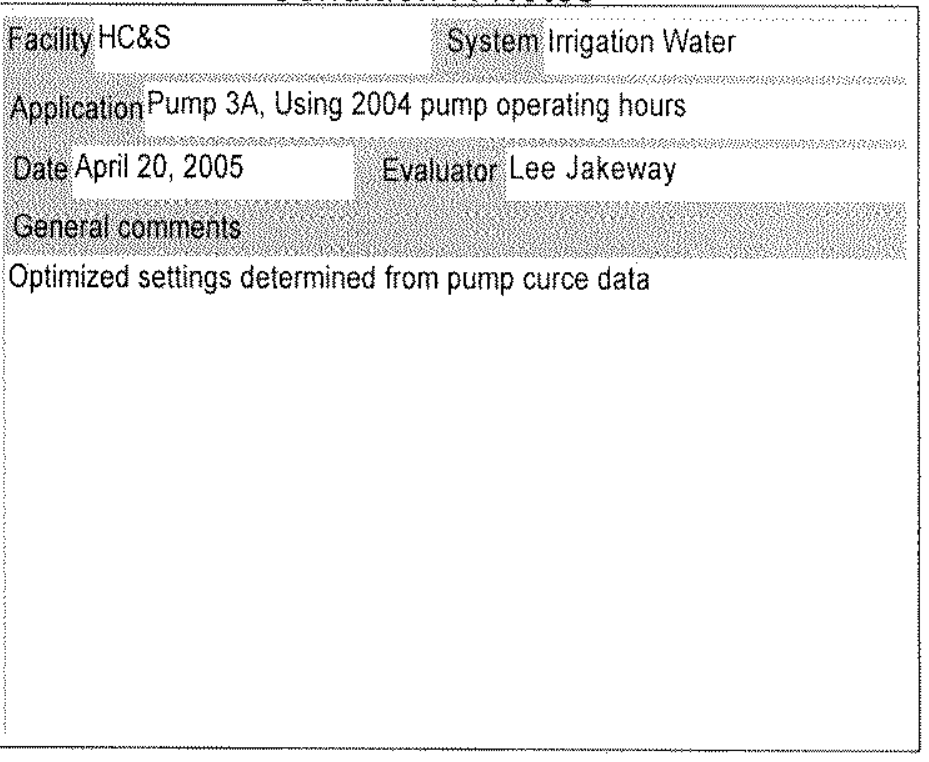

\section{Condition B Notes}

\section{Facility HC\&S System Irigation Water}

Application Pump 3A, Using 2004 pump operating hours

Date April 20,2005 Evaluator Lee Jakeway

General comments

Pump efficiency testing at Well 3 after pipeline replacement to Res. 82 Throttle valve at outlet of pump fully open. 


\section{This PSAT2004 analysis was printed at 10:50 AM on Saturday, January 07, 2006}

\section{Condition A}

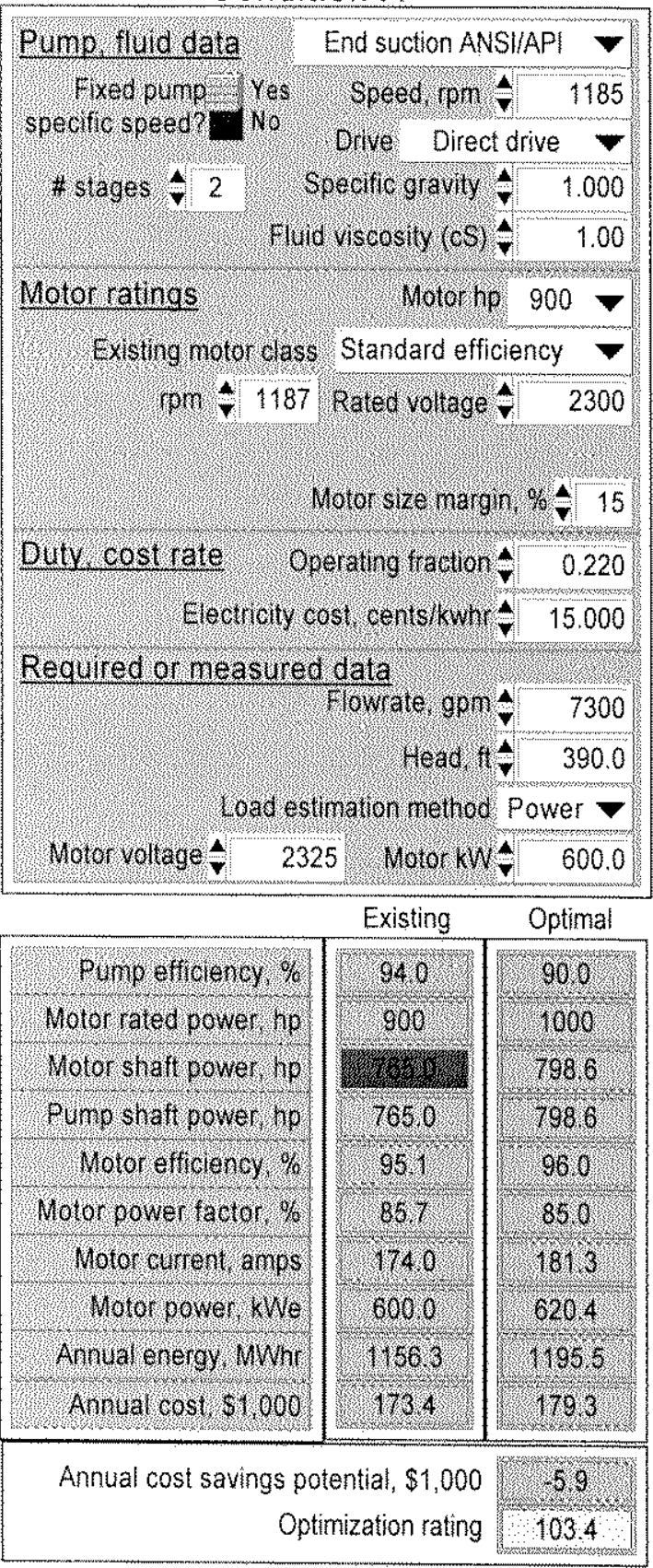

Condition B

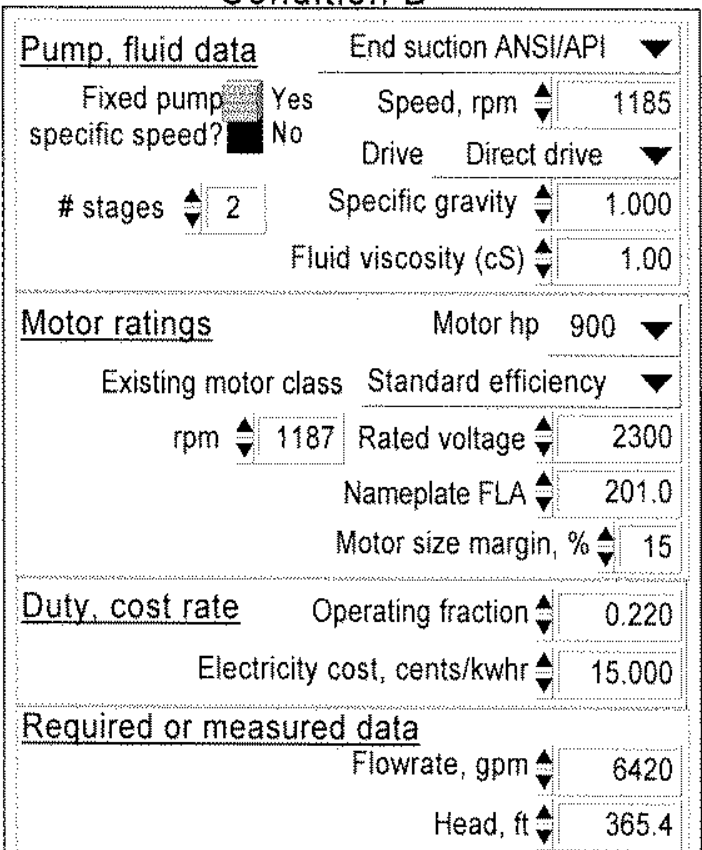

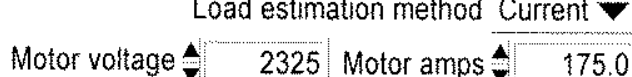
Existing Optimal

\begin{tabular}{|c|c|}
\hline Pump efficiency, $\%$ & 75.8 \\
\hline Motor rated power, hp & 900 \\
\hline Motor shaft power, ho & 781.9 \\
\hline Pump shaft power, hp & 781.9 \\
\hline Motor efficiency, $\%$ & 95.1 \\
\hline Motor power factor, $\%$ & 87.0 \\
\hline Motor current, amps & 175.0 \\
\hline Motor power, $\mathrm{kWe}$ & 613.3 \\
\hline Annual energy, MWhr & 1182.0 \\
\hline Annual cost, $\$ 1,000$ & 177.3 \\
\hline
\end{tabular}

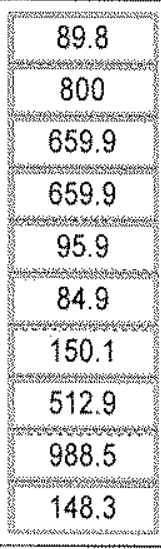

Annual cost savings potential, $\$ 1,000$ Optimization rating

\section{Condition A Notes}

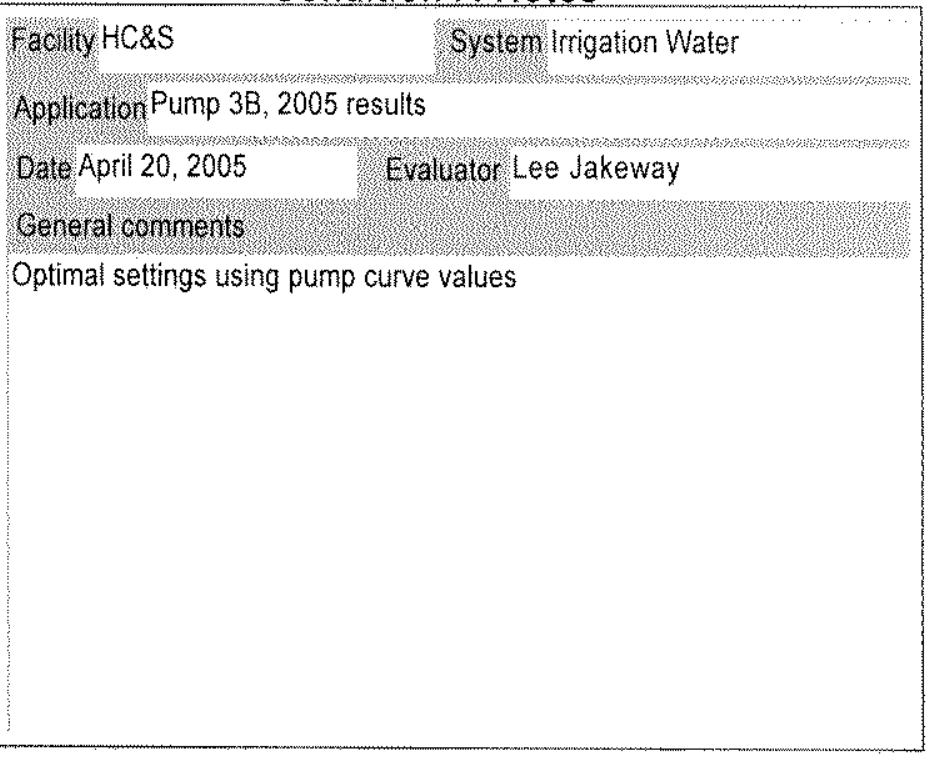

\section{Condition B Notes}

Facility HC\&S System Irrigation Water

Application Pump 3B, 2005 results

Date April 20, $2005 \quad$ Evaluator Lee Jakeway

General comments

Pump efficiency testing at Well 3 after pipeline change made

Throttle valve at outlet of pump fully open. Used 2004 operating hours to

determine operating fraction

Controlotron flow meter used 


\section{This PSAT2004 analysis was printed at 10:52 AM on Saturday, January 07, 2006}

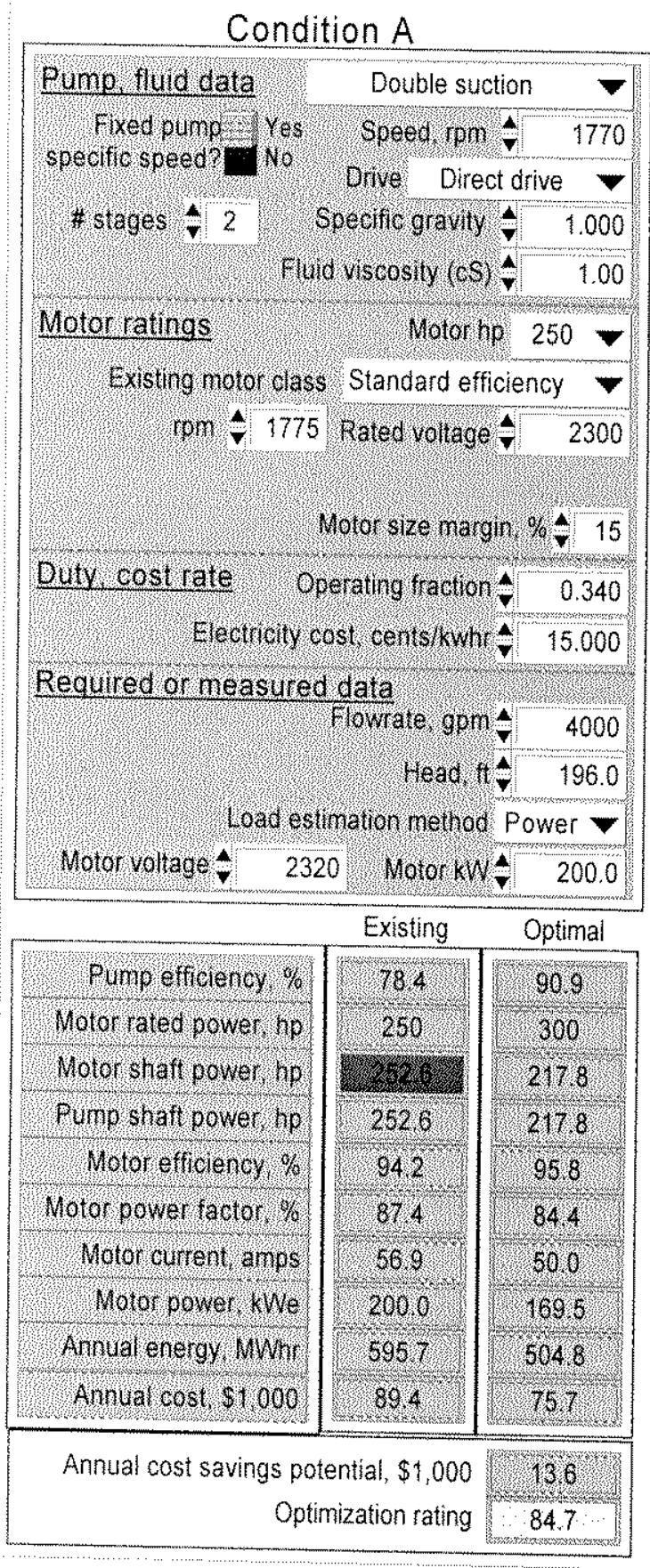

\section{Condition B}

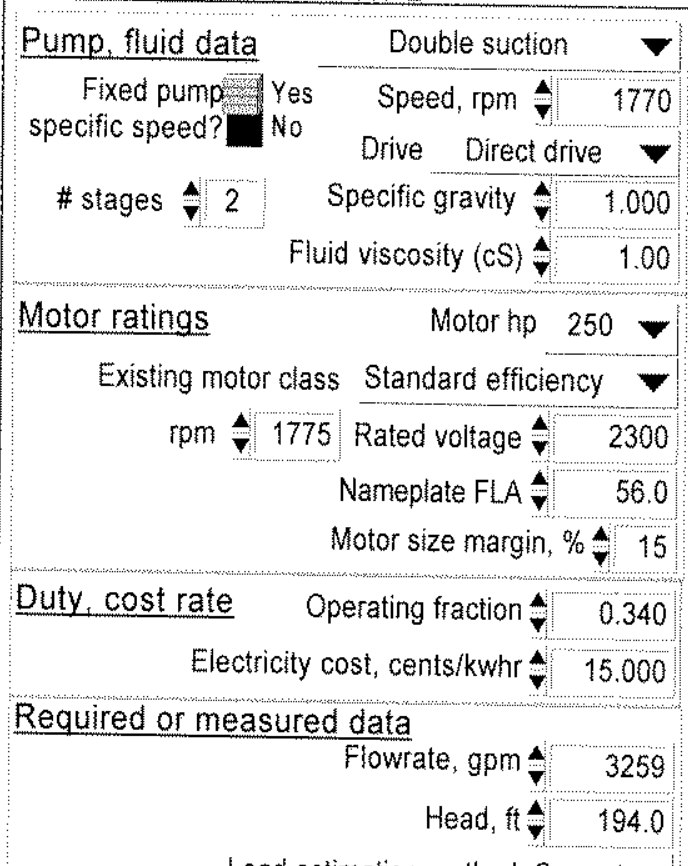

Load estimation method Current $\boldsymbol{\nabla}$

Motor voltage $4 \quad 2320$ Motor amps $\bar{\nabla} \quad 52.0$

\begin{tabular}{|c|c|c|}
\hline & Existing & Optimal \\
\hline Pump efficiency, $\%$ & 68.4 & 90.7 \\
\hline Motor rated power, hp & 250 & 250 \\
\hline Motor shaft power, hp & 233.3 & 176.0 \\
\hline Pump shaft power, hp & 233.3 & 176.0 \\
\hline Motor efficiency, $\%$ & 94.3 & 95.7 \\
\hline Motor power factor, $\%$ & 88.3 & 83.9 \\
\hline Motor current, amps & 52.0 & 40.7 \\
\hline Motor power, kWe & 184.6 & 137.1 \\
\hline Annual energy, MWhr & 549.8 & 408.3 \\
\hline Annual cost, $\$ 1,000$ & 82.5 & 61.2 \\
\hline \multirow{2}{*}{\multicolumn{2}{|c|}{ Annual cost savings potential, $\$ 1,000$}} & 21.2 \\
\hline & & 74.3 \\
\hline
\end{tabular}

\section{Condition A Notes}

Facily Well 1 Systern Well Pump

Aoplication Pump 1

Date November 11, 2003 Evaluator Lee Jakeway

General comnents

Optimal settings determined fromwell chart flow and head values

\section{Condition B Notes}

\section{Facility Well 1 \\ System Well Pump}

Application Pump 1

Date November 11, $2003 \quad$ Evaluator Lee Jakeway

General comments

Data collected from Well 1. Check valve and stop valve were part of

system at pump outlet. Flow measurement was taken using Panametric flow meter on $20^{\text {R }}$ cast iron pipe, 0.8 wall thickness, near reservoir 83 outlet. Electrical readings were taken from analog meters on the board. 


\section{This PSAT2004 analysis was printed at 10:55 AM on Saturday, January 07, 2006}

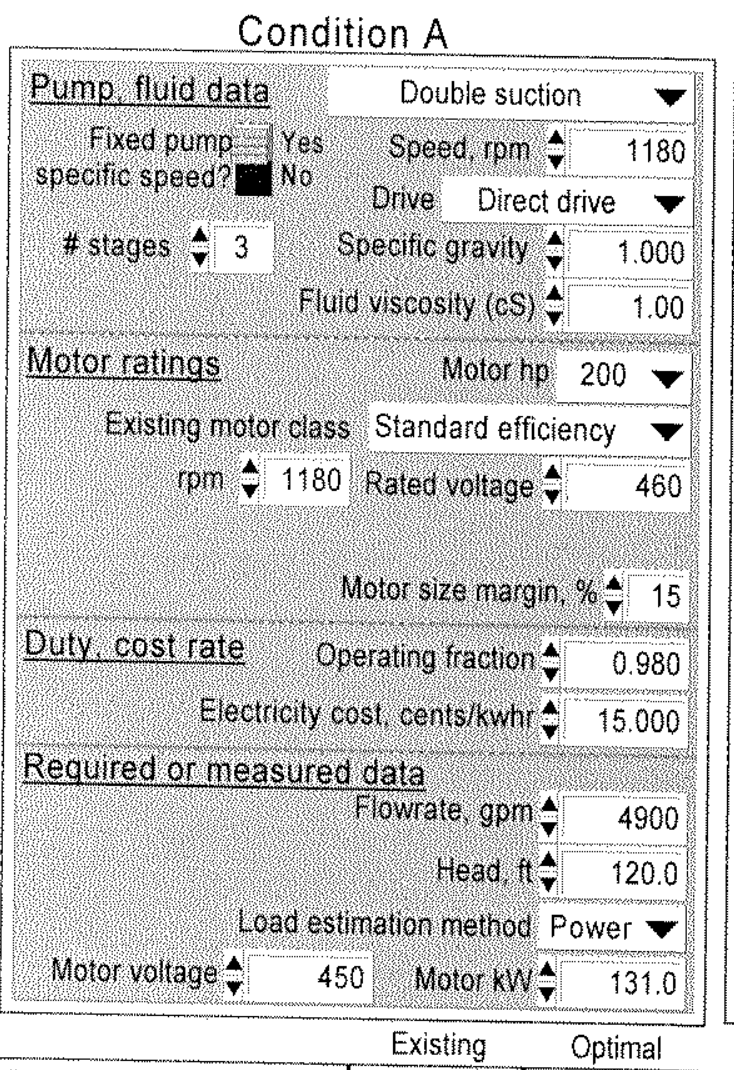

\section{Condition B}

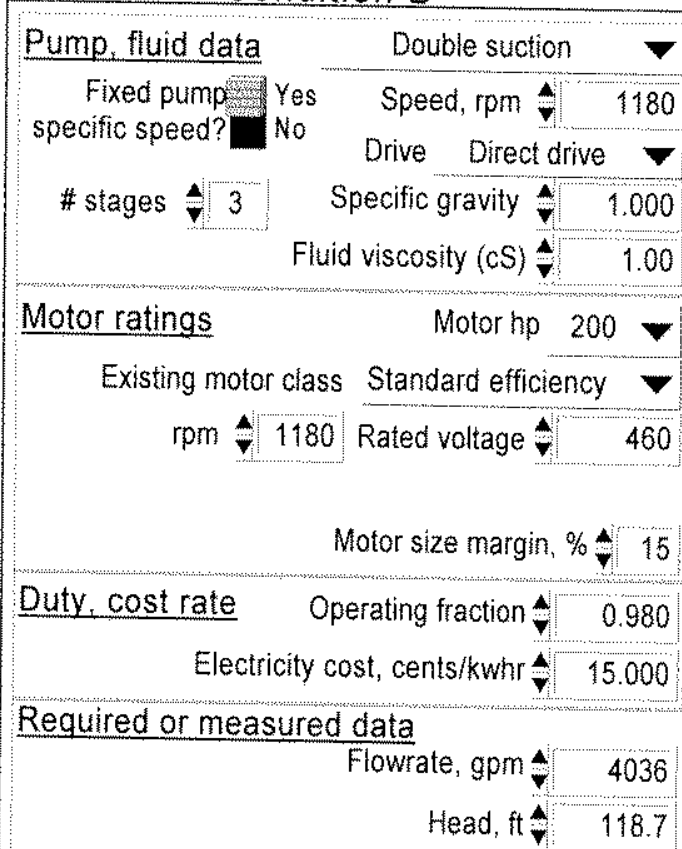

Load estimation method Power $\nabla$

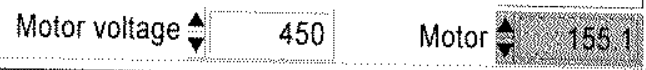

\begin{tabular}{|c|c|c|}
\hline & Existing & Optimal \\
\hline Pump efficiency, $\%$ & 61.9 & 90.1 \\
\hline Motor rated power, hp & 200 & 200 \\
\hline Motor shaft power, hp & 195.4 & 134.3 \\
\hline Pump shaft power, hp & 195.4 & 134.3 \\
\hline Motor efficiency, $\%$ & 94.0 & 95.0 \\
\hline Motor power factor, $\%$ & 83.9 & 81.2 \\
\hline Motor current, amps & 237.3 & 166.7 \\
\hline Motor power, kWe & 155.1 & 105.4 \\
\hline Annual energy, MWhr & 1331.5 & 905.1 \\
\hline Annual cost, $\$ 1,000$ & 199.7 & 135.8 \\
\hline \multirow{2}{*}{\multicolumn{2}{|c|}{ Annual cost savings potential, $\$ 1,000$}} & 64.0 \\
\hline & & 68.0 \\
\hline
\end{tabular}

Condition A Notes

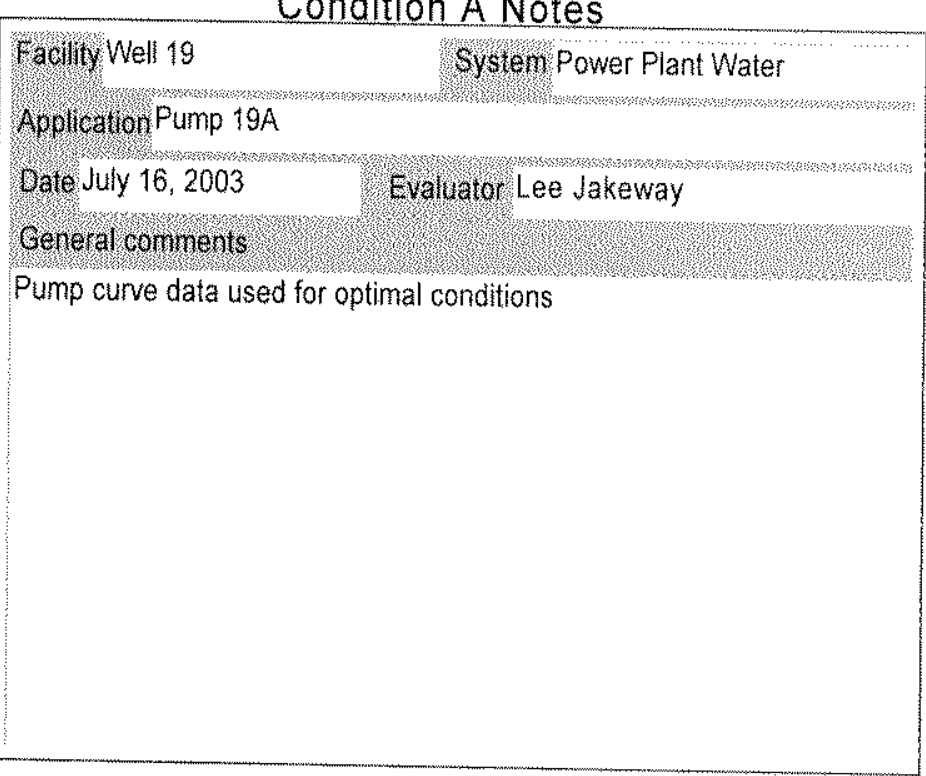

\section{Condition B Notes}

\section{Facility Well 19}

Application Pump 19A

Date July 16, $2003 \quad$ Evaluator Lee Jakeway

General comments

Data obtained when $19 \mathrm{~A}$ running singularly, 19B was down. No trouble experienced with power plant cooling. They were measuring about 15 psi going into TG5 generator cooler. Puno shat power, ho Motor effichency, of Molor power factor. $\%$ Motor current amps Moror power KWe Annula energy. Who Anulal cost $\$ 1,000$

Annual cost savings potential, $\$ 1,000$ Optimization rating 


\section{This PSAT2004 analysis was printed at 10:55 AM on Saturday, January 07, 2006}

Condition A

\begin{tabular}{|c|c|c|}
\hline Pump, nund data & Double suctio & on \\
\hline Yhed punp Yes & spece, rpni, $\mathbf{v}$ & 1180 \\
\hline specific speed? Wo & Dive Direct & drive $\nabla$ \\
\hline f slages 42 & pecific gravity & 1.000 \\
\hline 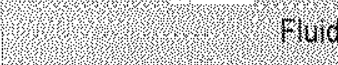 & hiscosity $(\cos )=$ & 1.00 \\
\hline Molor ralings & Notor no & $200 \nabla$ \\
\hline Existing notor class & Standard efficie & iency $\nabla$ \\
\hline prr 41180 & Rated voltage & 460 \\
\hline & Motor size margh & $\operatorname{rof} 415$ \\
\hline Duty cost rate & erating racions & 0.980 \\
\hline clectricty o & ost centskwhir & $\$ 15.000$ \\
\hline Required or neasured & data. & \\
\hline & flowrare gpms & 4900 \\
\hline & (t) & 120.0 \\
\hline $10 \mathrm{ad}$ est & maton nethod & Power \\
\hline Molor voliage a & Wotor wh & 128.0 \\
\hline & Existing & Optimal \\
\hline Pump efriciency \% & 92,1 & 91 1. \\
\hline Motor rated power. no & 200 & 200 \\
\hline Motor shatt power, ho & S & 1630 \\
\hline Pump siatt power ho & 1613 & 1630 \\
\hline Motor efficiency, ofo & 940 & 952 \\
\hline Motor power ractor ? & 83.2 & 830 \\
\hline Motor current amps & 1074 & 1072 \\
\hline Motor power. kWe & 1280 & 1276 \\
\hline Annual energy. nWh & 10988 & 10958 \\
\hline Annualcost $\$ 1.000$ & 1648 & 1644 \\
\hline Annual cost savings po & tential, $\$ 1,000$ & 06 \\
\hline & imization rating & 997 \\
\hline
\end{tabular}

\section{Condition B}

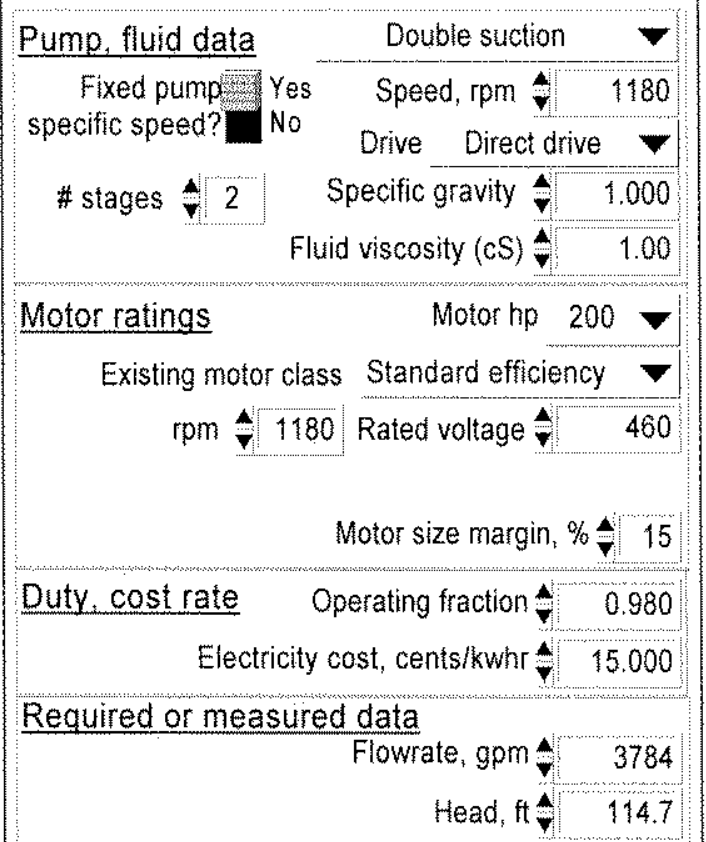

Load estimation method Power $\nabla$

Motor voltage $4 \quad 450 \quad$ Motor 15,2

\begin{tabular}{|c|c|c|}
\hline & Existing & Optimal \\
\hline Pump efficiency, $\%$ & 57.5 & 91.0 \\
\hline Motor rated power, hp & 200 & 150 \\
\hline Motor shaft power, hp & 190.5 & 120.5 \\
\hline Pump shaft power, hp & 190.5 & 120.5 \\
\hline Motor efficiency, \% & 94.0 & 95.1 \\
\hline Motor power factor, $\%$ & 83.8 & 82.6 \\
\hline Motor current, amps & 231.4 & 146.7 \\
\hline Motor power, kWe & 151.2 & 94.5 \\
\hline Annual energy, MWhr & 1298.0 & 811.0 \\
\hline Annual cost, $\$ 1,000$ & 194.7 & 121.6 \\
\hline \multicolumn{2}{|c|}{ Annual cost savings potential, $\$ 1,000$} & 73.1 \\
\hline \multicolumn{2}{|c|}{ Optimization rating } & 62.5 \\
\hline
\end{tabular}

\section{Condition A Notes}

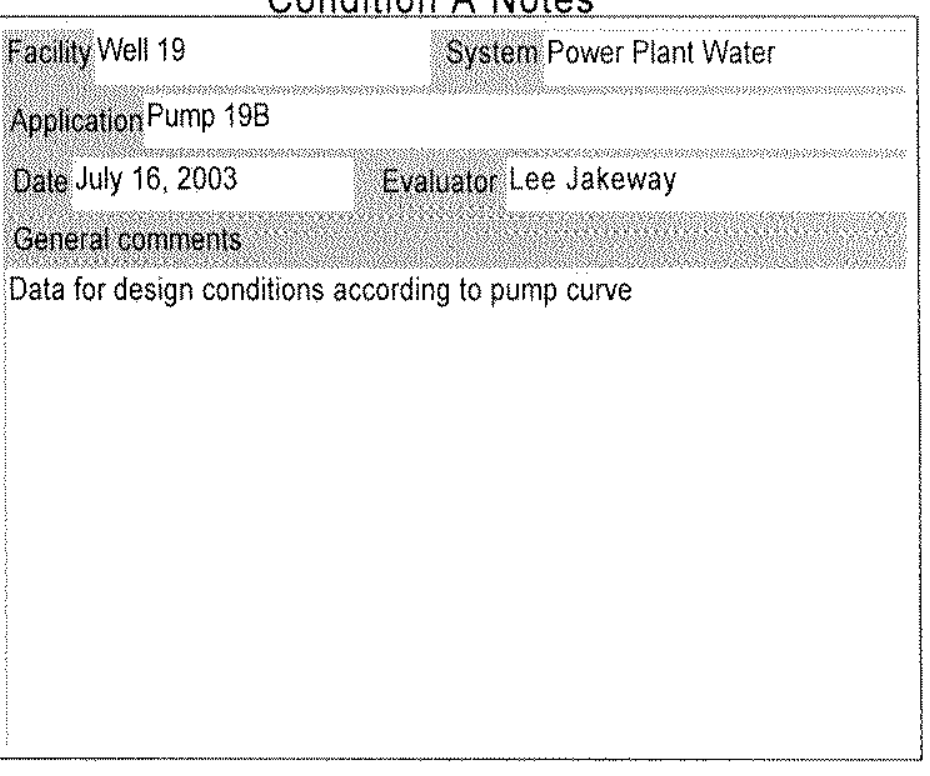

\section{Condition B Notes}

Facility Well $19 \quad$ System Power Plant Water

Application Pump 19B

Date July 16, $2003 \quad$ Evaluator Lee Jakeway

General comments

Data obtained when $19 \mathrm{~B}$ running singularly. Experienced trouble at first when some flow was going back to $19 \mathrm{~A}$ because of faulty check valve,

Problem was corrected by closing gate valve on 19A then taking

readings for $19 \mathrm{~B}$. 


\section{This PSAT2004 analysis was printed at 10:58 AM on Saturday, January 07, 2006}

\section{Condition A}

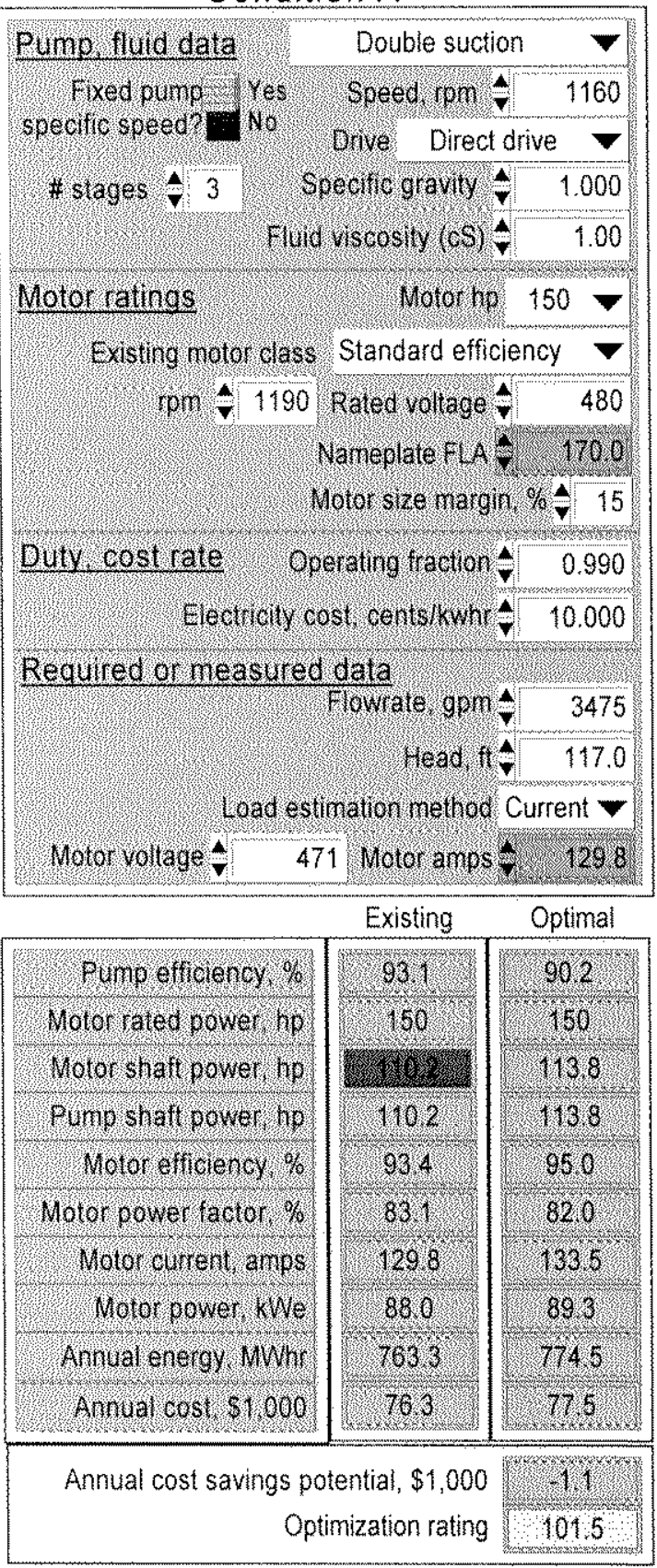

Condition B

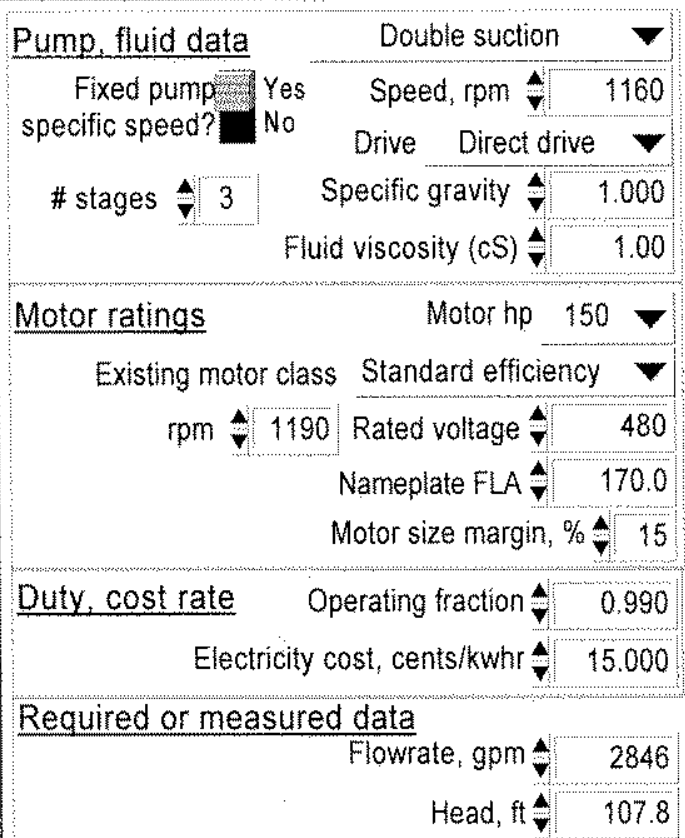

Load estimation method Current $\nabla$

Motor voltage $\boldsymbol{\nabla} \quad 471$ Motor amps $\boldsymbol{\nabla} 129.8$

\begin{tabular}{|c|c|c|}
\hline & Existing & Optimal \\
\hline Pump efficiency, $\%$ & 70.3 & 90.0 \\
\hline Motor rated power, hp & 150 & 100 \\
\hline Motor shaft power, hp & 110.2 & 86.1 \\
\hline Pump shaft power, hp & 110.2 & 86.1 \\
\hline Motor efficiency, $\%$ & 93.4 & 94.8 \\
\hline Motor power factor, $\%$ & 83.1 & 82.8 \\
\hline Motor current, amps & 129.8 & 100.2 \\
\hline Motor power, kWe & 88.0 & 67.7 \\
\hline Annual energy, MWhr & 763.3 & 587.1 \\
\hline Annual cost, $\$ 1,000$ & 114.5 & 88.1 \\
\hline \multirow{2}{*}{\multicolumn{2}{|c|}{$\begin{array}{r}\text { Annual cost savings potential, } \$ 1,000 \\
\text { Optimization rating }\end{array}$}} & 26.4 \\
\hline & & 76.9 \\
\hline
\end{tabular}

\section{Condition A Notes}

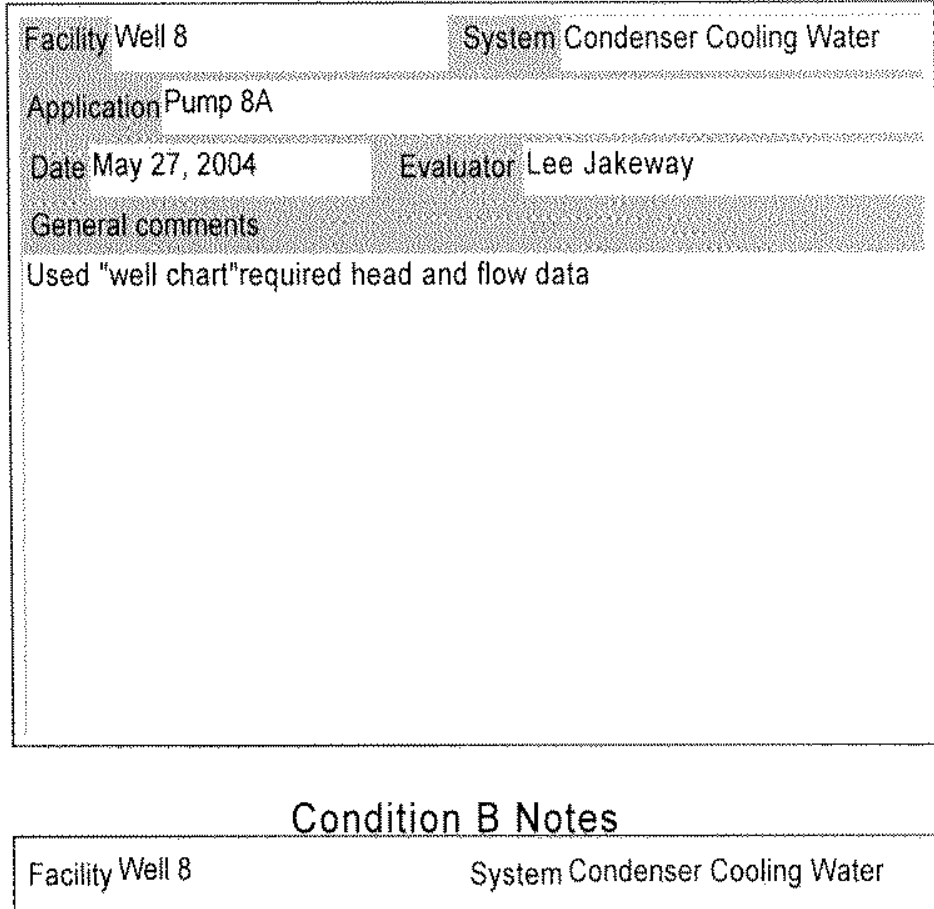

Application Pump $8 \mathrm{~A}$

Date May 27, $2004 \quad$ Evaluator Lee Jakeway

General comments

Pump $8 \mathrm{~A}$ flow determined by difference and prorated based on amperage reading with $8 \mathrm{~A}$ and $8 \mathrm{~B}$. Revised flow figure used from $7 /$ $28 / 04$ results 


\section{This PSAT2004 analysis was printed at 10:58 AM on Saturday, January 07, 2006}

Condition A

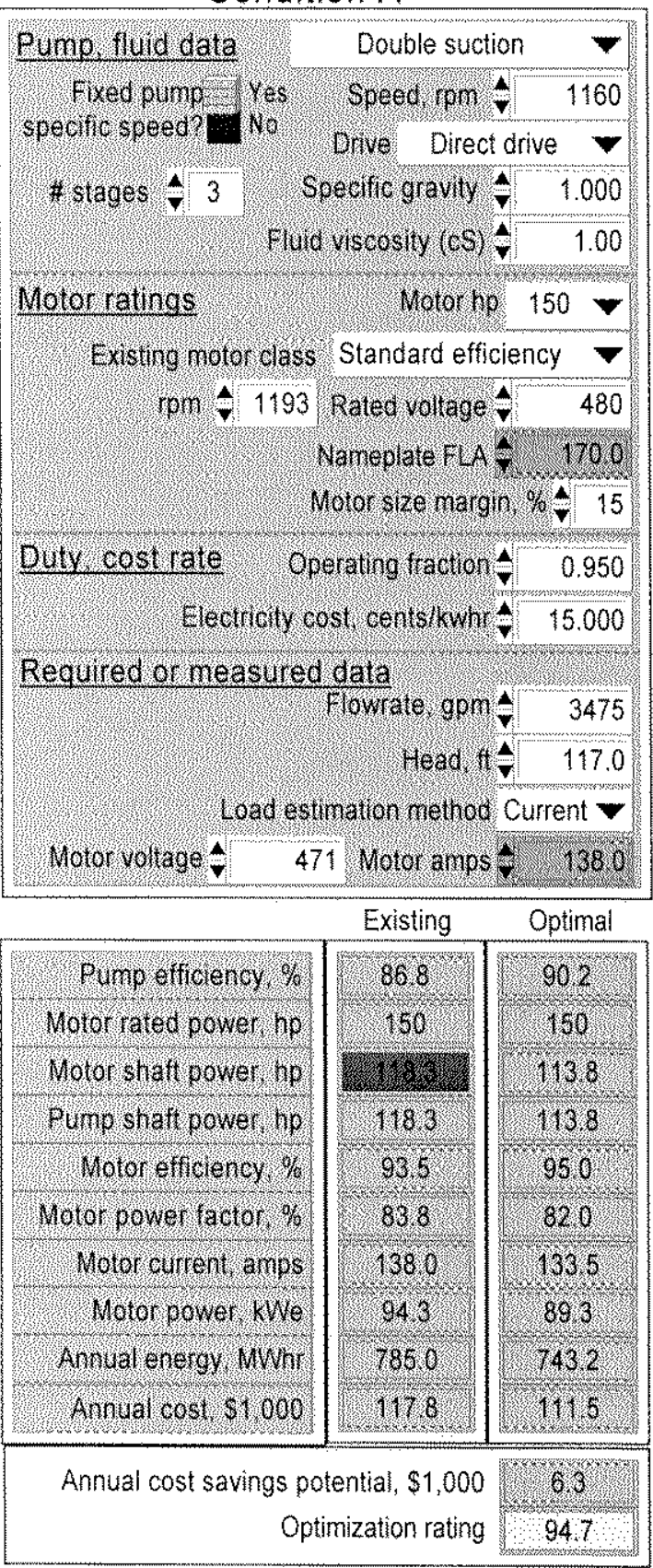

Condition B

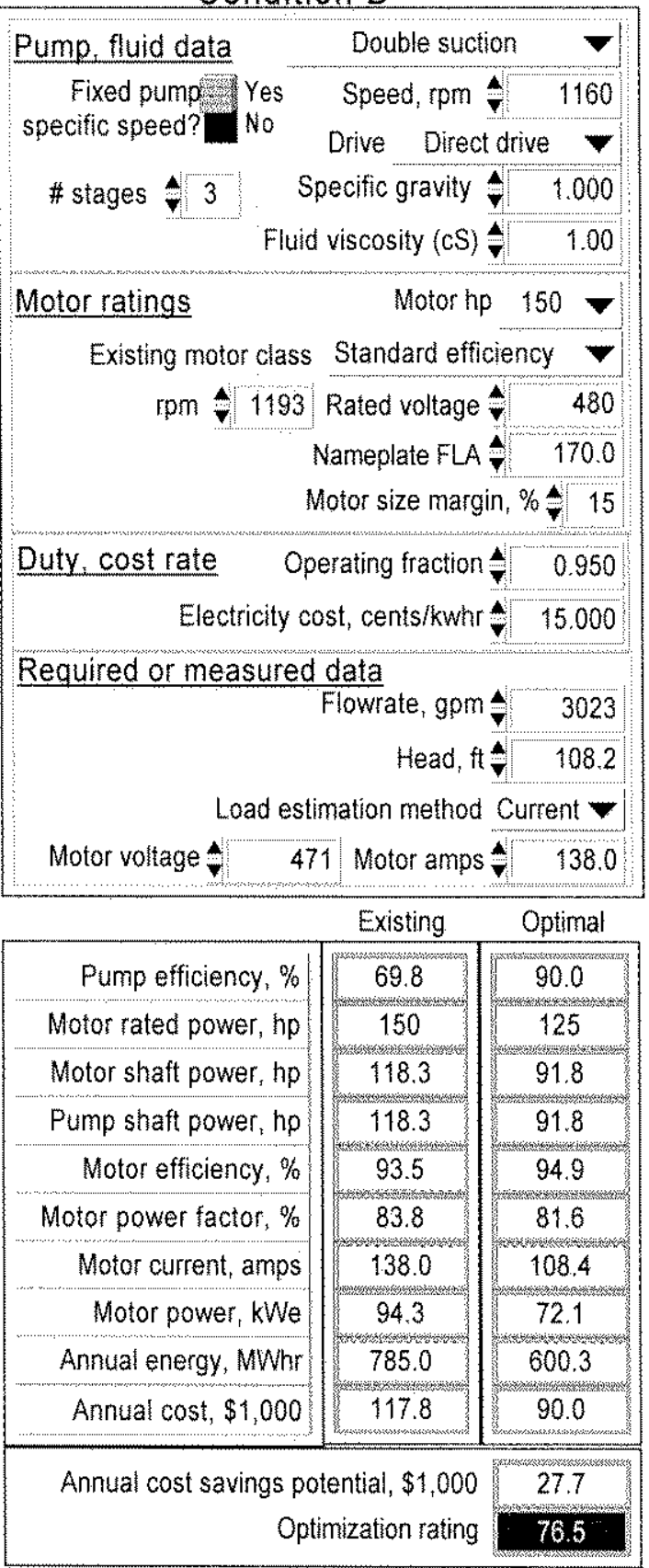

\section{Condition A Notes}

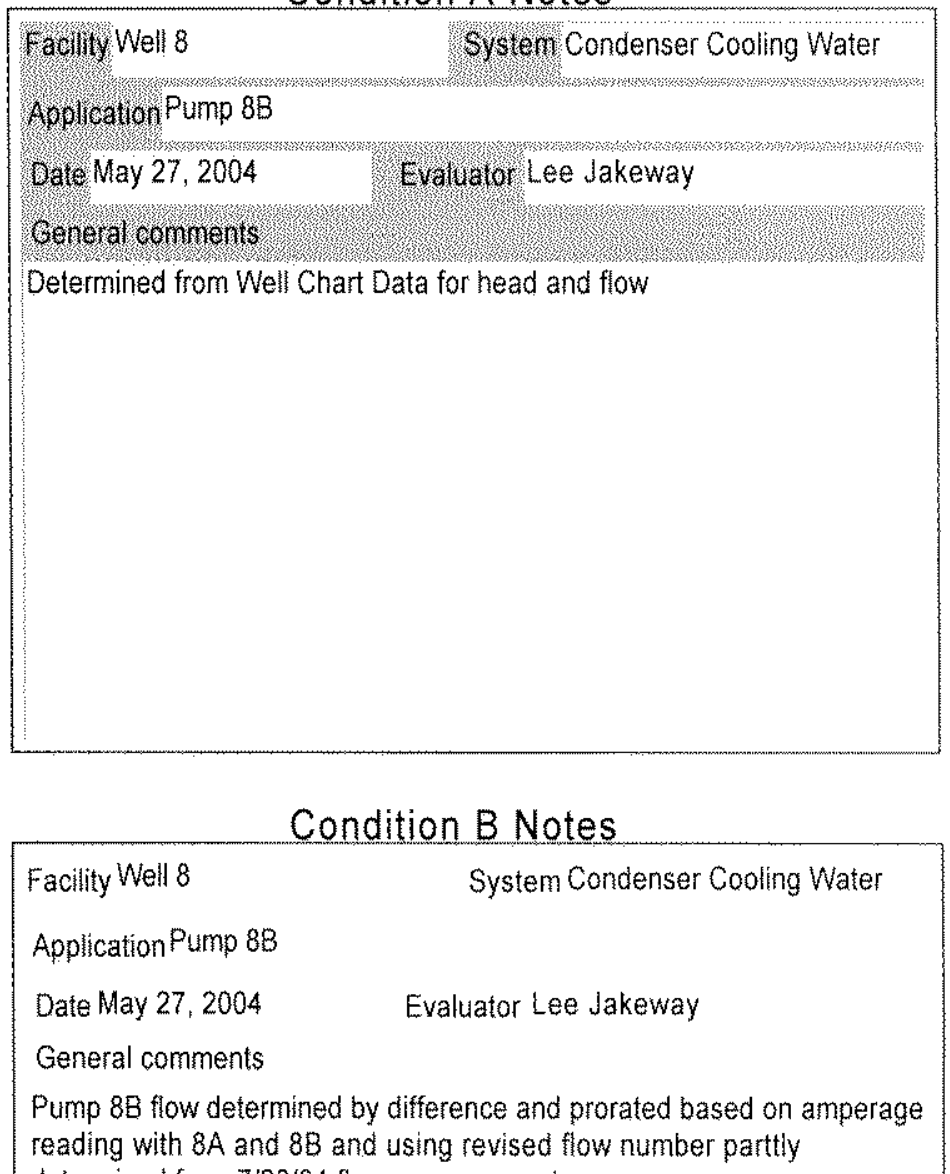

determined from $7 / 28 / 04$ flow measurements 


\section{This PSAT2004 analysis was printed at 10:58 AM on Saturday, January 07, 2006}

\section{Condition A}

\begin{tabular}{|c|c|c|}
\hline \multirow{5}{*}{ 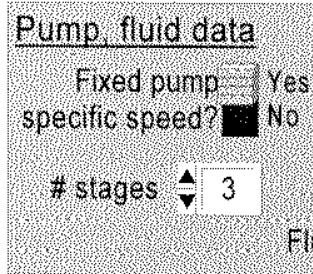 } & \multirow{2}{*}{\multicolumn{2}{|c|}{$\begin{array}{l}\text { Double suction } \\
\text { Soeed ron } \rightarrow-116\end{array}$}} \\
\hline & & \\
\hline & \multicolumn{2}{|c|}{ Drve Direct drive } \\
\hline & \multicolumn{2}{|c|}{ Specific gravity $\rightarrow \quad 1.000$} \\
\hline & \multicolumn{2}{|c|}{ Fluro viscosity $(\mathrm{cs}) \boldsymbol{4}$} \\
\hline Motor ratings & \multicolumn{2}{|c|}{ Motor hp 150} \\
\hline \multirow{2}{*}{\multicolumn{3}{|c|}{ 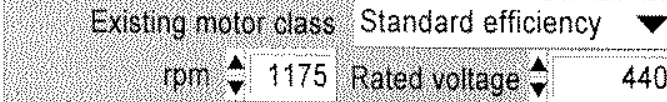 }} \\
\hline & & \\
\hline & \multicolumn{2}{|c|}{ 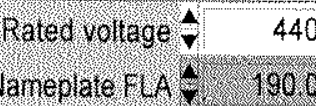 } \\
\hline & \multicolumn{2}{|c|}{ Motor size margin, o $\ 15$} \\
\hline \multirow{2}{*}{\multicolumn{3}{|c|}{ 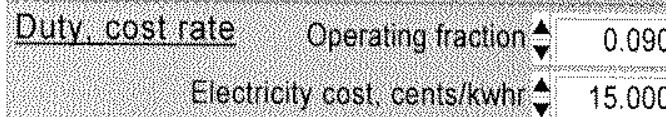 }} \\
\hline & & \\
\hline \multirow{5}{*}{\multicolumn{3}{|c|}{ 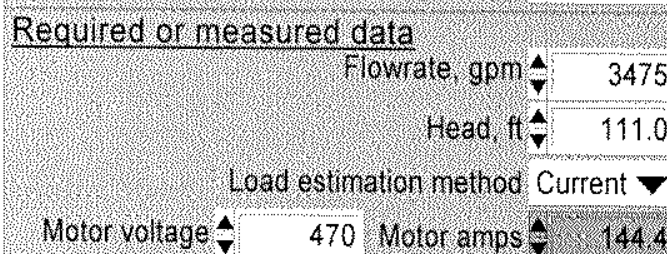 }} \\
\hline & & \\
\hline & & \\
\hline & & \\
\hline & & \\
\hline \multicolumn{3}{|c|}{ Existing } \\
\hline Punp efficiency. \% & 840 & 900 \\
\hline Motor rated power hp & 150 & 125 \\
\hline Notor shaft power hp & $21 x^{2}$ & 1082 \\
\hline Pump shaft power, hp & 1159 & 1082 \\
\hline Motor efficiency \% & 93.5 & 950 \\
\hline Motor power tactor \% & 787 & 812 \\
\hline Notor current, amps & 1444 & 1285 \\
\hline Motor power, kWe? & 925 & 84.9 \\
\hline Annual energy WWhr & 72.9 & 670 \\
\hline Annual cost $\$ 1,000$ & 10.9 & 100 \\
\hline Annual cost savings & ial, $\$ 1,000$ & 0.9 \\
\hline & nization rating & 91.9 \\
\hline
\end{tabular}

\section{Condition B}

\begin{tabular}{|c|c|c|c|c|}
\hline Pump, fluid data & & \multicolumn{2}{|c|}{ Double suction } & $\nabla$ \\
\hline \multirow{2}{*}{$\begin{array}{l}\text { Fixed pump } \\
\text { specific speed? }\end{array}$} & \multirow{2}{*}{$\begin{array}{l}\text { Yes } \\
\text { No }\end{array}$} & \multicolumn{2}{|c|}{ Speed $_{1} \mathrm{rpm} \mathbf{\nabla}$} & 1160 \\
\hline & & Drive & Direct drive & $\nabla$ \\
\hline \multirow[t]{2}{*}{ \# stages 43} & \multicolumn{3}{|c|}{ Specific gravity } & 1.000 \\
\hline & \multicolumn{3}{|c|}{ Fluid viscosity $(\mathrm{cS})$} & 1.00 \\
\hline
\end{tabular}

$$
\begin{aligned}
& \text { Motor ratings Motor hp } 150 \nabla \\
& \text { Flowrate, gpm } \mathbf{\gamma} 2177 \\
& \text { Head, } \mathrm{ft} \boldsymbol{\nabla} 107.8
\end{aligned}
$$

\begin{tabular}{|c|c|c|}
\hline & Existing & Optimal \\
\hline Pump efficiency, $\%$ & 51.1 & 89.8 \\
\hline Motor rated power, hp & 150 & 100 \\
\hline Motor shaft power, hp & 115.9 & 66.0 \\
\hline Pump shaft power, hp & 115.9 & 66.0 \\
\hline Motor efficiency, $\%$ & 93.5 & 94.7 \\
\hline Motor power factor, $\%$ & 78.7 & 75.4 \\
\hline Motor current, amps & 144.4 & 84.6 \\
\hline Motor power, $\mathrm{kWe}$ & 92.5 & 51.9 \\
\hline Annual energy, MWhr & 72.9 & 40.9 \\
\hline Annual cost, $\$ 1,000$ & 10.9 & 6.1 \\
\hline \multicolumn{2}{|c|}{ Annual cost savings potential, $\$ 1,000$} & 4.8 \\
\hline \multicolumn{2}{|c|}{ Optimization rating } & 56.2 \\
\hline
\end{tabular}

Load estimation method Current $\mathbf{\nabla}$

Motor voltage $\boldsymbol{\nabla} \quad 470$ Motor amps $\boldsymbol{\nabla} \quad 144.4$
Condition A Notes

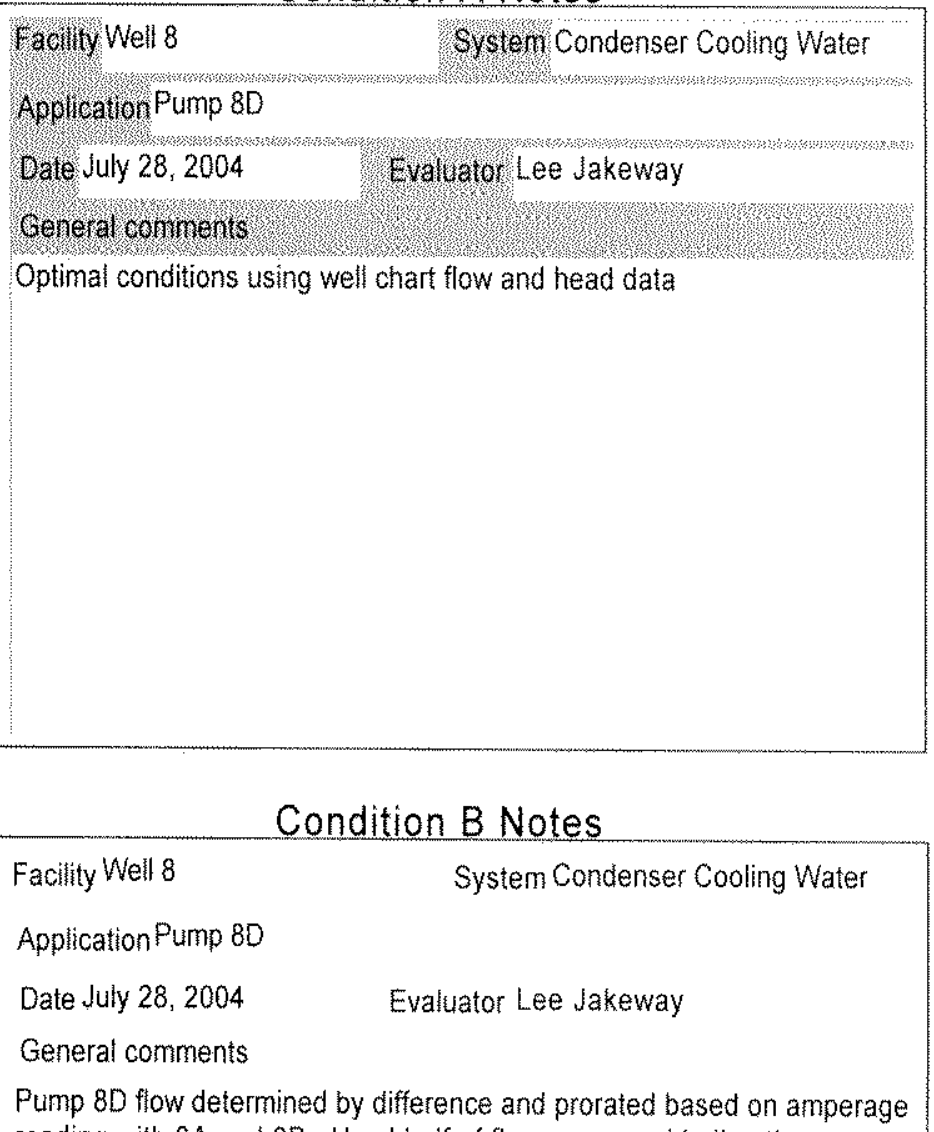

reading with $8 \mathrm{~A}$ and $8 \mathrm{~B}$. Used half of flow measured indirectly 


\section{This PSAT2004 analysis was printed at 11:00 AM on Saturday, January 07, 2006}

\section{Condition A}

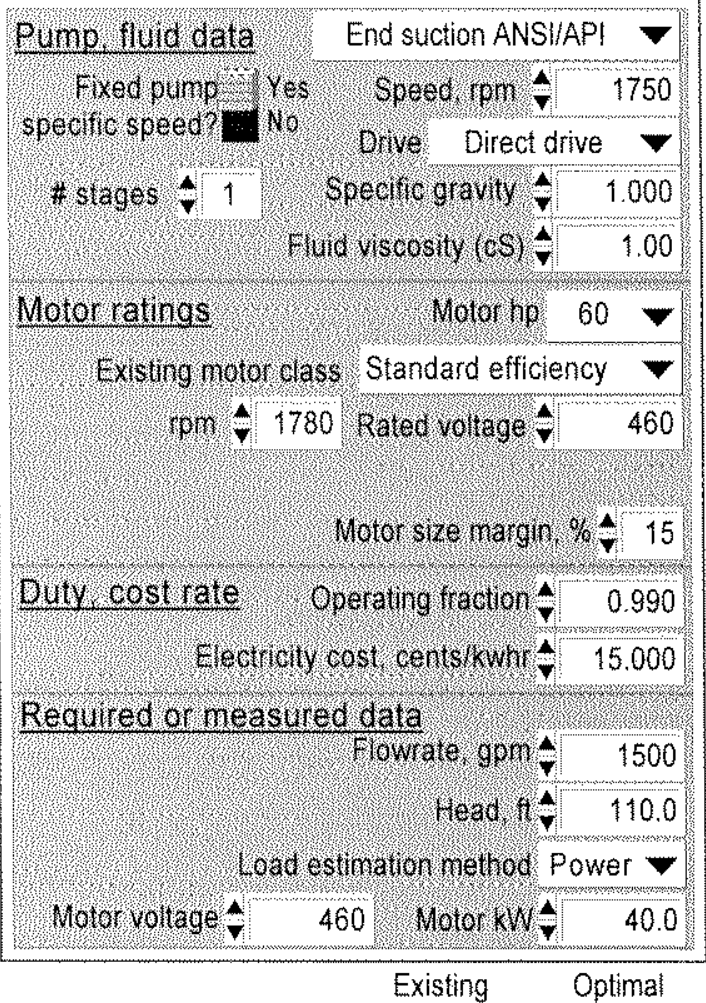

\begin{tabular}{|c|c|c|}
\hline & Existin & paris \\
\hline Pump eficiency. \% & 846 & 860 \\
\hline Motor rated power, ho & 60 & 60 \\
\hline Molor shatipower hp & C. & 48.4 \\
\hline Pump shati power, hp & 49.3 & 48.4 \\
\hline Notor efficiency \% & 919 & $944^{2}$ \\
\hline Notor power ractor, ch & 840 & 84,4 \\
\hline Motor current, amps & 50.7 & 668 \\
\hline Motor power kne & 40,0 & 38.3 \\
\hline Annual energy Whir & 3469 & 3318 \\
\hline Annual cost st 000 & 52.0 & 498 \\
\hline \multicolumn{2}{|c|}{ Annual cost savings potential, $\$ 1,000$} & 23 \\
\hline \multicolumn{2}{|c|}{ Optimization rating } & 957 \\
\hline
\end{tabular}

\section{Condition B}

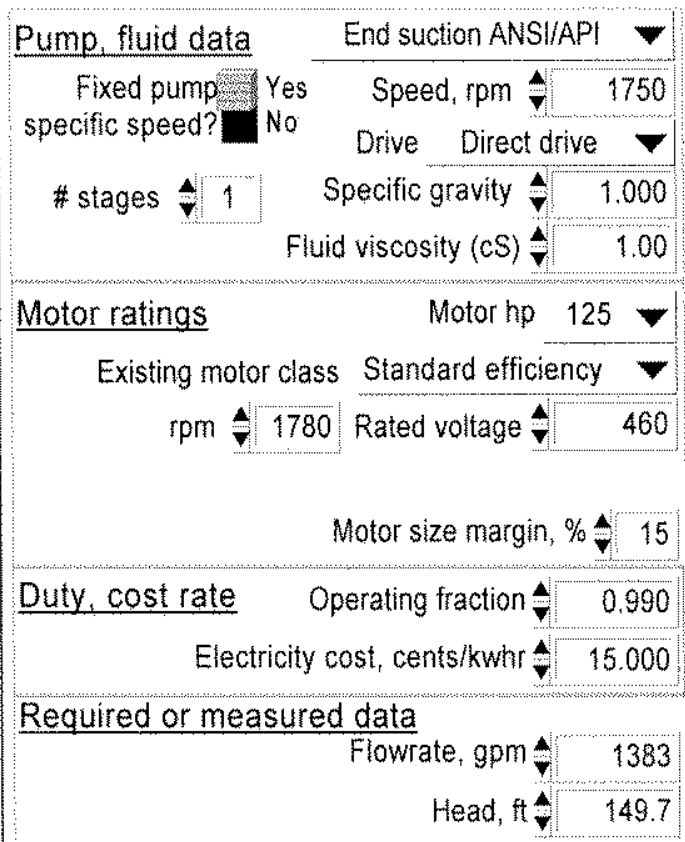

Load estimation method Power $\boldsymbol{\nabla}$

Motor voltage $\frac{1}{\nabla} \quad 460 \quad$ Motor 807

\begin{tabular}{|c|c|c|}
\hline & Existing & Optimal \\
\hline Pump efficiency, $\%$ & 69.3 & 85.0 \\
\hline Motor rated power, hp & 125 & 75 \\
\hline Motor shaft power, hp & 75.5 & 61.5 \\
\hline Pump shaft power, hp & 75.5 & 61.5 \\
\hline Motor efficiency, $\%$ & 92.8 & 94.7 \\
\hline Motor power factor, $\%$ & 81.9 & 85.0 \\
\hline Motor current, amps & 92.9 & 71.4 \\
\hline Motor power, kWe & 60.7 & 48.4 \\
\hline Annual energy, MWhr & 526.4 & 419.9 \\
\hline Annual cost, $\$ 1,000$ & 79.0 & 63.0 \\
\hline
\end{tabular}

Annual cost savings potential, $\$ 1,000$ Optimization rating

\section{Condition A Notes}

Pacily Equip No. $7717 \quad$ Systen Wet Scrubber
Applicaton Optimized wet scrubber system supply pump
Gate October $21,2004 \quad$ Evaluator Lee Jakeway
Gentrat cornments

using existing pump BEP for head, $110^{\prime}$ and estimated flow required for wet scrubber systems based on measured flow after Bir.
wet scrubber recycle system installed.

\section{Condition B Notes}

\section{Facility Equip No. $7717 \quad$ System Wet Scrubber}

Application Wet scrubber system supply pump

\section{Date October 21, $2004 \quad$ Evaluator Lee Jakeway}

General comments

Valve was nearly fully closed on Blr 3 supply and partially open on Blr 1\&2 supply with actual power readings that were taken shortly after using the multi-lin recorder over a 90 minute period. 


\section{This PSAT2004 analysis was printed at 11:03 AM on Saturday, January 07, 2006}

\section{Condition A}

\begin{tabular}{|c|c|c|}
\hline Pumo, filid data & Vertical turbine & $\nabla$ \\
\hline Fixed pump - yes & Specer ron $\oplus$ & 1785 \\
\hline specific speed? No & Drve Direct $\mathrm{d}$ & ive $\nabla$ \\
\hline 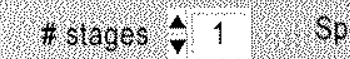 & encile gravity & 1.000 \\
\hline Pr. & Viscosity $(\operatorname{cs}) 4$ & 1.00 \\
\hline Molor ratings & Nolor hp & $250 \nabla$ \\
\hline Existing notor class & Energy efficient & $\checkmark$ \\
\hline $\operatorname{prr} 41775)^{2}$ & Rated roluge & 2300 \\
\hline (1) & lotor size nargi & \% 15 \\
\hline Duly, cost rate & erating raction $\mathbf{v}$ & 0.800 \\
\hline Electricity co & st. gentsikwhrt & 15.000 \\
\hline Required or measured & data & \\
\hline & Fowrate gpru & 8000 \\
\hline & Head d 14 & 100.0 \\
\hline Load estin & mation nethod $\mathrm{P}$ & ower $\nabla$ \\
\hline Motor voltage 4 & Notor kWA & 172.0 \\
\hline & Existing & Optimal \\
\hline Pump eficiency \% & 914 & 90.7 \\
\hline Motor rated power, ho & 250 & 300 \\
\hline Motor shatt power. hp. & 52 & 2228 \\
\hline Pump shart power. np & 221.0 & 2228 \\
\hline Notor efficency, o & 959 & 958 \\
\hline Molor power ractor of & 86.1 & 84.7 \\
\hline Motor current amps & 497 & 509 \\
\hline Molor power We & $17 \%$ & 173 \\
\hline Annual energy Whrr & 1205.4 & 12147. \\
\hline Annual cost st o00 & 1800 & $\begin{array}{l}182 \\
7 \\
2\end{array}$ \\
\hline Annual cost savings pot & tential, $\$ 1,000$ & 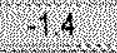 \\
\hline & imization rating & 100.8 \\
\hline
\end{tabular}

\section{Condition B}

\begin{tabular}{|c|c|c|c|c|}
\hline Pump fluid data & & \multicolumn{2}{|c|}{ Vertical turbine } & $\gamma$ \\
\hline \multirow{2}{*}{$\begin{array}{l}\text { Fixed pump } \\
\text { speciffc speed? }\end{array}$} & Yes & \multicolumn{2}{|c|}{ Speed, rpm $\widehat{\nabla}$} & 1785 \\
\hline & No & Drive & Direct drive & $\nabla$ \\
\hline \multirow[t]{2}{*}{ \# stages $\hat{\mathbf{Y}}$} & \multicolumn{3}{|c|}{ Specific gravity } & 1.000 \\
\hline & \multicolumn{3}{|c|}{ Fluid viscosity (cS) $\hat{\nabla}$} & 1.00 \\
\hline
\end{tabular}

Motor ratings Motorhp 250

Existing motor class Standard efficiency rpm $* 1775$ Rated voltage $\frac{\mathbf{\nabla}}{\mathbf{\gamma}} 2300$ Nameplate FLA 휘 $\quad 58.5$ Motor size margin, \% 15 Duty cost rate Operating fraction $\sqrt{\mathbf{V}} \quad 0.800$ Electricity cost, cents/kwhr $\boldsymbol{\nabla} \quad 15.000$ Required or measured data

$$
\begin{aligned}
& \text { Flowrate, gpm } 4750 \\
& \text { Head, } \mathrm{ft}-63.4
\end{aligned}
$$

Load estimation method Current $\boldsymbol{\nabla}$

\begin{tabular}{|c|c|c|}
\hline & Existing & Optimal \\
\hline Pump efficiency, $\%$ & 39.2 & 89.6 \\
\hline Motor rated power, hp & 250 & 100 \\
\hline Motor shaft power, hp & 194.3 & 84.9 \\
\hline Pump shaft power, hp & 194.3 & 84,9 \\
\hline Motor efficiency, $\%$ & 94.2 & 95.1 \\
\hline Motor power factor, $\%$ & 83.2 & 85.4 \\
\hline Motor current, amps & 46.0 & 19.4 \\
\hline Motor power, kWe & 153.8 & 66.6 \\
\hline Annual energy, MWhr & 1078.1 & 466.8 \\
\hline Annual cost, $\$ 1,000$ & 161.7 & 70.0 \\
\hline \multicolumn{2}{|c|}{ Annual cost savings potential, $\$ 1,000$} & 91.7 \\
\hline \multicolumn{2}{|c|}{ Optimization rating } & 43.3 \\
\hline
\end{tabular}

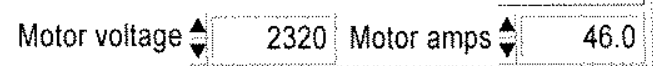

\section{Condition A Notes}

\section{Facility Pump No. $6170 \quad$ Systen Factory Vertical Pumps}

Application Spray pond recirculation system

Date September 28, 2005 Evaluator Lee Jakeway

Ceneral comnents

Optimized conditions using pump curve information. Pump no. 6170 is one of four vertical pumps that pump boiling house condenser cooling water back to the spray pond for cooling. Water returns to factory and is then pumped to boiling house evaporators and pans via booster pumps. Three other vertical pumps were operating during the test.

\section{Condition B Notes}

\section{Facility Pump No.6170 System Factory Vertical Pumps}

Application Spray pond recirculation system

Date September 28, 2005 Evaluator Lee Jakeway

General comments

Actual operating conditions. Pump no. 6170 is one of four vertical pumps that pump bolling house condenser cooling water back to the spray pond for cooling. Water returns to factory and is then pumped to boiling house evaporators and pans via booster pumps. Three other vertical pumps were operating during the test. Flow measured with flow meter using Doppler feature. Electrical readings taken from board panel.

Reportedly, pump shafts were shortened thus modifying pump output curve 


\section{This PSAT2004 analysis was printed at 11:04 AM on Saturday, January 07, 2006}

\section{Condition A}

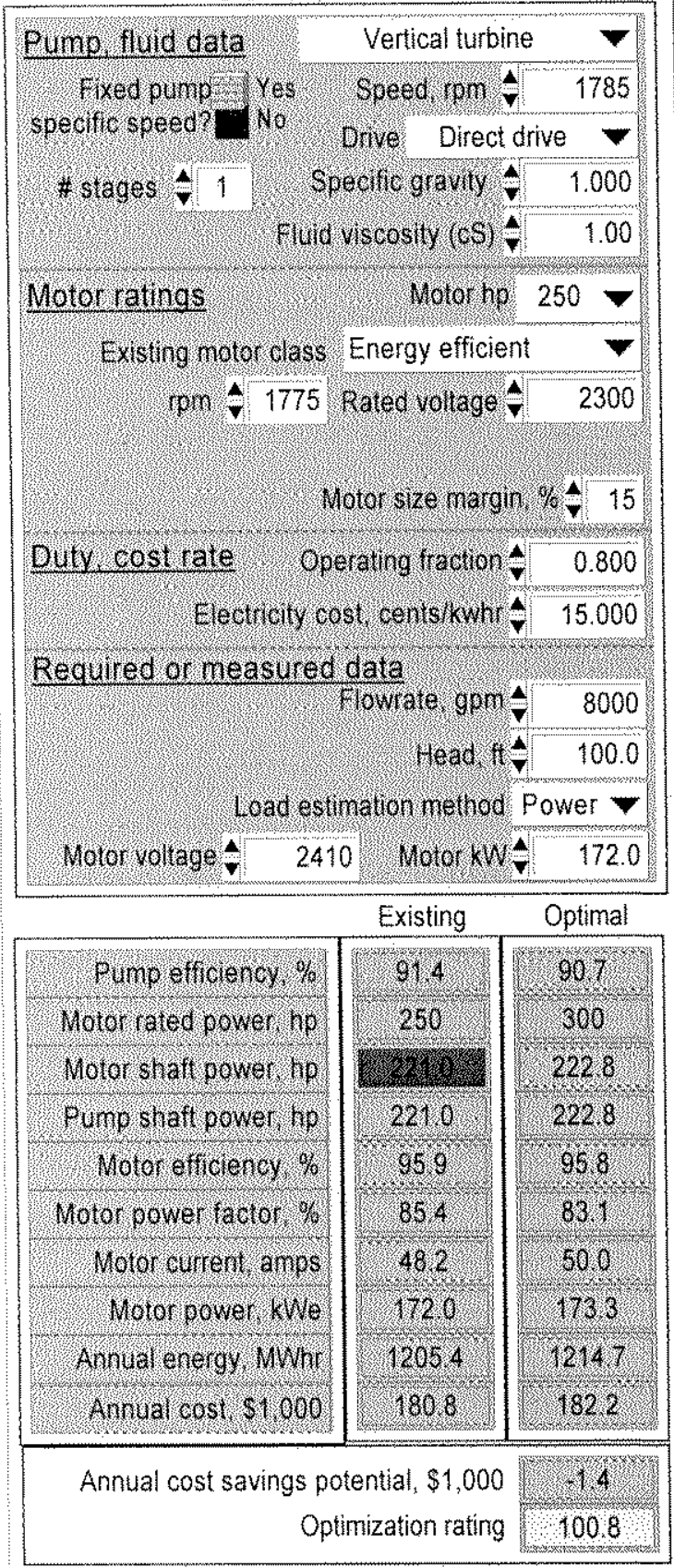

\section{Condition B}

\begin{tabular}{|c|c|c|c|c|}
\hline Pump, fluid data & & \multicolumn{3}{|c|}{ Vertical turbine } \\
\hline \multirow{2}{*}{$\begin{array}{c}\text { Fixed pump } \\
\text { specific speed? }\end{array}$} & Yes & \multicolumn{2}{|c|}{ Speed, rpm $\hat{\nabla}$} & 1785 \\
\hline & No & Drive & Direct drive & $\psi$ \\
\hline \multirow{2}{*}{ \# stages $\mathbf{v} 1$} & \multirow{2}{*}{\multicolumn{3}{|c|}{$\begin{array}{l}\text { Specific gravity } \hat{\nabla} \\
\text { uid viscosity (cS) }\end{array}$}} & 1.000 \\
\hline & & & & 1.00 \\
\hline
\end{tabular}

Motor ratings $\quad$ Motor hp 250

Existing motor class Standard efficiency

$\mathrm{rpm} * 1775$ Rated voltage $\boldsymbol{*} 2300$

Nameplate FLA $\mathbf{\nabla} \quad 58.5$

Motor size margin, $\%$ A 15

Duty, cost rate Operating fraction $\mathbb{\nabla} 0.800$ Electricity cost, cents $/ \mathrm{kwhr} \underset{\mathbf{\nabla}}{ } 15.000$

\section{Required or measured data}

$$
\begin{array}{r}
\text { Flowrate, gpm } \frac{4}{7} \quad 4500 \\
\text { Head, } \mathrm{ft} \approx \\
\mathbf{7}
\end{array}
$$

Load estimation method Current $\nabla$

\begin{tabular}{|c|c|c|}
\hline & Existing & Optimal \\
\hline Pump efficiency, $\%$ & 66.6 & 89.3 \\
\hline Motor rated power, $h p$ & 250 & 100 \\
\hline Motor shaft power, hp & 100.3 & 74.8 \\
\hline Pump shaft power, hp & 100.3 & 74.8 \\
\hline Motor efficiency, $\%$ & 92.5 & 95.1 \\
\hline Motor power factor, $\%$ & 69.2 & 82.5 \\
\hline Motor current, amps & 28.0 & 17.0 \\
\hline Motor power, kWe & 80.9 & 58.7 \\
\hline Annual energy, MWhr & 566.8 & 411.1 \\
\hline Annual cost, $\$ 1,000$ & 85.0 & 61.7 \\
\hline
\end{tabular}

Motor voltage $\boldsymbol{A} \quad 2410$ Motor amps $\frac{\hat{\boldsymbol{v}}}{\mathbf{v}} \quad 28.0$

Annual cost savings potential, $\$ 1,000$ Optimization rating

\section{Condition A Notes}

Facily Pump $6163 \quad$ Systern Factory Vertical Pumps

Application Spray pond recirculation system

Date September 28, 2005 Wvalualor Lee Jakeway

General somnuents

Optimal operating conditions using pump curve data. Pump no. 6163 is one of four vertical pumps that pumps boiling house condenser cooling water back to the spray pond for cooling. Water returns to factory and is then pumped to boiling house evaporators and pans via booster pumps. Three other vertical pumps were operating during the test.

\section{Condition B Notes}

\section{Facility Pump $6163 \quad$ System Factory Vertical Pumps}

Application Spray pond recirculation system

Date September 28,2005 Evaluator Lee Jakeway

General comments

Actual operating conditions. Pump no. 6163 is one of four vertical pumps that pumps boiling house condenser cooling water back to the spray pond for cooling. Water returns to factory and is then pumped to boiling house evaporators and pans via booster pumps. Three other vertical pumps were operating during the test. Pump flow estimated from 2002 combined flow data. Electrical readings obtained from board readings 


\section{This PSAT2004 analysis was printed at 11:04 AM on Saturday, January 07, 2006}

\section{Condition A}

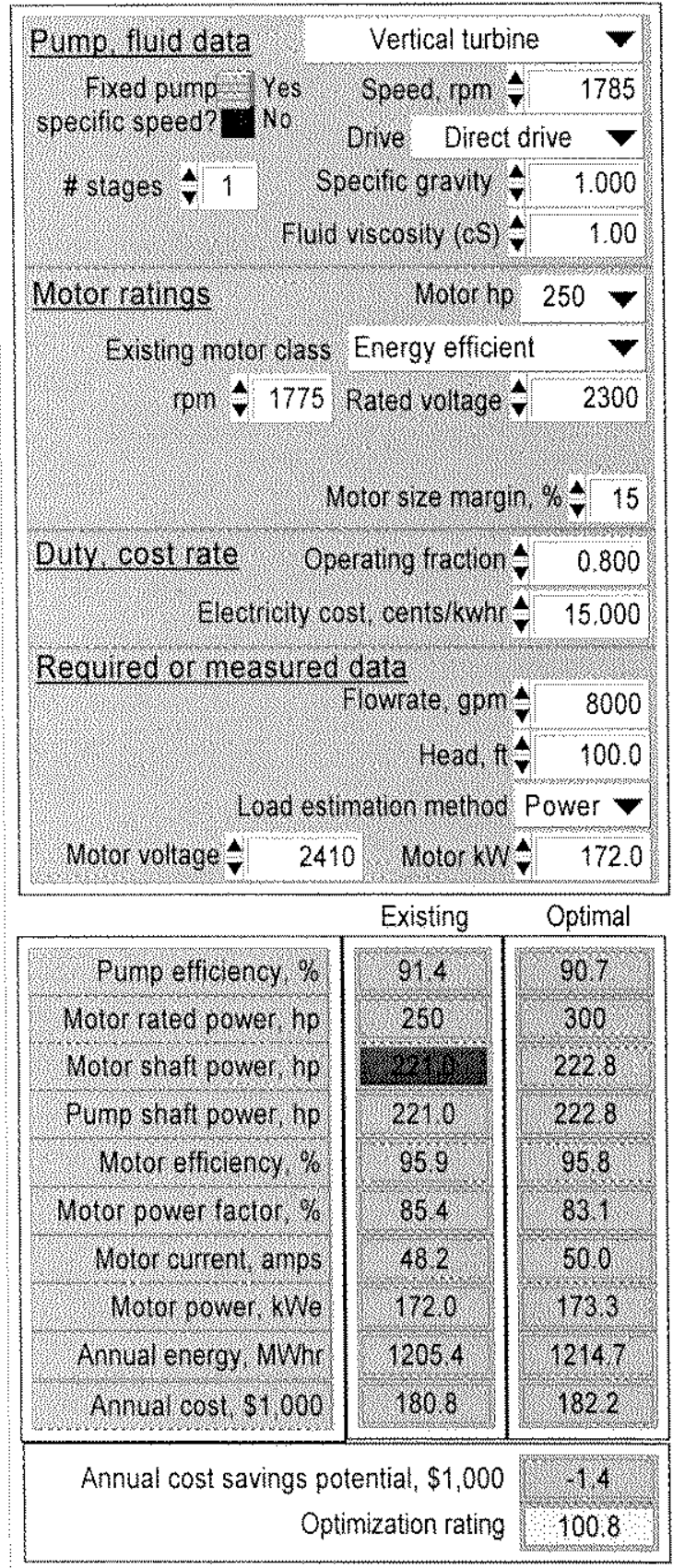

Condition B

\begin{tabular}{|c|c|c|c|c|}
\hline Pump, fluid data & & \multicolumn{2}{|c|}{ Vertical turbine } & $\nabla$ \\
\hline \multirow{2}{*}{$\begin{array}{c}\text { Fixed pump } \\
\text { specific speed? }\end{array}$} & Yes & \multicolumn{2}{|c|}{ Speed, rpm } & 1785 \\
\hline & No & Drive & Direct drive & $\nabla$ \\
\hline \multirow[t]{2}{*}{ \# stages $\|$} & & Specific & avity $\mathbf{v}$ & 1.000 \\
\hline & & lid visc & $(\mathrm{cS}) *$ & 1.00 \\
\hline
\end{tabular}

Motor ratings Motorhp $250 \nabla$

Existing motor class Standard efficiency $\mathrm{rpm} \hat{\boldsymbol{\gamma}} 1775$ Rated voltage $\sqrt{\mathrm{V}} \quad 2300$ Nameplate FLA $\mathbf{\nabla} \quad 58.5$ Motor size margin, \% 4 ㄴ 15

\begin{tabular}{lll} 
Duty, cost rate & Operating fraction $\mathbb{\nabla}$ & 0.800 \\
\hline
\end{tabular} Electricity cost, cents/kwhr $\mathbf{\Delta} \quad 15.000$ Required or measured data

$$
\begin{aligned}
& \text { Flowrate, gpm } \mathbf{\nabla} \quad 4500 \\
& \text { Head, ft } 49.6
\end{aligned}
$$

Load estimation method Current $\boldsymbol{\nabla}$

\begin{tabular}{|c|c|c|}
\hline & Existing & Optimal \\
\hline Pump efficiency, $\%$ & 26.8 & 88.5 \\
\hline Motor rated power, hp & 250 & 75 \\
\hline Motor shaft power, hp & 210.1 & 63.7 \\
\hline Pump shaft power, hp & 210.1 & 63.7 \\
\hline Motor efficiency, $\%$ & 94.3 & 94.7 \\
\hline Motor power factor, $\%$ & 83.0 & 84.3 \\
\hline Motor current, amps & 48.0 & 14.3 \\
\hline Motor power, kWe & 166.3 & 50.2 \\
\hline Annual energy, MWhr & 1165.1 & 351.5 \\
\hline Annual cost, $\$ 1,000$ & 174.8 & 52.7 \\
\hline
\end{tabular}

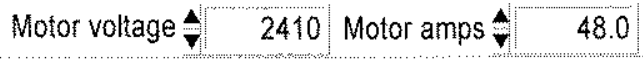

Annual cost savings potential, $\$ 1,000$ Optimization rating

\section{Condition A Notes}

Tacily Pump 6166 System Factory Vertical Pumps

Apolication Spray pond recirculation system

Dat september 28, 2005 , traluator Lee Jakeway

\section{Ceneral ormments}

Optimal operating conditions using pump curve data. Pump no. 6166 is one of four vertical pumps that pumps boiling house condenser cooling water back to the spray pond for cooling. Water returns to factory and is then pumped to boiling house evaporators and pans via booster pumps. Three other vertical pumps were operating during the test.

\section{Condition B Notes}

\section{Facility Pump $6166 \quad$ System Factory Vertical Pumps}

Application Spray pond recirculation system

Date September 28, 2005 Evaluator Lee Jakeway

General comments

Actual operating conditions. Pump no. 6166 is one of four vertical pumps that pumps bolling house condenser cooling water back to the spray pond for cooling. Water returns to factory and is then pumped to boiling house evaporators and pans via booster pumps. Three other vertical pumps were operating during the test. Pump flow was estimated based on 2002 operating data. Electrical readings were taken from the board 


\section{This PSAT2004 analysis was printed at 11:04 AM on Saturday, January 07, 2006}

\section{Condition A}

\begin{tabular}{|c|c|c|}
\hline Pump fluid data & Vertical turbin & $\nabla$ \\
\hline Fixed pump - yes & Speed rpm $\hat{\mathbf{V}}$ & 1785 \\
\hline $21.1 \mathrm{NO}$ & Dive Direct 0 & rive $\boldsymbol{\nabla}$ \\
\hline H stages $\div 1$ & becific gravily $\hat{\imath}$ & 1.000 \\
\hline fuid & viscosity $(\operatorname{cs}) \hat{y}$ & 1.00 \\
\hline Motor ratings & Notor ho & $250 \nabla$ \\
\hline Existing motor class & Energy efficien & $\nabla$ \\
\hline on 41775 & Rated voltage $\mathbf{y}$ & \\
\hline & totor size nargin & 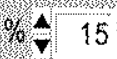 \\
\hline Duty cost rate & rating fraction & 0.800 \\
\hline Electricity co & st. centskwhr & 15.000 \\
\hline Required or measured & data & \\
\hline & Flowrate gpn 4 & 8000 \\
\hline & Head $\pi \hat{v}$ & 100.0 \\
\hline Load estin & mation method $\mathrm{f}$ & ower $\mathbf{r}$ \\
\hline Motor voltage -230 & 5 Notor $\mathrm{W}=$ & 172.0 \\
\hline & Existing & Optimal \\
\hline Punp efficiency, $\%$ & 914 & 907 \\
\hline Motor rated pover, hp & 250 & 300 \\
\hline Motor shaft power hp & Tritu & 2228 \\
\hline Pump shatt power hp & 2210 & 2228 \\
\hline Molor efficiency. \% & 959 & 958 \\
\hline Motor power factor. \% & 86.2 & 84.9 \\
\hline Motor current amps & 50.0 & 5111 \\
\hline Motor power, we & 1720 & 173.3 \\
\hline Annual energy MWh & 12054 & 12147 \\
\hline Annual cost \$1 000 & 180.8 & 1822 \\
\hline Annual cost savings pot & ential, $\$ 1,000$ & 1.4 \\
\hline & nization rating & 100.8 \\
\hline
\end{tabular}

\section{Condition B}

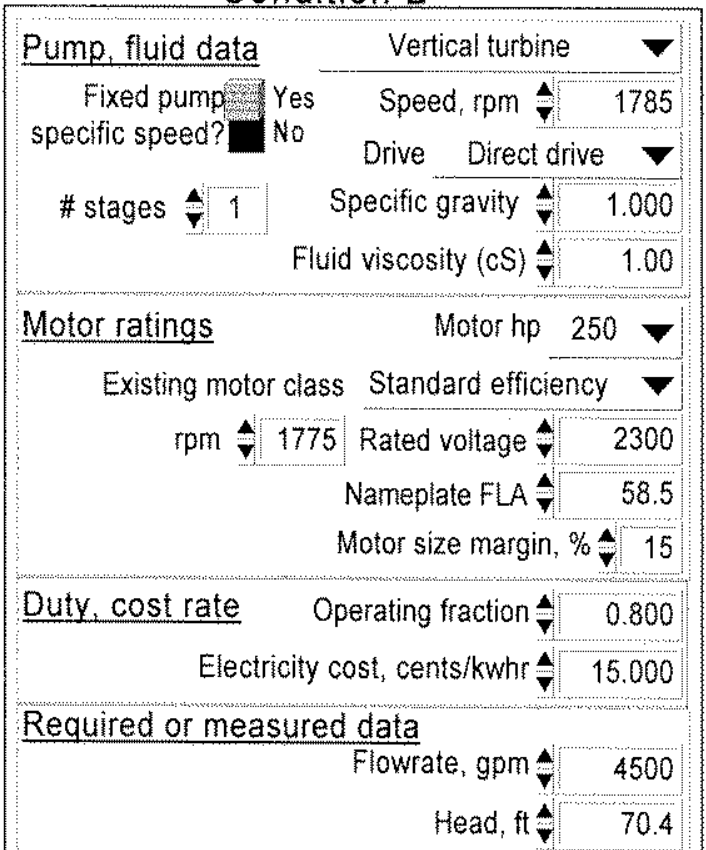

Load estimation method Current $\nabla$

\begin{tabular}{l|l|l|l|}
\hline Motor voltage & $\mathbf{\nabla}$ & 2305 & Motor amps \\
$\boldsymbol{\nabla}$ & 38.0 \\
\hline
\end{tabular}

\begin{tabular}{|c|c|c|}
\hline & Existing & Optimal \\
\hline Pump efficiency, $\%$ & 51.8 & 90.1 \\
\hline Motor rated power, hp & 250 & 125 \\
\hline Motor shaft power, hp & 154.3 & 88.8 \\
\hline Pump shaft power, hp & 154.3 & 88.8 \\
\hline Motor efficiency, $\%$ & 93.8 & 95.3 \\
\hline Motor power factor, $\%$ & 80.9 & 83.8 \\
\hline Motor current, amps & 38.0 & 20.8 \\
\hline Motor power, $\mathrm{kWe}$ & 122.7 & 69.5 \\
\hline Annual energy, MWhr & 859.6 & 486.8 \\
\hline Annual cost, $\$ 1,000$ & 128.9 & 73.0 \\
\hline
\end{tabular}

Annual cost savings potential, $\$ 1,000$ Optimization rating

\section{Condition A Notes}

Facilyy Pump $6168 \quad$ Systen Factory Vertical Pumps

Abplication Spray pond recirculation system

Date September 28, 2005 Evaluator Lee Jakeway

General cominents

Optimized conditions using pump curve dała. Pump no. 6168 is one of four vertical pumps that pumps boiling house condenser cooling water back to the spray pond for cooling. Water returns to factory and is then pumped to boiling house evaporators and pans via booster pumps. Three other vertical pumps were operating during the test.

\section{Facility Pump $6168 \quad$ System Factory Vertical Pumps}

Application Spray pond recirculation system

Date September 28, $2005 \quad$ Evaluator Lee Jakeway

General comments

Actual operating conditions. Pump no. 6168 is one of four vertical pumps that pumps boling house condenser cooling water back to the spray pond for cooling. Water returns to factory and is then pumped to boiling house evaporators and pans via booster pumps. Three other vertical pumps were operating during the test. Pump flow estimated and

averaged from 2002 flow data. 


\section{This PSAT2004 analysis was printed at 11:02 AM on Saturday, January 07, 2006}

\section{Condition A}

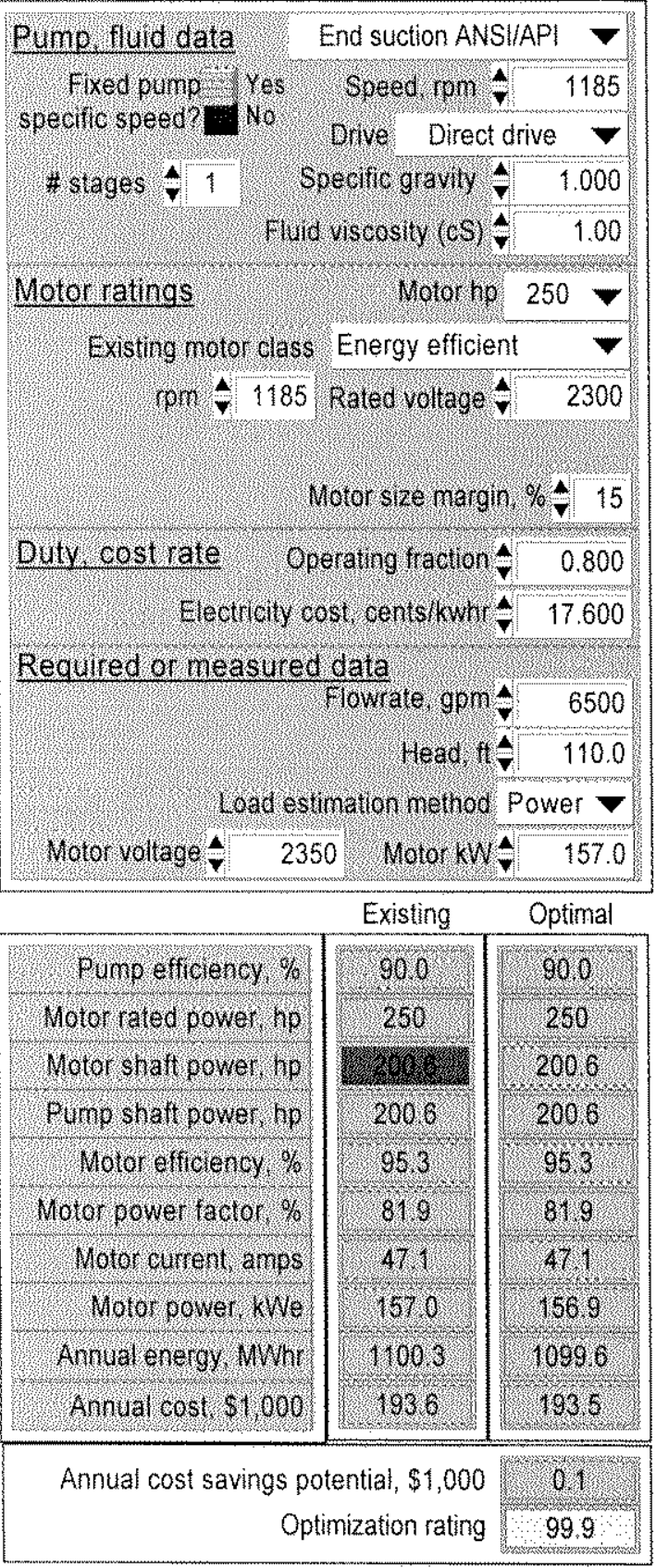

Condition B

\begin{tabular}{|c|c|c|c|}
\hline \multirow{3}{*}{$\begin{array}{l}\text { Pump, fluid data } \\
\text { Fixed pump } \\
\text { specific speed? }\end{array}$} & & \multicolumn{2}{|c|}{ End suction ANSIIAPI } \\
\hline & Yes & Speed, rpm & 1185 \\
\hline & & Direct drive & $\nabla$ \\
\hline \multirow[t]{2}{*}{ \# stages 41} & \multirow{2}{*}{\multicolumn{2}{|c|}{$\begin{array}{l}\text { Specific gravity } \widehat{\mathbf{v}} \\
\text { uid viscosity (cS) }\end{array}$}} & 1.000 \\
\hline & & & 1.00 \\
\hline
\end{tabular}

Motor ratings Motorhp $250 \nabla$

Existing motor class Standard efficiency $\mathrm{rpm} \hat{\boldsymbol{\nabla}} 1185$ Rated voltage $\hat{\boldsymbol{\nabla}} \quad 2300$ Nameplate FLA 화 $\quad 59.0$ Motor size margin, \% $\mathbf{\nabla} 15$

\begin{tabular}{|c|c|c|}
\hline Duty, cost rate & Operating fraction $\boldsymbol{\nabla}$ & 0.800 \\
\hline \multicolumn{2}{|c|}{ Electricity cost, cents/kwhr } & 17.600 \\
\hline
\end{tabular}
$\frac{\text { Required or measured data }}{\text { Flowrate, gpm }} * 4526$ Head, $\mathrm{ft}_{\mathbf{7}} \mathbf{1 2 8 . 9}$ Load estimation method Current $\mathbf{v}$ \begin{tabular}{ll|l|l|} 
Motor volfage & $\boldsymbol{\nabla}$ & 2350 & Motor amps \\
$\mathbf{y}$ & 49.0 \\
\hline
\end{tabular}

\begin{tabular}{|c|c|c|}
\hline & Existing & Optimal \\
\hline Pump efficiency, $\%$ & 70.8 & 89.2 \\
\hline Motor rated power, $h p$ & 250 & 200 \\
\hline Motor shaft power, hp & 208.1 & 165.3 \\
\hline Pump shaft power, hp & 208.1 & 165.3 \\
\hline Motor efficiency, $\%$ & 94.3 & 95.3 \\
\hline Motor power factor, $\%$ & 82.5 & 81.9 \\
\hline Motor current, amps & 49.0 & 38.8 \\
\hline Motor power, $\mathrm{kWe}$ & 164.6 & 129.4 \\
\hline Annual energy, MWhr & 1153.7 & 906.8 \\
\hline Annual cost, $\$ 1,000$ & 203.0 & 159.6 \\
\hline
\end{tabular}

Annual cost savings potential, $\$ 1,000$ Optimization rating

\section{Condition A Notes}

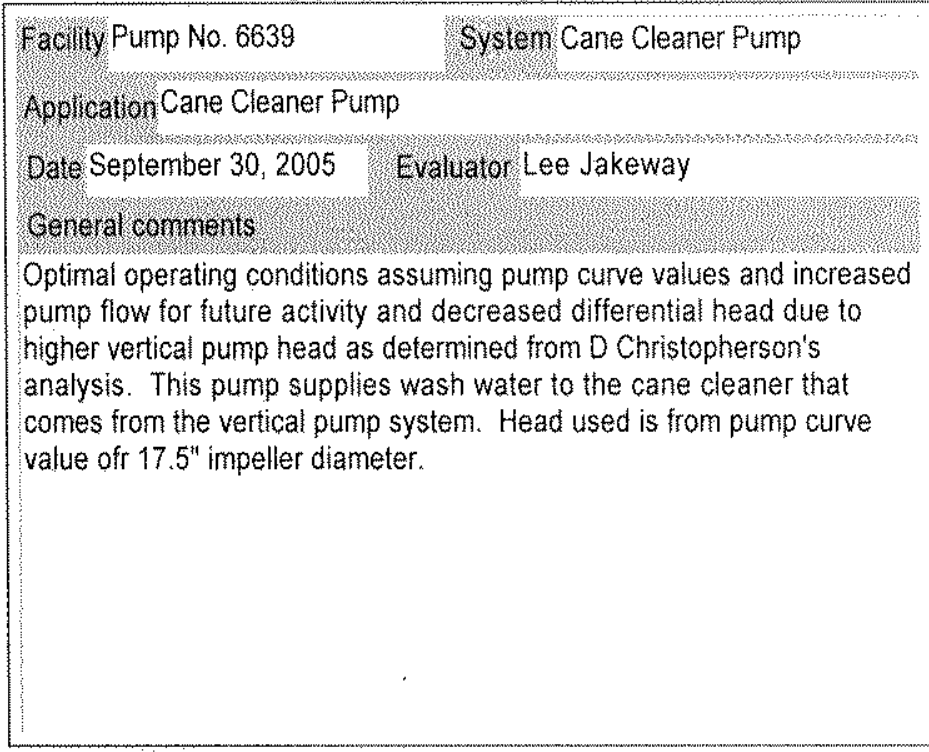

\section{Condition B Notes}

\section{Facility Pump No. $6639 \quad$ System Cane Cleaner Pump}

Application Cane Cleaner Pump

Date September 30, 2005 Evaluator Lee Jakeway

General comments

Actual operating conditions. This pump supplies wash water to the cane cleaner that comes from the vertical pump system.

Flow reading came from Suite Voyager and electrical readings were obtained from board readings 


\section{APPENDIX B}

\section{University of Hawaii Reports}




\title{
Steam Generation Efficiency Assessment Task 1 Deliverable Report
}

\author{
December 2004
}

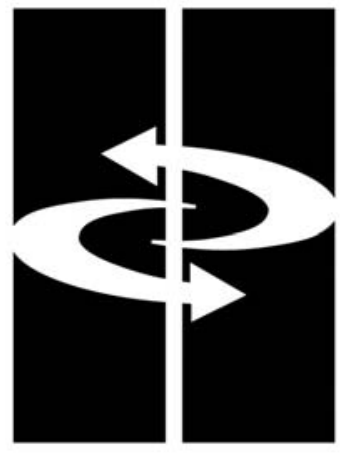

HAWAII NATURAL ENERGY INSTITUTE

School of Ocean and Earth Sciences and Technology

University of Hawaii at Manoa 


\title{
Steam Generation Efficiency Assessment Task 1 Deliverable Report
}

\author{
Scott Q. Turn \\ Vheissu I. Keffer \\ Hawaii Natural Energy Institute \\ University of Hawaii \\ Charles M. Kinoshita \\ Department of Molecular Biosciences and Bioengineering \\ University of Hawaii
}

\author{
Prepared for \\ Hawaiian Commercial \& Sugar, Co. \\ HC\&S Purchase Order No. 64137
}

December 2004

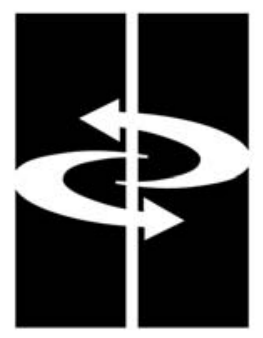

Hawaii Natural Energy Institute

School of Ocean and Earth Sciences and Technology

University of Hawaii 


\section{Contents}

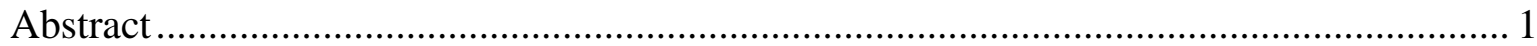

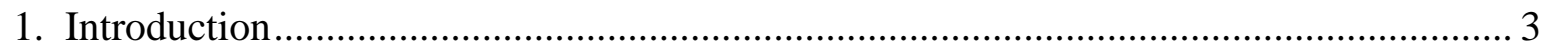

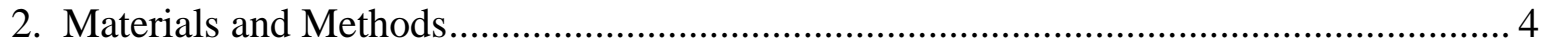

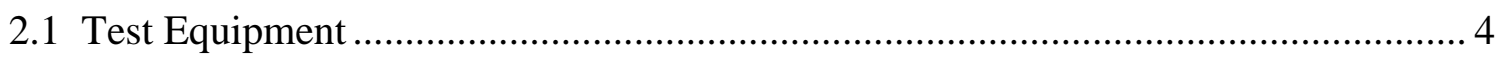

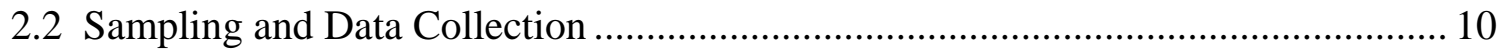

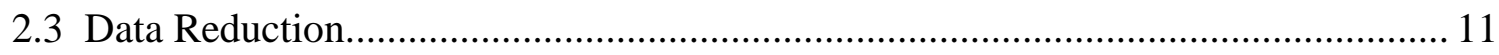

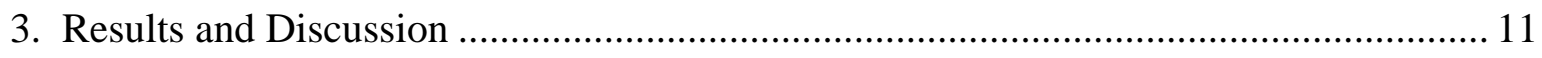

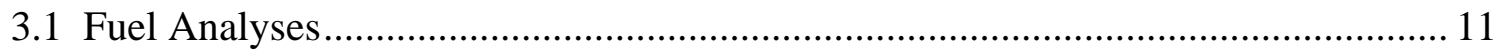

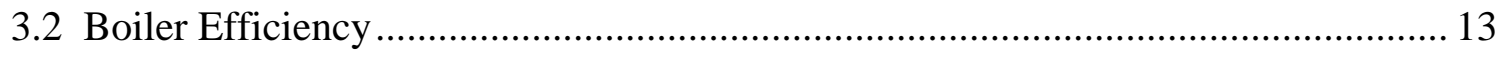

3.2.1 Measured Quantities ........................................................................ 13

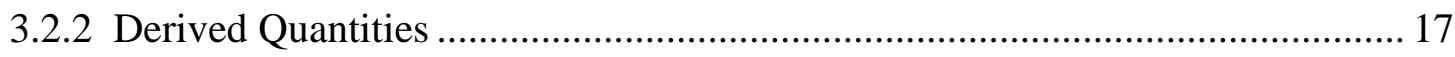

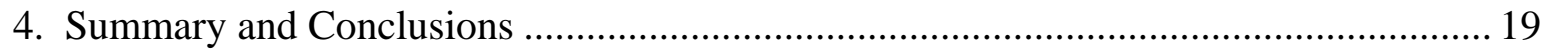

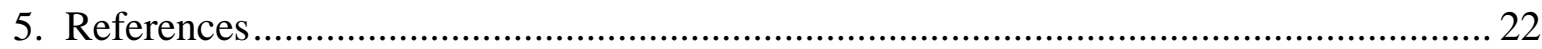

Appendix A: Fuel and Grate Ash Analysis Sheets

Appendix B: Tables of Flue Gas Composition and Temperature Averages from Individual Sampling Locations

Appendix C: Graphs of Flue Gas Composition and Temperature Measurements from Individual Sampling Locations

Appendix D: Graphs of HC\&S Wonderware Monitoring and Control Parameters 


\begin{abstract}
Measurements required to calculate steam generation efficiency were made on three generating units operated by Hawaiian Commercial \& Sugar Co. at its Puunene factory in September, 2003, and June, 2004. Tests were conducted on coal and bagasse for all three units and a single test firing fuel oil was conducted in Boiler 3. Measurements of flue gas temperature and composition at the exit of the air preheater were made during each test. Fuel and grate ash were sampled and analyses were performed following the test. Flue gases and temperatures were sampled using probe bundles inserted through access ports in the boiler walls. Each probe bundle was composed of three extraction tubes paired with Type $\mathrm{K}$ thermocouples. Boiler 1 was fitted with two probe bundles (a total of six sample extraction locations), whereas Boilers 2 and 3 were fitted with one probe bundle each. Data recorded during the test campaign were later reduced to average values and used to calculate steam generation efficiencies using the energy balance method.
\end{abstract}

Flue gas temperatures at the exit of the air preheater of the three boilers ranged from 180 to $248^{\circ} \mathrm{C}$. The lowest exit temperature was recorded for Boiler 3 during the fuel oil test. When bagasse and coal were fired, Boiler 1 had the lowest exit temperature and Boilers 2 and 3 were consistently higher by 15 and $\sim 30^{\circ} \mathrm{C}$, respectively. Exit temperatures of all boilers were higher when bagasse was fired, ranging from 220 to $248^{\circ} \mathrm{C}$ compared to 189 to $219^{\circ} \mathrm{C}$ for coal.

Gas composition measurements made after the air preheater showed carbon monoxide concentrations to be highly dependent on fuel type. Fossil fuels produced relatively low CO concentrations in the range of 10 to $90 \mathrm{ppmv}$. Bagasse tests exhibited elevated CO concentrations with averages ranging from 1,300 to 3,200 ppmv.

Excess air values calculated for coal tests ranged from $46 \%$ for Boiler 3, to $101 \%$ and $128 \%$ for Boilers 1 and 2, respectively. Excess air values calculated for Boilers 1, 2, and 3 operating on bagasse were 57,58 , and $17 \%$, respectively. The excess air value calculated for Boiler 3 using fuel oil was $99 \%$.

Calculated efficiencies for Boilers 1, 2, and 3 firing coal were 80.8, 76.1, and 82.4\%, respectively. Higher excess air values and flue gas exit temperatures were the main factors contributing to the lower efficiency of Boiler 2 compared to the other two units.

Calculated efficiencies for Boilers 1, 2, and 3 firing bagasse were 65.9, 63.2, and 67.2\%, respectively, markedly lower than those determined for coal. Boiler 2's lower efficiency resulted from higher flue gas temperature and CO concentrations than the other two units. Lower efficiencies observed when firing bagasse compared to coal are largely due to the different moisture contents of the two fuels, $48 \%$ for bagasse and 6 to $10 \%$ for coal.

Several opportunities to improve boiler efficiency are evident from the results of the test campaign. Reducing excess air, flue gas $\mathrm{CO}$ concentrations, and flue gas exit temperature all can contribute to increased efficiency, although the first two items may be more easily addressed in the near term. It is recognized that the ability to respond to these opportunities may largely be 
determined by the physical limitations of the boiler system's equipment and the constraints imposed by operating the units in conjunction with the Puunene sugar factory.

Potential cost savings from improvements in boiler efficiency were calculated. When all three steam generating units fire coal, a 1\% increase in efficiency in each of the three boilers would result in a savings of 9.5 tons of coal per day with an associated cost savings of $\$ 620$ per day. Similarly, when all three units fire bagasse, a 1\% improvement in efficiency would save 21.5 tons fuel (dry basis) per day and result in a cost savings of $\$ 750$ per day. 


\section{Introduction}

Increasing competition from abroad and lower prices for sugar and sugar products has taken its toll on the sugar industry in Hawaii. The challenges facing the remaining local producers are more formidable than ever. The increasing cost of energy has been added to these already strenuous challenges. To remain competitive and profitable in today's market it is imperative that Hawaii sugar producers use the most energy efficient production methods and run the most energy efficient processing facilities possible.

Hawaii’s largest remaining sugar producer, Hawaiian Commercial \& Sugar Co. (HC\&S), has undertaken a plantation wide energy efficiency assessment with cost share from the U.S. Department of Energy's (DOE) Office of Industrial Technology (OIT). This assessment includes analyses of the irrigation pumping systems, electrical distribution system, and sugar factory. The sugar factory assessment is composed of two parts, a boiler efficiency assessment for the cogeneration plant and a steam use assessment for the entire factory including the mill, processing plant, and cogeneration plant. The University of Hawaii (UH) was contracted to provide technical assistance on the boiler efficiency and steam assessment portions of the project. This report summarizes work completed by UH on the steam generator efficiency portion of the factory assessment.

HC\&S has three steam generation units at its Puunene sugar factory. All three are grate-fired, stoker-type units. Boilers 1 and 2 are identical and operate at 900 psi steam pressure and each has a rated capacity of $120 \mathrm{klb}$ steam $\mathrm{hr}^{-1}$. Flue gases from the two units are exhausted through a common wet scrubber and stack. Residue from their grates enters a common water quench and is removed by a belt conveyor. Boiler 3 operates at 425 psi steam pressure and is rated for 290 $\mathrm{klb}$ steam hr${ }^{-1}$. Flue gas from Boiler 3 is exhausted through a dedicated wet scrubber and stack and the grate residue is also removed using a dedicated water quench and conveyor system.

The two generally accepted methods of determining steam generator efficiency are detailed in ASME PTC 4-1998 Fired Steam Generators Performance Test Codes [1] and are referred to as the input/output (or direct ) method and the energy balance (or indirect) method, respectively. The direct method requires highly accurate measurement of all input and output flows, while the indirect method requires identification and determination of all losses.

The direct method requires measurement of fuel and steam flow rates, moisture content, fuel higher heating value, steam temperature and steam pressure. The effectiveness of the direct method is strongly dependent on the accurate measurement of the fuel flow rate, moisture content, and higher heating value. Steam temperature and pressure measurements have a weaker affect on the overall accuracy of the efficiency calculation.

The indirect method requires measurement of fuel higher heating value, moisture content, and ultimate analysis and flue gas exit temperature and composition. Unburned carbon losses must also be determined. Radiation losses and unaccounted losses must also be measured or calculated using manufacturer's specifications. The effectiveness of the indirect method is strongly dependent on the fuel higher heating value and the moisture content determination. Total accuracy is also moderately dependant on the ultimate analysis and flue gas exit 
temperature and composition. Losses estimated from manufacturer's specifications have a small effect on the accuracy of the overall efficiency determination [2].

Bagasse is the primary fuel used in all three boilers at HC\&S. Bagasse is a bulky fuel and, as such, is difficult to accurately meter, thus favoring the indirect method for determining boiler efficiency. The ASME test code [1] also recommends using the indirect method since it provides lower overall test uncertainty and can be corrected to standard or guarantee conditions. For these reasons the indirect method was chosen for this assessment.

HC\&S must conduct emission compliance tests on a yearly basis. During these tests the factory and boiler system are operated as steadily as possible and at, or near, full capacity. The steam generator efficiency assessments were schedule at the same time as the compliance tests to take advantage of this period of steady operation and the fuel analyses performed as part of the compliance determination.

\section{Materials and Methods}

\subsection{Test Equipment}

In advance of the test campaign, the UH project team made several trips to set up measurement sites on each of the HC\&S boilers. ASME's test code indicates that measurement of flue gas composition and temperature for calculation of boiler efficiency using the indirect method should be made immediately following the last heat recovery device. In the case of HC\&S boilers this point is located after the air preheater. ASME's test code suggests a sample matrix of 16 points, evenly spaced throughout the duct. Due to equipment and time limitations, and physical constraints, sampling at 16 points was not feasible.

Upon reviewing available access to the ducts following the air preheaters on Boilers 1, 2, and 3, it became clear that the sampling matrix would have to be scaled back. Boiler 1 had two accessible ports and Boilers 2 and 3 each had one accessible port. Prior to testing, each port was fitted with three stainless steel sample extraction tubes $(\mathrm{ID}=11.8 \mathrm{~mm}=0.465 ", \mathrm{OD}=12.7$ $\mathrm{mm}=0.50$ ") welded together to form a triangular probe bundle. The tubes were cut to different lengths and protruded into the flue gas flow with inlets located 2, 4, and $6 \mathrm{ft}(0.61,1.22$, and 1.83 $\mathrm{m})$ from the duct wall. Type $\mathrm{K}$ thermocouples were attached to each of the three tubes in the probe bundle to provide companion temperature readings at each inlet location. The tube bundle and thermocouple assembly was fitted through a 2" $(50.8 \mathrm{~mm})$ pipe cap that could be attached to ports located on the boiler wall. A diagram of the probe assembly is shown below in Figure 1. Figures 2 and 3 provide schematics of the probe sampling locations in the duct cross sections immediately following the air preheater for each boiler. Figure 4 presents a schematic of Boiler 1 indicating the sampling location and this is also representative of Boilers 2 and 3. 

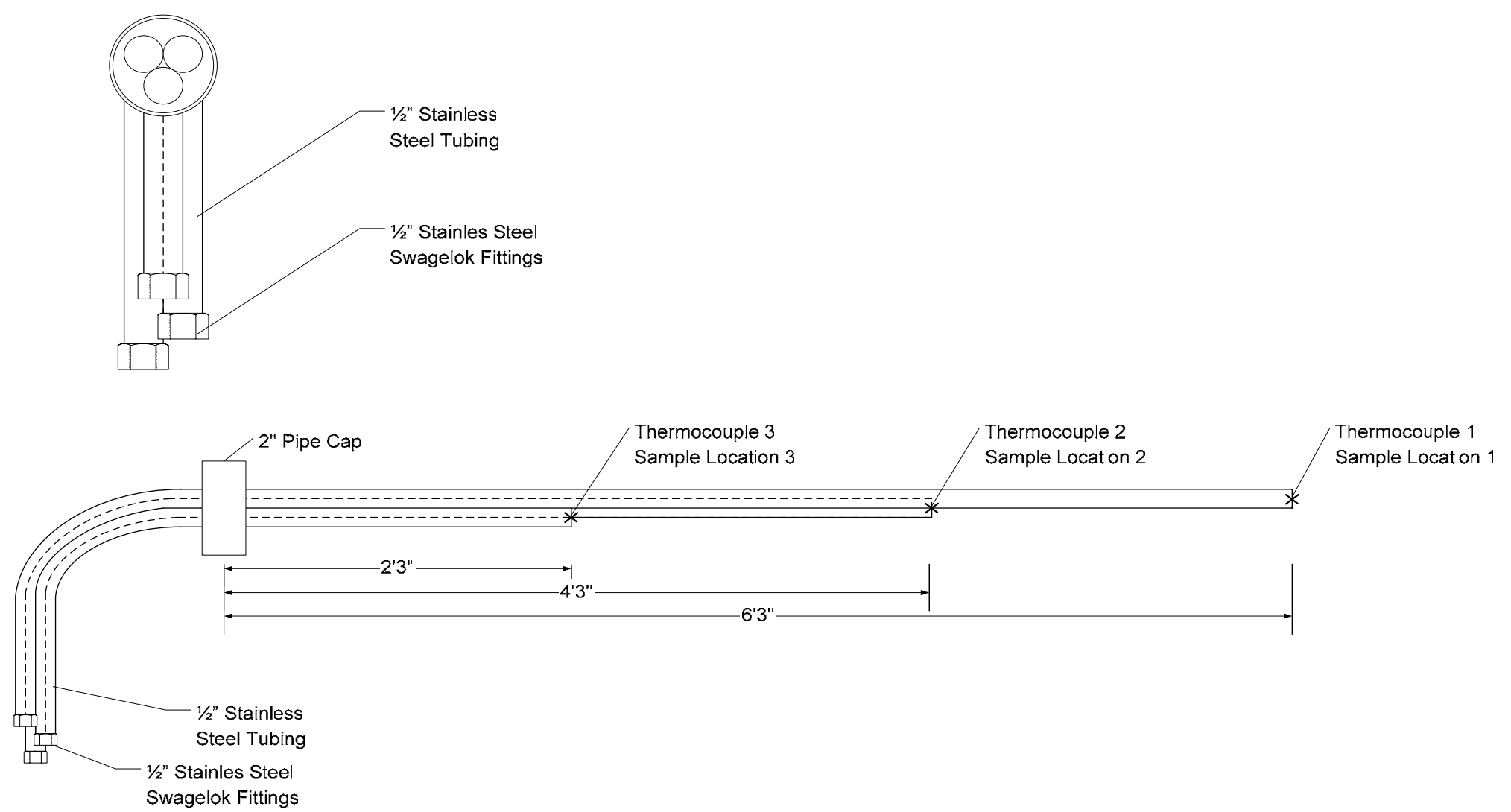

Figure 1. Schematic of probe bundle containing three stainless steel sample extraction tubes and thermocouple assemblies. 


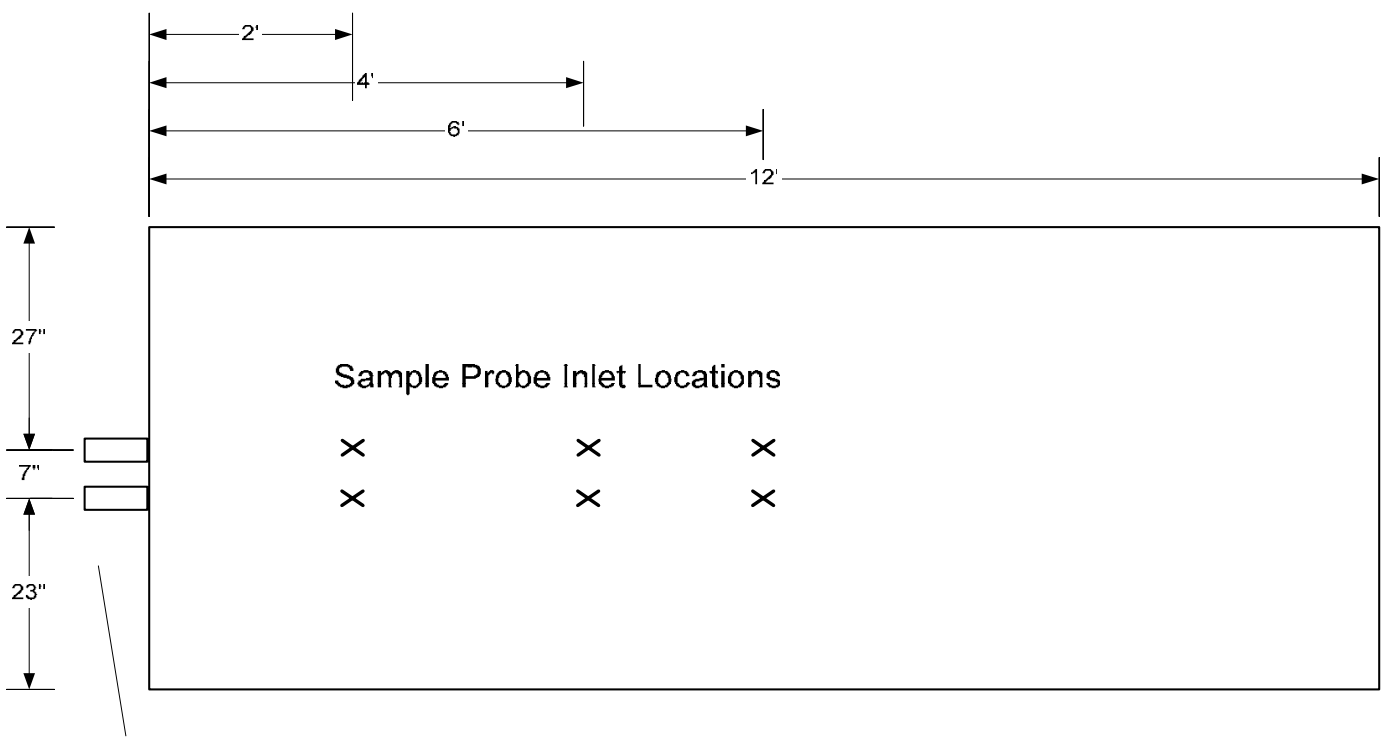

Ports in wall of Boiler 1

(a)

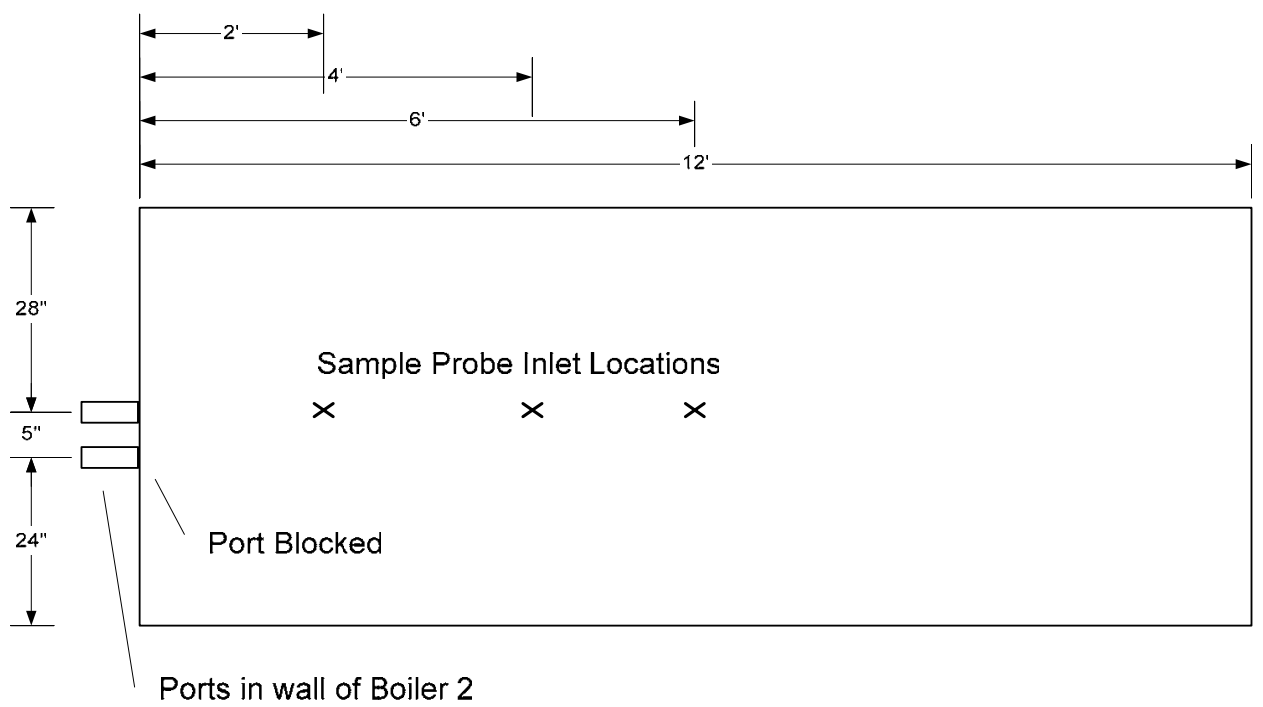

(b)

Figure 2. Cross sectional of duct downstream of the air preheater showing port and sample probe inlet locations. Boiler 1 is shown in (a) and Boiler 2 is shown in (b). 


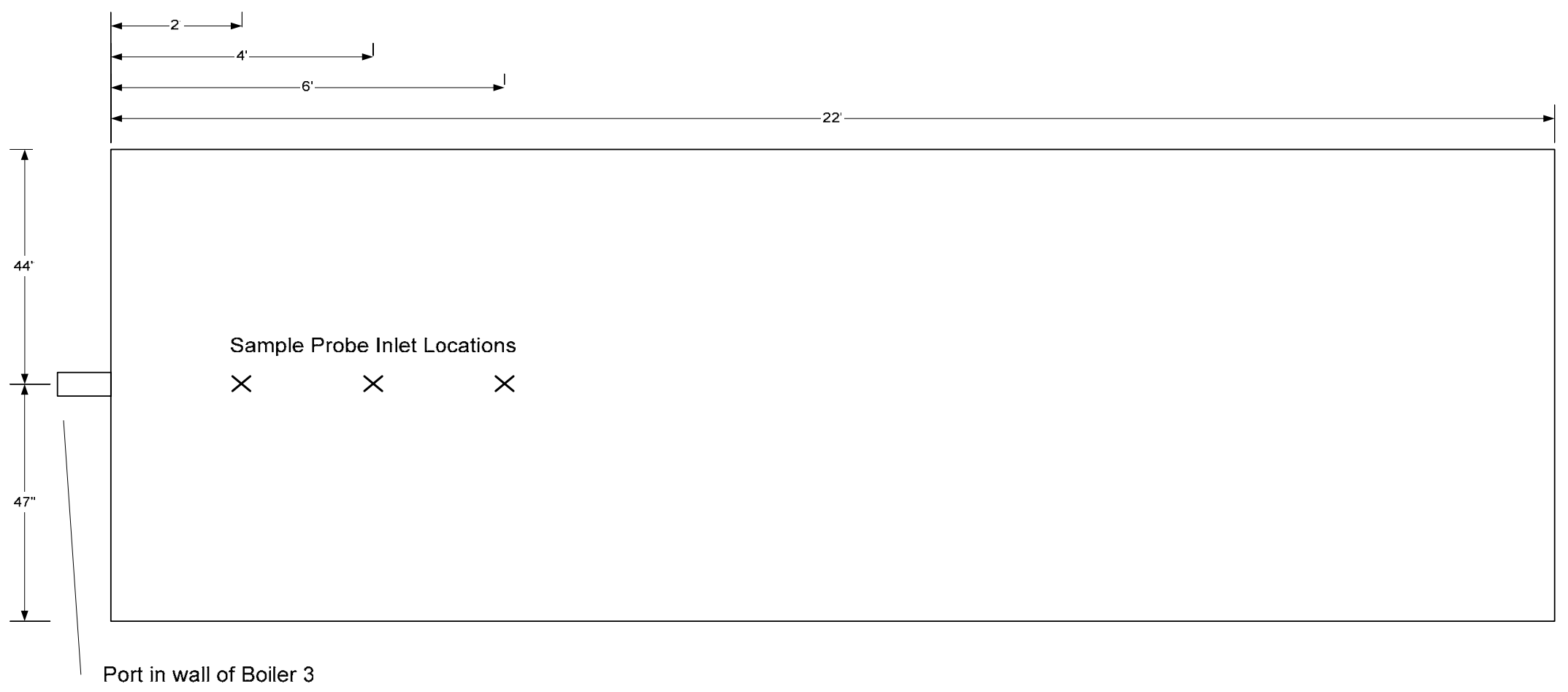

Figure 3. Cross sectional of duct in Boiler 3 downstream of the air preheater showing port and sample probe inlet locations. 


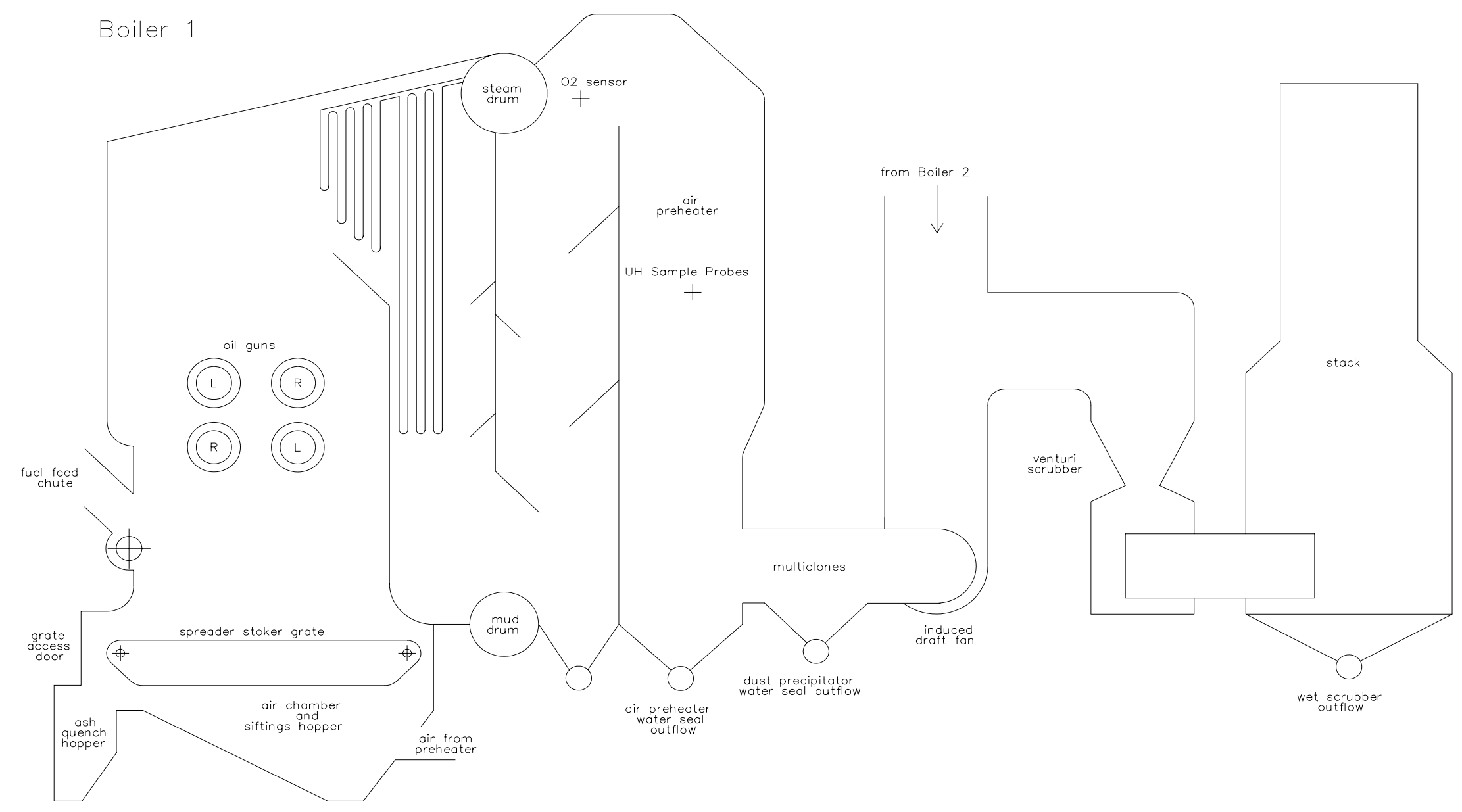

Figure 4. Schematic of Boiler 1 showing UH sample probe location after the air preheater. 
External to the boiler, the sample extraction tubes were bent $90^{\circ}$ and terminated with Swagelok fittings. The $90^{\circ}$ degree bend reduced the potential for kinks in the flexible sample tubes used between the probe and the sampling system. Three 20' $(6.1 \mathrm{~m})$ lengths of 0.5 " $(12.7 \mathrm{~mm})$ diameter, Teflon tubing were bundled together and attached to the sample extraction tubes. The sample lines allowed sensitive gas monitoring equipment and the sampling system to be located in a milder and more accessible environment than that found near the boiler walls.

In order to remove entrained particulate matter and water vapor from extracted flue gas, the sample stream was directed through a set of four impingers immersed in an ice bath and a silica gel desiccant bed. Particulate matter removal and dehumidification was necessary to ensure safe operation of down stream components; a diaphragm pump, a volumetric flow meter, and a portable gas analyzer (Horiba, Model PG-250). Flue gas was drawn through one sample extraction tube at a time and directed through the impinger system to remove particulate matter and condense water vapor. The cooled gas then passed through the desiccant bed, the diaphragm pump, and the volumetric flow meter before being exhausted to atmosphere, as shown in Figure 5. A slip stream of the cool dry gas drawn from the flow between the volumetric flow meter and exhaust was directed to the portable gas analyzer.

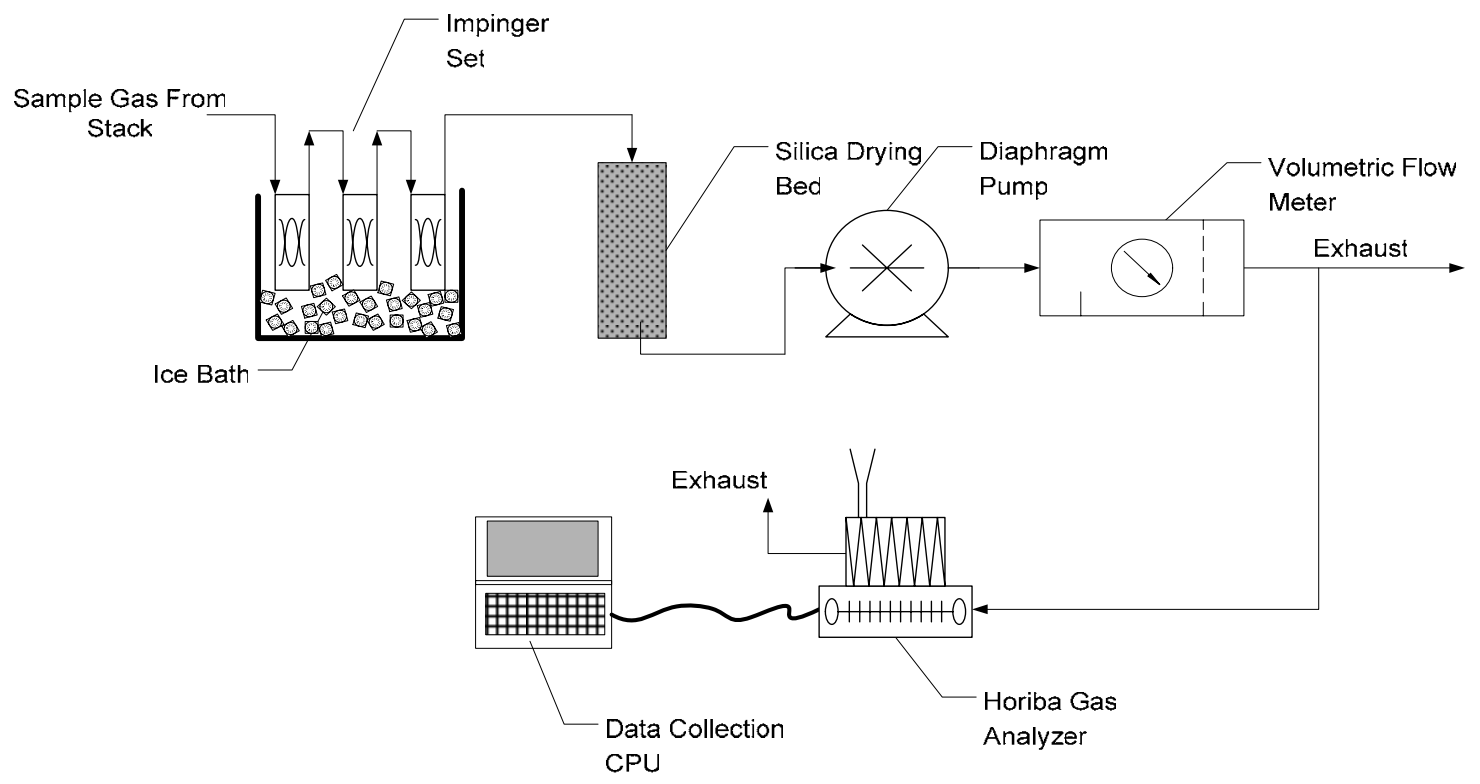

Figure 5. Sampling conditioning system used for analysis of flue gases downstream of the air preheater.

The Horiba PG-250 gas analyzer measures $\mathrm{NOx}, \mathrm{CO}, \mathrm{CO}_{2}, \mathrm{SO}_{\mathrm{x}}$, and $\mathrm{O}_{2}$. Ranges for these gases are 0 to 25/50/100/250/500/1000/2500 ppm for $\mathrm{NO}_{\mathrm{x}}, 0$ to 200/500/1000/3000/5000 ppm for $\mathrm{SO}_{\mathrm{x}}$, 0 to $200 / 500 / 1000 / 2000 / 5000 \mathrm{ppm}$ for CO, 0 to 5/10/20 vol \% for $\mathrm{CO}_{2}$ and 0 to 5/10/25 vol \% for $\mathrm{O}_{2}$. For coal and fuel oil tests the $0-500 \mathrm{ppm}$ range for $\mathrm{CO}$ was selected. The $0-5000 \mathrm{ppm}$ range for $\mathrm{CO}$ was selected for bagasse-fired tests. All calibration standards for the project were purchased from Matheson Trigas and had accuracy of $\pm 2 \%$. The composition of each calibration standard is listed in Table 1. 
Table 1. Gases used to calibrate the Horiba PG-250 gas analyzer

\begin{tabular}{ccc}
\hline Calibration Gas & $\begin{array}{c}\text { Concentration } \\
\text { (volume basis) }\end{array}$ & Balance Gas \\
\hline $\mathrm{O}_{2}$ & $21 \%$ & $\mathrm{~N}_{2}$ \\
$\mathrm{CO}_{2}$ & $12.5 \%$ & $\mathrm{~N}_{2}$ \\
$\mathrm{CO}$ & $500 \mathrm{ppm}$ & $\mathrm{N}_{2}$ \\
$\mathrm{CO}$ & $5000 \mathrm{ppm}$ & $\mathrm{N}_{2}$ \\
$\mathrm{SO}_{\mathrm{x}}$ & $250 \mathrm{ppm}$ & $\mathrm{N}_{2}$ \\
$\mathrm{NO}_{\mathrm{x}}$ & $250 \mathrm{ppm}$ & $\mathrm{N}_{2}$ \\
$\mathrm{~N}_{2}$ & $100 \%$ & None \\
\hline
\end{tabular}

Data from the Horiba were saved to a laptop computer running Horiba proprietary software and individual data points were recorded on a 5 s sampling interval.

Temperature data from the three Type K thermocouples (Omega Engineering, Stanford, CT) on the active probe bundle were sampled on 5 second intervals and recorded on a data logger (Campbell Scientific, Model 23X, Logan, UT).

\subsection{Sampling and Data Collection}

Efficiency assessment testing was scheduled for the week of September 22-27, 2003. Tests took place on Boiler 1 and 2 on September 22-23 and on Boiler 3 on September 25-27. September $24^{\text {th }}$ was used to move emission monitoring equipment between stacks. Flue gas composition data for the coal test on Boiler 2 was lost during the test campaign and a make-up test was completed on June 29, 2004. Although the June 29 test was not conducted in conjunction with a compliance test, test conditions were maintained as close to those of the September test as possible.

The compliance testing schedule called for one fuel to be tested on one of the stacks each day. Sampling equipment was assembled on location at the beginning of each day. Start up protocol for the Horiba analyzer includes a one hour warm up period after powering up the unit. This was followed by a daily leak check and calibration sequence.

Flue gas sampling was initiated after the gas analyzer calibration routine was completed and when system operators indicated that steady boiler operations had been attained. A minimum of three, 10 minute samples were drawn from each of the three extraction tubes in each probe bundle. To change the sampling point, sample lines were exchanged at the connection to the impinger set. Lines were capped when not in use. Sample gas flow rate was maintained in the range of 12 to $15 \mathrm{~L} \mathrm{~min}^{-1}$ (0.42 to $0.53 \mathrm{scfm}$ ).

For the September 2003 tests, three fuel and grate residue samples were collected per compliance test by HC\&S staff. Grate residue samples were collected only for the coal tests. Grate residues from Boilers 1 and 2 are commingled in a water quench and a single composite residue sample was obtained from the drag conveyor outfall. Bagasse residue is entrained in the flue gases and removed in the air pollution control equipment downstream and no residues were generated when firing fuel oil. Solid fuel samples were subjected to moisture, proximate, ultimate, and 
heating value analyses. Fuel oil samples were subjected only to moisture and the latter two analyses. Grate ash samples were subjected to moisture, total and organic carbon, combustibles, and loss on ignition. All samples were analyzed by Standard Laboratories Inc., Casper, Wyoming.

For the June 29 coal make-up test, University of Hawaii personnel collected fuel and grate residue samples for analysis. Samples from this test were subjected to the same battery of analyses, however the analyses were performed by Hazen Research, Golden, Colorado.

Data from sensors HC\&S uses to monitor and control the power plant are acquired, recorded, and managed using Wonderware software. Data from the week of the test campaign were requested and received from $\mathrm{HC} \& S$ personnel as one-minute averages for each boiler. Quantities included steam flow rates, temperatures, and pressures; steam blow down flow rates; boiler feedwater flow rates and temperatures; indicators of combustion air flow to grate locations and windboxes; indicators of induced draft, forced draft, and overfire air flows; fuel oil, bagasse, and coal flow rates; flue gas $\mathrm{O}_{2}$ concentrations; steam drum and header pressures; air preheater performance data; grate temperatures; and wet scrubber water flow rates.

\subsection{Data Reduction}

Averages and standard deviations were computed from the data collected at each of the flue gas sampling locations. A composite average and standard deviation of flue gas properties for each boiler operating on a given fuel were calculated from the individual sampling location averages. Results from analysis of the triplicate fuel and grate residue samples were averaged to produce a composite average for each boiler on each fuel.

\section{Results and Discussion}

\subsection{Fuel Analyses}

Average results of the fuel and grate ash analyses pertinent to efficiency calculations are summarized in Table 2. Lab reports of analyses for individual samples are presented in Appendix A. Consistency is generally good between samples of the same material acquired on different days. Results of the coal samples (06/29/04) analyzed by Hazen Research show slightly higher values for ash, volatile matter, higher heating value, $\mathrm{C}$, and $\mathrm{S}$, than results of the earlier coal analyses performed by Standard Laboratories. It is not apparent whether this is due to actual differences in the fuel, or rather the result of differences in analytical technique between the two laboratories. Coal moisture contents ranged from 6.6 to $10.7 \%$ wet basis. The higher value was recorded for the 9/26/03 test and was the result of rain on 9/23 and 9/24/03. Bagasse moisture was consistently $\sim 48 \%$ wet basis over all tests. 
Table 2. Average properties of fuels used, and residues generated, during the efficiency assessment test periods.

\begin{tabular}{|c|c|c|c|c|c|c|}
\hline & $\begin{array}{l}\text { Coal Test } \\
09-22-03\end{array}$ & $\begin{array}{c}\text { Bagasse Test } \\
09-23-03\end{array}$ & $\begin{array}{c}\text { Bagasse Test } \\
09-25-03\end{array}$ & $\begin{array}{l}\text { Coal Test } \\
09-26-03\end{array}$ & $\begin{array}{c}\text { Fuel Oil } \\
\text { (Bunker C) } \\
09-27-03\end{array}$ & $\begin{array}{l}\text { Coal Test } \\
06-29-04\end{array}$ \\
\hline Boiler(s) & $1 \& 2$ & $1 \& 2$ & 3 & 3 & 3 & 2 \\
\hline \multicolumn{7}{|l|}{ Fuel Analyses } \\
\hline No. of Analyses & 3 & 3 & 3 & 3 & 3 & 3 \\
\hline Moisture Content (\% wet basis) & $6.6 \pm 0.04$ & $48.1 \pm 0.7$ & $47.8 \pm 1.7$ & $10.7 \pm .1$ & $0 \pm 0$ & $6.8 \pm 0.9$ \\
\hline \multicolumn{7}{|l|}{ Proximate Analysis (\% dry basis) } \\
\hline Ash & $13.12 \pm 0.12$ & $1.84 \pm 0.29$ & $1.78 \pm .26$ & $13.55 \pm 0.05$ & $0.00 \pm 0.00$ & $14.64 \pm 0.60$ \\
\hline Volatiles & $41.06 \pm 0.25$ & $80.81 \pm 0.34$ & $80.78 \pm 0.31$ & $41.25 \pm 0.14$ & & $43.88 \pm 1.40$ \\
\hline Fixed C & $45.82 \pm 0.23$ & $17.35 \pm 0.23$ & $17.43 \pm 0.10$ & $45.2 \pm 0.10$ & & $41.48 \pm 1.36$ \\
\hline \multicolumn{7}{|l|}{ Higher Heating Value (dry basis) } \\
\hline $\mathrm{MJ} / \mathrm{kg}$ & $29.0 \pm 0.11$ & $19.0 \pm 0.15$ & $19.1 \pm 0.17$ & $28.9 \pm 0.07$ & $45.7 \pm 0.1$ & $29.6 \pm 0.19$ \\
\hline BTU/lb & $12,476 \pm 46$ & $8,167 \pm 64$ & $8,194 \pm 72$ & $12,437 \pm 32$ & $19,640 \pm 27$ & $12,725 \pm 83$ \\
\hline \multicolumn{7}{|l|}{ Ultimate Analysis (\% dry basis) } \\
\hline $\mathrm{C}$ & $70.15 \pm 0.16$ & $49.54 \pm 0.21$ & $49.58 \pm 0.22$ & $69.71 \pm 0.18$ & $86.46 \pm 0.41$ & $70.87 \pm 0.81$ \\
\hline $\mathrm{H}$ & $5.43 \pm 0.02$ & $5.69 \pm 0.05$ & $5.71 \pm 0.05$ & $5.39 \pm 0.07$ & $11.70 \pm 0.53$ & $5.36 \pm 0.16$ \\
\hline O (by difference) ${ }^{c}$ & $9.74 \pm 0.04$ & $42.66 \pm 0.25$ & $42.73 \pm 0.08$ & $9.78 \pm 0.20$ & $1.64 \pm 0.39$ & $7.48 \pm 0.70$ \\
\hline $\mathrm{N}$ & $1.08 \pm 0.02$ & $0.16 \pm 0.03$ & $0.13 \pm 0.03$ & $1.07 \pm 0.01$ & $0.02 \pm 0.02$ & $1.11 \pm 0.03$ \\
\hline S & $0.48 \pm 0.01$ & $0.11 \pm 0.03$ & $0.08 \pm 0.01$ & $0.49 \pm 0.01$ & $0.18 \pm 0.02$ & $0.54 \pm 0.01$ \\
\hline \multicolumn{7}{|l|}{ Residue Analysis } \\
\hline Number of Analyses & 3 & & & 3 & & 3 \\
\hline Organic Carbon (\% dry basis) & $2.58 \pm 0.46$ & & & $3.63 \pm 1.10$ & & $7.44 \pm 0.75$ \\
\hline
\end{tabular}




\subsection{Boiler Efficiency}

Table 3 summarizes the boiler efficiency data from the test campaign. The upper half of the table presents average values of relevant measured quantities. The averages from which the data in Table 3 were derived are presented in Appendix B. Plots of output from the Horiba gas analyzer and the Type $\mathrm{K}$ thermocouples for each of the tests are compiled in Appendix $\mathrm{C}$. The lower half of Table 3 presents data that were derived from the fuel properties and measured quantities

Data from the HC\&S Wonderware system is presented as graphs, for reference, in Appendix D.

\subsubsection{Measured Quantities}

Flue gas temperatures at the exit of the air preheater of the three boilers ranged from 180 to $248^{\circ} \mathrm{C}$. The lowest exit temperature was recorded for Boiler 3 during the fuel oil test. When bagasse and coal were fired, Boiler 1 had the lowest exit temperature and Boilers 2 and 3 were consistently higher by 15 and $\sim 30^{\circ} \mathrm{C}$, respectively. Exit temperatures of all boilers were higher when bagasse was fired, ranging from 220 to $248^{\circ} \mathrm{C}$ compared to 189 to $219^{\circ} \mathrm{C}$ for coal. (Note: Flue gas temperatures in degrees Fahrenheit are shown in the lower half of Table 3). For comparison, various historical temperature values (either predicted or measured) provided by the boiler manufacturer are summarized in Table 4. The acceptance test report prepared by Riley Stoker Corporation in 1956 when Boiler 1 was commissioned on bagasse indicates that at a steam flow of $66.8 \mathrm{Mg}$ per hour (147,000 lb per hr), the exit temperature at the air preheater was $224^{\circ} \mathrm{C}$. Similarly, Boiler 3 performance tests conducted by Foster Wheeler in 1978 using bagasse reported an air preheater exit temperature of $224^{\circ} \mathrm{C}$ at a steam flow rate of $131.1 \mathrm{Mg}$ per hour (289,000 lb per hr), a flow rate most comparable to the current test condition. From these data it can be concluded that the air preheater exit temperature of Boiler 1 is comparable to the value measured at the time of commissioning, but that values for Boilers 2 and 3 are roughly 15 to $25^{\circ} \mathrm{C}$ above values determined by the original equipment manufacturer.

Measurements of gas composition made after the air preheater found carbon monoxide concentrations to be highly dependent on fuel type. Fossil fuels produced relatively low CO concentrations in the range of 10 to 90 ppmv. Bagasse tests exhibited elevated CO concentrations with averages ranging from 1,300 to 3,200 ppmv. The averages for bagasse are necessarily underestimates of the true average value as CO concentration exceeded the maximum value on the 0 to 5,000 ppmv range of the gas analyzer on several occasions, producing an over range marker in the data file. Over range markers were replaced with values of 5,114 ppmv (maximum value for the 0 to 5,000 range before over range value is issued) so that an average could be computed.

Table 3 also includes flue gas $\mathrm{O}_{2}$ concentrations with values ranging from 3 to $12 \%$. Boilers 1 and 2 firing coal had values at the upper end of this range and both averaged $\sim 7.7 \% \mathrm{O}_{2}$ when fueled with bagasse. Boiler 3 exhibited the lowest average $\mathrm{O}_{2}$ concentration of $3.0 \%$ while operating on bagasse and this correlates with the high CO concentration (3200 ppmv) reported in the previous paragraph. Boiler 3 operating on coal and fuel oil produced flue gas $\mathrm{O}_{2}$ concentrations of $\sim 7 \%$. A comparison of the $\mathrm{O}_{2}$ concentrations measured by the Horiba and 
those recorded by the HC\&S Wonderware system from the $\mathrm{O}_{2}$ sensor installed in each boiler for monitoring and control purposes is shown in Figure 6. Differences ( $\% \mathrm{O}_{2}$, absolute) between the two measuring devices at their respective locations ranged from 3.4 to $4.6 \%$ for Boiler $1,5.7$ to 9.3\% for Boiler 2, and 0.3 to $2.0 \%$ for Boiler 3. Differences may be the result of calibration or air ingress in the ducting between the upstream $\mathrm{HC} \& \mathrm{~S} \mathrm{O}_{2}$ sensor and the downstream $\mathrm{UH}$ sampling location at the outlet of the air preheater. The two measurement locations on Boiler 1 are shown in Figure 4 and are representative of other two boilers.

$\mathrm{NO}_{\mathrm{x}}$ concentrations ranged from 50 to 227 ppmv with values generally correlating with fuel nitrogen content (fuel oil $<$ bagasse $<$ coal). 
Table 3. Averages of relevant measured and derived quantities from boiler efficiency tests at HC\&S.

\begin{tabular}{|c|c|c|c|c|c|c|c|}
\hline Boiler No. & 1 & 1 & 2 & 2 & 3 & 3 & 3 \\
\hline Test Date & $9 / 22 / 03$ & $9 / 23 / 03$ & $6 / 29 / 04$ & 9/23/03 & $9 / 25 / 03$ & $9 / 26 / 03$ & $9 / 27 / 03$ \\
\hline Fuel & Coal & Bagasse & Coal & Bagasse & Bagasse & Coal & \#2 Fuel Oil \\
\hline $\begin{array}{l}\text { Average Steam Flow Rate, klb/hr (from } \\
\text { HC\&S Wonderware system) }\end{array}$ & $105.9 \pm 4.5$ & $104.2 \pm 3.8$ & $104.0 \pm 8.6$ & $105.3 \pm 3.8$ & $259.7 \pm 19.4$ & $266.8 \pm 5.2$ & $241.8 \pm 6.5$ \\
\hline \multicolumn{8}{|l|}{ Measured Quantities $^{1}$} \\
\hline Fuel Moisture Content, \% wet basis & $6.6 \pm 0.04$ & $48.1 \pm 0.7$ & $6.8 \pm 0.9$ & $48.1 \pm 0.7$ & $47.8 \pm 1.7$ & $10.7 \pm 0.1$ & $0.0 \pm 0.0$ \\
\hline Flue Gas Air Preheater Exit Temperature, C & $189 \pm 6$ & $220 \pm 8$ & $219 \pm 8$ & $248 \pm 15$ & $235 \pm 25$ & $204 \pm 16$ & $180 \pm 11$ \\
\hline Flue Gas CO Concentration, ppmv & $83 \pm 11$ & $1311 \pm 758^{2}$ & $87 \pm 23$ & $2696 \pm 1368^{2}$ & $3156 \pm 1546^{2}$ & $10 \pm 3$ & $26 \pm 26$ \\
\hline Flue Gas $\mathrm{O}_{2}$ Concentration, $\%$ vol & $10.7 \pm 1.4$ & $7.6 \pm 1.9$ & $11.9 \pm 1.5$ & $7.7 \pm 2.5$ & $3.0 \pm 1.4$ & $6.8 \pm 0.6$ & $10.7 \pm 1.4$ \\
\hline Flue Gas $\mathrm{NO}_{\mathrm{x}}$ Concentration, ppmv & $127 \pm 20$ & $78 \pm 15$ & $180 \pm 31$ & $82 \pm 18$ & $119 \pm 18$ & $227 \pm 29$ & $55 \pm 10$ \\
\hline $\begin{array}{l}\text { Total Organic Carbon Content of Grate } \\
\text { Residue }^{3}, \% \\
\end{array}$ & $2.6 \pm 0.46$ & & $7.4 \pm 0.75$ & & & $3.6 \pm 1.10$ & \\
\hline \multicolumn{8}{|l|}{ Derived Quantities } \\
\hline Flue Gas Air Preheater Exit Temperature, F & 372 & 429 & 426 & 479 & 455 & 400 & 357 \\
\hline Flue Gas CO Concentration, \% vol & 0.008 & 0.131 & 0.009 & 0.270 & 0.316 & 0.001 & 0.003 \\
\hline Excess Air, \% & 101 & 57 & 128 & 58 & 17 & 46 & 99 \\
\hline $\begin{array}{l}\text { Fuel Efficiency, Indirect Method, } \\
\text { Uncorrected for Losses, \% }\end{array}$ & 83.3 & 68.5 & 79.6 & 66.5 & 70.3 & 85.0 & 84.0 \\
\hline $\begin{array}{l}\text { Efficiency Correction, Loss from Total } \\
\text { Organic } \\
\end{array}$ & 0.40 & & 1.34 & & & 0.60 & \\
\hline $\begin{array}{l}\text { Efficiency Correction, Loss from CO in the } \\
\text { Flue Gas, \% }\end{array}$ & 0.05 & 0.61 & 0.13 & 1.27 & 1.10 & 0.00 & 0.01 \\
\hline $\begin{array}{l}\text { Efficiency Correction, Loss from Surface } \\
\text { Radiation }{ }^{4}, \% \\
\end{array}$ & 0.5 & 0.5 & 0.5 & 0.5 & 0.5 & 0.5 & 0.5 \\
\hline $\begin{array}{r}\text { Efficiency Correction, Manufacturers } \\
\text { Unaccounted for Losses }{ }^{4}, \% \\
\end{array}$ & 1.5 & 1.5 & 1.5 & 1.5 & 1.5 & 1.5 & 1.5 \\
\hline $\begin{array}{l}\text { Fuel Efficiency, Indirect Method, Corrected } \\
\text { for Losses, \% }\end{array}$ & 80.8 & 65.9 & 76.1 & 63.2 & 67.2 & 82.4 & 82.0 \\
\hline \multicolumn{8}{|c|}{$\begin{array}{l}{ }^{1} \text { Error values equal to one standard deviation. } \\
{ }^{2} \text { Measured values exceeded instrument range, over range values replaced with 5,114 ppmv to calculate average and standard deviation } \\
{ }^{3} \text { Grate residue samples collected from tests using coal } \\
{ }^{4} \text { Based on past study of Puunene Boiler } 3 \text { conducted by Foster Wheeler in } 1978 \text { [4]. }\end{array}$} \\
\hline
\end{tabular}


Table 4. Historical original equipment manufacturer (OEM) air preheater exit gas temperature values by measurement and prediction

\begin{tabular}{lccccc}
\hline Unit & Fuel & Steam Flow Rate (klbs/hr) & $\mathrm{T}(\mathrm{F})$ & $\mathrm{T}(\mathrm{C})$ & Data Source $^{1}$ \\
\hline Boiler 1 & Baggase & 147.2 & 436 & 224 & 1 \\
Boiler 1 & Oil & 186.9 & 420 & 216 & 1 \\
Boiler 3 & Oil & 290.0 & 333 & 167 & 2 \\
Boiler 3 & Oil & 319.0 & 344 & 173 & 2 \\
Boiler 3 & Bagasse & 145.0 & 353 & 178 & 3 \\
Boiler 3 & Bagasse & 217.5 & 395 & 202 & 3 \\
Boiler 3 & Bagasse & 289.0 & 436 & 224 & 3 \\
Boiler 3 & Bagasse & 319.0 & 453 & 234 & 3 \\
Boiler 3 & Bagasse & 290.0 & 423 & 217 & 2 \\
Boiler 3 & Bagasse & 319.0 & 447 & 231 & 2 \\
\hline 1 1 indicates data from Riley Stoker acceptance test report [5] \\
2 indicates data from Foster Wheeler engineering prediction report [6] \\
3 indicates data from Foster Wheeler performance test report [7] \\
\hline \multicolumn{7}{l}{} \\
\hline \multicolumn{7}{l}{}
\end{tabular}

\section{$\square$ UH $\square$ HC\&S}

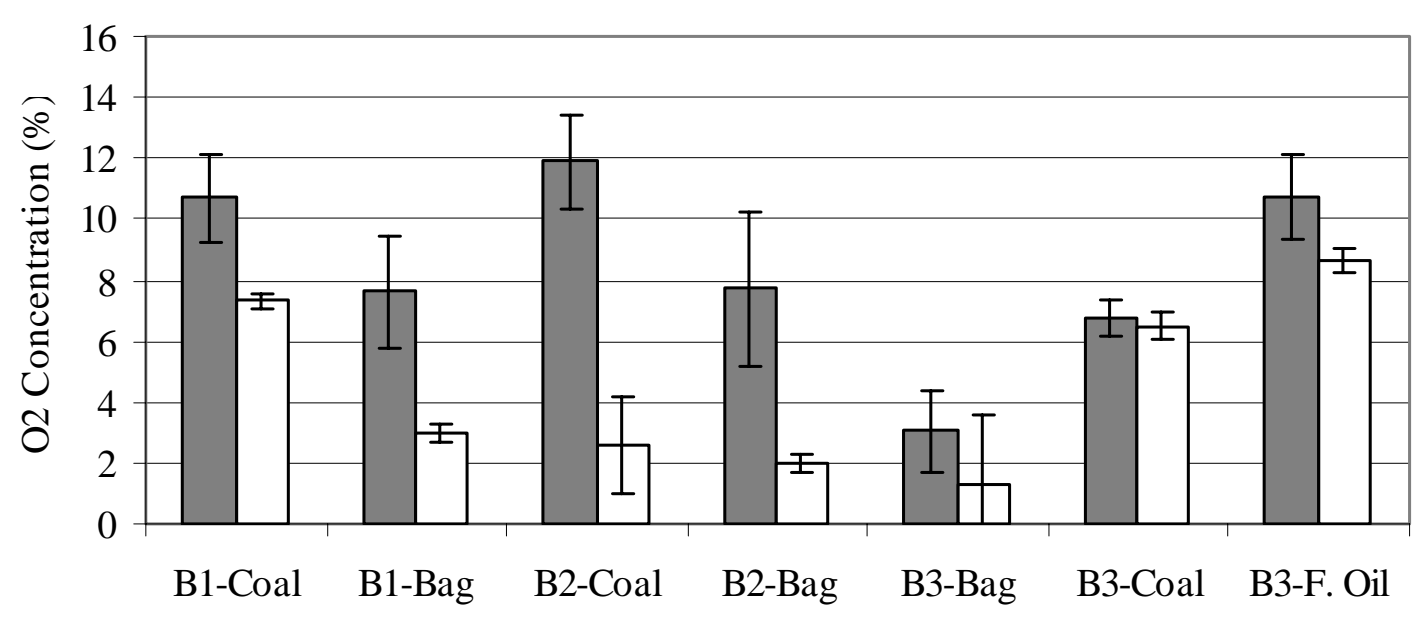

Figure 6. Comparison of average flue gas $\mathrm{O}_{2}$ concentrations recorded by the Horiba gas analyzer down stream of the air preheater and the $\mathrm{O}_{2}$ sensor installed in each boiler for monitoring and control purposes. B\# indicates boiler number. Coal, Bag, and F. Oil indicate that coal, bagasse, and fuel oil were fired during the measurement.

Results of the analysis of organic carbon concentrations in the grate residues are also presented in Table 3. It was assumed that firing fuel oil results in no residues and therefore no carbon losses. As noted in the previous section, grate samples were collected only from tests using coal 
since residues from bagasse were entrained from the grate and removed in the pollution control devices down stream. Carbon lost in this manner was not quantified in the present work. In cofiring tests conducted at HC\&S in 2002, samples were collected from the air preheater and dust precipitator water seal and wet scrubber effluents from Boiler 1 fired with a mixture of $25 \%$ coal, $13 \%$ fuel oil, and $62 \%$ bagasse. Analysis showed that the dust precipitator and wet scrubber removed large amounts of particulate matter that was composed of $~ 90 \%$ ash, indicating that the maximum organic carbon content of particulate matter would be $\sim 10 \%$ by difference. If measured, organic carbon content could be expected to be smaller than $10 \%$ since the combustible fraction contains other species. For example, the analysis of the grate residues collected from the current coal fired efficiency tests show that the organic carbon as a fraction of total combustibles ranges from 15 to $40 \%$. Based on this we can estimate that the organic carbon content of the particulate matter removed in the pollution control devices would be on the order of $5 \%$ and could be expected to reduce boiler efficiency accordingly, roughly in the range 0.1 to $0.2 \%$.

\subsubsection{Derived Quantities}

Excess air values reported in Table 3 were derived using the flue gas $\mathrm{O}_{2}$ concentration, the fuel composition, and chemical stoichiometry. Excess air is necessary to improve fuel conversion but too much contributes unnecessary thermal mass and increases air and flue gas handling requirements. Values calculated for coal ranged from $46 \%$ for Boiler 3, to $101 \%$ and $128 \%$ for Boilers 1 and 2, respectively. An excess air range of 30 to $60 \%$ is recommended for spreader stokers firing coal [3]. Similarly the value of excess air for all bagasse-fired units is recommended to be 25 to 35\% [3]. Excess air values calculated for Boilers 1, 2, and 3 operating on bagasse were 57, 58, and 17\%, respectively. Excess air values for fuel oil fired from registertype burners are recommended to be in a range of 5 to $10 \%$ [3]; the value calculated for Boiler 3 using fuel oil was $99 \%$.

Results of efficiency calculations using the indirect method are presented in Table 3.

Uncorrected values were calculated based on the $\mathrm{O}_{2}$ concentration and temperature of flue gas at the air preheater exit temperature and the fuel elemental composition and heating value. These values are corrected for losses in efficiency associated with organic carbon in the grate residue, carbon monoxide in the flue gas, surface radiation, and manufacturer's unaccounted for losses. Where appropriate, values for each of these losses are presented in Table 3. Assumed values for surface radiation losses and manufacturer's unaccounted for losses are based on values from past performance evaluations. Final boiler efficiencies are presented at the bottom of the table.

Calculated efficiencies for coal for Boilers 1, 2, and 3 were 80.8, 76.1, and 82.4\%, respectively. Several factors contributed to the lower efficiency of Boiler 2 when compared to the other two units. For the coal tests, Boiler 2 had the highest flue gas exit temperature and largest amount of excess air. The organic carbon content of the grate residue collected during testing of Boiler 2 was also higher than that from the other boiler tests. While this contributes to the lower boiler efficiency value, it is recognized that samples are mixtures of grate residues from Boilers 1 and 2, and Boiler 2 may not be wholly responsible for the elevated organic carbon content. CO in the flue gases from the three boilers ranged from 10 to $87 \mathrm{ppmv}$ and values for Boilers 1 and 2 were at the upper end of this range. 
Calculated efficiencies for Boilers 1, 2, and 3 firing bagasse were 65.9, 63.2, and 67.2\%, respectively, markedly lower than those determined for coal. The ranking of boilers in the order of decreasing efficiency was the same as with coal (Blr 3>Blr 1>Blr 2). In addition, the relative differences between units were also consistent for both fuels. Boiler 1 was $2 \%$ (relative) lower than Boiler 3 and Boiler 2 was $~ 7 \%$ (relative) lower than Boiler 3. The ranking of the boilers with regard to excess air (Blr 2>Blr 1>Blr 3) and exit temperature (Blr 2>Blr 3>Blr 1) followed the same order as those found for coal and these parameters are largely responsible for the differences in efficiency when comparing boilers fired on the same fuel. The elevated carbon monoxide levels when firing bagasse also reduced efficiency, with losses ranging from 0.62 to $1.29 \%$.

All three of the boilers exhibited similar reductions in efficiency when operated on bagasse relative to coal, becoming 17 to $18.5 \%$ (relative) less efficient. This can be attributed largely to the greater moisture content of bagasse, $48 \%$ compared to 6 to $10 \%$ for coal.

Boiler 3 was the only unit tested on No. 2 fuel oil and calculated efficiency was $82.0 \%$.

Sensitivity calculations were performed to provide an indication of the efficiency increases that could result from reductions in excess air or the flue gas temperature at the preheater exit.

Results are presented in Figure 7. Boiler 2 fueled with coal had the highest excess air measured during the test campaign at 128\%. Reducing the excess air from 128 to $45 \%$ (by decreasing the flue gas $\mathrm{O}_{2}$ concentration used in the calculation from 11.9 to 6.6\%) increased boiler efficiency from 76.1 to $81.4 \%$. Similarly, the highest average air preheater exit temperature, $248^{\circ} \mathrm{C}$, was recorded on Boiler 2 fueled with bagasse. Changing only the value of the exit temperature from 248 to $220^{\circ} \mathrm{C}\left(428^{\circ} \mathrm{F}\right)$ resulted in an increase in boiler efficiency from 63.2 to $65.1 \%$. Reducing $\mathrm{CO}$ in the flue gas and the organic carbon content of grate residues also will result in efficiency improvements. The calculated losses in efficiency from each are presented in Table 3 and provide upper limits to possible efficiency increases.

Boiler efficiency is defined as the energy embodied in the steam flowing out of the boiler, divided by the energy contained in the fuel flowing into the boiler at steady state. Potential savings from incremental improvements in boiler efficiency can be calculated by (1) holding the fuel flow rate constant, increasing the efficiency value, and then calculating the increased steam flow rate, or (2) holding the steam flow rate constant, increasing the efficiency value, and then calculating the decreased fuel flow rate. The former method was used to calculate fuel savings that could be expected from a 1\% improvement in boiler efficiency for each of the steam generating units.

The results of these calculations are presented in Table 5. In addition, associated savings were estimated using fuel unit costs provided by HC\&S and are included in Table 5. A 1\% improvement in operating efficiency using coal in Boilers 1 and 2 would save 4.3 tons coal (dry basis) per day. Similarly, a 1\% improvement in efficiency for Boiler 3 operating on coal would result in savings of 5.2 tons coal (dry basis) per day. At a coal unit cost of $\$ 65.22$ per ton, this would result in direct fuel savings worth $\$ 620$ per day when all three boilers fired coal. 


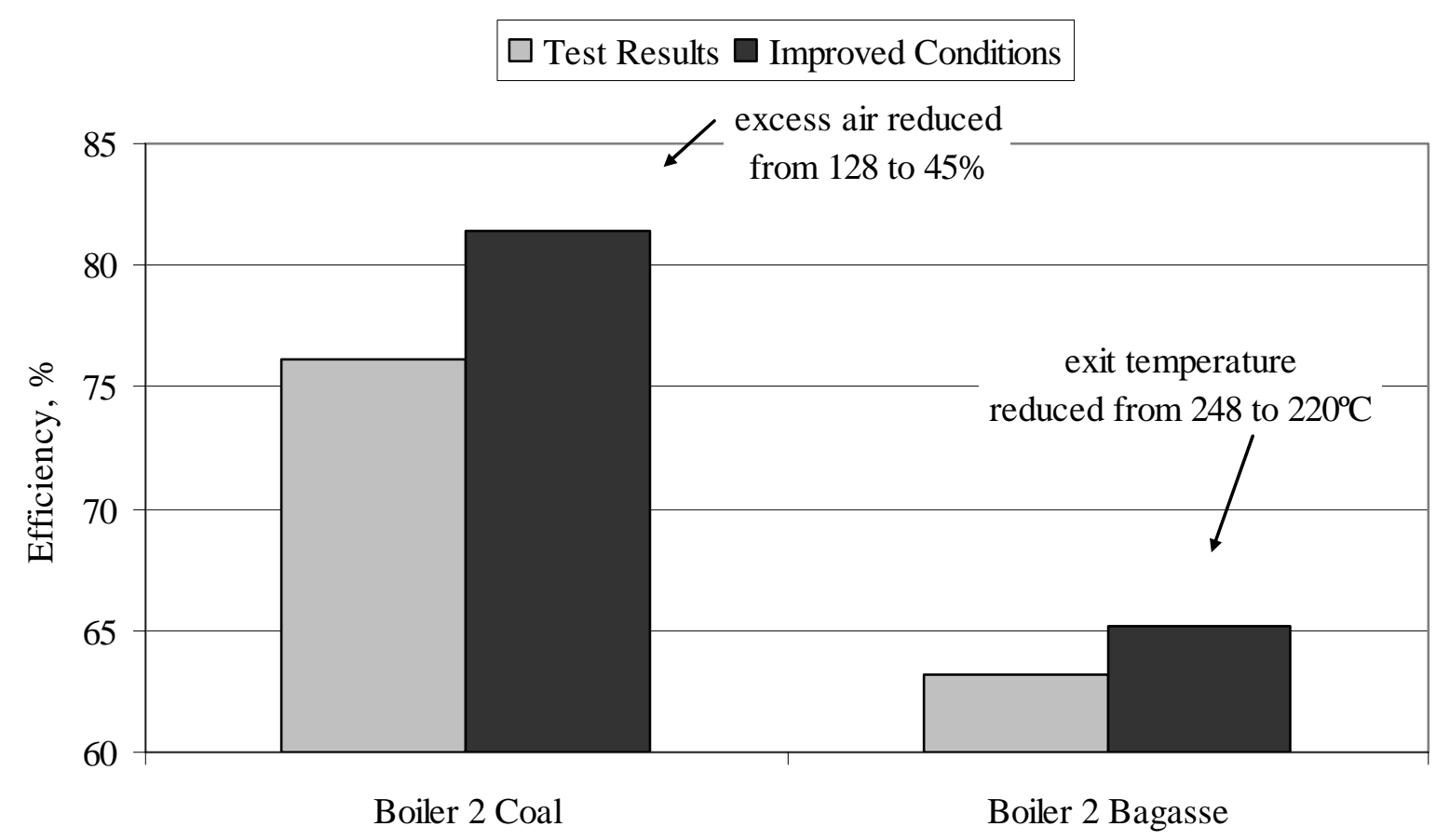

Figure 7. Sensitivity of boiler efficiency calculations to changes in values of excess air and flue gas temperature at the exit of the air preheater.

If all three boilers fire bagasse, a 1\% improvement in efficiency would save 21.5 tons (dry basis) per day. A value for bagasse, $\$ 34.78$ per ton (dry basis), was calculated based on the price of coal, the heating values of the two fuels, and the average boiler efficiencies for the two fuels. The latter was weighted based on steam flow. i.e. since Boiler 3 produces more steam it would contribute more to the average. The 21.5 tons of bagasse saved by efficiency improvements would result in a cost savings of $\sim \$ 750$ per day.

\section{Summary and Conclusions}

Measurements required to calculate steam generation efficiency were made on three generating units operated by Hawaiian Commercial \& Sugar Co. at its Puunene factory in September, 2003, and June, 2004. Tests were conducted on coal and bagasse for all three units and a single test firing fuel oil was conducted in Boiler 3. Measurements of flue gas temperature and composition at the exit of the air preheater were made during each test. Fuel and grate ash were sampled and analyses were performed following the test. Flue gases and temperatures were sampled using probe bundles inserted through access ports in the boiler walls. Each probe bundle was composed of three extraction tubes paired with Type K thermocouples. Boiler 1 was fitted with two probe bundles (a total of six sample extraction locations), whereas Boilers 2 and 3 were fitted with one probe bundle each. Data recorded during the test campaign were later reduced to average values and used to calculate steam generation efficiencies using the energy balance method. 
Flue gas temperatures at the exit of the air preheater of the three boilers ranged from 180 to $248^{\circ} \mathrm{C}$. The lowest exit temperature was recorded for Boiler 3 during the fuel oil test. When bagasse and coal were fired, Boiler 1 had the lowest exit temperature and Boilers 2 and 3 were consistently higher by 15 and $\sim 30^{\circ} \mathrm{C}$, respectively. Exit temperatures of all boilers were higher when bagasse was fired, ranging from 220 to $248^{\circ} \mathrm{C}$ compared to 189 to $219^{\circ} \mathrm{C}$ for coal.

Gas composition measurements made after the air preheater showed carbon monoxide concentrations to be highly dependent on fuel type. Fossil fuels produced relatively low CO concentrations in the range of 10 to 90 ppmv. Bagasse tests exhibited elevated CO concentrations with averages ranging from 1,300 to 3,200 ppmv.

Excess air values calculated for coal tests ranged from $46 \%$ for Boiler 3, to $101 \%$ and $128 \%$ for Boilers 1 and 2, respectively. Excess air values calculated for Boilers 1, 2, and 3 operating on bagasse were 57, 58, and 17\%, respectively. The excess air value calculated for Boiler 3 using fuel oil was $99 \%$.

Calculated efficiencies for Boilers 1, 2, and 3 firing coal were 80.8, 76.1, and 82.4\%, respectively. Higher excess air values and flue gas exit temperatures were the main factors contributing to the lower efficiency of Boiler 2 compared to the other two units.

Calculated efficiencies for Boilers 1, 2, and 3 firing bagasse were 65.9, 63.2, and 67.2\%, respectively, markedly lower than those determined for coal. Boiler 2's lower efficiency resulted from higher flue gas temperature and CO concentrations compared to the other two units. Compared to coal, lower efficiencies observed when firing bagasse were largely due to the different moisture contents of the two fuels, $48 \%$ for bagasse and 6 to $10 \%$ for coal.

Several opportunities to improve boiler efficiency are evident from the results of the test campaign. Reducing excess air, flue gas CO concentrations, and flue gas exit temperature all can contribute to increased efficiency although the first two items may be more easily addressed in the near term. It is recognized that the ability to respond to these opportunities may largely be determined by the physical limitations of the boiler system's equipment and the constraints imposed by operating the units in conjunction with the Puunene sugar factory.

Potential cost savings from improvements in boiler efficiency were calculated. When all three steam generating units fire coal, a $1 \%$ increase in efficiency in each of the three boilers would result in a savings of 9.5 tons of coal per day with an associated cost savings of $\$ 620$ per day. Similarly, when all three units fire bagasse, a $1 \%$ improvement in efficiency would save 21.5 tons fuel (dry basis) per day and result in a cost savings of $\$ 750$ per day. 
Table 5. Projected fuel savings that could result from a 1\% improvement in efficiency for each steam generating unit above efficiency values measured on the indicated test date.

\begin{tabular}{|c|c|c|c|c|c|c|c|}
\hline $\begin{array}{l}\text { Boiler No. } \\
\text { Test Date } \\
\text { Fuel }\end{array}$ & $\begin{array}{r}1 \\
9 / 22 / 03 \\
\text { Coal }\end{array}$ & $\begin{array}{r}1 \\
9 / 23 / 03 \\
\text { Bagasse }\end{array}$ & $\begin{array}{r}2 \\
6 / 29 / 04 \\
\text { Coal }\end{array}$ & $\begin{array}{r}2 \\
9 / 23 / 03 \\
\text { Bagasse }\end{array}$ & $\begin{array}{r}3 \\
9 / 25 / 03 \\
\text { Bagasse }\end{array}$ & $\begin{array}{r}3 \\
9 / 26 / 03 \\
\text { Coal }\end{array}$ & $\begin{array}{r}3 \\
9 / 27 / 03 \\
\# 2 \text { Fuel Oil }\end{array}$ \\
\hline Steam Flow rate from Wonderware system, klb/hr & 105.9 & 104.2 & 104.0 & 105.3 & 259.7 & 266.8 & 241.8 \\
\hline Steam Pressure from Wonderware system, psig & 900.0 & 899.7 & 898.7 & 898.9 & 421.6 & 426.8 & 418.7 \\
\hline Steam Pressure from Wonderware system, MPa & 6.2 & 6.2 & 6.2 & 6.2 & 2.9 & 2.9 & 2.9 \\
\hline Steam Temperature from Wonderware system, ${ }^{\circ} \mathrm{F}$ & 694.5 & 749.9 & 749.5 & 749.9 & 765.9 & 733.6 & 725.5 \\
\hline Steam Enthalpy, BTU/lbm & $1,326.6$ & $1,364.4$ & $1,364.2$ & $1,364.5$ & $1,396.4$ & $1,378.5$ & $1,374.5$ \\
\hline Steam Enthalpy Flow, MMBTU/hr & 140.5 & 142.2 & 141.9 & 143.7 & 362.7 & 367.7 & 332.4 \\
\hline Fuel Energy Flow, MM BTU/hr & 173.8 & 215.9 & 186.4 & 227.3 & 539.3 & 446.2 & 405.4 \\
\hline $\begin{array}{l}\text { Fuel Energy Flow with 1\% Increase in Efficiency, } \\
\text { MM BTU/hr }\end{array}$ & 171.7 & 212.7 & 184.0 & 223.7 & 531.4 & 440.8 & 400.5 \\
\hline $\begin{array}{l}\text { Fuel Energy Savings with 1\% increase in Efficiency, } \\
\text { MM BTU/hr }\end{array}$ & 2.1 & 3.2 & 2.4 & 3.5 & 7.9 & 5.3 & 4.9 \\
\hline Fuel Heating Value, BTU/lbm (dry basis) & 12,476 & 8,167 & 12,725 & 8,167 & 8,194 & 12,437 & 19,640 \\
\hline $\begin{array}{l}\text { Fuel Savings with } 1 \% \text { increase in efficiency, } \\
\text { ton/day (dry basis) }\end{array}$ & 2.0 & 4.7 & 2.3 & 5.2 & 11.6 & 5.2 & 3.0 \\
\hline $\begin{array}{l}\text { Fuel Savings with } 1 \% \text { increase in efficiency, } \\
\text { Mg per day (dry basis) }\end{array}$ & 1.9 & 4.3 & 2.1 & 4.7 & 10.5 & 4.7 & 2.7 \\
\hline Unit Cost of Fuel (\$/dry ton) & 65.22 & $34.78^{1}$ & 65.22 & $34.78^{1}$ & $34.78^{1}$ & 65.22 & $454.69^{2}$ \\
\hline $\begin{array}{l}\text { Fuel Cost Savings with } 1 \% \text { increase in efficiency, } \\
\$ / \text { day }\end{array}$ & 133 & 165 & 149 & 181 & 403 & 337 & 1,357 \\
\hline
\end{tabular}




\section{References}

1. Anon. 1999. Fired Steam Generators, Performance Test Codes, ASME PTC 4-1998. The American Society of Mechanical Engineers. NY, NY.

2. Kinoshita, C.M. 1992. Cogeneration in the Hawaiian Sugar Industry. Hawaii Natural Energy Institute, University of Hawaii.

3. Anon. 1978. Steam - Its Generation and Use. Babcock \& Wilcox. NY, NY.

4. Foster Wheeler. 1978. Data attached to December 5, 1978 letter from Willard M. Eller, HC\&S to R.H. Magee, Foster Wheeler Company.

5. Anon. 1956. Acceptance Test Report of The Electric Steel Foundry Company, Honolulu, T. H. Covering One Riley Steam Generating Unit Fired with a Traveling Grate Spreader Stoker and Pneumatic Distributors for Bagasse Fuel with Auxiliary Firing by Oil Installed at The Hawaiian Commercial \& Sugar Company, Puunene, Maui, Hawaii. Contract B2151 RST 5589. Riley Stoker Corporation, Worcester, Massachusetts.

6. Anon. 1972. Summary Performance Sheet for Engineering Prediction, Proposal 72-2149. Foster Wheeler Ltd., St. Catharines, Ontario.

7. Anon. 1978. Summary Performance Sheet. Foster Wheeler Ltd., St. Catharines, Ontario. 
Appendix A 


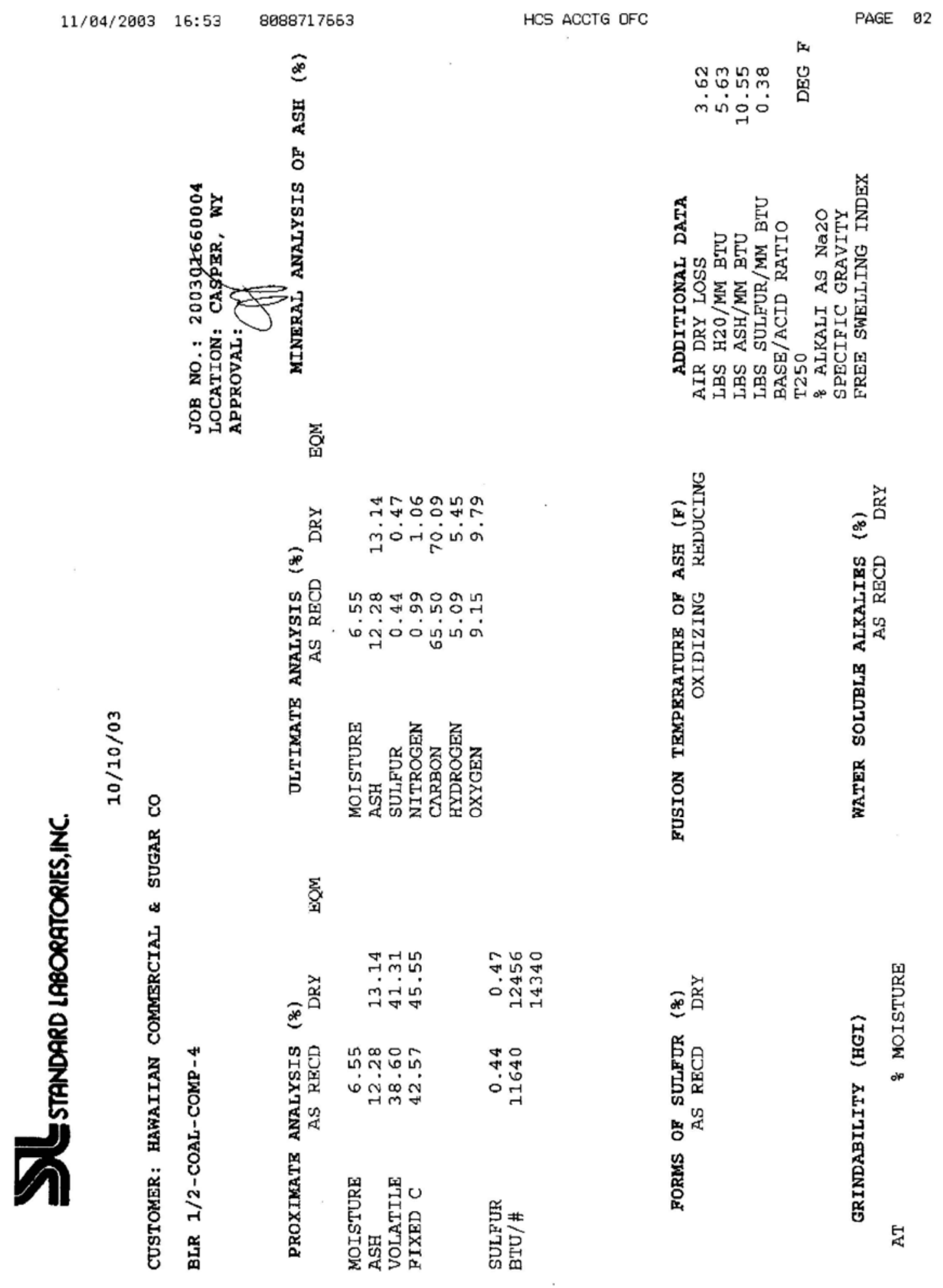




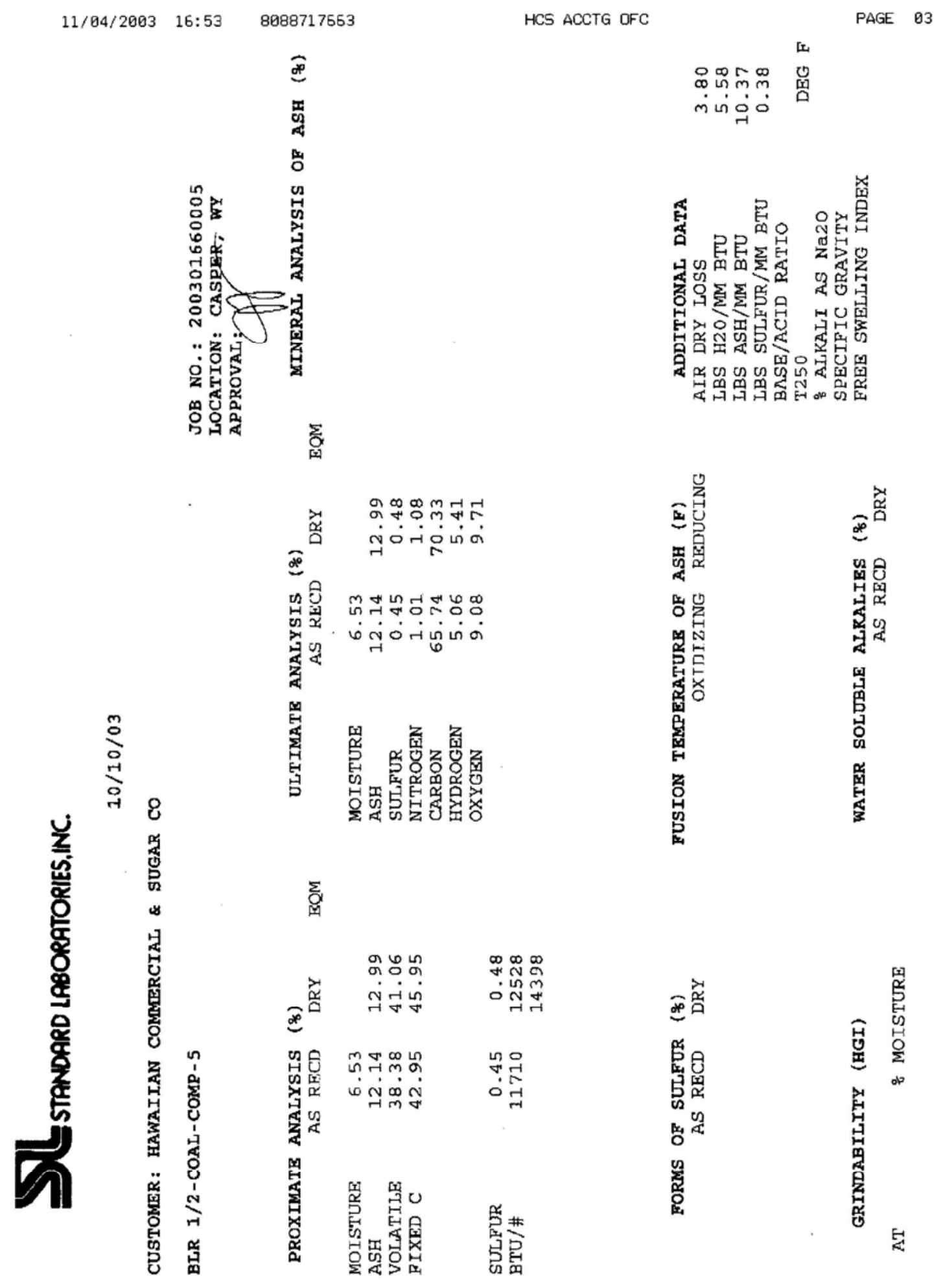




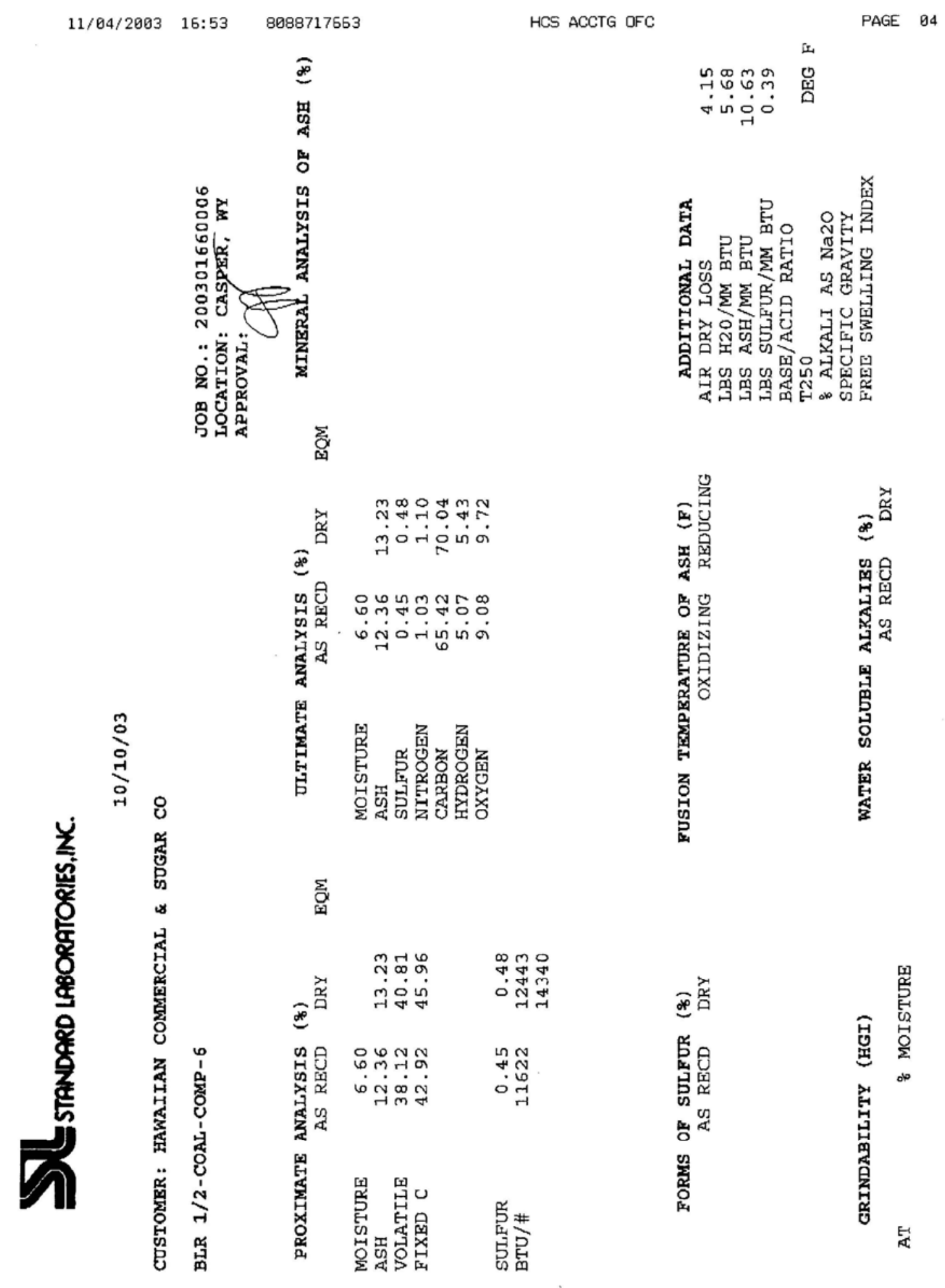




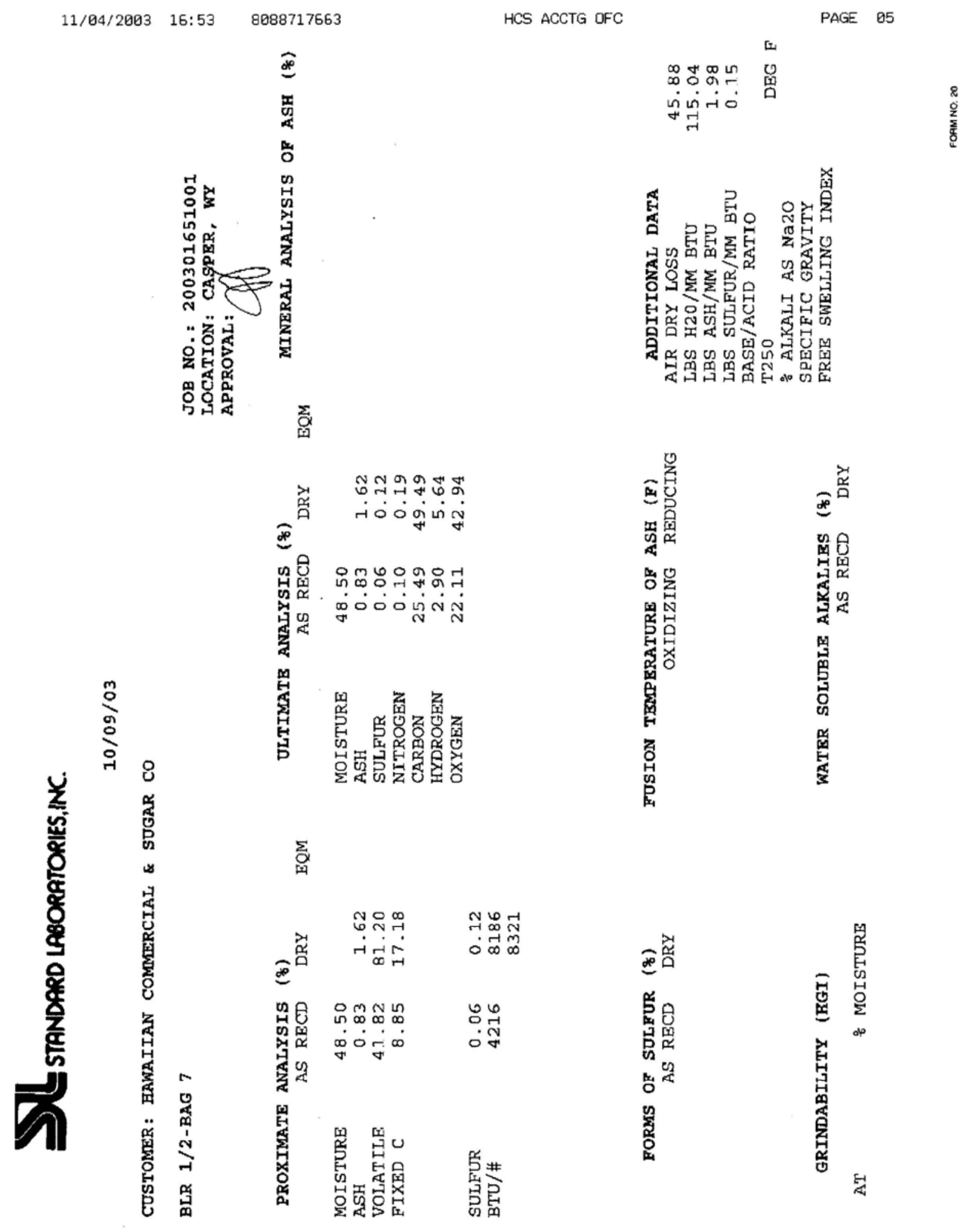




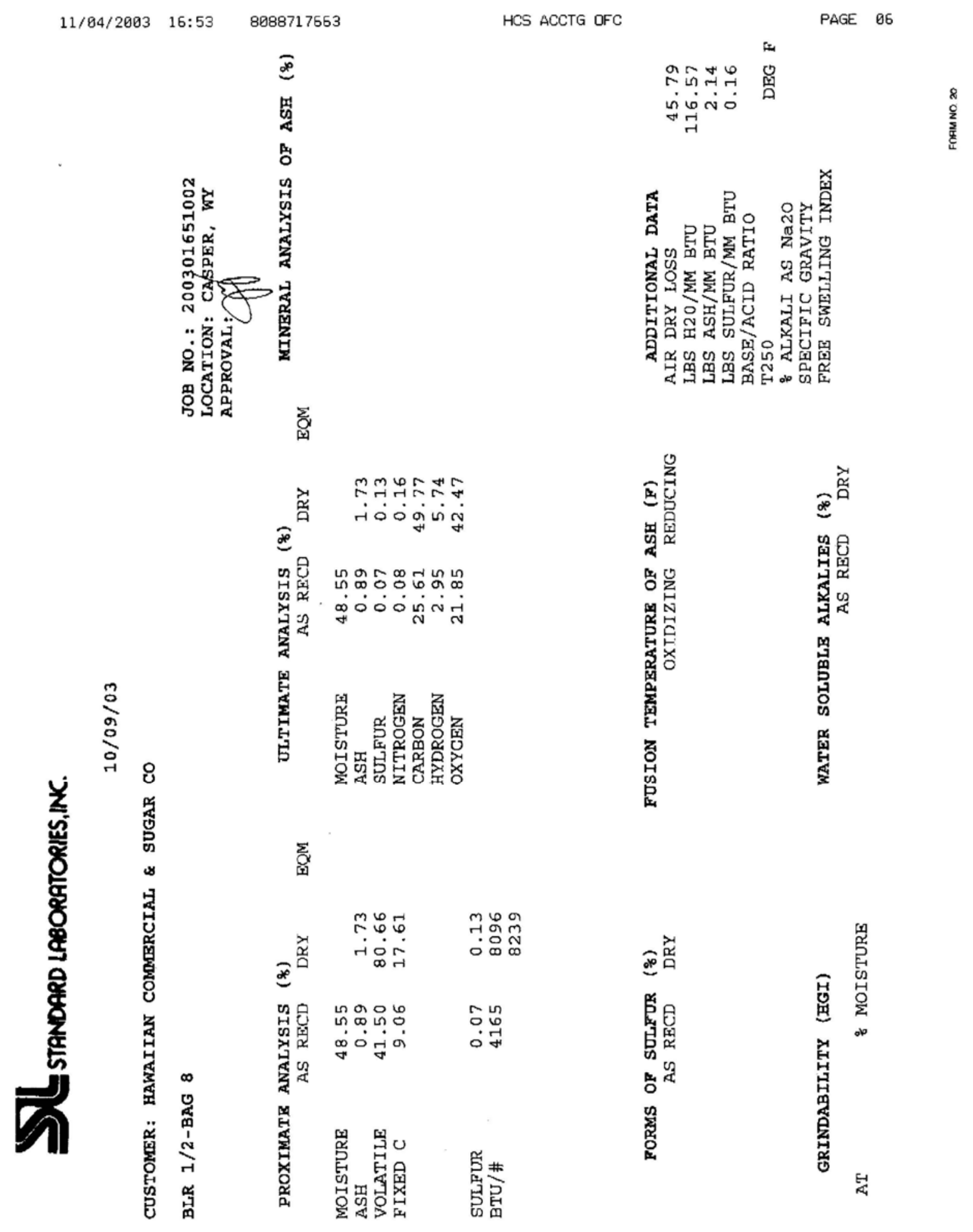




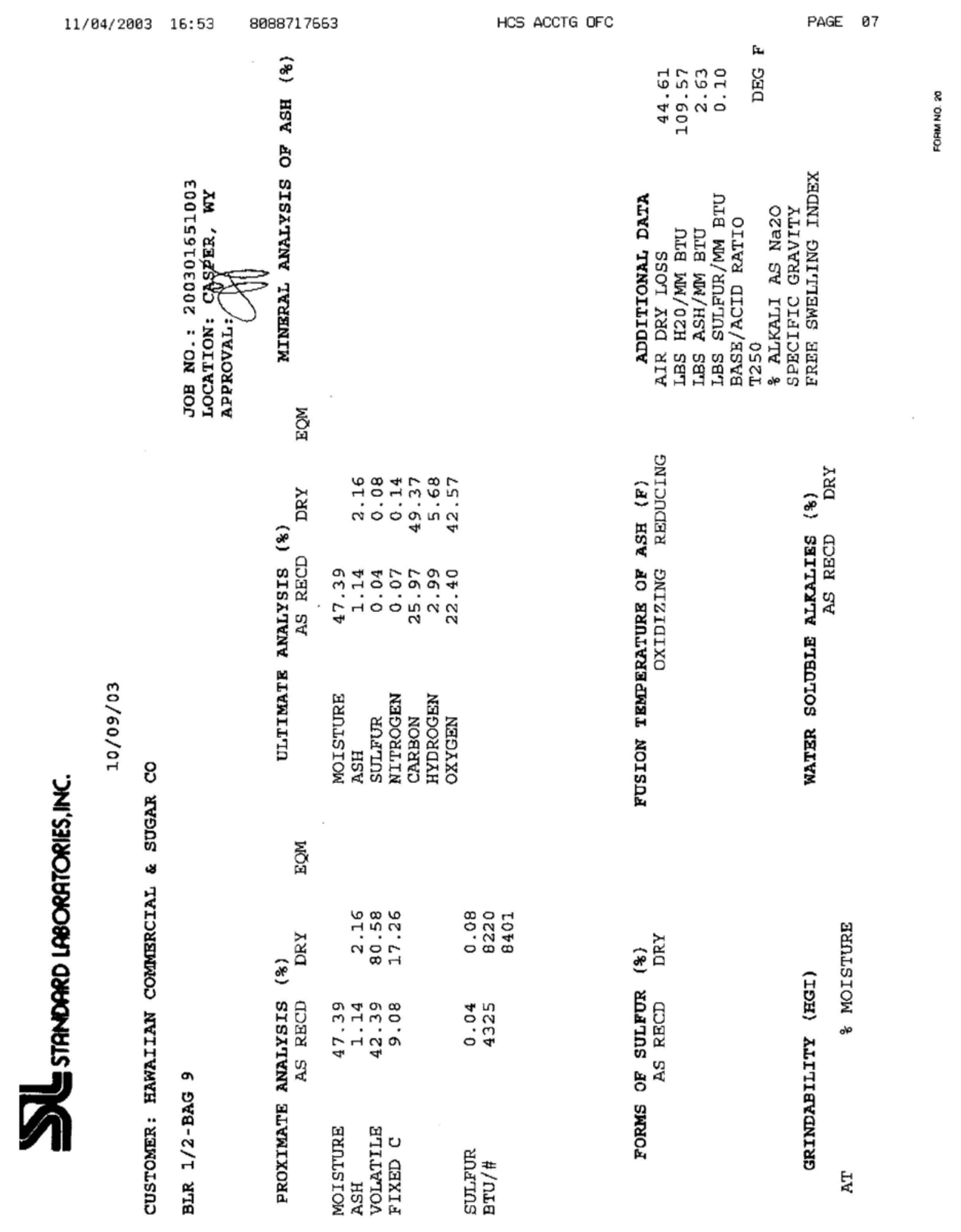




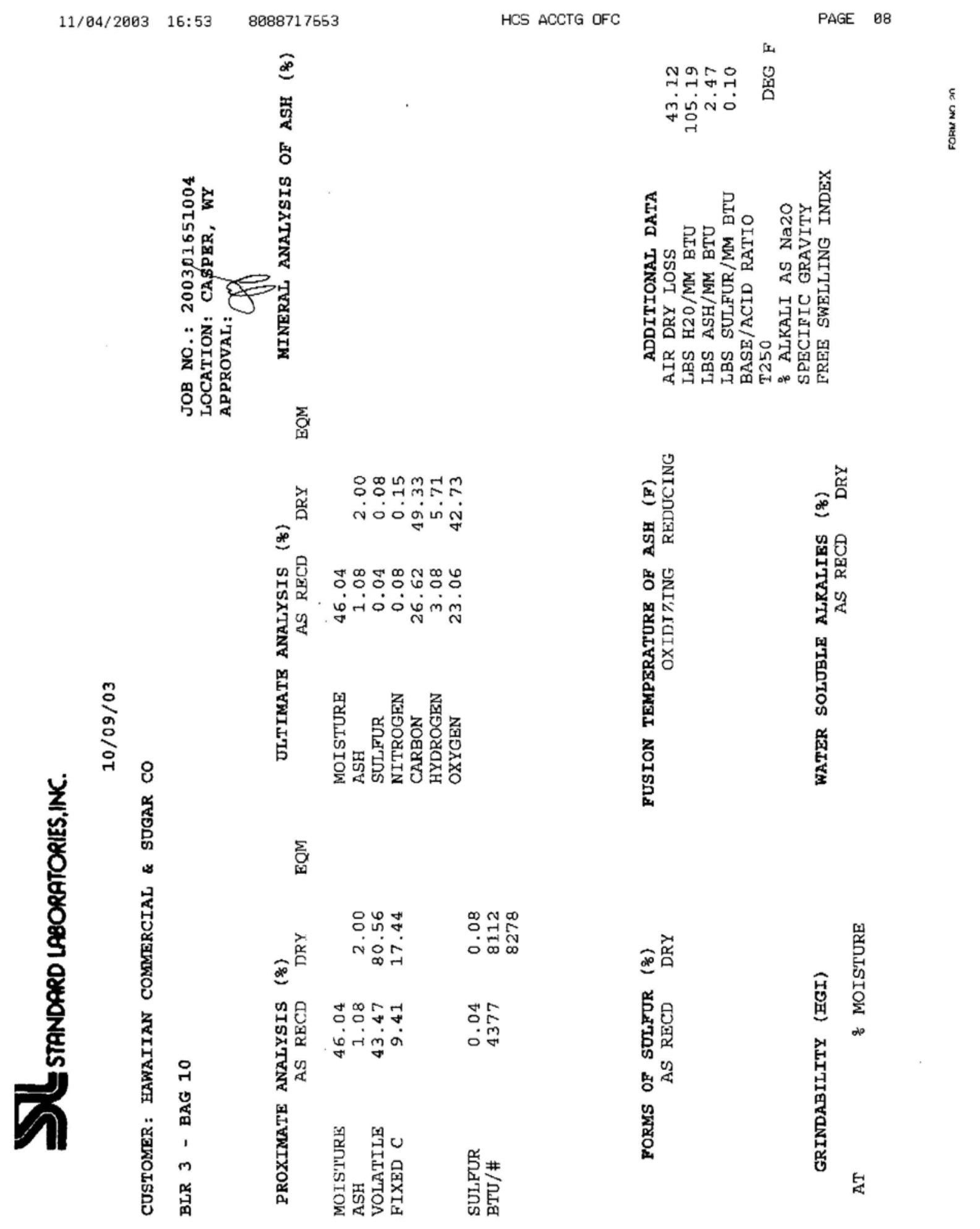




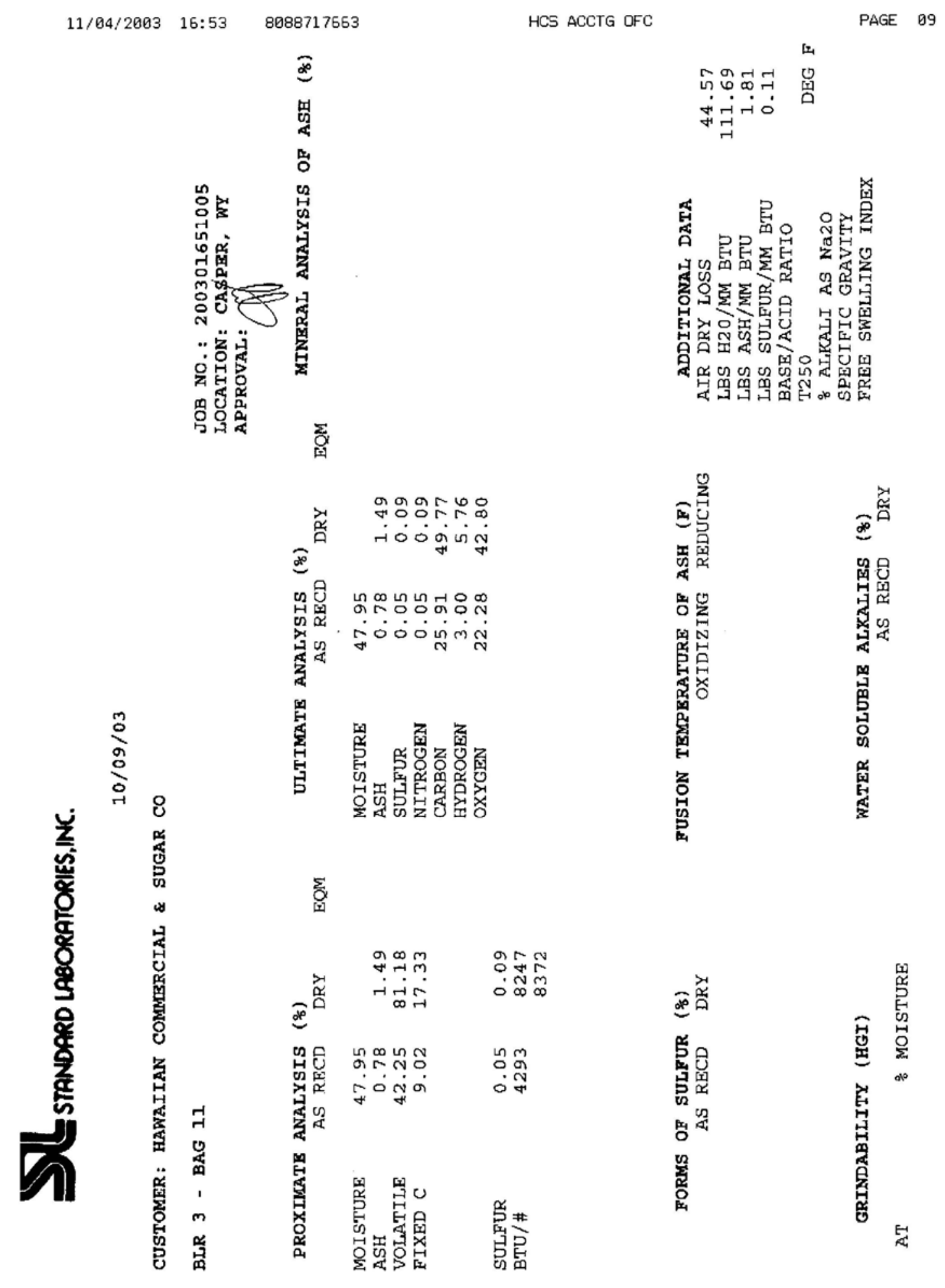




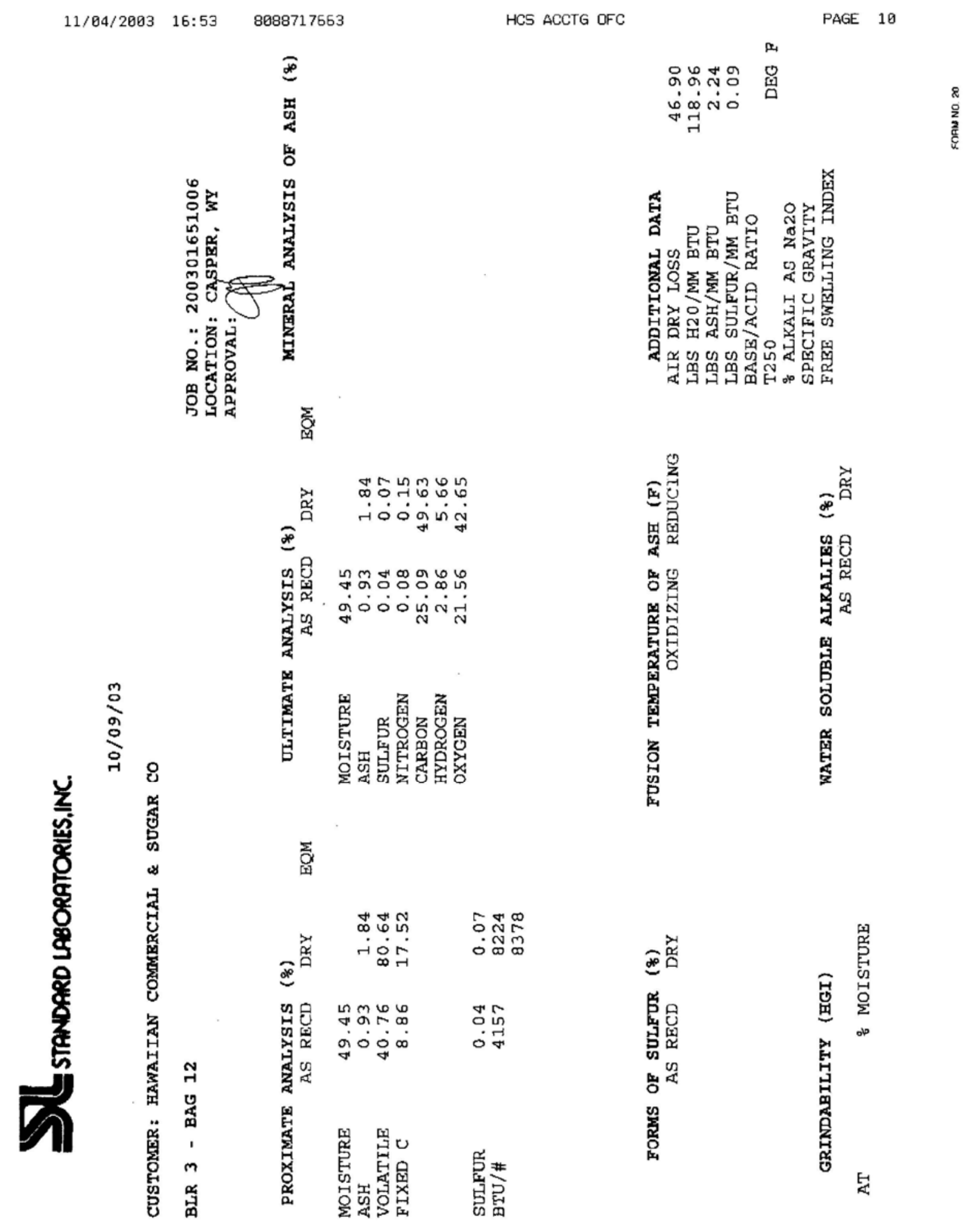




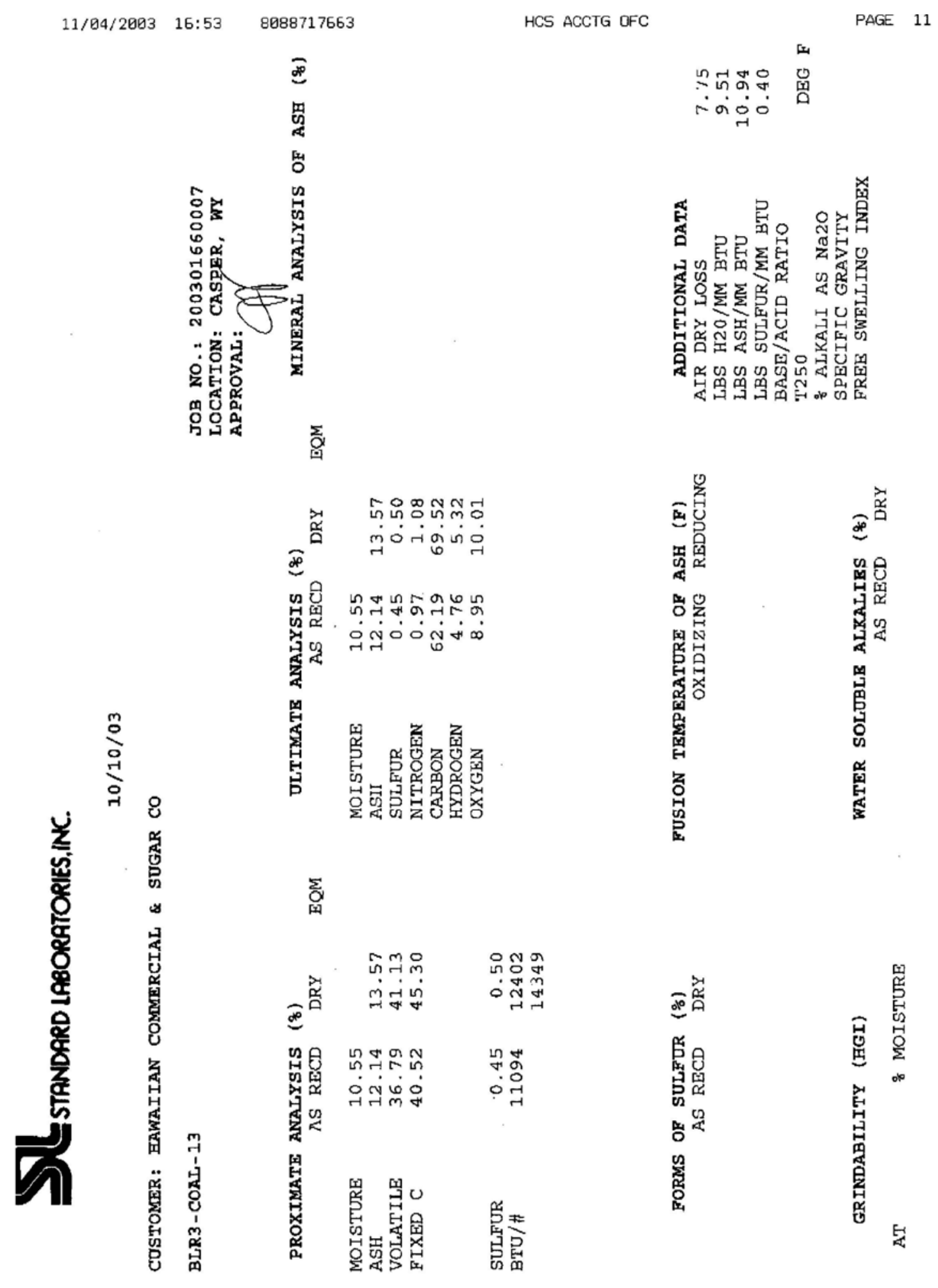




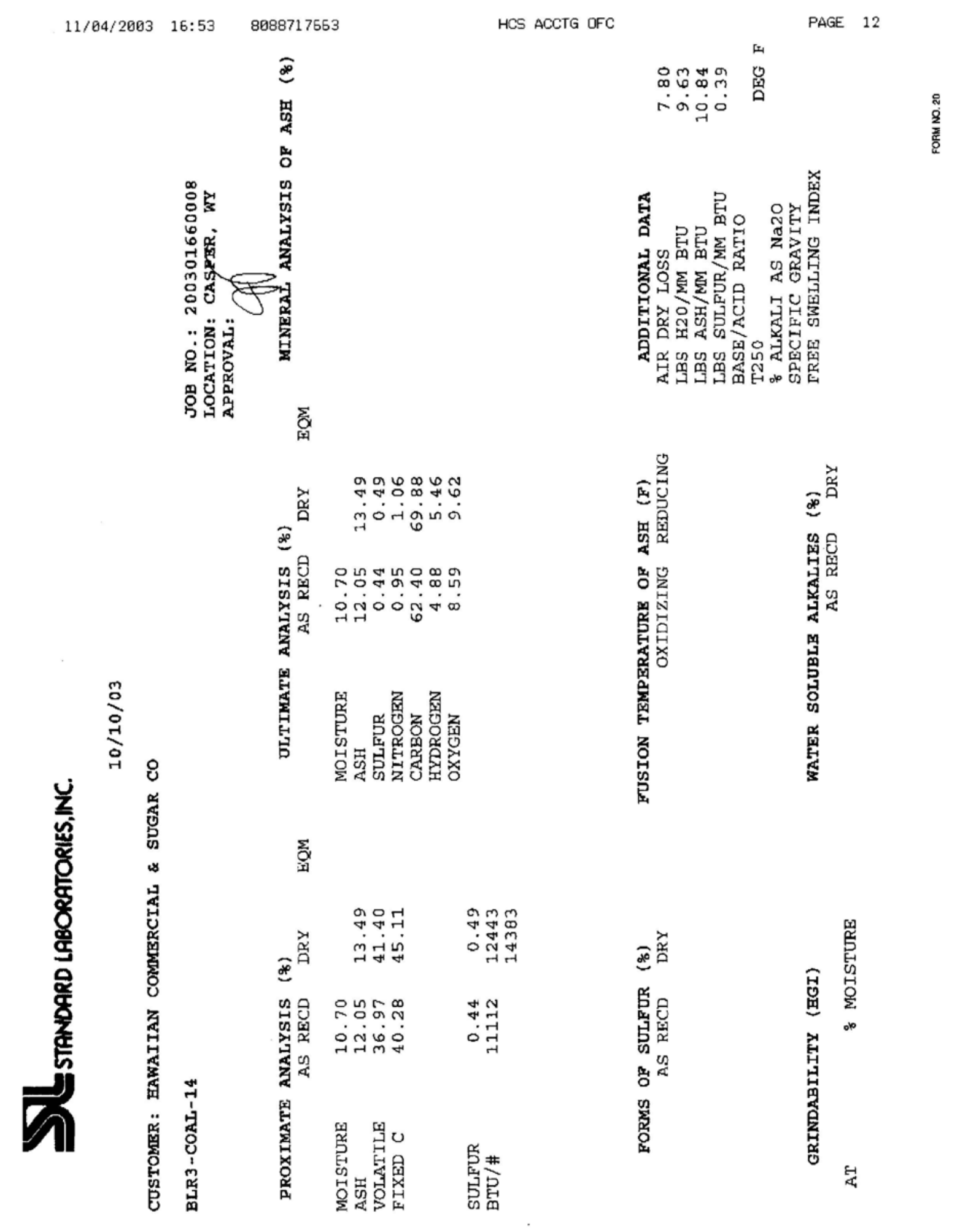




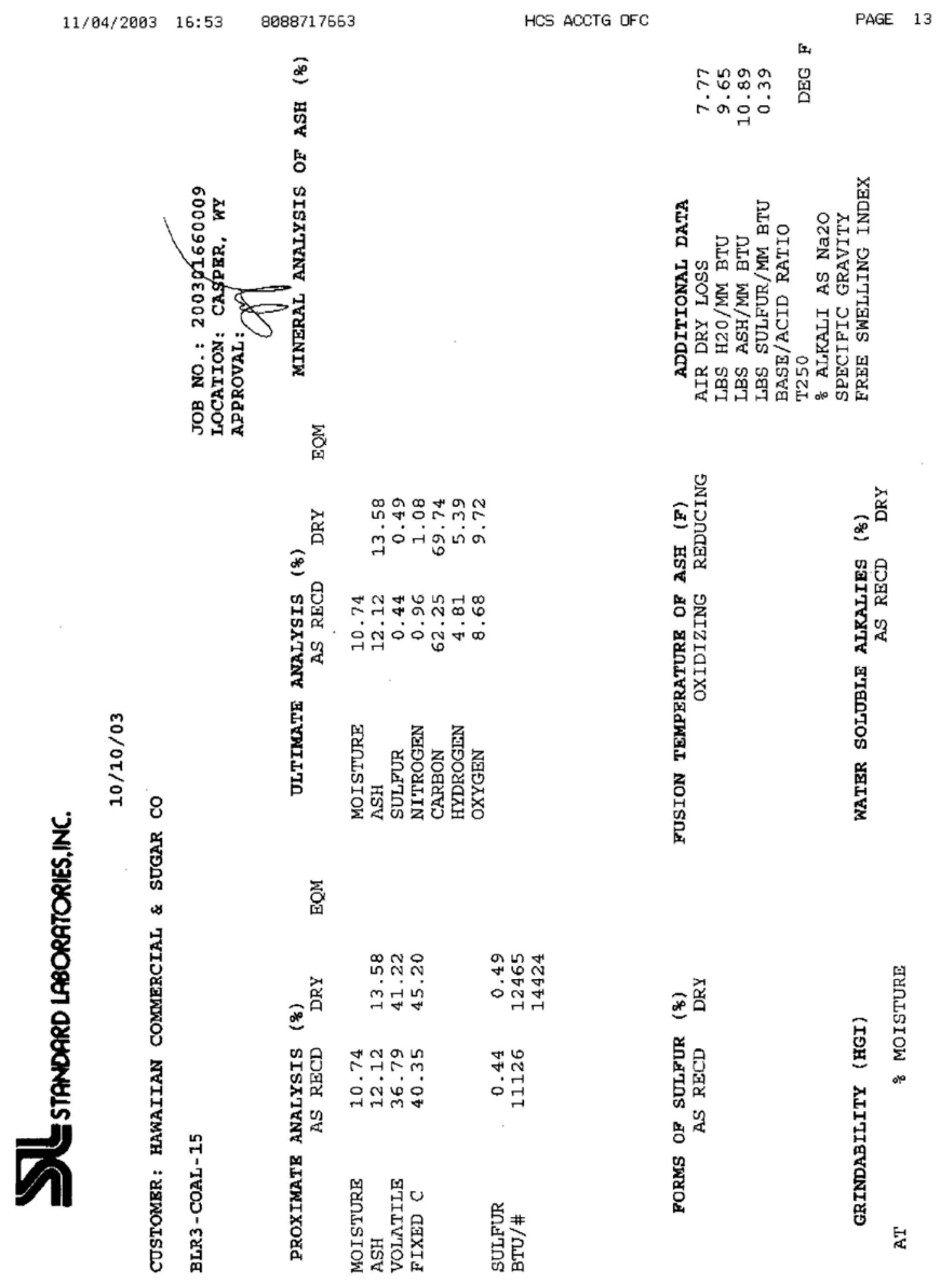




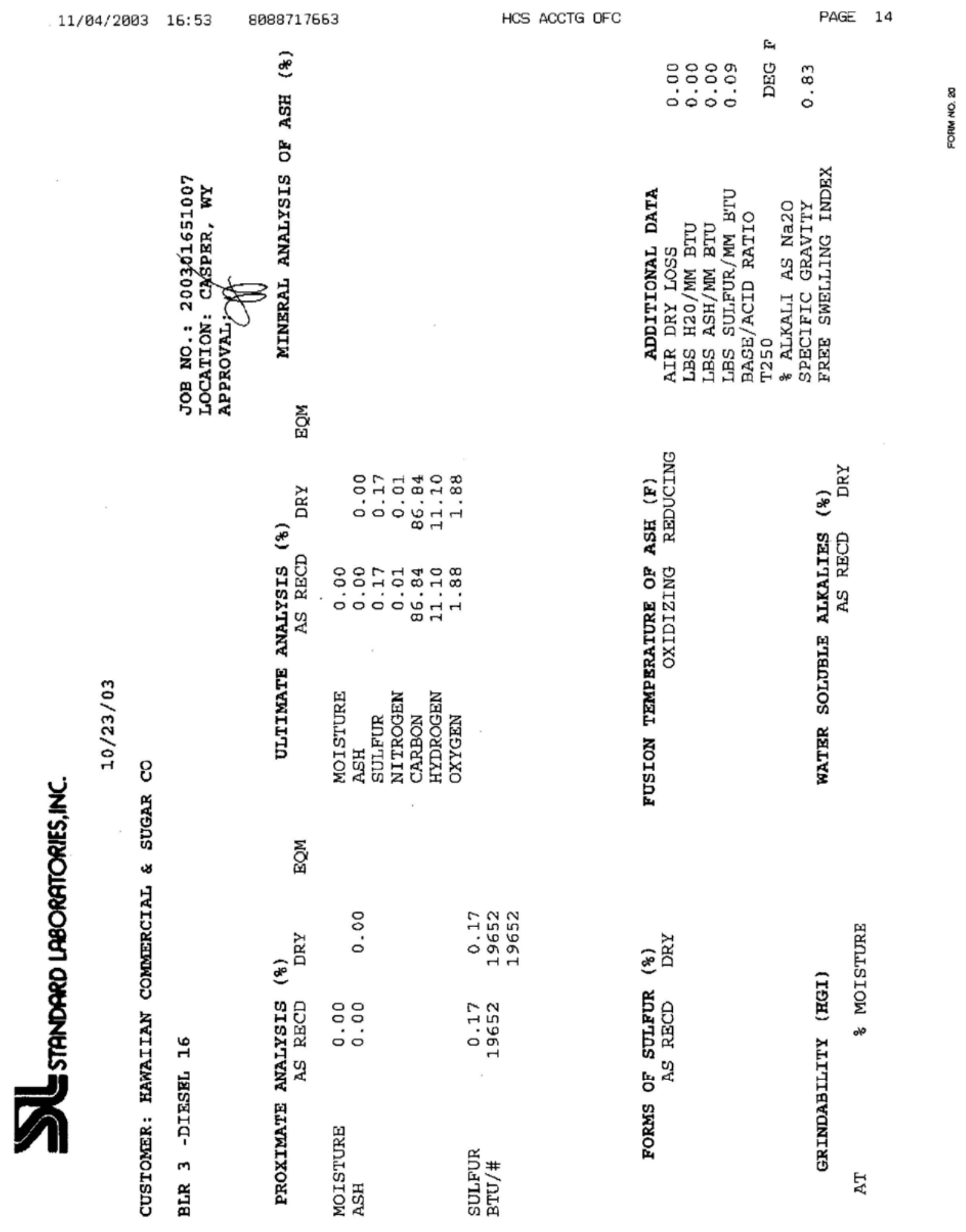




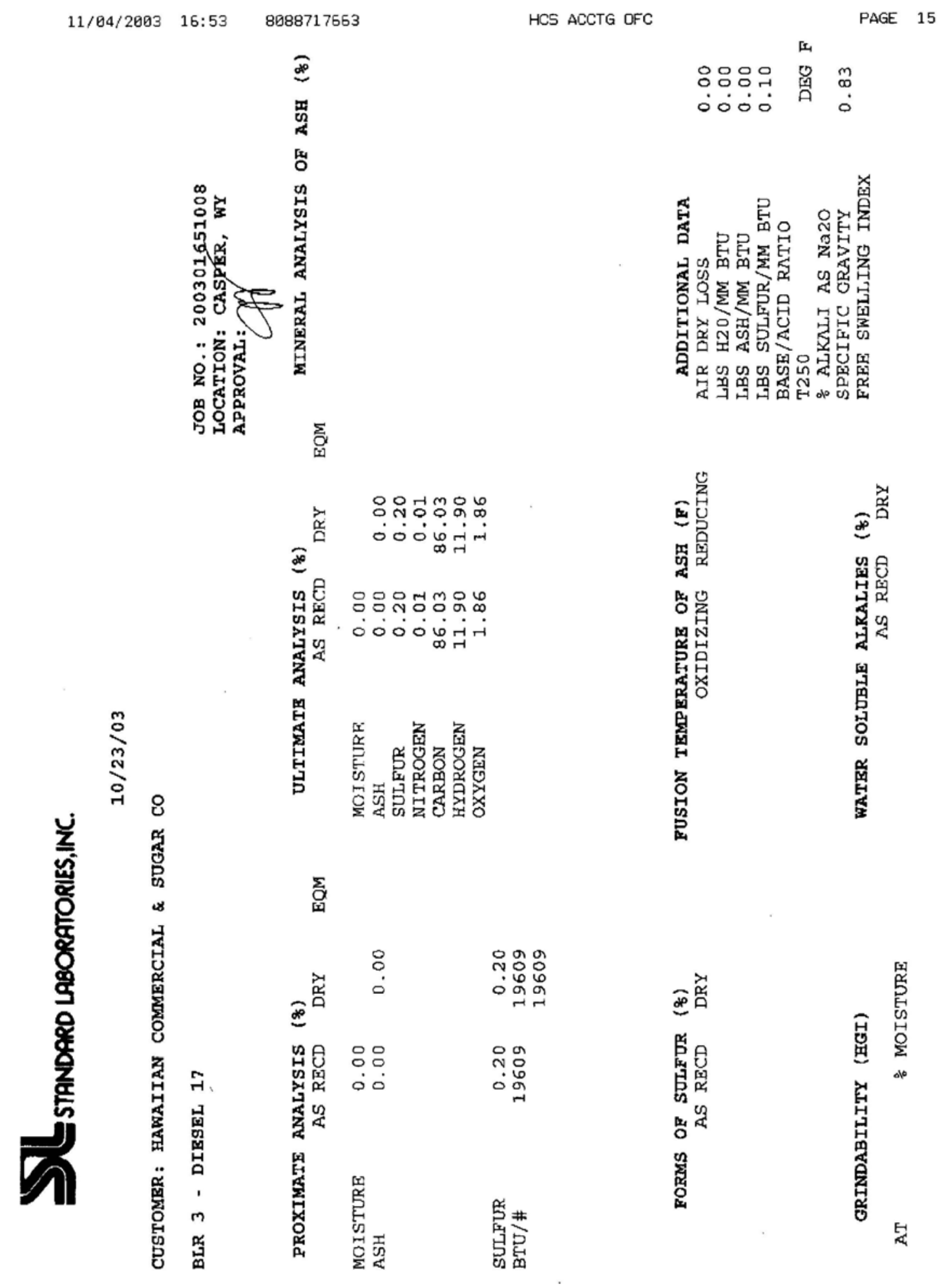




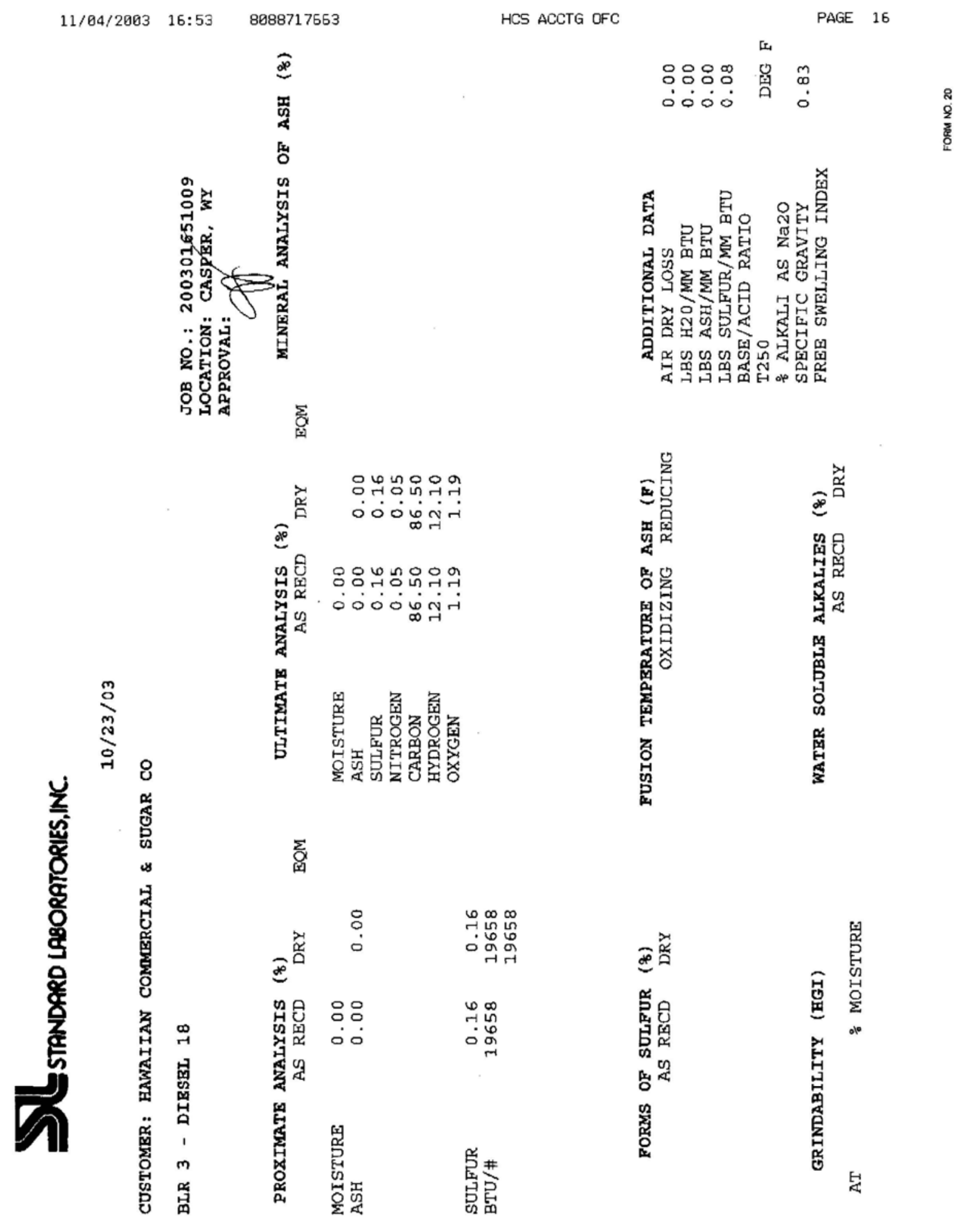




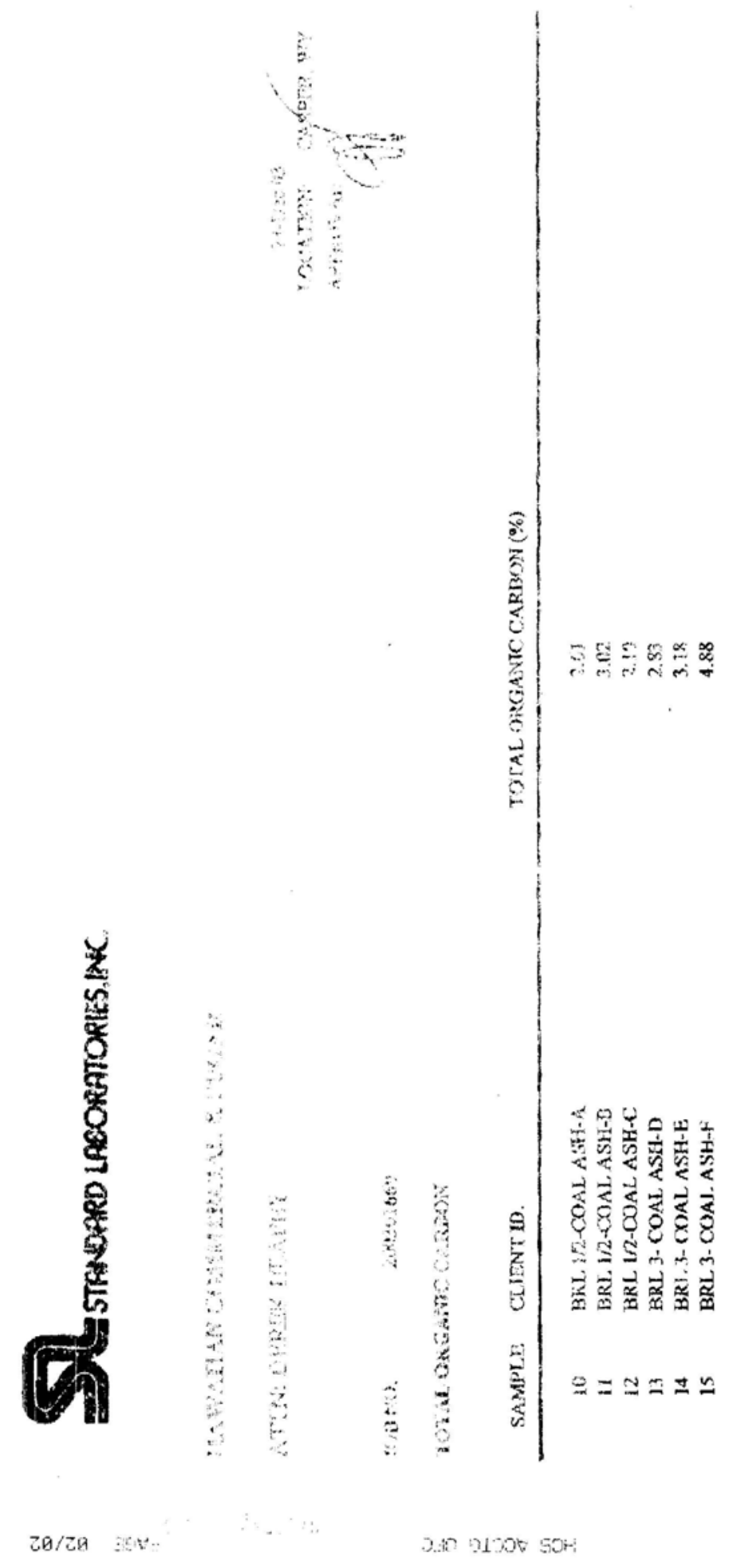

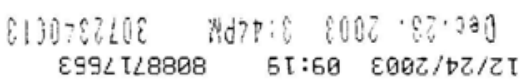




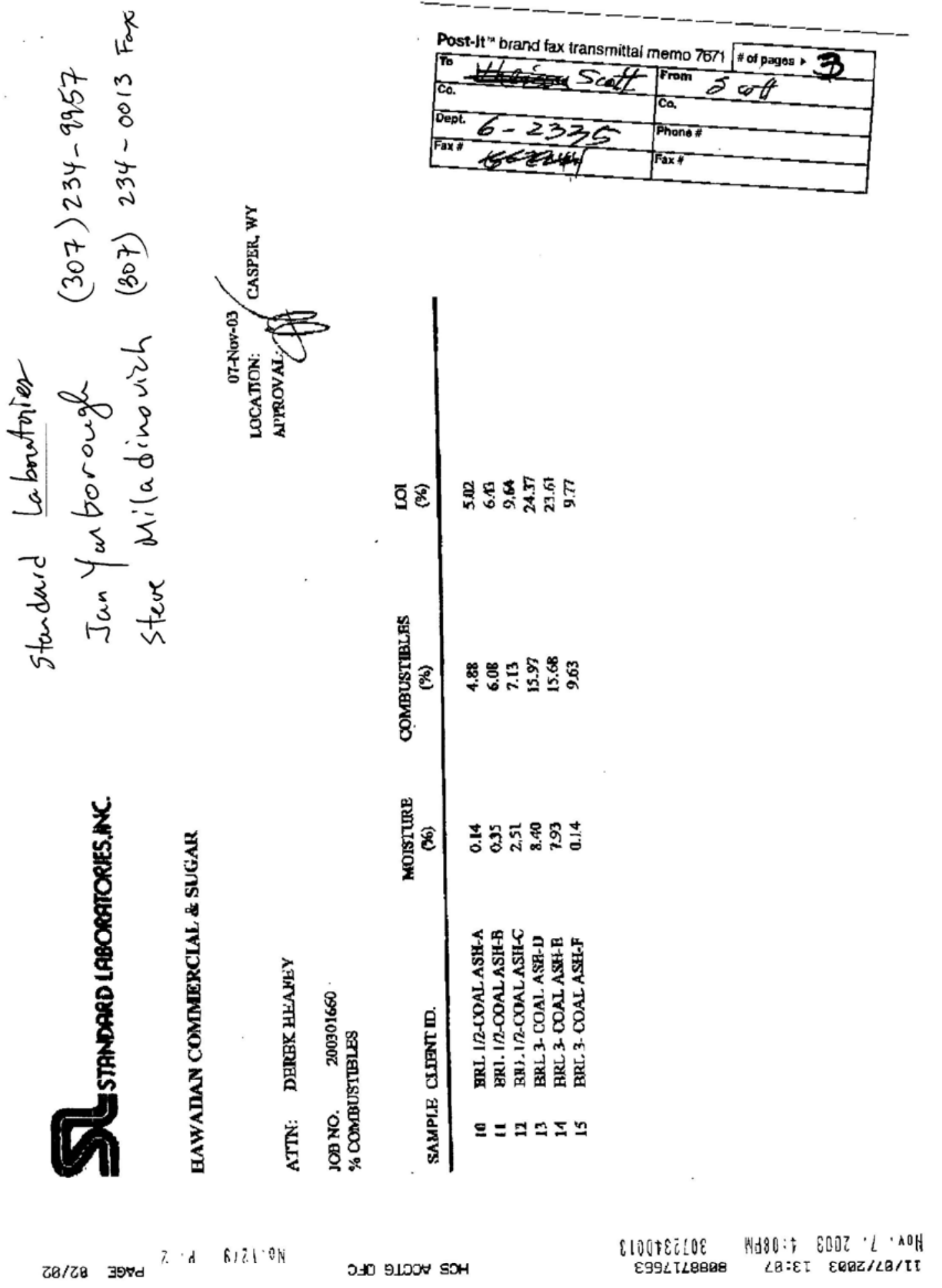




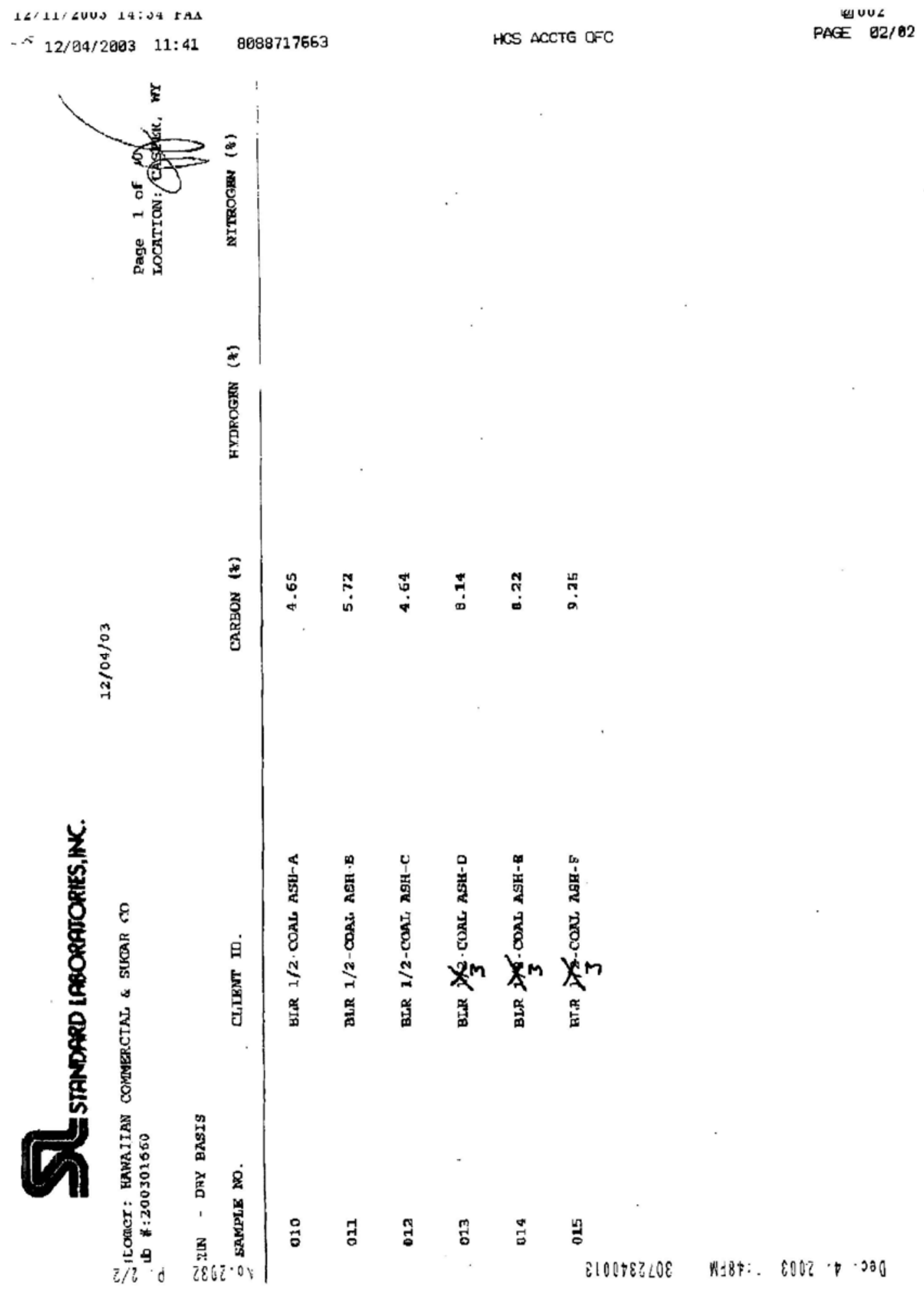




\section{June 29, 2004 Boile \#2 Efficiendy}

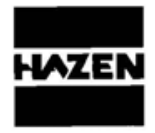

Hazen Research, Inc.

4601 Indiana St.

Golden, CO 80403 USA

Tel: (303) 279-4501

Fax: (303) 278-1528

Hawaii Natural Energy Institute

Scott Q. Turn

2540 Dole Street, Holmes Hall 246

Honolulu, Hawaii 96822

\section{Reporting \\ Basis >}

Proximate (\%)

As $\operatorname{Rec}{ }^{\prime} d$
Dry

0.00

14.58

45.46

39.96

100.00

$\frac{37.65}{100.00}$

0.50
11982
14086

Btu/lb (HHV)

MMF Btu/1b

MAF Btu/lb

Air Dry Loss (\%)

0.53

12717

15114

2.24

Ultimate (\%)

$\begin{array}{lrr}\text { Moisture } & 5.78 & 0.00 \\ \text { Carbon } & 66.27 & 70.34 \\ \text { Hydrogen } & 4.88 & 5.18 \\ \text { Nitrogen } & 1.03 & 1.09 \\ \text { Sulfur } & 0.50 & 0.53 \\ \text { Ash } & 13.74 & 14.58 \\ \text { Oxygen* } & 7.80 & 8.28 \\ \text { Total } & 100.00 & 100.00\end{array}$

Chlorine**

0.02

0.02

Forms of Sulfur (as S,\%)

$$
\begin{aligned}
& \text { Sulfate } \\
& \text { Pyritic } \\
& \text { Organic }
\end{aligned}
$$

Total

0.50

0.53

Date

HRI Project 009-444

HRI Series No. G64/04-1

Date Rec'd. 07/12/04

Cust. P.0.\#

Sample Identification

Coal \#1 06/29/04 10:20

Eqm Air Dry

Water Soluble Alkalies (\%)

$\mathrm{Na2O}$

K20

.00

3.62

67.79

4.99

1.05

0.51

14.05

$\overline{100.00}$

100.00

0.02

Lb. Alkali/MM Btu= 0.08

Lb. Ash/MM Btu= $\quad 11.46$

Lb. S02/MM Btu= 0.83

$\mathrm{HGI}=\quad$ a $\quad \%$ Moisture

As Rec'd. Sp.Gr. =

Free Swelling Index=

Report Prepared By:

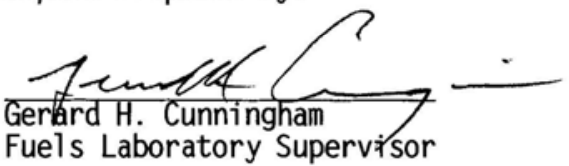

* 0xygen by Difference.

** Not usually reported as part of the ultimate analysis. 
Hawaii Natural Energy Institute Scott Q. Turn

2540 Dole Street, Holmes Hall 246

Honolulu, Hawaii 96822

Date July 282004

HRI Project 009-444

HRI Series No. G64/04-1

Date Rec'd.

Cust. P.0.\#

Sample Identification: Coal \#1 06/29/04 10:20

\section{Elemental Analysis of Ash (\%)}

SIO2

AL203

TI02

FE203

CAO

MGO

NA20

$\mathrm{K} 20$

P205

$\mathrm{S} 03$

Tota1
68.37

23.45

1.89

1.31

0.82

0.44

0.34

0.34

0.15

0.11

97.22

Ash Fusion Temperatures (Deg F)

Ash Viscosity Calculations *

$\begin{array}{lc}\text { Base Content (\%) } & 3.35 \\ \text { Acid Content (\%) } & 96.65 \\ \text { Dolomite Ratio } & 38.77 \\ \text { Base/Acid Ratio } & 0.03 \\ \text { Silica/Alumina Ratio } & 2.92 \\ \text { T(cv) (Deg F) } & \text { ND } * * \\ \text { T250 Temperature (Deg F) } & >2800 \\ \text { Equiv Silica Content (\%) } & 96.38 \\ \text { Viscosity from equiv } & \\ \text { Silica @ 2600 F (Poise) } & >999.99 \\ \text { Ash Type } & \text { HIGH RANK }\end{array}$

Slagging Type $=$ LOW
Fouling Type $=$ LOW

Initial Hemispherical Fluid

$\begin{array}{cc}\begin{array}{c}\text { Oxidizing } \\ \text { Atmosphere }\end{array} & \begin{array}{c}\text { Reducing } \\ \text { Atmosphere }\end{array} \\ 2700+ & 2700+\end{array}$

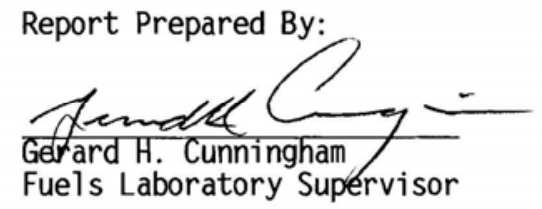

Note: The sample was calcined prior to elemental analysis.

* 'Fusibility-Viscosity of Lignite-Type Ash' . A.F. Duzy, 1965.

'Coal Ash Deposition Studies and Application to Boiler Design', R.C. Attig and A.F. Duzy, 1969.

'Relationship of Coal-Ash Viscosity to Chemical Composition', W.L. Sage and J.B. McIlroy, 1960.

'Fuel and Ash Characterization and its Effect on the Design of Industrial Boilers', Vecci, et al, 1978.

** OTL=0utside Table Limits. ND=Not Determined. 


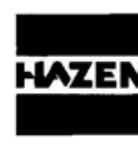

Hazen Research, Inc.

4601 Indiana St.

Golden, CO 80403 USA

Tel: (303) 279-4501

Fax: (303) 278-1528

Hawaii Natural Energy Institute Scott Q. Turn

2540 Dole Street, Holmes Hall 246

Honolulu, Hawaii 96822

\section{Reporting \\ Basis >}

As Rec'd

Proximate $(\%)$

Moisture
Ash
Volatile
Fixed C
Total

7.05

13.08

40.31

39.56

100.00

Sulfur
Btu/lb (HHV)
MMF Btu/lb

MAF Btu/1b

Air Dry Loss

(\%)

0.51

11909

13882

Ultimate (\%)

$\begin{array}{lr}\text { Moisture } & 7.05 \\ \text { Carbon } & 66.7 \\ \text { Hydrogen } & 5.12 \\ \text { Nitrogen } & 1.06 \\ \text { Sulfur } & 0.51 \\ \text { Ash } & 13.08 \\ \text { Oxygen* } & 6.44 \\ \text { Total } & 100.00\end{array}$

Chlorine**

0.03

4.42

0.00

14.07

43.36

42.57

100.00

0.54

12812

15128

14909

7.05

66.74

5.12

3.08

44

Forms of Sulfur (as S,\%)

Sulfate
Pyritic
Organic

Total

0.51

Water Soluble Alkalies (\%)

$\mathrm{Na2O}$

$\mathrm{K} 20$

0.54
Date July 282004

HRI Project 009.444

HRI Series No. G64/04-2

Date Rec'd. 07/12/04

Cust. P.0.\#

Sample Identification

Coal \#2 06/29/04 11:35

Eqm

Air Dry
2.75

13.68

42.17

$\overline{100.00}$

41.40

100.00

0.53

12459

\begin{tabular}{rr}
2.75 \\
69.83 \\
5.35 \\
1.11 \\
0.53 \\
13.68 \\
6.75 \\
\hline $100.00 \quad \frac{6.00}{100}$ \\
& 0.03
\end{tabular}

Lb. Alkali/MM Btu= 0.07

Lb. Ash/MM Btu= 10.98

Lb. SO2/MM Btu= 0.85 $\mathrm{HGI}=\quad$ a $\quad$ Moisture As Rec'd. Sp.Gr. = Free Swelling Index=

Report Prepared By:

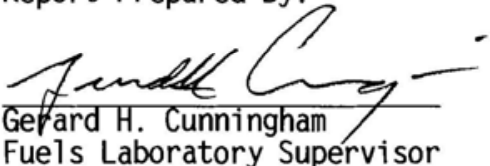

* 0xygen by Difference.

** Not usually reported as part of the ultimate analysis. 


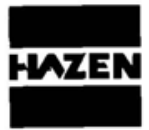

Hazen Research, Inc.

4601 Indiana St.

Golden, CO 80403 USA

Tel: (303) 279-4501

Fax: (303) 278-1528

Hawaii Natural Energy Institute

Scott Q. Turn

2540 Dole Street, Holmes Hall 246

Honolulu, Hawaii 96822

\section{Elemental Analysis of Ash (\%)}

\begin{tabular}{lr} 
SI02 & 67.5 \\
AL203 & 24.2 \\
TI02 & 2.03 \\
FE203 & 1.7 \\
CAO & 0.8 \\
MGO & 0.47 \\
NA20 & 0.4 \\
K20 & 0.27 \\
P205 & 0.1 \\
SO3 & 0.19 \\
\hline
\end{tabular}

Total 97.88

Ash Viscosity Calculations *

$\begin{array}{lc}\text { Base Content (\%) } & 3.84 \\ \text { Acid Content (\%) } & 96.16 \\ \text { Dolomite Ratio } & 34.93 \\ \text { Base/Acid Ratio } & 0.04 \\ \text { Silica/Alumina Ratio } & 2.79 \\ \text { T(cv) (Deg F) } & \text { ND } * \star \\ \text { T250 Temperature (Deg F) } & >2800 \\ \text { Equiv Silica Content (\%) } & 95.64 \\ \text { Viscosity from equiv } & \\ \text { Silica a 2600 F (Poise) } & >999.99 \\ \text { Ash Type } & \text { HIGH RANK }\end{array}$

.12

0.19

HIG RANK
Date July 282004

HRI Project 009-444

HRI Series No. G64/04-2

Date Rec'd. 07/12/04

Cust. P.0.\#

Sample Identification:

Coal \#2 06/29/04 11:35
Ash Fusion Temperatures (Deg F)

$\begin{array}{lc}\text { Oxidizing } & \text { Reducing } \\ \text { Atmosphere } & \text { Atmosphere }\end{array}$

Initial

$2700+$

$2700+$

Softening

Hemispherical

Fluid

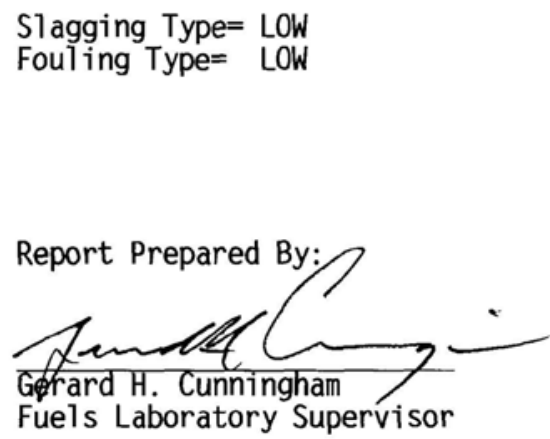

Note: The sample was calcined prior to elemental analysis.

* 'Fusibility-Viscosity of Lignite-Type Ash'. A.F. Duzy, 1965.

'Coal Ash Deposition Studies and Application to Boiler Design',

R.C. Attig and A.F. Duzy, 1969.

'Relationship of Coal-Ash Viscosity to Chemical Composition',

W.L. Sage and J.B. McIlroy, 1960.

'Fuel and Ash Characterization and its Effect on the Design

of Industrial Boilers', Vecci, et al, 1978.

** OTL=0utside Table Limits. ND=Not Determined. 


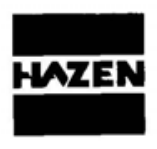

Hazen Research, Inc. 4601 Indiana St.

Golden, CO 80403 USA

Tel: (303) 279-4501

Fax: (303) 278-1528

Hawaii Natural Energy Institute Scott Q. Turn

2540 Dole Street, Holmes Hall 246

Honolulu, Hawaij 96822

\section{Reporting \\ Basis >}

Proximate (\%)

$\begin{array}{lr}\text { Moisture } & 7.49 \\ \text { Ash } & 14.13 \\ \text { Volatile } & 39.60 \\ \text { Fixed C } & 38.78 \\ \text { Total } & 100.00\end{array}$

Sulfur $\quad 0.51$

Btu/1b (HHV) 11700

MMF Btu/lb 13822

MAF Btu/lb

Air Dry Loss (\%)

As $\operatorname{Rec}^{\prime} \mathrm{d}$

Ultimate (\%)

Moisture
Carbon
Hydrogen
Nitrogen
Sulfur
Ash
Oxygen*
Total

7.49

65.20

4.99

1.02

0.51

14.13

6.66

100.00

Chlorine**

0.02

4.59

0.00

15.27

42.81

41.92

100.00

0.55

12647

15167

14927

0.00

70.48

5.39

1.10

0.55

15.27

7.21

100.00

0.02

Date July 282004

HRI Project 009-444

HRI Series No. G64/04-2

Date Rec'd.

Cust. P.0.\#

Sample Identification

Coal \#3 06/24/04 12:06

$$
\cdot
$$

Eqm

Air Dry

$\begin{array}{rr} & 3.04 \\ & 14.81 \\ 41.51 \\ 100.00 \quad \frac{40.64}{100.00} \\ & 0.53 \\ & 12262\end{array}$

$\begin{array}{rr} & 3.04 \\ & 68.34 \\ 5.23 \\ 1.07 \\ 0.53 \\ 14.81 \\ 6.98 \\ 100.00 \quad \frac{6}{10000} \\ & 0.02\end{array}$

Forms of Sulfur (as S, \%)

Sulfate
Pyritic
Organic

Total

0.51

Water Soluble Alkalies (\%)

$\mathrm{Na2O}$

K20
Lb. Alkali/MM Btu= 0.06

Lb. Ash/MM Btu= 12.08

Lb. S02/MM Btu= 0.86

$\mathrm{HGI}=\quad$ a $\quad \%$ Moisture

As Rec'd. Sp.Gr. =

Free Swelling Index=

0.55
Report Prepared By:

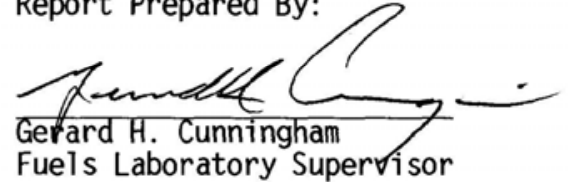

* 0xygen by Difference.

** Not usually reported as part of the ultimate analysis. 


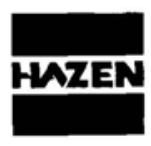

Hazen Research, Inc. 4601 Indiana St.

Golden, CO 80403 USA

Tel: (303) 279-4501

Fax: (303) 278-1528

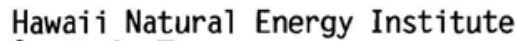
Scott Q. Turn

2540 Dole Street, Holmes Hall 246

Honolulu, Hawaij 96822

Elemental Analysis of Ash (\%)

\begin{tabular}{lr} 
SI02 & 68.41 \\
AL203 & 24.06 \\
TI02 & 1.86 \\
FE203 & 1.24 \\
CA0 & 0.58 \\
MG0 & 0.29 \\
NA20 & 0.27 \\
K20 & 0.24 \\
P205 & 0.09 \\
S03 & 0.09 \\
\hline & \\
Total & 97.13
\end{tabular}

Ash Viscosity Calculations *

$\begin{array}{lc}\text { Base Content (\%) } & 2.70 \\ \text { Acid Content (\%) } & 97.30 \\ \text { Dolomite Ratio } & 33.21 \\ \text { Base/Acid Ratio } & 0.03 \\ \text { Silica/Alumina Ratio } & 2.84 \\ \text { T(cv) (Deg F) } & \mathrm{ND} \star \star \\ \text { T250 Temperature (Deg F) } & >2800 \\ \text { Equiv Silica Content (\%) } & 97.01 \\ \text { Viscosity from equiv } & \\ \text { Silica @ 2600 F (Poise) } & >999.99 \\ \text { Ash Type } & \text { HIGH RANK }\end{array}$

Date July 282004

HRI Project 009.444

HRI Series No. G64/04-2

Date Rec'd.

Cust. P.0.\#

Sample Identification:

Coal \#3 06/24/04 12:06
Ash Fusion Temperatures (Deg F)

Initial

Softening

Hemispherical

Fluid

$\begin{array}{cr}\text { Oxidizing } & \begin{array}{c}\text { Reducing } \\ \text { Atmosphere }\end{array} \\ 2700+ & 2700+ \\ & \\ & \end{array}$

Slagging Type $=$ LOW
Fouling Type $=$ LOW

Report Prepared By:

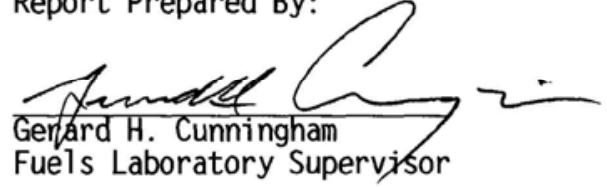

Note: The sample was calcined prior to elemental analysis.

* 'Fusibility-Viscosity of Lignite-Type Ash'. A.F. Duzy, 1965.

'Coal Ash Deposition Studies and Application to Boiler Design',

R.C. Attig and A.F. Duzy, 1969.

'Relationship of Coal-Ash Viscosity to Chemical Composition',

W.L. Sage and J.B. McIlroy, 1960.

'Fuel and Ash Characterization and its Effect on the Design

of Industrial Boilers', Vecci, et al, 1978.

** OTL=0utside Table Limits. ND=Not Determined. 


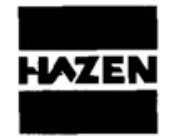

Hazen Research, Inc.

4601 Indiana St.

Golden, CO 80403 USA

Tel: (303) 279-4501

Fax: (303) 278-1528

Hawaii Natural Energy Institute

Date:

PROJ. \#

July 28, 2004

Scott Q. Turn

CTRL \#

009-444

REC'D

07/12/04

2540 Dole Street, Holmes Hall

Honolulu, Hawaii 96822

\begin{tabular}{lccc}
\hline $\begin{array}{l}\text { Sample } \\
\text { Number }\end{array}$ & $\begin{array}{c}\text { Sample } \\
\text { Identification }\end{array}$ & $\begin{array}{c}\text { Chlorine in } \\
\text { Ash, \% }\end{array}$ & $\begin{array}{c}\text { Carbon Dioxide } \\
\text { in Ash, \% }\end{array}$ \\
\hline G64/04-1 & Coal \#1 10:20 & 0.04 & 0.08 \\
G64/04-2 & Coal \#2 11:35 & 0.02 & 0.02 \\
G64/04-3 & Coal \#3 12:06 & $<0.01$ & 0.04
\end{tabular}

All samples were dated 06/29/04.

The samples were ashed at 800 degrees Celsius prior to analysis.

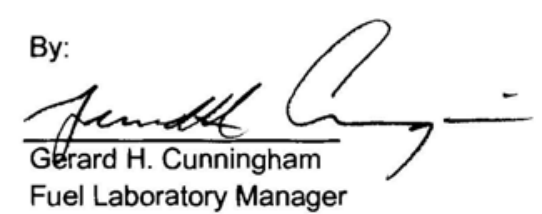




\section{FAZEN}

\section{Hazen Research, Inc.}

4601 Indiana St.

Golden, CO 80403 USA

Tel: (303) 279-4501

Fax: (303) 278-1528

Hawaii Natural Energy Institute

Date:

PROJ. \#

July 28,2004

G64/04

Scott Q. Turn

2540 Dole Street, Holmes Hall

Honolulu, Hawaii 96822
REC'D 07/12/04

Sample Number: G64/04-4

Sample Identification: Ash \#1 06/29/04 10:20

$\begin{array}{ll}\text { Air Dry Loss, \% } & 39.44 \\ \text { LOI @ } 800 \text { C, \% } & 44.59\end{array}$

Carbon Forms

Air Dry Basis

$\begin{array}{ll}7.98 & 4.83 \\ 0.03 & 0.02 \\ 7.95 & 4.81\end{array}$

Total Carbon, $\%$

Carbon Dioxide as C, \%

7.95

0.02

Organic Carbon, \%*

Sample Number: G64/04-5

Sample Identification: Ash \#2 06/29/04 11:35

Air Dry Loss, $\%$

42.02

LOI@ 800 C, \%

46.62

\section{Carbon Forms}

Air Dry Basis

As Received Basis

Total Carbon, \%

Carbon Dioxide as C, \%

Organic Carbon, \%*

$\begin{array}{ll}7.73 & 4.48 \\ 0.01 & 0.01 \\ 7.72 & 4.48\end{array}$

Sample Number: G64/04-6

Sample Identification: Ash \#3 06/29/04 12:06

Air Dry Loss, \%

LOI@ 800 C, \%

42.52

46.55

Carbon Forms

Air Dry Basis

As Received Basis

Total Carbon, $\%$

Carbon Dioxide as C, \%

Organic Carbon, \%*

* by difference

The LOI values are reported on an "as received" basis.

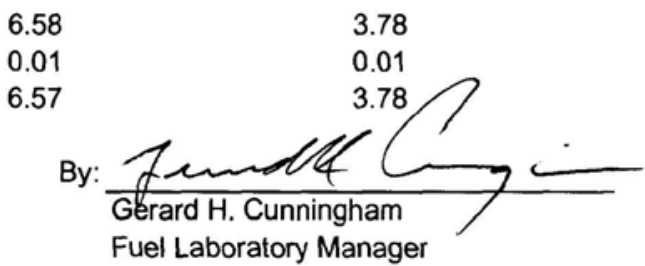




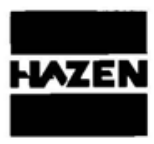

Hazen Research, Inc.

4601 Indiana St.

Golden, CO 80403 USA

Tel: (303) 279-4501

Fax: (303) 278-1528

Hawaii Natural Energy Institute

Scott Q. Turn

2540 Dole Street, Holmes Hall

Date: $\quad$ August 24, 2004

PROJ. \# 009-444

CTRL \# G64/04

Honolulu, Hawaii 96822

Sample Number: G64/04-4

Sample Identification: Ash \#1 06/29/04 10:20

Air Dry Loss, $\%$

39.44

LOI@ 800 C, \%

44.59

$\begin{array}{lccr}\text { Carbon Forms } & \text { Dry Basis } & \text { Air Dry Basis } & \text { As Received B } \\ \text { Total Carbon,\% } & 8.01 & 7.98 & 4.83 \\ \text { Carbon Dioxide as C, \% } & 0.03 & 0.03 & 0.02 \\ \text { Organic Carbon, \%* } & 7.98 & 7.95 & 4.81\end{array}$

Sample Number: G64/04-5

Sample Identification: Ash \#2 06/29/04 11:35

Air Dry Loss, \%

42.02

LOI @ 800 C, \%

46.62

$\begin{array}{lccr}\text { Carbon Forms } & \text { Dry Basis } & \text { Air Dry Basis } & \text { As Received B } \\ \text { Total Carbon,\% } & 7.76 & 7.73 & 4.48 \\ \text { Carbon Dioxide as C, \% } & 0.01 & 0.01 & 0.01 \\ \text { Organic Carbon, \%* } & 7.75 & 7.72 & 4.48\end{array}$

Sample Number: G64/04-6

Sample Identification: Ash \#3 06/29/04 12:06

Air Dry Loss, \%

LOI @ 800 C, \%

Carbon Forms

Total Carbon, $\%$

Carbon Dioxide as C, \%

Organic Carbon, \%*

* by difference

The LOI values are reported on an "as received" basis.

6.60
0.01
6.59

42.52

46.55

Air Dry Basis

As Received Basis

6.58

0.01

3.78

6.57

3.78

By:

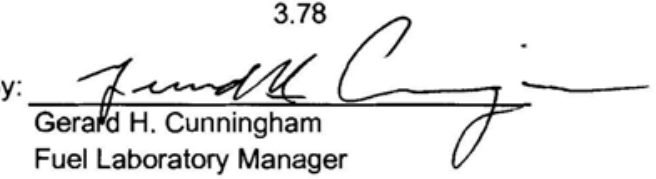


Appendix B

B-1 
Table B1. Ten minute averages of test parameters for Boiler 1 firing coal, September 22, 2003.

\begin{tabular}{|c|c|c|c|c|c|c|}
\hline Time and Location & $\begin{array}{c}\text { Average } \\
\text { ppm-CO }\end{array}$ & $\begin{array}{c}\text { St Dev } \\
\text { CO }\end{array}$ & $\begin{array}{c}\text { Average } \\
\text { \%CO2 }\end{array}$ & $\begin{array}{c}\text { Average } \\
\text { \%O2 }\end{array}$ & $\begin{array}{c}\text { St Dev } \\
\text { O2 }\end{array}$ & $\begin{array}{c}\text { Average } \\
\text { Temp. (C) }\end{array}$ \\
\hline 10:02 Location 1-1 & 82.4 & 5.5 & 10.7 & 8.8 & 0.4 & 195.3 \\
\hline 10:12 Location 1-2 & 78.7 & 3.9 & 9.5 & 10.2 & 0.2 & 189.4 \\
\hline 10:22 Location 1-3 & 96.0 & 3.6 & 9.0 & 10.7 & 0.2 & 176.3 \\
\hline 10:32 Location 2-1 & 74.9 & 9.7 & 10.0 & 9.5 & 0.4 & 194.5 \\
\hline 10:42 Location 2-2 & 90.7 & 6.0 & 8.2 & 11.6 & 0.3 & 188.3 \\
\hline 10:52 Location 2-3 & 93.7 & 4.0 & 6.9 & 13.2 & 0.2 & 187.7 \\
\hline & & & & & & \\
\hline 11:04 Location 1-1* & 80.9 & 9.3 & 9.7 & 9.7 & 1.2 & 194.7 \\
\hline 11:12 Location 1-2 & 78.9 & 5.1 & 9.6 & 10.1 & 0.3 & 190.2 \\
\hline 11:22 Location 1-3 & 89.8 & 7.2 & 9.2 & 10.5 & 0.1 & 176.4 \\
\hline 11:32 Location 2-1 & 65.7 & 6.9 & 10.2 & 9.3 & 0.2 & 195.3 \\
\hline 11:42 Location 2-2 & 84.0 & 4.7 & 8.6 & 11.3 & 0.2 & 189.6 \\
\hline 11:52 Location 2-3 & 91.1 & 3.4 & 6.7 & 13.4 & 0.1 & 187.8 \\
\hline * 4min period & & & & & & \\
\hline & & & & & & \\
\hline 12:04 Location 1-1 & 69.7 & 5.4 & 10.5 & 9.0 & 0.3 & 196.0 \\
\hline 12:14 Location 1-2 & 78.7 & 2.6 & 9.2 & 10.5 & 0.2 & 189.4 \\
\hline 12:24 Location 1-3 & 96.3 & 6.3 & 8.9 & 10.9 & 0.3 & 176.7 \\
\hline 12:34 Location 2-1 & 70.1 & 3.1 & 9.8 & 9.7 & 0.3 & 194.7 \\
\hline 12:51 Location 2-2 & 82.5 & 4.5 & 8.5 & 11.3 & 0.2 & 189.6 \\
\hline 13:01 Location 2-3 & 91.8 & 4.1 & 6.8 & 13.3 & 0.1 & 188.5 \\
\hline & & & & & & \\
\hline Average across & & & & & & \\
\hline locations & 83.1 & & 9.0 & 10.7 & & 188.9 \\
\hline
\end{tabular}


Table B2. Ten minute averages of test parameters for Boiler 2 firing coal, June 29, 2004.

\begin{tabular}{|l|c|c|c|c|c|c|}
\hline Time and Location & $\begin{array}{c}\text { Average } \\
\text { ppm-CO }\end{array}$ & $\begin{array}{c}\text { St Dev } \\
\text { CO }\end{array}$ & $\begin{array}{c}\text { Average } \\
\text { \%CO2 }\end{array}$ & $\begin{array}{c}\text { Average } \\
\text { \%O2 }\end{array}$ & $\begin{array}{c}\text { St Dev } \\
\text { O2 }\end{array}$ & $\begin{array}{c}\text { Average } \\
\text { Temp. (C) }\end{array}$ \\
\hline 10:12 Location 1-1 & 55.8 & 5.2 & 9.4 & 10.0 & 0.3 & 221.7 \\
\hline 11:05 Location 1-2 & 87.3 & 3.3 & 8.7 & 10.9 & 0.5 & 227.7 \\
\hline 11:15 Location 1-3 & 108.7 & 3.0 & 5.7 & 14.5 & 0.3 & 207.4 \\
\hline & & & & & & \\
\hline 11:25 Location 1-1 & 65.4 & 5.9 & 8.6 & 11.0 & 0.4 & 218.1 \\
\hline 11:35 Location 1-2 & 82.2 & 5.0 & 8.7 & 11.0 & 0.3 & 228.8 \\
\hline 11:45 Location 1-3 & 122.8 & 5.7 & 6.5 & 13.6 & 0.5 & 208.3 \\
\hline & & & & & & \\
\hline 11:55 Location 1-1 & 67.1 & 2.7 & 8.5 & 11.2 & 0.2 & 219.1 \\
\hline 12:05 Location 1-2 & 73.4 & 3.3 & 8.5 & 11.2 & 0.3 & 228.7 \\
\hline 12:15 Location 1-3 & 117.8 & 7.1 & 6.4 & 13.7 & 0.3 & 207.9 \\
\hline & & & & & & \\
\hline $\begin{array}{l}\text { Averages across } \\
\text { locations }\end{array}$ & 86.7 & 4.6 & 7.9 & 11.9 & 0.3 & 218.6 \\
\hline
\end{tabular}

Table B3. Ten minute averages of test parameters for Boiler 1 firing bagasse, September 23, 2003.

\begin{tabular}{|c|c|c|c|c|c|c|}
\hline Time and Location & $\begin{array}{c}\text { Average } \\
\text { ppm-CO }\end{array}$ & $\begin{array}{c}\text { St Dev } \\
\text { CO }\end{array}$ & $\begin{array}{c}\text { Average } \\
\text { \%CO2 }\end{array}$ & $\begin{array}{c}\text { Average } \\
\text { \%O2 }\end{array}$ & $\begin{array}{c}\text { St Dev } \\
\text { O2 }\end{array}$ & $\begin{array}{c}\text { Average } \\
\text { Temp. (C) }\end{array}$ \\
\hline 09:17 Location 1-1 & 1358.0 & 384.6 & 15.2 & 5.4 & 4.3 & 229.1 \\
\hline 09:27 Location 1-2 & 761.5 & 147.6 & 13.0 & 7.6 & 0.2 & 219.9 \\
\hline 09:37 Location 1-3 & 1064.1 & 227.6 & 12.5 & 8.1 & 0.3 & 202.0 \\
\hline 09:47 Location 2-1 & 906.5 & 193.1 & 14.3 & 6.3 & 0.2 & 225.6 \\
\hline 09:57 Location 2-2 & 899.2 & 91.7 & 12.2 & 8.5 & 0.2 & 220.4 \\
\hline 10:07 Location 2-3 & 670.2 & 61.2 & 9.2 & 11.6 & 0.1 & 215.5 \\
\hline & & & & & & \\
\hline 10:17 Location 1-1 & 761.4 & 92.8 & 14.7 & 6.0 & 0.2 & 226.8 \\
\hline 10:27 Location 1-2 & 936.5 & 185.2 & 13.5 & 7.2 & 0.3 & 219.5 \\
\hline 10:37 Location 1-3 & 1306.0 & 219.7 & 12.9 & 7.8 & 0.2 & 203.8 \\
\hline 10:47 Location 2-1 & 1141.2 & 267.6 & 14.5 & 6.0 & 0.3 & 227.4 \\
\hline 10:57 Location 2-2 & 1220.3 & 287.3 & 12.4 & 8.2 & 0.3 & 223.1 \\
\hline 11:07 Location 2-3 & 2819.3 & 287.3 & 10.5 & 10.0 & 0.3 & 220.8 \\
\hline & & & & & & \\
\hline 11:34 Location 1-1 & 2559.3 & 988.9 & 15.7 & 4.7 & 0.6 & 232.6 \\
\hline 11:49 Location 1-2 & 2253.4 & 744.2 & 14.4 & 6.1 & 0.4 & 223.8 \\
\hline 11:59 Location 1-3 & 999.0 & 225.0 & 12.4 & 8.1 & 0.6 & 205.8 \\
\hline 12:09 Location 2-1 & 1199.4 & 497.5 & 14.2 & 6.2 & 0.5 & 227.7 \\
\hline 12:19 Location 2-2 & 1500.8 & 519.8 & 12.6 & 8.0 & 0.3 & 224.4 \\
\hline 12:29 Location 2-3 & 1234.3 & 298.1 & 9.5 & 11.2 & 0.5 & 219.3 \\
\hline & & & & & & \\
\hline Averages across & 1310.6 & & 13.0 & 7.6 & & 220.4 \\
\hline locations & & & & & & \\
\hline
\end{tabular}


Table B4. Ten minute averages of test parameters for Boiler 2 firing bagasse, September 23, 2003.

\begin{tabular}{|c|c|c|c|c|c|c|}
\hline Time and Location & $\begin{array}{c}\text { Average } \\
\text { ppm-CO }\end{array}$ & $\begin{array}{c}\text { St Dev } \\
\text { CO }\end{array}$ & $\begin{array}{c}\text { Average } \\
\text { \%CO2 }\end{array}$ & $\begin{array}{c}\text { Average } \\
\text { \%O2 }\end{array}$ & $\begin{array}{c}\text { St Dev } \\
\text { O2 }\end{array}$ & $\begin{array}{c}\text { Average } \\
\text { Temp. (C) }\end{array}$ \\
\hline 13:19 Location 3-1 & 4014.1 & 1066.1 & 13.9 & 6.4 & 0.7 & 247.4 \\
\hline 13:34 Location 3-2 & 2666.2 & 1447.4 & 14.5 & 5.8 & 1.2 & 266.9 \\
\hline 13:44 Location 3-3 & 1972.5 & 490.3 & 9.8 & 10.9 & 0.3 & 232.1 \\
\hline & & & & & & \\
\hline 13:54 Location 3-1 & 1893.1 & 407.7 & 13.3 & 7.1 & 0.4 & 246.5 \\
\hline 14:04 Location 3-2 & 3806.3 & 1820.4 & 14.7 & 5.3 & 2.4 & 265.9 \\
\hline 14:14 Location 3-3 & 2272.7 & 790.0 & 9.9 & 10.8 & 0.5 & 231.1 \\
\hline & & & & & & \\
\hline 14:24 Location 3-1 & 1418.0 & 488.3 & 13.0 & 7.5 & 0.3 & 245.6 \\
\hline 14:34 Location 3-2 & 3179.2 & 768.8 & 15.5 & 5.0 & 0.2 & 267.3 \\
\hline 14:44 Location 3-3 & 3043.1 & 1507.3 & 9.6 & 10.9 & 0.9 & 230.4 \\
\hline & & & & & & \\
\hline $\begin{array}{c}\text { Averages across } \\
\text { locations }\end{array}$ & 2696.1 & & 12.7 & 7.7 & & 248.1 \\
\hline
\end{tabular}


Table B5. Ten minute averages of test parameters for Boiler 3 firing bagasse, September 25, 2003.

\begin{tabular}{|c|c|c|c|c|c|c|}
\hline Time and Location & $\begin{array}{l}\text { Average } \\
\text { ppm-CO }\end{array}$ & $\begin{array}{l}\text { St Dev } \\
\text { CO }\end{array}$ & $\begin{array}{l}\text { Average } \\
\% \mathrm{CO} 2\end{array}$ & $\begin{array}{l}\text { Average } \\
\% \mathrm{O} 2\end{array}$ & $\begin{array}{l}\text { St Dev } \\
\text { O2 }\end{array}$ & $\begin{array}{l}\text { Average } \\
\text { Temp. (C) }\end{array}$ \\
\hline 8:57 Location 3-1 & 3162.5 & 1475.3 & 18.3 & 2.3 & 0.7 & 237.6 \\
\hline 9:07 Location 3-2 & 2662.6 & 1254.7 & 17.8 & 2.9 & 0.7 & 251.1 \\
\hline 9:17 Location 3-3 & 2861.3 & 435.7 & 13.8 & 5.4 & 1.1 & 211.4 \\
\hline 9:27 Location 3-1 & 4432.1 & 804.6 & 18.6 & 1.8 & 0.5 & 240.2 \\
\hline 9:37 Location 3-2 & 3281.5 & 1868.7 & 17.9 & 2.4 & 1.3 & 248.6 \\
\hline 10:14 Location 3-1 & 5114.0 & 0.0 & 19.4 & 0.7 & 0.2 & 250.9 \\
\hline 10:35 Location 3-2* & 4374.0 & 855.1 & 18.5 & 2.0 & 0.5 & 261.7 \\
\hline 10:59 Location 3-1 & 4984.3 & 409.7 & 19.0 & 1.5 & 0.3 & 256.1 \\
\hline \multicolumn{7}{|l|}{ *12min period } \\
\hline 12:58 Location 3-2 & 4519.1 & 909.2 & 17.9 & 2.6 & 0.4 & 250.1 \\
\hline 13:08 Location 3-1 & 2440.1 & 952.6 & 17.0 & 3.6 & 0.5 & 245.9 \\
\hline 13:18 Location 3-3 & 2413.6 & 957.4 & 16.0 & 4.7 & 0.7 & 211.2 \\
\hline 13:28 Location 3-1 & 4788.5 & 426.7 & 18.3 & 2.1 & 0.3 & 249.9 \\
\hline 13:38 Location 3-2 & 5114.0 & 0.0 & 18.6 & 1.7 & 0.2 & 257.8 \\
\hline 13:48 Location 3-3 & 2043.0 & 656.1 & 17.0 & 3.7 & 0.3 & 208.9 \\
\hline 13:58 Location 3-1 & 1986.6 & 551.6 & 17.9 & 2.7 & 0.2 & 236.6 \\
\hline 14:08 Location 3-2 & 2383.7 & 843.7 & 17.0 & 3.6 & 0.5 & 231.9 \\
\hline 14:18 Location 3-3 & 1237.6 & 244.3 & 14.9 & 5.6 & 0.3 & 195.7 \\
\hline 14:28 Location 3-1 & 2287.9 & 993.7 & 17.4 & 3.2 & 0.4 & 230.1 \\
\hline 14:38 Location 3-2 & 2040.4 & 800.4 & 17.2 & 3.5 & 0.4 & 231.4 \\
\hline 14:48 Location 3-3 & 984.1 & 327.4 & 15.6 & 5.0 & 0.4 & 194.4 \\
\hline $\begin{array}{l}\text { Averages across } \\
\text { locations }\end{array}$ & 3155.5 & & 17.4 & 3.0 & & 235.1 \\
\hline
\end{tabular}


Table B6. Ten minute averages of test parameters for Boiler 3 firing coal, September 26, 2003.

\begin{tabular}{|c|c|c|c|c|c|c|}
\hline Time and Location & $\begin{array}{c}\text { Average } \\
\text { ppm-CO }\end{array}$ & $\begin{array}{c}\text { St Dev } \\
\text { CO }\end{array}$ & $\begin{array}{c}\text { Average } \\
\text { \%CO2 }\end{array}$ & $\begin{array}{c}\text { Average } \\
\% \text { O2 }\end{array}$ & $\begin{array}{c}\text { St Dev } \\
\text { O2 }\end{array}$ & $\begin{array}{c}\text { Average } \\
\text { Temp. (C) }\end{array}$ \\
\hline 10:18 Location 3-1 & 8.6 & 1.0 & 11.7 & 7.6 & 0.2 & 212.6 \\
\hline 10:28 Location 3-2 & 14.0 & 1.9 & 12.5 & 6.7 & 0.2 & 214.1 \\
\hline 10:38 Location 3-3 & 12.7 & 1.8 & 12.4 & 6.7 & 0.4 & 179.9 \\
\hline & & & & & & \\
\hline 10:48 Location 3-1 & 7.6 & 1.1 & 12.1 & 7.0 & 0.4 & 211.8 \\
\hline 10:58 Location 3-2 & 15.7 & 1.1 & 13.3 & 5.7 & 0.2 & 213.8 \\
\hline 11:08 Location 3-3 & 14.1 & 4.6 & 12.6 & 6.5 & 0.3 & 178.9 \\
\hline & & & & & & \\
\hline 11:18 Location 3-1 & 8.4 & 0.9 & 11.7 & 7.6 & 0.2 & 213.0 \\
\hline & & & & & & \\
\hline 13:03 Location 3-1 & 7.1 & 2.3 & 12.3 & 6.9 & 0.3 & 218.3 \\
\hline 13:13 Location 3-2 & 11.9 & 0.8 & 13.0 & 6.1 & 0.2 & 214.6 \\
\hline 13:23 Location 3-3 & 10.3 & 1.4 & 12.5 & 6.7 & 0.3 & 181.3 \\
\hline & & & & & & \\
\hline 13:33 Location 3-1 & 6.6 & 0.8 & 11.8 & 7.4 & 0.2 & 214.0 \\
\hline 13:43 Location 3-2 & 12.2 & 0.7 & 12.9 & 6.2 & 0.2 & 212.9 \\
\hline 13:53 Location 3-3 & 8.5 & 1.0 & 12.5 & 6.6 & 0.3 & 179.0 \\
\hline & & & & & & \\
\hline 14:03 Location 3-1 & 6.8 & 0.6 & 11.8 & 7.4 & 0.1 & 214.3 \\
\hline & & & & & & \\
\hline $\begin{array}{c}\text { Averages across } \\
\text { locations }\end{array}$ & 10.3 & & 12.3 & 6.8 & & 204.2 \\
\hline
\end{tabular}


Table B7. Ten minute averages of test parameters for Boiler 3 firing fuel oil, September 27, 2003.

\begin{tabular}{|l|c|c|c|c|c|c|}
\hline Time and Location & $\begin{array}{c}\text { Average } \\
\text { ppm-CO }\end{array}$ & $\begin{array}{c}\text { St Dev } \\
\text { CO }\end{array}$ & $\begin{array}{c}\text { Average } \\
\text { \%CO2 }\end{array}$ & $\begin{array}{c}\text { Average } \\
\text { \%O2 }\end{array}$ & $\begin{array}{c}\text { St Dev } \\
\text { O2 }\end{array}$ & $\begin{array}{c}\text { Average } \\
\text { Temp. (C) }\end{array}$ \\
\hline 10:04 Probe 3-2 & 22.6 & 9.8 & 9.8 & 7.5 & 0.4 & 183.7 \\
\hline 10:14 Probe 3-3 & -11.1 & 1.7 & 9.6 & 8.0 & 0.3 & 159.8 \\
\hline 10:24 Probe 3-1 & -5.2 & 2.0 & 8.8 & 8.8 & 1.1 & 179.5 \\
\hline 10:34 Probe 3-2 & -4.1 & 6.1 & 8.6 & 9.4 & 0.5 & 182.9 \\
\hline 10:44 Probe 3-3 & -11.3 & 2.5 & 8.9 & 8.7 & 0.4 & 160.4 \\
\hline 10:54 Probe 3-1 & -13.2 & 0.7 & 9.4 & 8.1 & 0.2 & 180.2 \\
\hline & & & & & & \\
\hline 11:52 Probe 3-1* & 11.9 & 4.6 & 7.6 & 10.7 & 0.2 & 186.1 \\
\hline 12:00 Probe 3-2 & 35.6 & 9.8 & 6.9 & 11.5 & 0.2 & 193.4 \\
\hline 12:10 Probe 3-3 & 11.6 & 4.7 & 7.0 & 11.5 & 0.4 & 168.0 \\
\hline 12:20 Probe 3-1 & 17.6 & 3.8 & 7.3 & 11.0 & 0.1 & 185.3 \\
\hline 12:30 Probe 3-2 & 52.6 & 12.2 & 6.6 & 12.1 & 0.1 & 192.6 \\
\hline 12:40 Probe 3-3 & 26.2 & 5.8 & 7.3 & 11.0 & 0.1 & 167.5 \\
\hline 12:50 Probe 3-1 & 50.5 & 15.3 & 6.7 & 11.8 & 0.2 & 184.8 \\
\hline 13:00 Probe 3-2 & 20.7 & 8.1 & 7.6 & 10.6 & 0.2 & 191.6 \\
\hline 13:10 Probe 3-3 & 35.3 & 10.5 & 6.8 & 11.9 & 0.5 & 167.6 \\
\hline * 4 min period & & & & & & \\
\hline & & & & & & \\
\hline 13:26 Probe 3-1 & 28.0 & 10.0 & 7.6 & 10.7 & 0.3 & 186.1 \\
\hline 13:36 Probe 3-2 & 63.8 & 11.3 & 6.9 & 11.7 & 0.2 & 193.1 \\
\hline 13:46 Probe 3-3 & 26.8 & 11.5 & 6.8 & 11.8 & 0.3 & 167.3 \\
\hline 13:56 Probe 3-1 & 17.9 & 8.6 & 7.8 & 10.4 & 0.2 & 186.5 \\
\hline 14:06 Probe 3-2 & 54.5 & 8.0 & 6.9 & 11.7 & 0.2 & 193.6 \\
\hline 14:16 Probe 3-3 & 18.7 & 22.7 & 6.7 & 11.9 & 2.4 & 168.6 \\
\hline 14:26 Probe 3-1 & 38.9 & 33.4 & 5.5 & 13.6 & 4.1 & 185.6 \\
\hline 14:36 Probe 3-2 & 87.8 & 13.5 & 6.9 & 11.6 & 0.2 & 193.9 \\
\hline 14:46 Probe 3-3 & 38.4 & 10.4 & 6.8 & 11.7 & 0.3 & 168.8 \\
\hline & & & & & & \\
\hline Averages across & & & & & & \\
locations & 25.6 & & 7.5 & 10.7 & & 180.3 \\
\hline
\end{tabular}


Appendix C 
$\times$ Tref $-\mathrm{T} 1 \cdot \mathrm{T} 2+\mathrm{T} 3 * \mathrm{~T} 4 \Delta \mathrm{T} 5 \square \mathrm{T} 6$

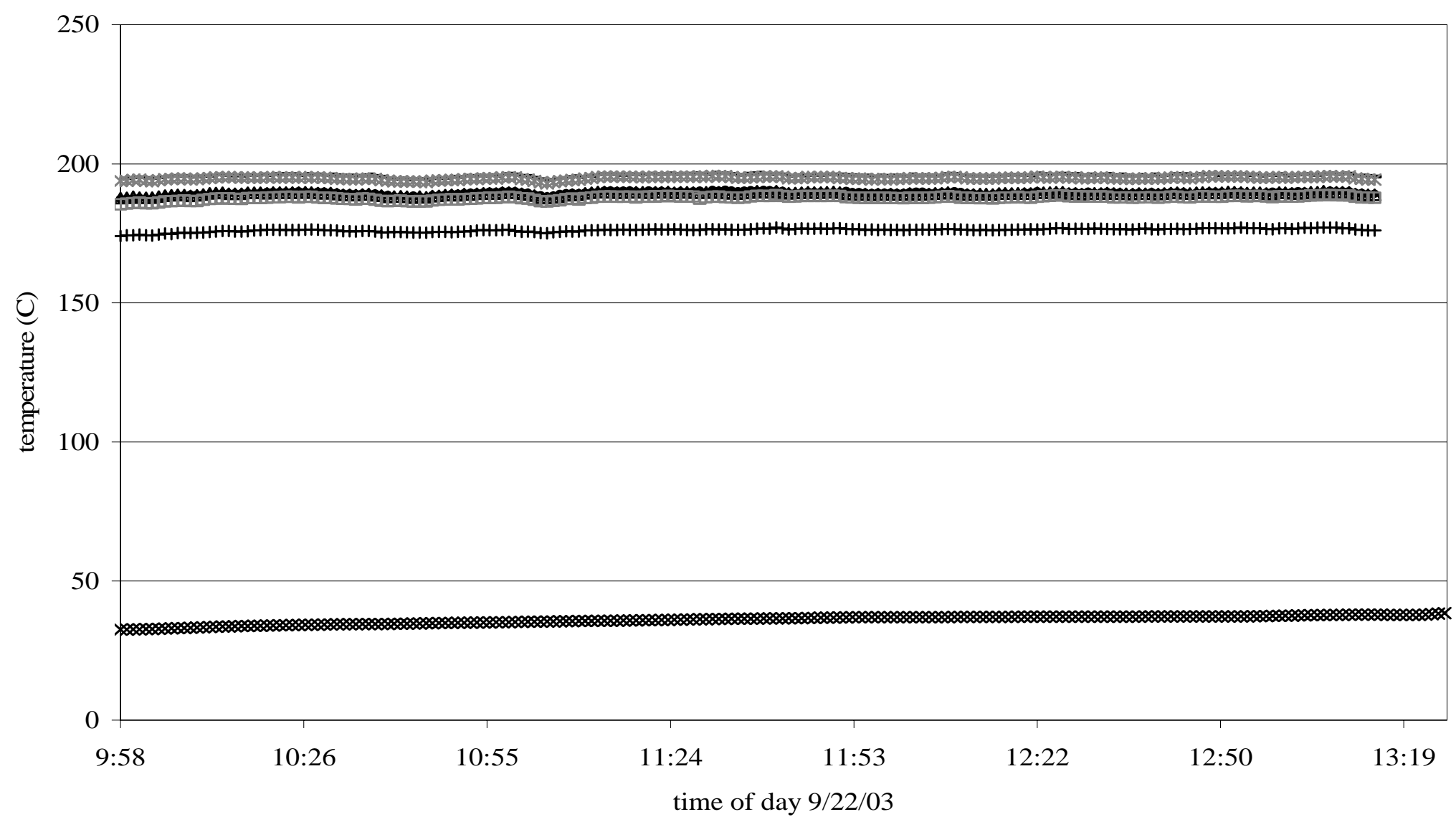

Figure C1. Temperatures recorded downstream of the air preheater in Boiler 1 firing coal on September 22, 2003. Temperatures T1 though T6 are from Type $\mathrm{K}$ thermocouples located in the flue gas. Tref is an indicator of ambient temperature. 
$\times$ Tref $-\mathrm{T} 1 \cdot \mathrm{T} 2+\mathrm{T} 3 * \mathrm{~T} 4 \Delta \mathrm{T} 5 \square \mathrm{T} 6$

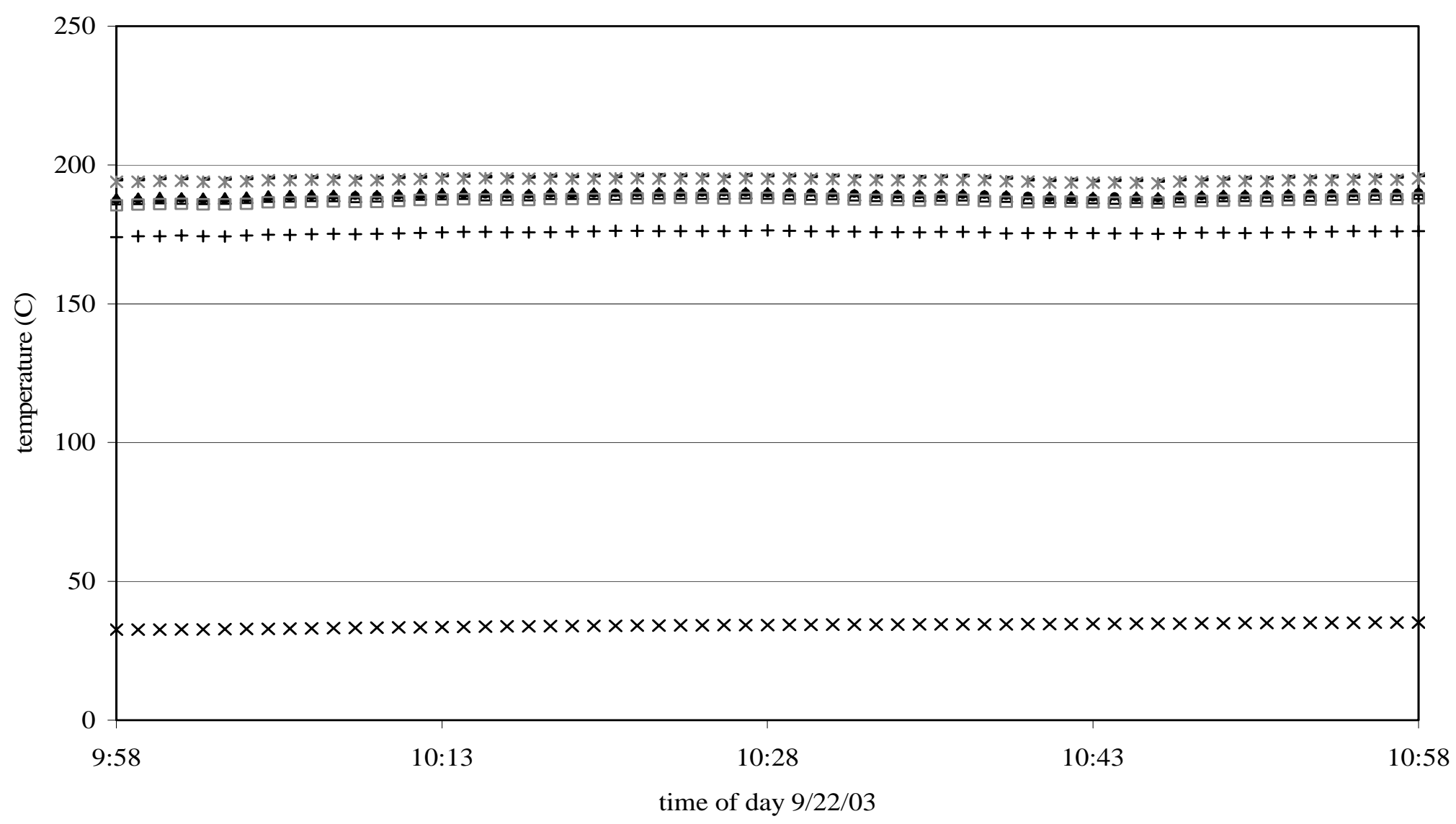

Figure C2. Temperatures recorded downstream of the air preheater in Boiler 1 firing coal on September 22, 2003, first sample period. Temperatures T1 though T6 are from Type K thermocouples located in the flue gas. Tref is an indicator of ambient temperature. 
- $\mathrm{CO} 2+\mathrm{O} 2$

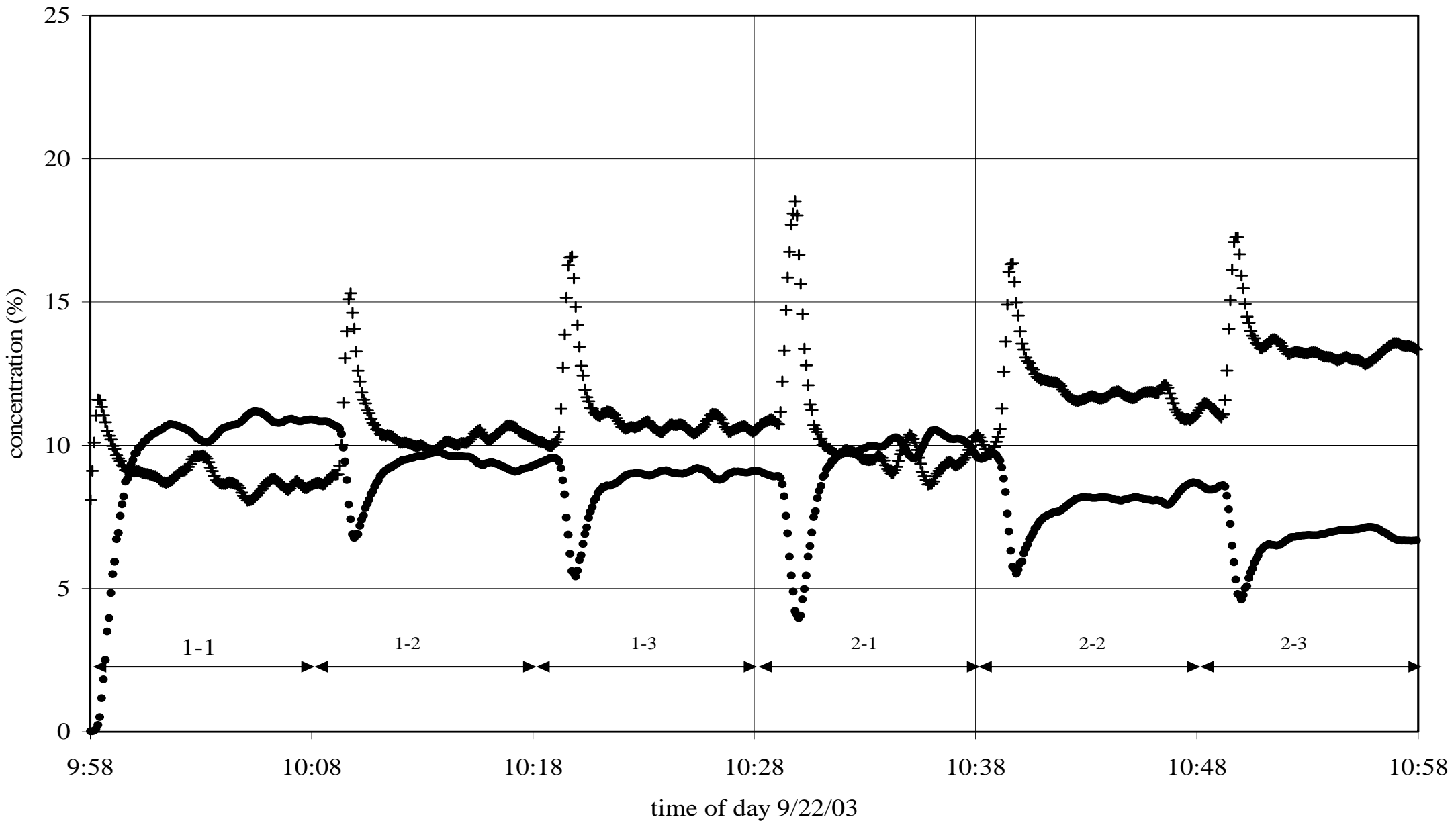

Figure C3. $\mathrm{O}_{2}$ and $\mathrm{CO}_{2}$ concentrations recorded downstream of the air preheater in Boiler 1 firing coal on September 22, 2003, first sample period. 


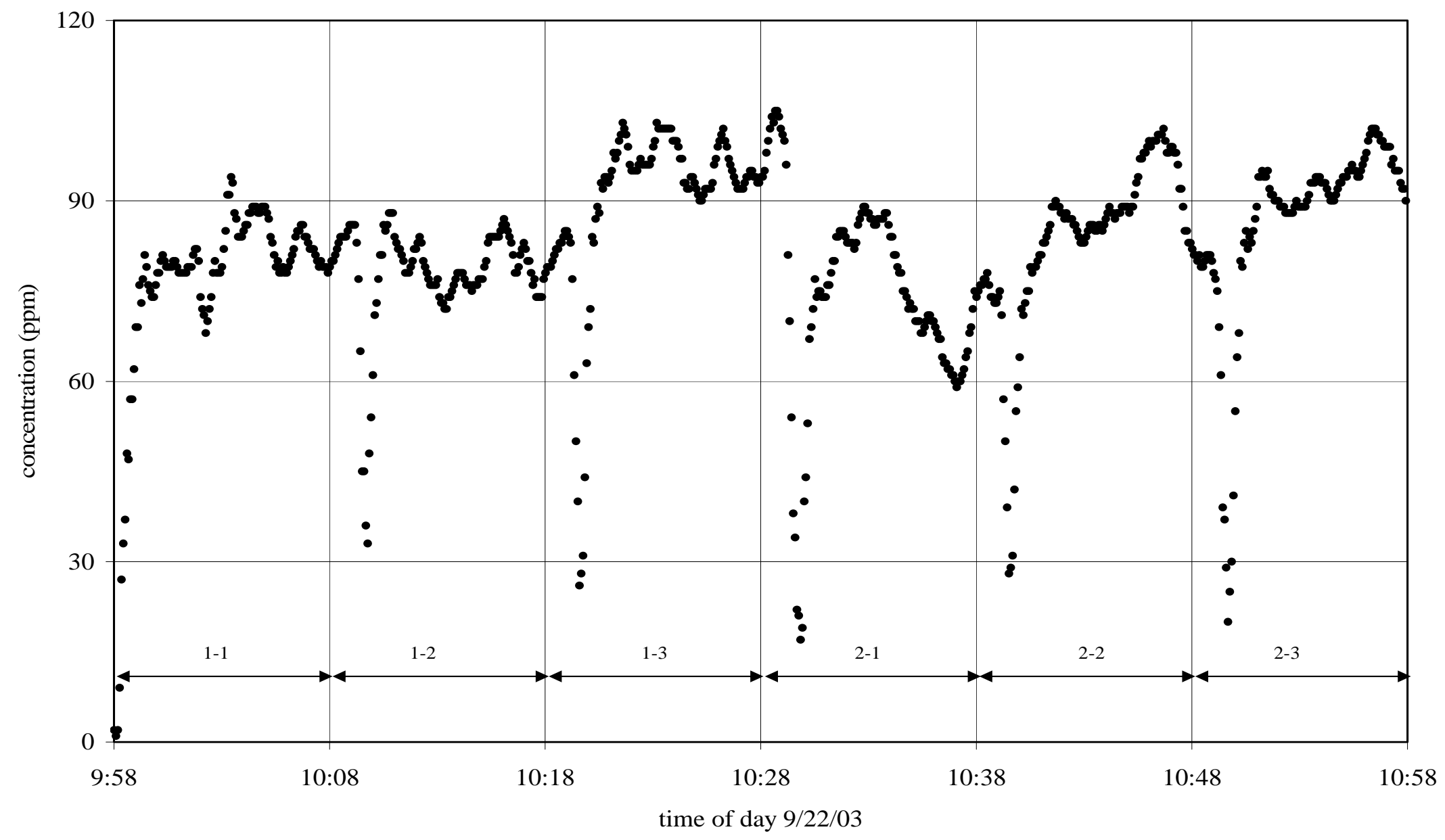

Figure C4. CO concentrations recorded downstream of the air preheater in Boiler 1 firing coal on September 22, 2003, first sample period. 


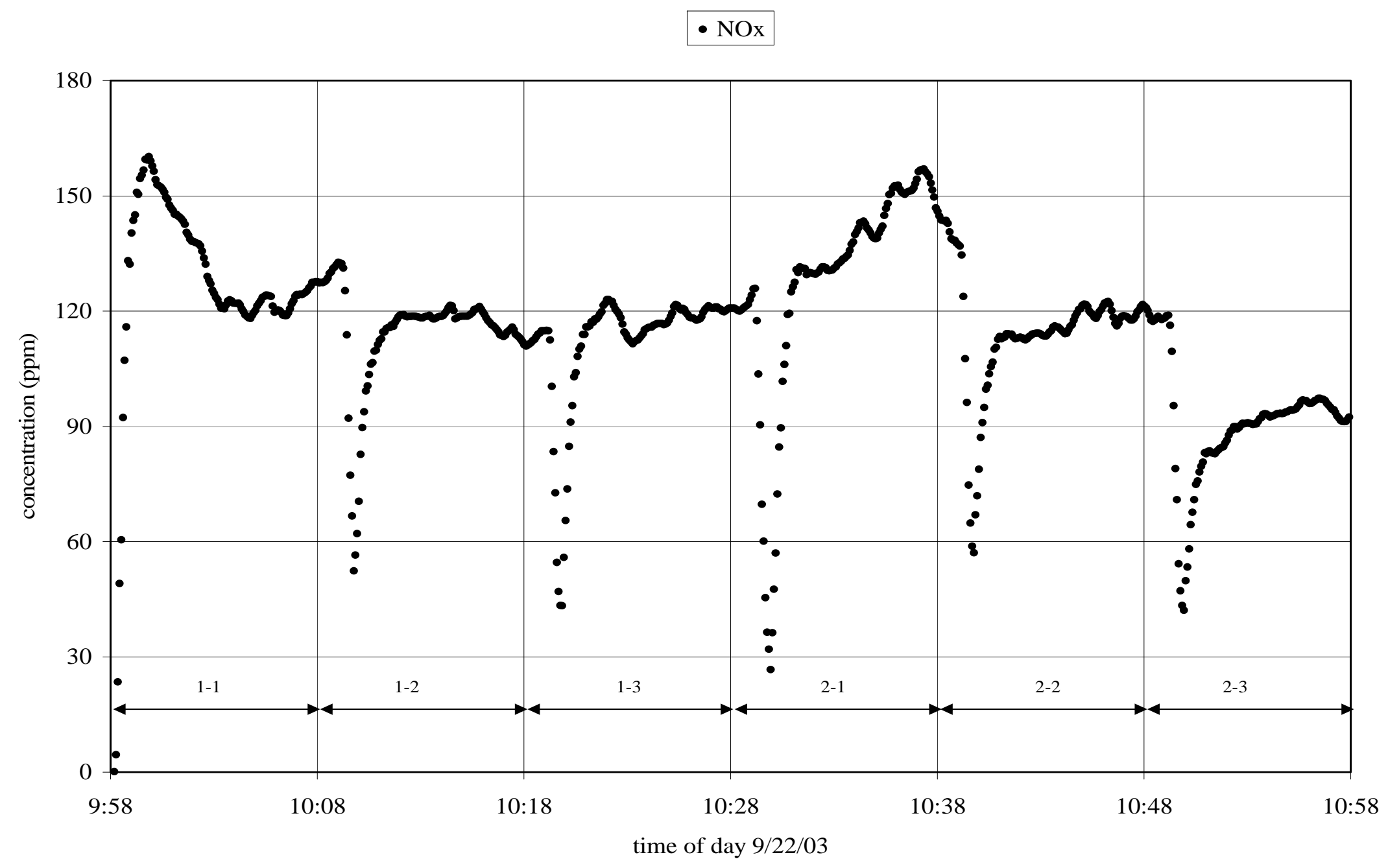

Figure C5. NOx concentrations recorded downstream of the air preheater in Boiler 1 firing coal on September 22, 2003, first sample period. 
$\times$ Tref $-\mathrm{T} 1 \cdot \mathrm{T} 2+\mathrm{T} 3 * \mathrm{~T} 4 \Delta \mathrm{T} 5 \square \mathrm{T} 6$

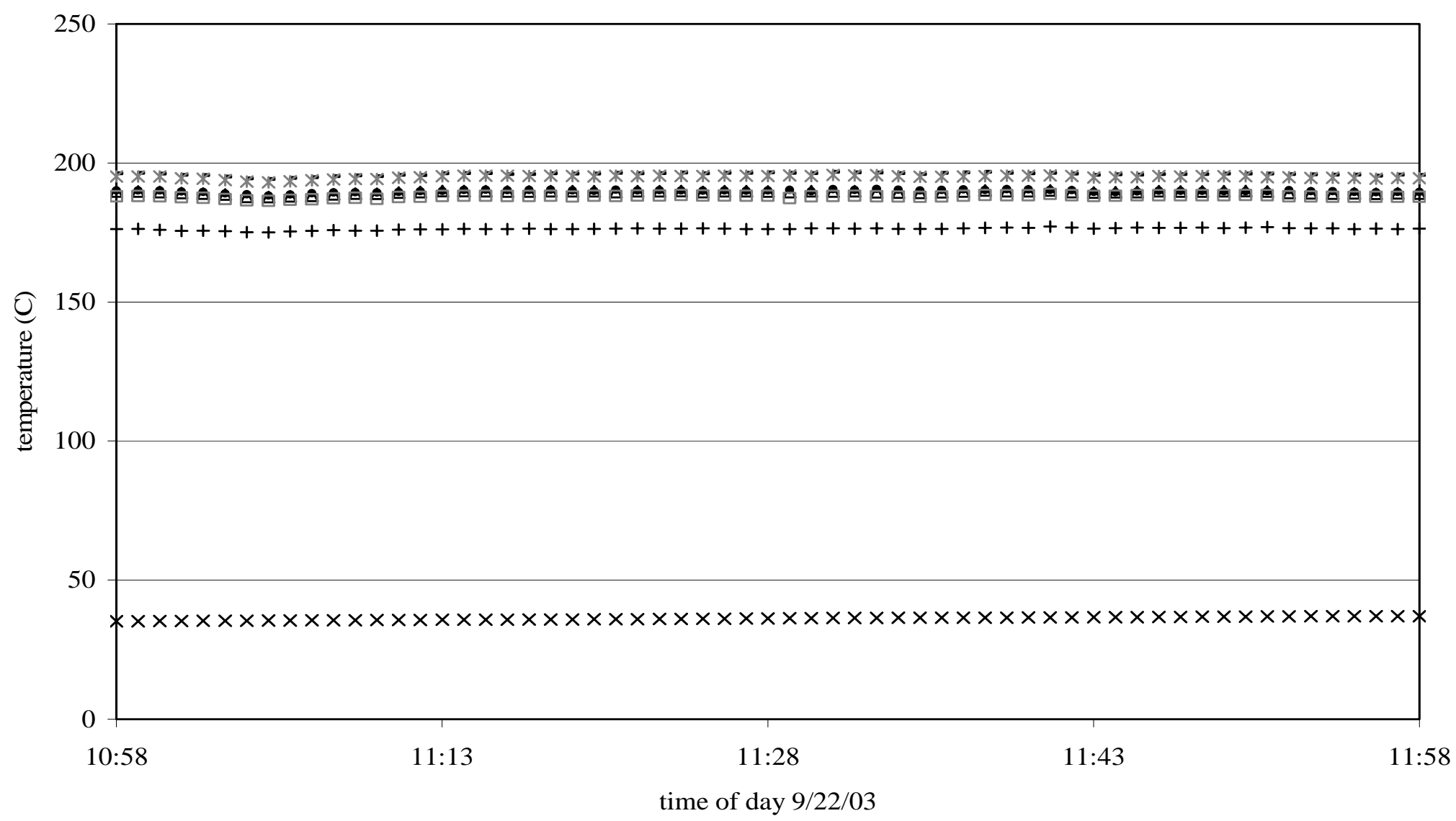

Figure C6. Temperatures recorded downstream of the air preheater in Boiler 1 firing coal on September 22, 2003, second sample period. Temperatures T1 though T6 are from Type K thermocouples located in the flue gas. Tref is an indicator of ambient temperature. 
- $\mathrm{CO} 2+\mathrm{O} 2$

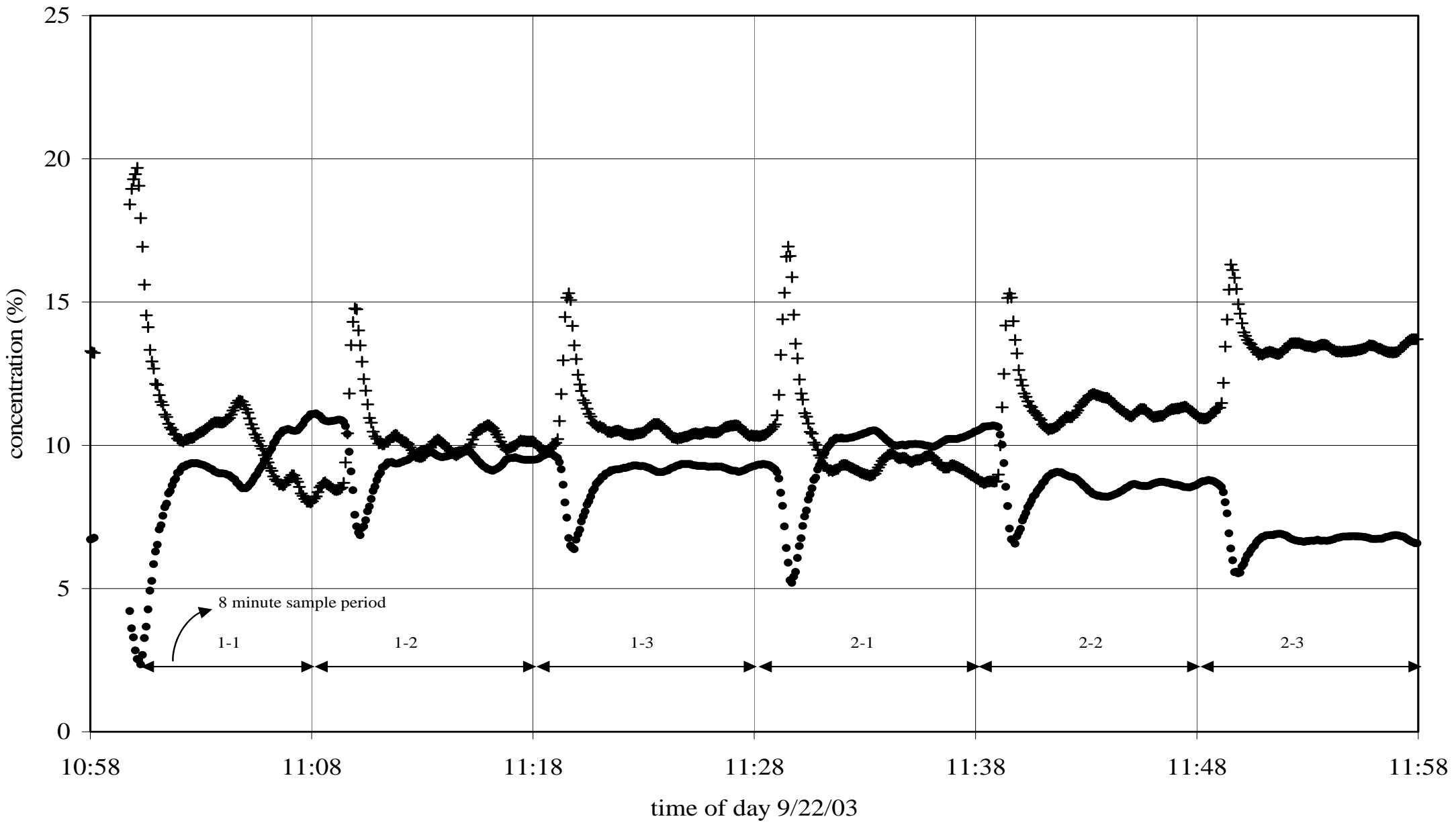

Figure C7. $\mathrm{O}_{2}$ and $\mathrm{CO}_{2}$ concentrations recorded downstream of the air preheater in Boiler 1 firing coal on September 22, 2003, second sample period. 


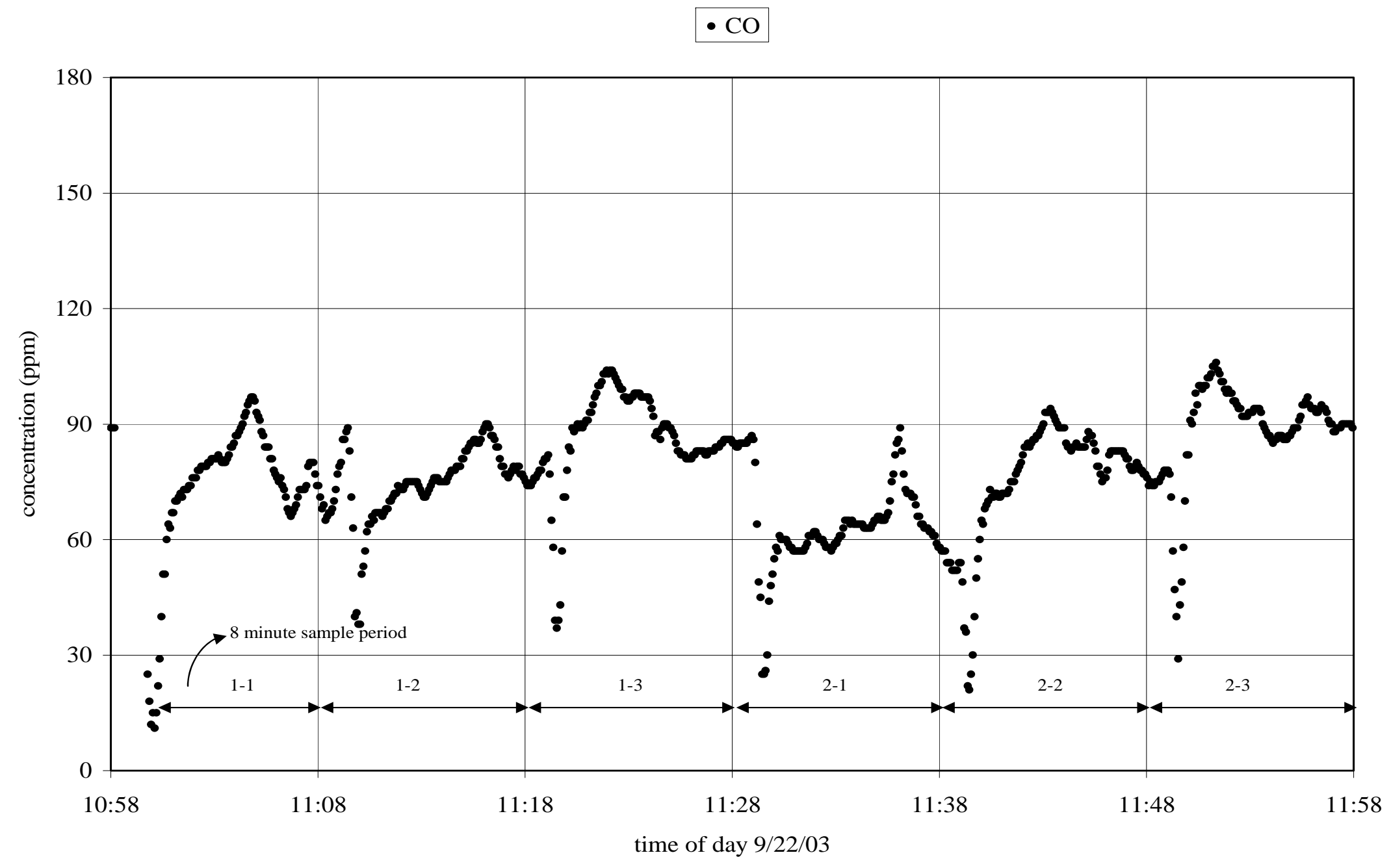

Figure C8. CO concentrations recorded downstream of the air preheater in Boiler 1 firing coal on September 22, 2003, second sample period. 


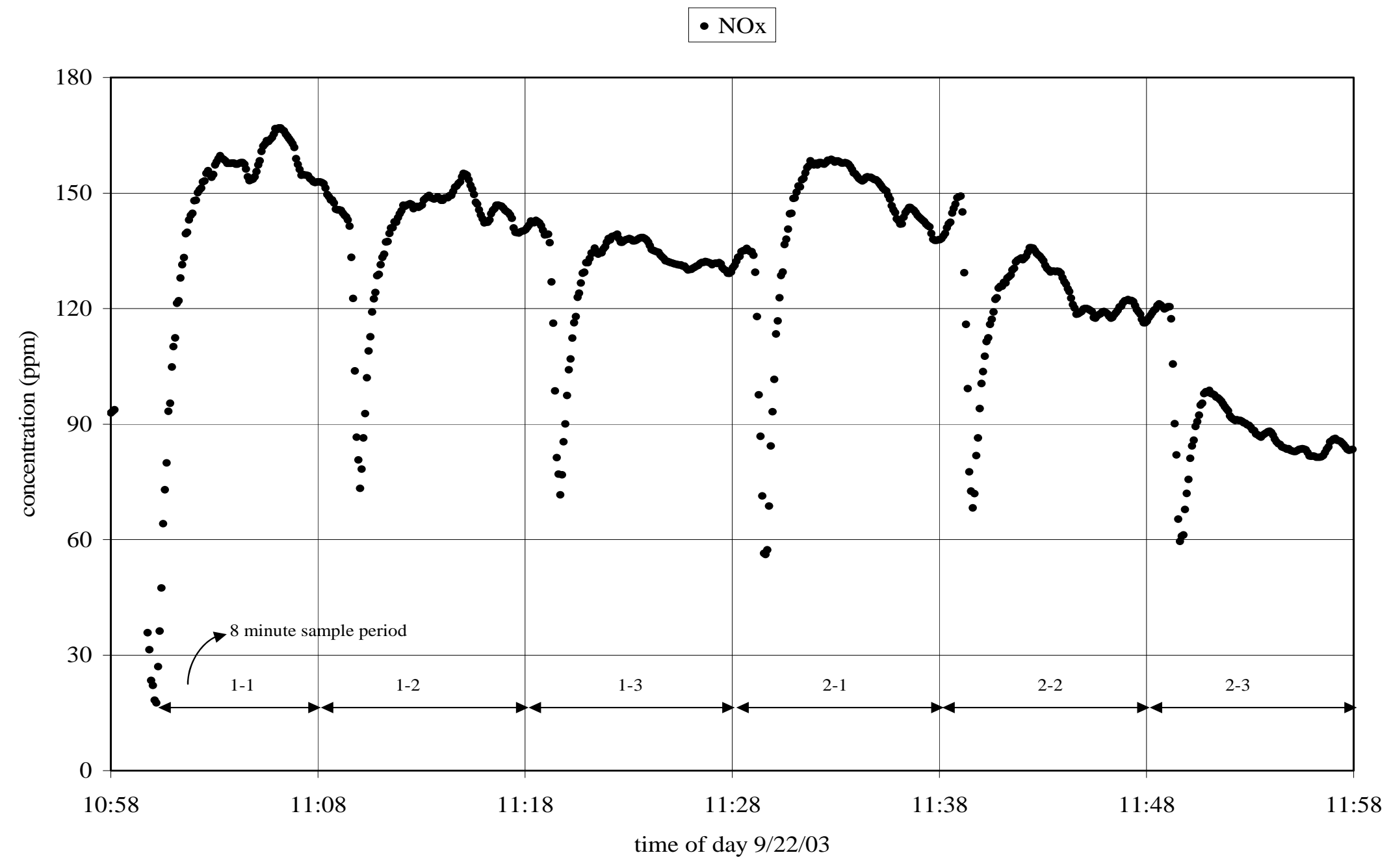

Figure C9. NOx concentrations recorded downstream of the air preheater in Boiler 1 firing coal on September 22, 2003, second sample period. 
$\times$ Tref $-\mathrm{T} 1 \bullet \mathrm{T} 2+\mathrm{T} 3 * \mathrm{~T} 4 \Delta \mathrm{T} 5 \square \mathrm{T} 6$

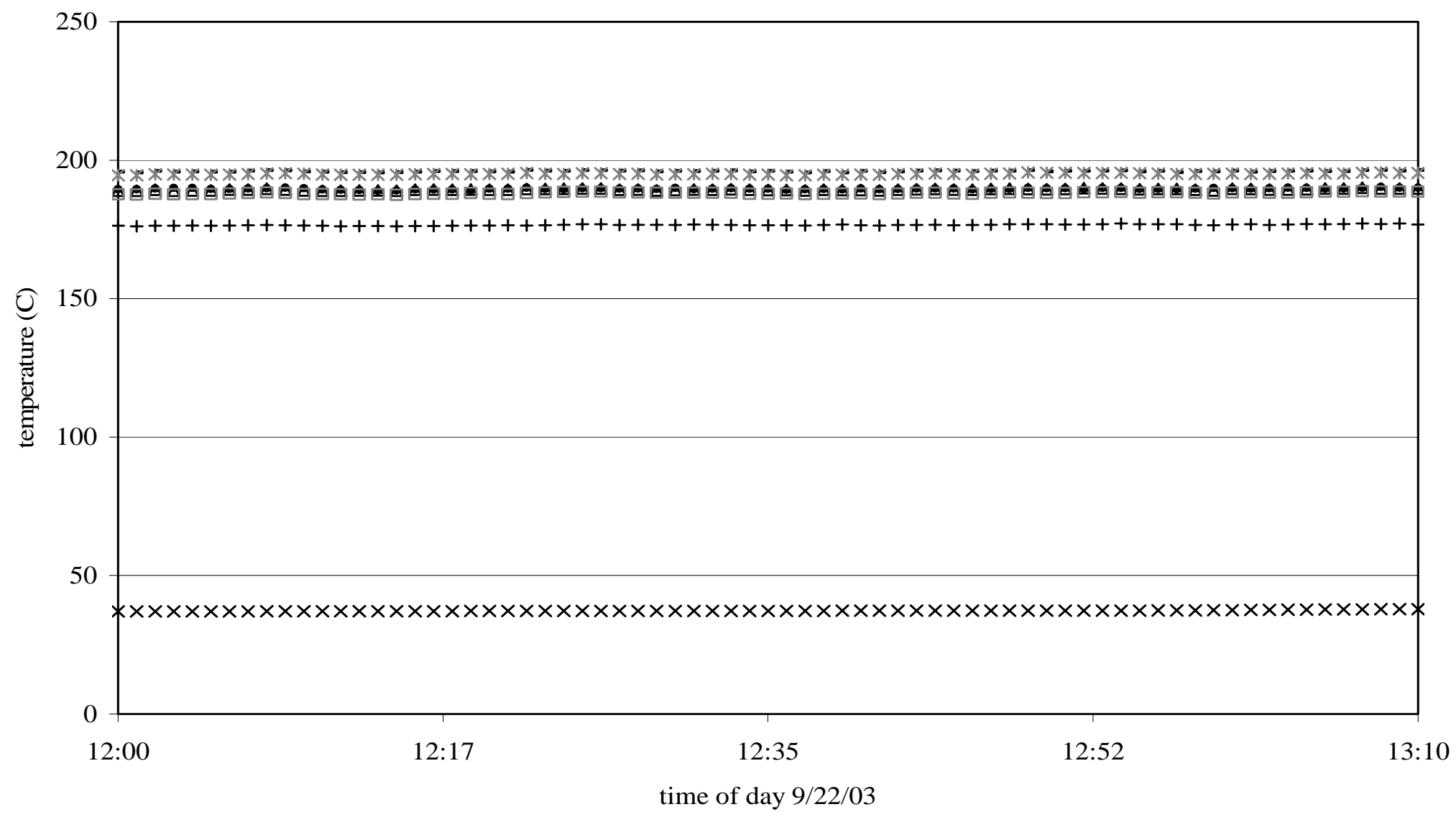

Figure C10. Temperatures recorded downstream of the air preheater in Boiler 1 firing coal on September 22, 2003, third sample period. Temperatures T1 though T6 are from Type K thermocouples located in the flue gas. Tref is an indicator of ambient temperature. 
- $\mathrm{CO} 2+\mathrm{O} 2$

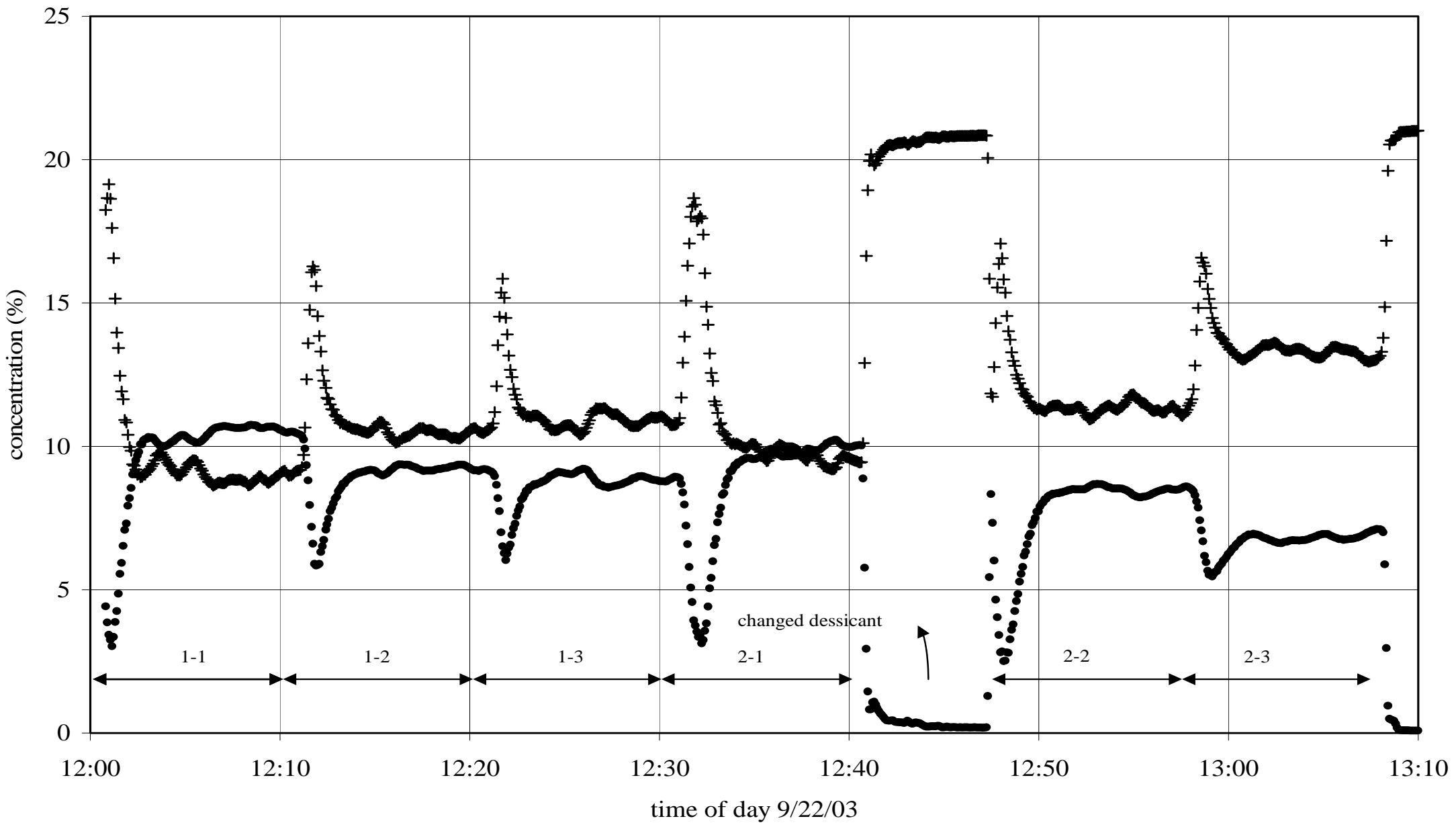

Figure C11. $\mathrm{O}_{2}$ and $\mathrm{CO}_{2}$ concentrations recorded downstream of the air preheater in Boiler 1 firing coal on September 22, 2003, third sample period. 


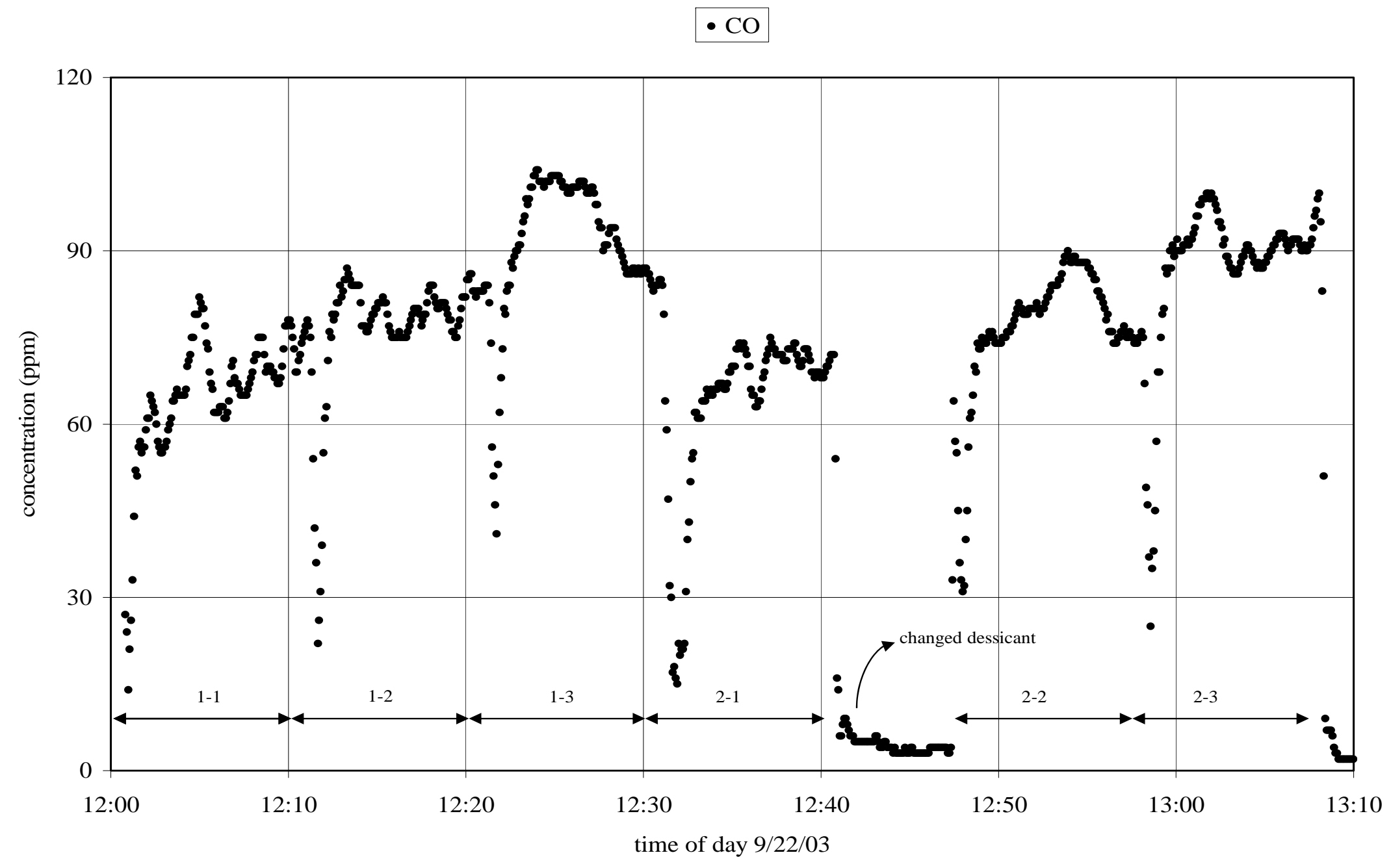

Figure C12. CO concentrations recorded downstream of the air preheater in Boiler 1 firing coal on September 22, 2003, third sample period. 


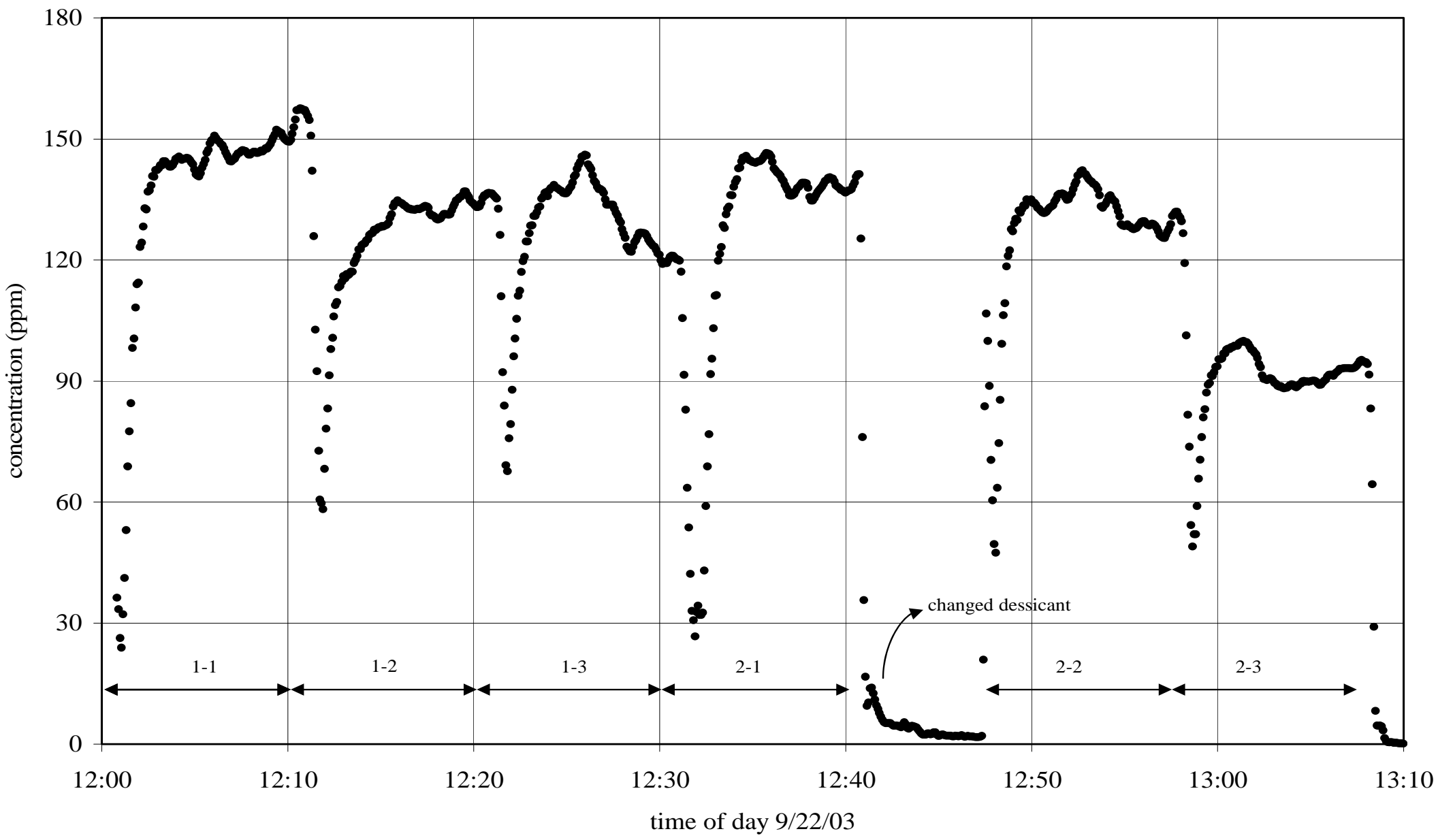

Figure C13. NOx concentrations recorded downstream of the air preheater in Boiler 1 firing coal on September 22, 2003, third sample period. 


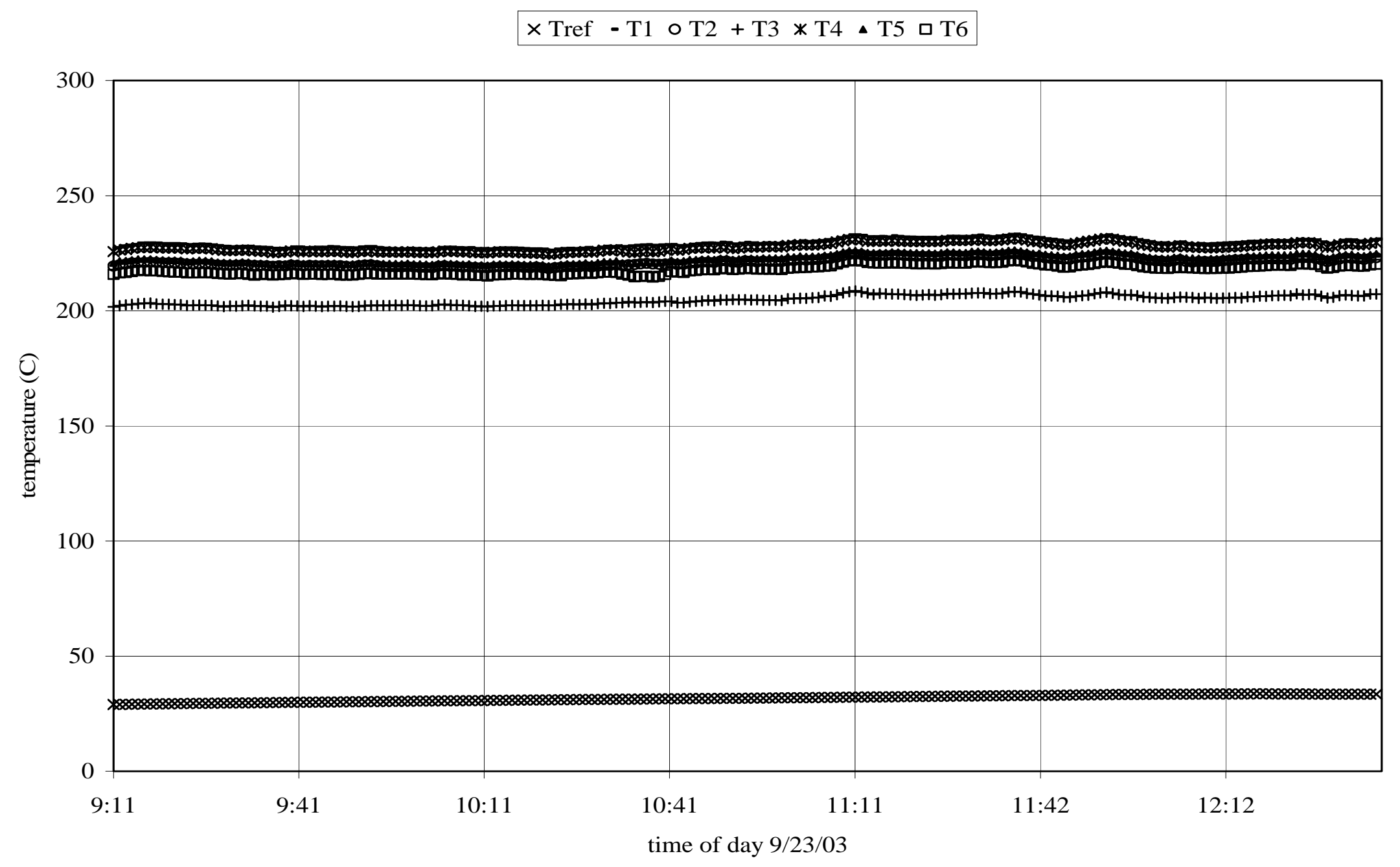

Figure C14. Temperatures recorded downstream of the air preheater in Boiler 1 firing bagasse on September 23, 2003. Temperatures T1 though T6 are from Type K thermocouples located in the flue gas. Tref is an indicator of ambient temperature. 


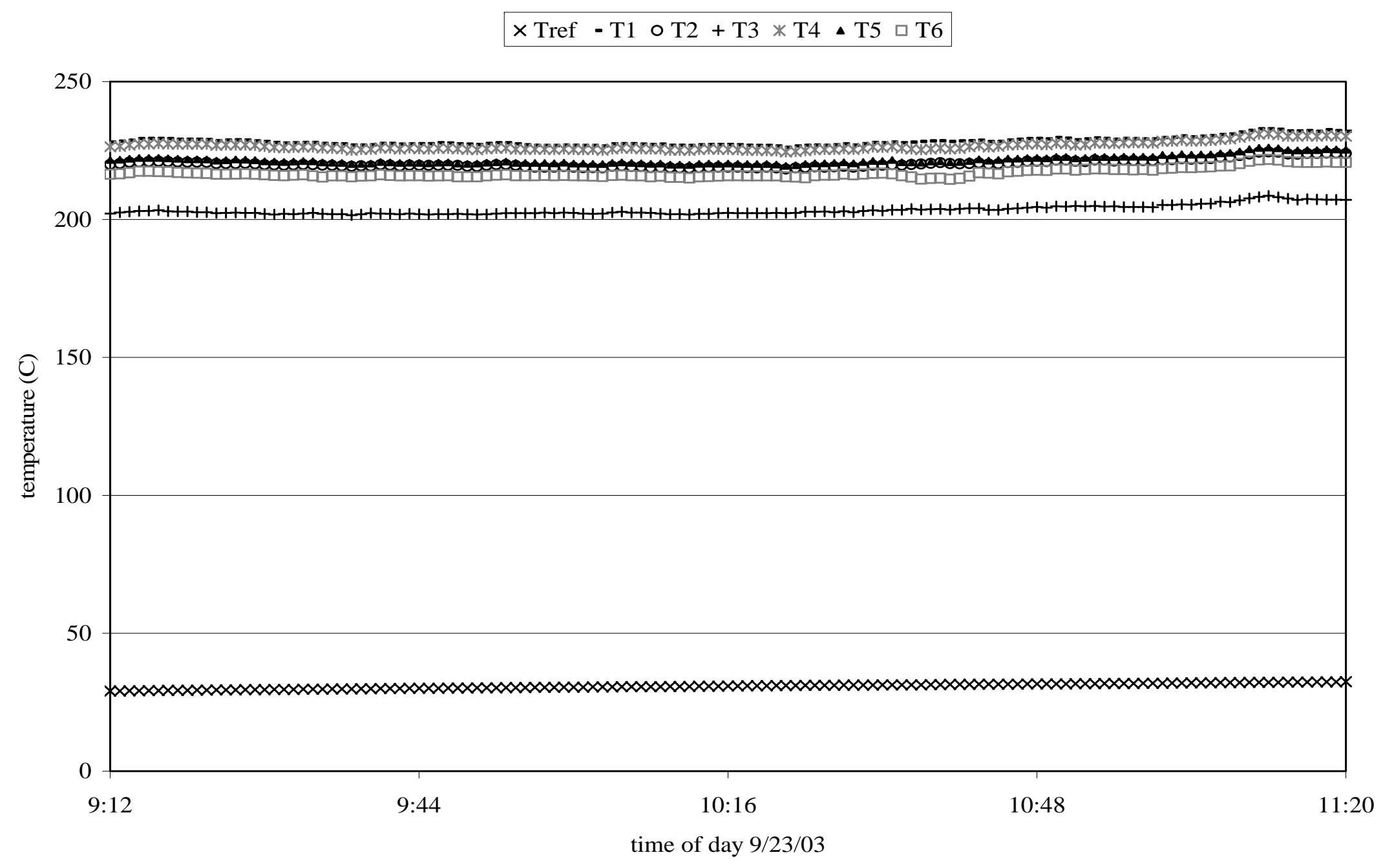

C15. Temperatures recorded downstream of the air preheater in Boiler 1 firing bagasse on September 23, 2003, first sample period. Temperatures T1 though T6 are from Type K thermocouples located in the flue gas. Tref is an indicator of ambient temperature. 
- $\mathrm{CO} 2+\mathrm{O} 2$

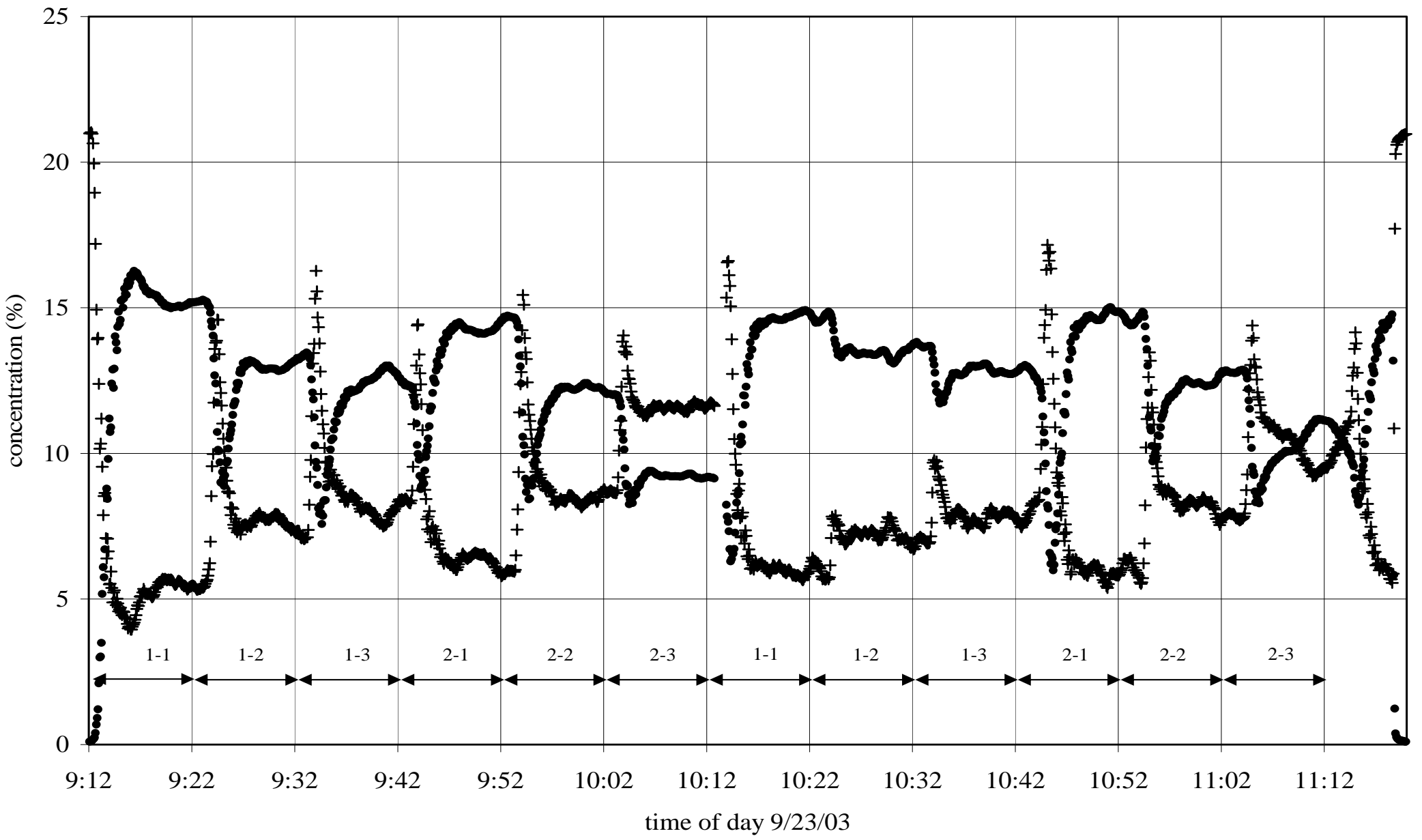

Figure C16. $\mathrm{O}_{2}$ and $\mathrm{CO}_{2}$ concentrations recorded downstream of the air preheater in Boiler 1 firing bagasse on September 23, 2003, first and second sample period. 


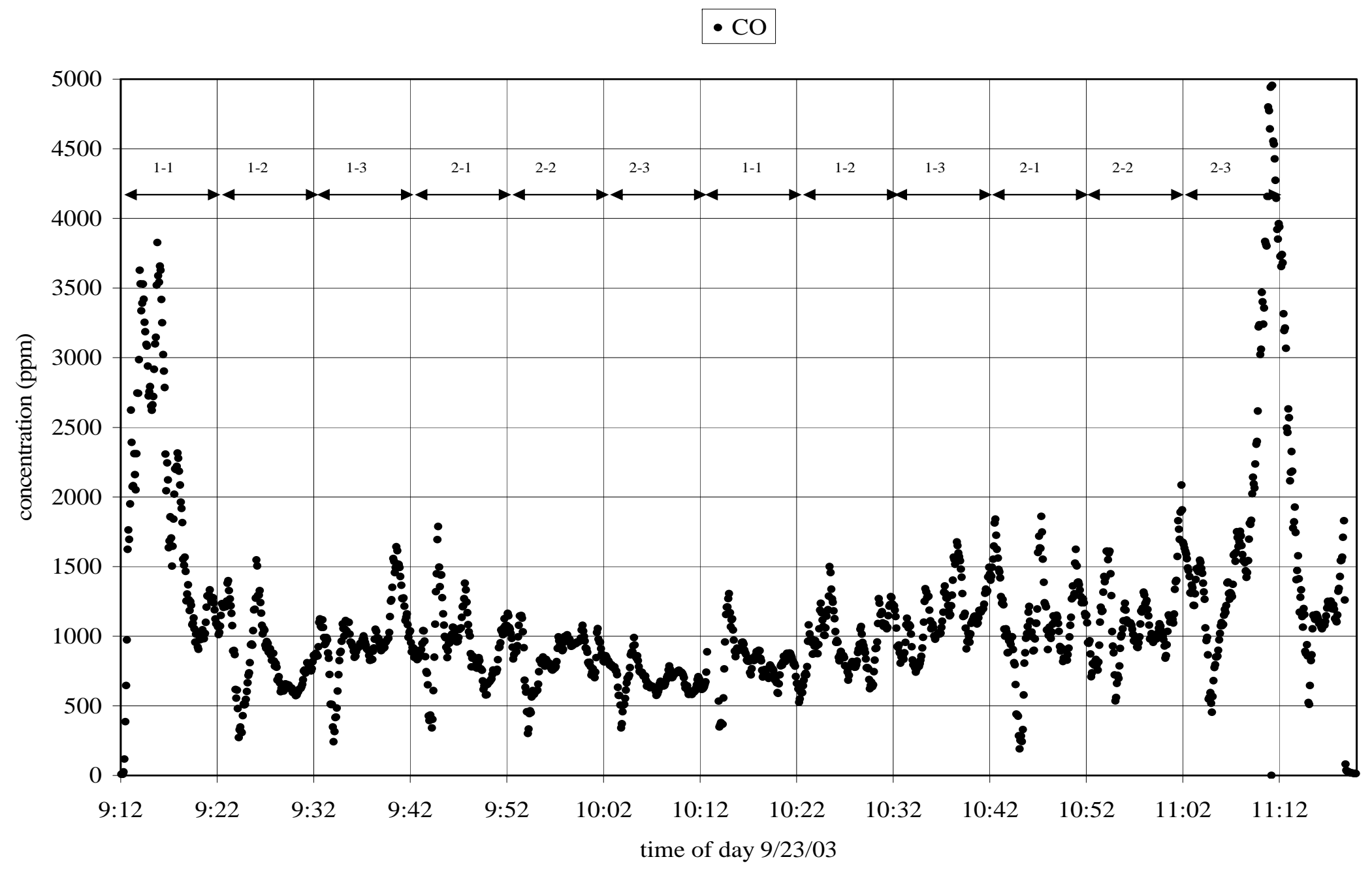

Figure Figure C17. CO concentrations recorded downstream of the air preheater in Boiler 1 firing bagasse on September 23, 2003, first and second sample period. 


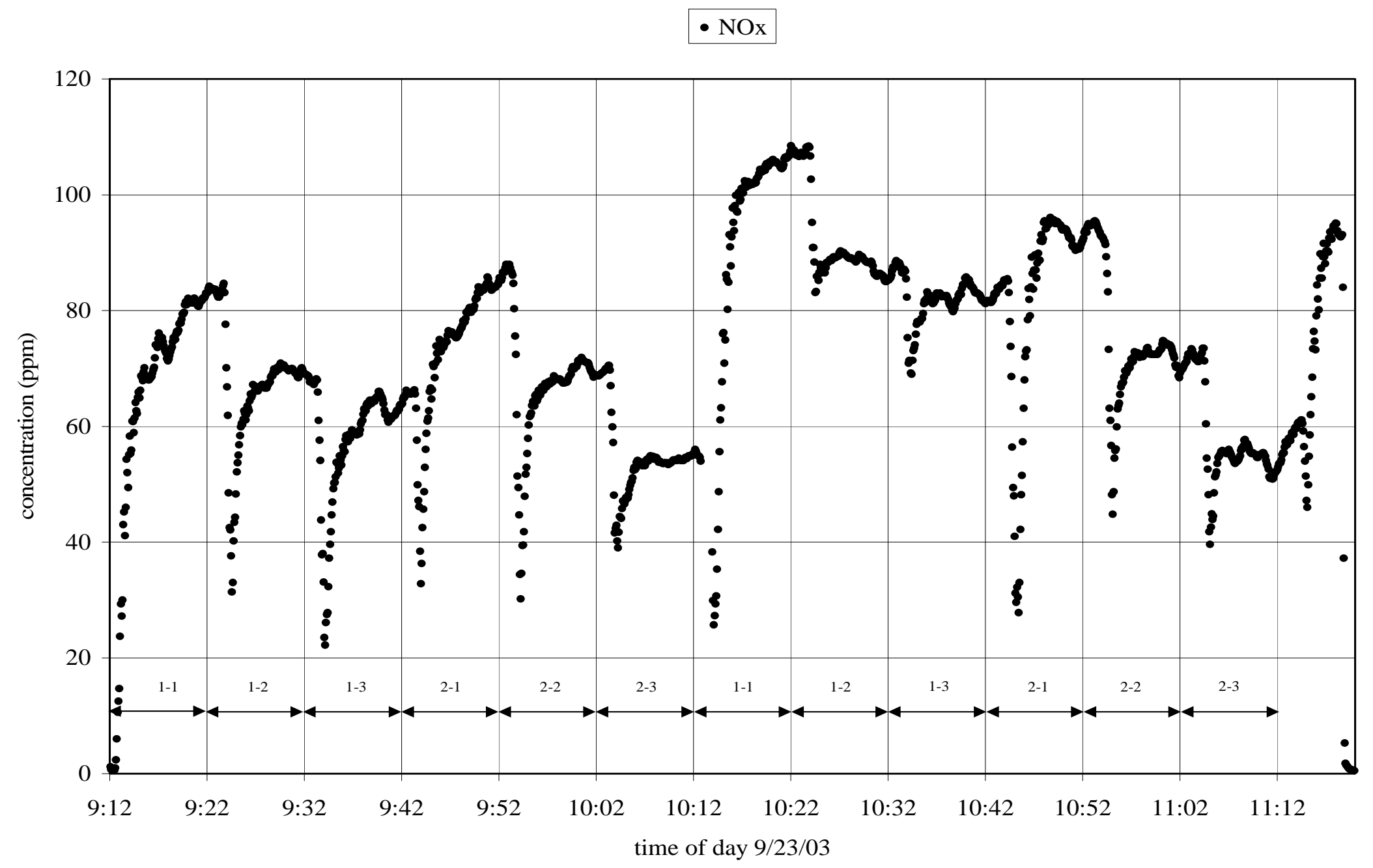

Figure C18. NOx concentrations recorded downstream of the air preheater in Boiler 1 firing bagasse on September 23, 2003, first and second sample period. 


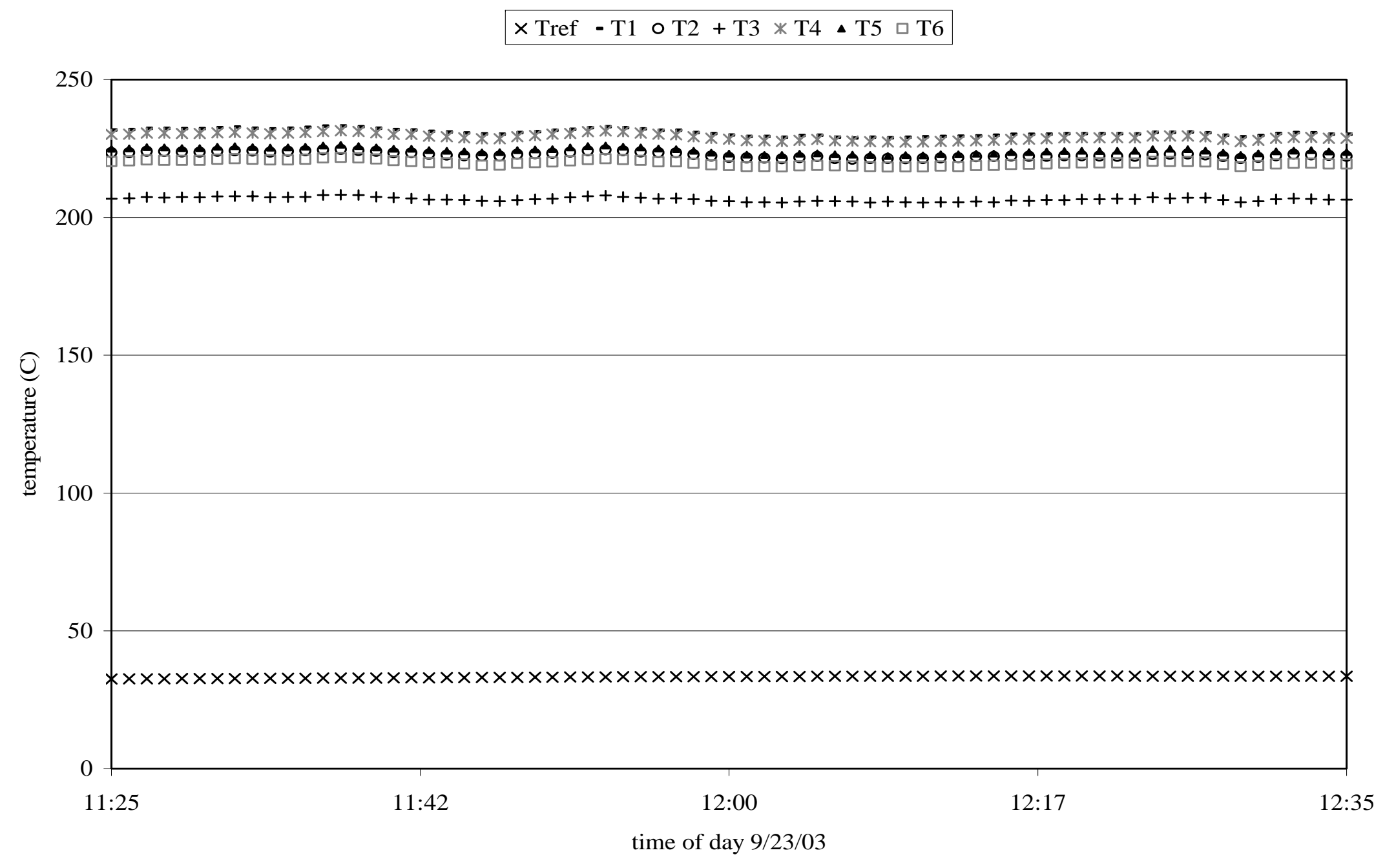

Figure C19. Temperatures recorded downstream of the air preheater in Boiler 1 firing bagasse on September 23, 2003, second sample period. Temperatures T1 though T6 are from Type K thermocouples located in the flue gas. Tref is an indicator of ambient temperature. 


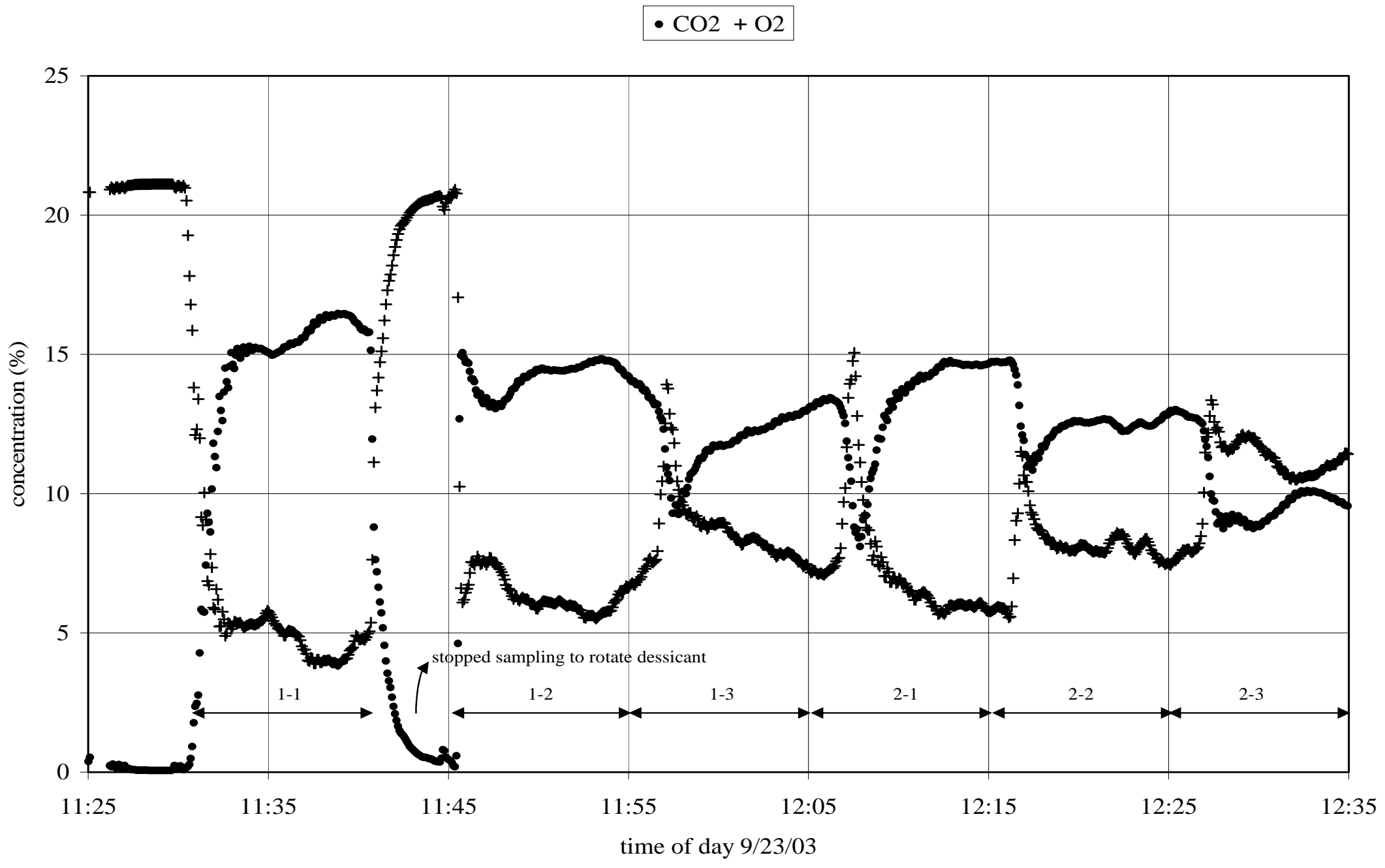

Figure C20. $\mathrm{O}_{2}$ and $\mathrm{CO}_{2}$ concentrations recorded downstream of the air preheater in Boiler 1 firing bagasse on September 23, 2003, third sample period. 


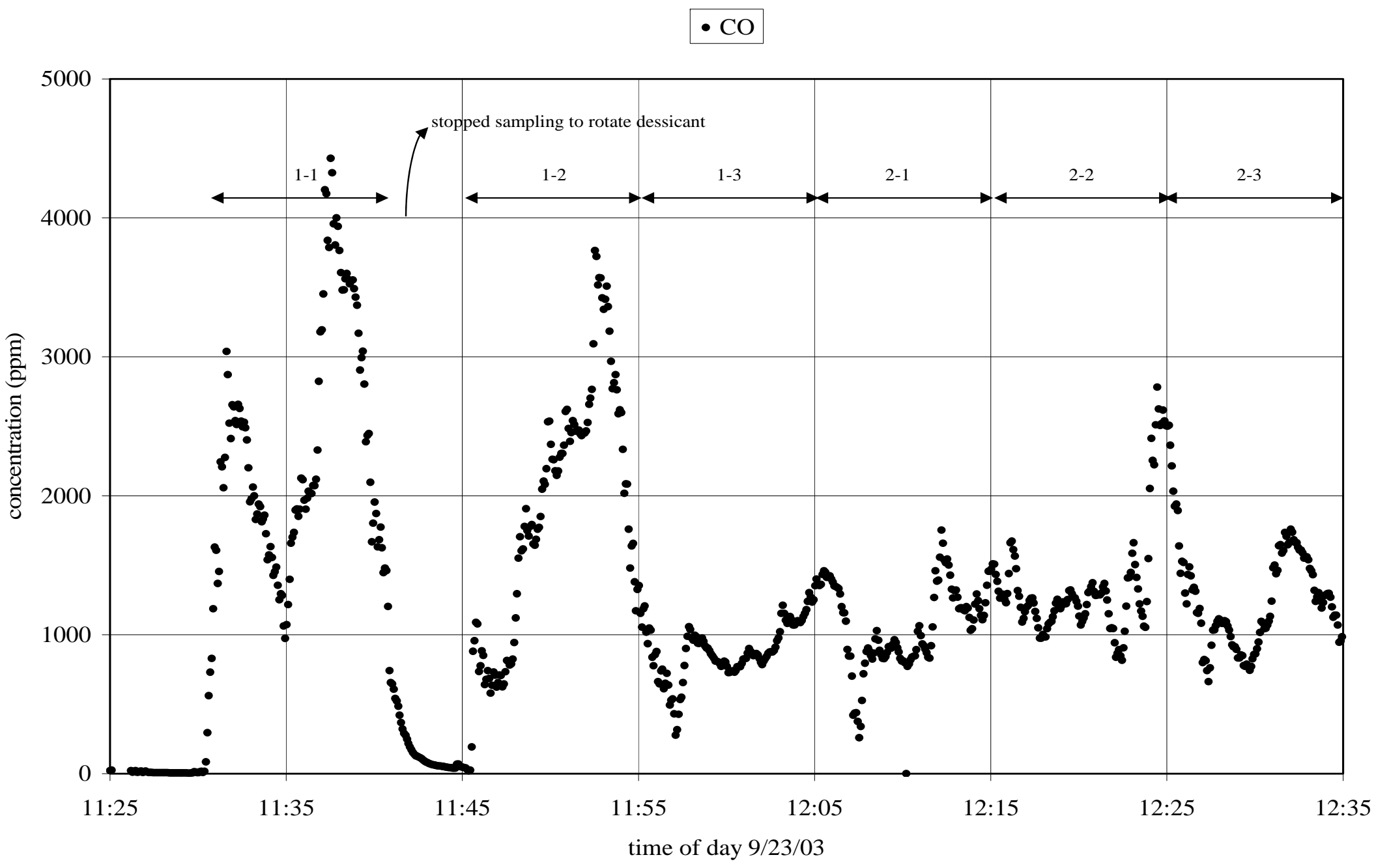

Figure C21. CO concentrations recorded downstream of the air preheater in Boiler 1 firing bagasse on September 23, 2003, third sample period. 


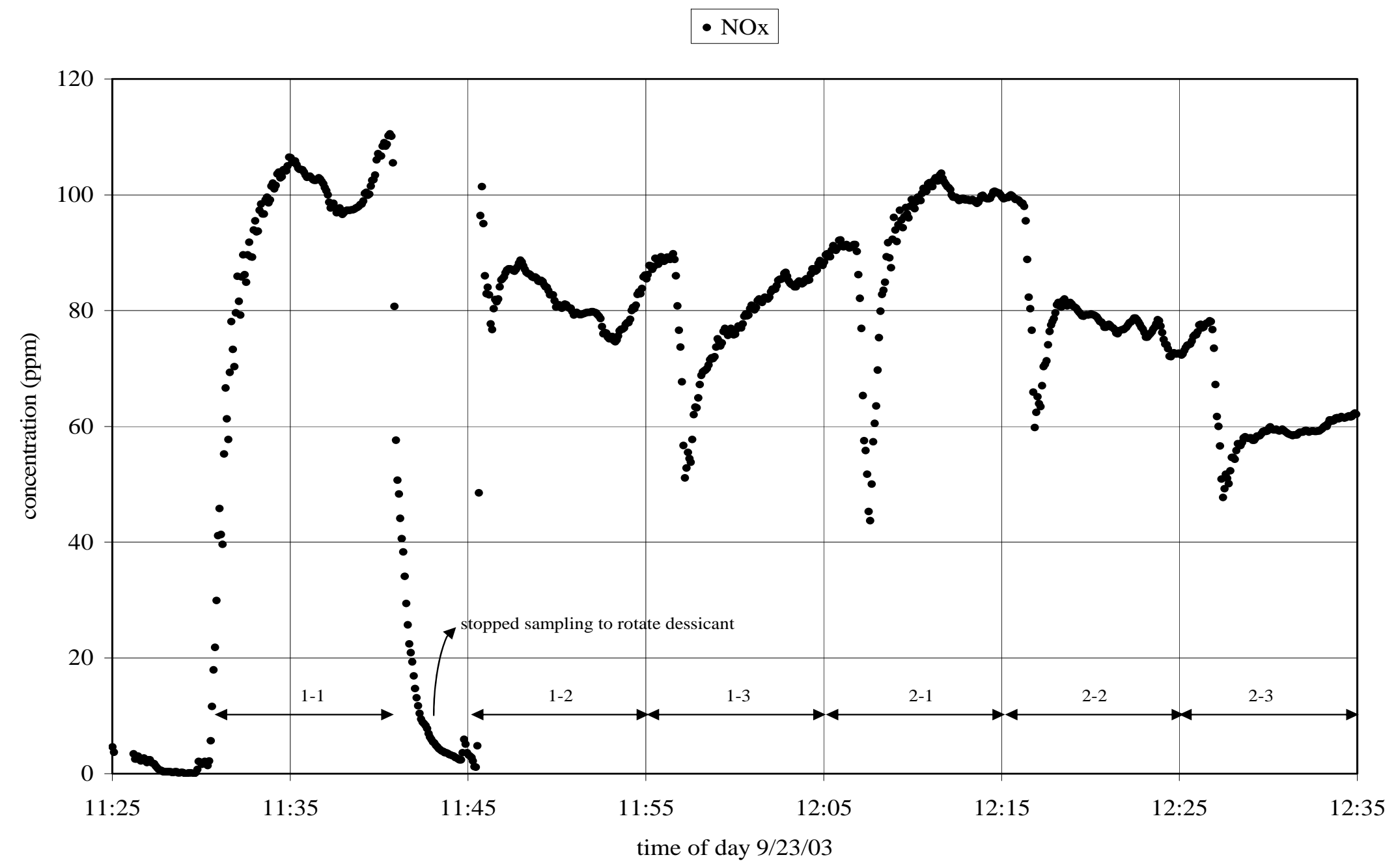

Figure C22. NOx concentrations recorded downstream of the air preheater in Boiler 1 firing bagasse on September 23, 2003, third sample period. 


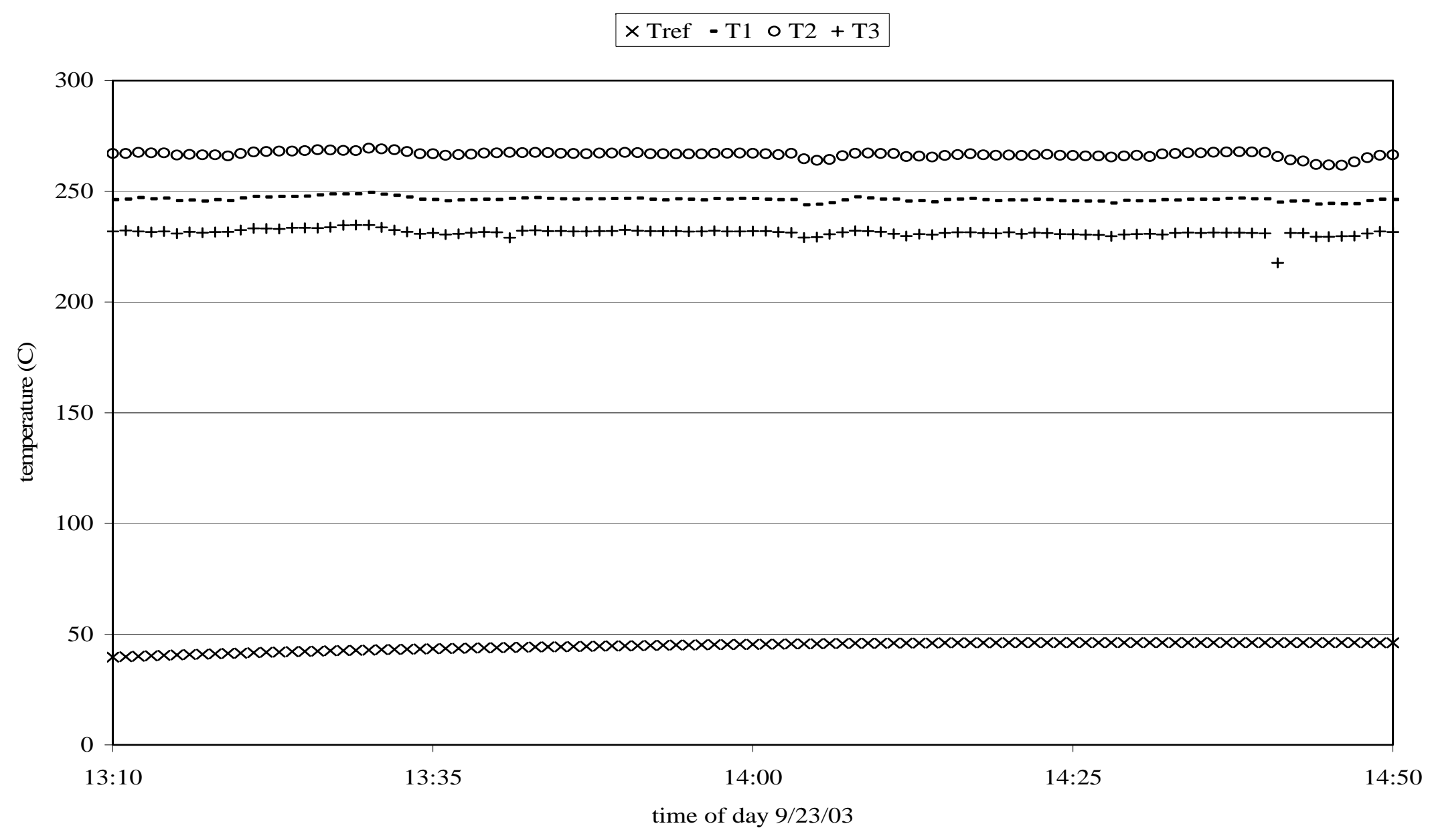

Figure C23. Temperatures recorded downstream of the air preheater in Boiler 2 firing bagasse on September 23, 2003, first sample period. Temperatures T1 though T3 are from Type K thermocouples located in the flue gas. Tref is an indicator of ambient temperature 


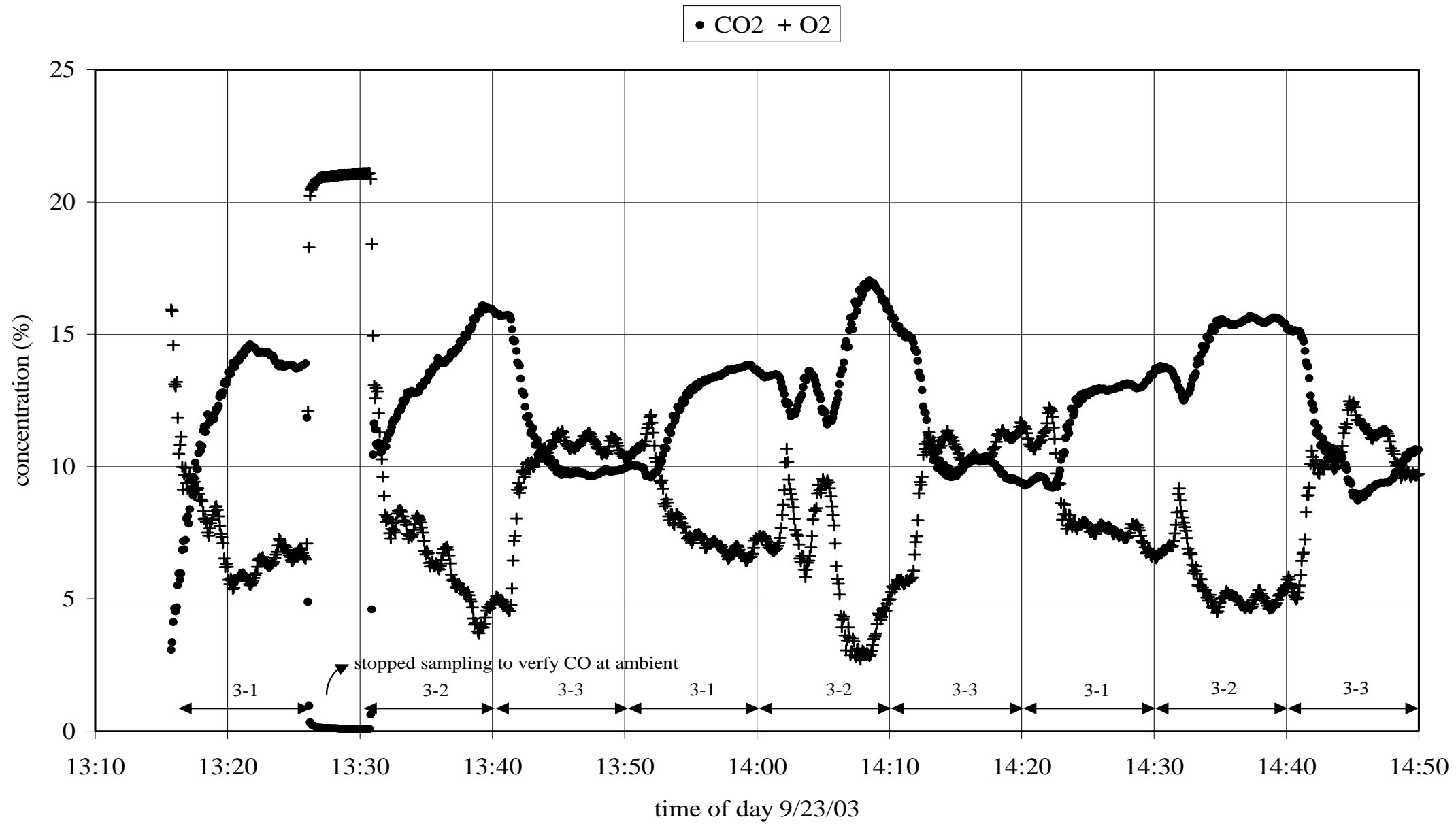

Figure C24. $\mathrm{O}_{2}$ and $\mathrm{CO}_{2}$ concentrations recorded downstream of the air preheater in Boiler 2 firing bagasse on September 23, 2003, three sample periods. 


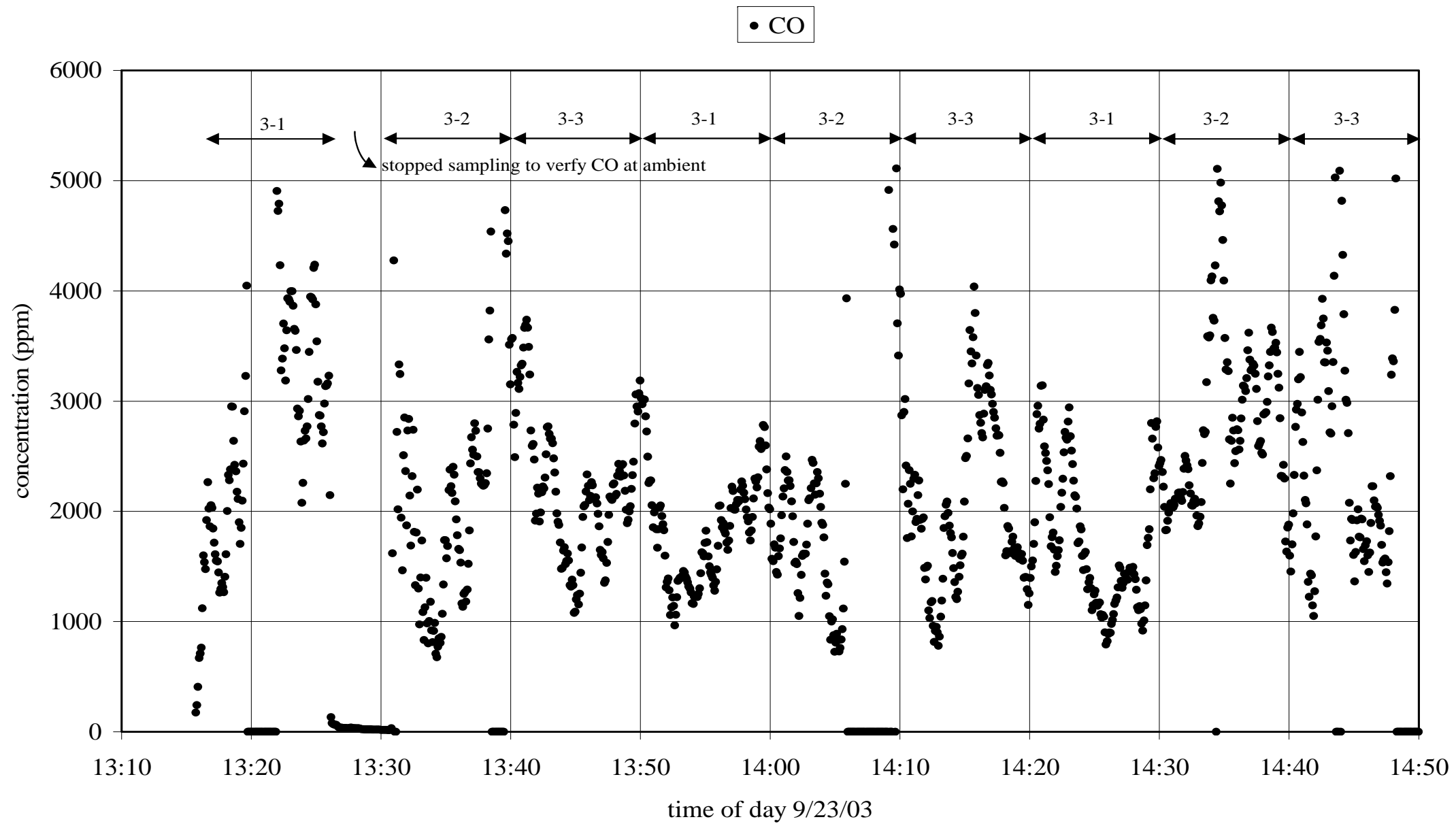

Figure C25. CO concentrations recorded downstream of the air preheater in Boiler 2 firing bagasse on September 23, 2003, three sample periods. 
- $\mathrm{NOx}$

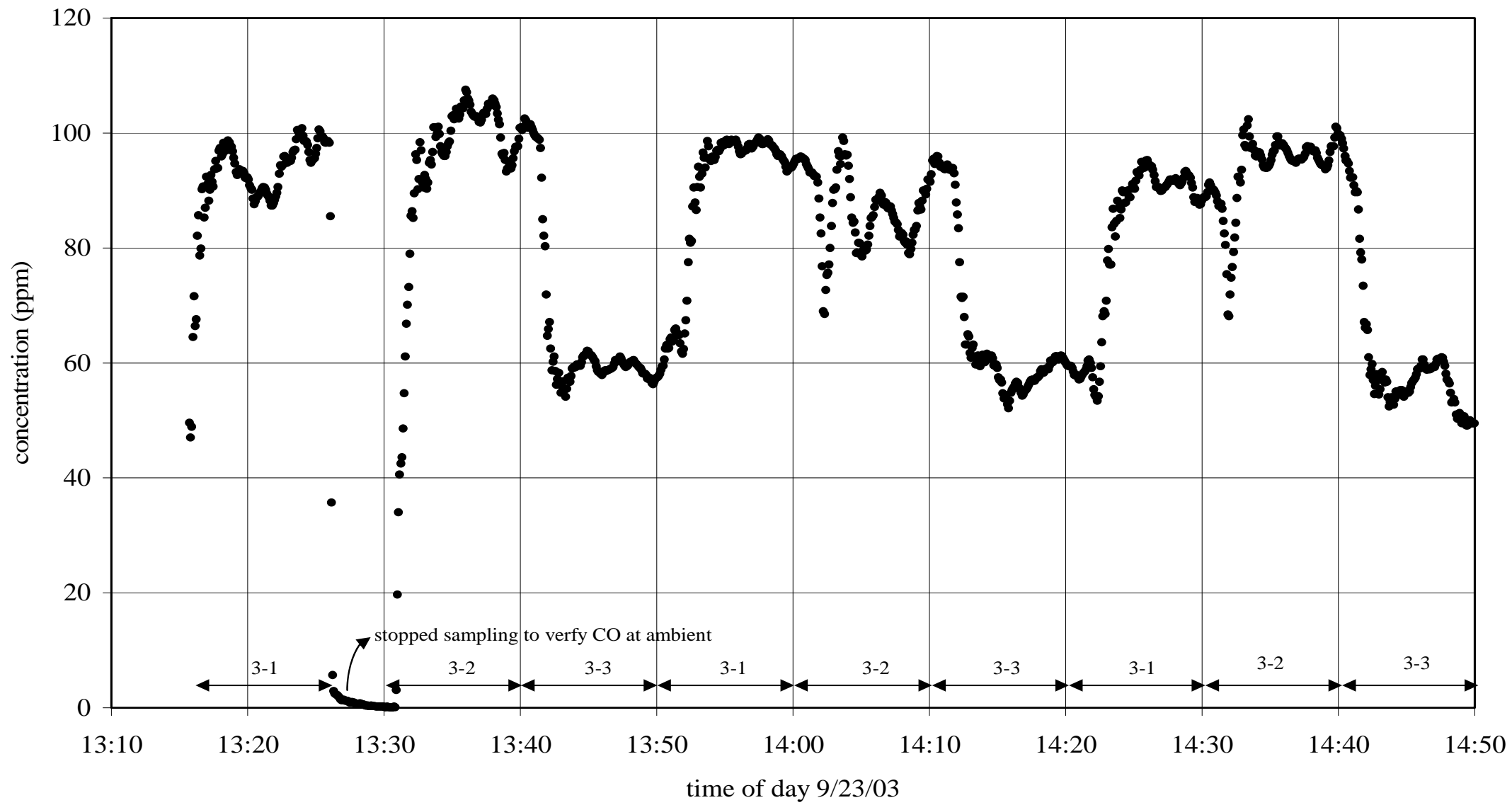

Figure C26. NOx concentrations recorded downstream of the air preheater in Boiler 2 firing bagasse on September 23, 2003, three sample periods. 


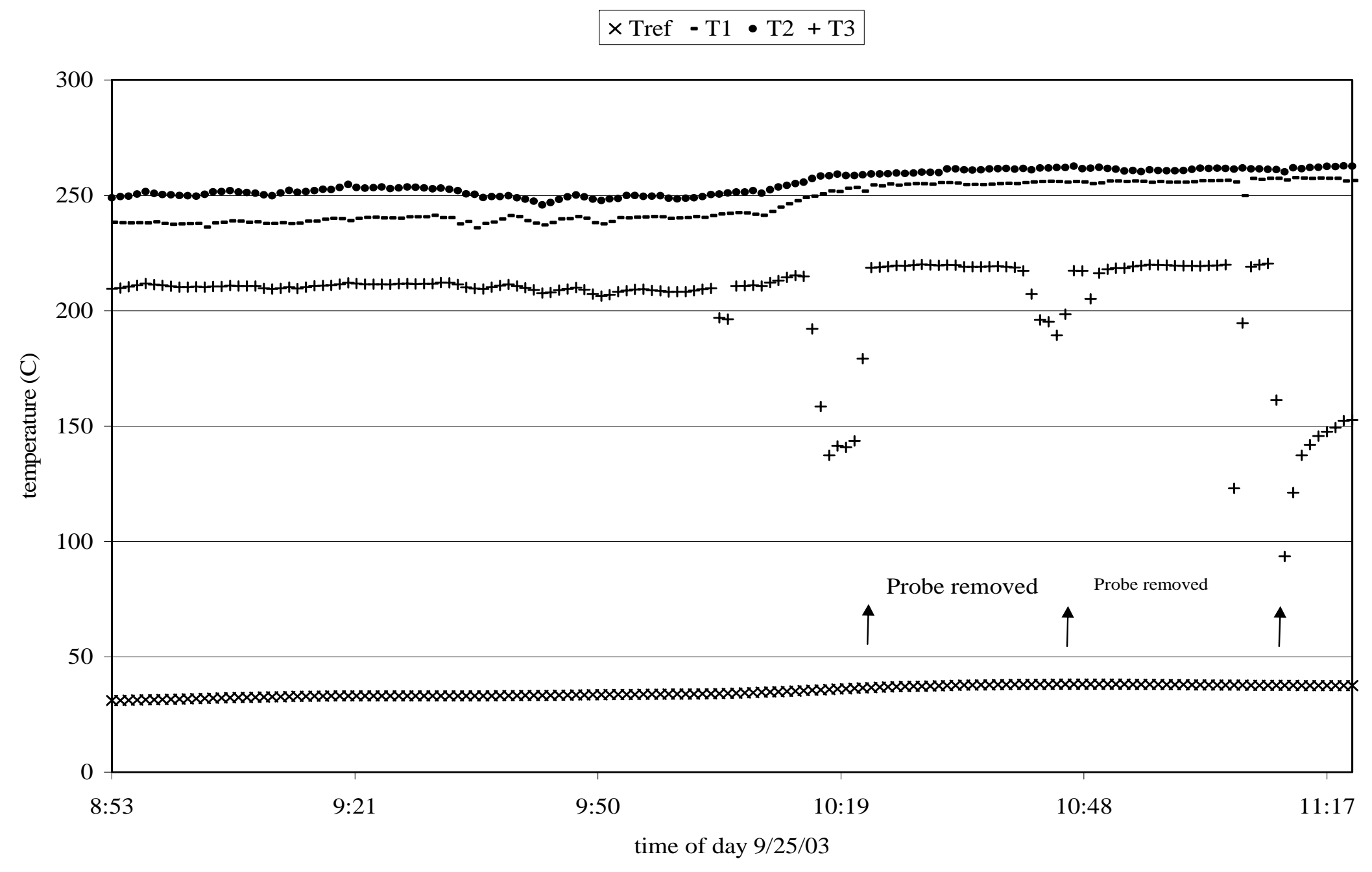

Figure C27. Temperatures recorded downstream of the air preheater in Boiler 3 firing bagasse on September 25, 2003. Temperatures T1 though T3 are from Type K thermocouples located in the flue gas. Tref is an indicator of ambient temperature. 


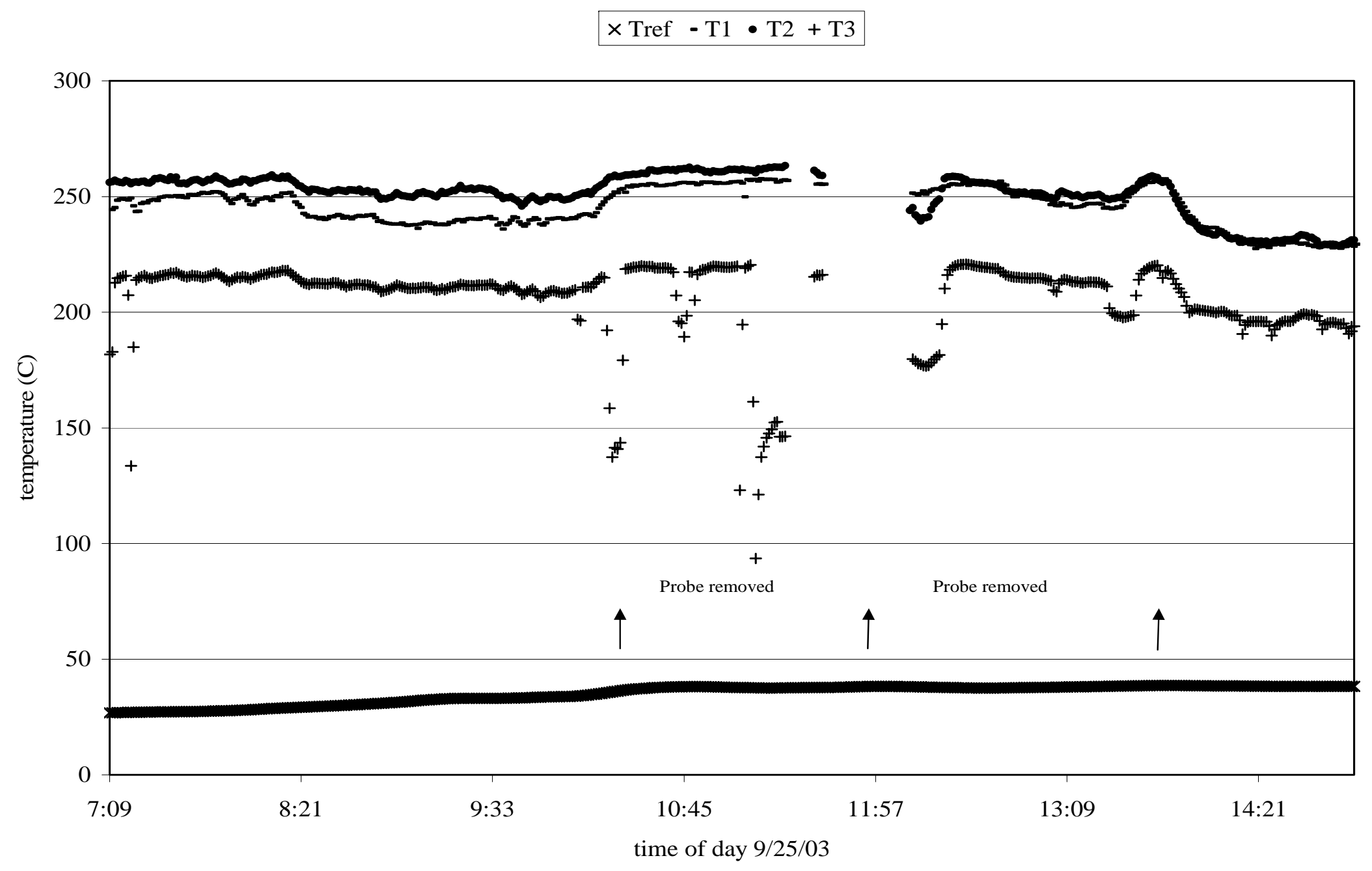

Figure C28. Temperatures recorded downstream of the air preheater in Boiler 3 firing bagasse on September 25, 2003, first sample period. Temperatures T1 though T3 are from Type K thermocouples located in the flue gas. Tref is an indicator of ambient temperature. 


\section{$\cdot \mathrm{CO} 2+\mathrm{O} 2$}

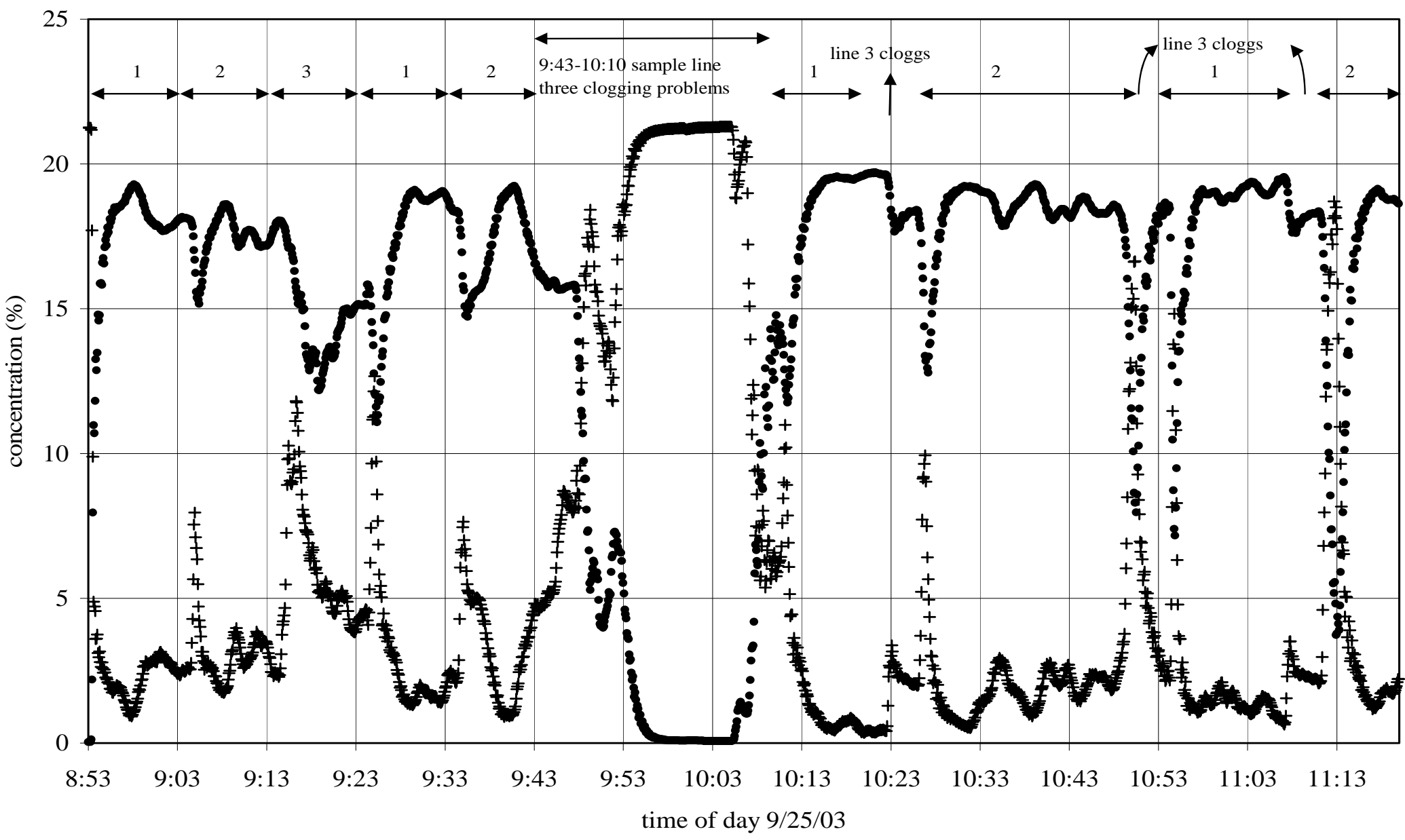

Figure Figure C29. $\mathrm{O}_{2}$ and $\mathrm{CO}_{2}$ concentrations recorded downstream of the air preheater in Boiler 3 firing bagasse on September 25, 2003, first sample period. 
- $\mathrm{CO}$

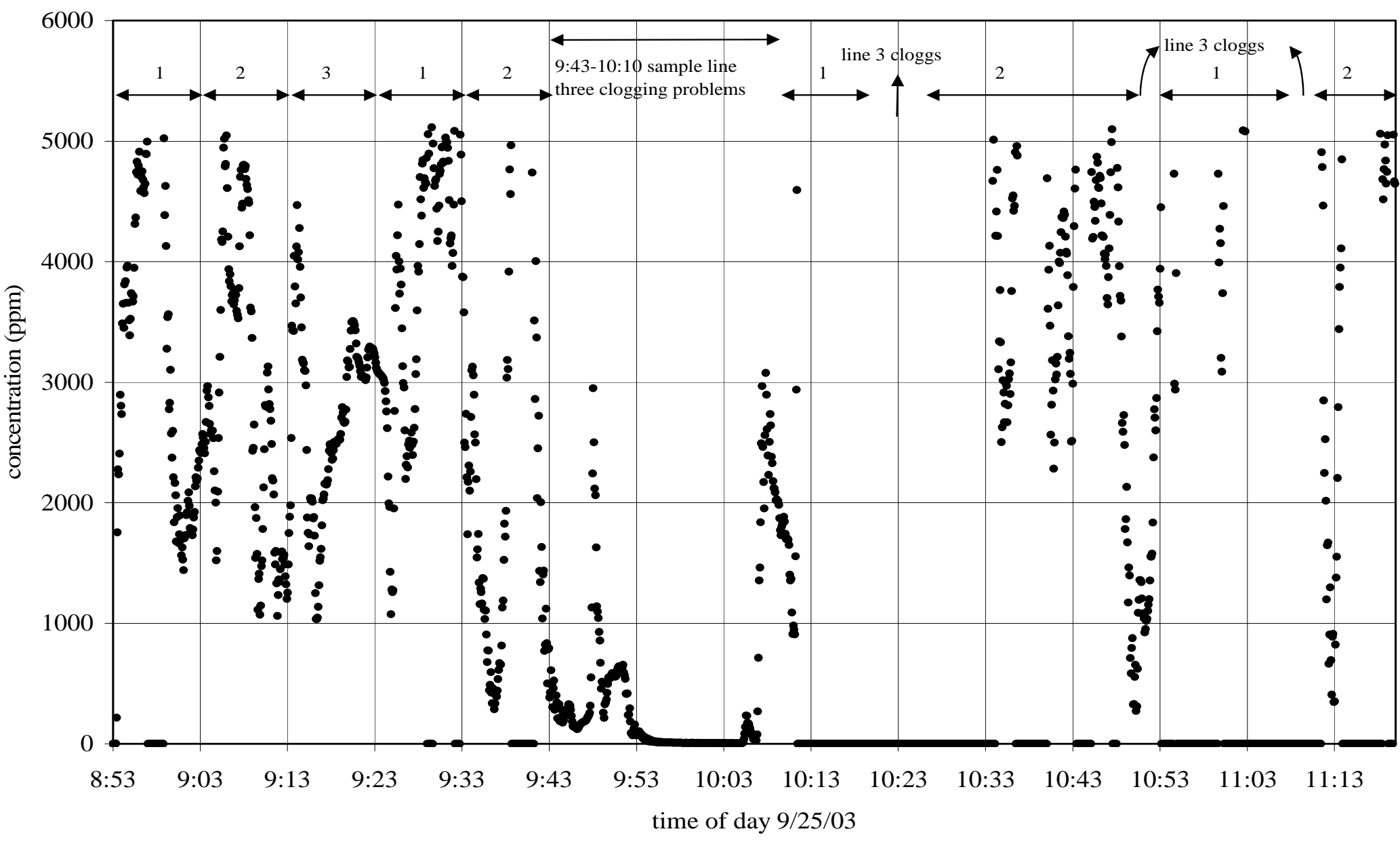

Figure C30. CO concentrations recorded downstream of the air preheater in Boiler 3 firing bagasse on September 25, 2003, first sample period. 


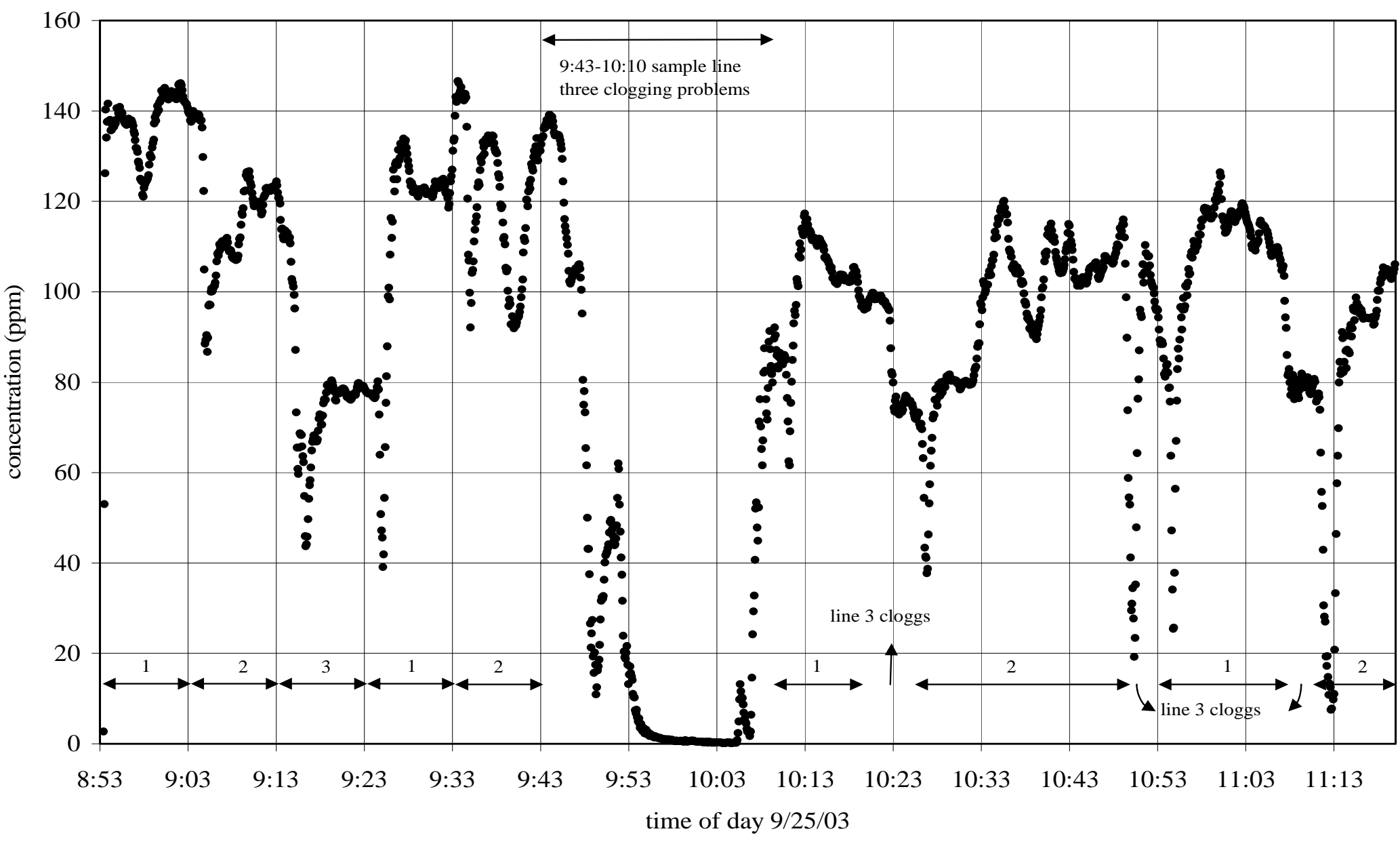

Figure C31. NOx concentrations recorded downstream of the air preheater in Boiler 3 firing bagasse on September 25, 2003, first sample period. 


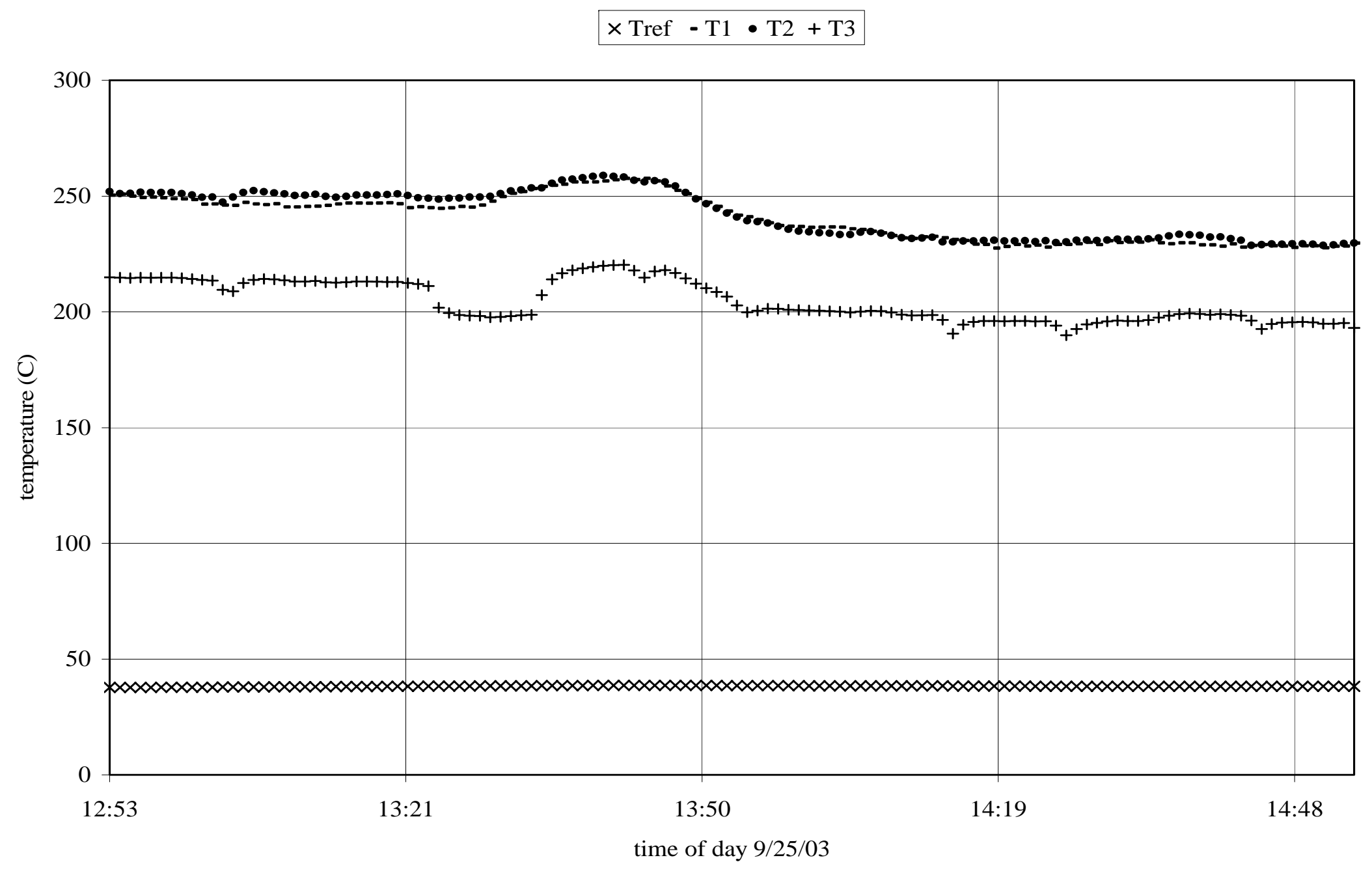

Figure C32. Temperatures recorded downstream of the air preheater in Boiler 3 firing bagasse on September 25, 2003, second sample period. Temperatures T1 though T3 are from Type K thermocouples located in the flue gas. Tref is an indicator of ambient temperature. 
- $\mathrm{CO} 2+\mathrm{O} 2$

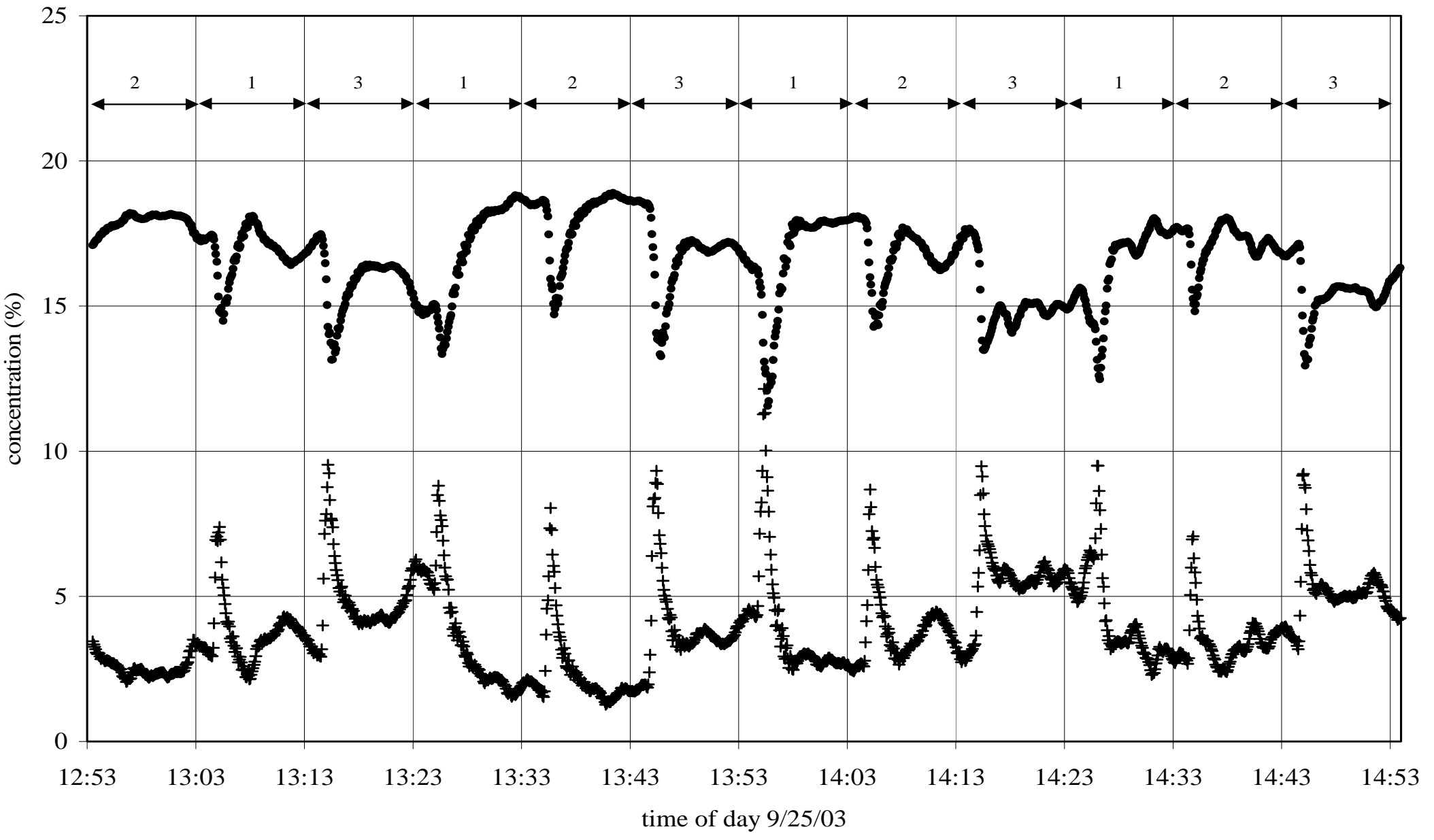

Figure C33. $\mathrm{O}_{2}$ and $\mathrm{CO}_{2}$ concentrations recorded downstream of the air preheater in Boiler 3 firing bagasse on September 25, 2003, second sample period. 


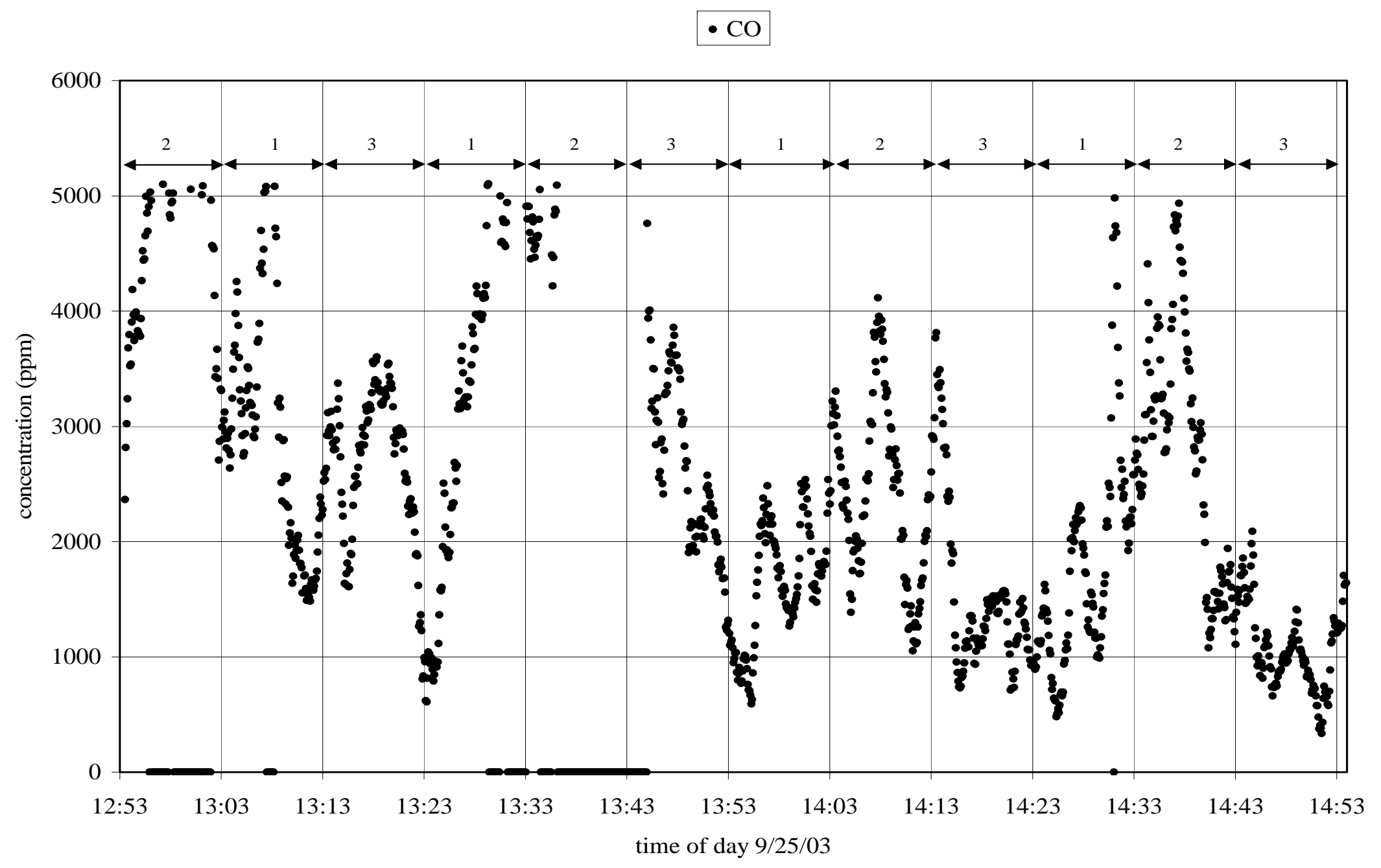

Figure C34. CO concentrations recorded downstream of the air preheater in Boiler 3 firing bagasse on September 25, 2003, second sample period. 


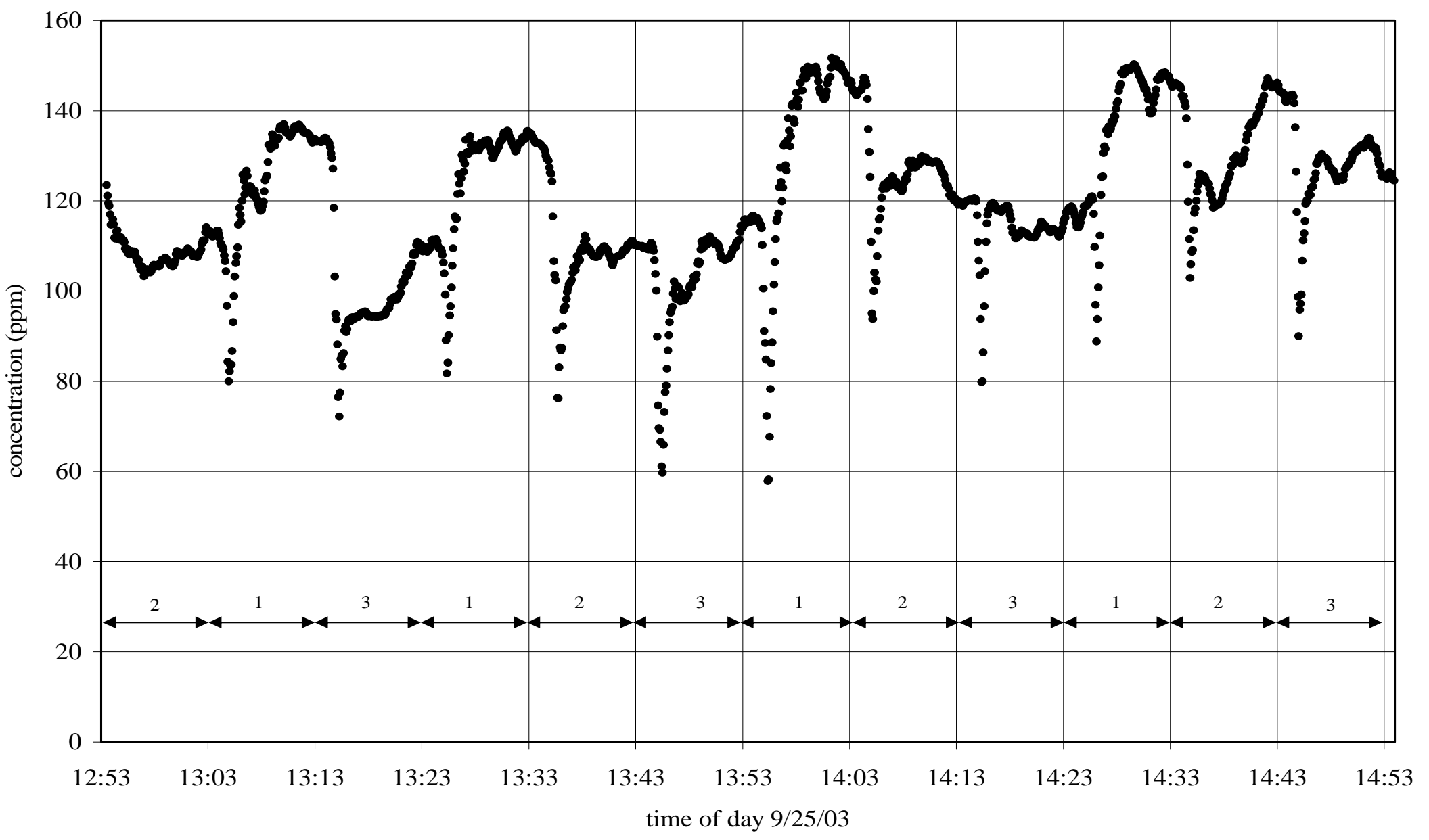

Figure C35. NOx concentrations recorded downstream of the air preheater in Boiler 3 firing bagasse on September 25, 2003, second sample period. 


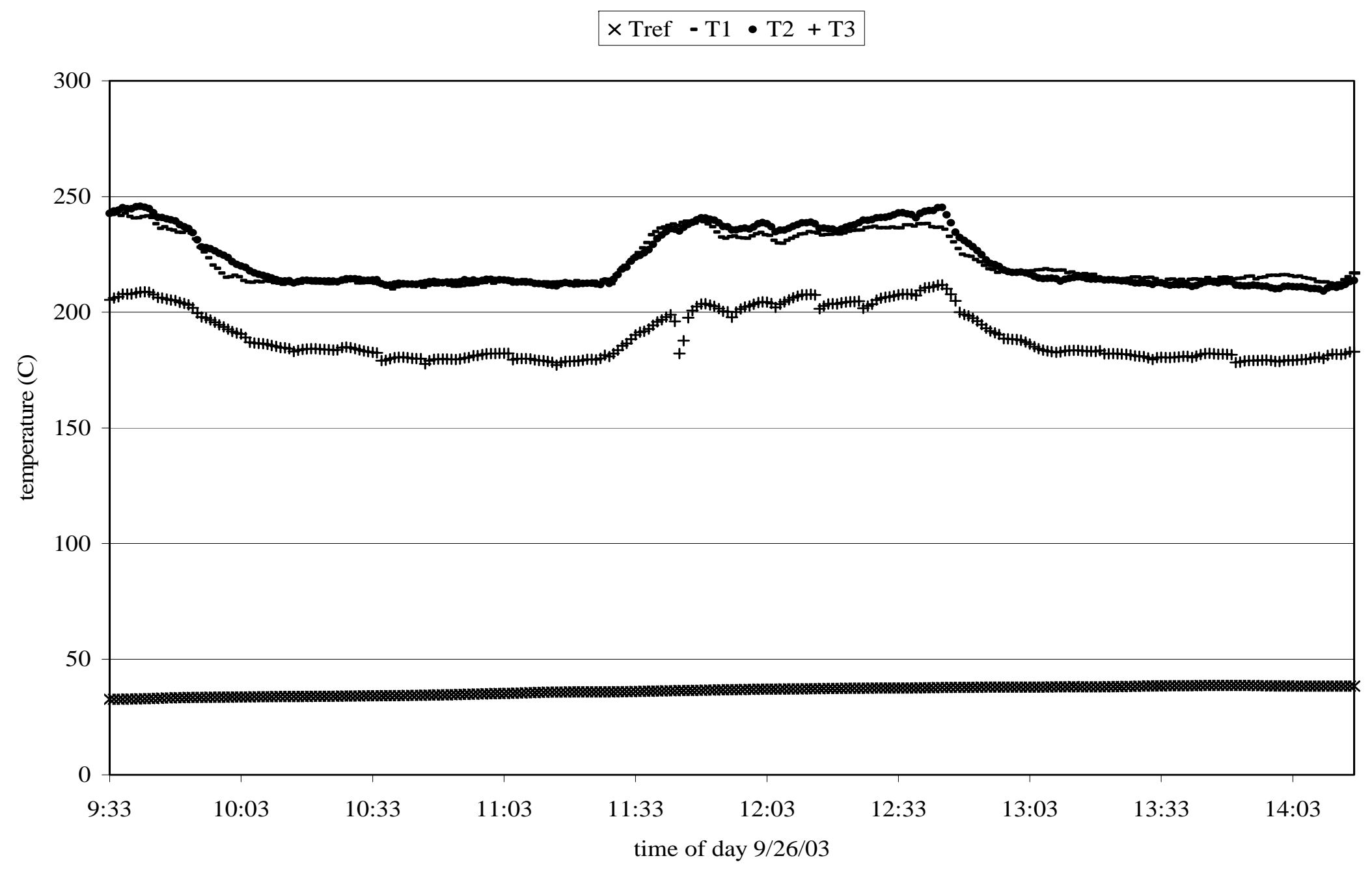

Figure C36. Temperatures recorded downstream of the air preheater in Boiler 3 firing coal on September 26, 2003. Temperatures T1 though T3 are from Type K thermocouples located in the flue gas. Tref is an indicator of ambient temperature. 
$\times$ Tref $-\mathrm{T} 1 \cdot \mathrm{T} 2+\mathrm{T} 3$

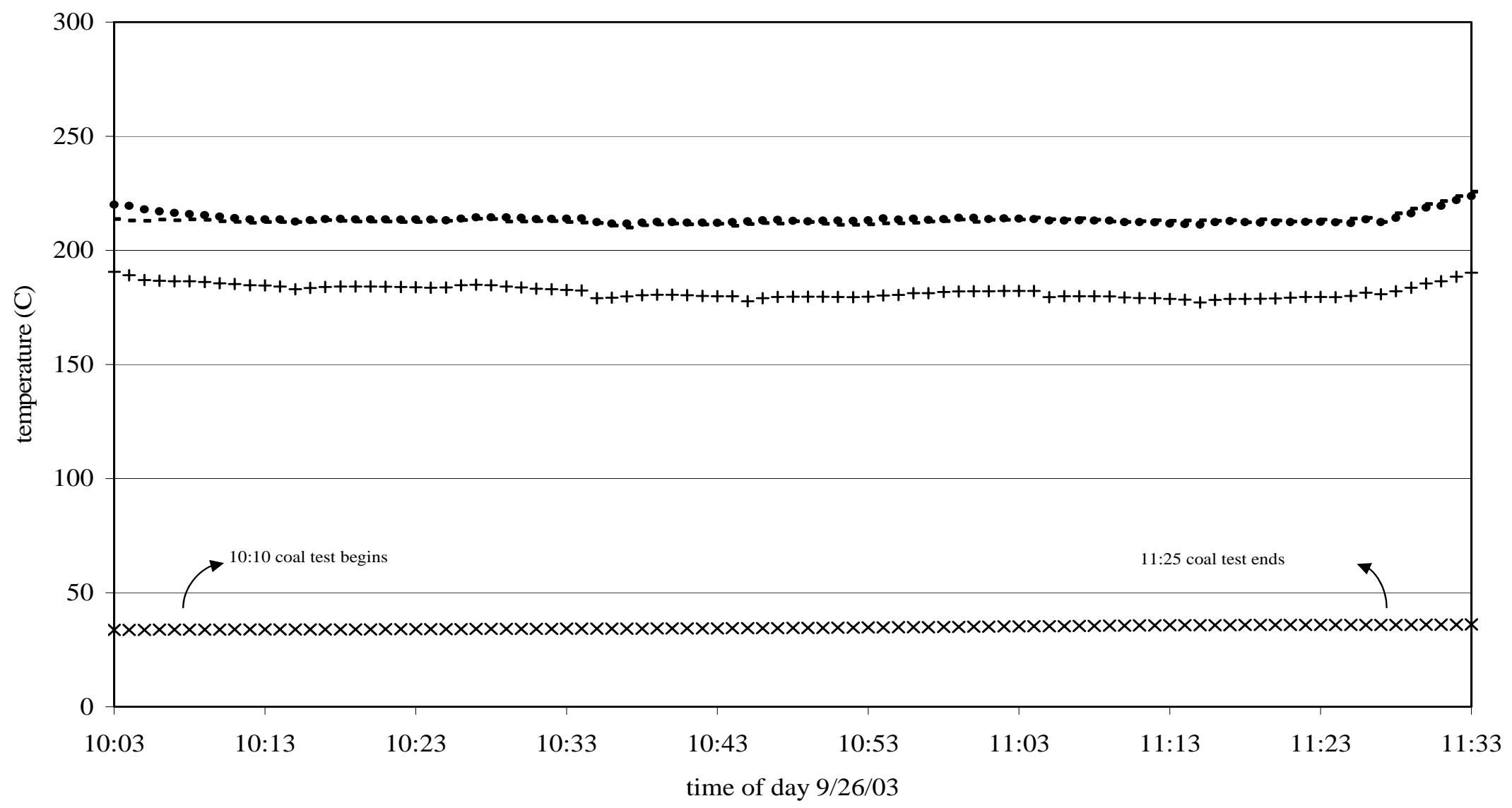

Figure C37. Temperatures recorded downstream of the air preheater in Boiler 3 firing coal on September 26, 2003, first sample period. Temperatures T1 though T3 are from Type K thermocouples located in the flue gas. Tref is an indicator of ambient temperature. 
- $\mathrm{CO} 2+\mathrm{O} 2$

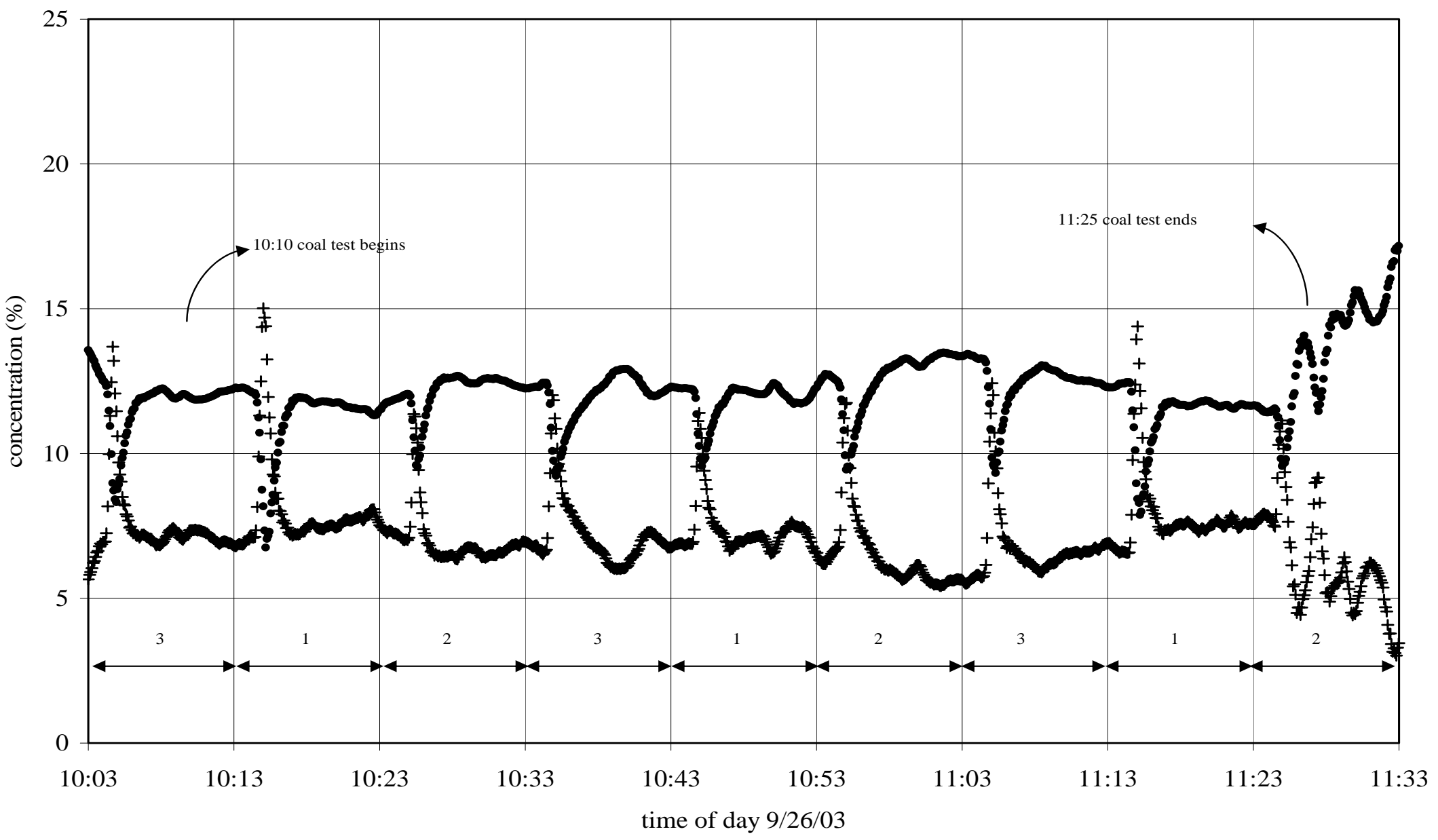

Figure C39. $\mathrm{O}_{2}$ and $\mathrm{CO}_{2}$ concentrations recorded downstream of the air preheater in Boiler 3 firing coal on September 26, 2003, first sample period. 


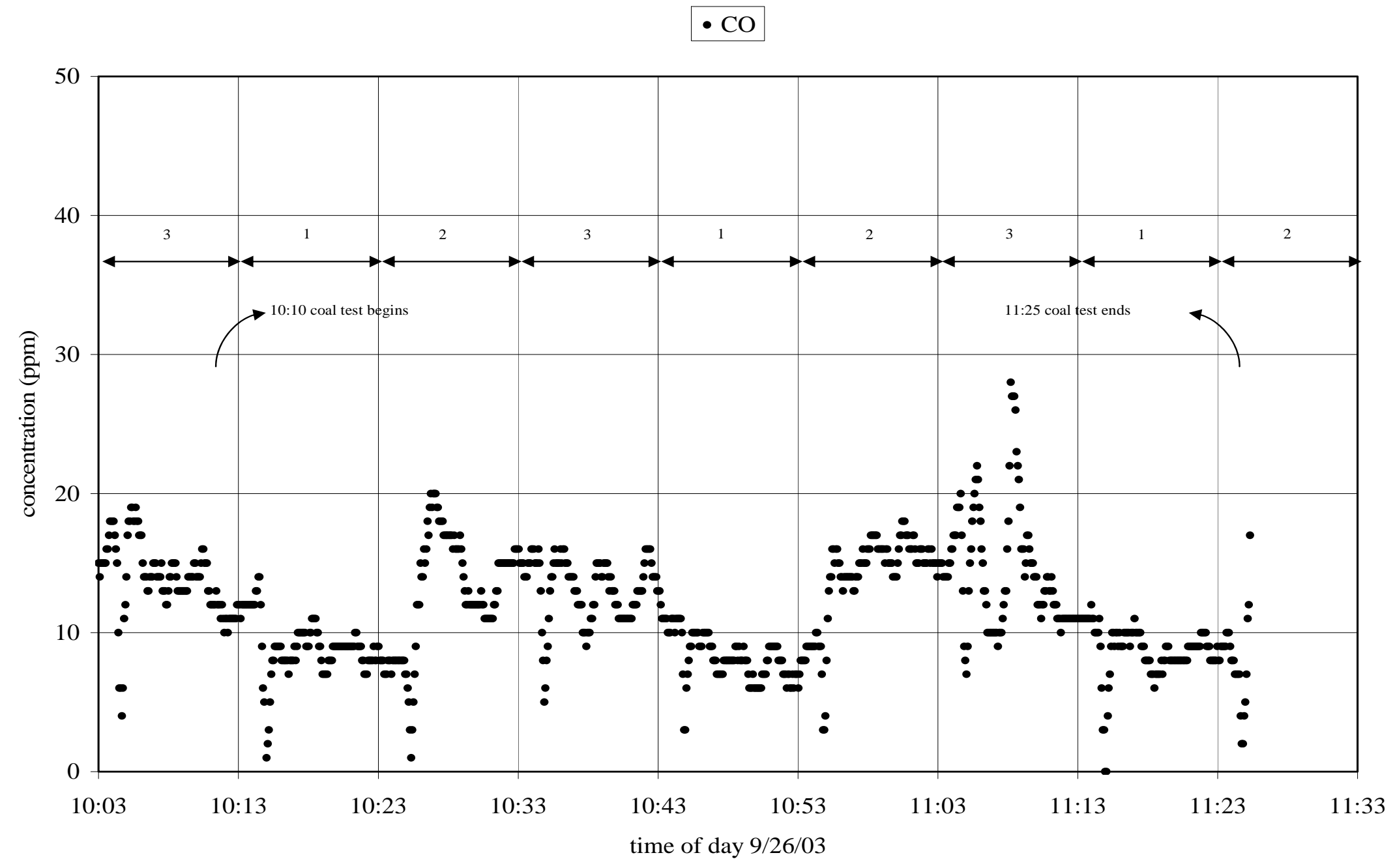

Figure C40. CO concentrations recorded downstream of the air preheater in Boiler 3 firing coal on September 26, 2003, first sample period. 


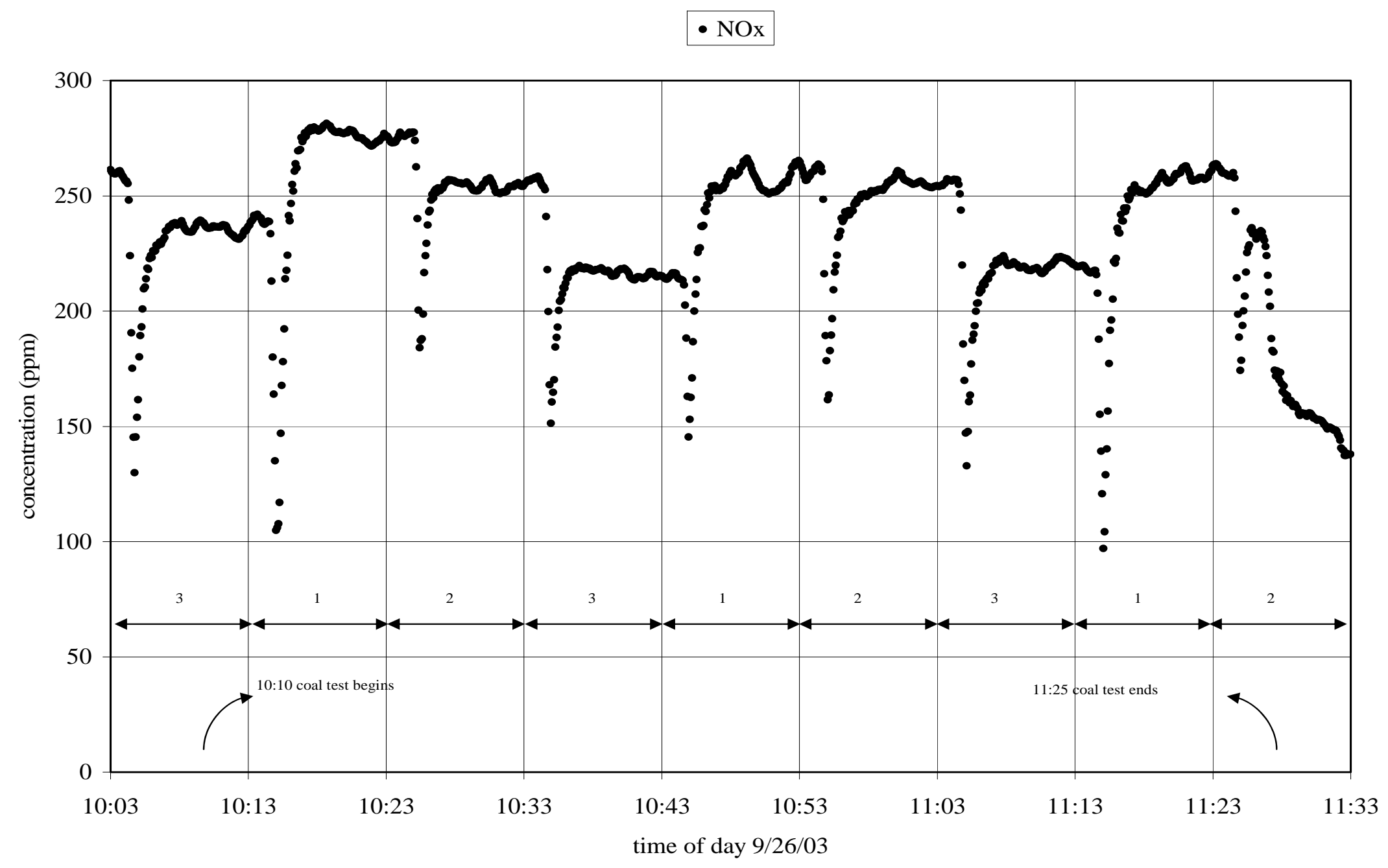

Figure C41. NOx concentrations recorded downstream of the air preheater in Boiler 3 firing coal on September 26, 2003, first sample period. 


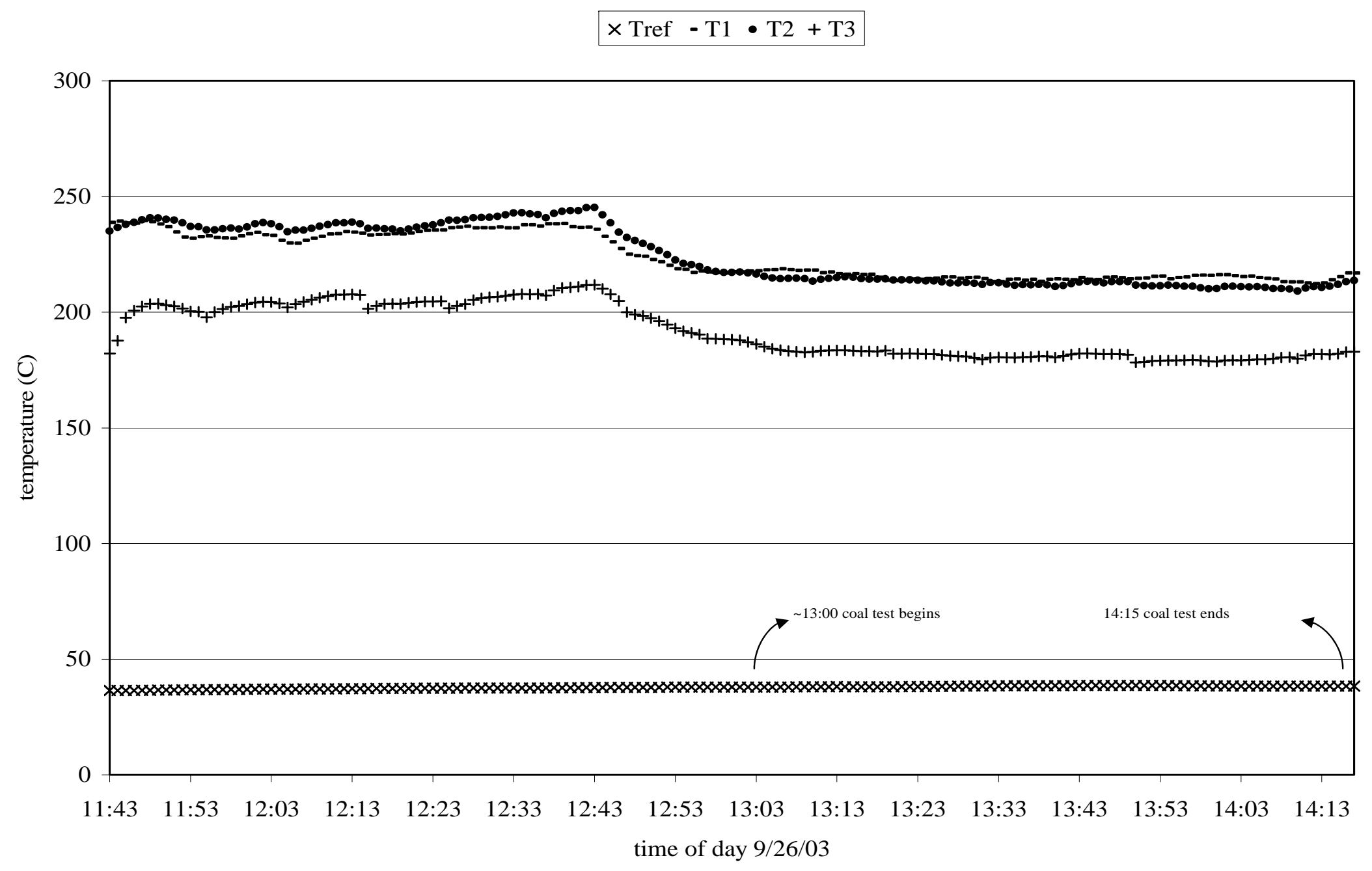

Figure C41. Temperatures recorded downstream of the air preheater in Boiler 3 firing coal on September 26, 2003, second sample period. Temperatures T1 though T3 are from Type K thermocouples located in the flue gas. Tref is an indicator of ambient temperature. 


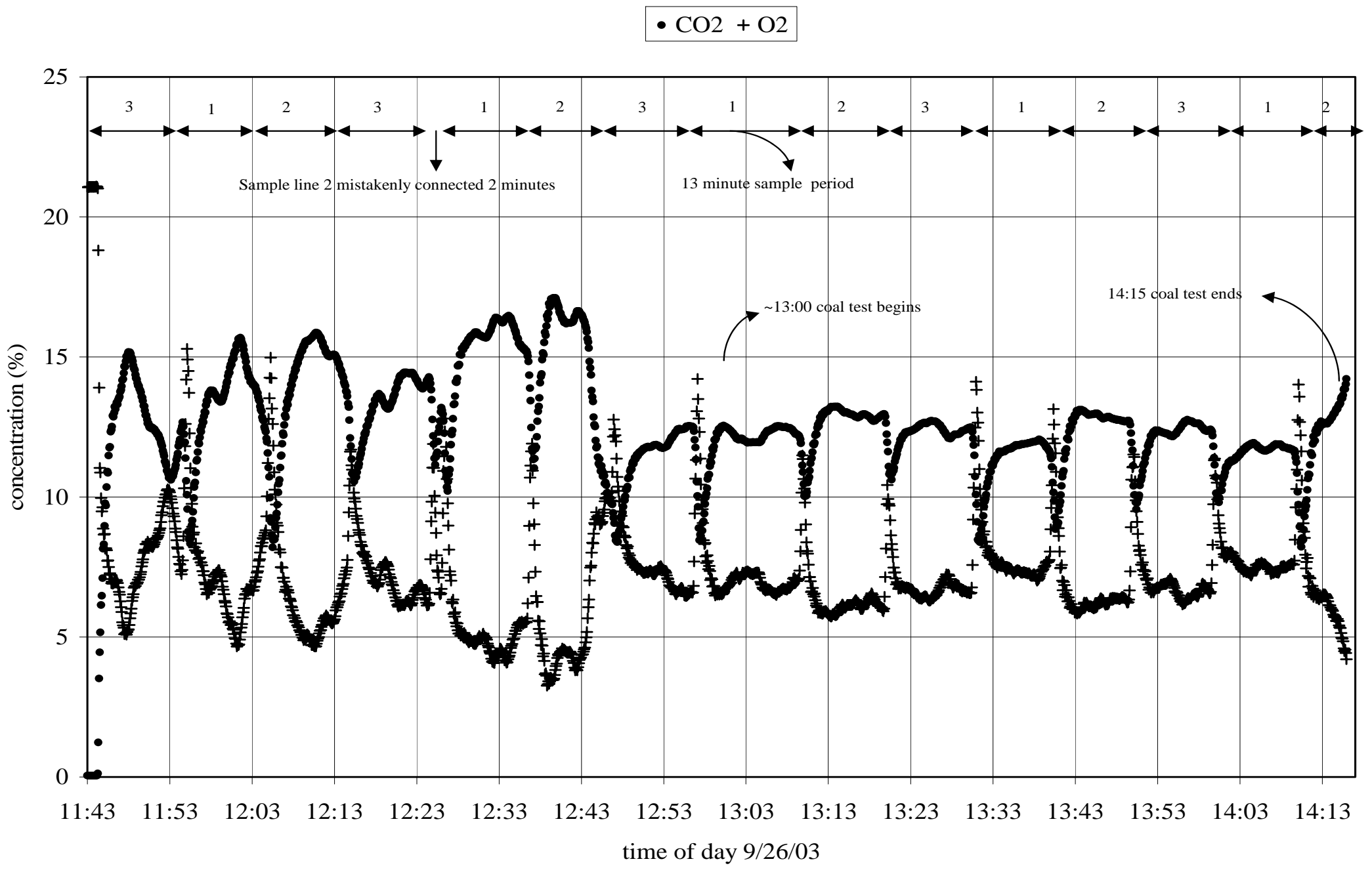

Figure C42. $\mathrm{O}_{2}$ and $\mathrm{CO}_{2}$ concentrations recorded downstream of the air preheater in Boiler 3 firing coal on September 26, 2003, second sample period. 


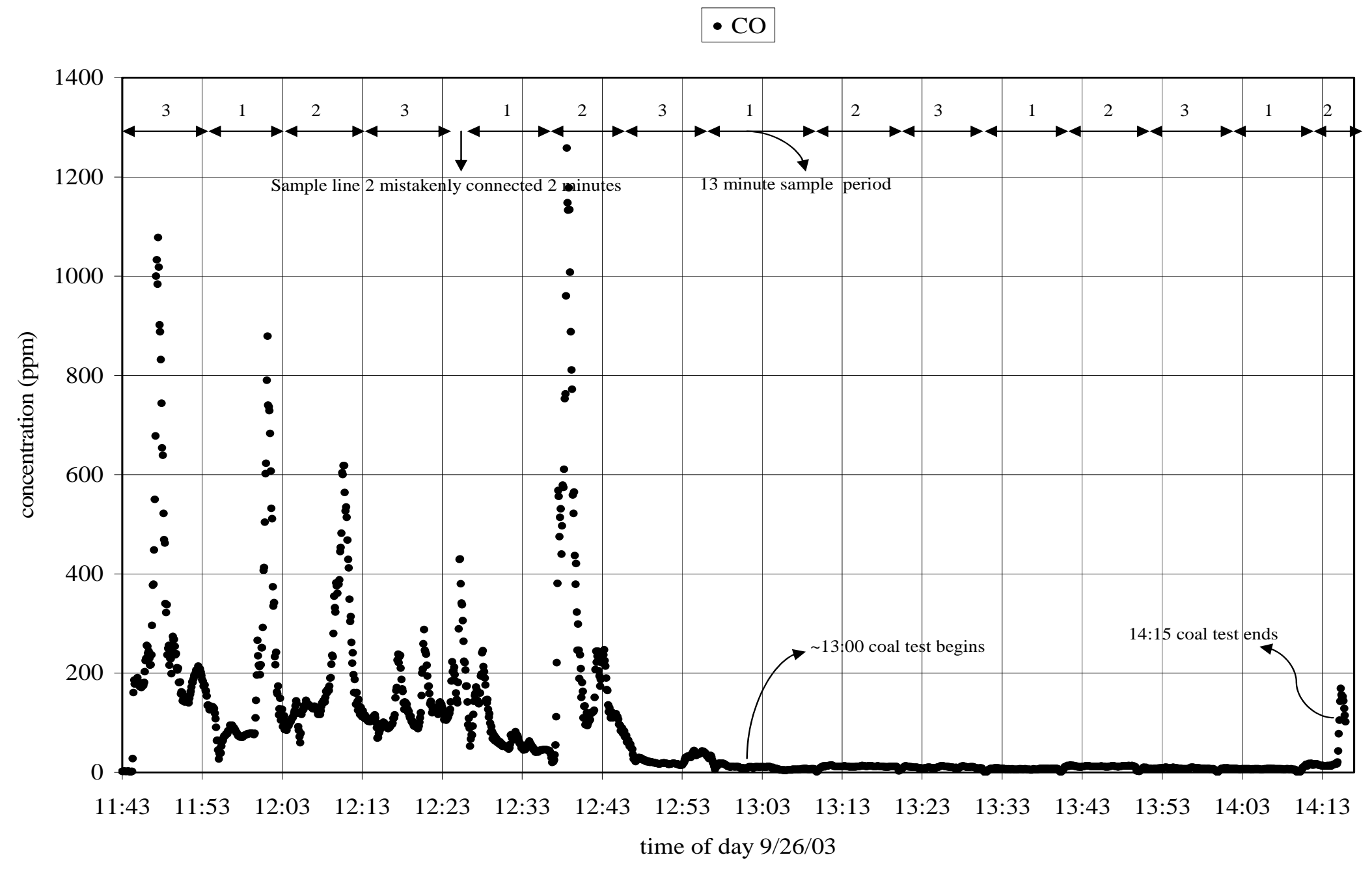

Figure C43. CO concentrations recorded downstream of the air preheater in Boiler 3 firing coal on September 26, 2003, second sample period. 


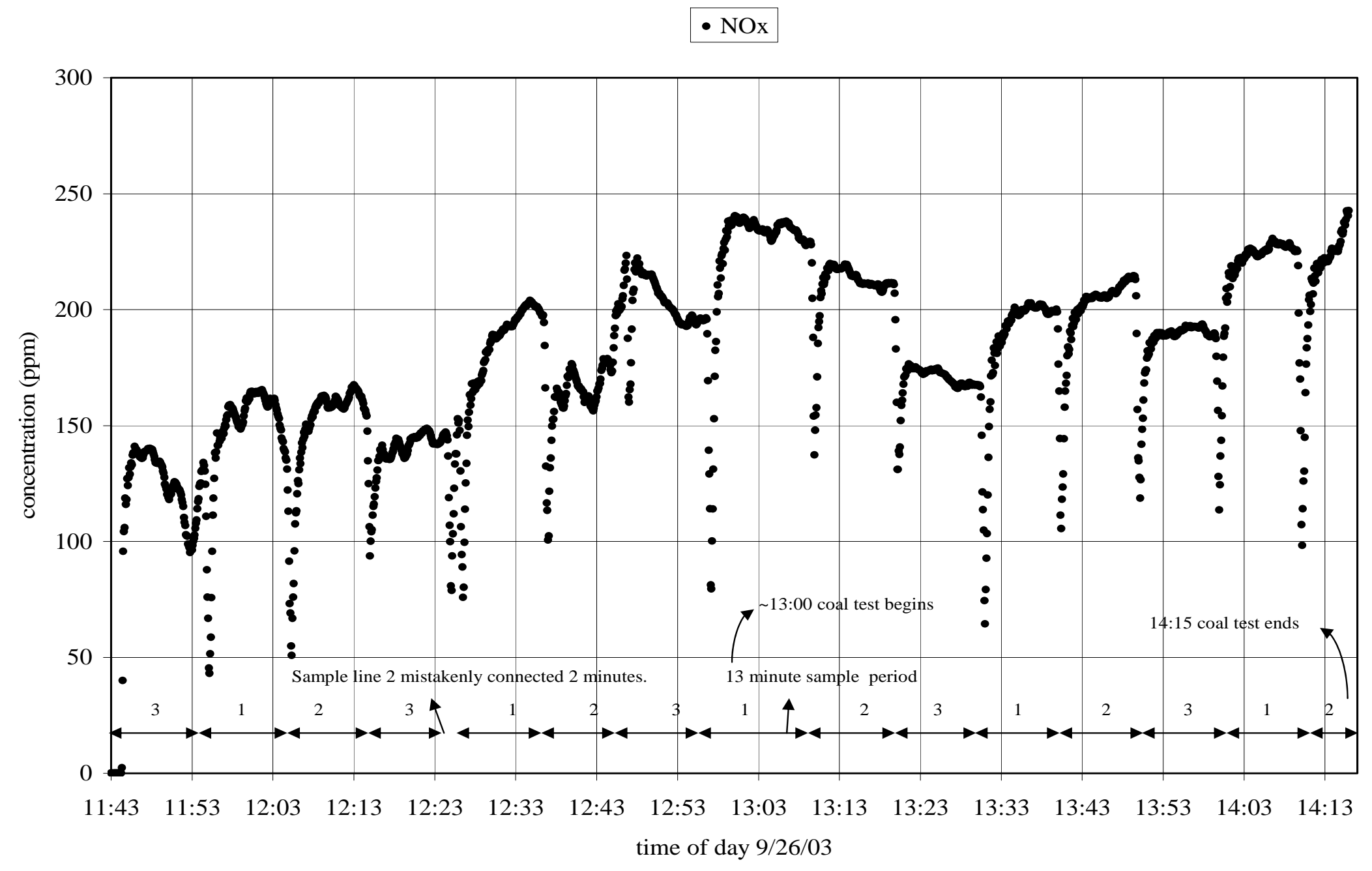

Figure C44. NOx concentrations recorded downstream of the air preheater in Boiler 3 firing coal on September 26, 2003, second sample period. 
$\times$ Tref $\bullet \mathrm{T} 1-\mathrm{T} 2+\mathrm{T} 3$

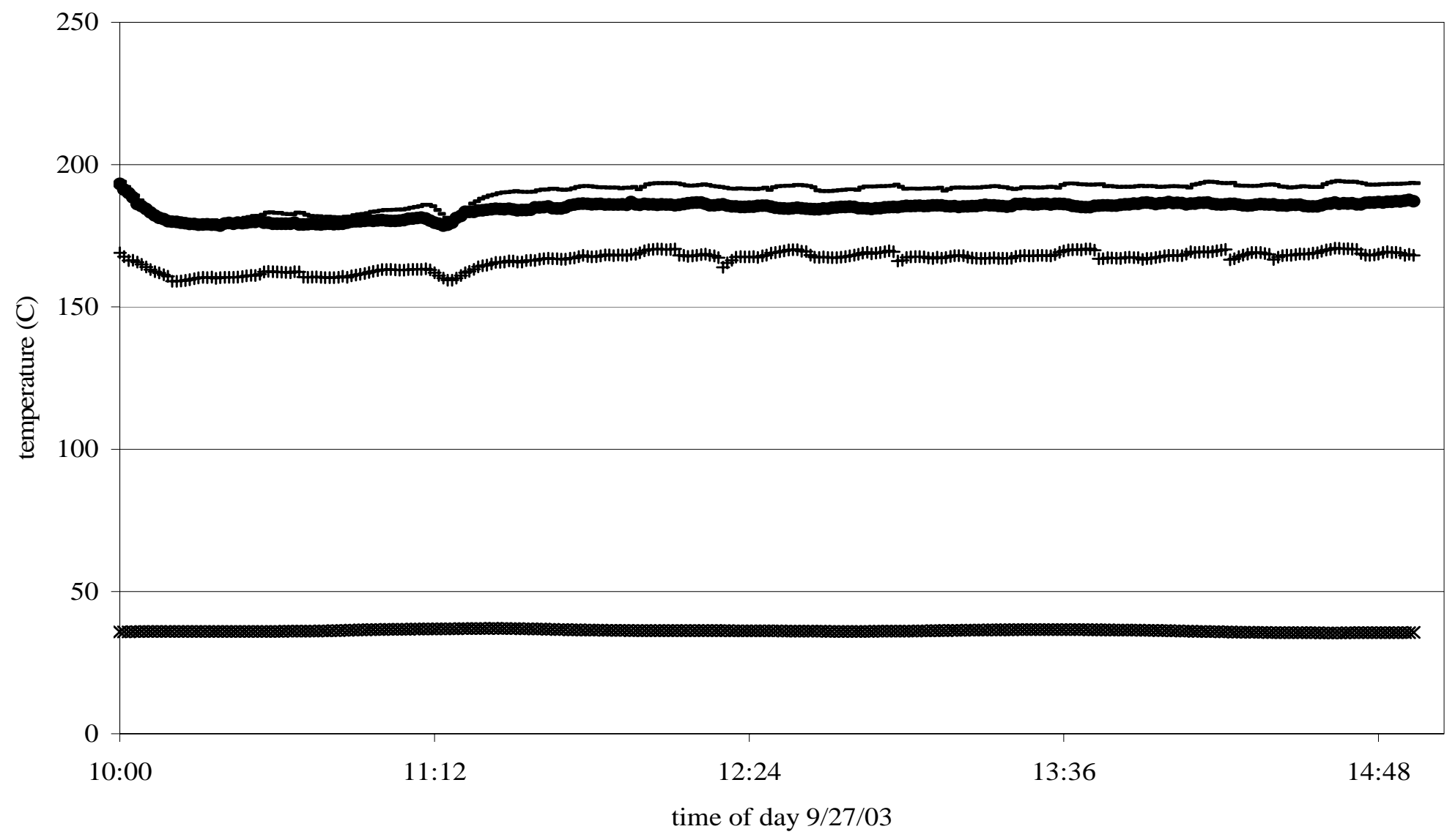

Figure C45. Temperatures recorded downstream of the air preheater in Boiler 3 firing oil on September 27, 2003. Temperatures T1 though T3 are from Type K thermocouples located in the flue gas. Tref is an indicator of ambient temperature. 
$\times$ Tref $-\mathrm{T} 1 \cdot \mathrm{T} 2+\mathrm{T} 3$

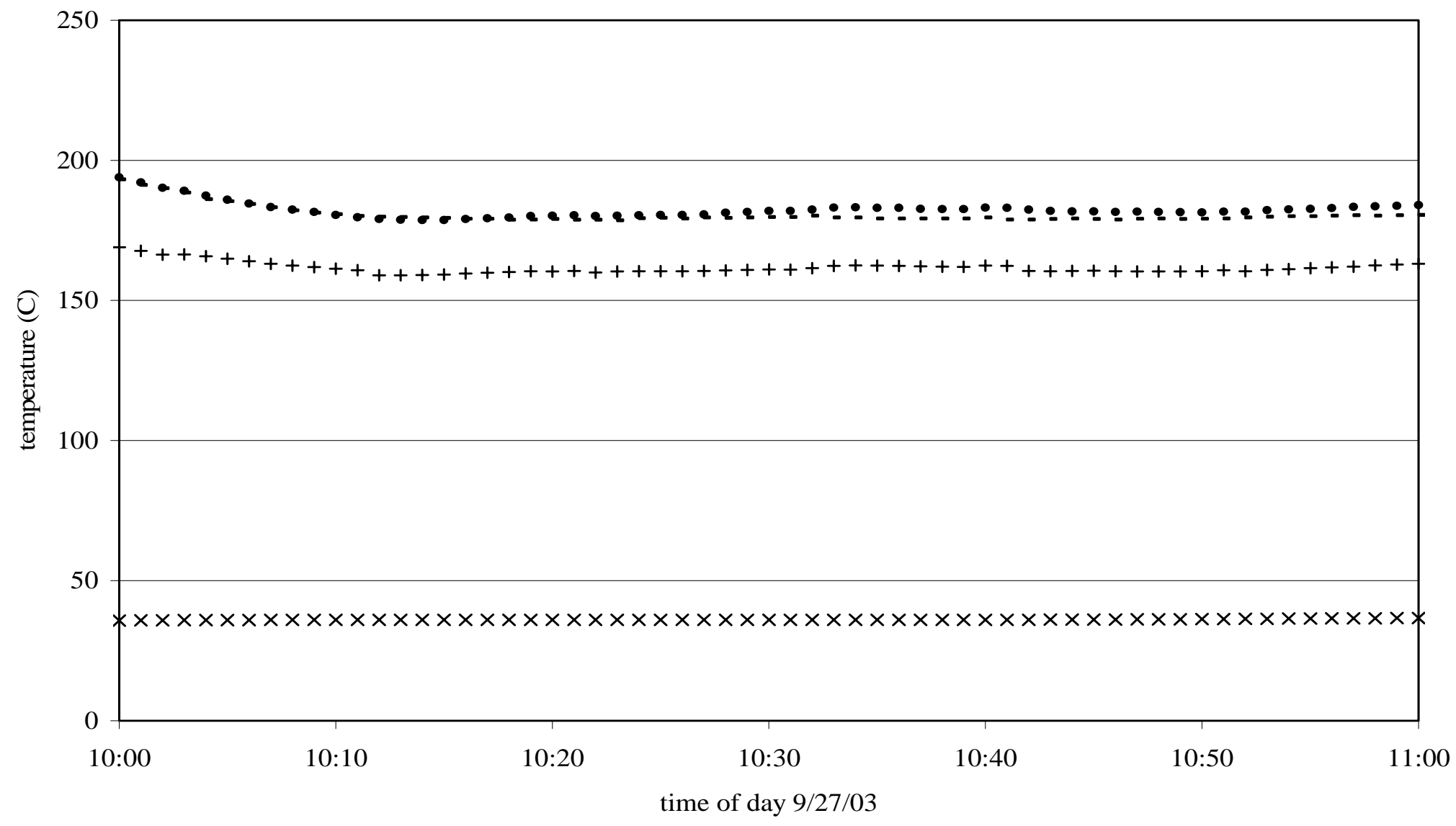

Figure C46. Temperatures recorded downstream of the air preheater in Boiler 3 firing oil on September 27, 2003, first sample period. Temperatures T1 though T3 are from Type K thermocouples located in the flue gas. Tref is an indicator of ambient temperature. 
- $\mathrm{CO} 2+\mathrm{O} 2$

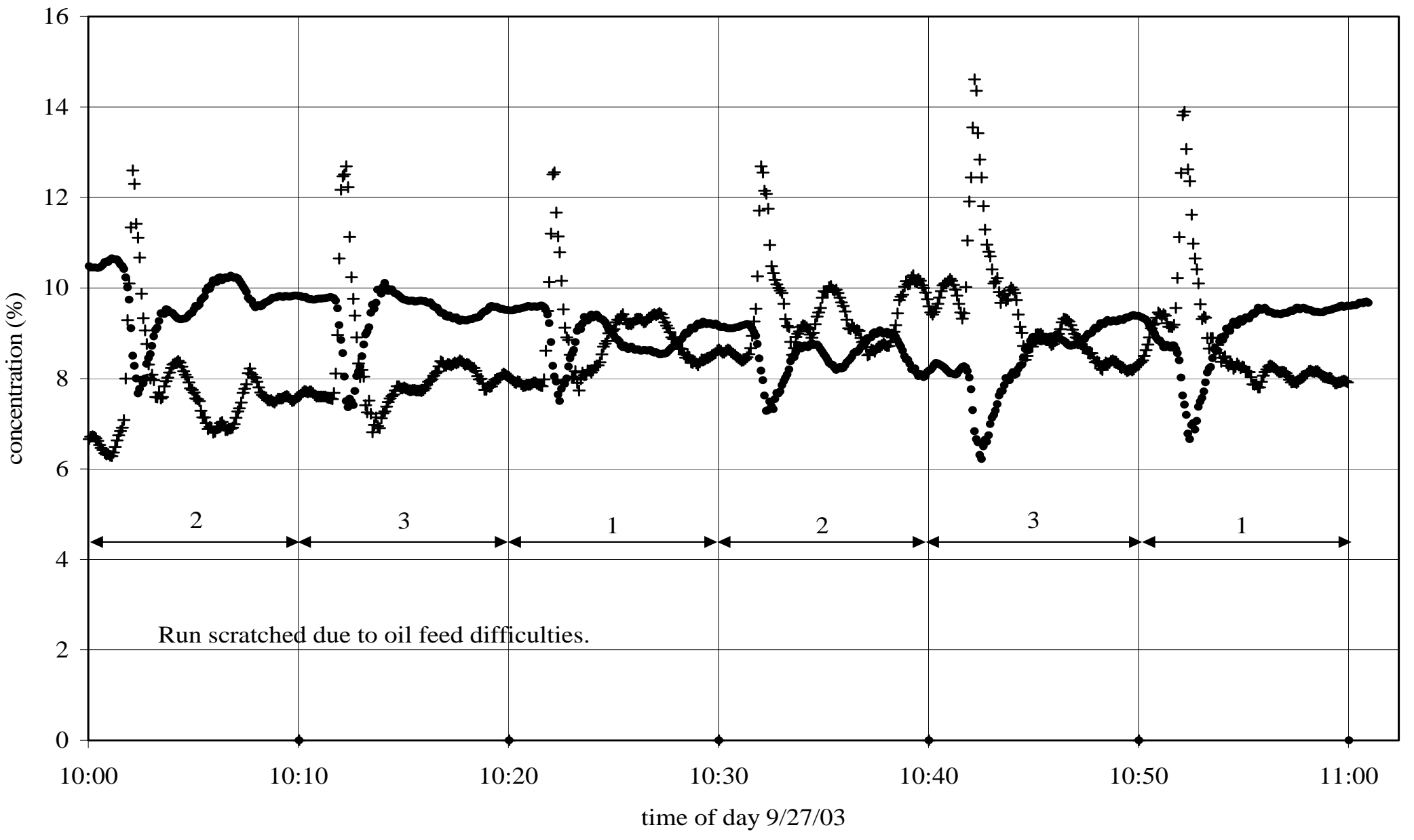

Figure C47. $\mathrm{O}_{2}$ and $\mathrm{CO}_{2}$ concentrations recorded downstream of the air preheater in Boiler 3 firing oil on September 27, 2003, first sample period. 


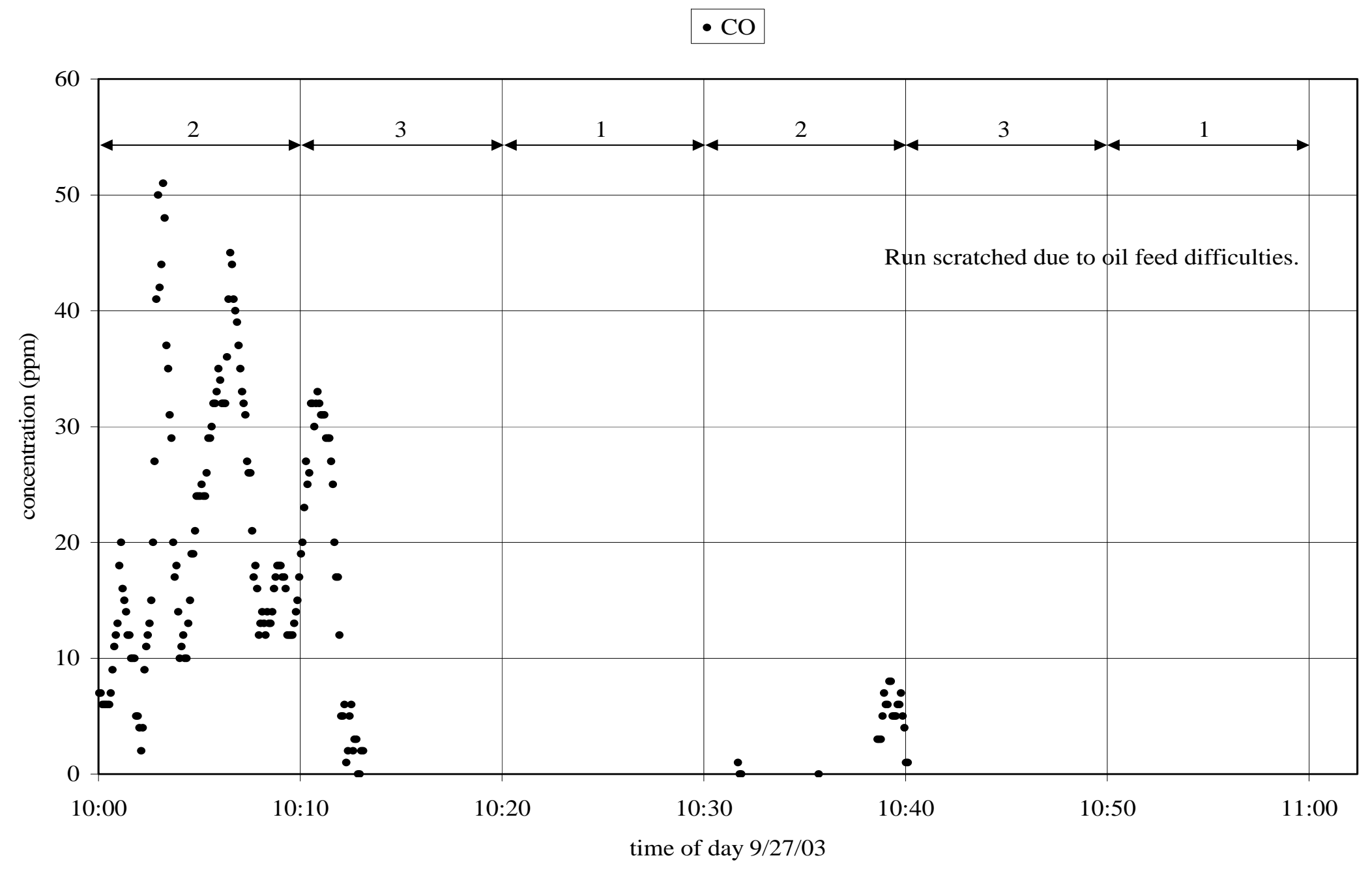

Figure C48. CO concentrations recorded downstream of the air preheater in Boiler 3 firing oil on September 27, 2003, first sample period. 


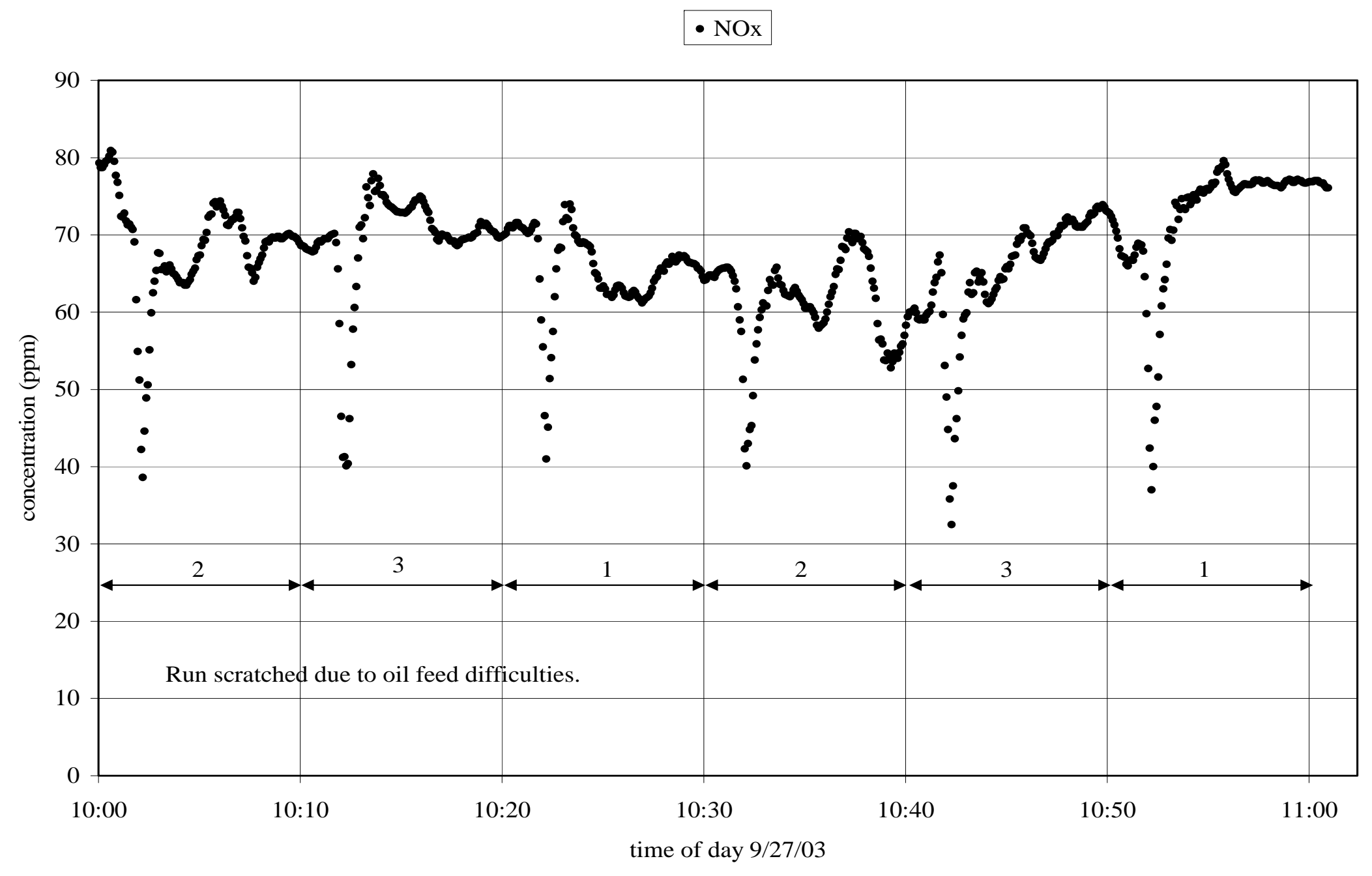

Figure C49. NOx concentrations recorded downstream of the air preheater in Boiler 3 firing oil on September 27, 2003, first sample period. 


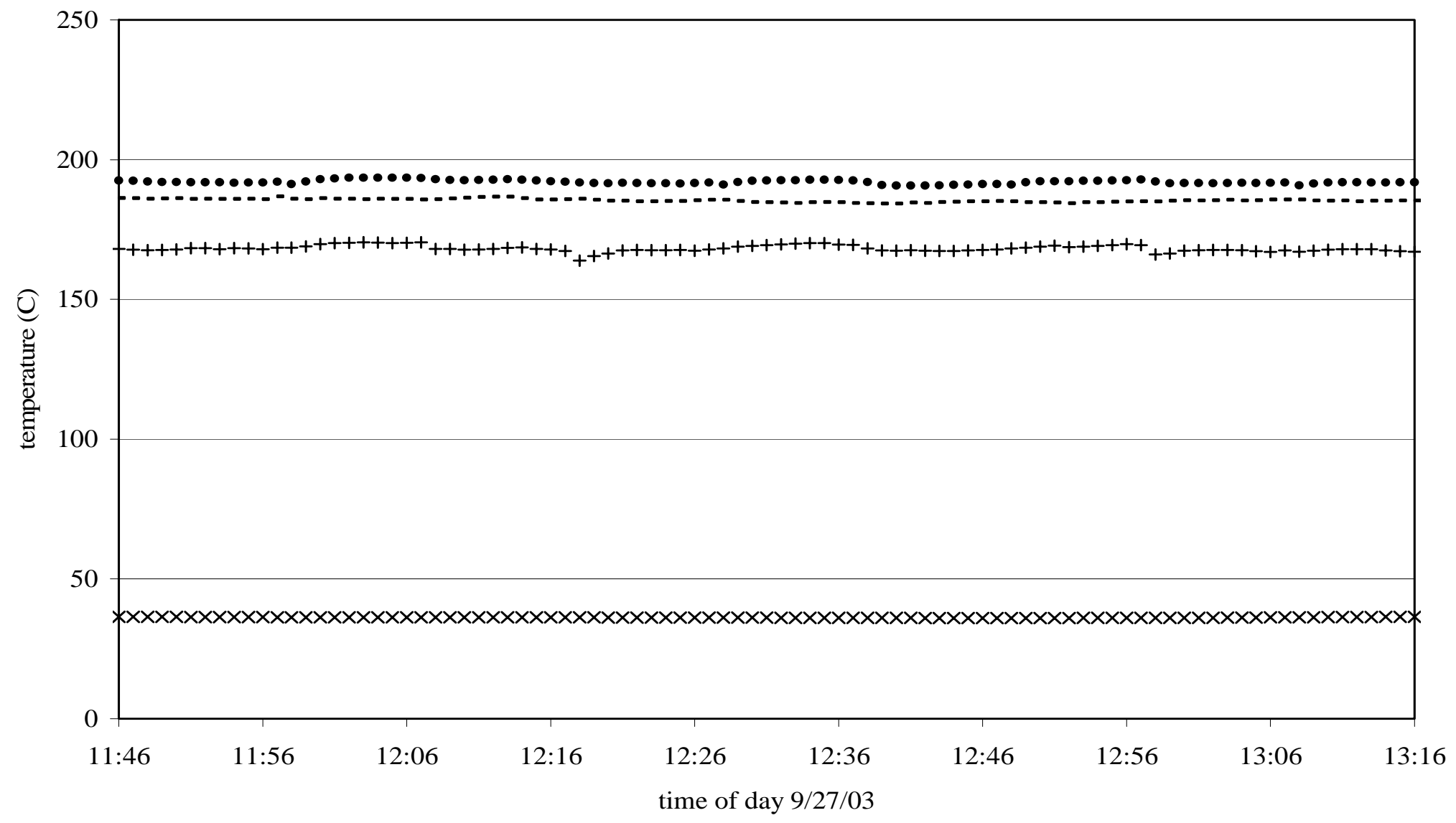

Figure C50. Temperatures recorded downstream of the air preheater in Boiler 3 firing oil on September 27, 2003, second sample period. Temperatures T1 though T3 are from Type K thermocouples located in the flue gas. Tref is an indicator of ambient temperature. 


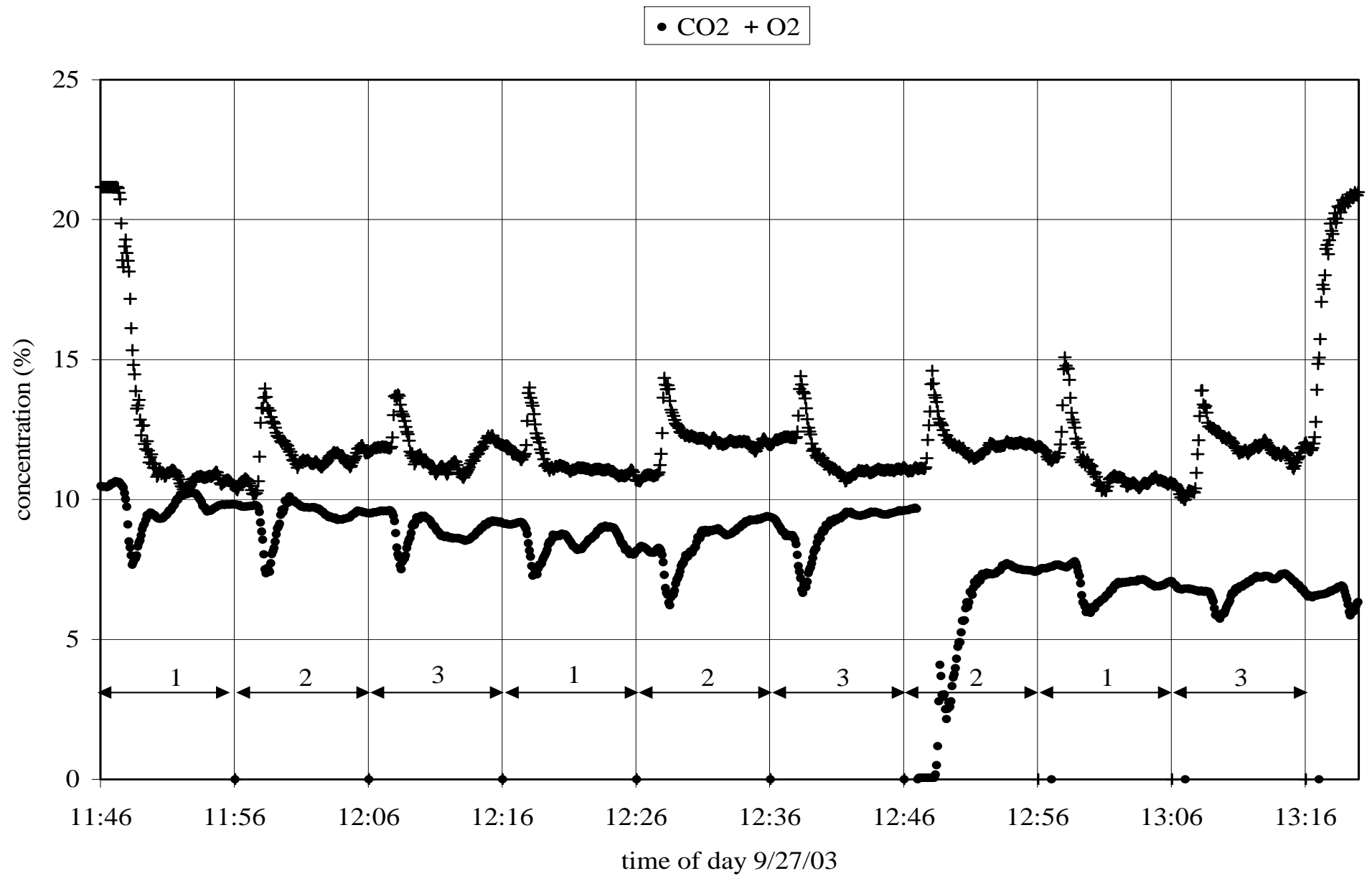

Figure C51. $\mathrm{O}_{2}$ and $\mathrm{CO}_{2}$ concentrations recorded downstream of the air preheater in Boiler 3 firing oil on September 27, 2003, second sample period. 


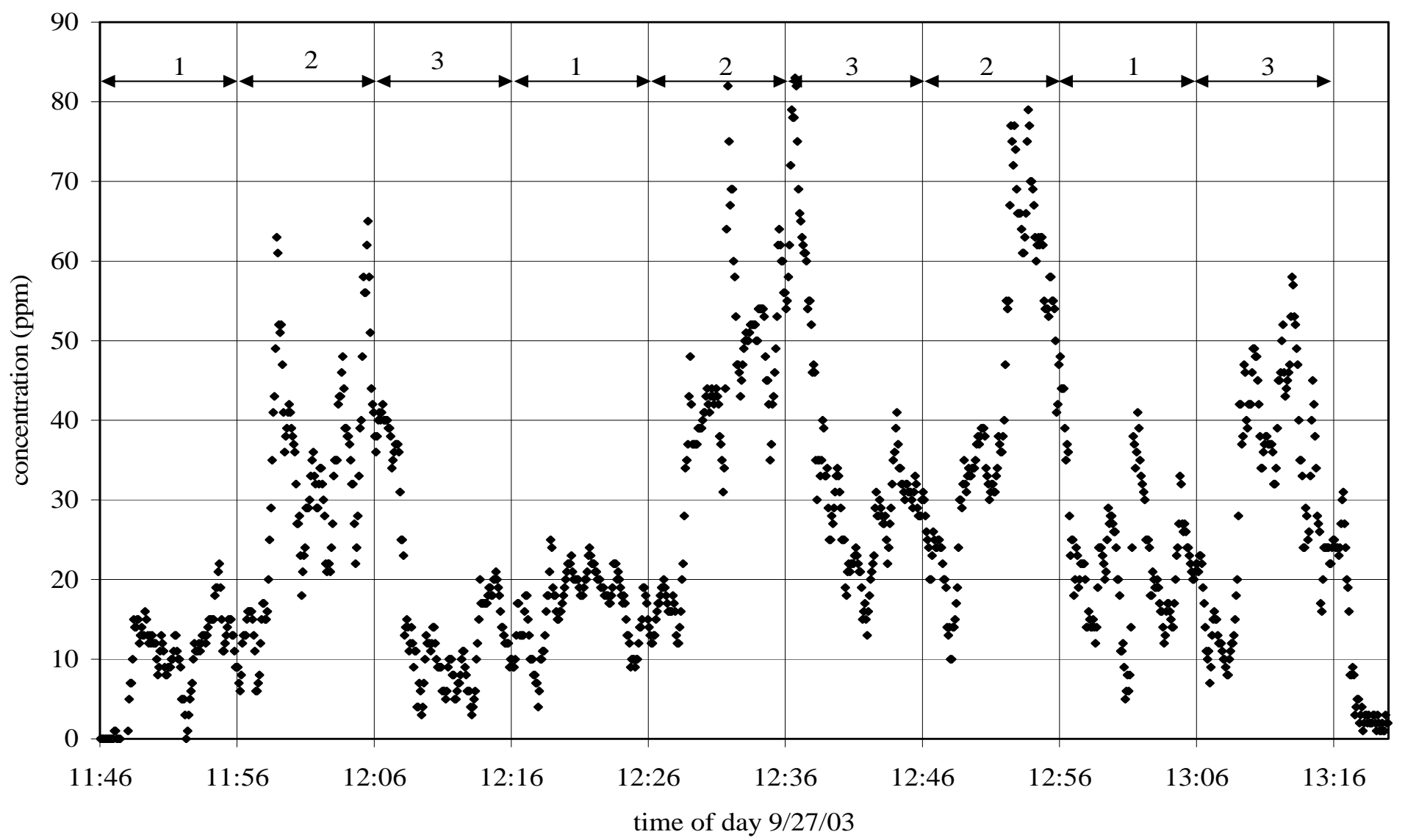

Figure C52. CO concentrations recorded downstream of the air preheater in Boiler 3 firing oil on September 27, 2003, second sample period. 


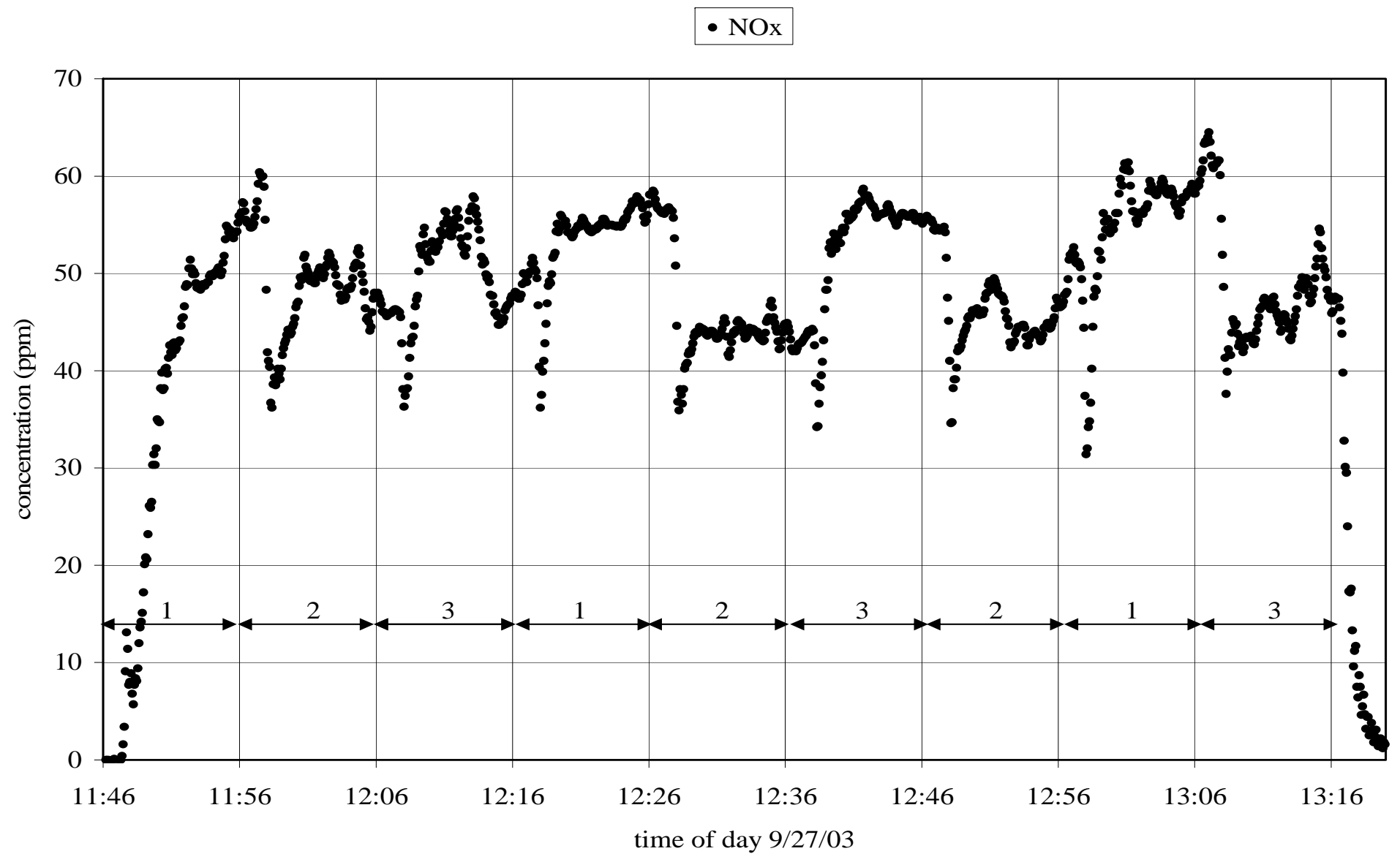

Figure C53. NOx concentrations recorded downstream of the air preheater in Boiler 3 firing oil on September 27, 2003, second sample period. 
$\times$ Tref $-\mathrm{T} 1 \cdot \mathrm{T} 2+\mathrm{T} 3$

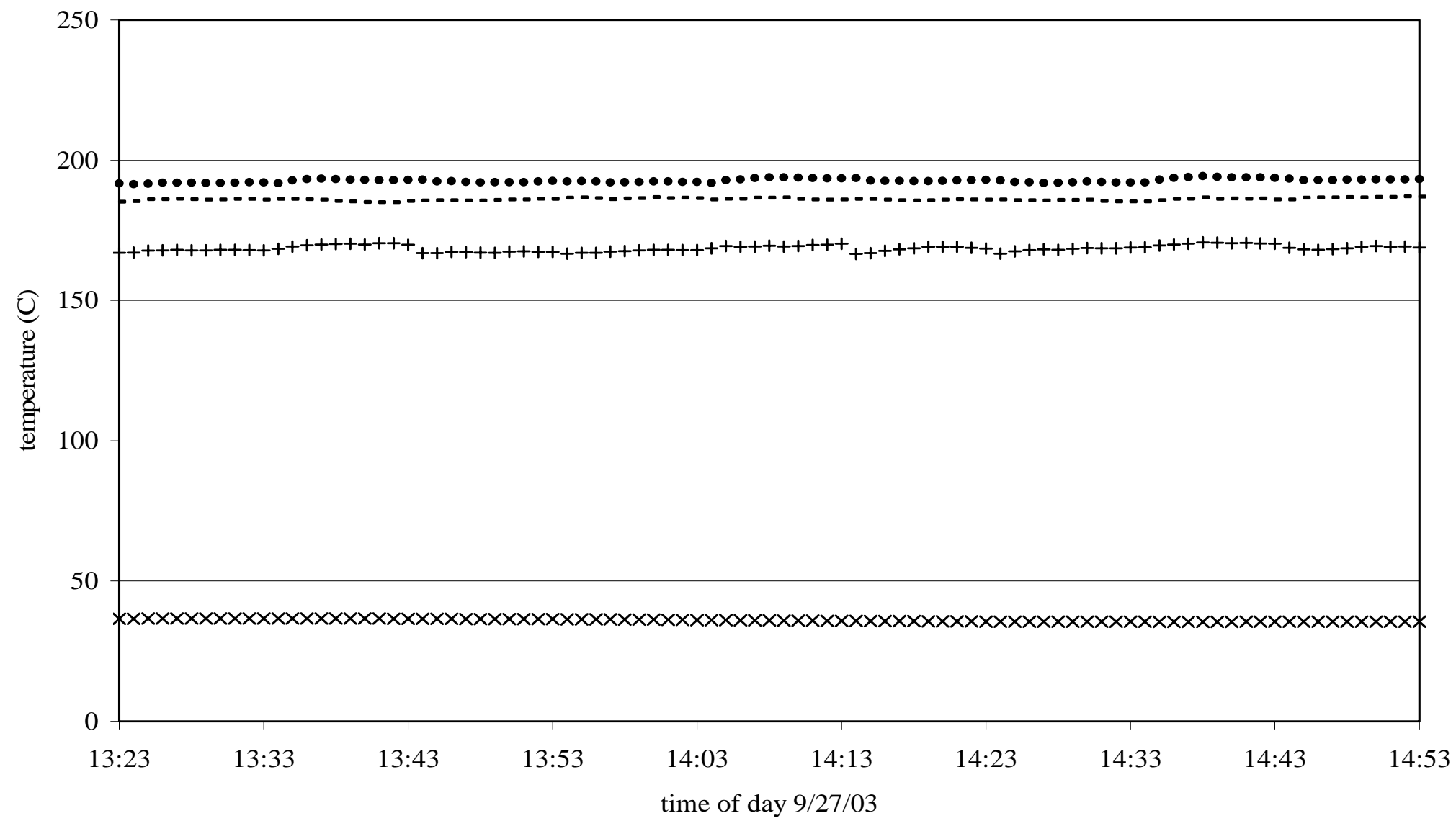

Figure C54. Temperatures recorded downstream of the air preheater in Boiler 3 firing oil on September 27, 2003, third sample period. Temperatures T1 though T3 are from Type K thermocouples located in the flue gas. Tref is an indicator of ambient temperature. 
- $\mathrm{CO} 2+\mathrm{O} 2$

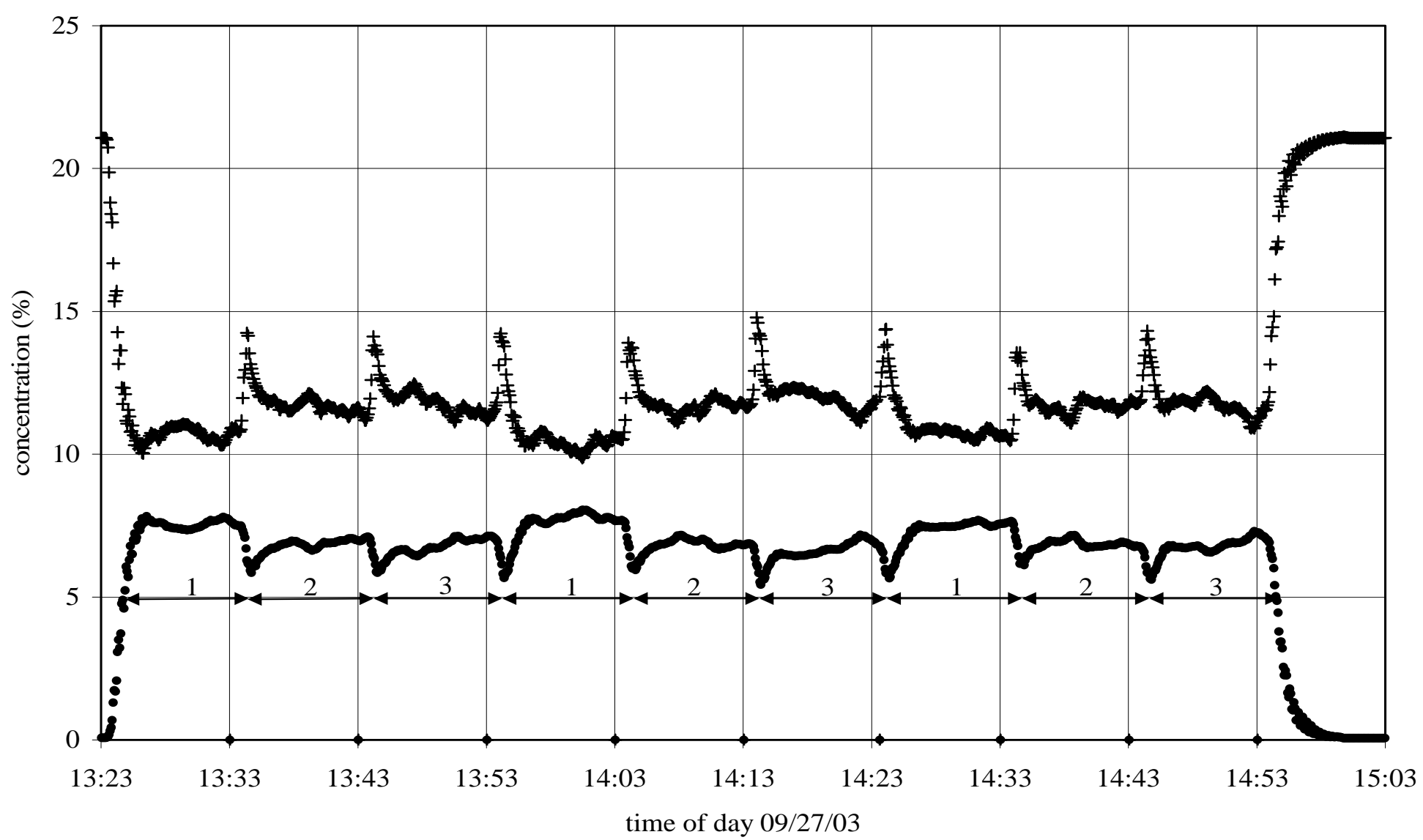

Figure C55. $\mathrm{O}_{2}$ and $\mathrm{CO}_{2}$ concentrations recorded downstream of the air preheater in Boiler 3 firing oil on September 27, 2003, third sample period. 
- $\mathrm{CO}$

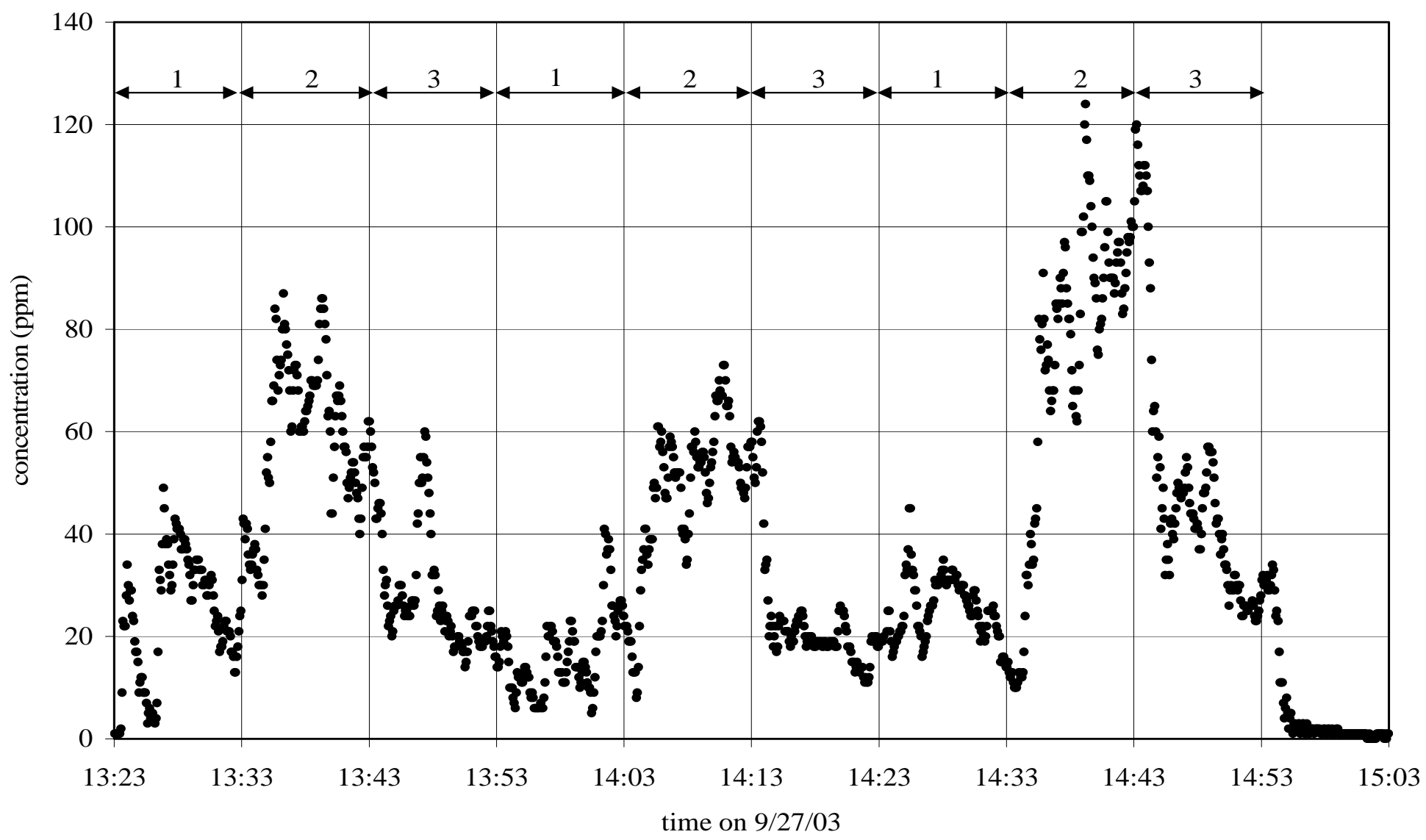

Figure C56. CO concentrations recorded downstream of the air preheater in Boiler 3 firing oil on September 27, 2003, third sample period. 


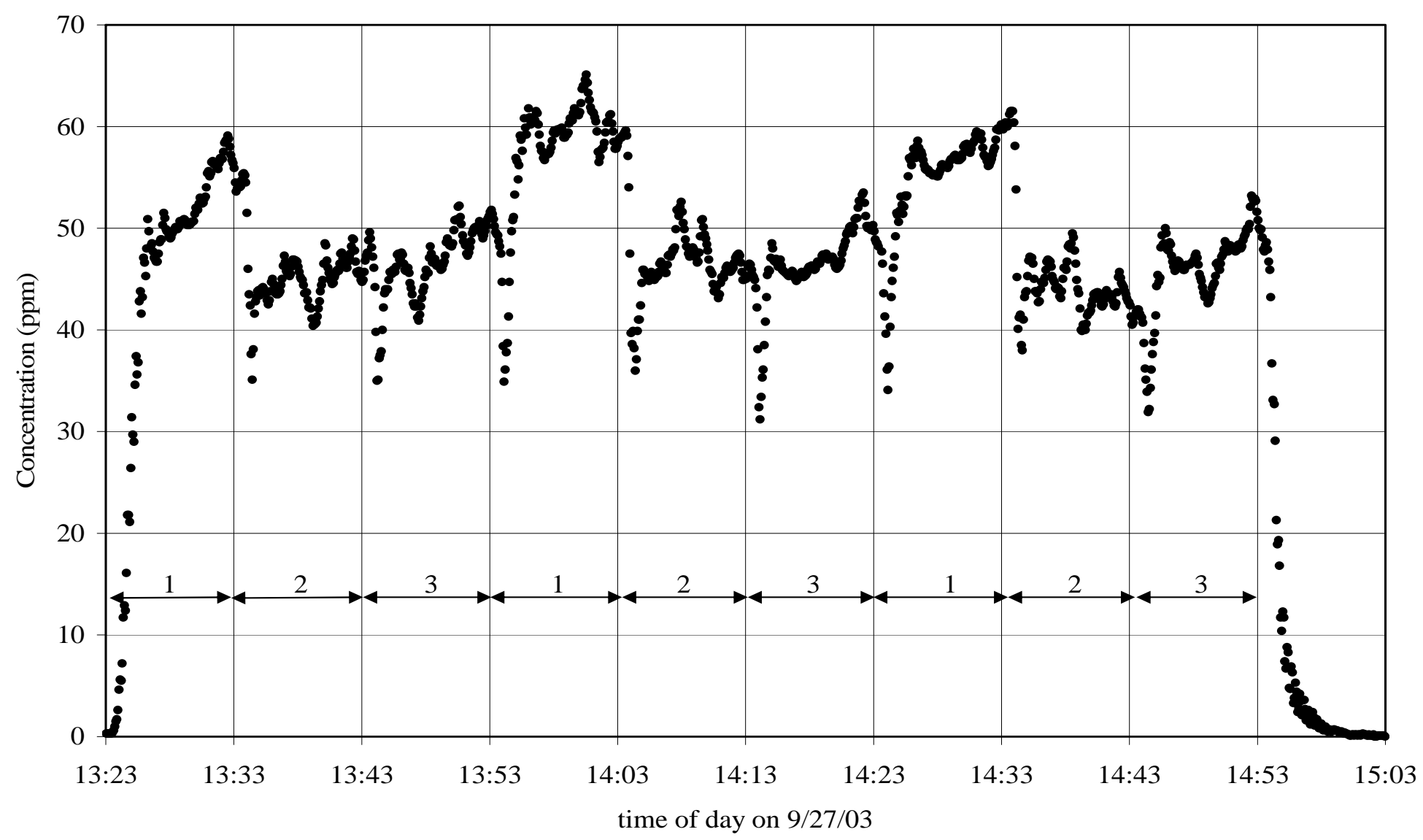

Figure C57. NOx concentrations recorded downstream of the air preheater in Boiler 3 firing oil on September 27, 2003, third sample period. 


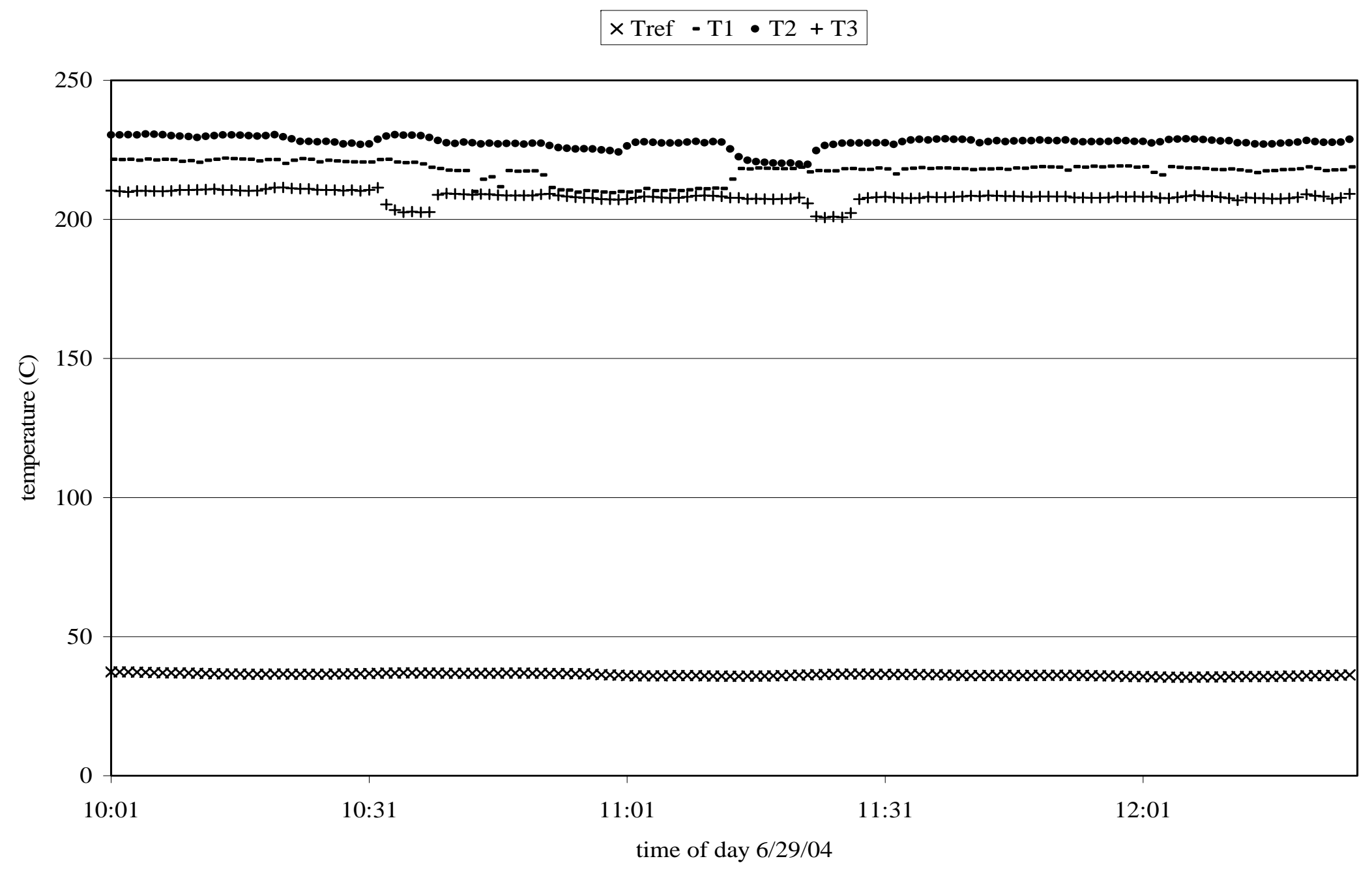

Figure C58. Temperatures recorded downstream of the air preheater in Boiler 2 firing coal on June 29, 2004. Temperatures T1 though T3 are from Type K thermocouples located in the flue gas. Tref is an indicator of ambient temperature. 


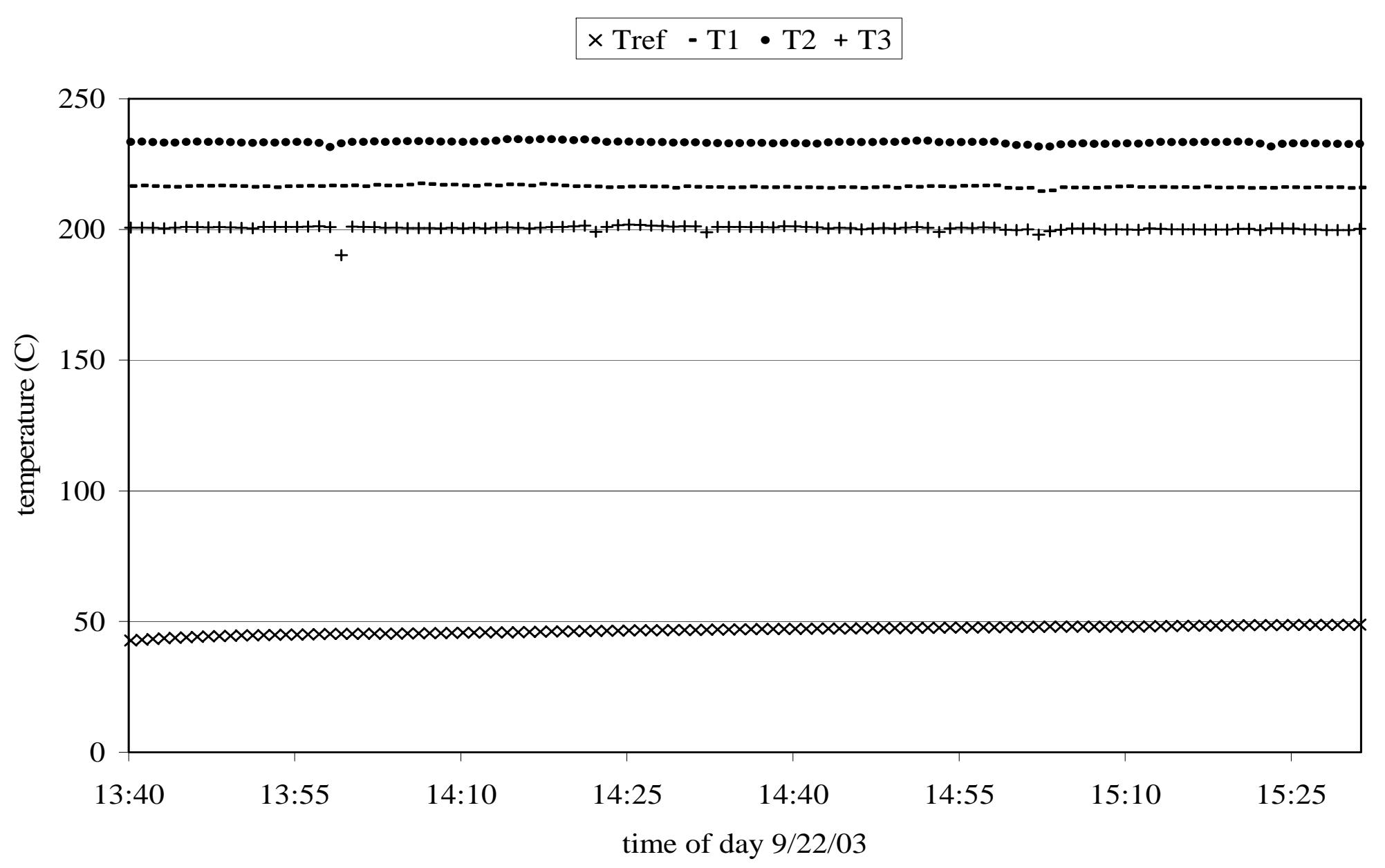

Figure C59. Temperatures recorded downstream of the air preheater in Boiler 2 firing coal on June 29, 2004, first sample period. Temperatures T1 though T3 are from Type K thermocouples located in the flue gas. Tref is an indicator of ambient temperature. 
- $\mathrm{CO} 2+\mathrm{O} 2$

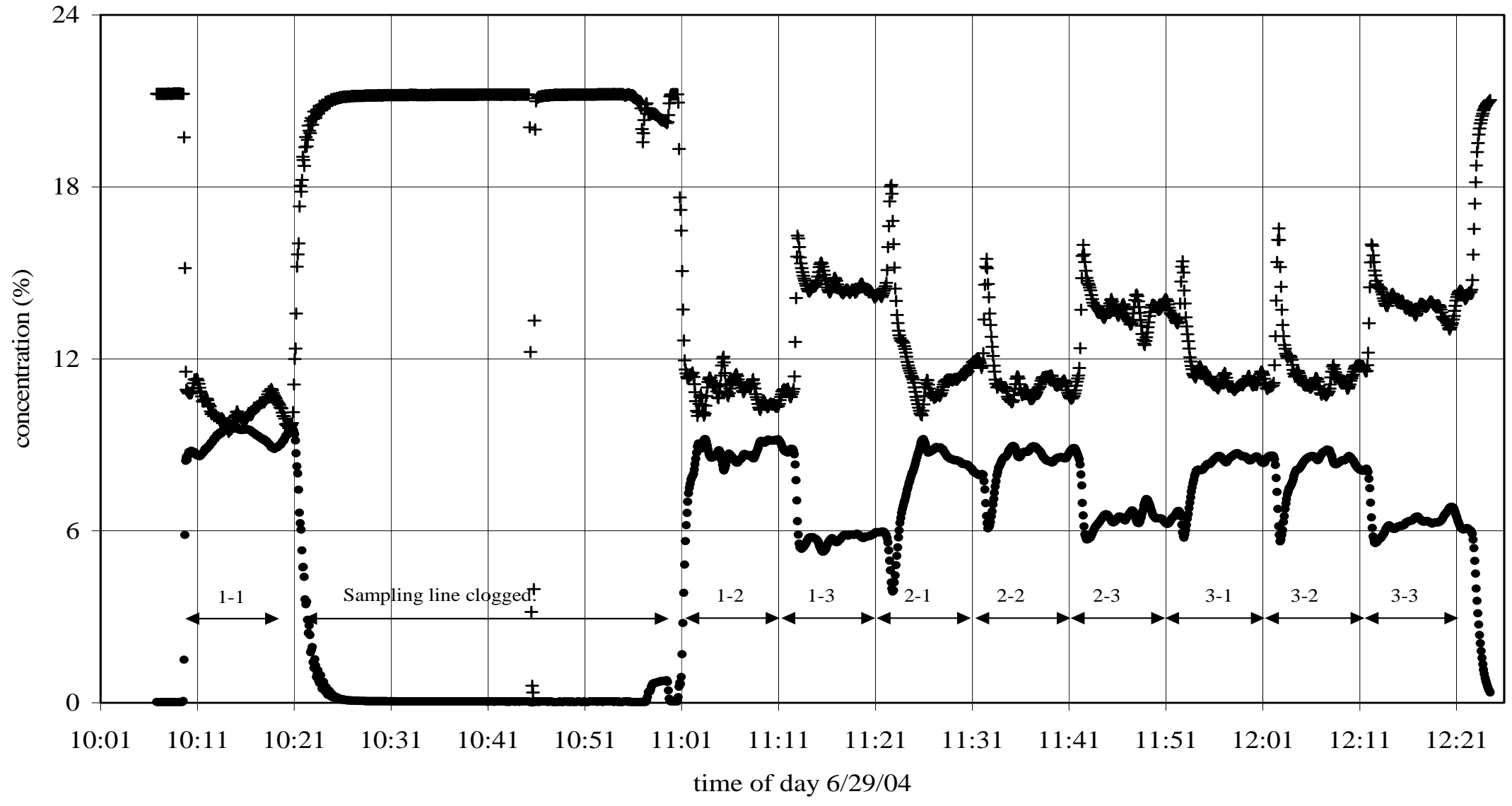

Figure C60. $\mathrm{O}_{2}$ and $\mathrm{CO}_{2}$ concentrations recorded downstream of the air preheater in Boiler 2 firing coal on June 29, 2004, first sample period. 
- ppm-CO

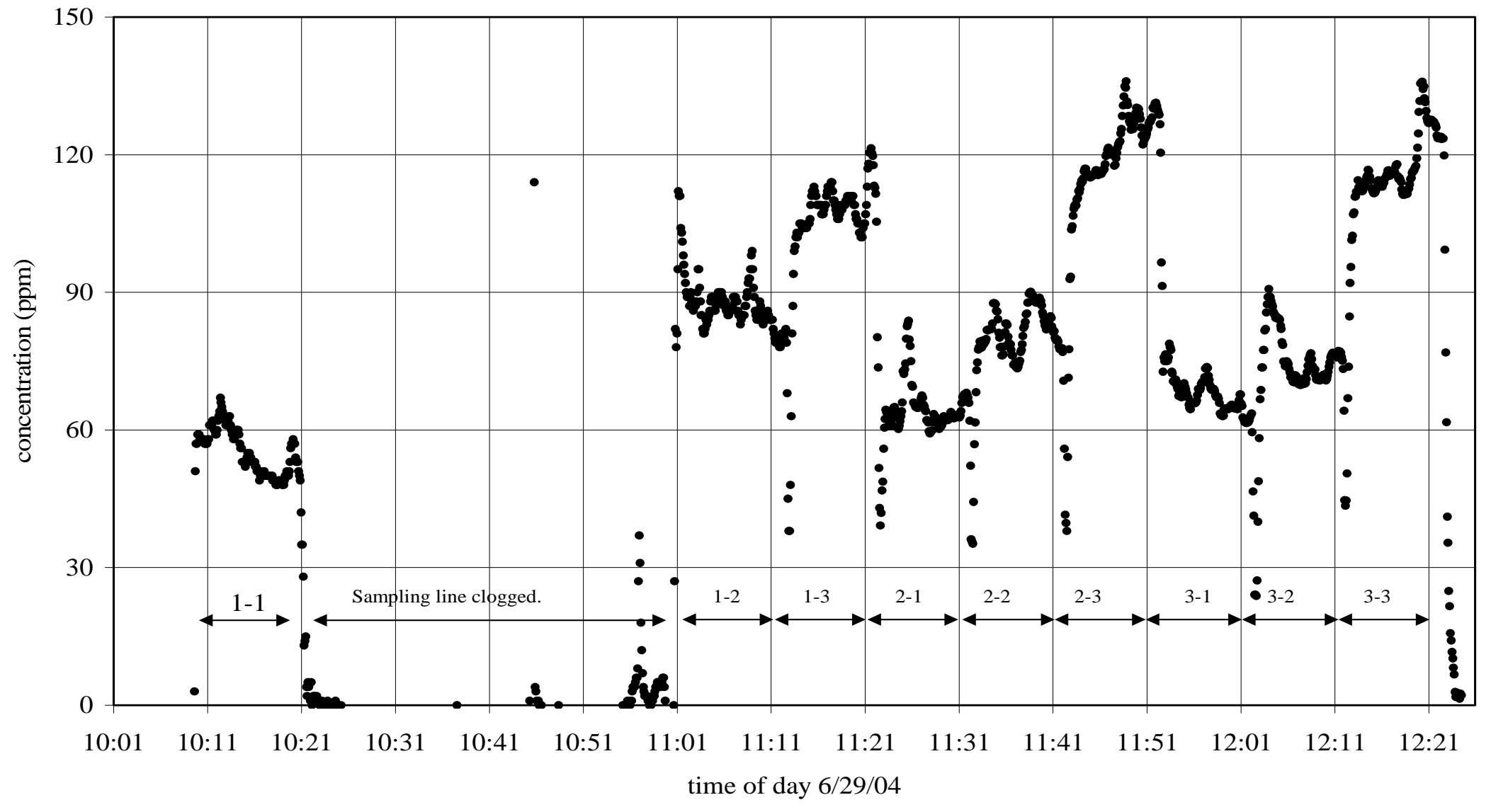

Figure C61. CO concentrations recorded downstream of the air preheater in Boiler 2 firing coal on June 29, 2004, first sample period. 


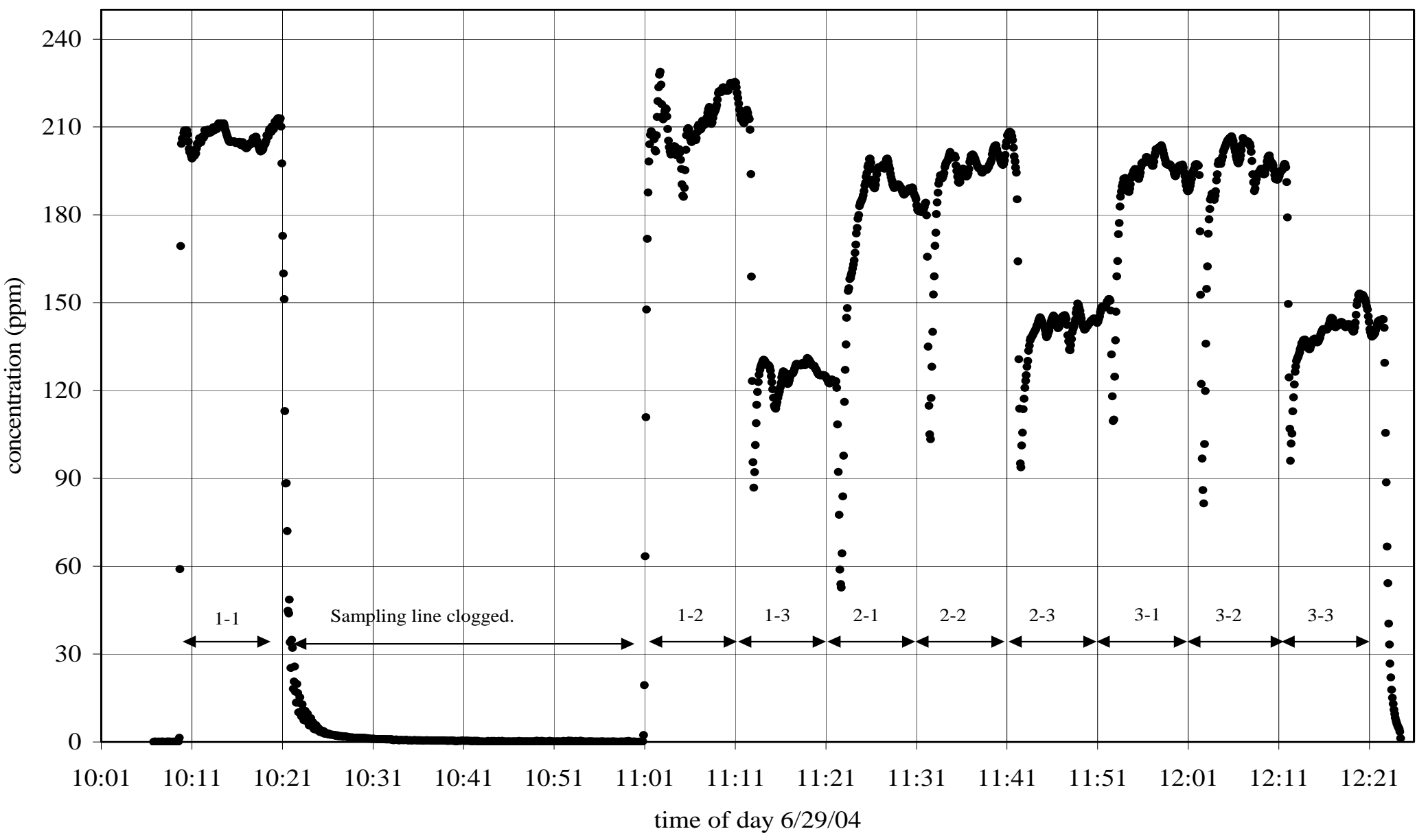

Figure C62. NOx concentrations recorded downstream of the air preheater in Boiler 2 firing coal on June 29, 2004, first sample period. 
Appendix D

D-1 


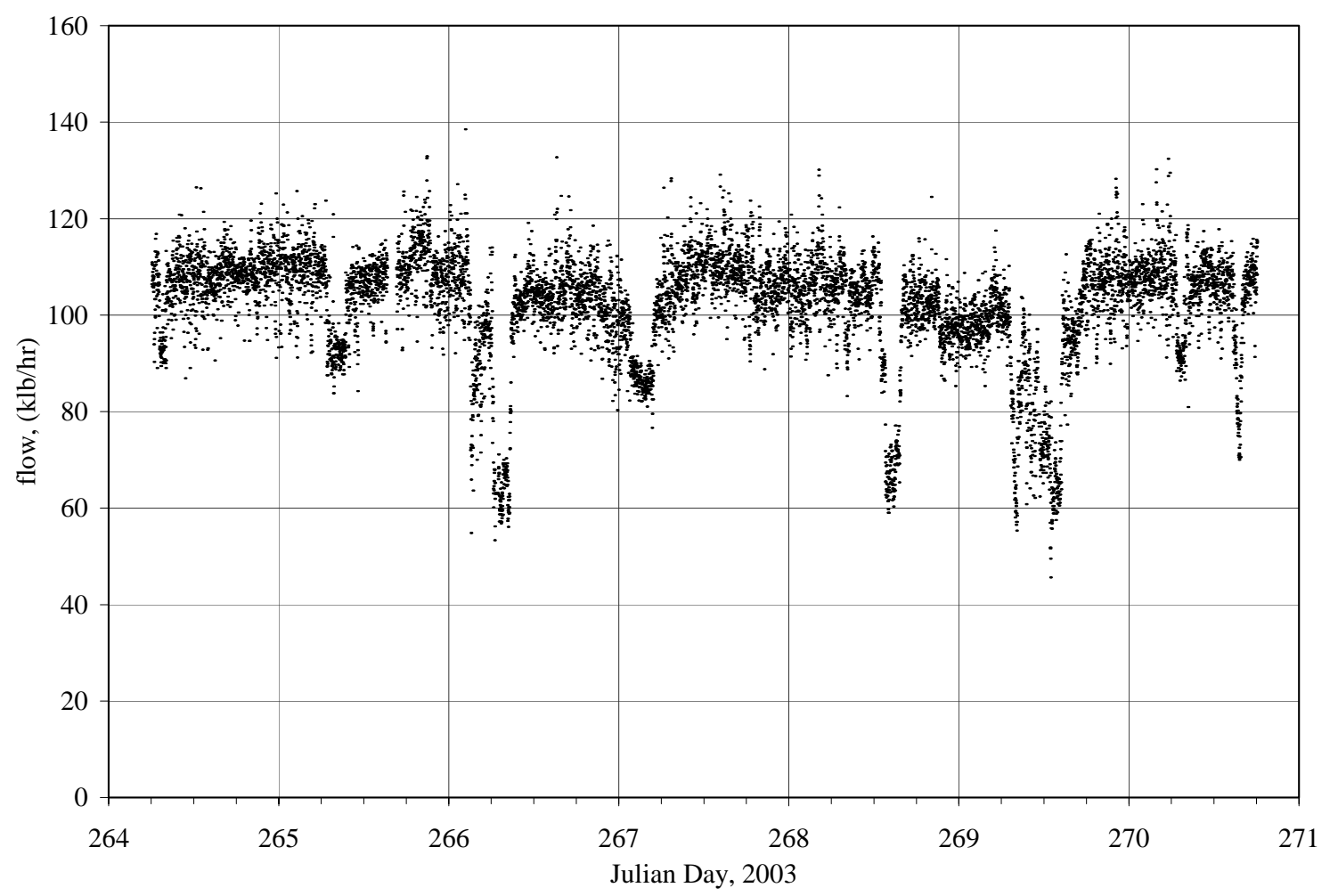

Figure D1. Steam flow rate, Boiler 1. Julian Day 264 = September 21, 2003.

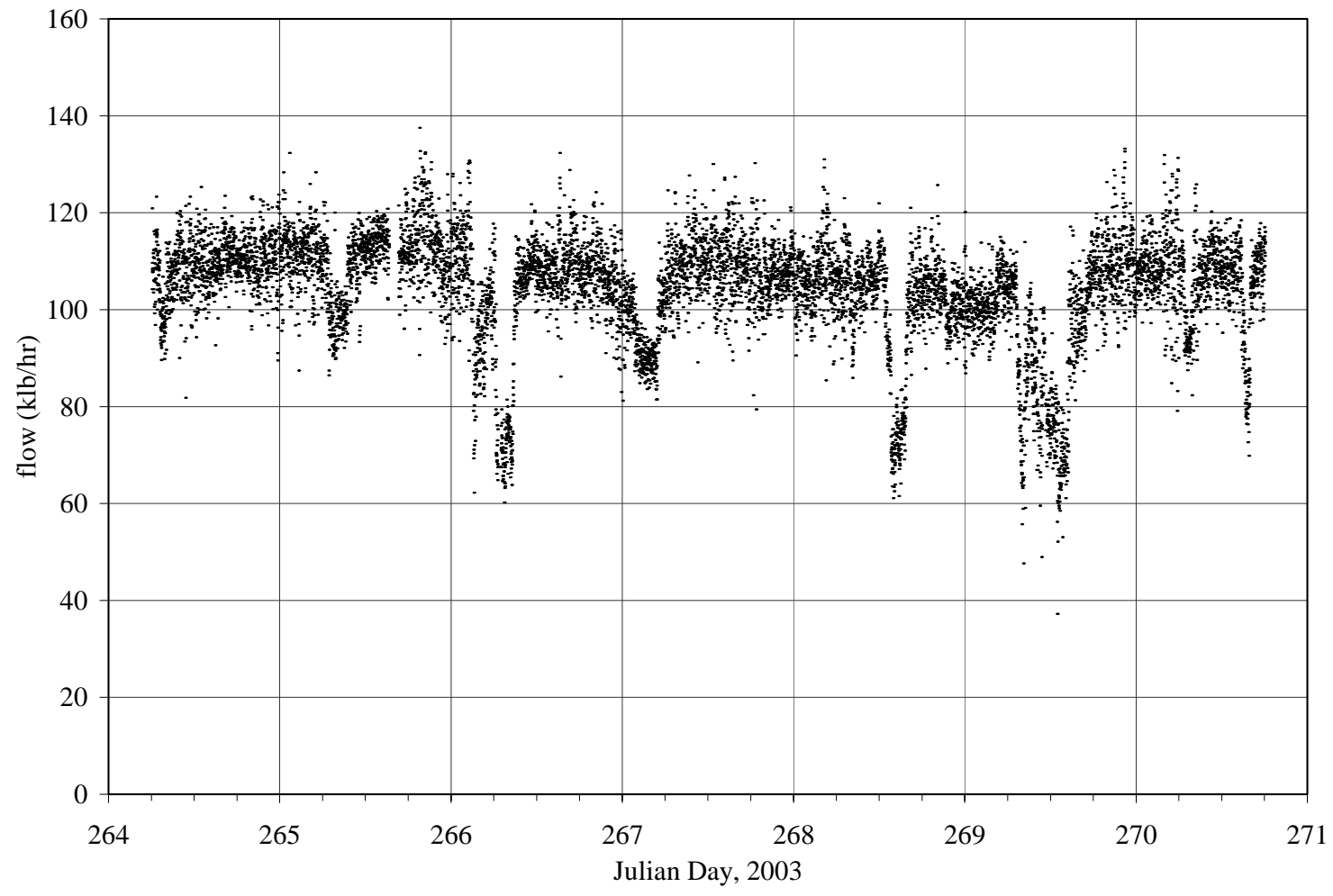

Figure D2. Feed water flow rate, Boiler 1. Julian Day 264 = September 21, 2003. 


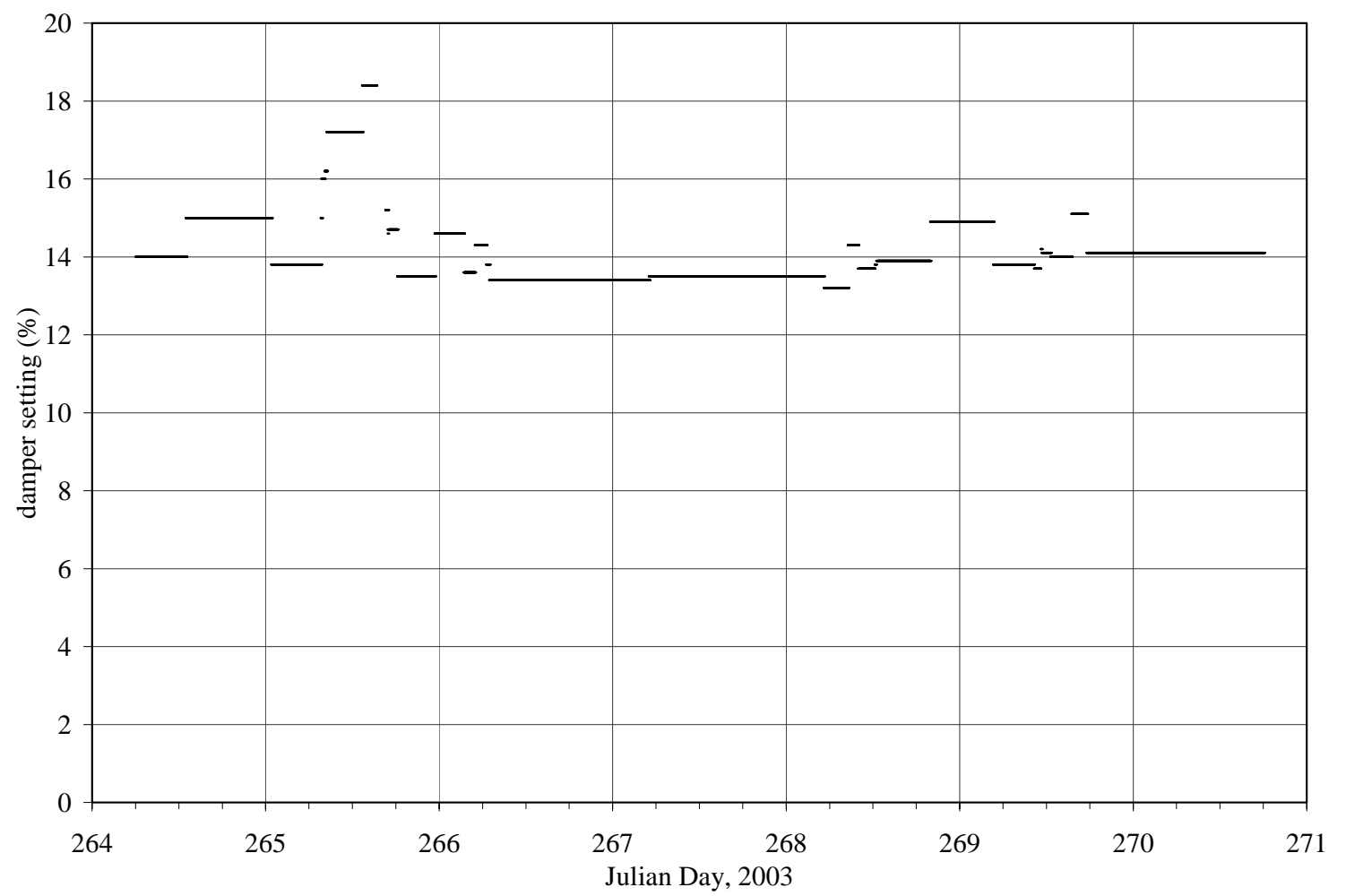

Figure D3. Right grate air flow rate, Boiler 1. Julian Day 264 = September 21, 2003.

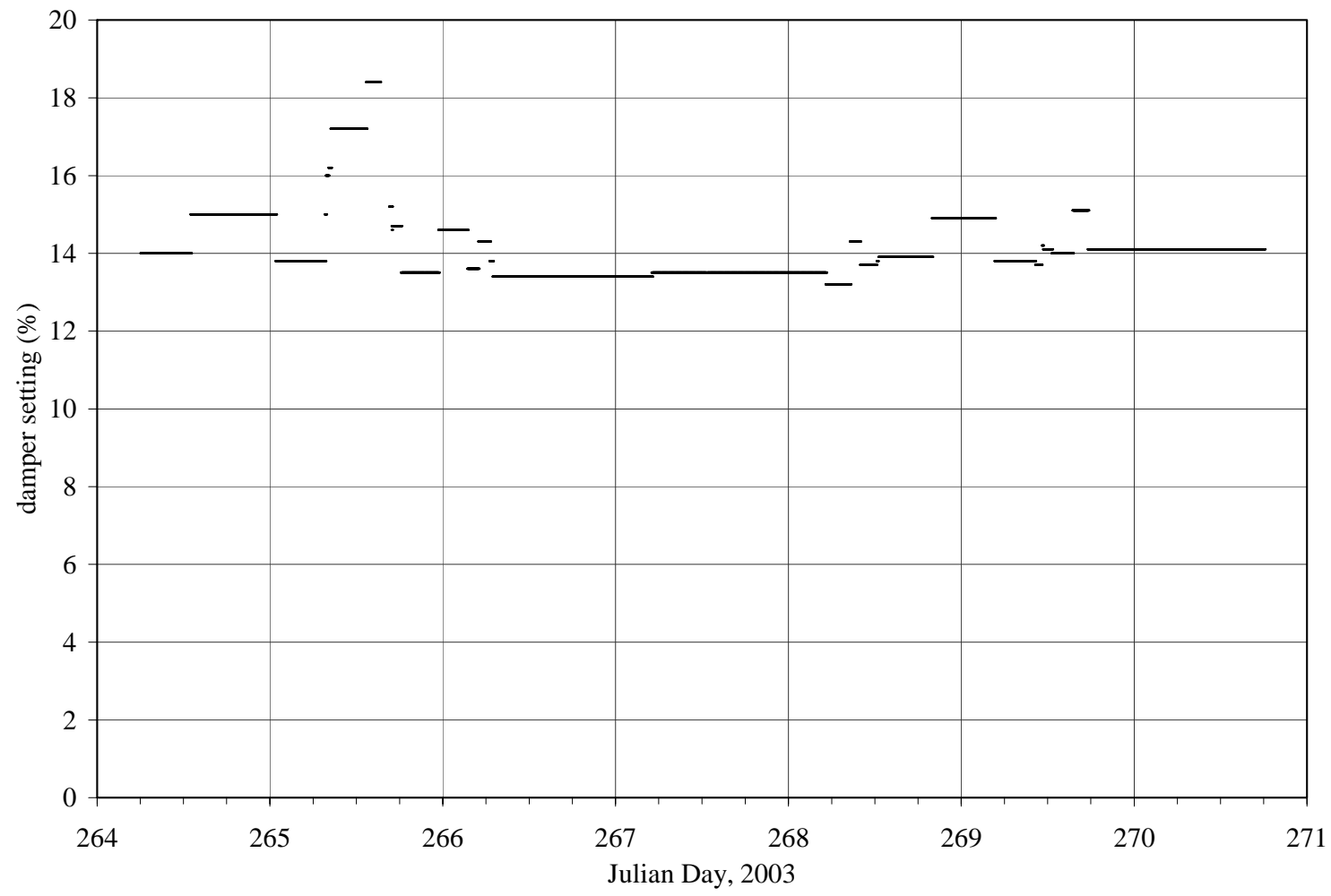

Figure D4. Left grate air flow rate, Boiler 1. Julian Day 264 = September 21, 2003. 


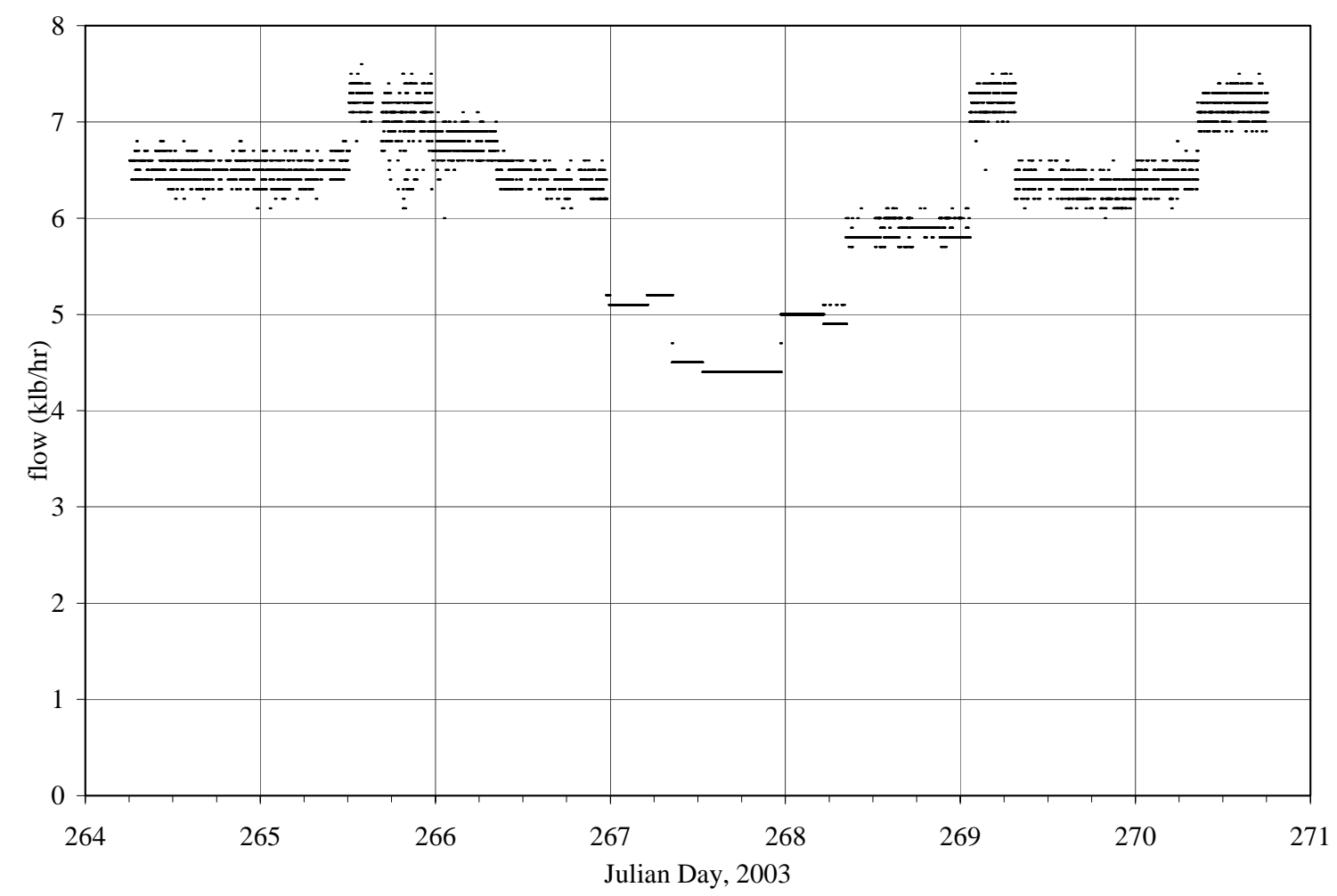

Figure D5. Continuous blowdown flow rate, Boiler 1. Julian Day 264 = September 21, 2003.

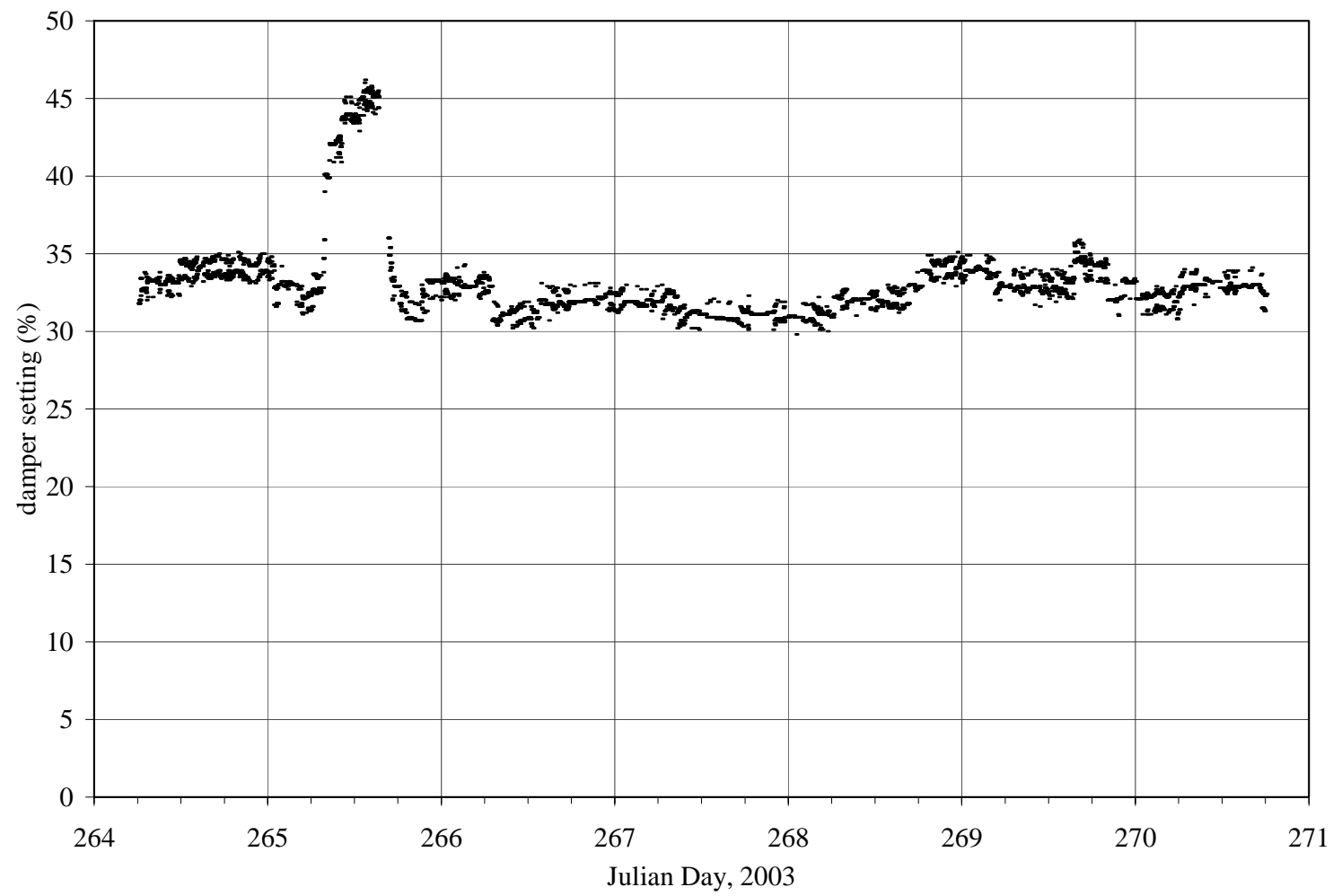

Figure D6. Grate air flow rate, Boiler 1. Julian Day 264 = September 21, 2003. 


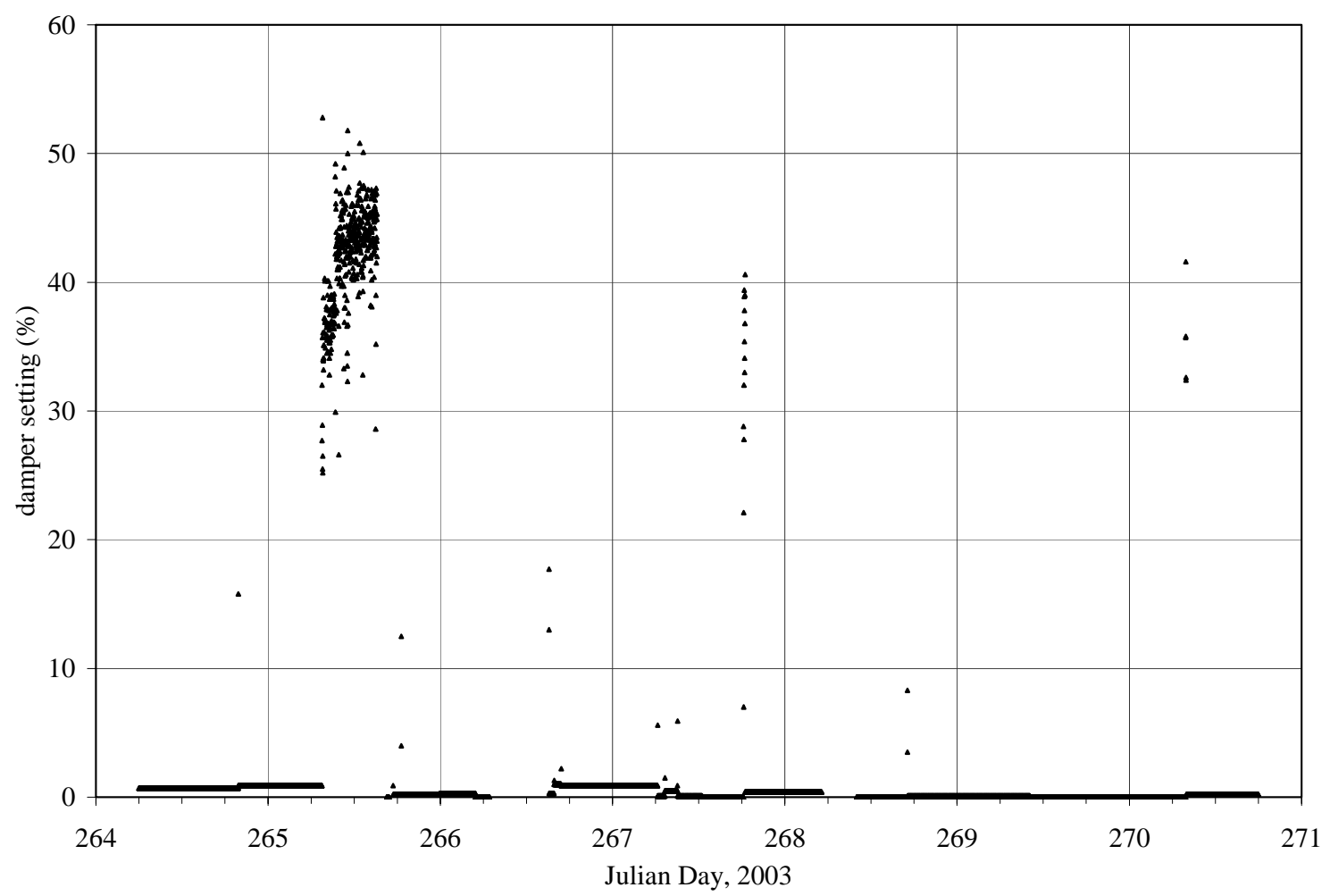

Figure D7. Coal flow rate, Boiler 1. Julian Day 264 = September 21, 2003.

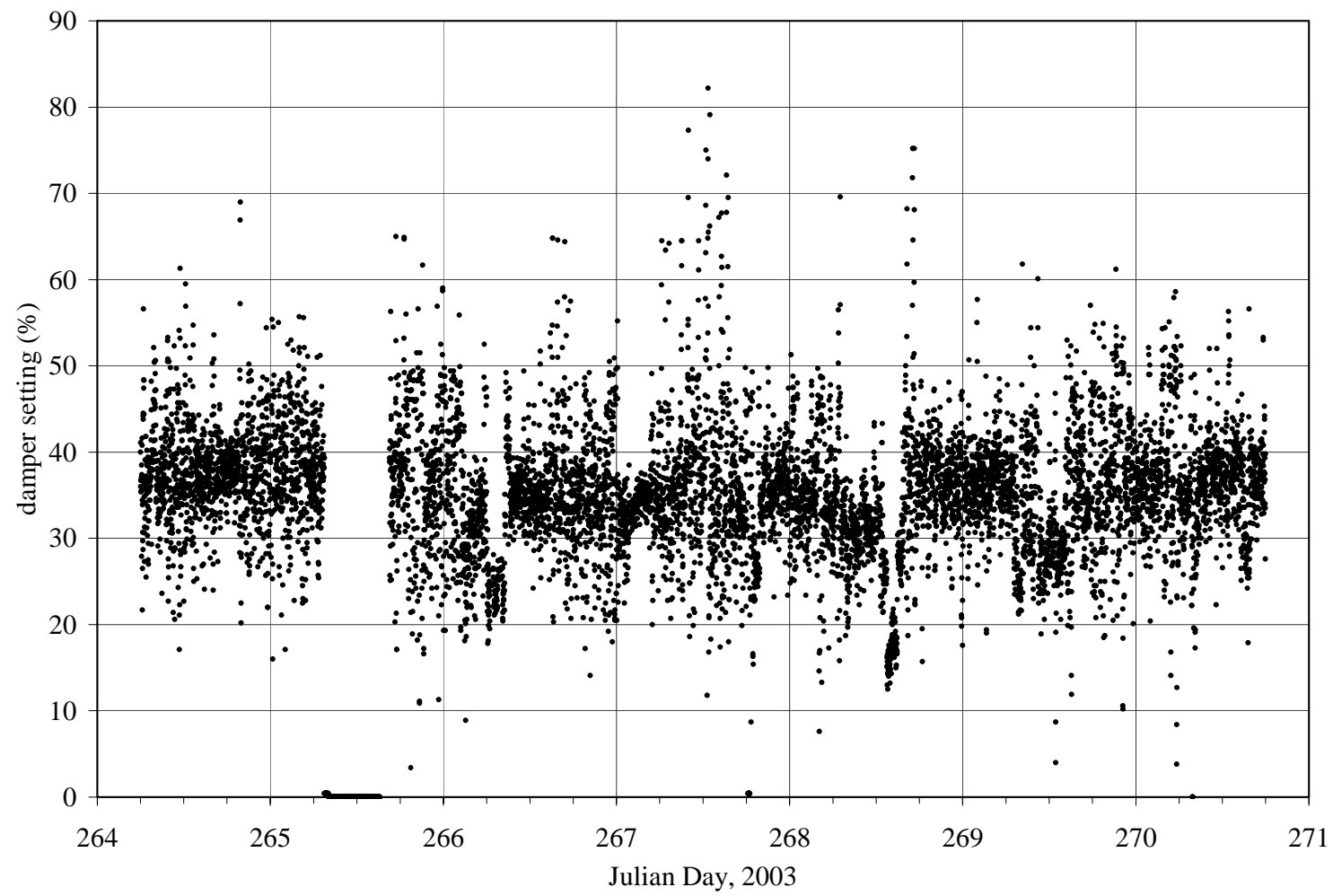

Figure D8. Bagasse feed rate, Boiler 1. Julian Day 264 = September 21, 2003. 


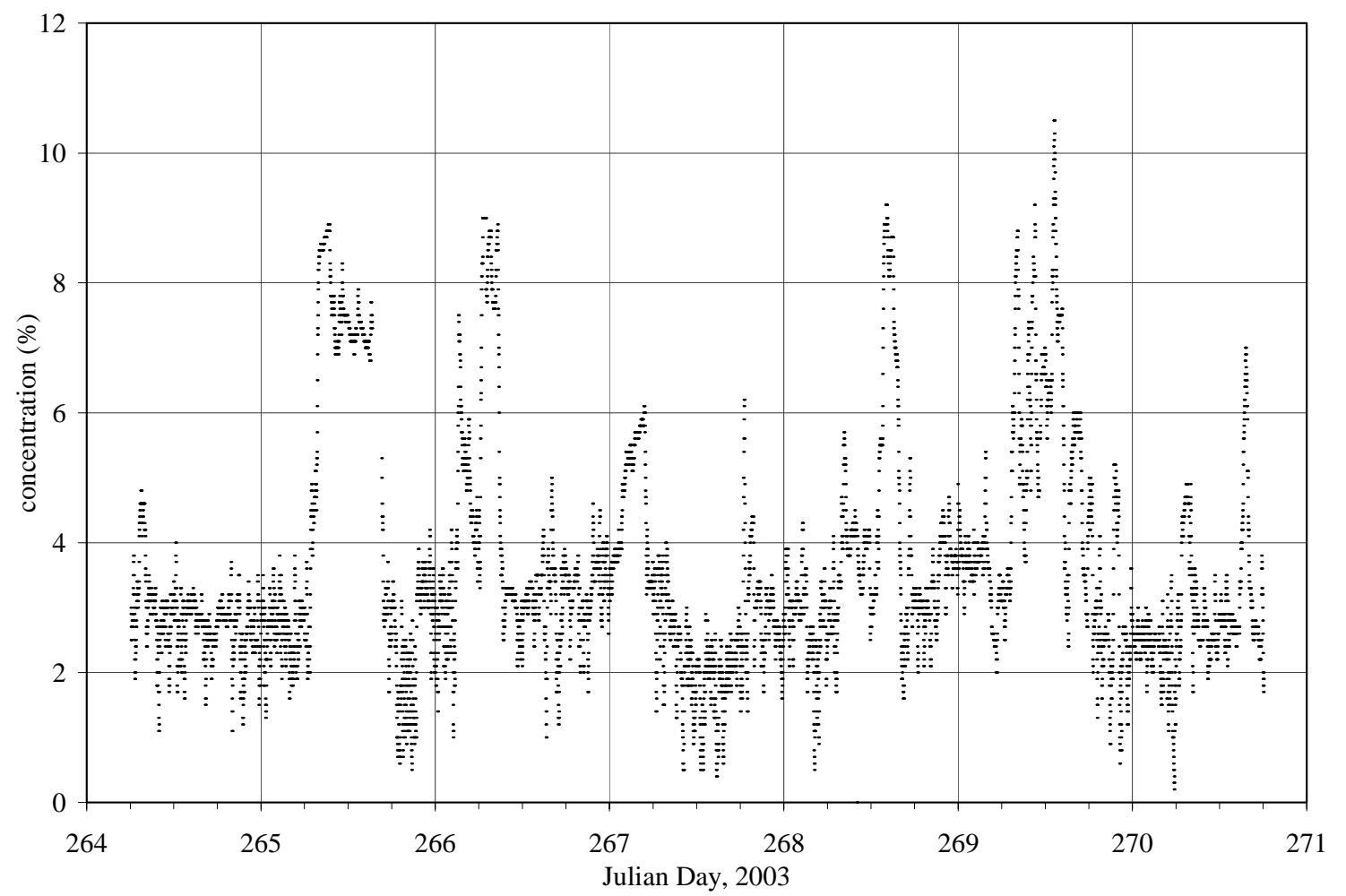

Figure D9. $\mathrm{O}_{2}$ concentration, Boiler 1. Julian Day 264 = September 21, 2003.

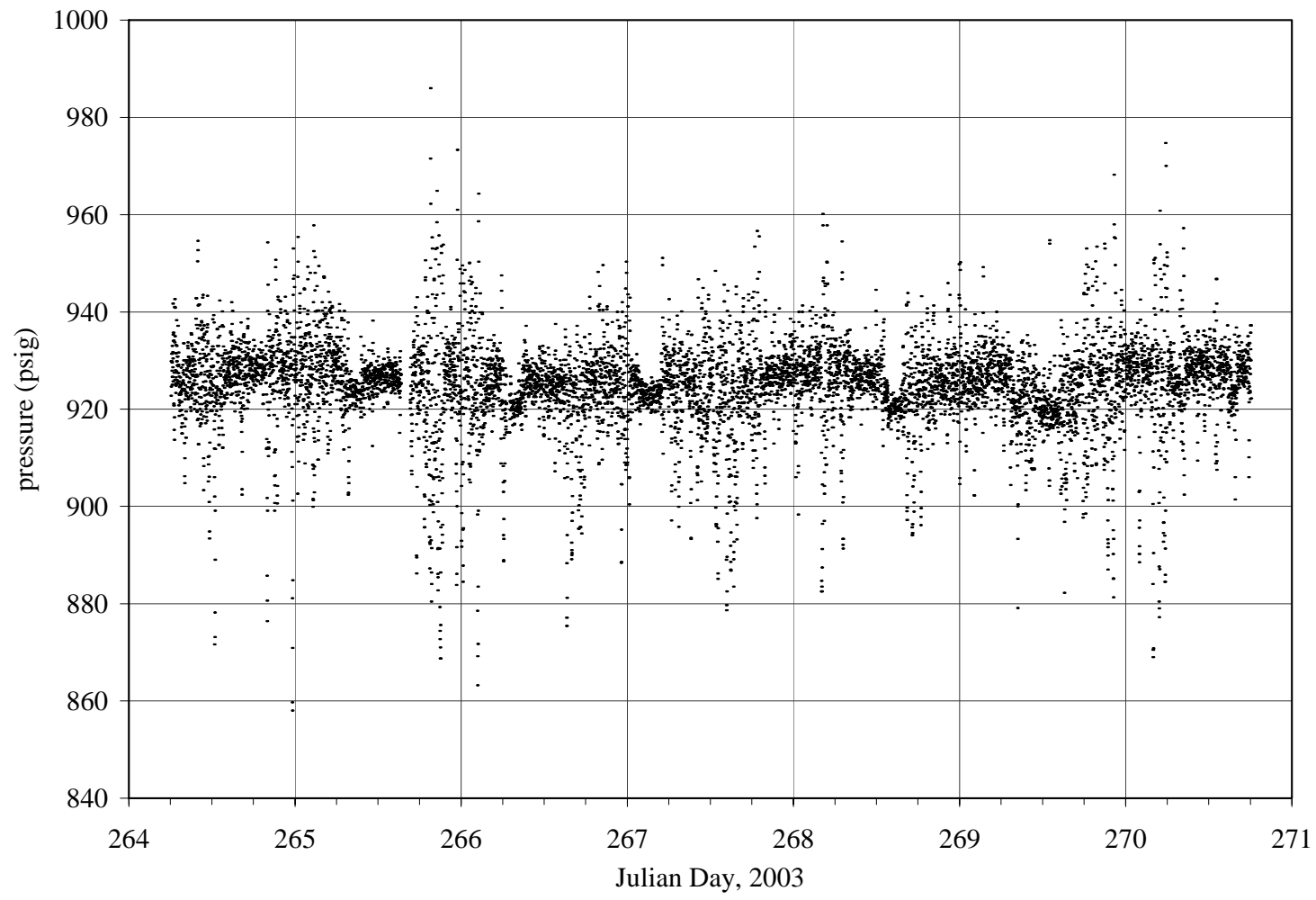

Figure D10. Drum pressure, Boiler 1. Julian Day 264 = September 21, 2003. 


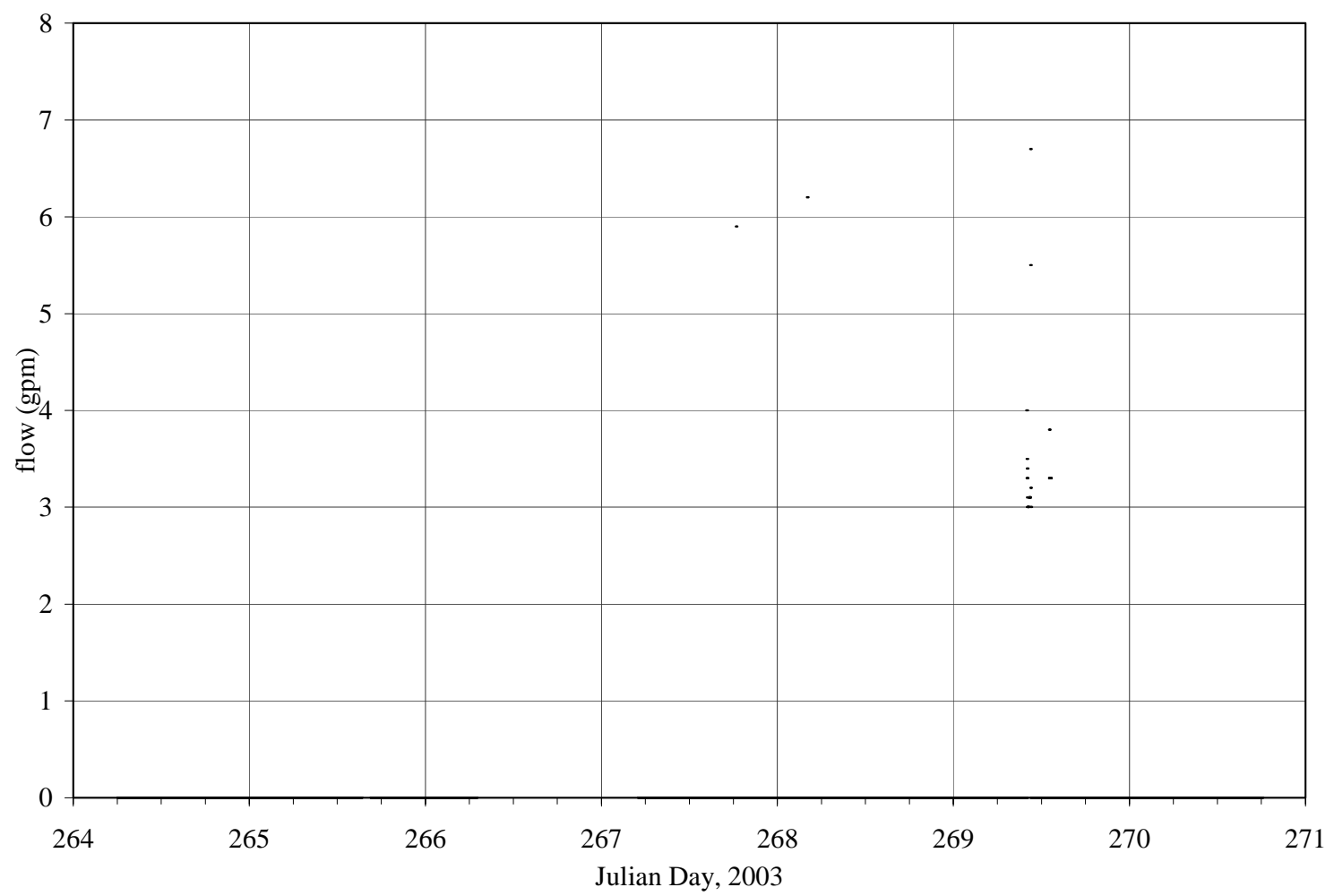

Figure D11. Fuel Oil flow rate, Boiler 1. Julian Day 264 = September 21, 2003.

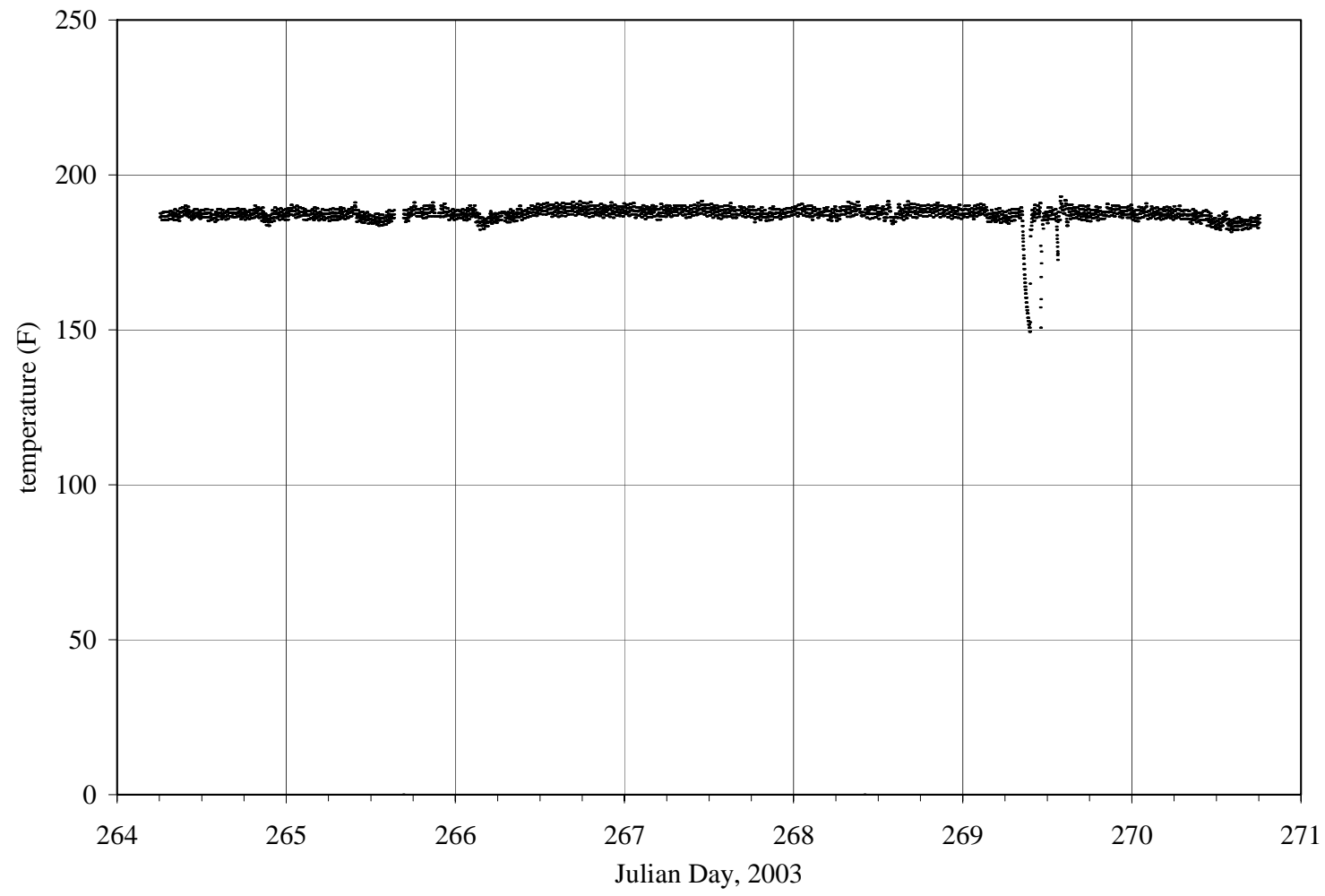

Figure D12. Fuel Oil temperature, Boiler 1. Julian Day 264 = September 21, 2003. 


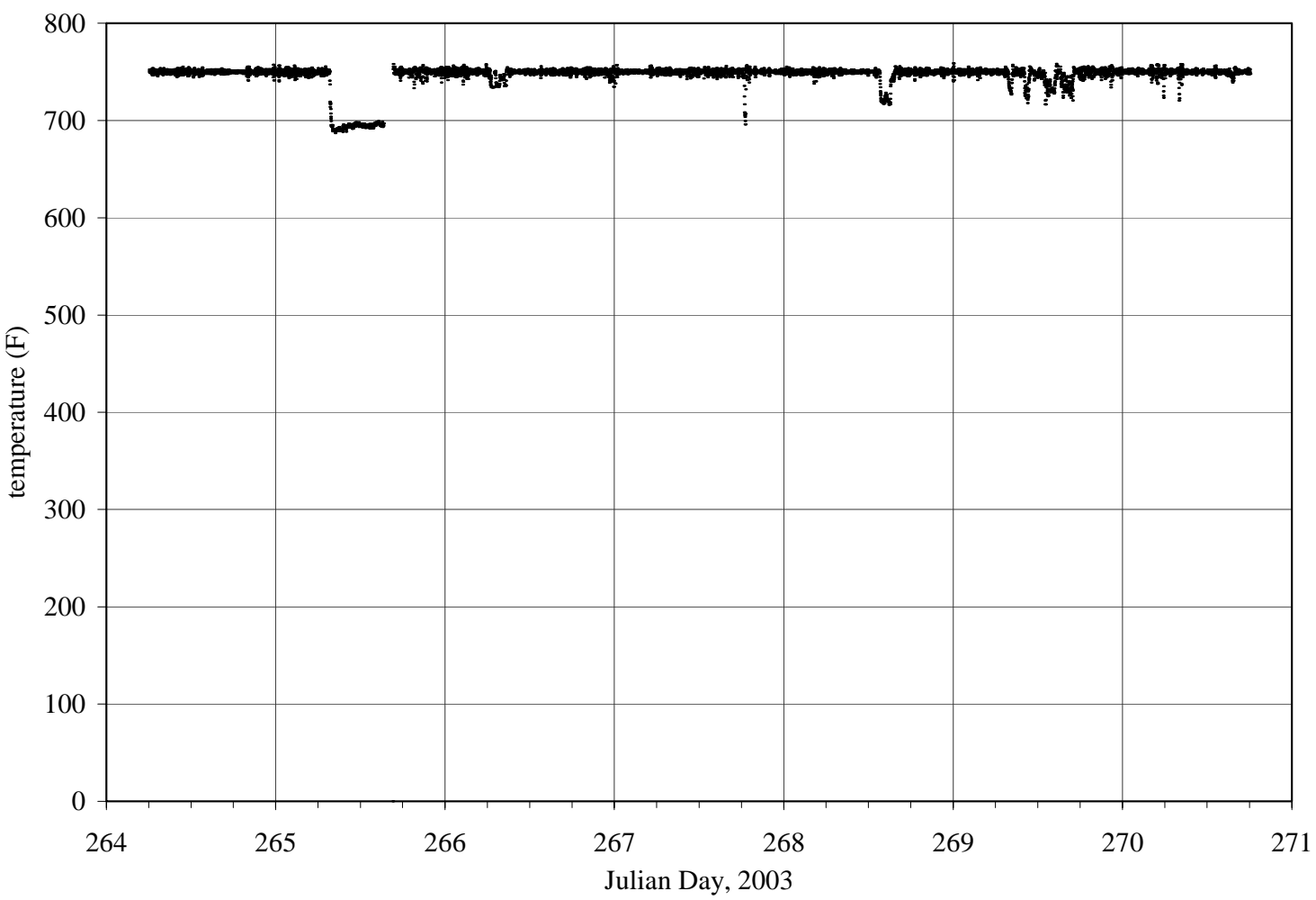

Figure D13. Steam temperature, Boiler 1. Julian Day 264 = September 21, 2003. 


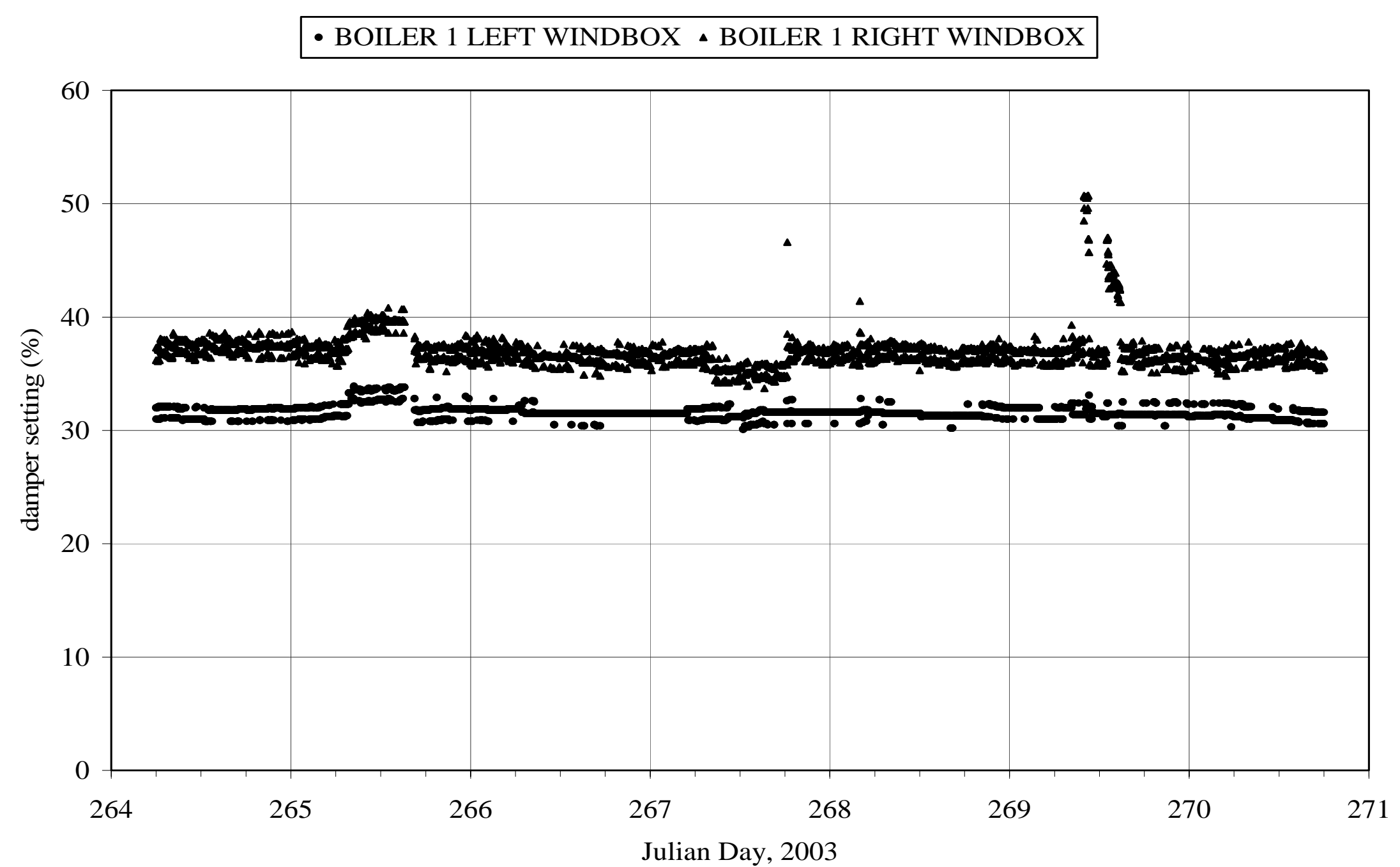

Figure D14. Air flow rates in left and right windbox, Boiler 1. Julian Day 264 = September 21, 2003. 


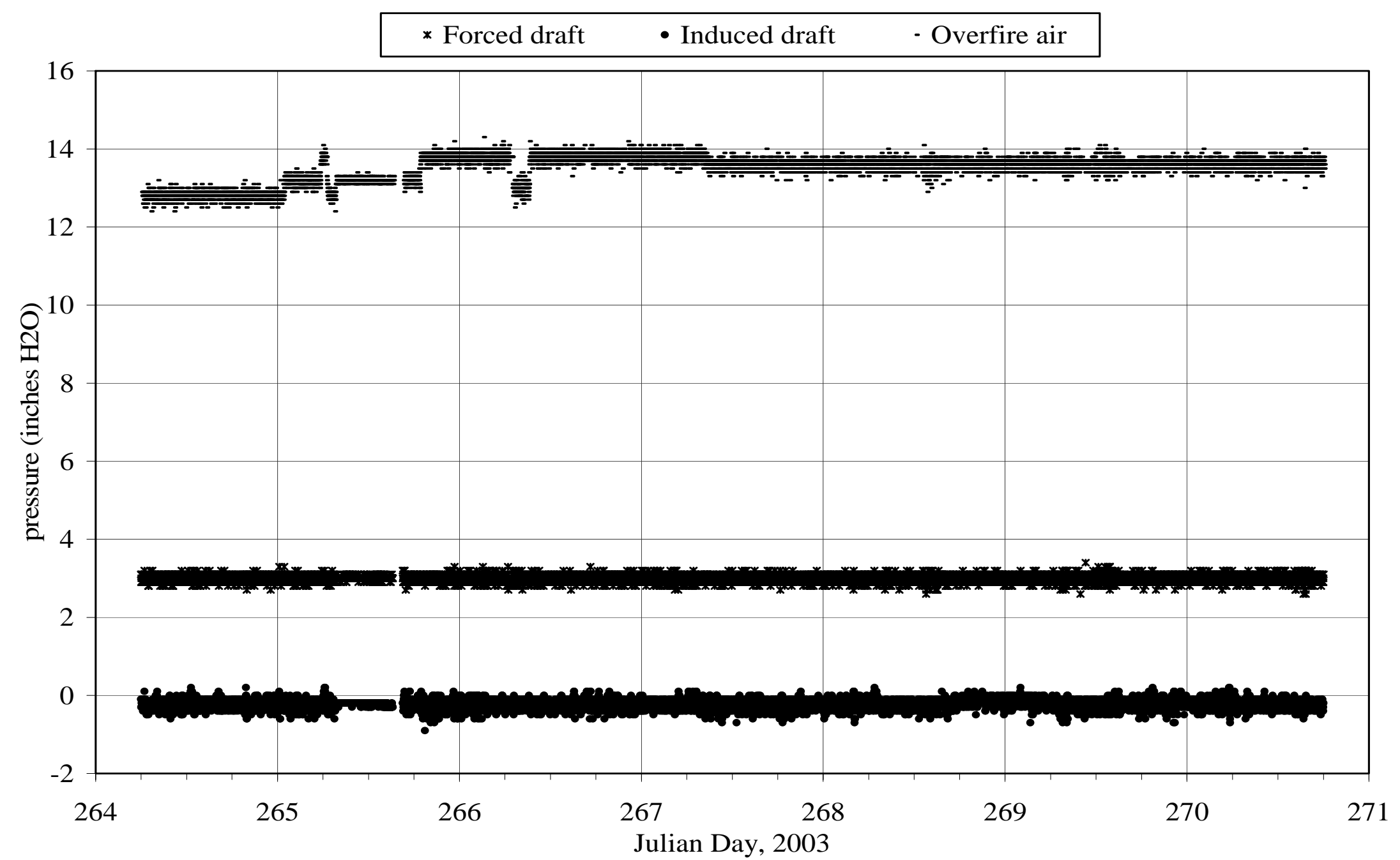

Figure D15. Forced draft, induced draft and overfire air pressure, Boiler 1. Julian Day 264 = September 21, 2003. 


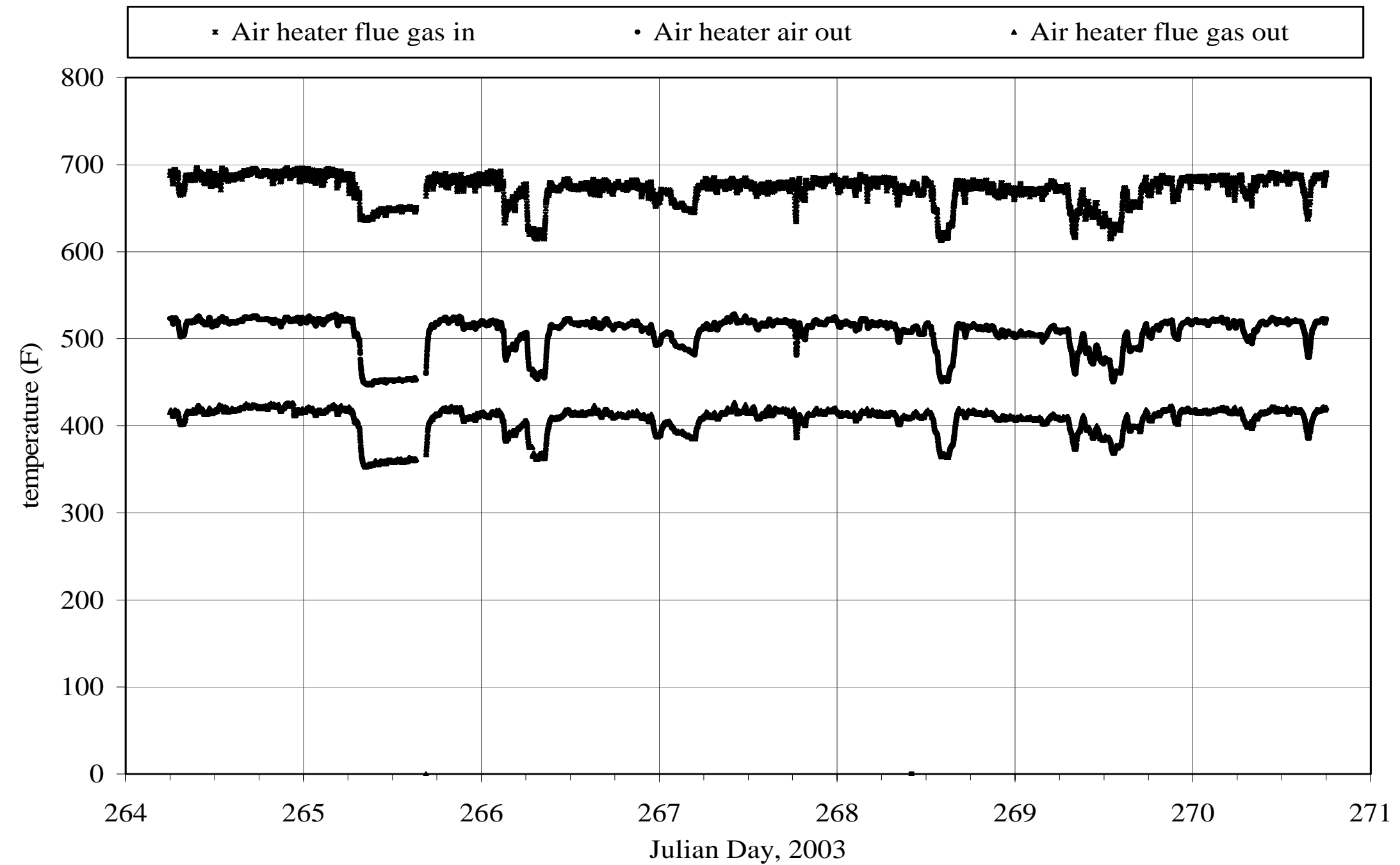

Figure D16. Air heater, flue gas in, flue gas out and air out temperature, Boiler 1. Julian Day $264=$ September 21, 2003. 


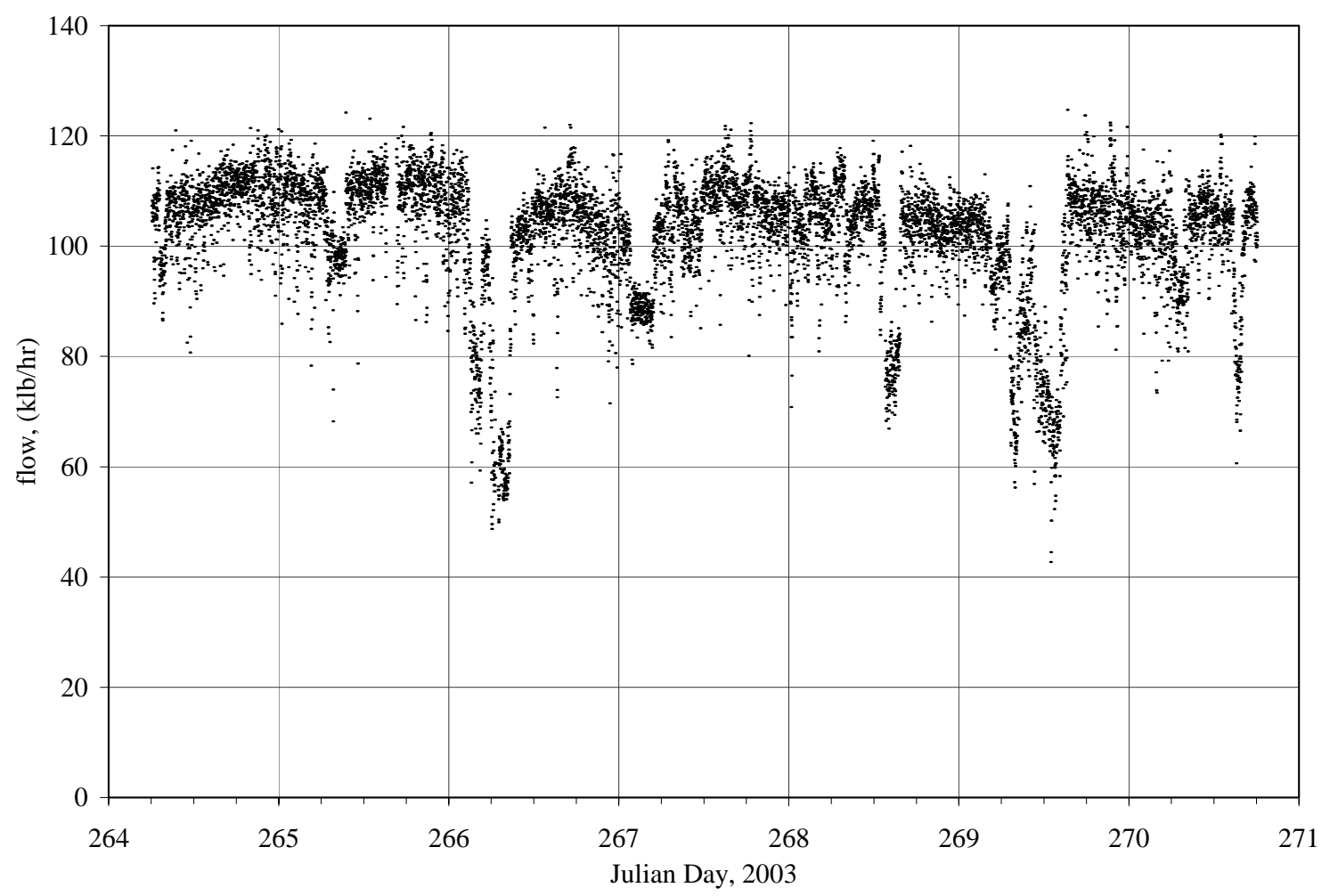

Figure D17. Steam flow rate, Boiler 2. Julian Day 264 = September 21, 2003.

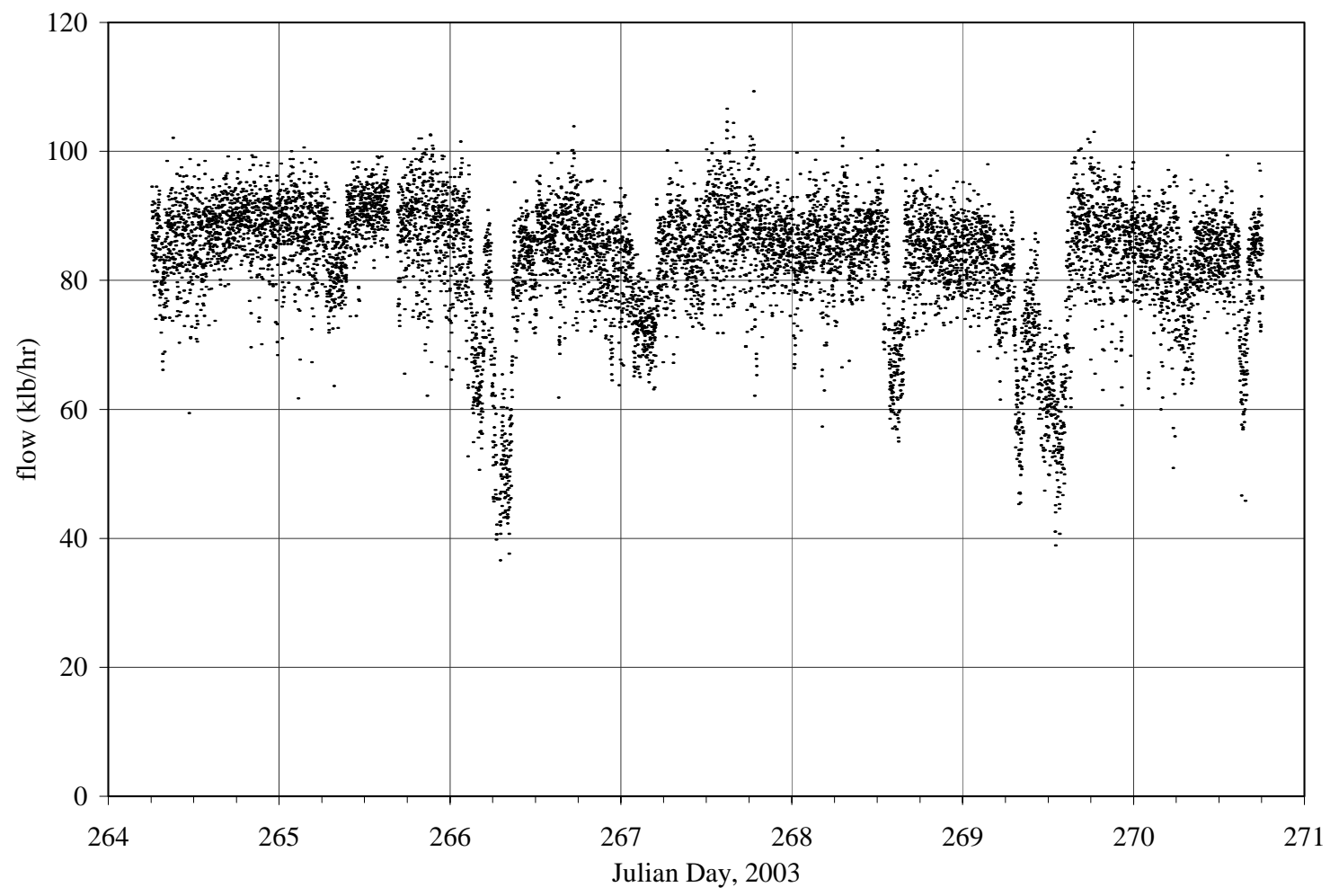

Figure D18. Feed water flow rate, Boiler 2. Julian Day 264 = September 21, 2003. 


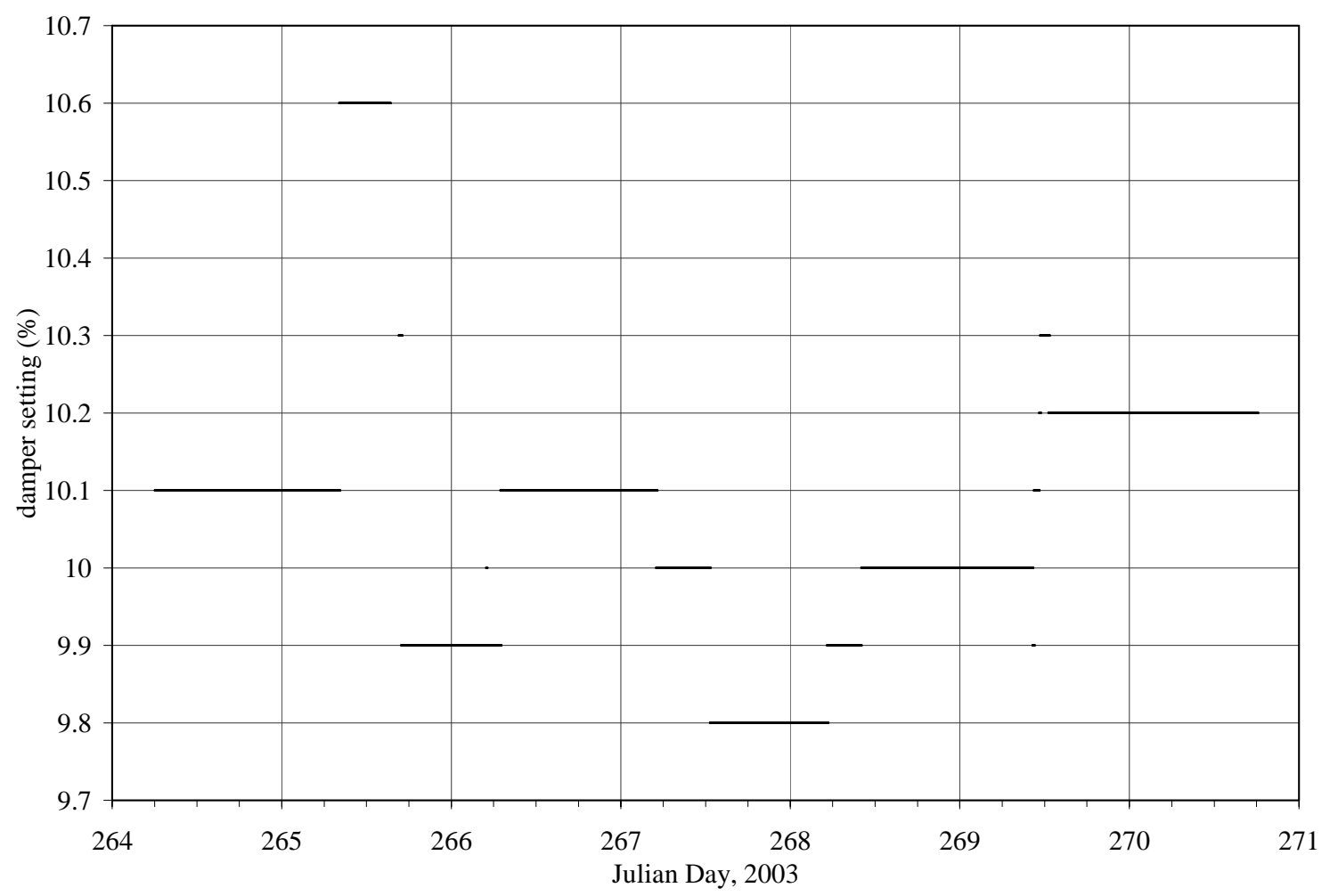

Figure D19. Right grate air flow rate, Boiler 2. Julian Day 264 = September 21, 2003.

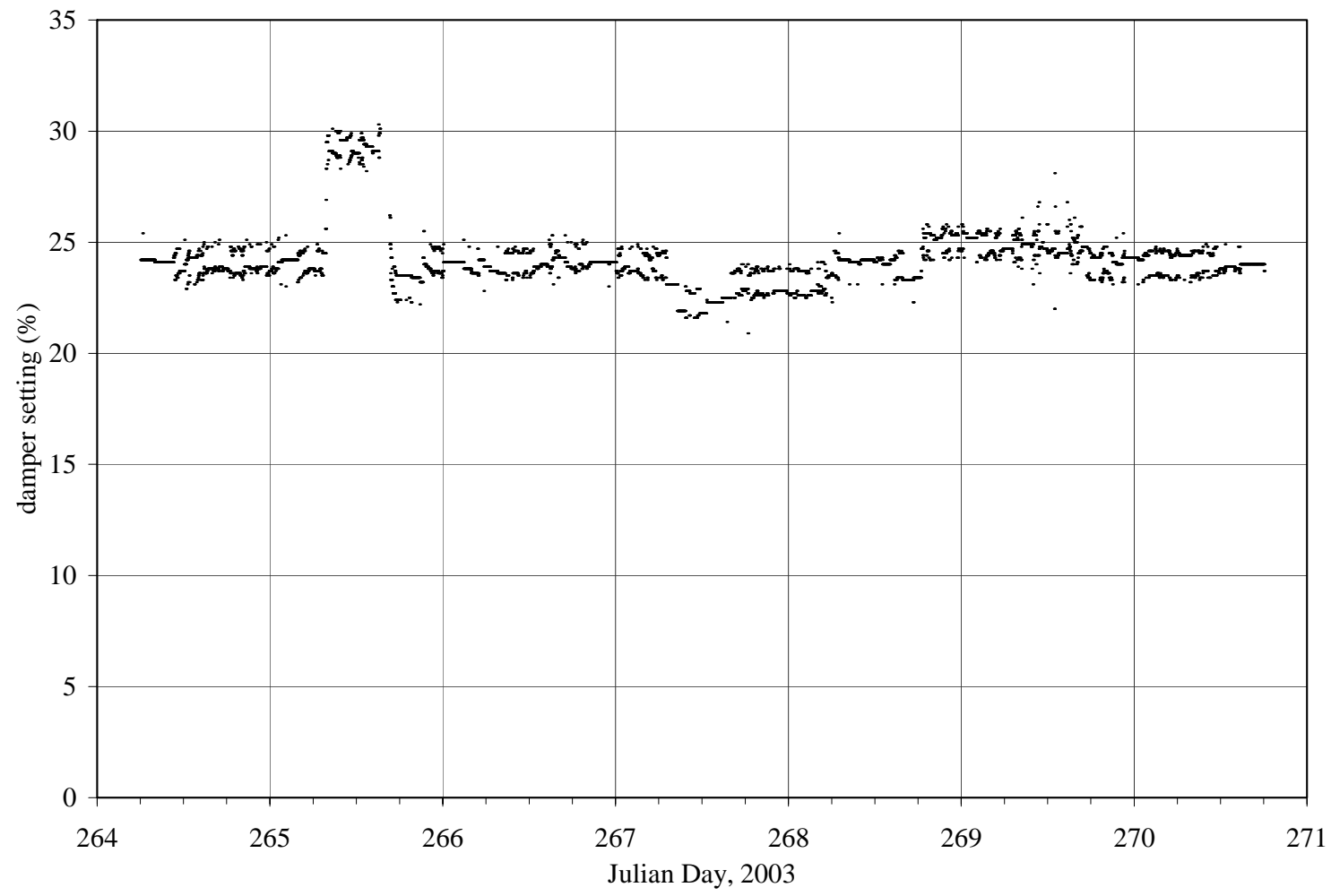

Figure D20. Left grate air flow rate, Boiler 2. Julian Day 264 = September 21, 2003. 


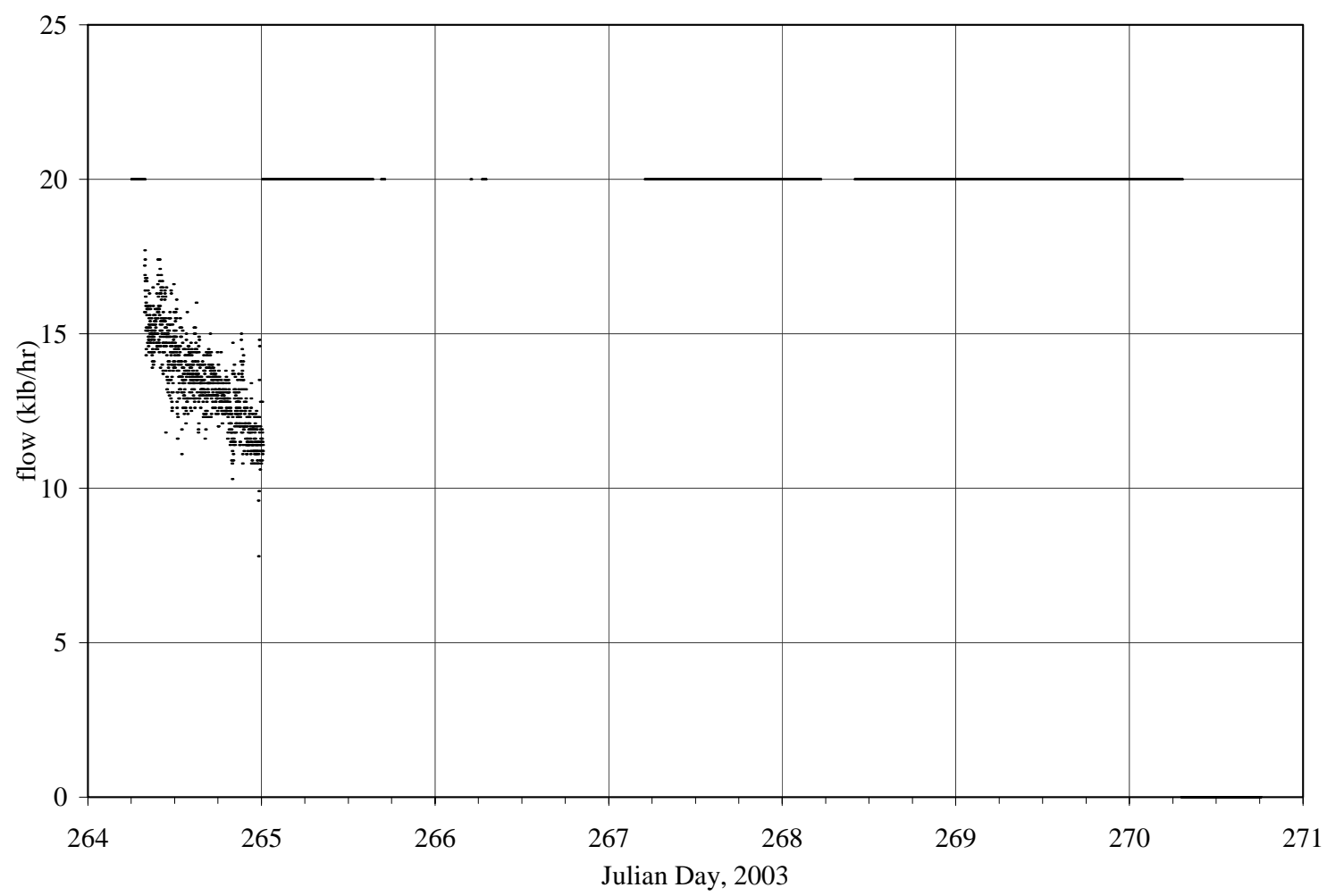

Figure D21. Continuous blowdown flow rate, Boiler 2. Julian Day $264=$ September 21, 2003.

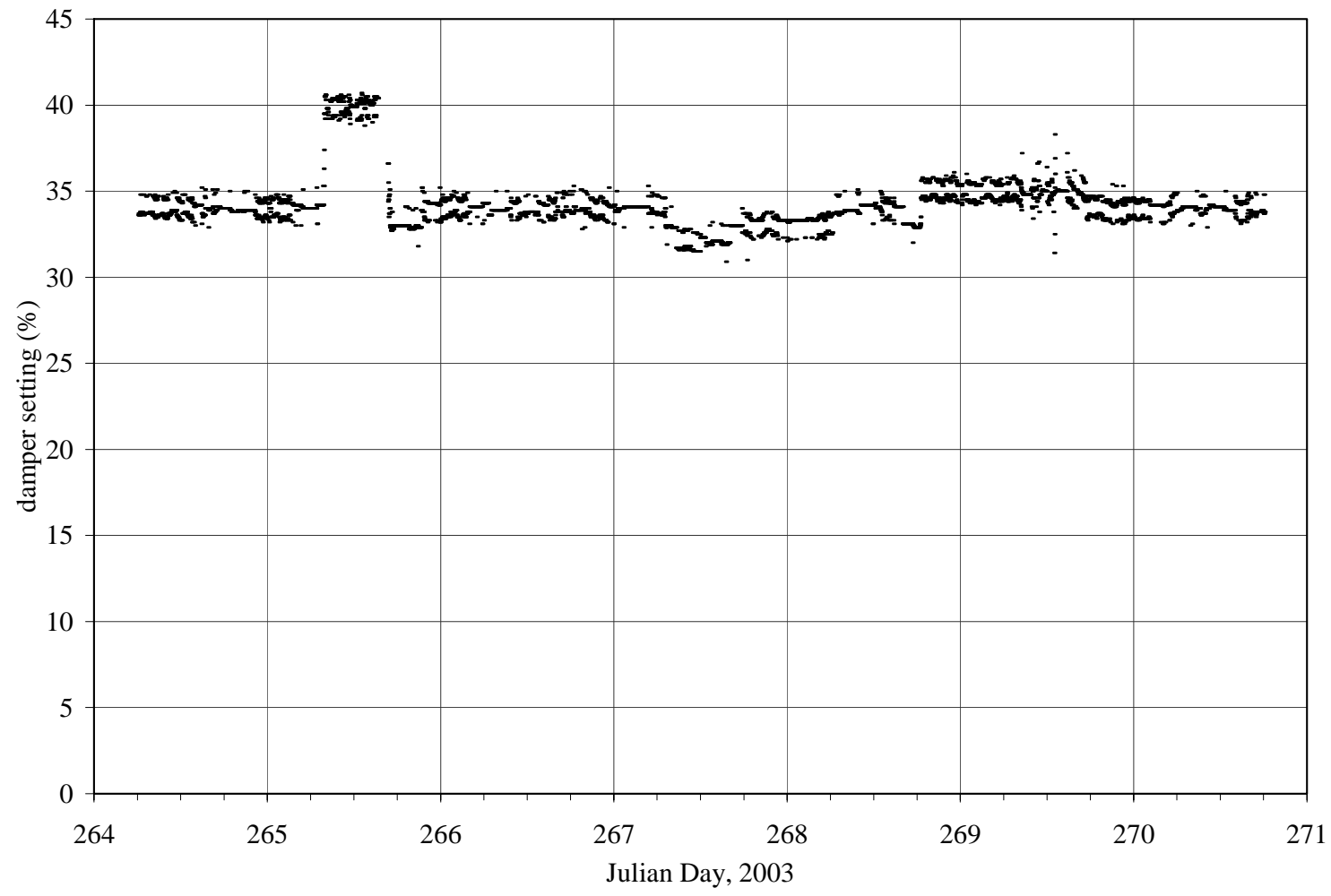

Figure D22. Grate air flow rate, Boiler 2. Julian Day 264 = September 21, 2003. 


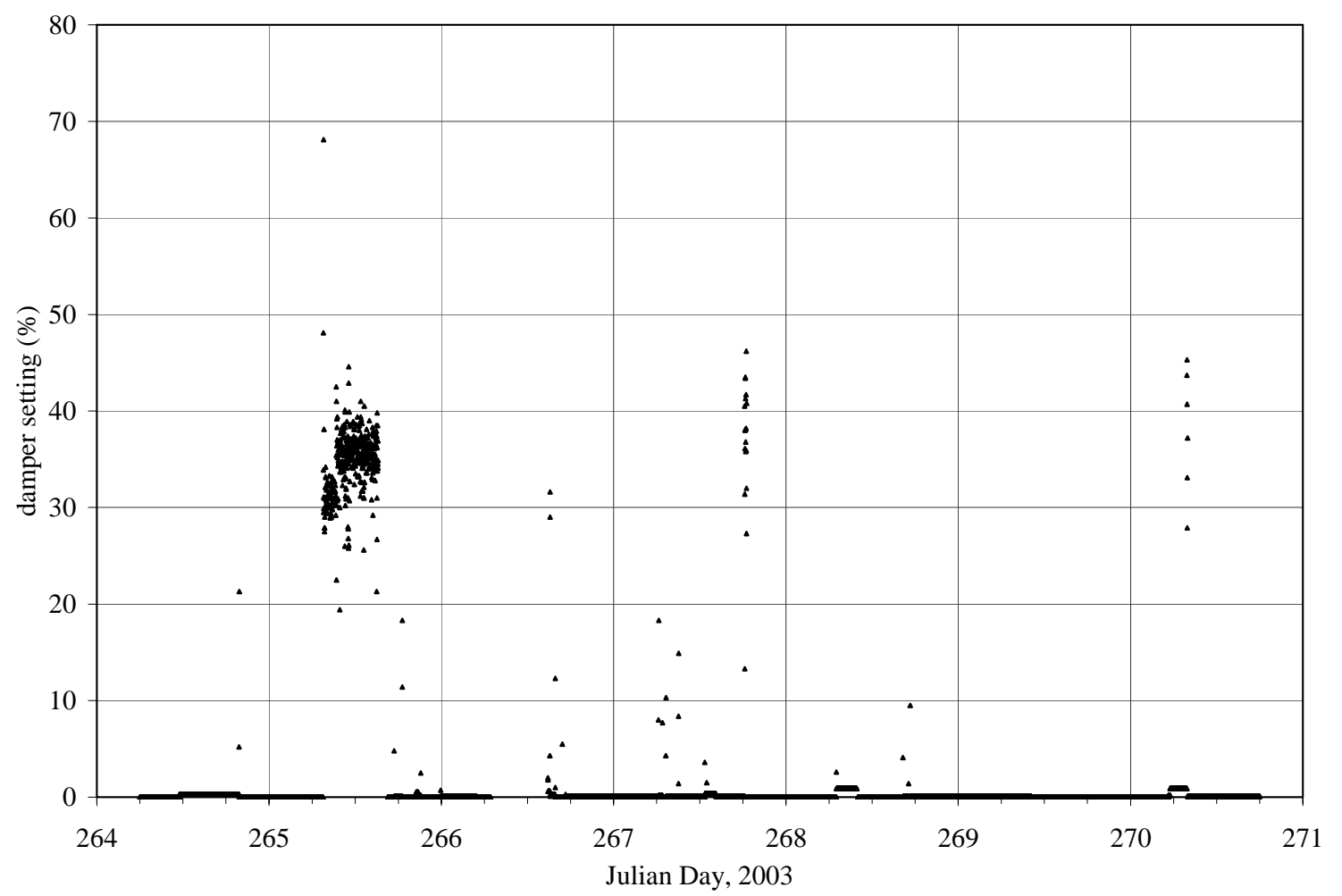

Figure D23. Coal flow rate, Boiler 2. Julian Day 264 = September 21, 2003.

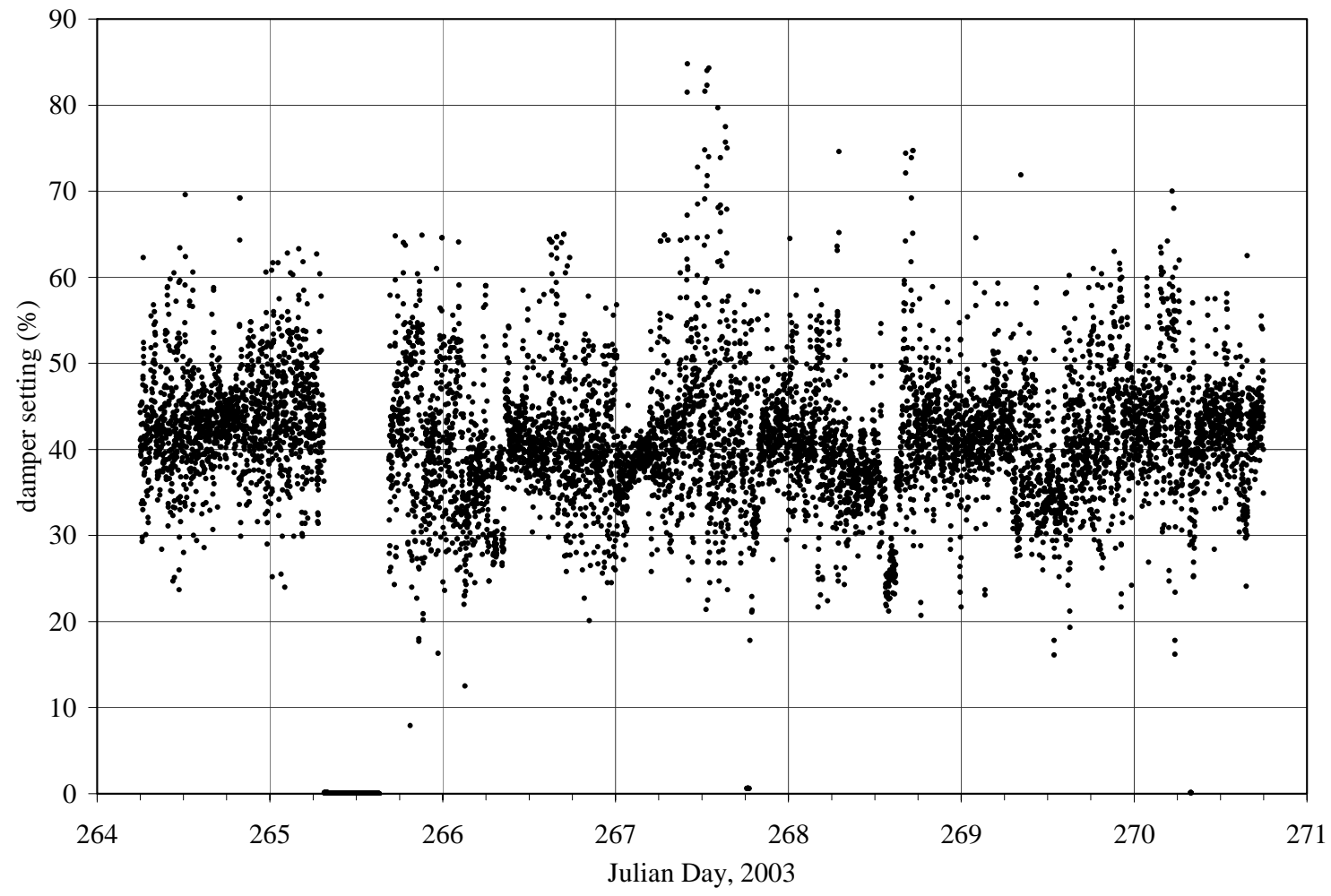

Figure D24. Bagasse feed rate, Boiler 2. Julian Day 264 = September 21, 2003. 


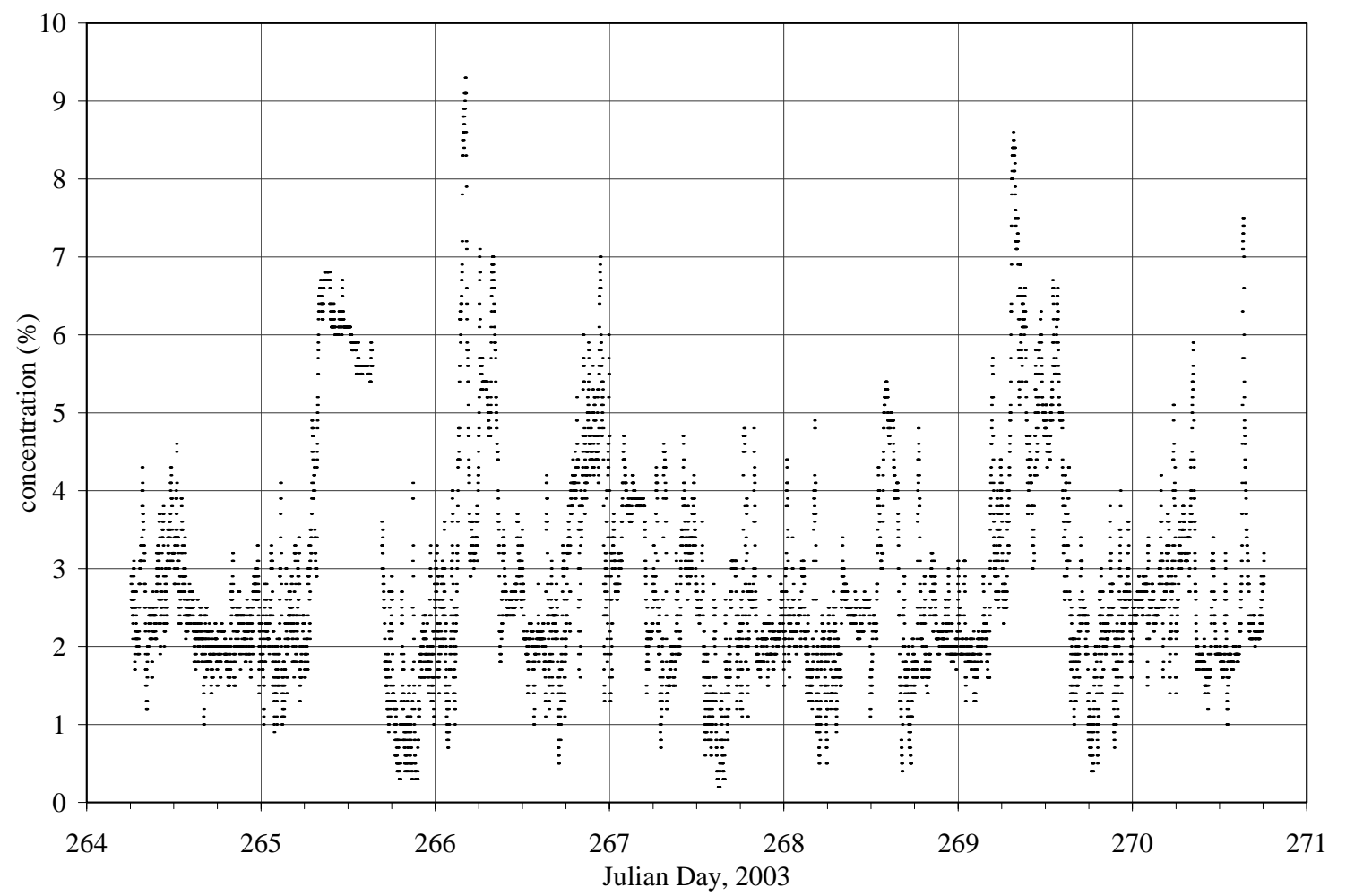

Figure D25. $\mathrm{O}_{2}$ concentration, Boiler 2. Julian Day 264 = September 21, 2003.

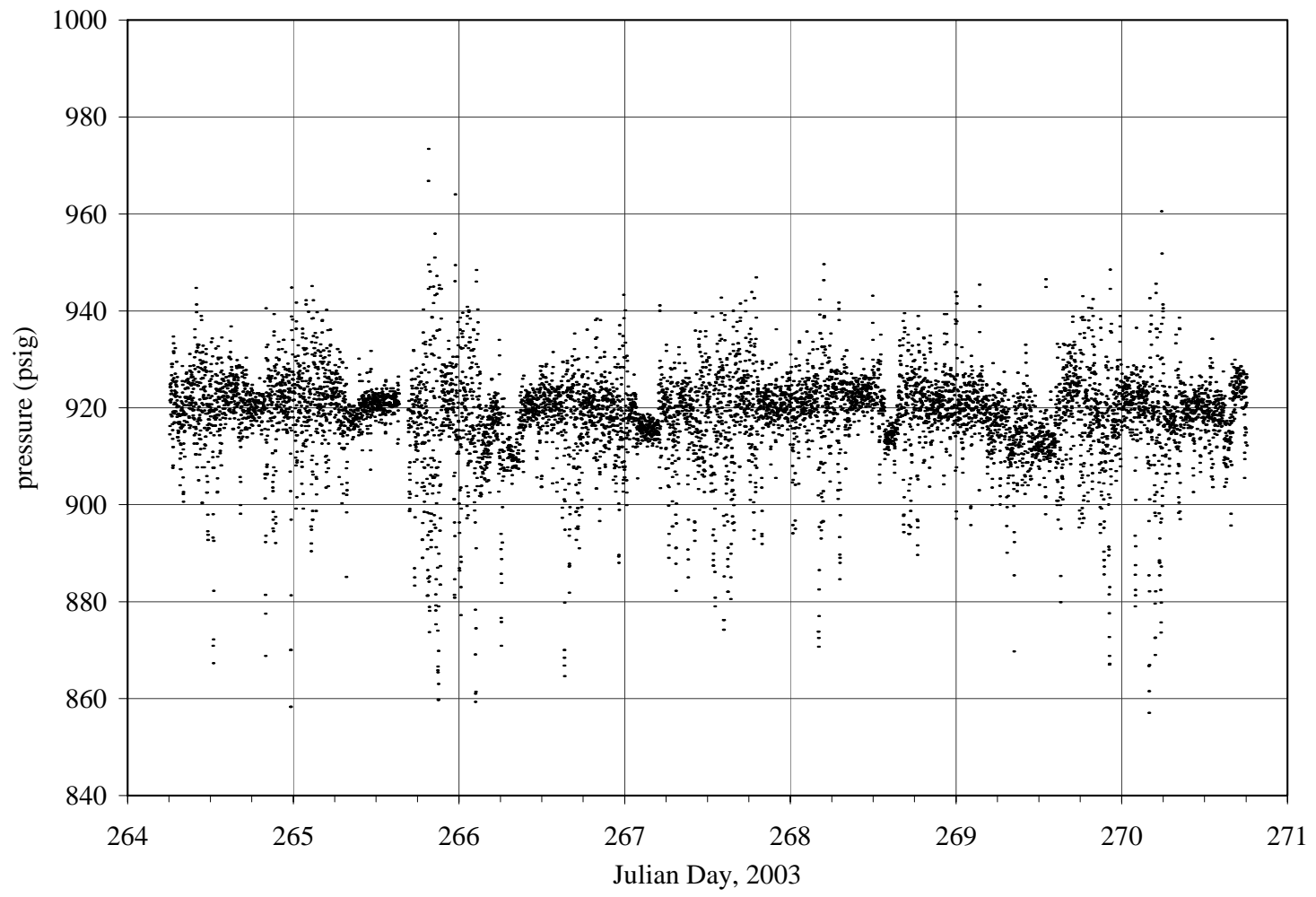

Figure D26. Drum pressure, Boiler 2. Julian Day 264 = September 21, 2003. 


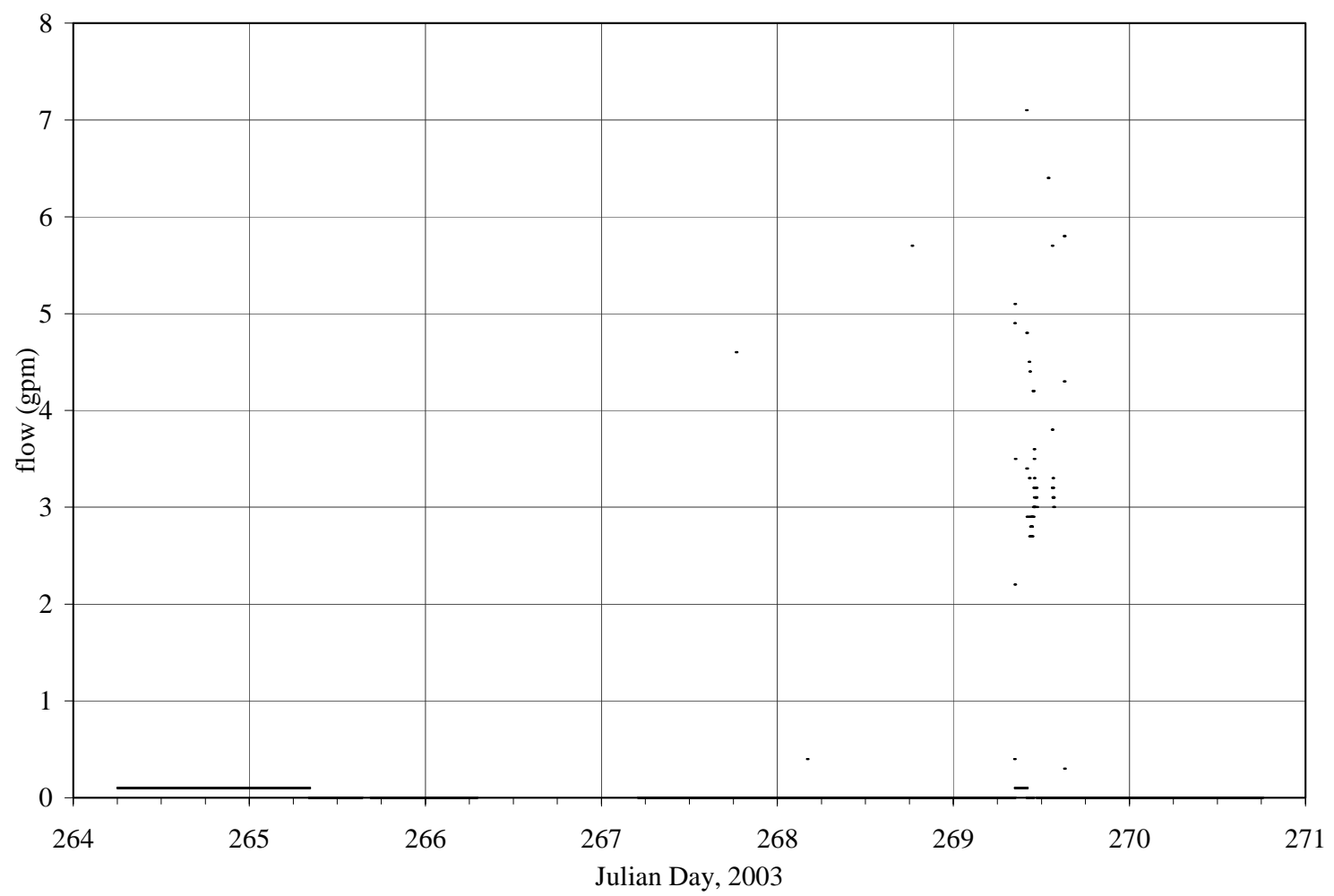

Figure D27. Fuel Oil flow rate, Boiler 2. Julian Day 264 = September 21, 2003.

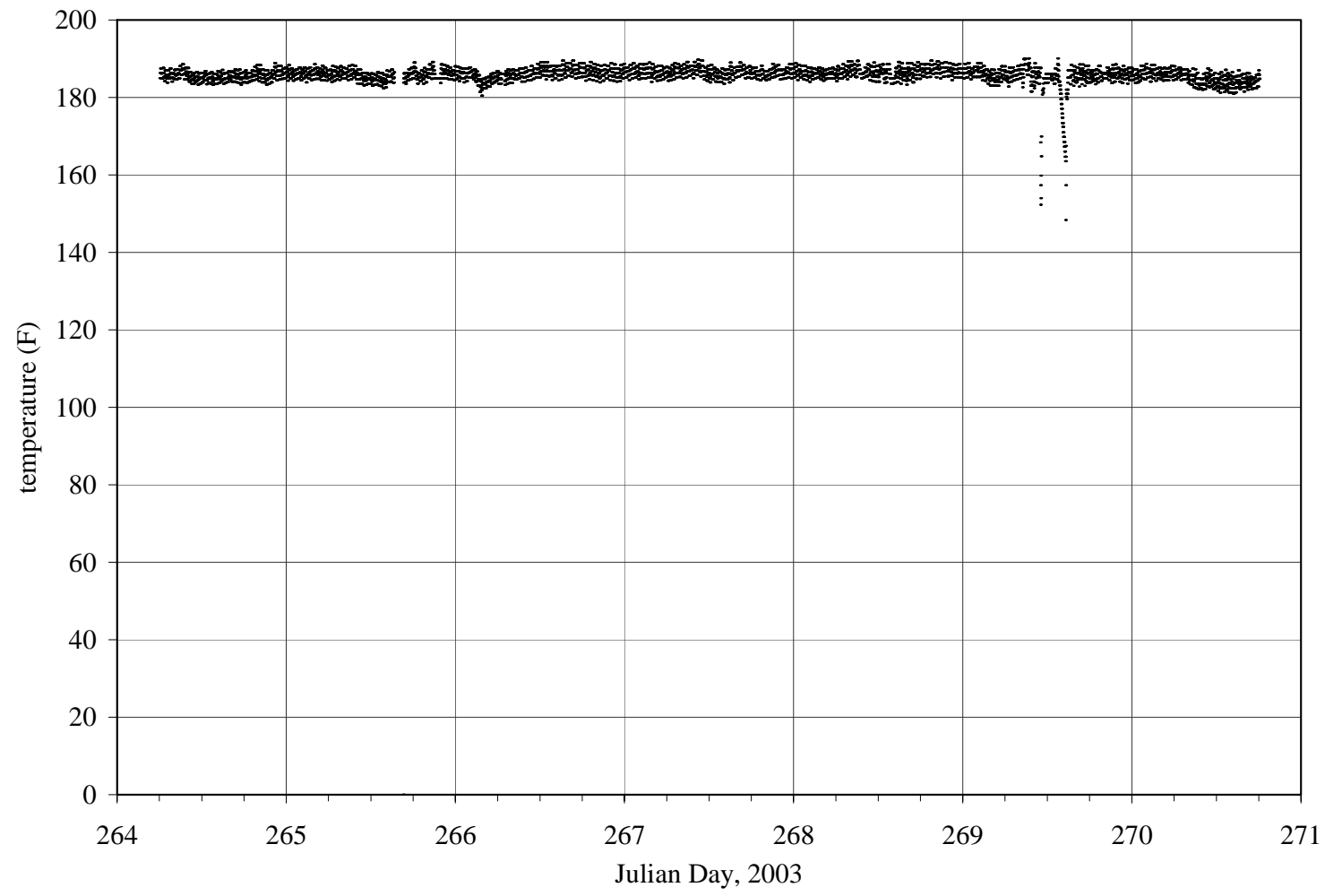

Figure D28. Fuel Oil temperature, Boiler 2. Julian Day 264 = September 21, 2003. 


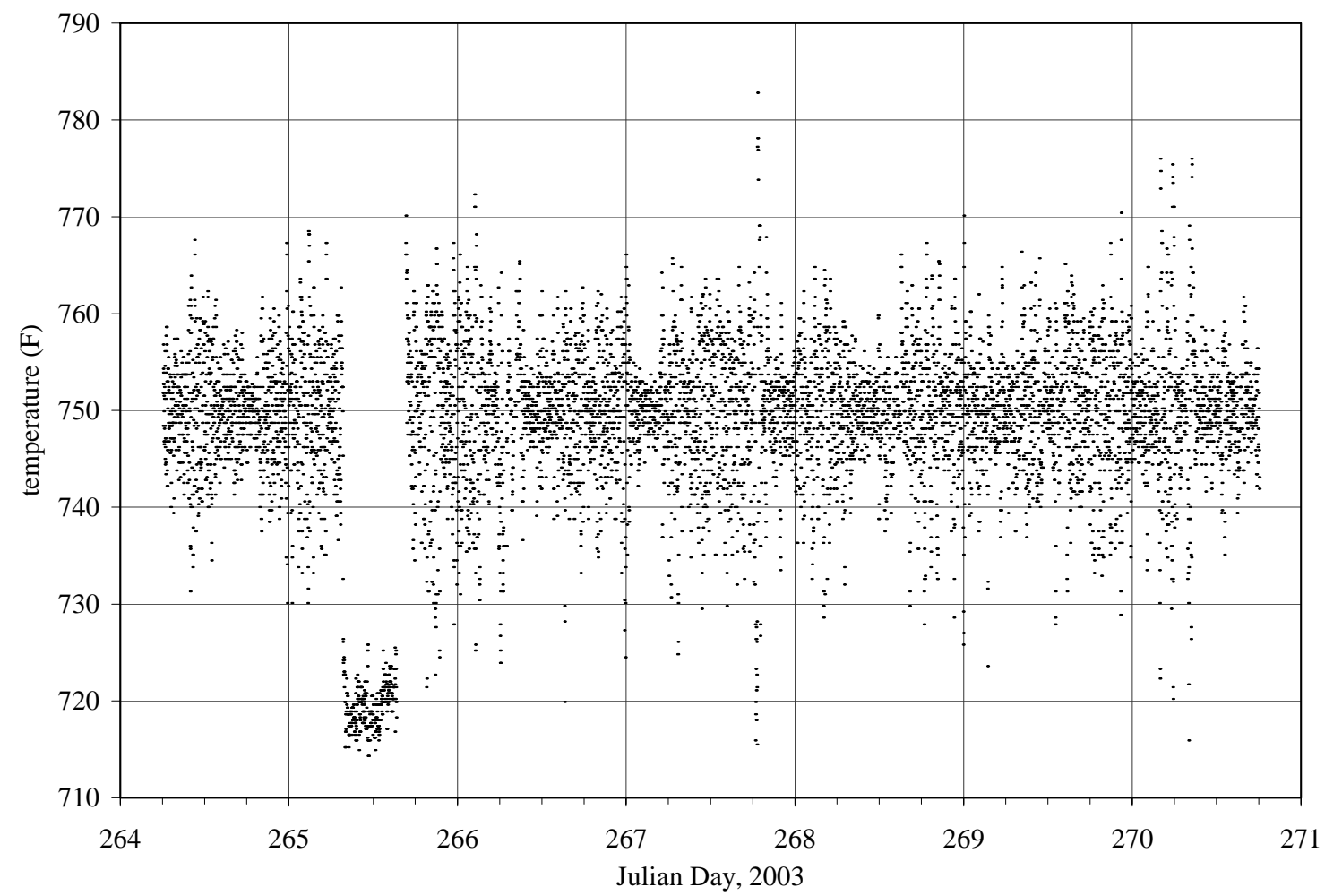

Figure D29. Steam temperature, Boiler 2. Julian Day 264 = September 21, 2003. 


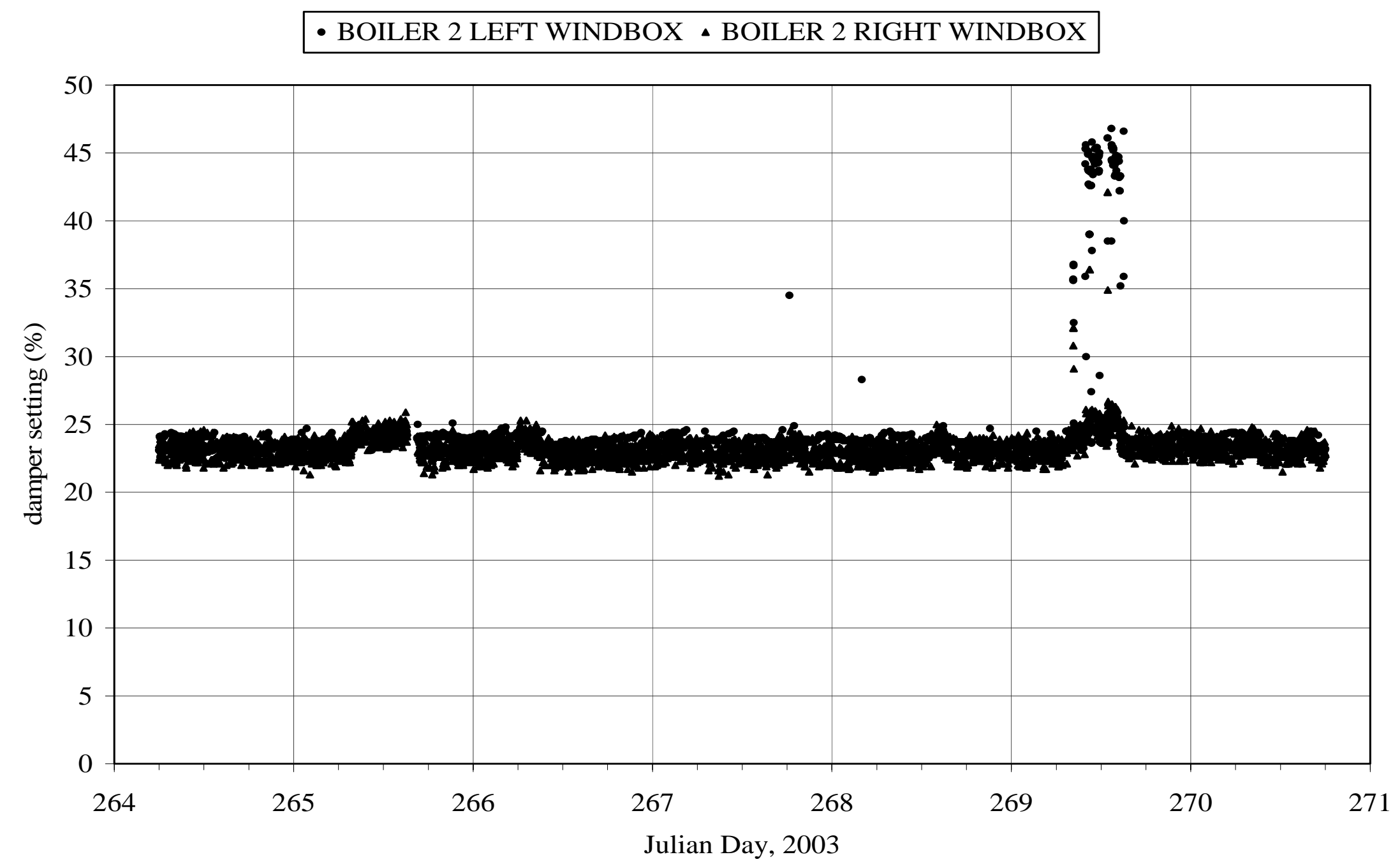

Figure D30. Air flow rates in left and right windbox, Boiler 2. Julian Day 264 = September 21, 2003. 


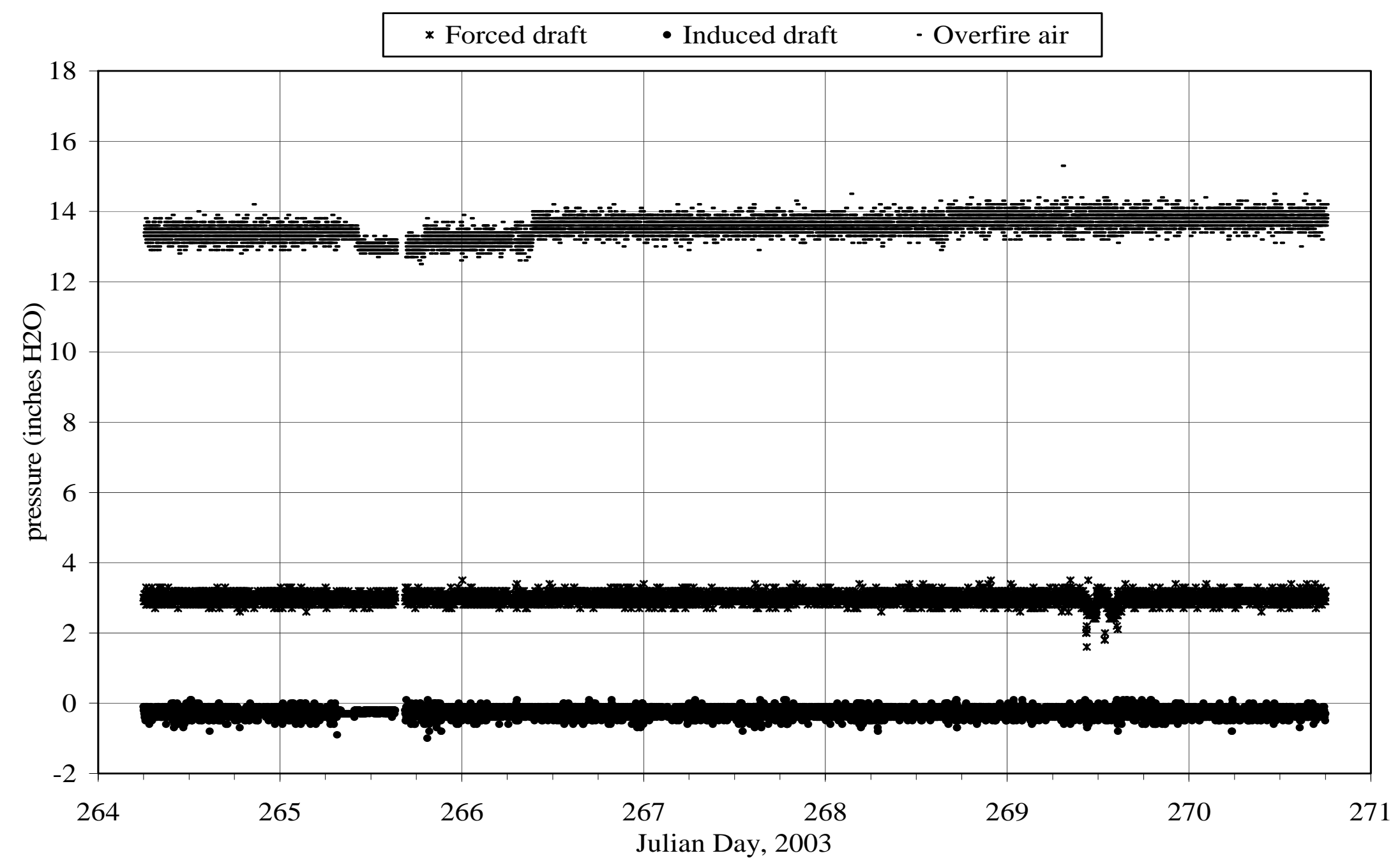

Figure D31. Forced draft, induced draft and overfire air pressure, Boiler 2. Julian Day 264 = September 21, 2003. 


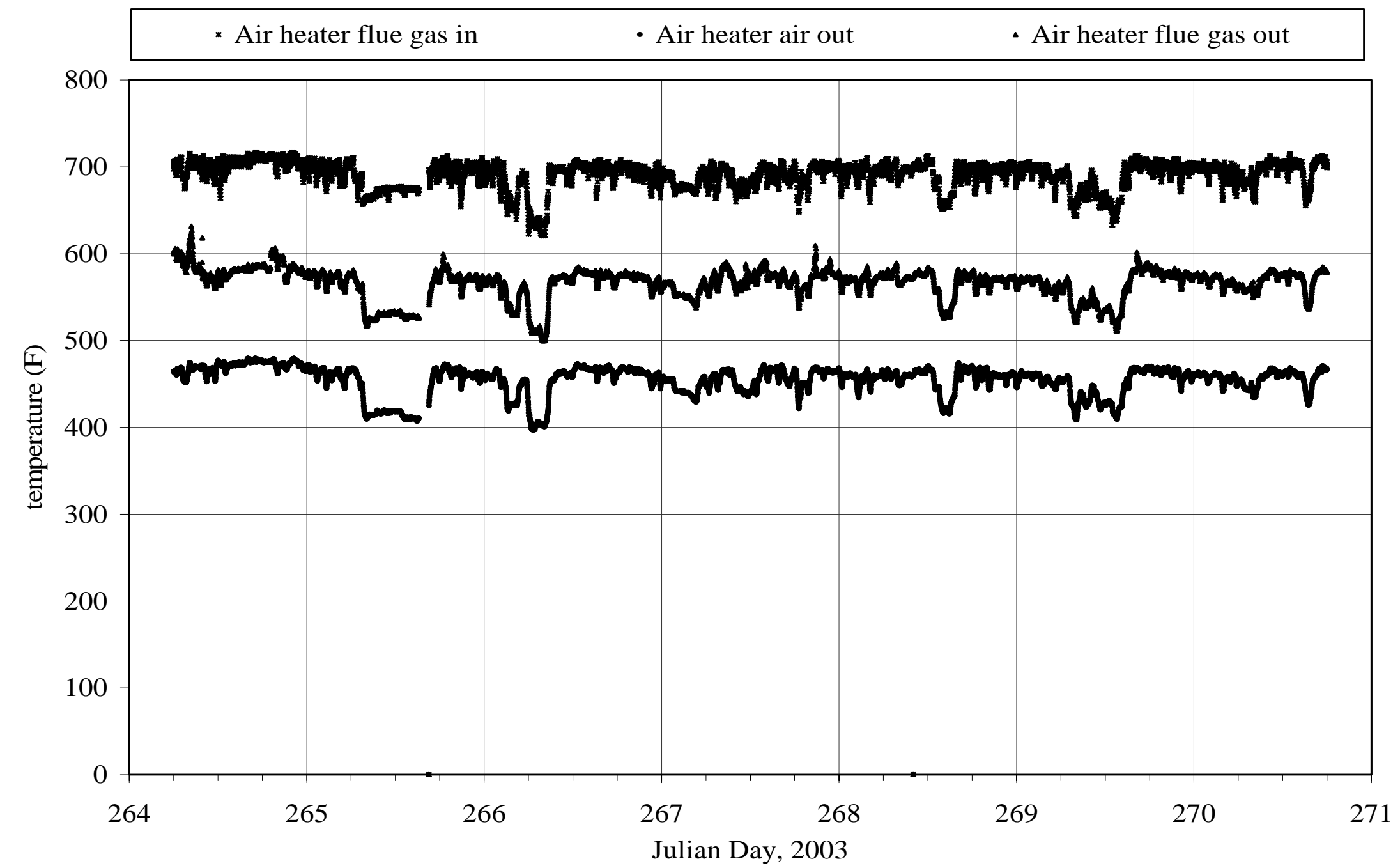

Figure D32. Air heater, flue gas in, flue gas out and air out temperature, Boiler 2. Julian Day $264=$ September 21, 2003. 


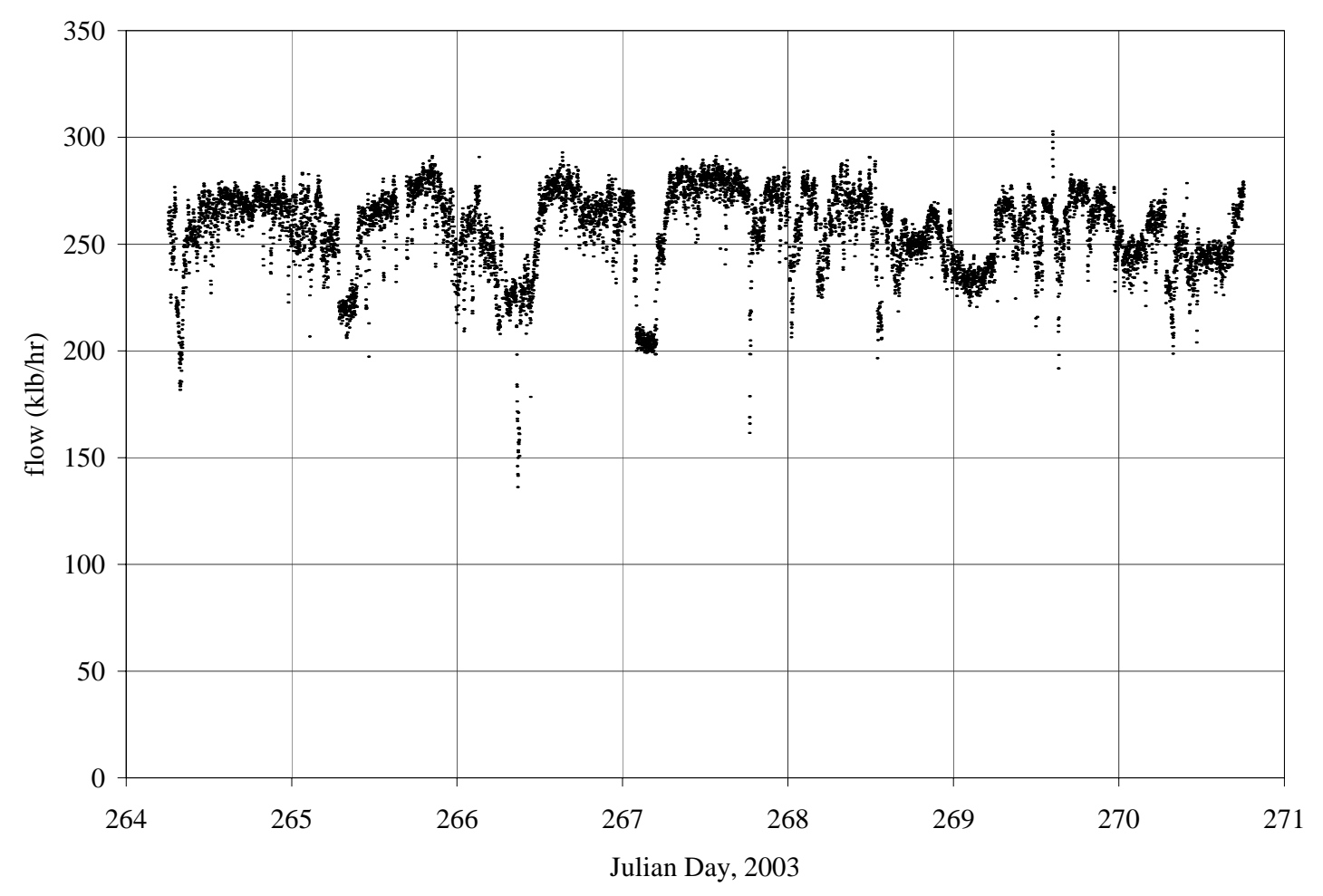

Figure D33. Steam flow rate, Boiler 3. Julian Day 264 = September 21, 2003.

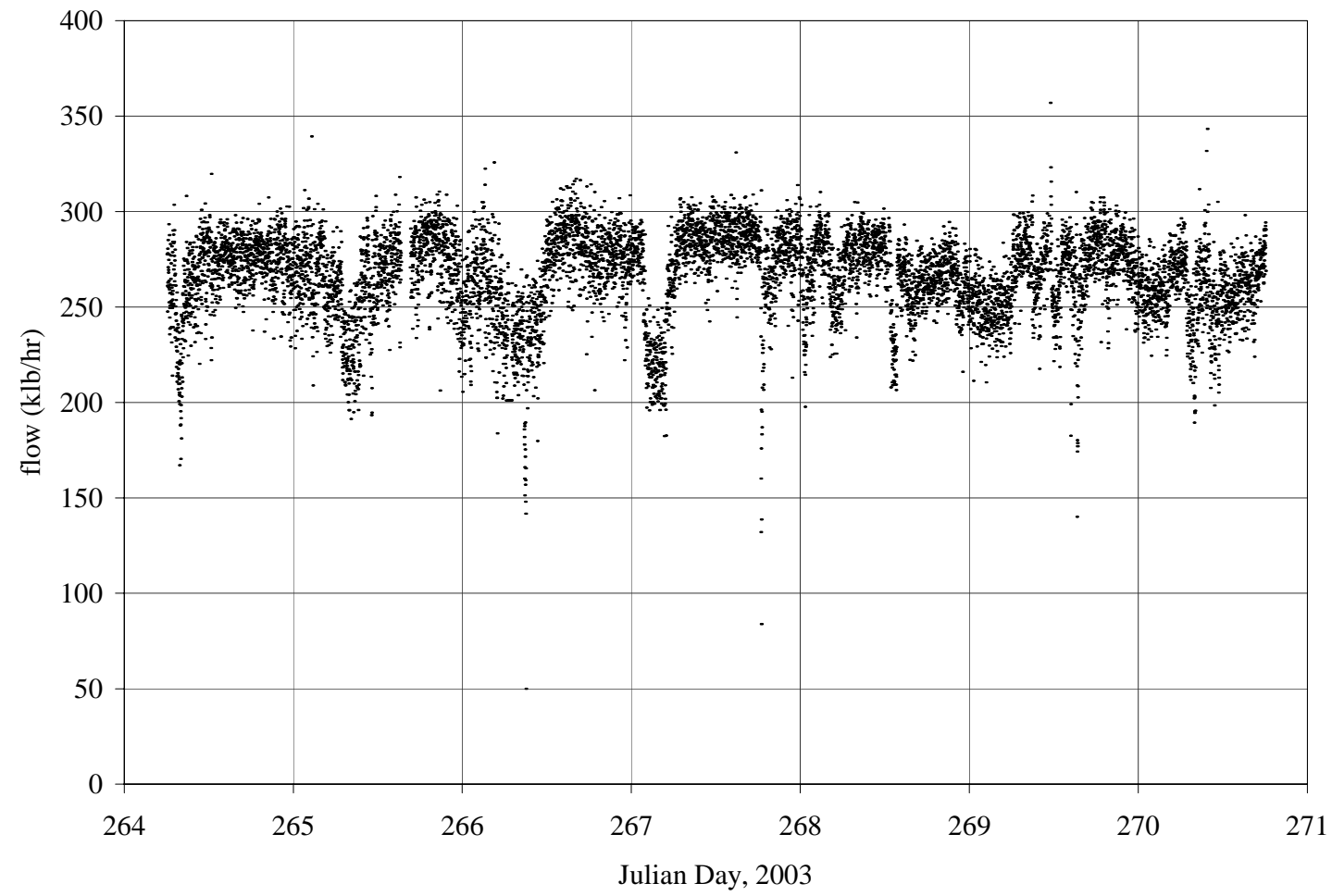

Figure D34. Feed water flow rate, Boiler 3. Julian Day 264 = September 21, 2003. 


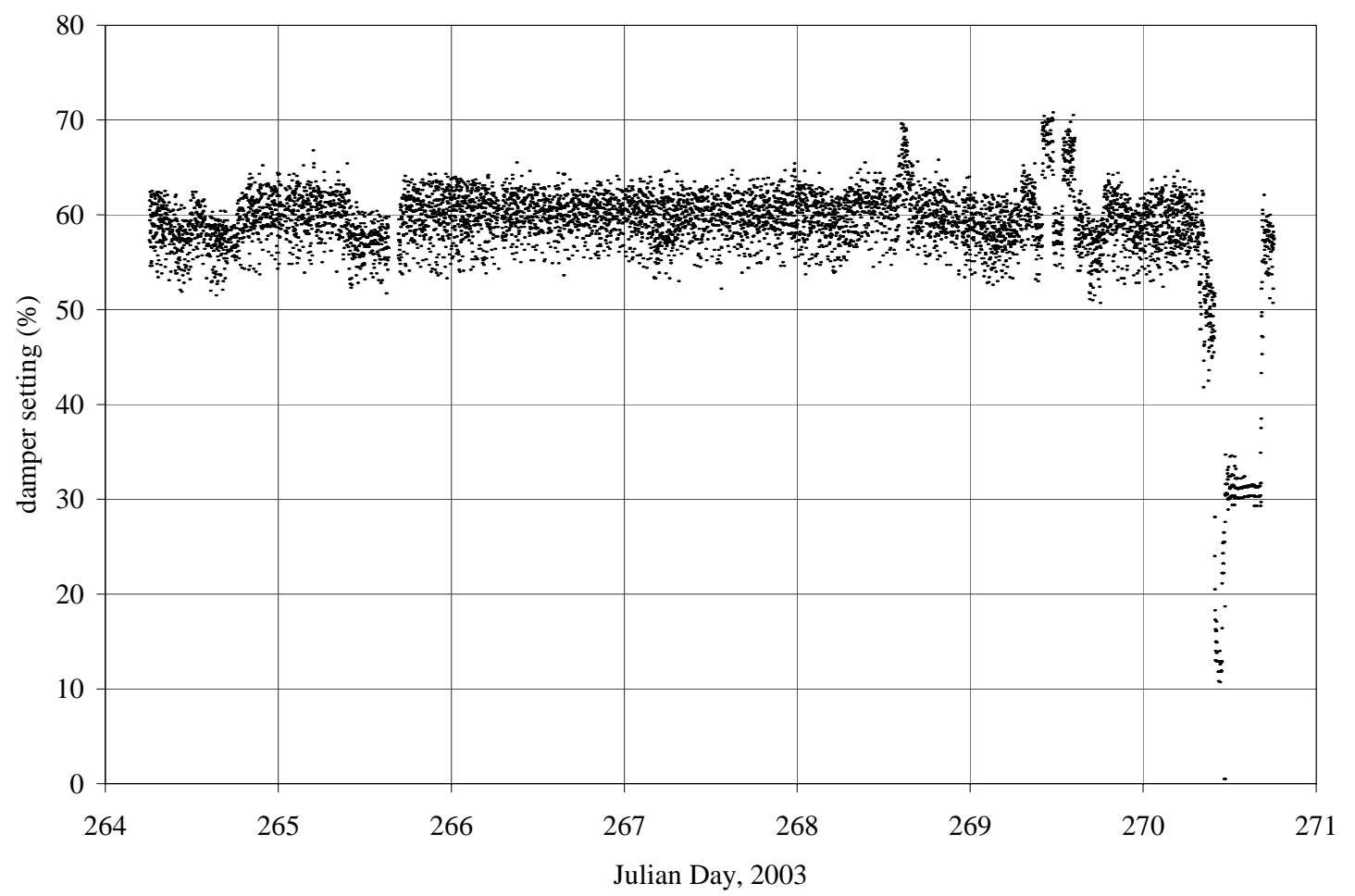

Figure D35. Right grate air flow rate, Boiler 3. Julian Day 264 = September 21, 2003.

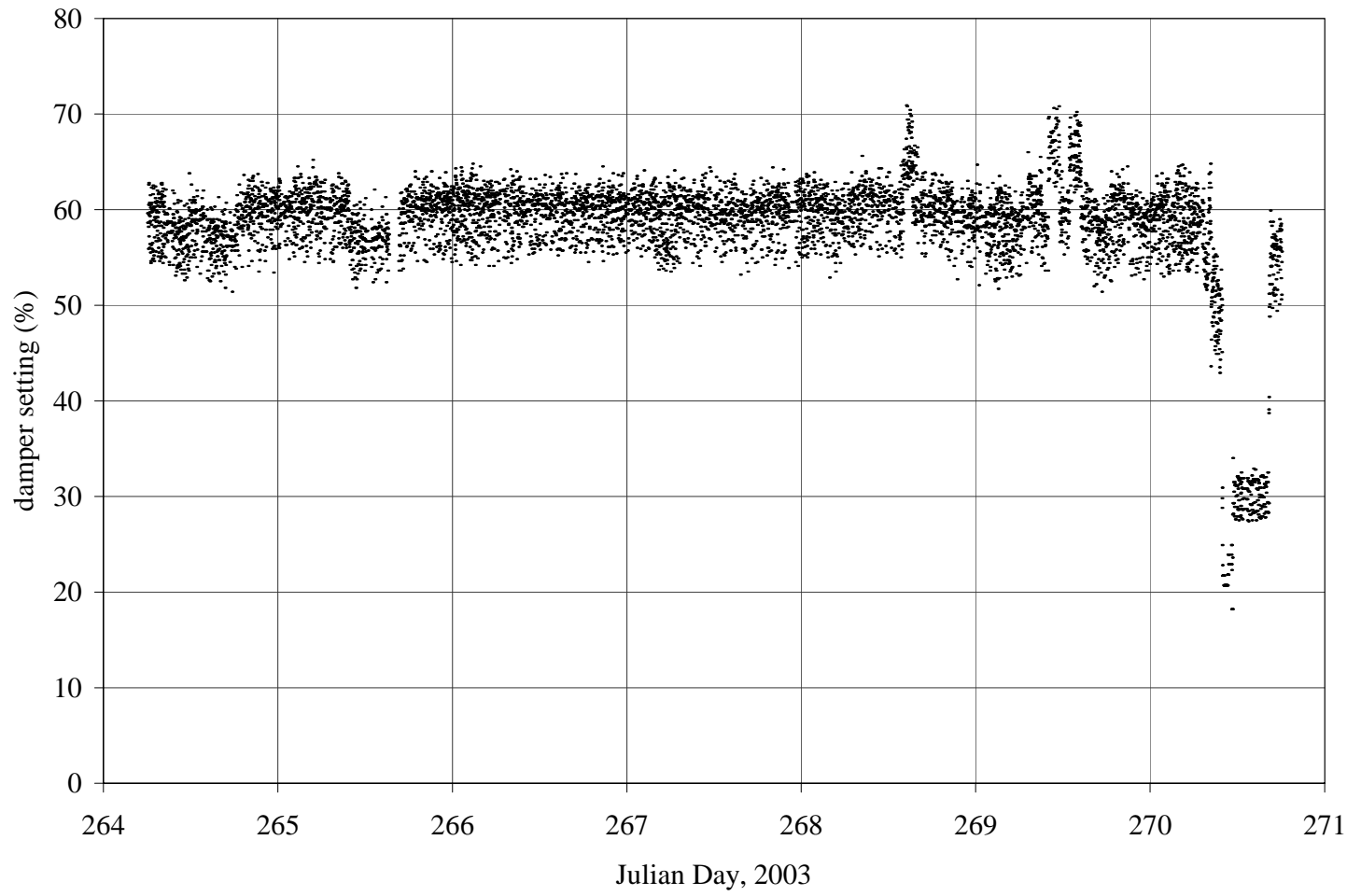

Figure D36. Left grate air flow rate, Boiler 3. Julian Day 264 = September 21, 2003. 


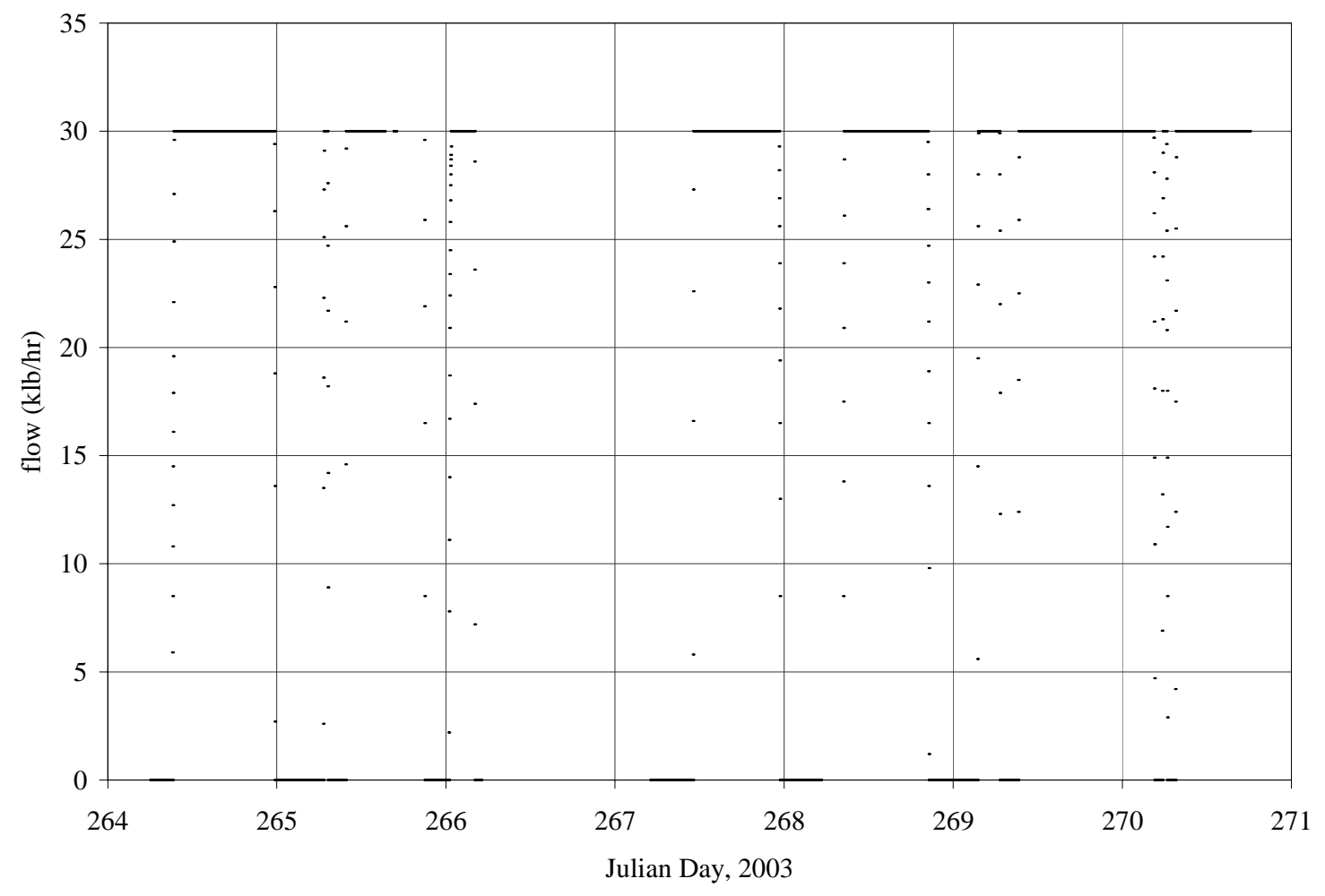

Figure D37. Continuous blowdown flow rate, Boiler 3. Julian Day $264=$ September 21, 2003.

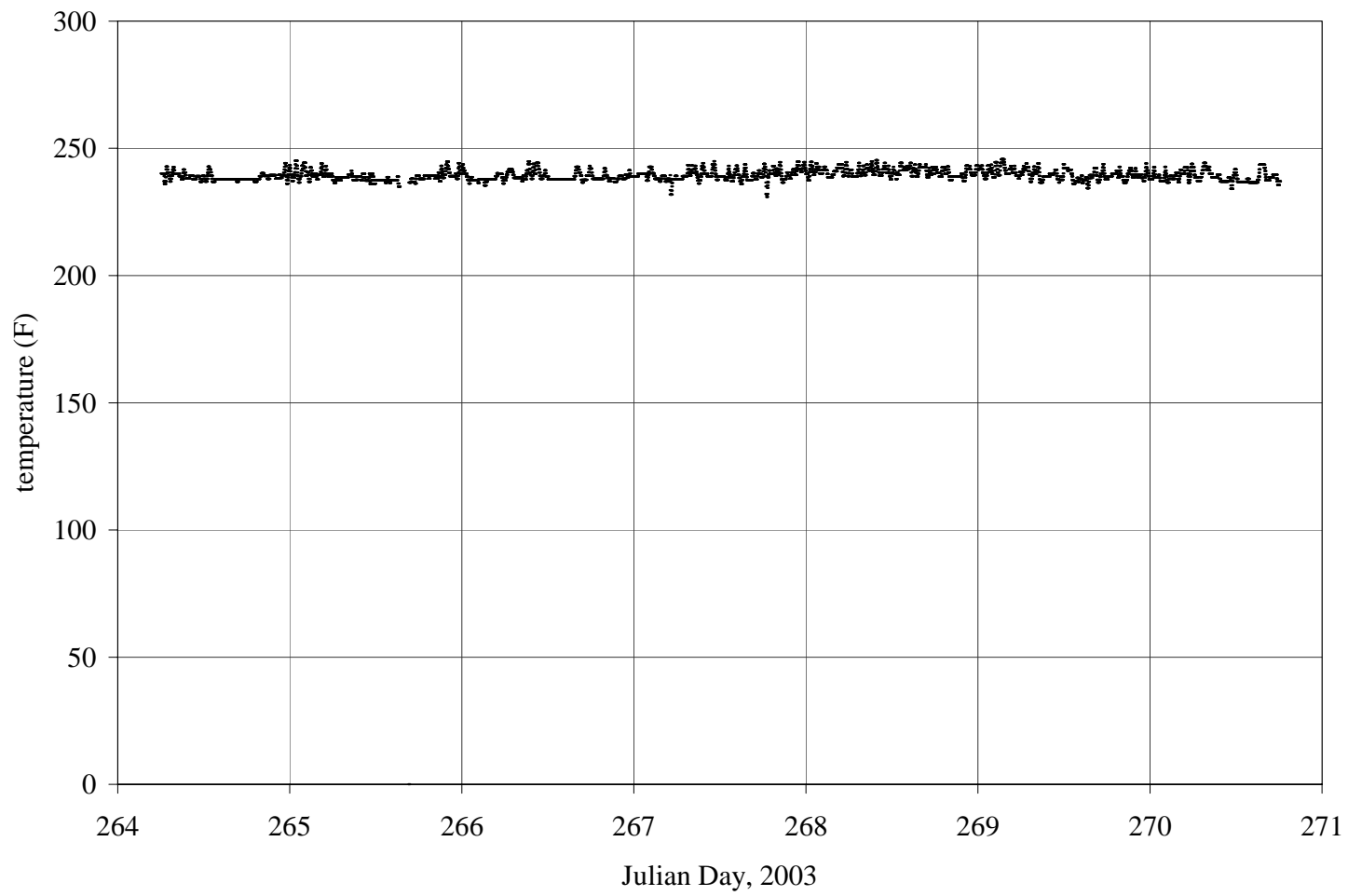

Figure D38. Feedwater temperature, Boiler 3. Julian Day 264 = September 21, 2003. 


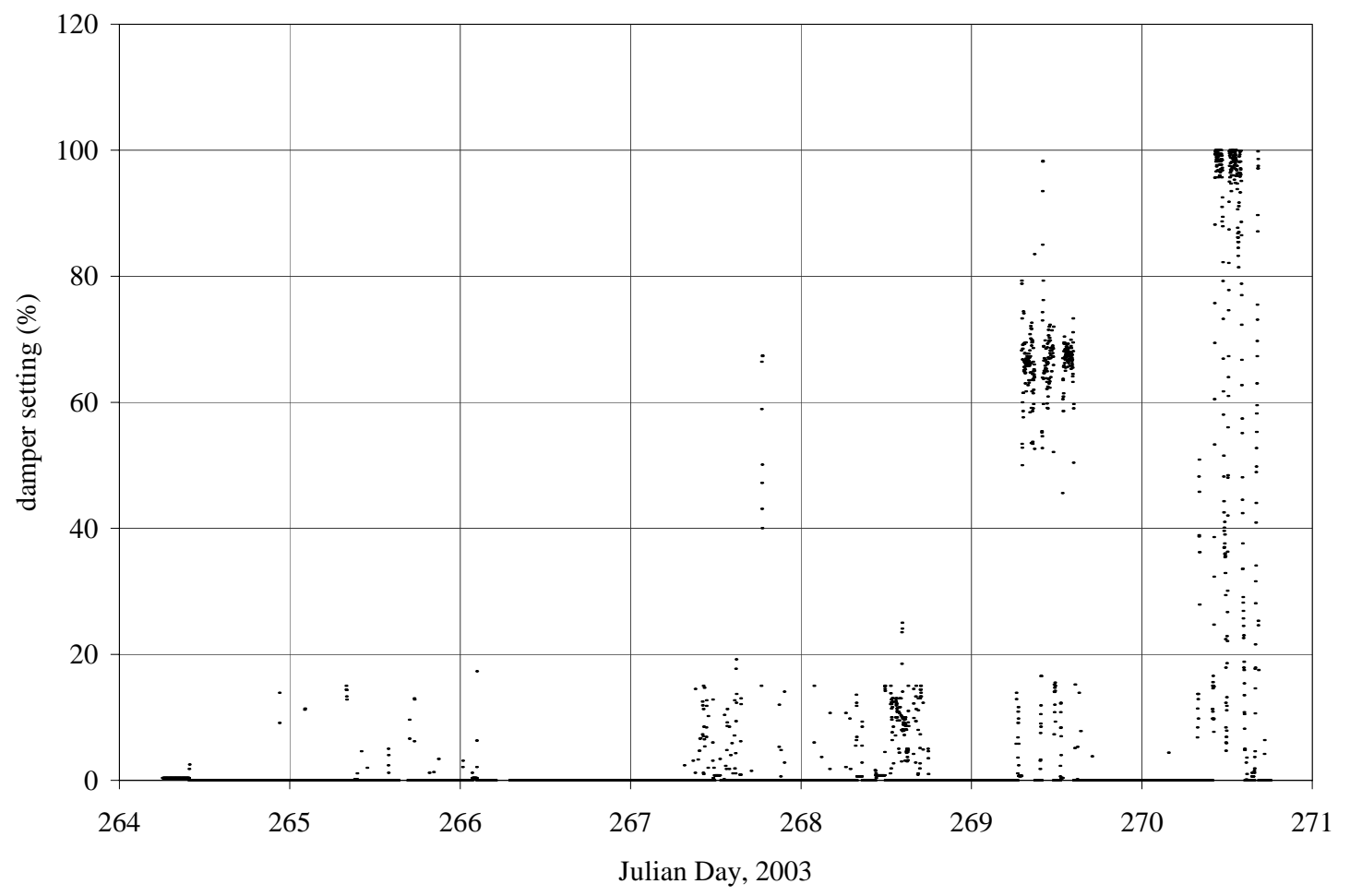

Figure D39. Coal flow rate, Boiler 3. Julian Day 264 = September 21, 2003.

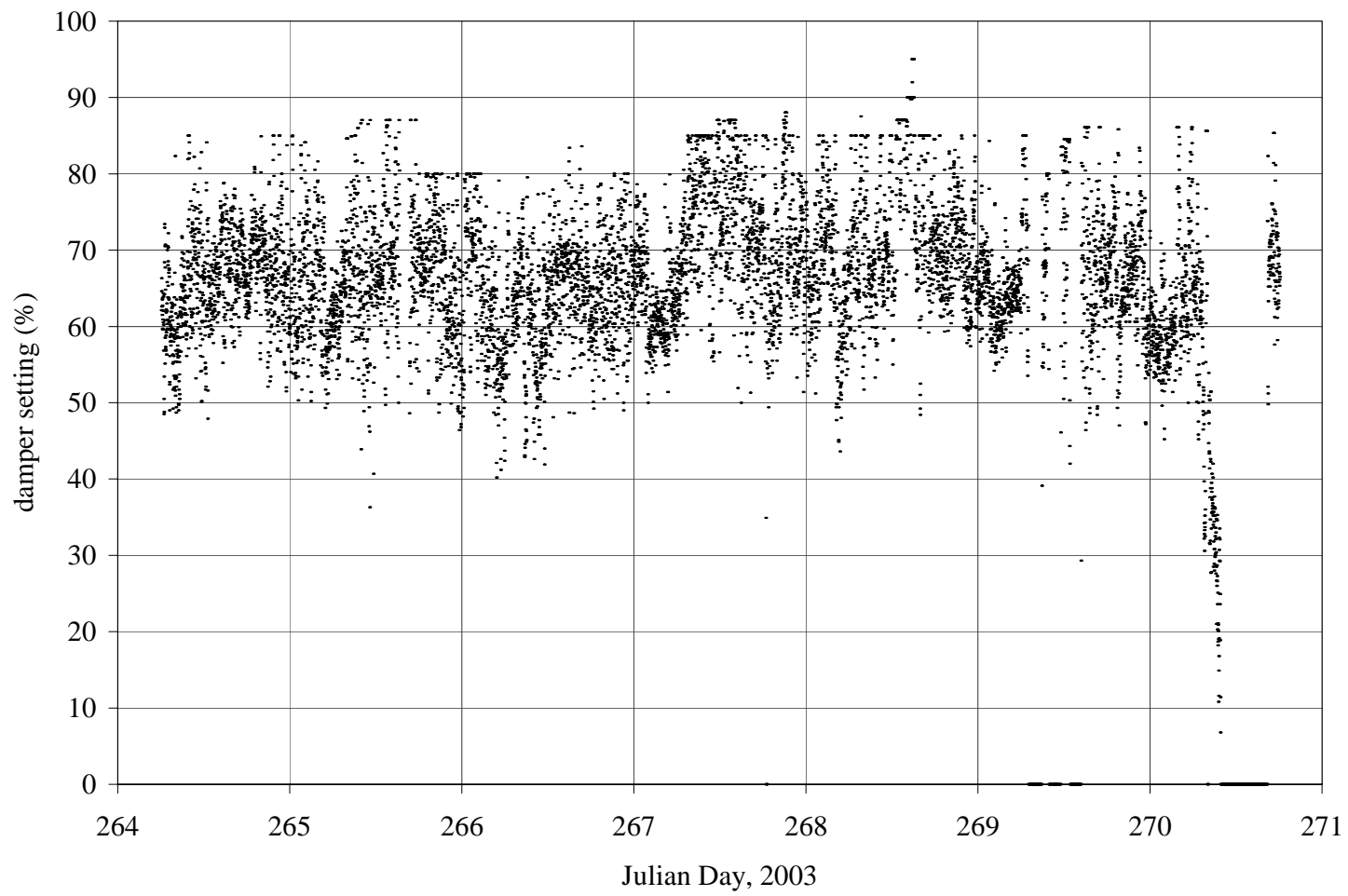

Figure D40. Bagasse feed rate, Boiler 3. Julian Day 264 = September 21, 2003. 


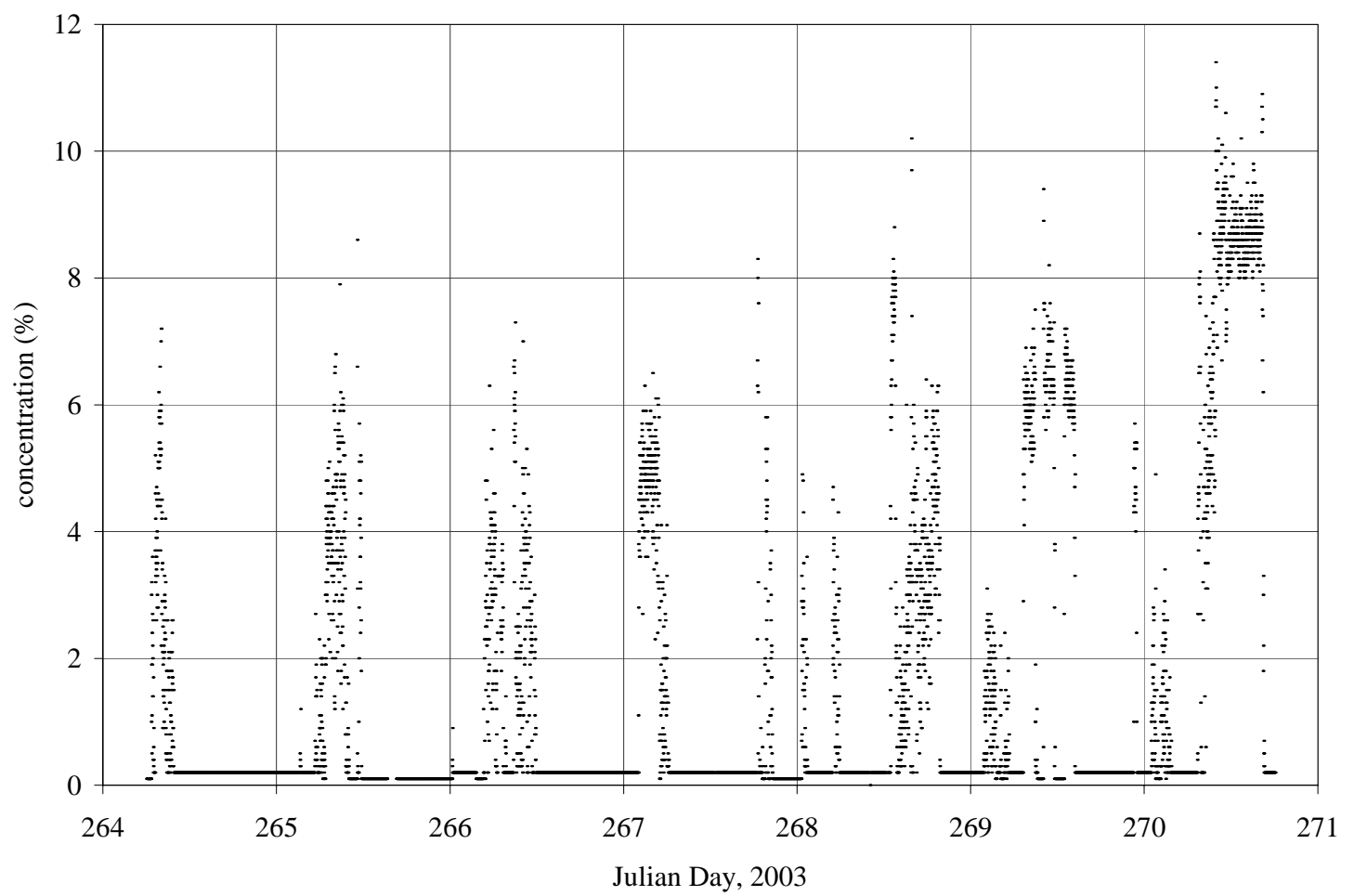

Figure D41. $\mathrm{O}_{2}$ concentration, Boiler 3. Julian Day 264 = September 21, 2003.

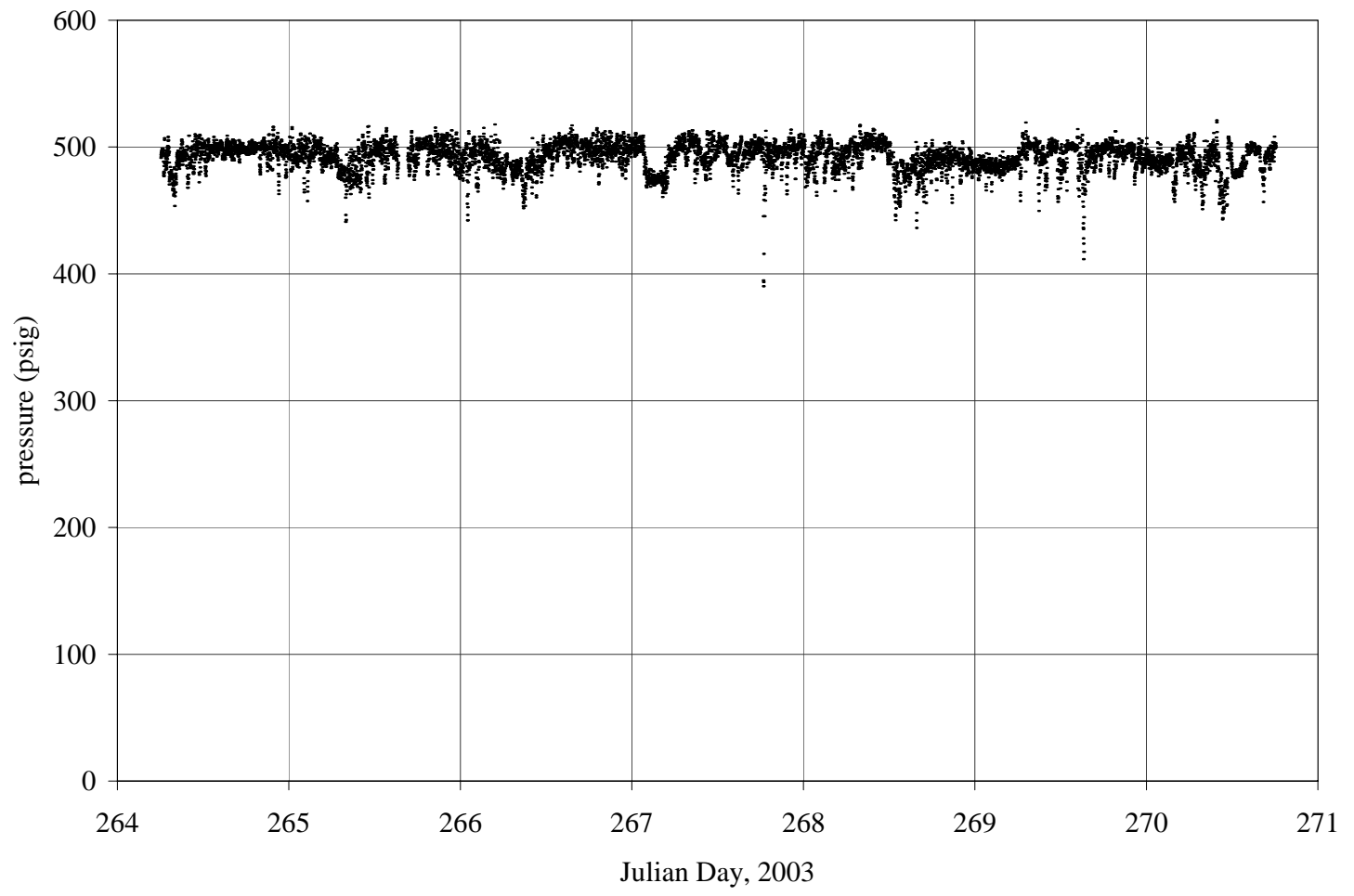

Figure D42. Drum pressure, Boiler 3. Julian Day 264 = September 21, 2003. 


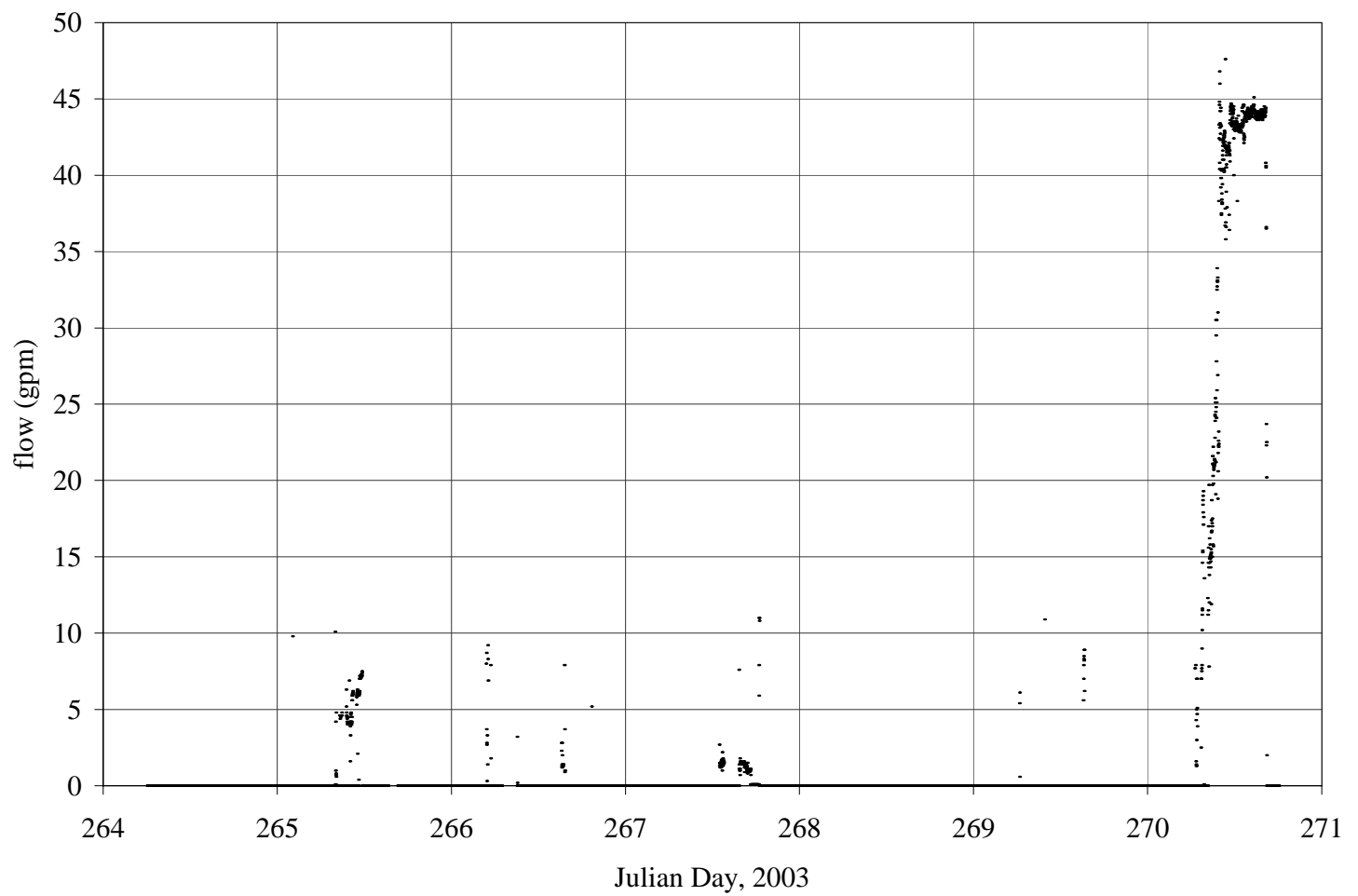

Figure D43. Fuel Oil flow rate, Boiler 3. Julian Day 264 = September 21, 2003.

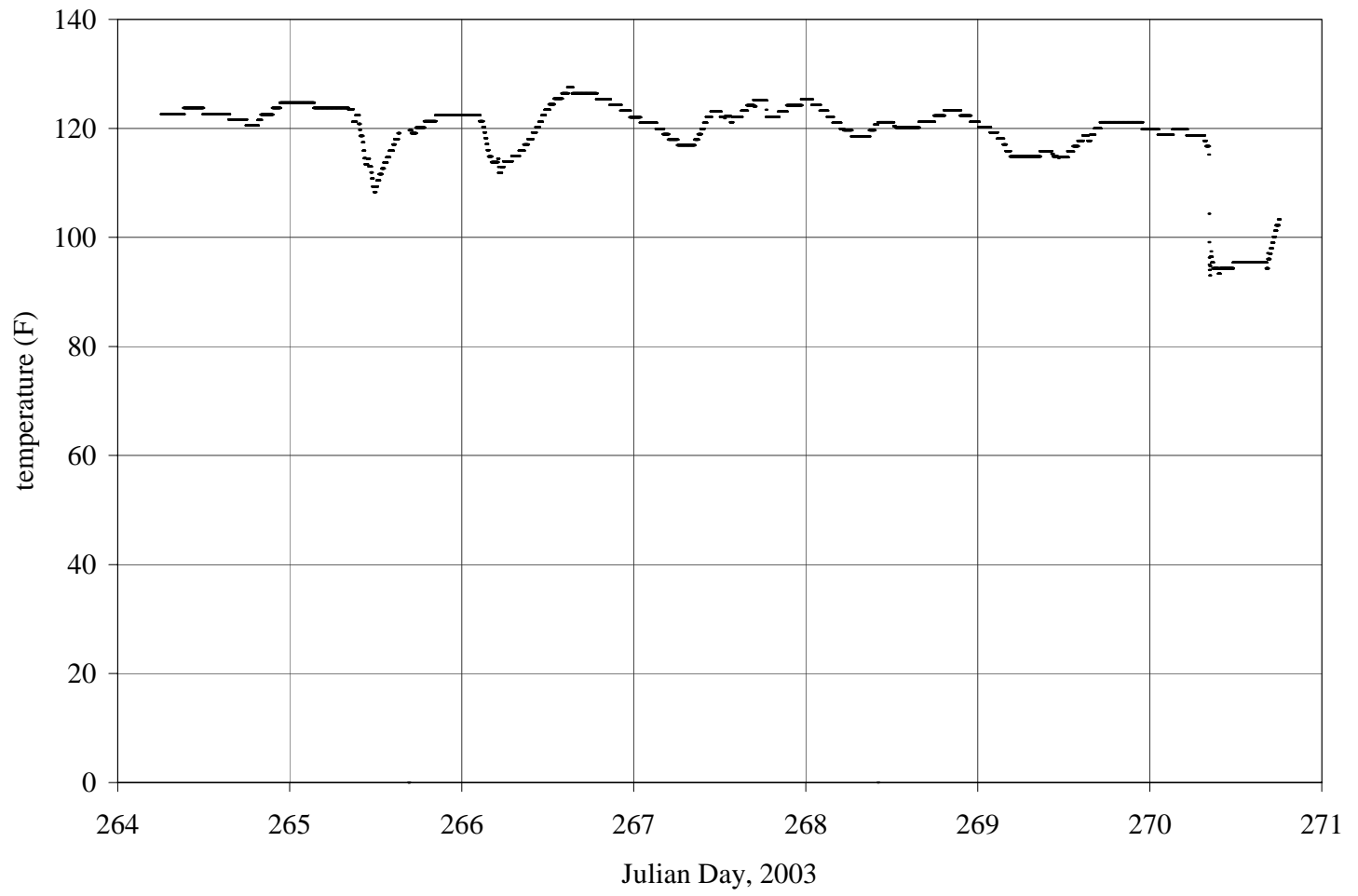

Figure D44. Fuel Oil temperature, Boiler 3. Julian Day 264 = September 21, 2003. 


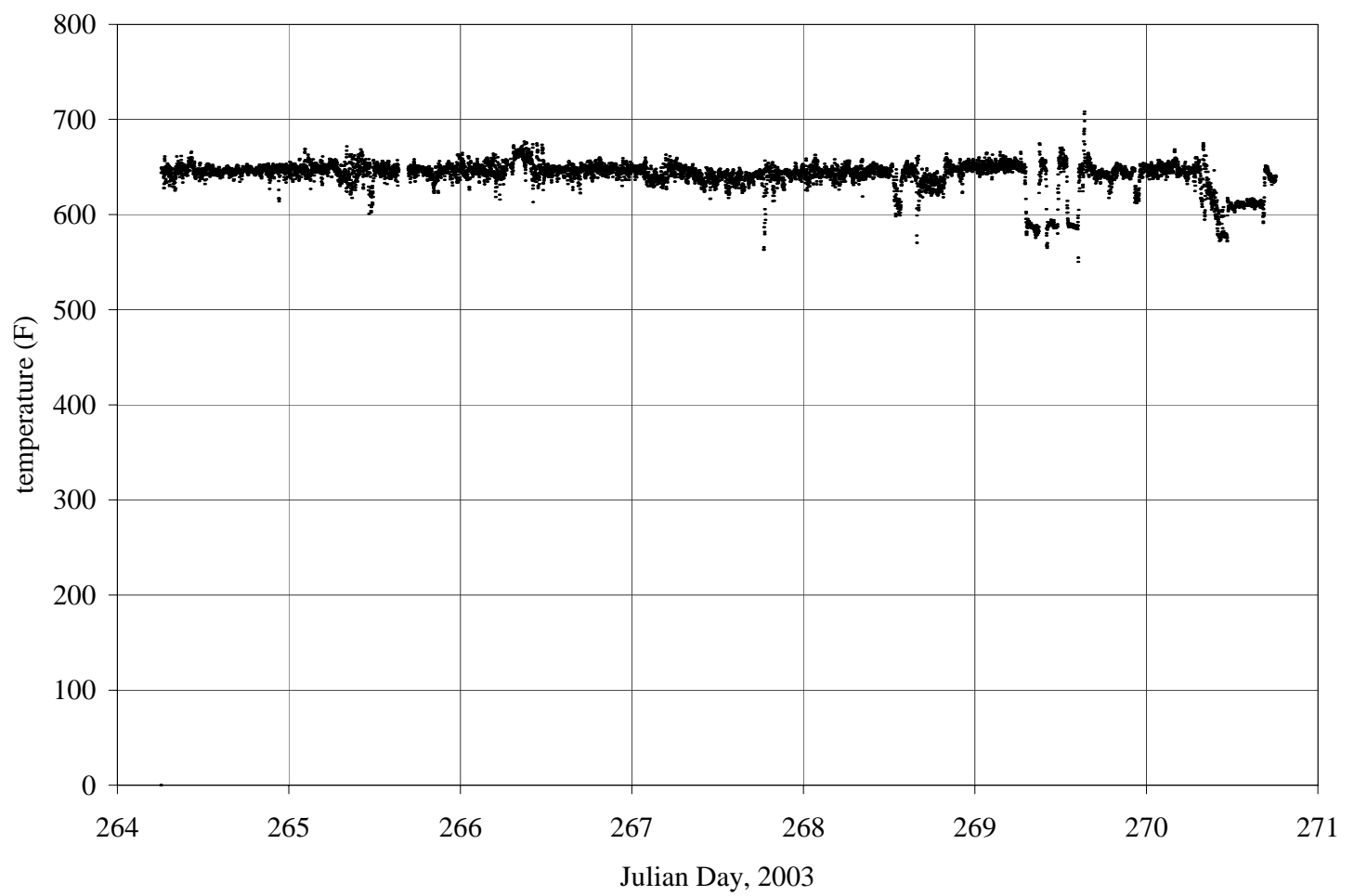

Figure D45. Primary steam temperature, Boiler 3. Julian Day $264=$ September 21, 2003.

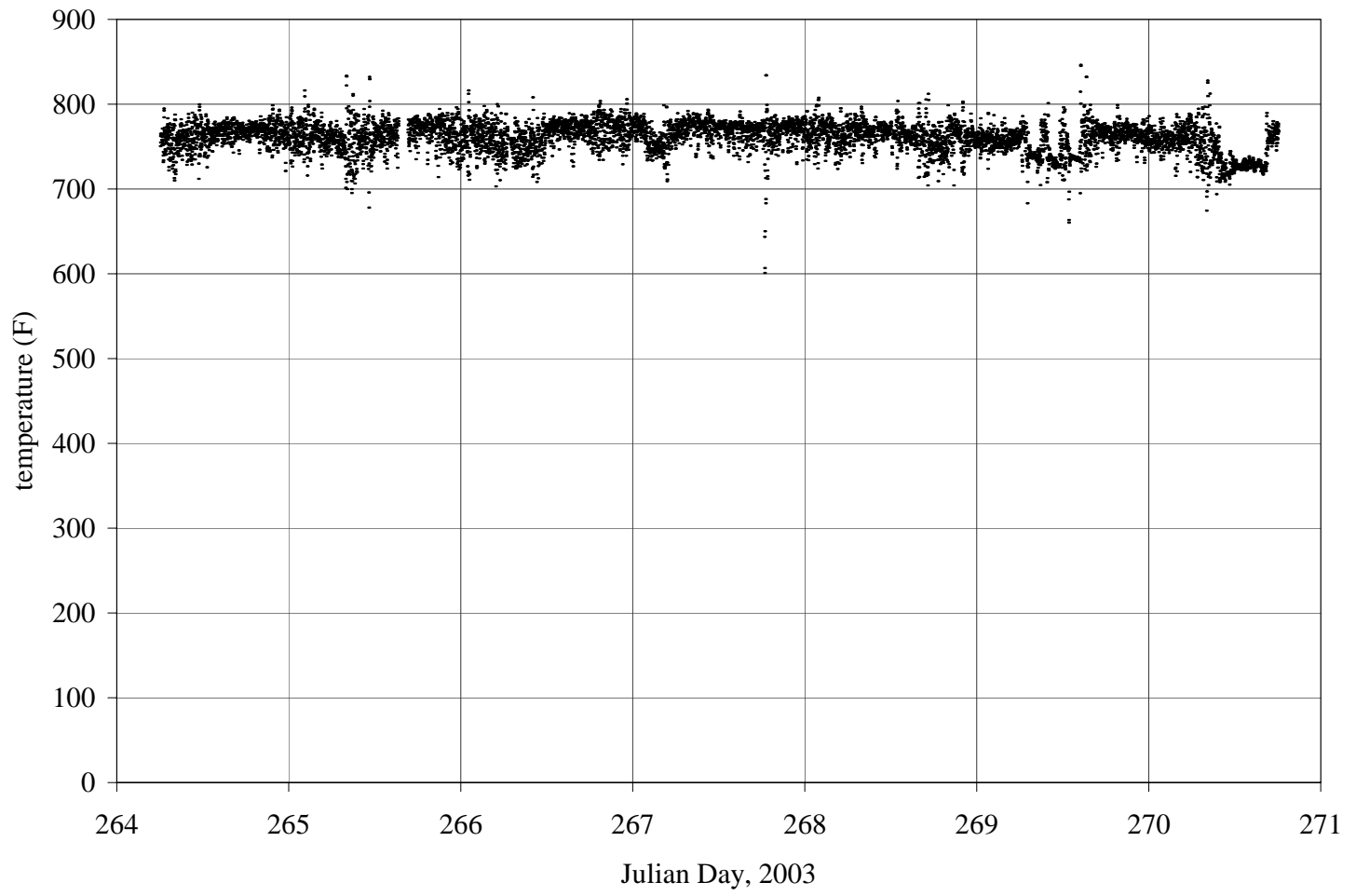

Figure D46. Final steam temperature, Boiler 3. Julian Day 264 = September 21, 2003. 


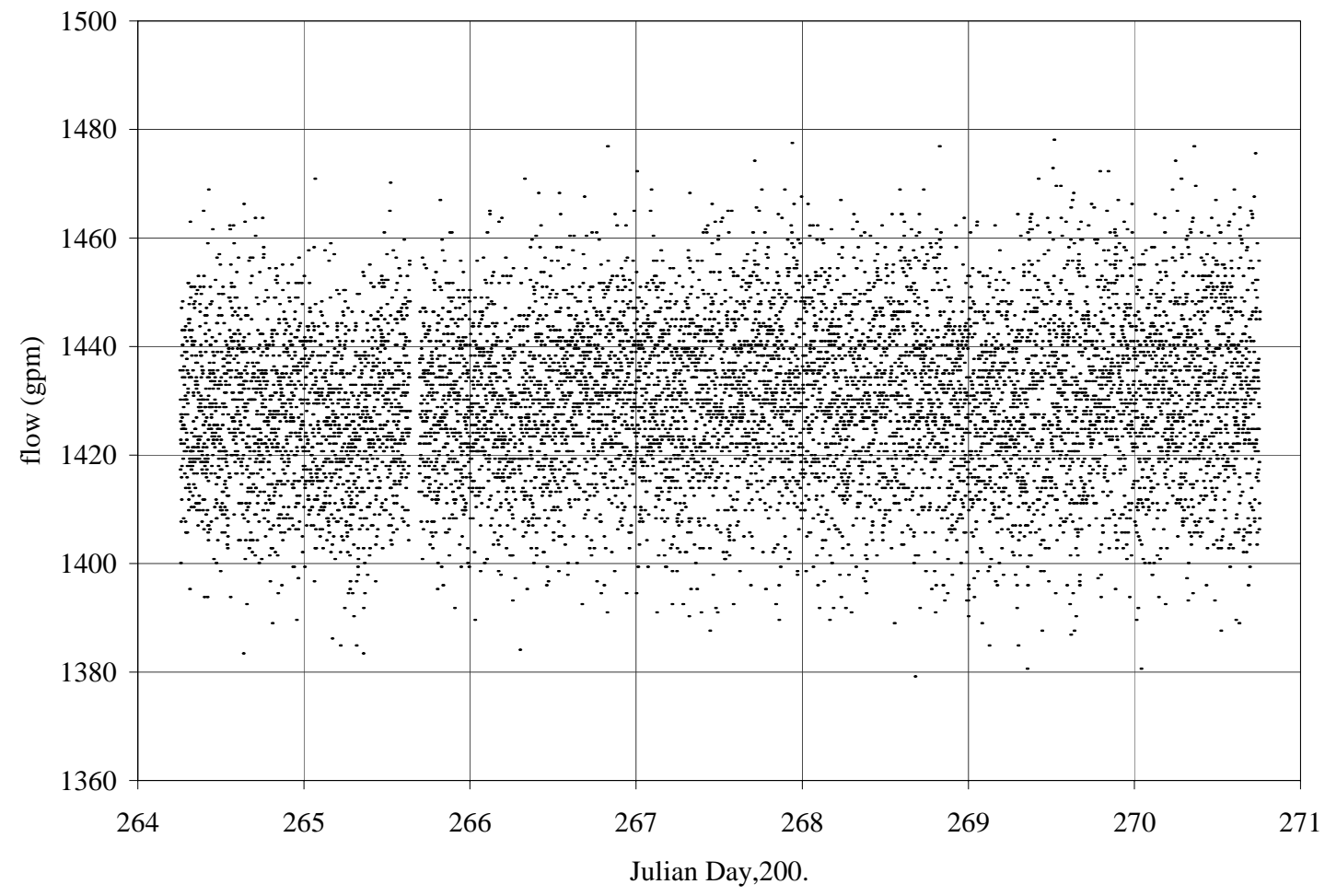

Figure D47. Wet scrubber flow rate, Boiler 3. Julian Day $264=$ September 21, 2003.

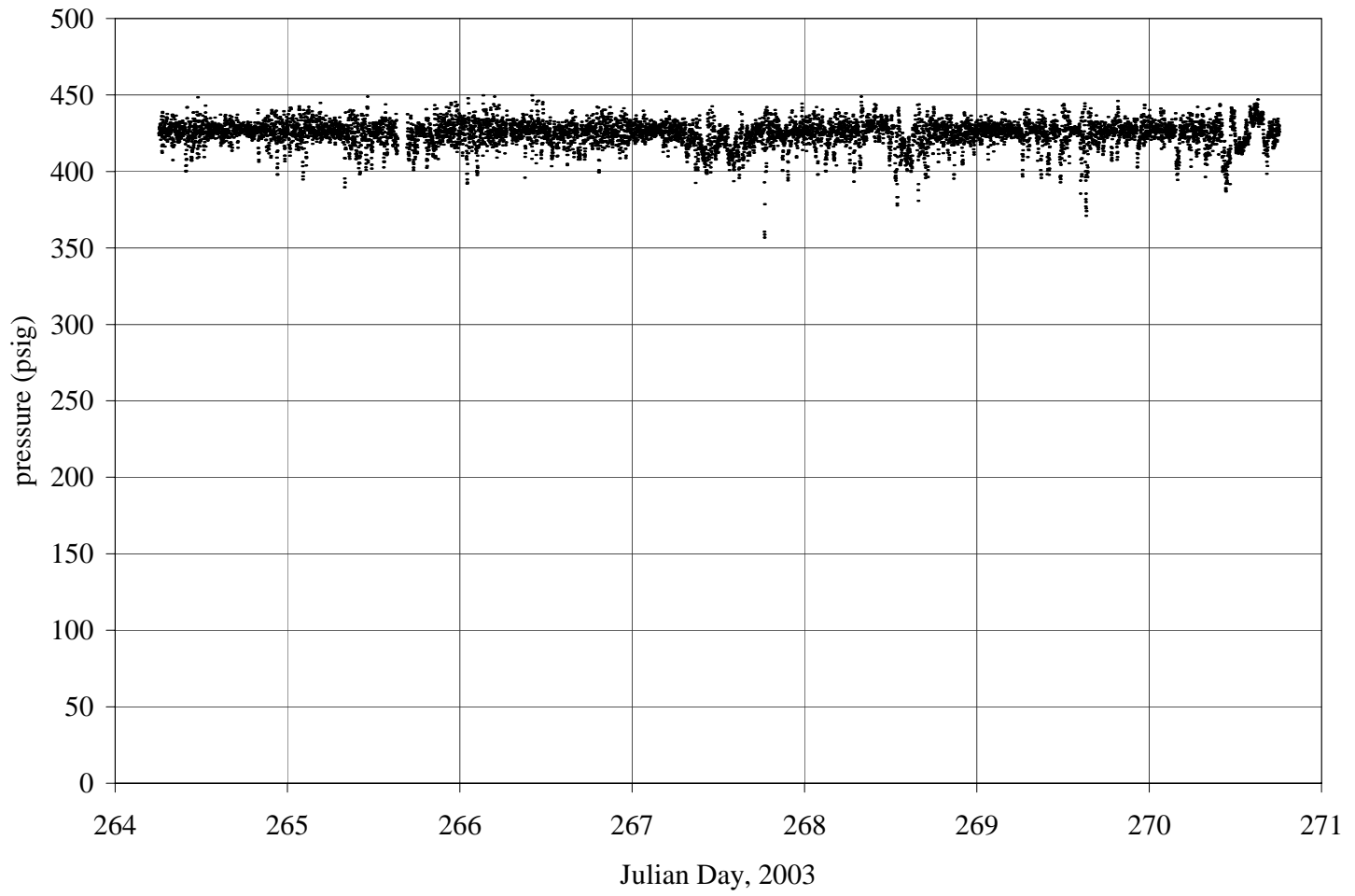

Figure D48. Steam header pressure, Boiler 3. Julian Day 264 = September 21, 2003. 


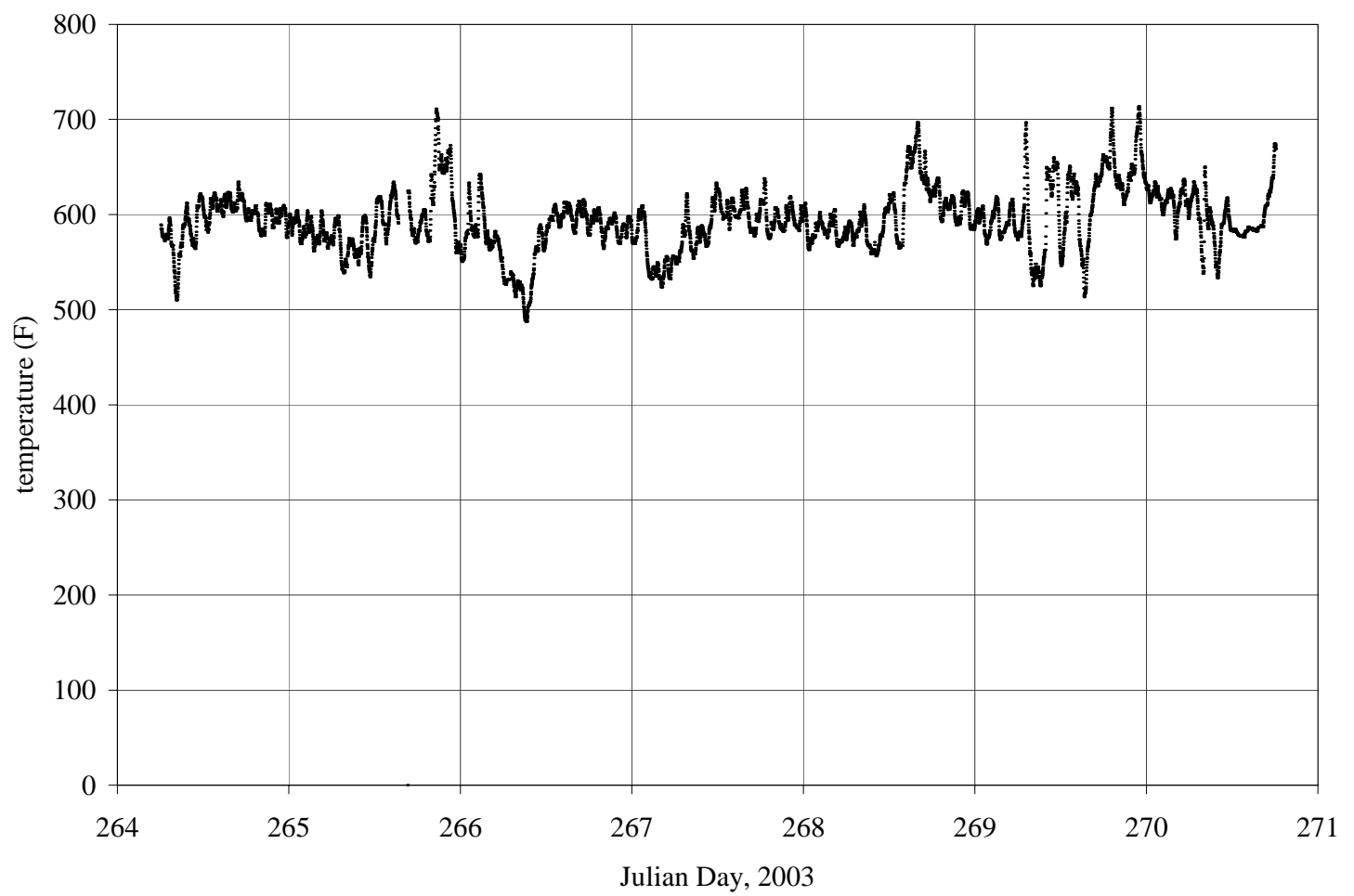

Figure D49. Grate temperature at thermocouple \#1, Boiler 3. Julian Day $264=$ September 21, 2003.

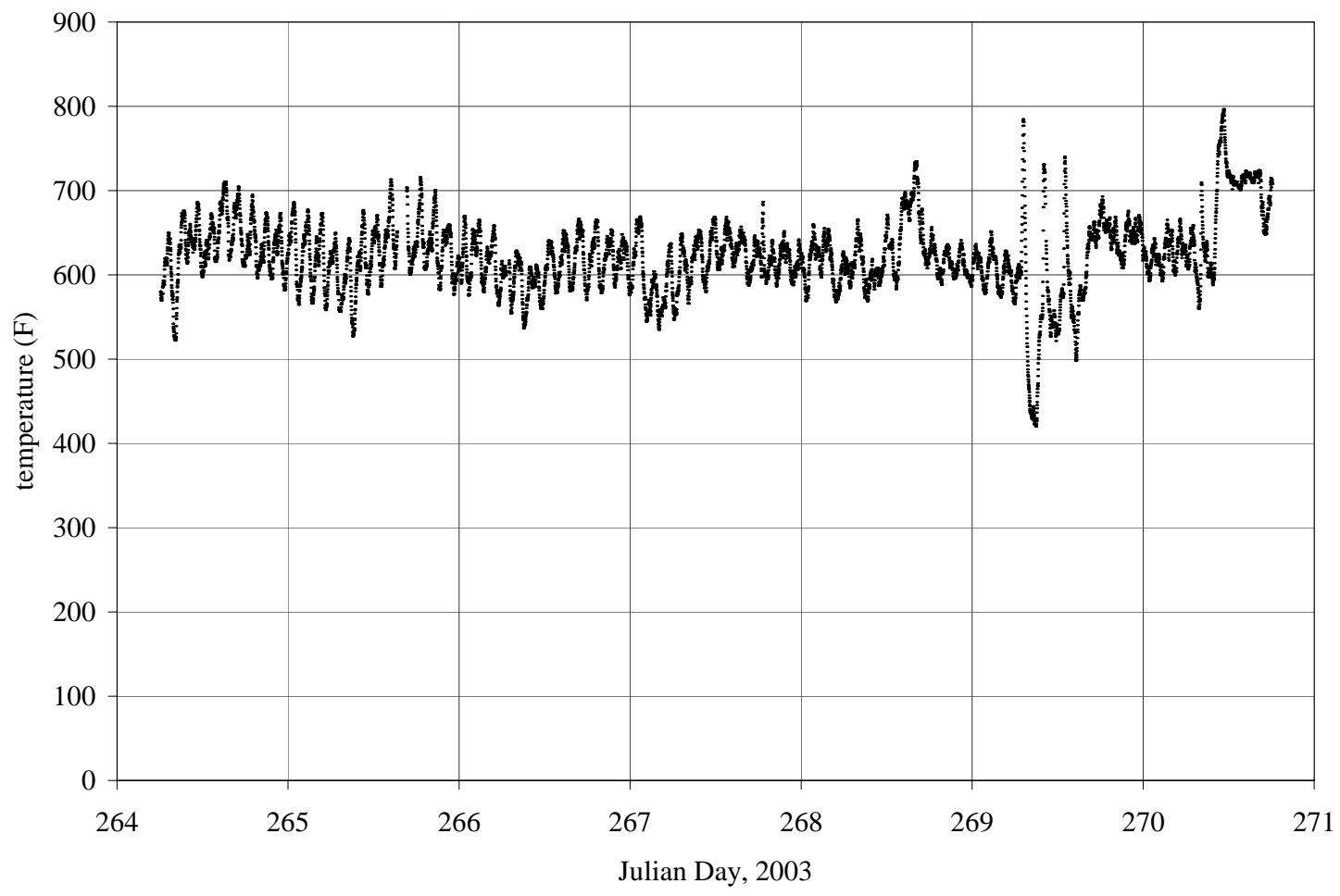

Figure D50. Grate temperature at thermocouple \#2, Boiler 3. Julian Day $264=$ September 21, 2003. 


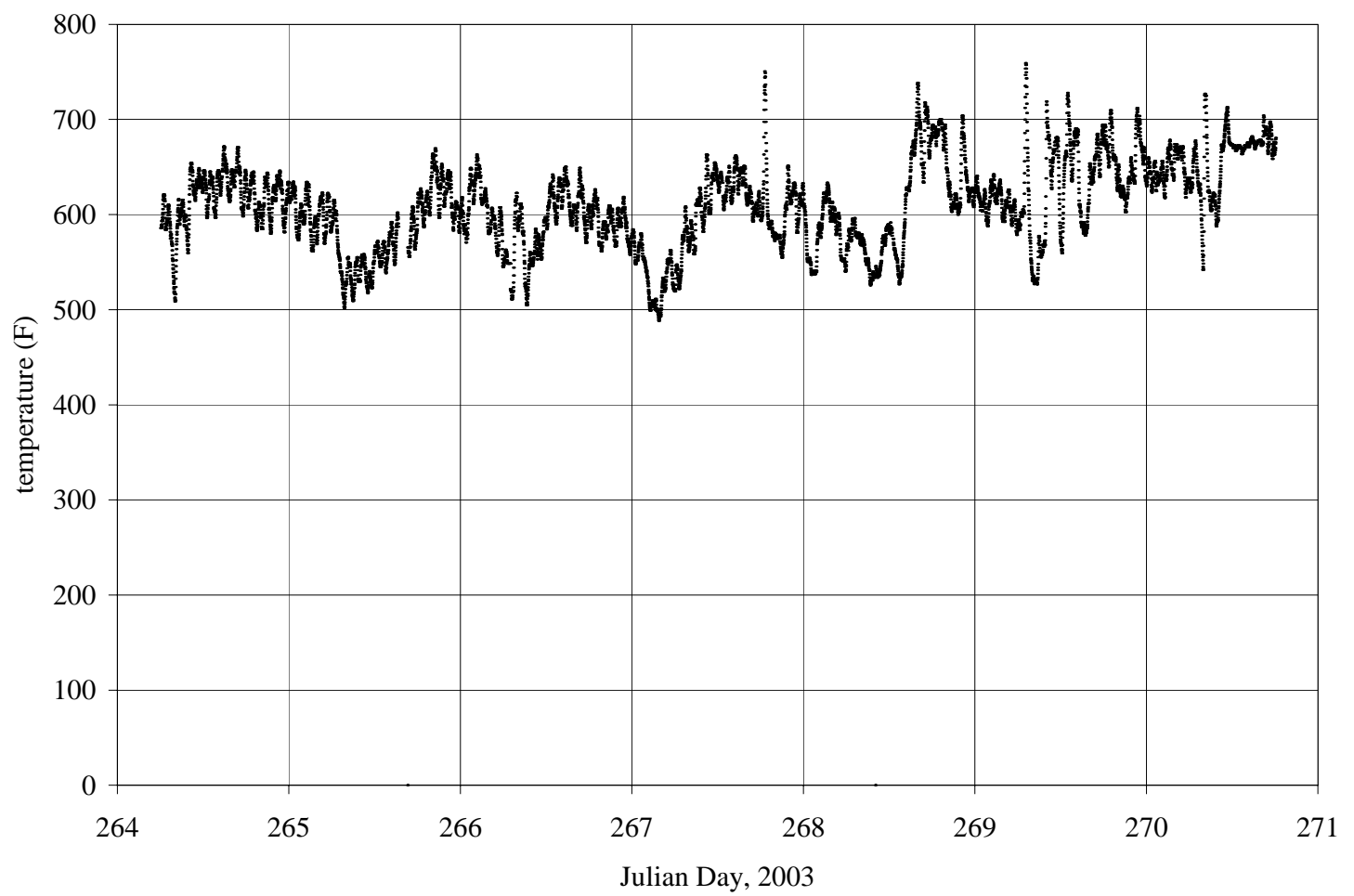

Figure D51. Grate temperature at thermocouple \#3, Boiler 3. Julian Day $264=$ September 21, 2003.

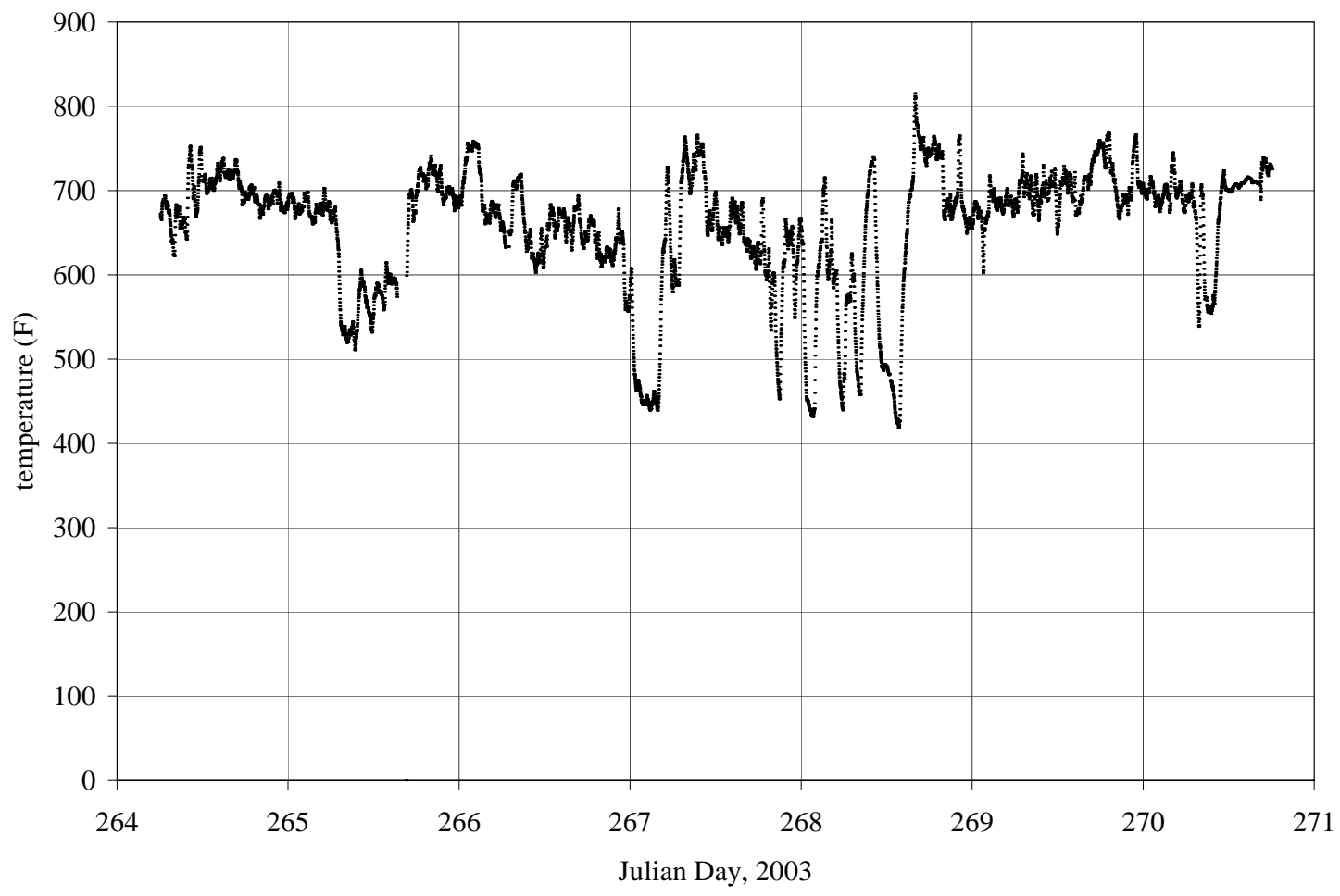

Figure D52. Grate temperature at thermocouple \#4, Boiler 3. Julian Day $264=$ September 21, 2003. 


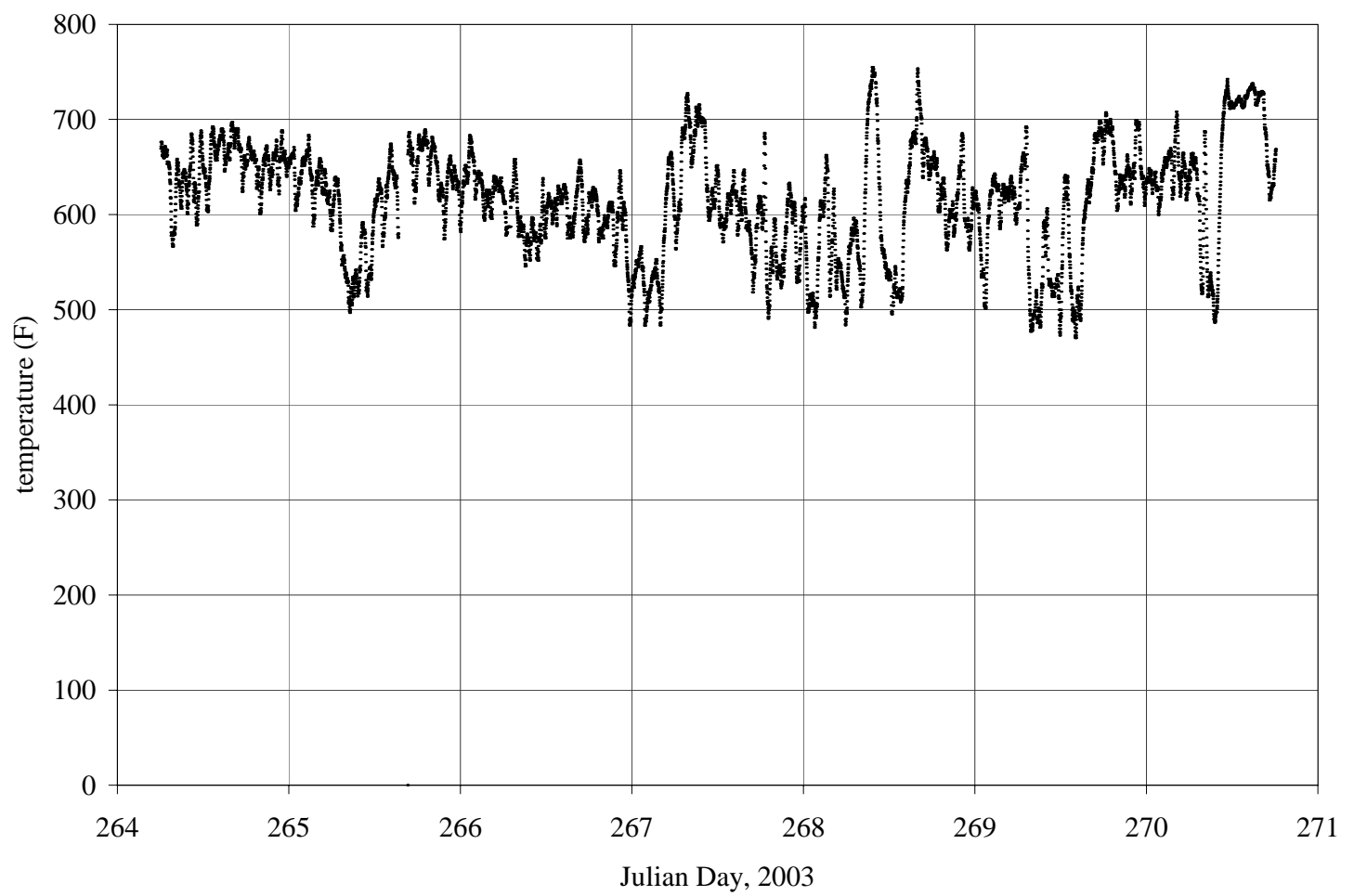

Figure D53. Grate temperature at thermocouple \#5, Boiler 3. Julian Day $264=$ September 21, 2003.

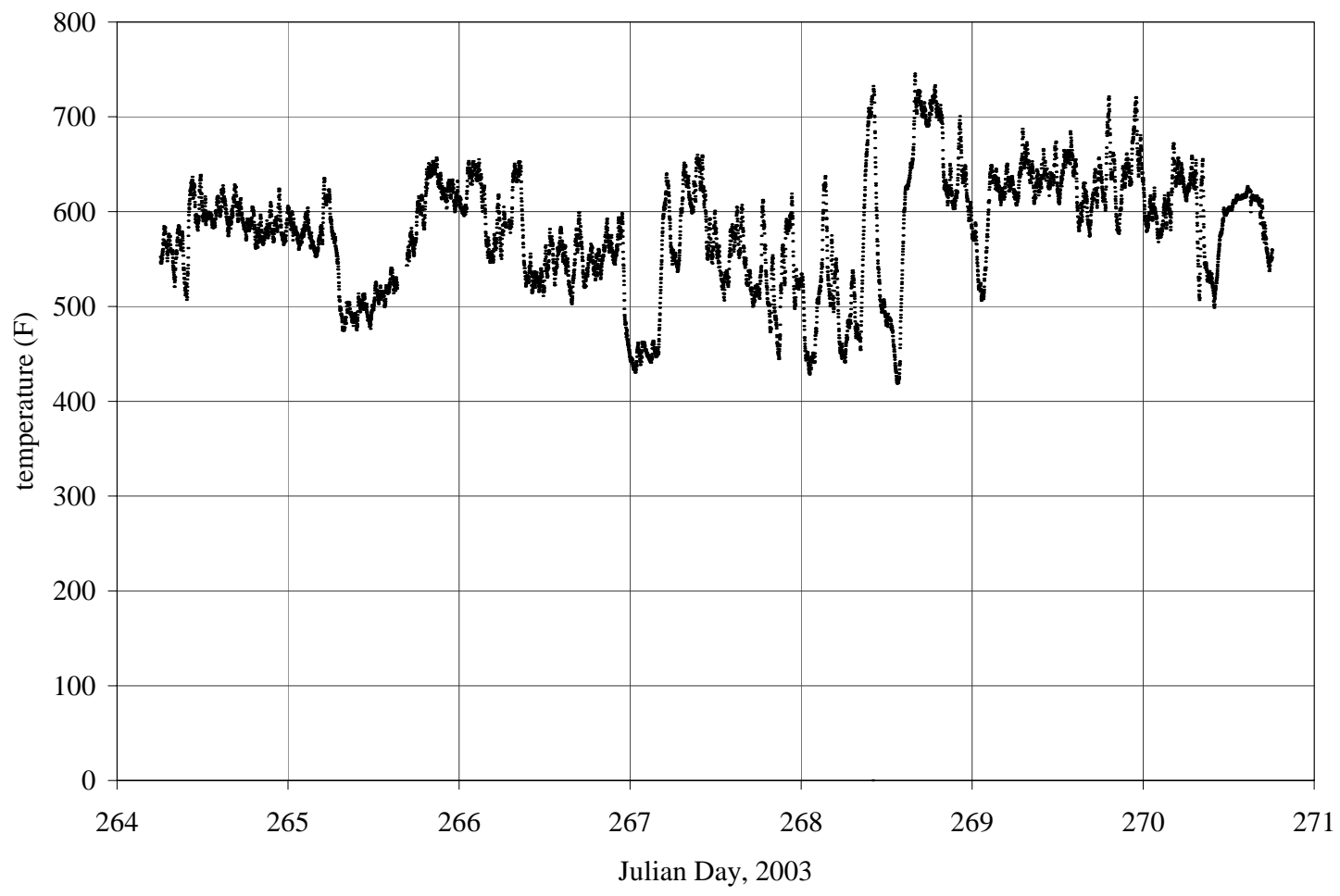

Figure D54. Grate temperature at thermocouple \#6, Boiler 3. Julian Day $264=$ September 21, 2003. 


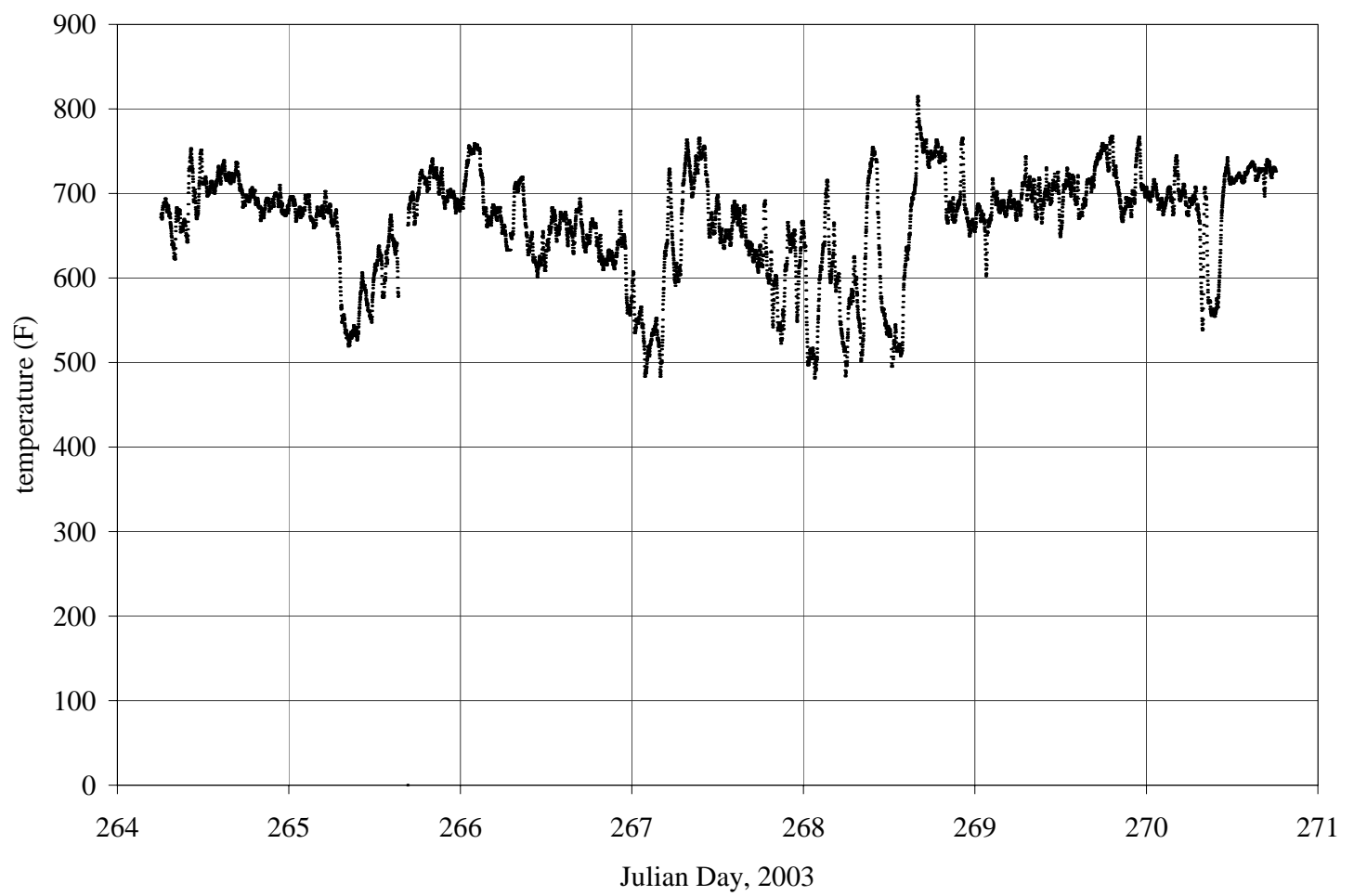

Figure D55. Air heater right bypass temperature, Boiler 3. Julian Day $264=$ September 21, 2003.

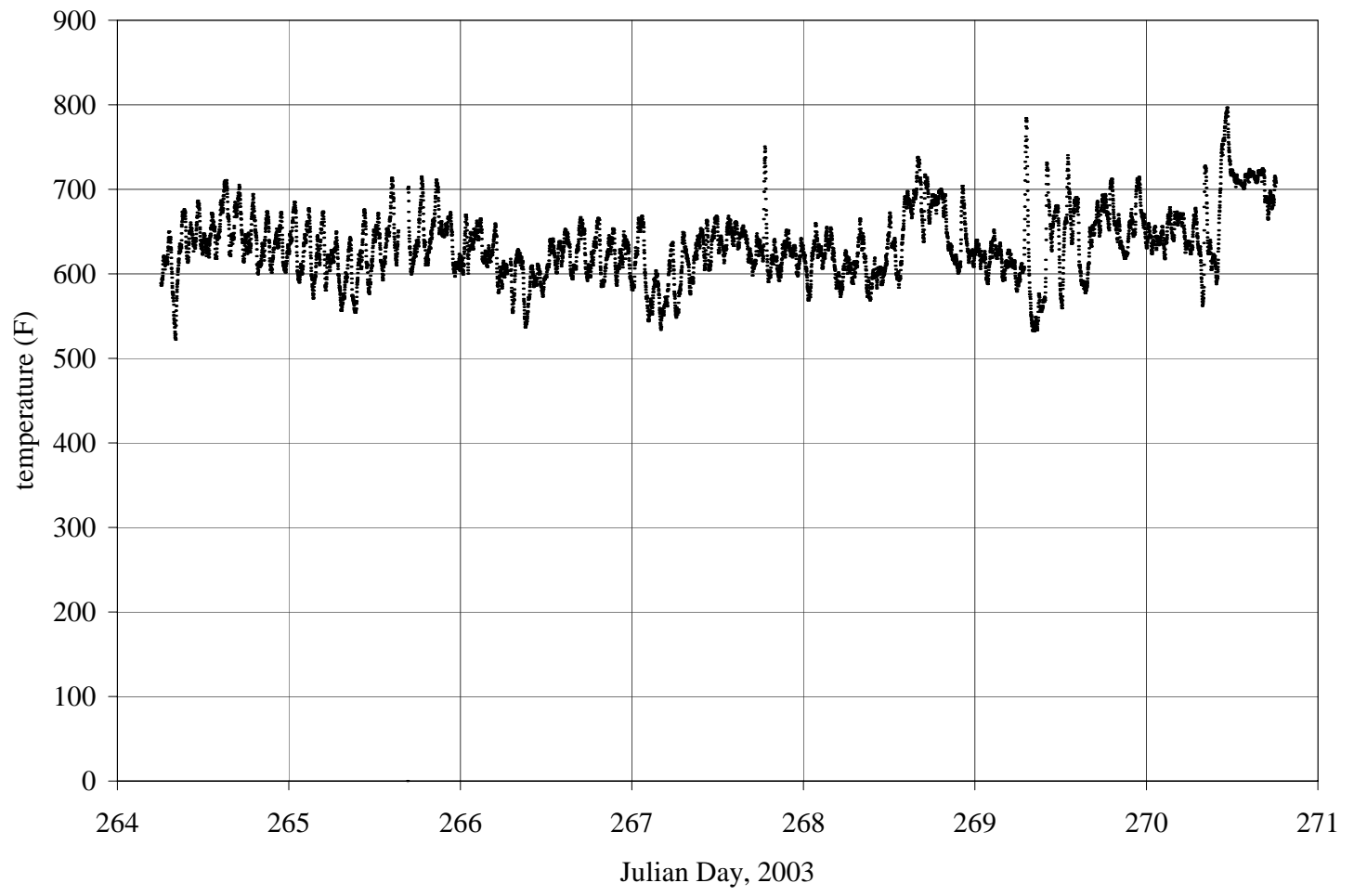

Figure D56. Air heater left bypass temperature, Boiler 3. Julian Day $264=$ September 21, 2003.. 


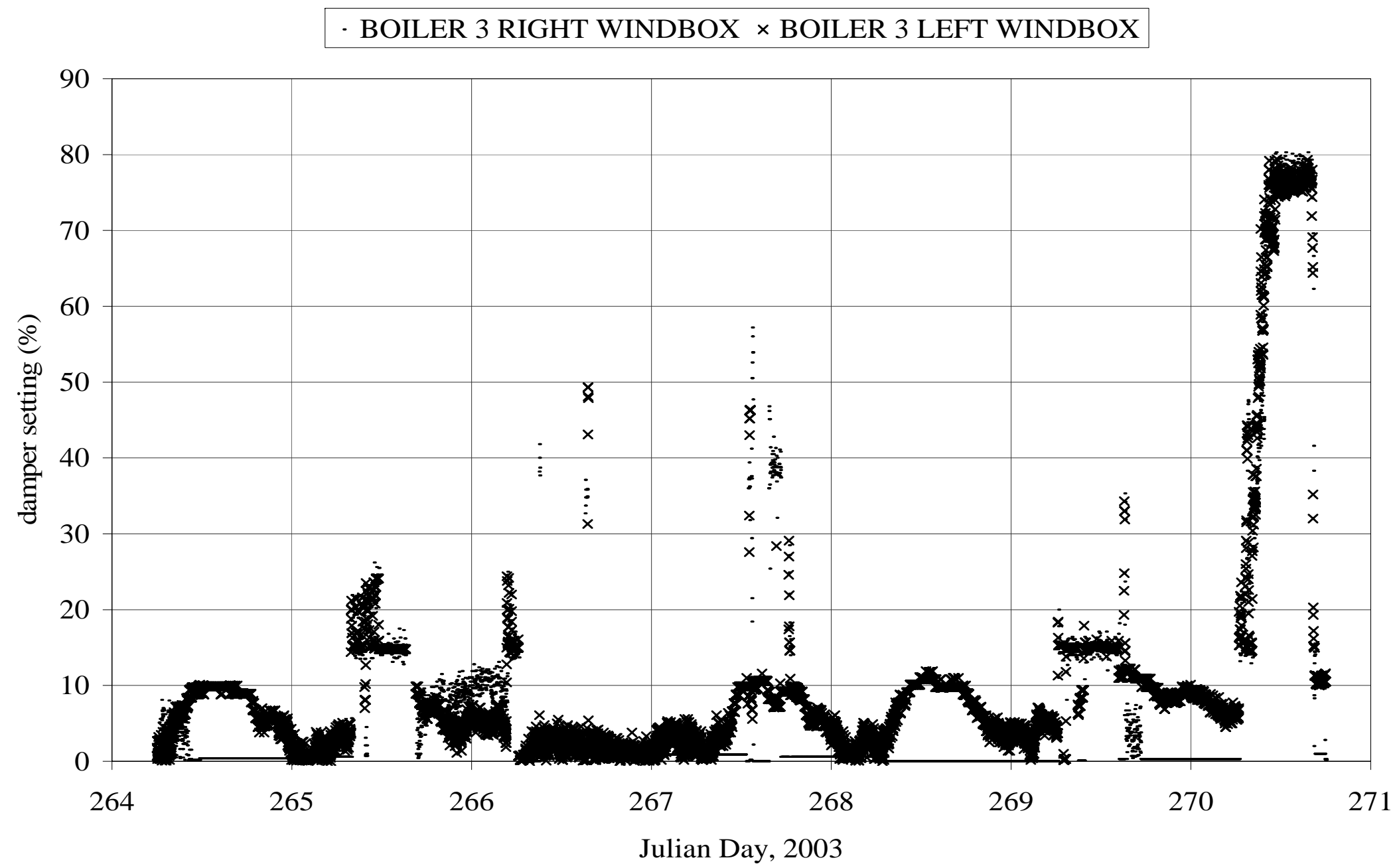

Figure D58. Air flow rates in left and right windbox, Boiler 3. Julian Day 264 = September 21, 2003. 


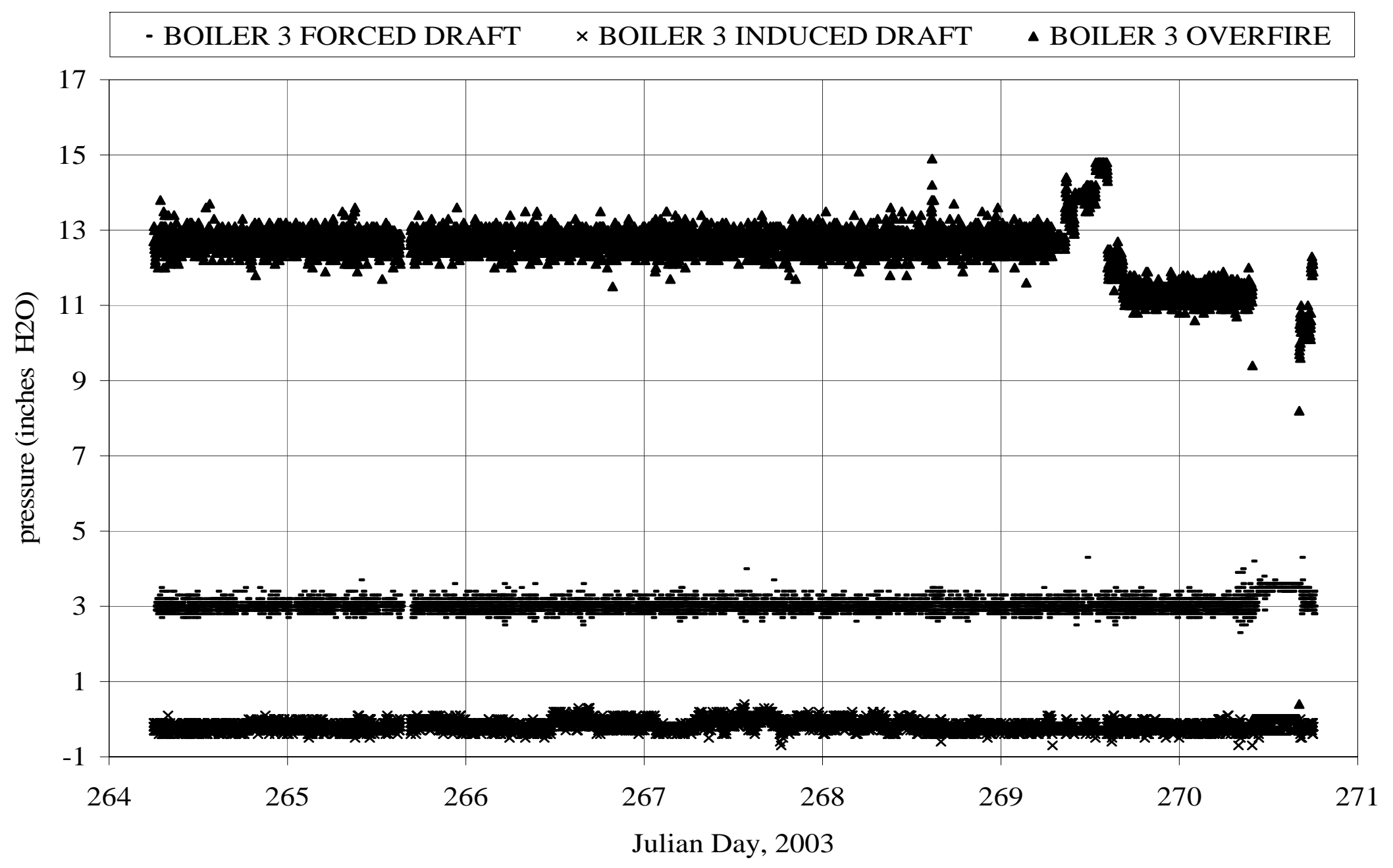

Figure D59. Forced draft, induced draft and overfire air pressure, Boiler 3. Julian Day 264 = September 21, 2003. 


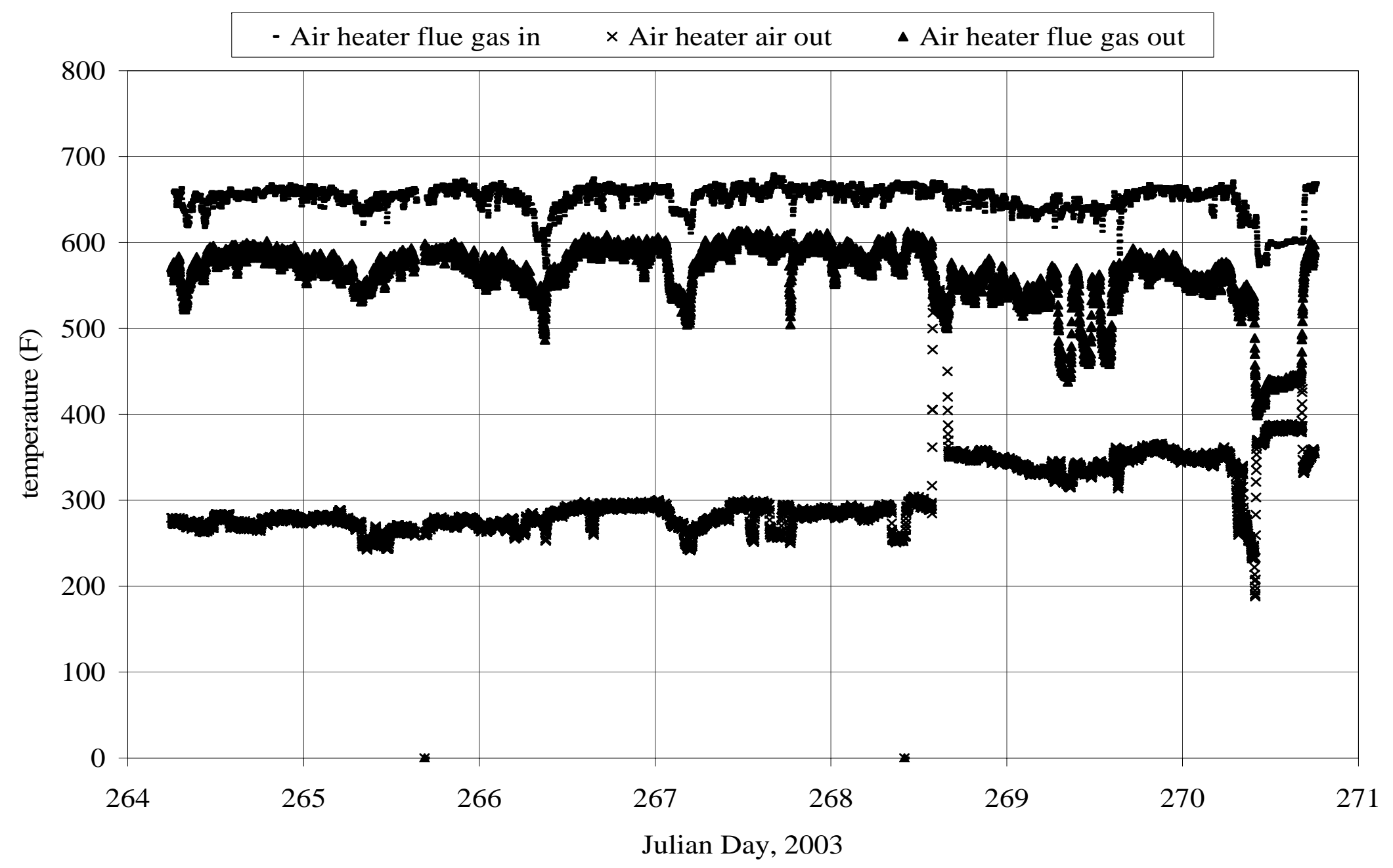

Figure D60. Air heater, flue gas in, flue gas out and air out temperature, Boiler 3. Julian Day 264 = September 21, 2003. 


\section{Integrated Sugar Factory Steam System Evaluation Task 2 Deliverable Report}

September 2005

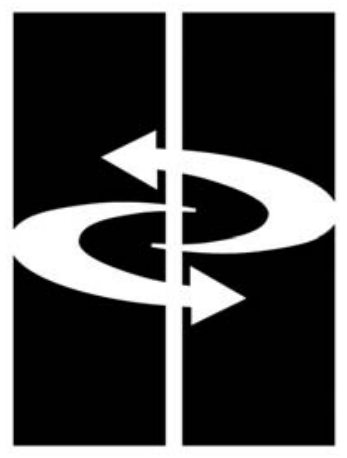

HAWAII NATURAL ENERGY INSTITUTE

School of Ocean and Earth Sciences and Technology

University of Hawaii at Manoa 


\title{
Integrated Sugar Factory Steam System Evaluation Task 2 Deliverable Report
}

\author{
Vheissu I. Keffer \\ Scott Q. Turn \\ Hawaii Natural Energy Institute \\ University of Hawaii \\ Charles M. Kinoshita \\ Department of Molecular Biosciences and Bioengineering \\ University of Hawaii
}

Prepared for

Hawaiian Commercial \& Sugar, Co.

HC\&S Purchase Order No. 64137

September 2005

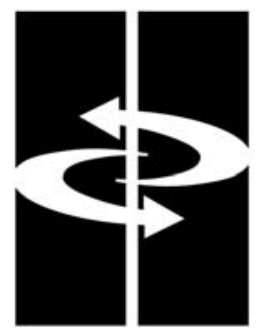

Hawaii Natural Energy Institute

School of Ocean and Earth Sciences and Technology

University of Hawaii 


\section{Contents}

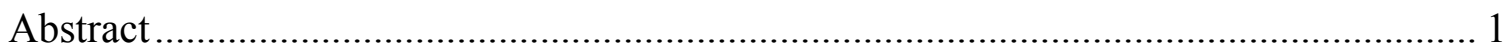

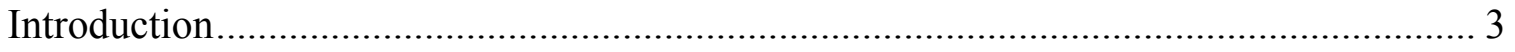

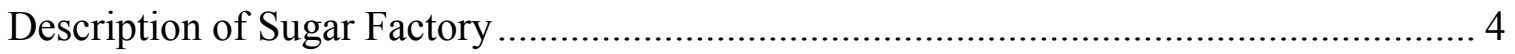

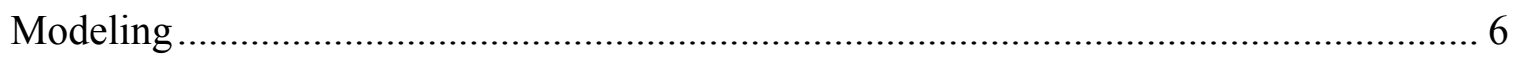

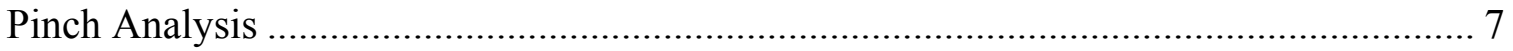

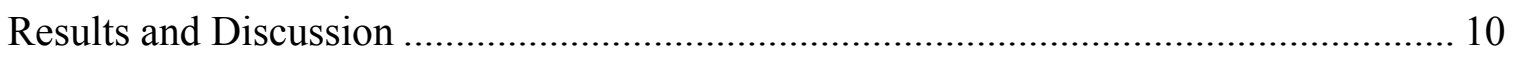

Second Evaporator Vapor to Pans ..................................................................... 11

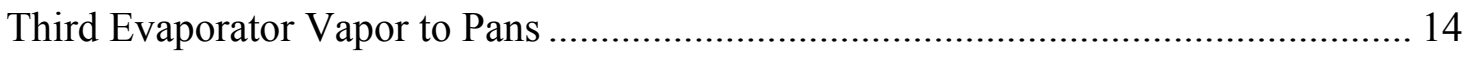

Plate Type Mixed Juice Heaters ...................................................................... 15

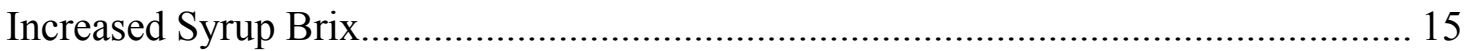

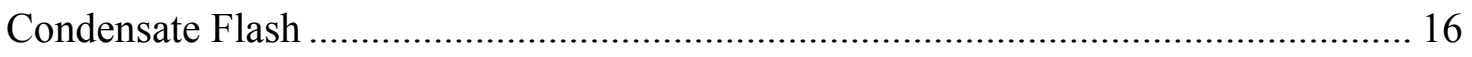

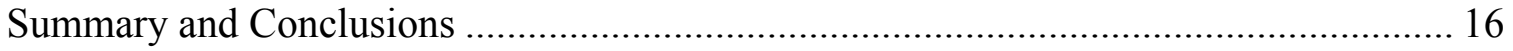

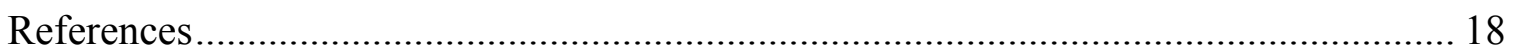

Appendix: Aspen Flow Sheets for HC\&S Puunene Sugar Factory 


\begin{abstract}
Hawaii's largest sugar producer, Hawaiian Commercial \& Sugar Co. (HC\&S), has undertaken a plantation wide energy efficiency assessment with cost share from the U.S. Department of Energy's (DOE) Office of Industrial Technology (OIT). This assessment includes analyses of the irrigation pumping system, electrical distribution system, and sugar factory. University of Hawaii (UH) project participants developed a comprehensive model of the steam system in the HC\&S sugar factory using Aspen Plus computer modeling software in order to conduct investigations into steam use. A pinch analysis was conducted to guide an investigation into energy saving modifications to factory equipment and operations.
\end{abstract}

The HC\&S sugar factory at Puunene is a modern and efficient facility with electrical power generation of approximately 80 to $85 \mathrm{kWh} /$ ton of cane (tc) during periods of steady operation. While this range is high compared to sugar producers in other parts of the world where levels of 10-30 kWh/tc or less are common, levels of $90-100+\mathrm{kWh} / \mathrm{tc}$ are thought to be attainable. Steam consumption for evaporating sugarcane juice and boiling sugar at HC\&S is in the range of 800-850 lbs steam/ tc. Experts feel that the most efficient factories should be able to operate on $650 \mathrm{lbs}$ steam/tc or less. Pinch analysis and Aspen Plus modeling software were used to investigate modifications that might reduce the factory steam-to-cane ratio and increase electricity export at HC\&S.

Simulation results for five scenarios were discussed with HC\&S personnel to determine feasibility based on operating constraints. Although simulation results for all five scenarios showed notable savings over the base case, only two of the five proposed changes were deemed possible within the operational constraints of the factory. Results from all five simulations were included for reference should conditions become more favorable for their implementation.

The two scenarios deemed possible within operating constraints were (1) operating the pan boiling system on second vapor rather than first vapor, and (2) adding the capability to flash condensates from evaporators 2, 3, and 4. Model results for using second vapor to supply pan boiling demands show a reduction in exhaust steam demand of about 21,000 pounds per hour which corresponds to a $65 \mathrm{lb} / \mathrm{tc}$ reduction in steam:cane ratio. If this steam was condensed in an existing turbo-generator at HC\&S (TG4) it would increase electrical power generation by $0.95 \mathrm{MW}$. The model shows a decrease of nearly $6 \%$ for heat exchange surface area required in the evaporator train, offset by a $10 \%$ increase in required heat exchange surface area for the pans.

Modeling results for adding the capability to flash condensates from evaporators 3 and 4 show a potential steam savings equivalent to 8,000 pounds of exhaust steam which corresponds to a $25 \mathrm{lb} / \mathrm{tc}$ reduction in steam:cane ratio. Condensing this steam in TG4 would increase electrical power generation by approximately $0.36 \mathrm{MW}$. Model results indicate a net increase of less than half a percent in evaporator heat exchange surface area. Heat exchange surface area would need to be increased in the first three effects and reduced in the fourth and fifth effects. A negative consequence of this modification is an 
increase in evaporator train condenser load, which would require increased cooling water supply and pumping capacity.

The combined modifications to the operation and equipment at HC\&S Puunene sugar factory could decrease the steam:cane ratio by $12 \%$. If the steam saved were converted to electricity present electricity export could be increased by $11 \%$. 


\section{Introduction}

Hawaii's largest sugar producer, Hawaiian Commercial \& Sugar Co. (HC\&S), has undertaken a plantation wide energy efficiency assessment with cost share from the U.S. Department of Energy's (DOE) Office of Industrial Technology (OIT). This assessment includes analyses of the irrigation pumping system, electrical distribution system, and sugar factory. The sugar factory assessment includes a steam generation efficiency assessment for the power plant and a steam utilization assessment of the factory including the mill, processing plant, and cogeneration plant. The University of Hawaii (UH) was contracted to provide technical assistance on these latter two tasks. This report summarizes work completed by UH on the steam utilization portion of the factory assessment.

Sugar cane processing yields three primary product streams; raw sugar, molasses, and bagasse. Raw sugar is sold as turbinado and to refiners while molasses is marketed as cattle feed supplement. Bagasse, the fibrous byproduct that remains after cane has been milled, is used as fuel in boilers to produce steam for electricity generation and factory processes. HC\&S produces electricity in excess of their in-house demand, allowing them to export electricity to the utility grid.

Bagasse is a renewable energy resource and power generated from bagasse is considered to be nominally greenhouse-gas neutral. Utilization of a renewable energy resource for power generation helps reduce Hawaii's dependence on imported oil, enhances the local economy, and has environmental benefits. Electricity sold to the local utility helps the utility company meet renewable portfolio standards that have been targeted by the State of Hawaii. Environmental benefits, rising energy costs, and a highly competitive sugar market encourage maximization of electricity export.

Exportable electricity can be increased in two ways; bagasse can be converted into steam (and subsequently electricity) more efficiently, or, steam and electricity use in the sugar factory can be reduced, thus increasing the amount of exportable power. An index of sugar factory electricity generation is commonly reported in terms of $\mathrm{kWh}$ per ton of cane (tc) processed. This index is affected by both steam generation efficiency and steam use efficiency in the factory. Steam use efficiency is often reported as steam:cane ratio and has units of $\mathrm{lb}$ steam/tc. A typical value of steam:cane ratio for sugar factories world wide is $1000 \mathrm{lb} / \mathrm{tc}$ [1,2]. A value of steam:cane ratio under $1000 \mathrm{lb} / \mathrm{tc}$ is obtained by employing steam saving measures.

During periods of steady operation, HC\&S's Puunene sugar factory generates about 80$85 \mathrm{kWh}$ per ton cane processed. While this number is high when compared with cane sugar producers from other parts of the world, some room for improvement exists. Maximum attainable electricity generation from an efficient sugar factory running at full capacity is in the range of 90 to $100+\mathrm{kWh} / \mathrm{tc}$ processed [2]. Steam consumed in cane processing at HC\&S is in the range of $800-850 \mathrm{lb} / \mathrm{tc}$. While HC\&S's steam:cane ratio is well below average accepted levels it does not yet approach minimum values. Experts 
feel that the most efficient factories should be able to operate on $650 \mathrm{lbs}$ steam/tc or less [3].

HC\&S's Puunene sugar factory currently employs most of the conventional steam saving measures found in the cane sugar industry and has been an industry leader in cogeneration. As such, examining the factory configuration and operating conditions of other sugar producers for areas of potential efficiency gains was not of great interest. Instead, computer analysis techniques were used to evaluate the steam use efficiency and how proposed modifications to the factory would impact steam requirements.

Sugar factories are complex operations, but advances in process modeling software now make it possible to generate accurate working models. If sufficient input parameters are provided, these models can be used to assess the entire process and identify areas where improvements might be feasible. Proposed process and operational modifications can be modeled to quantify improvements and identify potential impacts on the larger system. Results from these models combined with operator input and company goals can then be used to make decisions on plant upgrades.

The initial evaluation of HC\&S's Puunene sugar factory was accomplished using the Advanced $\underline{\text { System for }}$ Process ENgineering (ASPEN) PLUS ${ }^{\circledR}$ commercial software package from Aspen Technology Inc. (Cambridge, MA). Further analysis was carried out using a pinch analysis program, Aspen Pinch. Five areas where operational or equipment modifications could lead to more efficient use of process steam were identified. After consultation with HC\&S personnel, only two of the proposed modifications were found to function within operational constraints imposed by the existing sugar factory. The details of the modeling effort and results are presented in the remainder of this report.

\section{Description of Sugar Factory}

The HC\&S sugar factory was originally constructed in 1901. Over the last century improvements and modifications have occurred at regular intervals. In 1957, HC\&S installed, what was then, the largest bagasse-fired boiler system in the world. This boiler system remains in place today but has been updated and augmented with a third boiler that was commissioned in 1977. In the late 1980's the entire factory was computerized, making it one of the first sugar factories to do so. Today, HC\&S continues to innovate and update its factory and processing facilities [4].

Over the last 20 years the number of sugar plantations in Hawaii has declined dramatically as a result of increased competition from lower cost producers in other nations. Today, sugar is grown at two remaining plantations on Maui and Kauai, HC\&S and Gay \& Robinson (G\&R), respectively. Throughout the state-wide decline of the sugar industry, HC\&S has upgraded and modified its Puunene factory to increase capacity as other mills have closed. Today it processes about 1.6 million tons of net cane annually, producing over 200,000 tons of raw sugar and more than 70,000 tons of molasses [5]. 
The layout of the Puunene factory can be most easily described by breaking it into three main processes, steam generation, cane milling and juice boiling. Flow sheets depicting the three main process areas are presented in the Appendix.

In the power plant, steam is produced by burning sugarcane bagasse and/or coal in three generating units (Boiler 1, Boiler 2, and Boiler 3) and then fed to two turbo generators, TG4 and TG5. The boilers are all spreader stokers equipped with traveling grates.

Boilers 1 and 2 were purchased from Riley Stoker Corporation and are identical, with rated capacities of $125,000 \mathrm{lb}$ steam/hr and $900 \mathrm{psig}$ steam pressure. Flue gases from the two units are exhausted through a common wet scrubber and stack. Residue from their grates enters a common water quench and is removed by belt conveyor. Boiler 3 was purchased from Foster Wheeler, operates at 425 psig steam pressure, and is rated for $350,000 \mathrm{lb}$ steam $/ \mathrm{hr}$. Flue gas from Boiler 3 is exhausted through a dedicated wet scrubber and stack and the grate residue is also removed using a water quench and conveyor system

Electricity is generated using two extraction condensing turbines, TG4 and TG5. TG5 operates on 900 psig steam and has a maximum continuous rating of 200,000 lb steam $/ \mathrm{hr}$. The extraction pressure for TG5 is 425 psig. TG4 operates on 425 psig steam, has a maximum continuous rating of $400,000 \mathrm{lb} / \mathrm{hr}$, and steam is extracted at a pressure of 15 psig.

The mill line separates cane stalks into juice and fiber through a process of shredding and crushing. Cane from the fields is washed to clean off soil residues and then undergoes several particle size reductions before being crushed to expel the juice. The mill line at HC\&S has a 5,000 hp, steam-driven Walkers shredder with 96 hammers. The shredder is followed by four, $1,000 \mathrm{hp}$, steam-driven, six roll, light duty mills equipped with pressure feeders. The first mill has inlet dimensions of $84 " \mathrm{x} 43$ " with the subsequent mills having dimensions of 78" $\mathrm{x} 43^{\prime \prime}$. Turbines power the shredder and the mills; the shredder runs on 900 psig steam while the mills operate using 425 psig steam. An electric driven, 750 hp knife-set running at $880 \mathrm{rpm}$ precedes the shredder.

In the boiling house, juice from the milling process is concentrated into a thick syrup before undergoing crystallization and separation that results in two product streams, molasses and raw sugar. The boiling house at HC\&S employs three mixed juice heaters, a clarified juice heater, a quintuple effect evaporation train, and a pan evaporation set operating on the B magma boiling system. The quintuple effect evaporator train includes three first effect evaporators operating in series on exhaust (15 psig) steam followed by second, third, fourth and fifth effect evaporators, each running on vapor from the previous effect. The combined heat exchange surface area of all seven evaporators is 155,900 square feet. The juice heaters have a total heat exchange surface area of 21,800 square feet and operate using exhaust steam. The first and second mixed juice heaters operate on second and third vapor respectively, while the tertiary mixed juice heater and clarified juice heater operate on first vapor. 
The evaporator pan system includes $\mathrm{A}$ and $\mathrm{C}$ continuous pans and batch pans for $\mathrm{B}$ magma, B seed, low grade seed, and food grade sugar. Total evaporator pan heat exchange surface area is 49,400 square feet. The $\mathrm{A}$ and $\mathrm{C}$ continuous pans each have a heat exchange surface area of 10,342 square feet. The seed pans have heat exchange surface areas of 4,283 and 5,270 square feet for B seed and low grade seed, respectively. B magma batch pans have a combined heat exchange surface area of 9,000 square feet and the food grade pans have a combined area of 10,162 square feet. During periods of maximum utilization the A continuous pan is augmented with a pair of 4,061 square foot batch pans.

\section{Modeling}

The first step in assessing steam utilization at HC\&S's Puunene sugar factory was to develop a base model using the Aspen Plus computer simulation software. Since Aspen plus is primarily used in the petrochemical industry it does not include many of the unit operations found in a sugar factory, however it is flexible enough that equivalent substitutes can be developed based on existing unit operation models. The Aspen Plus solution algorithm assumes continuous, steady state processes. It should be emphasized that modeling is carried out only for continuous operations. Scenarios involving process interruptions, pan and evaporator steam outs, and scheduled down time cannot be accounted for in the Aspen models.

Establishing the factory layout and creating a preliminary flow sheet were necessary precursors to developing a working model. Working with HC\&S personnel an outline of the factory flow sheet was developed. Difficulty was encountered while trying to establish pan boiling system parameters. Portions of the pan boiling system operate using batch processing, and recycling and reboiling are utilized but are not explicitly measured. Dilution and reprocessing further complicate the process. Modifications to operating parameters occur in real time and from batch to batch making it a challenge to arrive at a set of representative operating parameters.

The black box nature of the pan boiling system necessitated making gross assumptions in the pan portion of the Aspen model. Using known inlet and outlet flows and input from HC\&S personnel, a rough but workable model was developed for the pan boiling section of the factory. Following the completion of the flow sheet, input for operating parameters was gathered. Establishing a representative set of operating parameters involved examination of data logs, onsite measurements, and extensive discussion with factory personnel.

In cases where required inputs could not be measured, estimates or calculated values were used. When calculation or estimation was not easily accomplished, average values were obtained from the literature. Estimated and calculated parameters included heat transfer coefficients in heaters evaporators and pans, vapor bleeding to pans and mixed juice heaters, massecuite and pan input flows, pressure losses, and thermal losses. 
Arriving at a representative set of values was also complicated by the vagaries of sugar processing. Variations in weather, field conditions, cane quality, milling throughput, boiler operation, evaporator performance, heat exchanger fouling, hydroelectric availability, and water treatment can influence the cane processing rate and boiling house efficiency. Arriving at a model that would simulate periods of high capacity operation $(<90 \%)$ required careful study of the factory operation. After extensive consultation with HC\&S personnel a representative set of base case operating parameters was established as shown in Table 1.

Initial model runs were completed using the operating parameters shown in Table 1. Wonderware data for the factory that were logged during boiler efficiency testing in September, 2003, were used for model verification. Further adjustments were made to optimize the model before investigating equipment and process changes. Areas of potential process change were identified with the help of pinch analysis.

\section{Pinch Analysis}

Pinch analysis is a tool for examining energy supply and demand balances in complex processes. Pinch analysis was developed in the early 1980's as a method to simplify the design of energy recovery systems. Since that time the technology has matured and a number of computer programs have been developed to enhance its application and ease of use. Despite these advances, pinch analysis has not been widely employed outside of a narrow group of industrial sectors, most notably petroleum refining and more recently steel and paper manufacturing. Raw sugar manufacturing from cane is an area where pinch technology has had limited penetration. Some examples of its application have been published, but raw sugar manufacture from cane largely remains a new application for pinch analysis [6].

Pinch analysis provides a systematic method for analyzing process energy demands using the first and second laws of thermodynamics. The first step in pinch analysis is to identify and quantify all process streams that undergo temperature changes. Process streams that require heating are listed as "cold streams" and streams that require cooling are listed as "hot streams." Values of specific heat, supply temperature, target temperature, and mass flow rate are tabulated for all hot and cold streams.

The next step is to display the tabulated process stream data graphically. It is useful to define a heat capacity flow rate $(\mathrm{CP})$ as the product of flow rate in $\mathrm{lb} / \mathrm{sec}$ and specific heat in BTU $/ \mathrm{lb}^{\circ} \mathrm{F}$. This new value, $\mathrm{CP}$, with units of BTU $/ \mathrm{sec}^{\circ} \mathrm{F}$ can be used to easily graph enthalpy change in process streams. Temperature is plotted against enthalpy in plots called composite curves. The process composite curve contains both hot and cold composite curves.

In the process composite curve, the hot curve appears above the cold curve. In most processes, the variation in $\mathrm{CP}$ values for different streams results in kinked composite curves as depicted in Figure 1. The smallest vertical distance between the hot and cold curves is called the minimum temperature approach (DTmin). This point represents a 
bottleneck in heat recovery and is also referred to as the pinch point or pinch temperature. The DTmin value can be adjusted, shifting the curves farther apart, leading to lower process-to-process heat exchange and higher utility requirements. For a given DTmin, minimum hot and cold utility requirements can be determined and are indicated by the extent to which the hot and cold ends of the composite curve do not overlap.

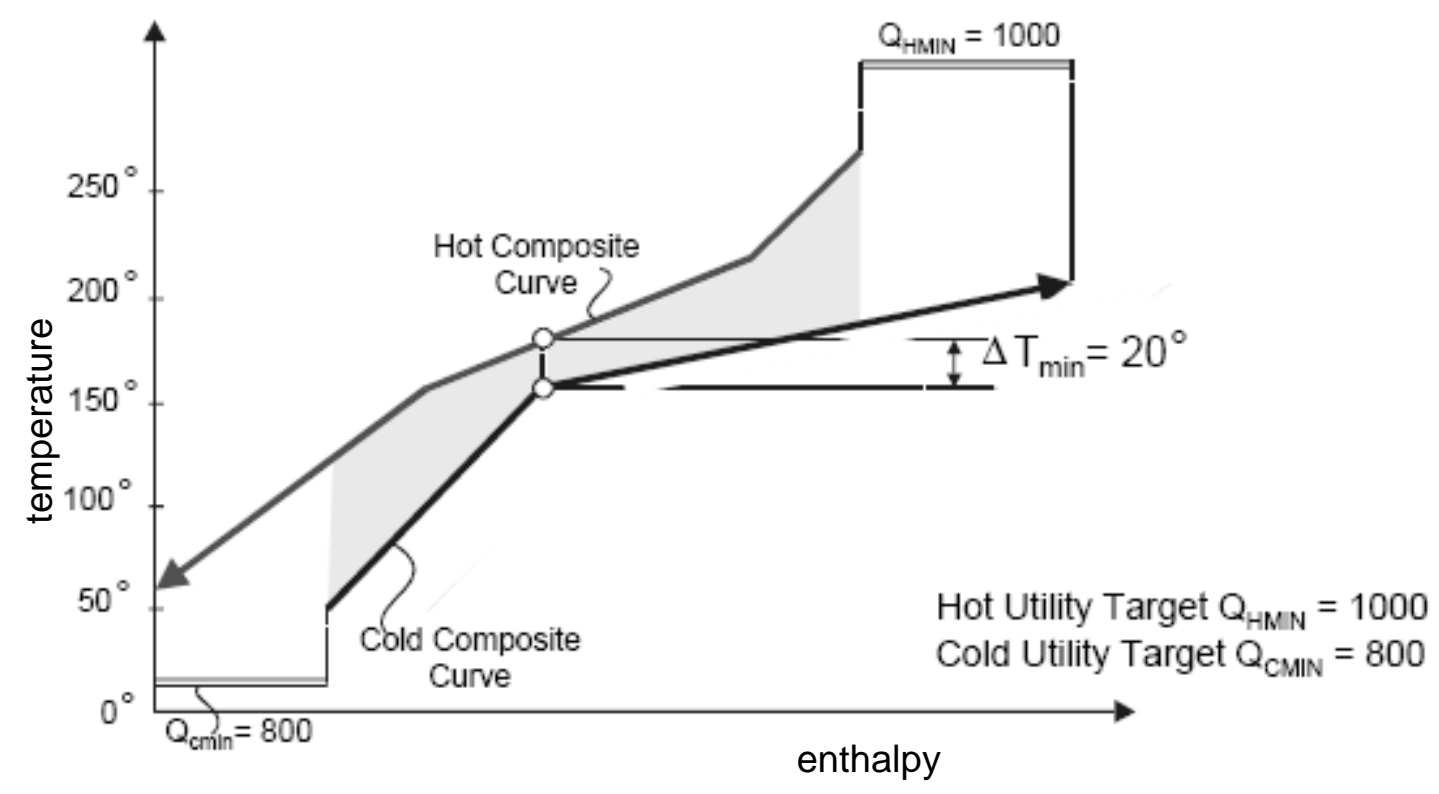

Figure 1. Example of a process composite curve generated with Aspen pinch showing hot and cold composite curves.

Using the process composite curve, minimum energy requirements can be determined and modifications to the process heat exchange network can be developed. Various programming tools are available to aid in heat exchange network design and process modification decisions. Additional information on pinch analysis and pinch techniques is available in numerous references $[7,8,9]$.

One of the reasons pinch analysis has not been widely adopted in the cane sugar industry is the unique thermal energy use profile of sugar factories. Industries in which pinch analysis is commonly applied involve heating and cooling of process streams over a range of temperatures. Sugar processing, on the other hand, involves primarily the evaporation of large quantities of water and has few if any high temperature product streams that require cooling. The temperature composite curve for a typical sugar factory shows limited opportunity for modifying heat exchange networks to improve efficiency. 
Table1. Base case operating parameters for HC\&S Puunene sugar factory.

\begin{tabular}{|c|c|c|c|c|c|}
\hline Factory Parameter & Units & Value & Factory Parameter & Units & Value \\
\hline Mixed Juice Flow & $\mathrm{lb} / \mathrm{hr}$ & $800,000^{b}$ & 3rd Vapor Bleed to MJH1 & $\mathrm{lb} / \mathrm{hr}$ & $13,500^{b}$ \\
\hline Mixed Juice Brix & brix & $14.4^{\mathrm{a}}$ & Syrup Brix & brix & $65^{b}$ \\
\hline Mixed Juice Purity & & $86.72^{\mathrm{a}}$ & Apan Feed Brix & brix & $70^{b}$ \\
\hline Mixed Juice Inlet Temperature & $\mathrm{F}$ & $110^{\mathrm{b}}$ & Apan Feed & $\mathrm{lb} / \mathrm{hr}$ & $200,250^{C}$ \\
\hline First Mixed Juice Heater Outlet Temperature & $\mathrm{F}$ & $168^{b}$ & Asugar Yield & $\%$ & $54.82^{a}$ \\
\hline Second Mixed Juice Heater Outlet Temperature & $\mathrm{F}$ & $187^{b}$ & Amassecuite Brix & brix & $91.2^{\mathrm{a}}$ \\
\hline Third Mixed Juice Heater Outlet Temperature & $\mathrm{F}$ & $214^{\mathrm{b}}$ & Bpan Feed Brix & brix & $70^{b}$ \\
\hline Clarified Juice Heater Inlet Temperature & $\mathrm{F}$ & $200^{b}$ & Bpan Feed & $\mathrm{lb} / \mathrm{hr}$ & $100,515^{c}$ \\
\hline Clarified Juice Heater Outlet Temperature & $\mathrm{F}$ & $221^{b}$ & Bsugar Yield & $\%$ & $43.5^{a}$ \\
\hline Evaporator 1A Inlet Steam Pressure & psia & $21.7^{\mathrm{b}}$ & Bmassecuite Brix & brix & $92.9^{\mathrm{a}}$ \\
\hline Evaporator 1A Inlet Steam Temperature & $\mathrm{F}$ & $268^{b}$ & Cpan Feed Brix & brix & $70^{\mathrm{b}}$ \\
\hline Evaporator $1 \mathrm{~A}$ Operating Pressure & psia & $19.5^{b}$ & Cpan Feed & $\mathrm{lb} / \mathrm{hr}$ & $66,062^{c}$ \\
\hline Evaporator 1B Inlet Steam Pressure & psia & $24.6^{\mathrm{b}}$ & Cmassecuite Brix & brix & \\
\hline Evaporator 1B Inlet Steam Temperature & $\mathrm{F}$ & $268^{b}$ & Seedpan Feed Brix & brix & $70^{b}$ \\
\hline Evaporator 1B Operating Pressure & psia & $19.5^{b}$ & Seedpan Feed & $\mathrm{lb} / \mathrm{hr}$ & $66,062^{c}$ \\
\hline Evaporator $1 \mathrm{C}$ Inlet Steam Pressure & psia & $26.4^{\mathrm{b}}$ & Seedmassecuite Brix & brix & \\
\hline Evaporator $1 \mathrm{C}$ Inlet Steam Temperature & $\mathrm{F}$ & $268^{b}$ & FGpan Feed Brix & brix & $70^{\mathrm{b}}$ \\
\hline Evaporator 1C Operating Pressure & psia & $19.5^{b}$ & Fgpan Feed & $\mathrm{lb} / \mathrm{hr}$ & $66,062^{c}$ \\
\hline Evaporator 2 Inlet Steam Pressure & psia & $19.5^{\mathrm{b}}$ & FGsugar Yield & $\%$ & \\
\hline Evaporator 2 Inlet Steam Temperature & $\mathrm{F}$ & $227^{b}$ & Fgmassecuite Brix & brix & \\
\hline Evaporator 2 Operating Pressure & psia & $13^{\mathrm{b}}$ & Molasses Yield & $\mathrm{lb} / \mathrm{hr}$ & \\
\hline Evaporator 3 Inlet Steam Pressure & psia & $13^{b}$ & Apan Heat Exchange Surface Area & $\mathrm{ft}^{2}$ & $10,342^{b}$ \\
\hline Evaporator 3 Inlet Steam Temperature & $\mathrm{F}$ & $214^{b}$ & Bpan Heat Exchange Surface Area & $\mathrm{ft}^{2}$ & $9,000^{b}$ \\
\hline Evaporator 3 Operating Pressure & psia & $8.8^{b}$ & Cpan Heat Exchange Surface Area & $\mathrm{ft}^{2}$ & $10,342^{b}$ \\
\hline Evaporator 4 Inlet Steam Pressure & psia & $8.8^{b}$ & Seedpan Heat Exchange Surface Area & $\mathrm{ft}^{2}$ & $9,554^{b}$ \\
\hline Evaporator 4 Inlet Steam Temperature & $\mathrm{F}$ & $187^{\mathrm{b}}$ & FGpan Heat Exchange Surface Area & $\mathrm{ft}^{2}$ & $10,162^{b}$ \\
\hline Evaporator 4 Operating Pressure & psia & $5^{b}$ & Pan Operating Pressure & psia & $2.2^{\mathrm{b}}$ \\
\hline Evaporator 5 Inlet Steam Pressure & psia & $5^{b}$ & 900psia Steam Flow & $\mathrm{lb} / \mathrm{hr}$ & $200,000^{b}$ \\
\hline Evaporator 5 Inlet Steam Temperature & $\mathrm{F}$ & $165^{b}$ & 425psia Steam Flow & $\mathrm{lb} / \mathrm{hr}$ & $400,000^{6}$ \\
\hline Evaporator 5 Operating Pressure & psia & $2.2^{b}$ & 30psia Steam Flow & $\mathrm{lb} / \mathrm{hr}$ & $260,000^{b}$ \\
\hline 1st Vapor Bleed to Pans & $\mathrm{lb} / \mathrm{hr}$ & $117,000^{b}$ & 900psia Steam to Shredder & $\mathrm{lb} / \mathrm{hr}$ & $39,500^{b}$ \\
\hline 1st Vapor Bleed to MJH3 & $\mathrm{lb} / \mathrm{hr}$ & & 425psia Steam to Mills & $\mathrm{lb} / \mathrm{hr}$ & $55,000^{b}$ \\
\hline 1st Vapor Bleed to CJH & $\mathrm{lb} / \mathrm{hr}$ & & 425/30 PRV Flow & $\mathrm{lb} / \mathrm{hr}$ & $12,000^{b}$ \\
\hline 2nd Vapor Bleed to Cpan & $\mathrm{lb} / \mathrm{hr}$ & & 425/150 PRV Flow & $\mathrm{lb} / \mathrm{hr}$ & $15,000^{b}$ \\
\hline 2nd Vapor Bleed to MJH2 & $\mathrm{Ib} / \mathrm{hr}$ & & Clarifier Tank Temperature Loss & $\mathrm{F}$ & $-14^{\mathrm{b}}$ \\
\hline
\end{tabular}


The limitations of pinch analysis for sugar factories are discussed by Thompson of Sugar Technologies International LTD [9]. Opportunities for heat recovery in sugar factories are limited to streams below the pinch temperature as no hot streams requiring cooling exist above the pinch. One of the opportunities for heat recovery below the pinch is preheating boiler air and boiler feed water with exhaust vapor or condensate. These heat recovery methods are rarely used as they are often uneconomical and/or difficult to implement.

Pinch analysis also identifies latent heat loads placed on the utility steam as an opportunity for energy savings. In the case of a sugar factory, this involves reducing the amount of water evaporated in the lower efficiency evaporation pans. This can be accomplished by increasing the inlet brix concentration, resulting in more water being evaporated in the preceding, highly efficient, multiple-effect evaporator train. Reducing the amount of additional water that is added during pan processing can also reduce heating loads. Operating at higher brix levels requires high purity juice which is mostly a function of field conditions and to a lesser extent, milling operations. While it is theoretically possible to operate at higher concentrations, 70-73 brix rather than 65 brix, the potential operational difficulties that could result from running at these levels may outweigh gains.

Continuous operation at elevated brix is extremely difficult to maintain. Fluctuation in purity levels force operators to work with a margin of safety during batch operations to avoid problems with false graining, conglomeration, inversion, and coloring. Within the current processing parameters, pan operations are conducted at brix levels that facilitate smooth operation and maintain a sufficient margin of error for unexpected process fluctuations. While operating at higher levels has been reported elsewhere, especially in the beet sugar industry, the feasibility of operating at these elevated levels must be confirmed in practice. Modeling showing potential gains resulting from operating at higher brix is discussed in the results section of this report.

The final area that pinch can be useful is in guiding evaporator configuration and process steam distribution. Quintuple effect evaporation trains such as the one utilized at HC\&S are highly efficient and do not lend themselves to analysis using pinch. Literature on pinch analysis for multiple effect evaporation systems suggests a decomposition approach that involves separating the evaporators from the process heating network. Matching the utility loads to the vapor streams in the evaporator train is then carried out to maximize efficiency. Employing this technique led to the identification of several areas where modifications could result in more efficient use of process steam $[9,10,11]$.

\section{Results and Discussion}

Scenarios identified in the pinch analysis were investigated using the Aspen Plus model. Results from runs of modified cases were compared against the base case and changes in steam demand, heat exchange surface areas and steam flows were noted. Where savings were realized, it was assumed that any increase in available steam (resulting from a decrease in steam demand) would be used to generate electricity for export. This may not always be the best choice, as increasing processing throughput or utilizing electricity to provide additional power to pump irrigation water might yield better economic returns depending on market conditions. 
Scenario analysis began with examination of HC\&S's evaporator train using the decomposition method. The first composite curve for utility loads, shown in Figure 2, was generated using Aspen Pinch. Unlike Figure 1, shown in the pinch analysis section, Figure 2 shows the unique energy use profile of a sugar factory. Note that no hot streams exist above the pinch point to provide heating. All the process heating above the pinch temperature must be provided by hot utility streams, in HC\&S's case, bled vapor from the evaporator train.

Temperature levels of the vapors available from the evaporation train in its current configuration were overlaid to produce Figure 3. As shown in the figure, nearly half of the utility heating demand is consumed in the pans. While some steam is used to raise the temperature of the syrup, the majority of the energy demand in the pans is for water evaporation, thus the long plateau at $152^{\circ} \mathrm{F}$, the vaporization temperature of water at 25.5 inches of mercury vacuum. In the base case model, the evaporation pans are operated primarily with first vapor, the exception being the Cseed continuous pan which operates on second vapor.

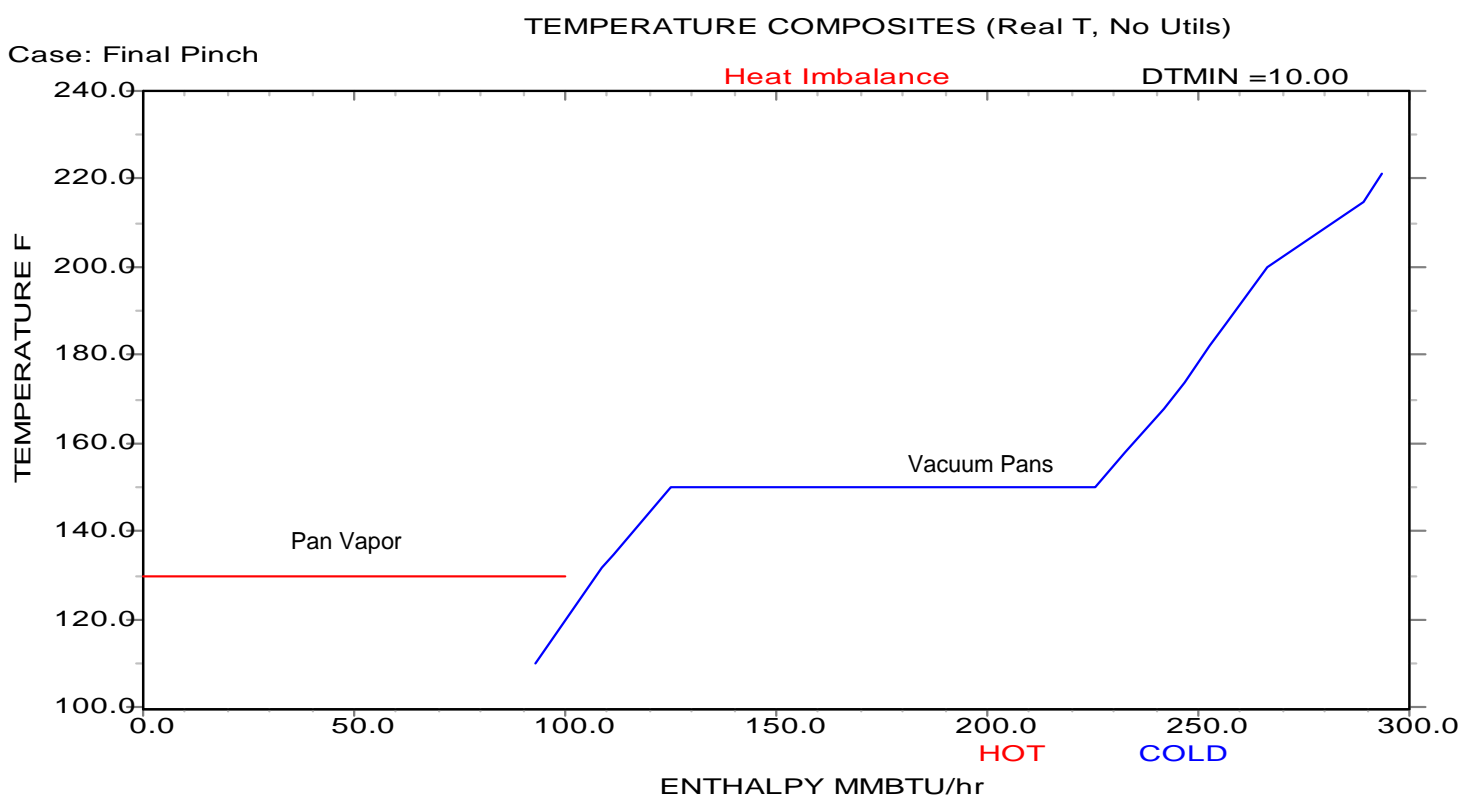

Figure 2. Composite curve for utilities at HC\&S Puunene sugar factory.

Looking at Figure 3, it appears that the $70^{\circ} \mathrm{F}$ temperature difference between first vapor and the temperature needed to evaporate water at 25.5 inches of mercury vacuum is much greater than necessary; however operational constraints reduce the available portion of this difference substantially. The syrup is boiled in large calandria pans and a boiling point rise in the range of $20-30^{\circ} \mathrm{F}$ is observed. Furthermore, the required temperature approach in shell and tube heater exchangers typically ranges from $10-20^{\circ} \mathrm{F}$. This gives a required temperature difference between the steam and the syrup of $30-50^{\circ} \mathrm{F}$ and it would appear that this could be satisfied using second 
vapor $\left(210^{\circ} \mathrm{F}-152^{\circ} \mathrm{F}=58^{\circ} \mathrm{F}\right)$, thereby reducing the demand for first vapor. This opportunity was investigated and modeled in Aspen. Results show that using second vapor to meet pan boiling demands would reduce exhaust steam demand by about 21,000 pounds per hour which corresponds to a $65 \mathrm{lb} / \mathrm{tc}$ reduction in steam:cane ratio. If this steam were condensed in TG4 it would produce $0.95 \mathrm{MW}$ of additional electricity.

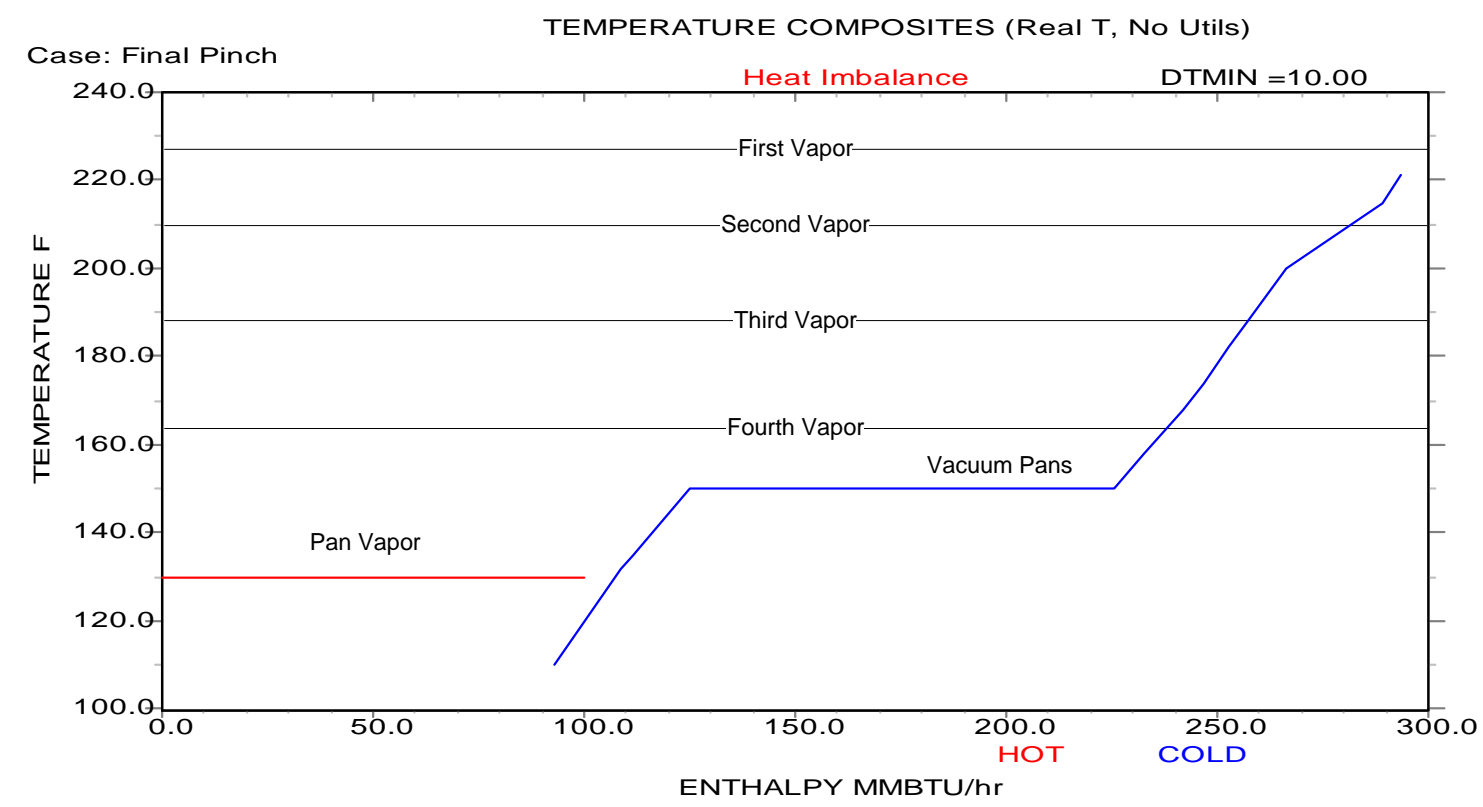

Figure 3. Composite curve for utilities at HC\&S Puunene sugar factory with pan outlet vapor temperature overlay.

Using second vapor would require heat exchange surface area modification in both the pans and the evaporators. Modeling shows a reduction in required heat exchange area for the first effect of the evaporation train with a corresponding increase in area for the second effect. Some reduction is also seen in the third, fourth, and fifth effects. Overall, the model results show the total heat exchange area required for the evaporation train would decrease by almost $6 \%$ and that the pan heat exchange surface area would need to be increased by approximately $10 \%$. Model results for heat exchanger specifications can be found in Table 2 and 3.

Piping for routing second vapor to the pans has been installed, but is currently unused, except in the case of the continuous $\mathrm{C}$ pan, where it provides second vapor for evaporation. Second vapor has been tested in the batch pans but resulted in slower heating rates and extended processing times. First vapor is favored under the current factory configuration because it allows operators to cycle the batch bans quickly. Replacing batch pans with continuous pans for B, seed, and food grade sugars, or adding heat exchange surface area to the present batch pans, would allow operators to maintain high cycle rates while utilizing second vapor.

Switching to continuous pans rather than increasing the surface area of the existing batch pans would help reduce the amount of additional surface area required to successfully operate the pans using second vapor. Capital costs and disruption to operations that would result from switching 
from batch to continuous pans would be significant. At present this modification may not represent an adequate return on investment, however the potential for significant energy savings warrants closer consideration.

Table 2. Evaporator heat exchanger specifications and model results.

\begin{tabular}{|c|c|c|c|c|c|c|c|c|}
\hline Evaporator & Value & $\begin{array}{l}\text { Base } \\
\text { Case }\end{array}$ & $\begin{array}{c}2^{\text {nd }} \text { Vapor } \\
\text { to Pans }\end{array}$ & $\begin{array}{c}3^{\text {rd }} \\
\text { Vapor } \\
\text { to Pans }\end{array}$ & $\begin{array}{c}\text { Vapor to } \\
\text { Mixed Juice } \\
\text { Heaters }\end{array}$ & $\begin{array}{c}\text { Flash } \\
\text { Condensate }\end{array}$ & $\begin{array}{l}70 \text { Brix } \\
\text { Syrup }\end{array}$ & Units \\
\hline \multirow{3}{*}{$1 \mathrm{~A}$} & Area & 32,910 & 30,256 & 27,305 & 31,795 & 31,994 & 31,994 & $\mathrm{ft}^{2}$ \\
\hline & $\mathrm{U}$ & 113 & 113 & 113 & 113 & 113 & 113 & $\begin{array}{l}\mathrm{Btu} / \mathrm{hr}- \\
\mathrm{ft}^{2}-{ }^{\circ} \mathrm{F}\end{array}$ \\
\hline & $\Delta$ Area & 0 & $-2,654$ & $-5,605$ & $-1,757$ & $-1,115$ & -916 & $\mathrm{ft}^{2}$ \\
\hline & & & & & & & & \\
\hline \multirow[t]{3}{*}{$1 \mathrm{~B}$} & Area & 21,084 & 19,372 & 17,471 & 19,951 & 20,363 & 20,494 & $\mathrm{ft}^{2}$ \\
\hline & $\mathrm{U}$ & 240 & 240 & 240 & 240 & 240 & 240 & $\begin{array}{l}\mathrm{Btu} / \mathrm{hr}- \\
\mathrm{ft}^{2}-{ }^{\circ} \mathrm{F}\end{array}$ \\
\hline & $\Delta$ Area & 0 & $-1,712$ & $-3,613$ & $-1,133$ & -721 & -590 & $\mathrm{ft}^{2}$ \\
\hline \multirow[t]{3}{*}{$1 \mathrm{C}$} & Area & 11,806 & 10,648 & 9595 & 11,165 & 11,398 & 11,271 & $\mathrm{ft}^{2}$ \\
\hline & $\mathrm{U}$ & 160 & 160 & 160 & 160 & 160 & 160 & $\begin{array}{l}\mathrm{Btu} / \mathrm{hr}- \\
\mathrm{ft}^{2}-{ }^{\circ} \mathrm{F}\end{array}$ \\
\hline & $\Delta$ Area & 0 & $-1,158$ & $-2,211$ & -641 & -408 & -535 & $\mathrm{ft}^{2}$ \\
\hline \multirow[t]{3}{*}{2} & Area & 24,689 & 48,424 & 42,150 & 26,424 & 22,493 & 25,931 & $\mathrm{ft}^{2}$ \\
\hline & $\mathrm{U}$ & 315 & 315 & 315 & 315 & 315 & 315 & $\begin{array}{l}\mathrm{Btu} / \mathrm{hr}- \\
\mathrm{ft}^{2}-{ }^{\circ} \mathrm{F}\end{array}$ \\
\hline & $\Delta$ Area & 0 & 23,735 & 17,461 & 1,735 & $-2,196$ & 1,242 & $\mathrm{ft}^{2}$ \\
\hline \multirow[t]{3}{*}{3} & Area & 22,572 & 17,546 & 40,872 & 22,820 & 21,511 & 23,792 & $\mathrm{ft}^{2}$ \\
\hline & $\mathrm{U}$ & 198 & 198 & 198 & 198 & 198 & 198 & $\begin{array}{l}\mathrm{Btu} / \mathrm{hr}- \\
\mathrm{ft}^{2}-{ }^{\circ} \mathrm{F}\end{array}$ \\
\hline & $\Delta$ Area & 0 & $-5,026$ & 18,300 & 248 & $-1,061$ & 1,220 & $\mathrm{ft}^{2}$ \\
\hline \multirow[t]{3}{*}{4} & Area & 21,516 & 15,004 & 8,177 & 27,782 & 22,582 & 23,173 & $\mathrm{ft}^{2}$ \\
\hline & $\mathrm{U}$ & 277 & 277 & 277 & 277 & 277 & 277 & $\begin{array}{l}\mathrm{Btu} / \mathrm{hr}- \\
\mathrm{ft}^{2}-{ }^{\circ} \mathrm{F}\end{array}$ \\
\hline & $\Delta$ Area & 0 & $-6,512$ & $-13,339$ & $-6,266$ & 1,066 & 1,769 & $\mathrm{ft}^{2}$ \\
\hline \multirow[t]{3}{*}{5} & $\Delta$ Area & 21,273 & 14,195 & 7,411 & 17,026 & 26,369 & 23,285 & $\mathrm{ft}^{2}$ \\
\hline & & 140 & 140 & 140 & 140 & 140 & 140 & $\begin{array}{l}\mathrm{Btu} / \mathrm{hr}- \\
\mathrm{ft}^{2}-{ }^{\circ} \mathrm{F}\end{array}$ \\
\hline & Area & 0 & $-7,078$ & $-13,862$ & $-4,247$ & 5,096 & 2,012 & $\mathrm{ft}^{2}$ \\
\hline & & & & & & & & \\
\hline
\end{tabular}


Table 3. Pan heat exchanger specifications and model results.

\begin{tabular}{|c|c|c|c|c|c|c|}
\hline Pan & Value & $\begin{array}{l}\text { Base } \\
\text { Case }\end{array}$ & $\begin{array}{l}2^{\text {nd }} \\
\text { Vapor to } \\
\text { Pans }\end{array}$ & $\begin{array}{l}3^{\text {rd }} \\
\text { Vapor to } \\
\text { Pans }\end{array}$ & $\begin{array}{l}70 \text { Brix } \\
\text { Syrup }\end{array}$ & Units \\
\hline \multirow[t]{3}{*}{$\mathrm{A}$} & Area & 10,348 & 11,391 & 15,041 & 8,174 & $\mathrm{ft}^{2}$ \\
\hline & U & 87.5 & 87.5 & 87.5 & 87.5 & $\mathrm{Btu} / \mathrm{hr}-\mathrm{ft}^{2}-{ }^{\circ} \mathrm{F}$ \\
\hline & $\Delta$ Area & 0 & 1,043 & 4,693 & $-2,174$ & $\mathrm{ft}^{2}$ \\
\hline \multirow[t]{3}{*}{$\mathrm{B}$} & Area & 8,946 & 9,971 & 13,916 & 8,946 & $\mathrm{ft}^{2}$ \\
\hline & $\mathrm{U}$ & 57 & 57 & 57 & 57 & $\mathrm{Btu} / \mathrm{hr}-\mathrm{ft}^{2}-{ }^{\circ} \mathrm{F}$ \\
\hline & $\Delta$ Area & 0 & 1,025 & 4,970 & 0 & $\mathrm{ft}^{2}$ \\
\hline \multirow[t]{3}{*}{$\mathrm{C}$} & Area & 9,608 & 10,902 & 16,946 & 9,608 & $\mathrm{ft}^{2}$ \\
\hline & $\mathrm{U}$ & 37 & 37 & 37 & 37 & $\mathrm{Btu} / \mathrm{hr}-\mathrm{ft}^{2}-{ }^{\circ} \mathrm{F}$ \\
\hline & $\Delta$ Area & 0 & 1294 & 7,338 & 0 & $\mathrm{ft}^{2}$ \\
\hline \multirow[t]{3}{*}{ Seed } & Area & 10,379 & 10,379 & 15,778 & 10,379 & $\mathrm{ft}^{2}$ \\
\hline & U & 37 & 37 & 37 & 37 & $\mathrm{Btu} / \mathrm{hr}-\mathrm{ft}^{2}-{ }^{\circ} \mathrm{F}$ \\
\hline & $\Delta$ Area & 0 & 0 & 5399 & 0 & $\mathrm{ft}^{2}$ \\
\hline \multirow[t]{3}{*}{ Food Grade } & Area & 10,100 & 11,450 & 17,877 & 10,100 & $\mathrm{ft}^{2}$ \\
\hline & $\mathrm{U}$ & 35 & 35 & 35 & 35 & $\mathrm{Btu} / \mathrm{hr}-\mathrm{ft}^{2}-{ }^{\circ} \mathrm{F}$ \\
\hline & $\Delta$ Area & 0 & 1,350 & 7,777 & 0 & $\mathrm{ft}^{2}$ \\
\hline
\end{tabular}

\section{Third Evaporator Vapor to Pans}

The use of third vapor for pan boiling was also modeled, although HC\&S personnel did not deem it to be a viable option due to operational/space constraints in the factory. Model results for using third vapor to drive pan boiling show additional savings over second vapor of 22,000 pounds of exhaust steam per hour which corresponds to a $69 \mathrm{lb} / \mathrm{tc}$ reduction in steam:cane ratio. If this steam were condensed in TG4 it would produce an additional $0.99 \mathrm{MW}$. The total increased electricity production gained by shifting from first vapor (base case) to third vapor would be $1.95 \mathrm{MW}$. The total reduction in steam:cane ratio would be $134 \mathrm{lb} / \mathrm{tc}$.

Using third vapor in the boiling pans would require more extensive modification to heat exchange surface areas than shifting to second vapor. Model results show that use of third vapor could lead to a reduction in total heat exchange area of nearly $12 \%$ in the evaporators. Increases in surface area for the second and third effects would be offset by reductions in first, fourth and fifth effects. Operating the pans on third vapor would require replacement of all batch pans with continuous pans to facilitate heat transfer at lower temperature differences. Model results show required pan heat exchange surface area increasing by nearly $60 \%$. Operating the boiling pans on third vapor would likely leave little room for error during the sensitive crystallization process. In addition, the infrastructure required to route low pressure third vapor steam to the pans in 
already tight factory space might require a major retrofit. Model results for heat exchanger area under this scenario are summarized in Tables 2 and 3.

\section{Plate Type Mixed Juice Heaters}

The third scenario investigated potential modifications to the mixed juice heaters. Once again, using steam from later evaporator effects can result in steam savings. HC\&S employs shell and tube type, mixed juice heaters. This design operates reliably and rarely has fouling problems but requires higher approach temperatures than plate type exchangers. The lower temperature approach values allowable with plate heat exchangers would enable the mixed juice heaters at HC\&S to be heated with lower temperature steam. Using plate heat exchangers, the first mixed juice heater could operate on fourth vapor, the second mixed juice heater on third vapor, and the third mixed juice heater and the clarified mixed juice heater could be shifted to second vapor. Modeling results for these modifications show an exhaust steam savings of 18,000 lbs/hr which corresponds to a $57 \mathrm{lb} / \mathrm{tc}$ reduction in steam:cane ratio. If this steam was condensed in TG4, electricity generation could increase by approximately $0.82 \mathrm{MW}$. The feasibility of adding plate heat exchangers is low however, because fouling problems and reduced reliability could outweigh potential energy gains. Model results for heat exchanger area under this scenario are summarized in Tables 2 and 4.

\section{Increased Syrup Brix}

As discussed in the pinch analysis section, raising the syrup brix level at the exit of the evaporators could reduce latent heat loads on the pans. Model evaluation of elevated brix shows reduced first vapor demand in the pans, however, operational difficulties that might ensue in the crystallization process cannot be fully represented in the model. Results show that an additional 2,900 pounds of exhaust steam would be required in the evaporators to increase syrup density to 70 brix. A syrup feed of 70 brix mixed with Bsugar remelt would produce an inlet brix of 73 brix to the A continuous vapor pan. Under these conditions first vapor demand in the A pan would decrease by $10,000 \mathrm{lbs} / \mathrm{hr}$. This is equivalent to a reduction in exhaust steam demand of $8,000 \mathrm{lbs} / \mathrm{hr}$. The net savings of exhaust steam from this modification would be about 5,000 $\mathrm{lbs} / \mathrm{hr}$ which corresponds to a $16 \mathrm{lb} / \mathrm{tc}$ reduction in steam:cane ratio. Condensing this steam in TG4 would result in increased electricity generation of approximately $0.23 \mathrm{MW}$. 
Table 4. Mixed juice heater heat exchanger specifications and model results.

\begin{tabular}{|c|c|c|c|c|}
\hline $\begin{array}{l}\text { Mixed } \\
\text { Juice } \\
\text { Heater }\end{array}$ & Value & Base Case & $\begin{array}{l}\text { Vapor to } \\
\text { Mixed Juice } \\
\text { Heaters }\end{array}$ & Units \\
\hline \multirow[t]{3}{*}{1} & Area & 4,612 & 8,641 & $\mathrm{ft}^{2}$ \\
\hline & $\mathrm{U}$ & 190 & 190 & $\mathrm{Btu} / \mathrm{hr}-\mathrm{ft}^{2}-{ }^{\circ} \mathrm{F}$ \\
\hline & $\Delta$ Area & 0 & 4,029 & $\mathrm{ft}^{2}$ \\
\hline \multirow[t]{3}{*}{2} & Area & 6,771 & 16,839 & $\mathrm{ft}^{2}$ \\
\hline & $\mathrm{U}$ & 63 & 63 & $\mathrm{Btu} / \mathrm{hr}-\mathrm{ft}^{2}{ }^{\circ}{ }^{\circ} \mathrm{F}$ \\
\hline & $\Delta$ Area & 0 & 10,068 & $\mathrm{ft}^{2}$ \\
\hline \multirow[t]{3}{*}{3} & Area & 5,154 & 9,030 & $\mathrm{ft}^{2}$ \\
\hline & $\mathrm{U}$ & 165 & 165 & $\mathrm{Btu} / \mathrm{hr}-\mathrm{ft}^{2}-{ }^{\circ} \mathrm{F}$ \\
\hline & $\Delta$ Area & 0 & 3,876 & $\mathrm{ft}^{2}$ \\
\hline \multirow[t]{3}{*}{ Clarified } & Area & 5,232 & 5,232 & $\mathrm{ft}^{2}$ \\
\hline & $\mathrm{U}$ & 215 & 215 & $\mathrm{Btu} / \mathrm{hr}-\mathrm{ft}^{2}-^{\circ} \mathrm{F}$ \\
\hline & $\Delta$ Area & 0 & & $\mathrm{ft}^{2}$ \\
\hline
\end{tabular}

\section{Condensate Flash}

Flashing condensate is a common steam saving method. At HC\&S, flashing is carried out on first and second effect condensate. The feasibility of flashing all condensates to subsequent effects was investigated using the Aspen model. Model results show a potential steam savings equivalent to 8,000 pounds of exhaust steam which corresponds to a $25 \mathrm{lb} / \mathrm{tc}$ reduction in steam:cane ratio. Condensing this steam in TG4 would result in increased electricity generation of approximately $0.36 \mathrm{MW}$. This modification would result in small changes to the required heat exchange surface area in the evaporation train. Model results show a net increase of less than one-half percent with reductions in the first three effects and increases in the fourth and fifth. This modification would increase evaporator train condenser load, an unwanted side effect.

\section{Summary and Conclusions}

Pursuant to the plantation wide energy efficiency assessment project proposed for HC\&S on Maui, UH project participants developed a comprehensive model of the HC\&S sugar factory using Aspen Plus computer modeling software. A pinch analysis was conducted to guide investigation into energy saving modifications to factory equipment and operations.

The HC\&S sugar factory at Puunene is a modern and efficient facility with electrical power generation of approximately 80 to $85 \mathrm{kWh} / \mathrm{tc}$ during periods of steady operation. While this range is high compared to sugar producers in other parts of the world where levels of 10-30 $\mathrm{kWh} / \mathrm{tc}$ or less are common, levels of $90-100+\mathrm{kWh} / \mathrm{tc}$ are believed to be attainable. Steam 
consumption for sugar boiling is in the range of 800-850 lb steam/tc. Experts believe that the most efficient factories should be able to operate on $650 \mathrm{lb}$ steam/tc or less [3]. Pinch analysis and Aspen Plus modeling software were used to investigate modifications that might improve boiling house steam:cane ratio and electricity export at HC\&S.

Simulation results for five scenarios were discussed with HC\&S personnel to determine their feasibility. Although simulation results for all five scenarios showed notable savings over the base case, only two of the five proposed changes were deemed possible within the operational constraints of the factory.

The two scenarios deemed possible within operating constraints were (1) operating the pan boiling system on second vapor rather than first vapor, and (2) adding the capability to flash condensates from evaporators 3 and 4 . Modeling the use of second vapor to supply pan boiling demands shows a reduction in exhaust steam demand of about 21,000 pounds per hour which corresponds to a $65 \mathrm{lb} / \mathrm{tc}$ reduction in steam:cane ratio. If this steam were condensed in TG4, it would increase electrical power generation by $0.95 \mathrm{MW}$. Using second vapor would require heat exchange surface area modification in both the pans and the evaporators. Overall, the model shows a decrease of nearly $6 \%$ for heat exchange surface area required in the evaporation train, offset by a $10 \%$ increase in required heat exchange surface area for the pans.

Modeling the addition of the capability to flash condensates from evaporators 3 and 4 shows a potential steam savings equivalent to 8,000 pounds of exhaust steam per hour, which corresponds to a $25 \mathrm{lb} / \mathrm{tc}$ reduction in steam:cane ratio. Condensing this steam in TG4 would increase electrical power generation by approximately $0.36 \mathrm{MW}$. Model results also indicate a net increase of less than $0.5 \%$ in required heat exchange surface area, with reductions in the first three effects offset by increases in the fourth and fifth effects. A negative consequence of this modification is an increase in evaporator train condenser load possibly offsetting some of the potential gain.

HC\&S exports up to $12 \mathrm{MW}$ to the local utility. The $1.32 \mathrm{MW}$ increase in electric generation from the combined effect of the above modifications would increase exportable electricity by nearly $11 \%$. Steam:cane ratio would be reduced by about $12 \%$ from a range of $800-850 \mathrm{lb} / \mathrm{tc}$ to a range of $710-760 \mathrm{lb} / \mathrm{tc}$. 


\section{References}

1. Hugot, E. 1972. Handbook of Cane Sugar Engineering. Elsevier Publishing Company, New York, NY.

2. Kinoshita, C.M. 1991. Proceeding of the International Conference on Energy from Sugarcane, Potential for Cane Energy. Winrock International, Arlington, VA.

3. Paturau, J.M. 1986. The Cane Sugar Factory of the Future. Sugar Y Azucar.

4. Anon. 2005. HC\&S History-The Company. Hawaii Commercial and Sugar, (http://www.hcsugar.com/hist_hcs.html).

5. Somera, B.J. and Wu, K.K. 2004. Factory Report for 2003. Hawaii Agriculture Research Center. Aiea, HI. (not for citation or use outside of the HI sugar industry)

6. Ghurair, J., Singh, G. 1999. Al Khaleej Sugar an Energy Efficient Refinery. Sugar Industry Technologists Meeting, Estoril, Portugal.

7. Linnhoff, B., Hindmarsh, E. 1982. The Pinch Design Method For Heat Exchanger Networks. Chemical Engineering Science.

8. Sahdev, M. Pinch Technology: Basics For Beginners. Cheresources, (http://www.cheresources.com/pinchtech7.shtml).

9. Thomson, Phil. 2000. Pinch Technology Explained. Sugar Knowledge International Ltd. England.

10. Urbaniec, K., Zalweski, P., and Klemes, J. 2000. A Decomposition Approach for the Retrofit of Energy Systems in the Sugar Industry. Applied Thermal Engineering.

11. Westphalen, D.L. and Maciel, M.R. 2000. Pinch Analysis of Evaporation Systems. Brazilian Journal of Chemical Engineering, Sao Paulo, Brazil.

12. Anon. 2003. Period Summary of Factory Results. Hawaii Agriculture Research Center. Aiea, HI.

13. Kinoshita, C.M. 1992. Cogeneration in the Hawaiian Sugar Industry. Hawaii Natural Energy Institute, University of Hawaii, Honolulu, HI.

14. Chen, J. 1985. Cane Sugar Handbook 11th Edition. John Wiley and Sons, New York, NY. 


\section{Appendix}




\section{Aspen flow sheet for HC\&S boiling house}

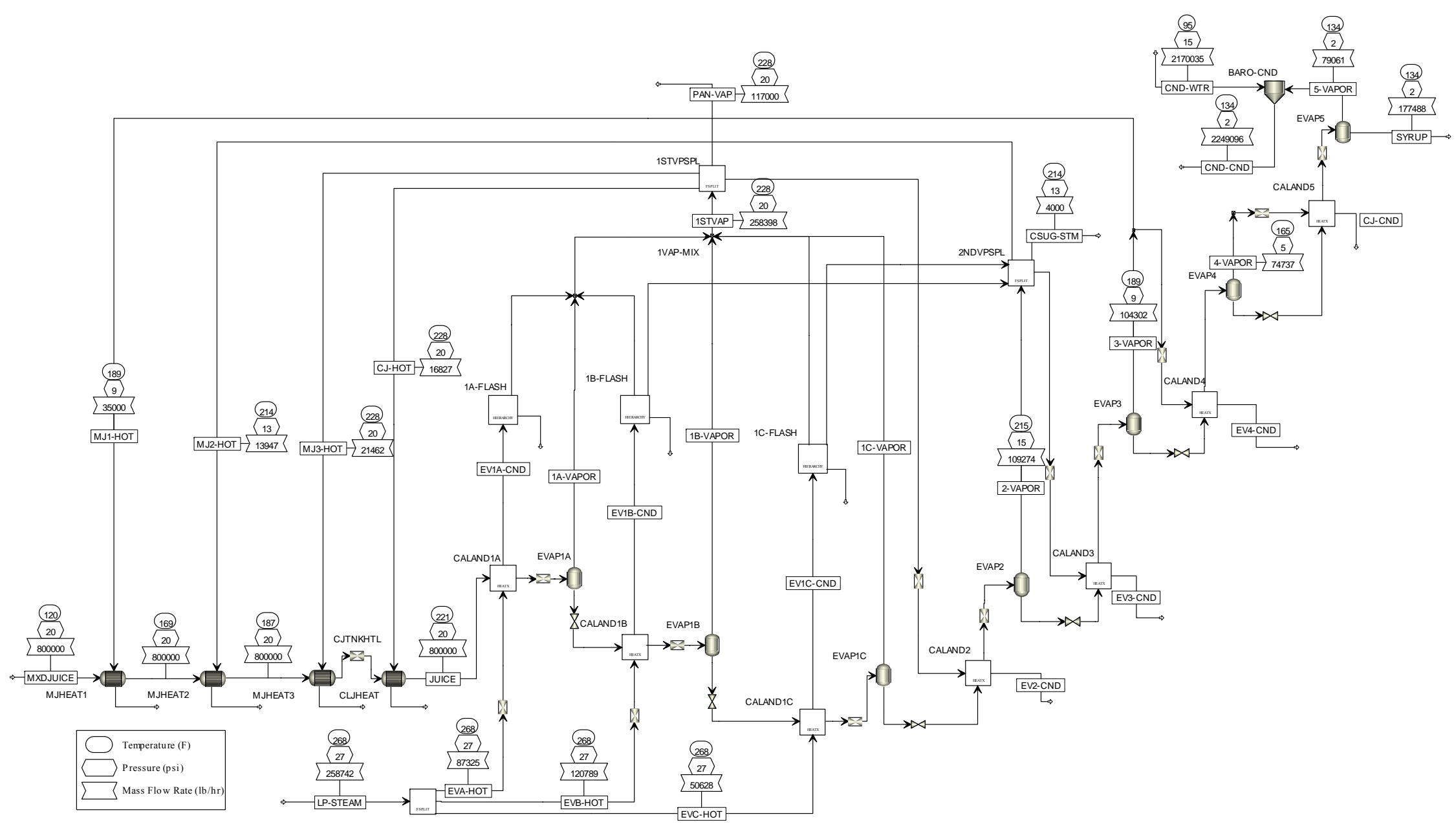




\section{Aspen flow sheet for HC\&S power plant}

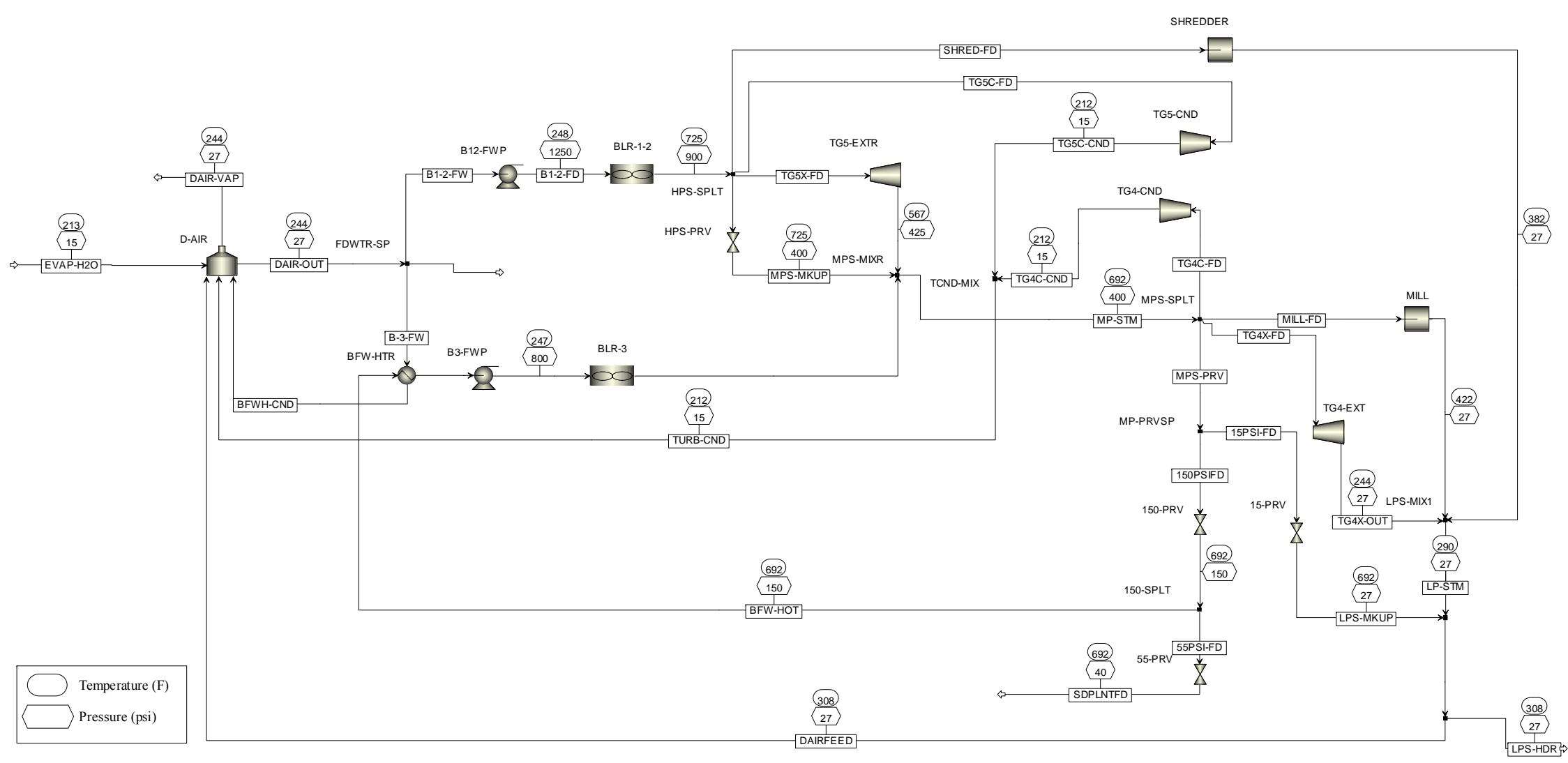


Aspen flow sheet for HC\&S pan boiling system

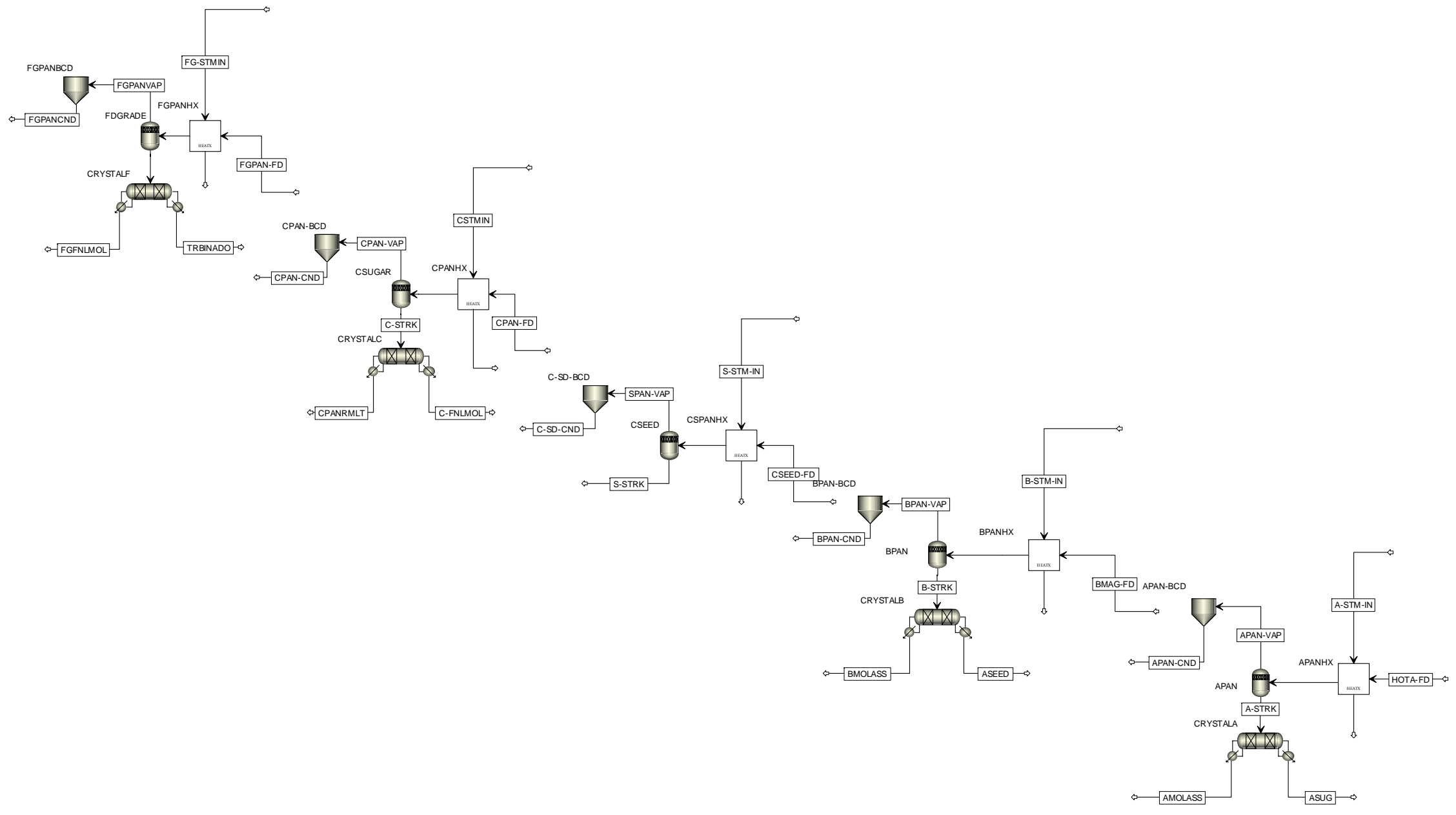




\section{APPENDIX C}

Steam Line Insulation Survey Results 
Missing Insulation Survey

Puunene Power Plant

\begin{tabular}{|c|c|c|c|c|}
\hline UNIT & LOCATION & DESCRIPTION & $\begin{array}{l}\text { APPROX } \\
\text { LINE } \\
\text { TEMP. }\end{array}$ & $\begin{array}{l}\text { ANNUAL HEAT } \\
\text { LOSS - MMbtu }\end{array}$ \\
\hline \multirow[t]{23}{*}{$\begin{array}{c}\text { Boiler } \\
\mathbf{3}\end{array}$} & & $\begin{array}{l}450 \text { \# steam line - 14" dia., } \\
\text { missing } 40 \text {, }\end{array}$ & 750 & $4,537.6$ \\
\hline & $10^{\text {th }}$ Floor & $\begin{array}{l}\text { 4" line, missing 20' (off } \\
\text { steam drum) }\end{array}$ & 750 & 791.4 \\
\hline & $9^{\text {th }}$ Floor & $\begin{array}{l}\text { 4" line, missing 10' (off } \\
\text { steam drum) }\end{array}$ & 750 & 395.7 \\
\hline & $\begin{array}{l}8^{\text {th }} \text { Floor - } \\
\text { Steam Drum } \\
\text { Level }\end{array}$ & $\begin{array}{l}18^{\prime \prime} \text { line, missing 20' - (two } \\
\text { relief valves) }\end{array}$ & 750 & $2,892.0$ \\
\hline & & 1" line, missing 25 & 750 & 348.7 \\
\hline & & 2" line, missing 5 & 750 & 113.6 \\
\hline & $7^{\text {th }}$ Floor & $2 \frac{1}{2 \prime \prime}$ line, missing $10^{\prime}$ & 750 & 267.2 \\
\hline & & $3 / 4 "$ line, missing $25^{\prime}$ & 350 & 81.0 \\
\hline & & $21 / 2 "$ line, missing 5 & 350 & 33.4 \\
\hline & $\begin{array}{l}6^{\text {th }} \text { Floor- } \\
\text { South side }\end{array}$ & $2 \frac{1}{2}{ }^{\prime \prime}$ line, missing 5 & 750 & 133.6 \\
\hline & & 4" line, missing 5 ' & 750 & 197.8 \\
\hline & $\begin{array}{l}5^{\text {th }} \text { Floor - } \\
\text { Mud Drum } \\
\text { Level }\end{array}$ & 2" line, missing 150' & 350 & 870.0 \\
\hline & $4^{\text {th }}$ Floor & 2" line, missing $10^{\prime}$ & 350 & 58.0 \\
\hline & & 12" line, missing 5 & 350 & 116.1 \\
\hline & & $2 \frac{1}{2}{ }^{\prime \prime}$ line, missing 15 & 350 & 100.4 \\
\hline & $\begin{array}{l}\text { @ Flash } \\
\text { Tank }\end{array}$ & 8" line, missing 5' & 750 & 358.6 \\
\hline & & $\begin{array}{l}3 \frac{1 / 2 "}{2} \text { line, missing } 20^{\prime}- \\
\text { includes } 3^{\text {rd }} \text { floor for this } \\
\text { line }\end{array}$ & 350 & 173.2 \\
\hline & & $\begin{array}{l}\text { 4" line, missing 20' - into } \\
\text { Boiler }\end{array}$ & 750 & 791.4 \\
\hline & $3^{\text {rd }}$ Floor & $\begin{array}{l}\text { 4" line, missing } 15 \text { ' vertical; } \\
32 \text { ' horiz. - off flash tank }\end{array}$ & 350 & 418.6 \\
\hline & $2^{\text {nd }}$ Floor & $\begin{array}{l}\text { 10" line, missing 6' - near } \\
\text { DA } 3\end{array}$ & 750 & 529.0 \\
\hline & & $\begin{array}{l}\text { Misc. } 2 \frac{1}{1} 2^{\prime \prime} \text { lines, missing } \\
300^{\prime} \text { (est) }\end{array}$ & 350 & $2,341.5$ \\
\hline & & $\begin{array}{l}\text { Misc. } 3 \frac{1}{1} / 2 \text { lines, missing } \\
\text { 200' (est) }\end{array}$ & 350 & 1732.0 \\
\hline & & $15^{\prime \prime}$ line, missing 20 & 350 & 505.6 \\
\hline
\end{tabular}




\begin{tabular}{|c|c|c|c|c|}
\hline & $\begin{array}{l}1^{\text {st }} \text { Floor - } \\
\text { Feed Pump } \\
\text { Area }\end{array}$ & $\begin{array}{l}18 " \text { line, } 20 \text { ' horiz. }-15 \\
\text { vert. (along TG } 5 \text { Building) }\end{array}$ & 150 & 2,035 \\
\hline & & $\begin{array}{l}10 " \text { line, missing } 36 \text { ' (non- } \\
\text { continous) }\end{array}$ & 350 & 716.8 \\
\hline & & $\begin{array}{l}\text { 4" lines, missing } 40 \text { ' } \\
\text { (to pumps) }\end{array}$ & 530 & 794.8 \\
\hline & & Misc. 1" lines, missing 150' & 530 & $1,114.5$ \\
\hline & $\begin{array}{l}\text { Deareator } \\
\text { tank }\end{array}$ & 10" line, missing 10' & 350 & 199.1 \\
\hline & & 4" line, missing 20' & 350 & 152.4 \\
\hline & & $8 "$ line, missing $15^{\prime}$ & 350 & 246.1 \\
\hline & & 2" line, missing $40^{\prime}$ & 350 & 232.0 \\
\hline & & $2 \frac{1}{2}=$ line, missing $15^{\prime}$ & 350 & 100.4 \\
\hline & $2^{\text {nd }}$ Floor & $\begin{array}{l}\text { Square 22" x 22" duct, } \\
\text { missing } 35 \text {, }\end{array}$ & 1200 & $24,183.6$ \\
\hline $\begin{array}{l}\text { Boilers } \\
1 \& 2\end{array}$ & & $\begin{array}{l}8^{\prime \prime} \text { line, missing } 70 \text { ' (900 \# } \\
\text { steam line - multiple } \\
\text { locations) }\end{array}$ & 750 & $5,019.7$ \\
\hline & $6^{\text {th }}$ floor & 2" line, missing 20' & 750 & 377.0 \\
\hline & & $\begin{array}{l}18 \text { " pressure relief lines, } \\
\text { missing } 40^{\prime}\end{array}$ & 750 & 578.4 \\
\hline & & 2" lines, missing $150^{\prime}$ & 750 & $3,408.0$ \\
\hline & $5^{\text {th }}$ floor & none & & \\
\hline & $4^{\text {th }}$ Floor & $8 "$ line, missing 10 & 750 & 717.1 \\
\hline & & $\begin{array}{l}6 " \text { line, missing } 20 \text { ' vert.- } 2 \text { ' } \\
\text { horiz. }\end{array}$ & 530 & 612.0 \\
\hline & & $\begin{array}{l}2 \frac{1}{1} 2^{\prime} \text { line, missing } 30^{\prime} \text { vert. - } \\
20^{\prime} \text { horiz. }\end{array}$ & 530 & 600.6 \\
\hline & & $2 \frac{1 / 2}{2}$ line, missing $15^{\prime}$ & 530 & 163.5 \\
\hline & & $\begin{array}{l}\text { 2" line, missing 40' (B2 } \\
\text { North side) }\end{array}$ & 320 & 200.4 \\
\hline & & 12" line missing 35 , & 350 & 510.8 \\
\hline & & $\begin{array}{l}8 " \text { line, missing } 70 \text { '(steam } \\
\text { drum lines: both boilers) }\end{array}$ & 350 & 1,022 \\
\hline & & $\begin{array}{l}20 " \text { line, missing } 20^{\prime} \text { (dead } \\
\text { head?) }\end{array}$ & 530 & $1,549.2$ \\
\hline & $3^{\text {rd }}$ Floor & $\begin{array}{l}\text { 8" line, missing 10' (dead } \\
\text { head?), }\end{array}$ & 320 & 139.9 \\
\hline & & 3" line, missing 25 & 530 & 402.0 \\
\hline & & 18 "line, missing 50' (vert.) & 320 & 1285.5 \\
\hline & $2^{\text {nd }}$ Floor & 8" line, missing 4' (to drum) & 350 & 65.6 \\
\hline & & 1" line, missing 15, & 350 & 33.5 \\
\hline & $1^{\text {st }}$ Floor & 4" line, missing $30^{\prime}$ & 250 & 120.6 \\
\hline & & $8 "$ line, missing 15, & 350 & 246.1 \\
\hline
\end{tabular}




\begin{tabular}{|c|c|c|c|c|}
\hline & & 2" line, missing $30^{\prime}$ & 250 & 63.6 \\
\hline & & 1" line, missing 25 ' & 250 & 29.5 \\
\hline \multirow[t]{11}{*}{ Boiler 1} & $3^{\text {rd }}$ Floor & $\begin{array}{l}10 \text { " line, missing 3' vert. } \\
20 \text { ' horiz. }\end{array}$ & 530 & 933.8 \\
\hline & $2^{\text {nd }}$ Floor & $8 "$ line, missing 15 & 350 & 246.1 \\
\hline & & 2" line, missing 4" & 350 & 23.2 \\
\hline & & $\begin{array}{l}\text { 8" line, missing 4' } \\
\text { (manifold?) }\end{array}$ & 250 & 30.8 \\
\hline & & 12" line, missing 12' & 350 & 278.6 \\
\hline & $\begin{array}{l}\text { Adj. to TG } \\
\text { Building }\end{array}$ & $4^{\prime \prime}$ line, missing $30^{\circ}$ & 250 & 120.6 \\
\hline & & 8" line, missing 10' & 250 & 90.2 \\
\hline & De-aerator & $12^{\prime \prime}$ line, missing 20' & 250 & 252.2 \\
\hline & & 10" line, missing 18' & 250 & 195.7 \\
\hline & & $\begin{array}{l}10 \text { " line, missing 5' (loop @ } \\
\text { north end) }\end{array}$ & 750 & 440.8 \\
\hline & & $10^{\prime \prime}$ line, missing 8 ' & 750 & 705.2 \\
\hline \multirow[t]{9}{*}{ TG 4} & $4^{\text {th }}$ Floor & 1" line, missing 5' & 750 & 57.4 \\
\hline & & 5"line, missing 6" & 750 & 28.7 \\
\hline & Gen Rm. & 2" line, missing 1' & 750 & 20.1 \\
\hline & & 6" line, missing 10' & 750 & 561.8 \\
\hline & & 4" line, missing 2' & 750 & 79.1 \\
\hline & $2^{\text {nd }}$ Floor & 1" line, missing 15 & 750 & 172.2 \\
\hline & & $\begin{array}{l}\text { 1" line, missing 13' (off } \\
\text { large line) }\end{array}$ & 530 & 96.6 \\
\hline & & $8^{\prime \prime}$ line, missing 20' & 750 & $1,392.2$ \\
\hline & $1^{\text {st }}$ Floor & $30^{\prime \prime}$ line, missing 10 & 250 & 268.0 \\
\hline \multirow[t]{2}{*}{ TG 3} & $1^{\text {st }}$ Floor & $\begin{array}{l}30 " \text { line, missing } 10^{\prime} \text { (dead } \\
\text { head) }\end{array}$ & 250 & 268.0 \\
\hline & & 36" line, missing 40' & 250 & 1310.4 \\
\hline $\begin{array}{l}\text { TG } 5 \\
\text { Bldg. }\end{array}$ & & $\begin{array}{l}\text { 10" line, missing 5' (roof } \\
\text { line) }\end{array}$ & 750 & 440.8 \\
\hline \multicolumn{4}{|c|}{ Total Annual Heat Loss } & 71,542.6 MMBTU \\
\hline
\end{tabular}

71,542.6 MMBTU $=2,981$ tons coal $(24.0 \mathrm{mmbtu} /$ ton $)$ or 8,876 tons bagasse ( 8.06 $\mathrm{mmbtu} /$ ton) or $12,442 \mathrm{bbls}$ diesel $(5.75 \mathrm{mmbtu} / \mathrm{bbl})$

2,981 tons coal $=\$ 208,670(\$ 70.00 /$ ton $)$

12,442 bbls diesel $=\$ 808,730(\$ 65.00 / \mathrm{bbl})$ 